\title{
ATLAS series of Shuttle Missions
}
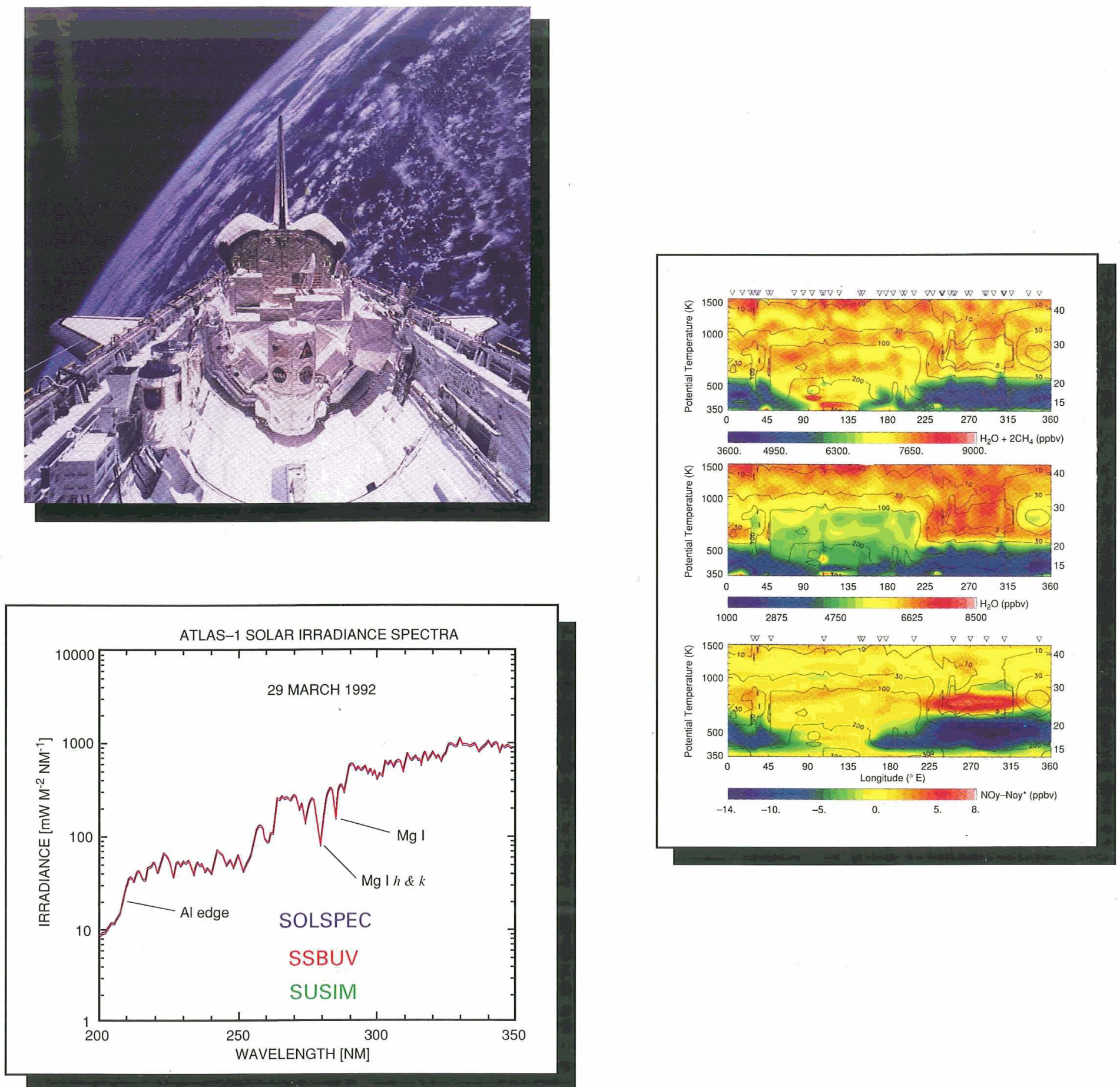

From Geophysical Research Letters

Volume 23, Number 17 


\title{
ATLAS series of Shuttle Missions
}

\author{
From Geophysical Research Letters \\ Volume 23, Number 17
}

\section{PUBLISHED BY AMERICAN GEOPHYSICAL UNION}

Cover. From the ATLAS series of space shuttle missions. Top left: Photograph taken by the space shuttle orbiter Atlantis of the ATLAS 3 and SSBUV payloads during the STS 66 flight of November 1994. The ATLAS payload is on the structure in the center of the photograph. On the orbiter sill to the left are the two cannisters housing the SSBUV experiment. (Photograph courtesy of NASA.) Lower left: Solar ultraviolet spectral irradiance from the ATLAS 1 mission as measured by the instruments
SOLSPEC, SSBUV, and SUSIM. (Courtesy of the respective instrument teams. See the paper by Cebula et al., this issue.) Right: Longitudinal plots of selected atmospheric properties as measured by the ATMOS instrument during the ATLAS 3 mission (sunrise occultations) in a latitude band between $64.5^{\circ} \mathrm{S}$ and $72.4^{\circ} \mathrm{S}$. Top: Total hydrogen $\left[\mathrm{H}_{2} \mathrm{O}+2 \mathrm{CH}_{4}\right]$. Center: Water vapor $\left[\mathrm{H}_{2} \mathrm{O}\right]$. Bottom: Active nitrogen $\left[N O_{y}\right]$ difference from that expected without denitrification $\left[N \mathrm{~N}^{*}\right]$. 
SPECIAL SECTION: ATLAS

The ATLAS series of shuttle missions (Paper 96GL02228) 2285 Jack A. Kaye and Timothy L. Miller

Observations of the solar irradiance in the 200- to 350-nm interval during the ATLAS 1 mission: A comparison among three sets of measurements-SSBUV, SOLSPEC, and SUSIM (Paper 96GL01109) 2289 R. P. Cebula, G. O. Thuillier, M. E. VanHoosier, E. Hilsenrath, M. Herse, G. E. Brueckner, and P. C. Simon

SOLCON solar constant observations from the ATLAS missions (Paper 96GL01878) 2293 Dominique Crommelynck, Alain Fichot, Vicente Domingo, and Robert Lee III

Observations of the lunar geometric albedo during the ATLAS 3 mission (Paper 96GL01122) 2297 S. J. Janz, E. Hilsenrath, R. P. Cebula, and T. J. Kelly

A comparison of ozone measurements made by the ATMOS, MAS, and SSBUV instruments during ATLAS 1, 2, and 3 (Paper 96GL01024) 2301

D. L. Kriebel, R. M. Bevilacqua, E. Hilsenrath, M. Gunson, G. K. Hartmann, M. Abrams, M. Daehler, T. A. Pauls, M. Newchurch, C. P. Aellig, and M. C. Bories

Ozone change from 1992 to 1993 as observed from SSBUV on the ATLAS 1 and ATLAS 2 missions (Paper 96GL01120) 2305

E. Hilsenrath, P. A. Newman, R. P. Cebula, P. W. DeCamp, T. J. Kelly, and L. Coy

Distinctive ozone structure in the high-latitude stratosphere: Measurements by the millimeter-wave atmospheric sounder (Paper 96GL01044) 2309

J. J. Olivero, T. A. Pauls, R. M. Bevilacqua, D. Kriebel, M. Daehler, M. L. Richards, N. Kämpfer, A. Berg, and C. Stodden

Measurements of $\mathrm{O}_{3}, \mathrm{H}_{2} \mathrm{O}$, and $\mathrm{ClO}$ in the middle atmosphere using the millimeter-wave atmospheric sounder (MAS) (Paper 96GL01475) 2313

G. K. Hartmann, R. M. Bevilacqua, P. R. Schwartz, N. Kämpfer, K. F. Künzi, C. P. Aellig, A. Berg, W. Boogaerts, B. J. Connor, C. L. Croskey, M. Daehler, W. Degenhardt, H. D. Dicken, D. Goldizen, D. Kriebel, J. Langen, A. Loidl, J. J. Olivero, T. A. Pauls, S. E. Puliafito, M. L. Richards, C. Rudin, J. J. Tsou, W. B. Waltman, G. Umlauft, and R. Zwick

MAS measurements of the latitudinal distribution of water vapor and ozone in the mesosphere and lower thermosphere (Paper 96GL01119) 2317

R. M. Bevilacqua, D. L. Kriebel, T. A. Pauls, C. P. Aellig, D. E. Siskind, M. Daehler, J. J. Olivero,

S. E. Puliafito, G. K. Hartmann, N. Kämpfer, A. Berg, and C. L. Croskey

Latitudinal distribution of upper stratospheric $\mathrm{ClO}$ as derived from space-borne microwave spectroscopy (Paper 96GL01215) 2321

C. P. Aellig, N. Kämpfer, C. Rudin, R. M. Bevilacqua, W. Degenhardt, P. Hartogh, C. Jarchow, K. Künzi, J. J. Olivero, C. Croskey, J. W. Waters, and H. A. Michelsen

Space-borne $\mathrm{H}_{2} \mathrm{O}$ observations in the Arctic stratosphere and mesosphere in the spring of 1992 (Paper 96GL01571) 2325

C. P. Aellig, J. Bacmeister, R. M. Bevilacqua, M. Daehler, D. Kriebel, T. Pauls, D. Siskind, N. Kämpfer, J. Langen, G. Hartmann, A. Berg, J. H. Park, and J. M. Russell III

Zeeman splitting of the 61-gigahertz oxygen $\left(\mathrm{O}_{2}\right)$ line in the mesosphere (Paper 96GL01043) 2329 G. K. Hartmann, W. Degenhardt, M. L. Richards, H. J. Liebe, G. A. Hufford, M. G. Cotton, R. M. Bevilacqua, J. J. Olivero, N. Kämpfer, and J. Langen

The atmospheric trace molecule spectroscopy (ATMOS) experiment: Deployment on the ATLAS Space Shuttle missions (Paper 96GL01569) 2333

M. R. Gunson, M. M. Abbas, M. C. Abrams, M. Allen, L. R. Brown, T. L. Brown, A. Y. Chang, A. Goldman, F. W. Irion, L. L. Lowes, E. Mahieu, G. L. Manney, H. A. Michelsen, M. J. Newchurch, C. P. Rinsland, R. J. Salawitch, G. P. Stiller, G. C. Toon, Y. L. Yung, and R. Zander

On the assessment and uncertainty of atmospheric trace gas burden measurements with high-resolution infrared solar occultation spectra from space by the ATMOS experiment (Paper 96GL01794) 2337

M. C. Abrams, A: Y. Chang, M. R. Gunson, M. M. Abbas, A. Goldman, F. W Irion, H. A. Michelsen,

M. J. Newchurch, C. P. Rinsland, G. P. Stiller, and R. Zander 
Trace gas transport in the Arctic vortex inferred from ATMOS ATLAS 2 observations during April 1993 (Paper 96GL00705) 2341

M. C. Abrams, G. L. Manney, M. R. Gunson, M. M. Abbas, A. Y. Chang, A. Goldman, F. W. Irion,

H. A. Michelsen, M. J. Newchurch, C. P. Rinsland, R. J. Salawitch, G. P. Stiller, and R. Zander

ATMOS/ATLAS 3 observations of long-lived tracers and descent in tine Antarctic vortex in November 1994 (Paper 96GL00704) 2345

M. C. Abrams, G. L. Manney, M. R. Gunson, M. M. Abbas, A. Y. Chang, A. Goldman, F. W. Irion,

H. A. Michelsen, M. J. Newchurch, C. P. Rinsland, R. J. Salawitch, G. P. Stiller, and R. Zander

Trends of OCS, HCN, SF6, CHClF 2 (HCFC-22) in the lower stratosphere from 1985 and 1994 atmospheric trace molecule spectroscopy experiment measurements near $30^{\circ} \mathrm{N}$ latitude (Paper 96GL01234) 2349

C. P. Rinsland, E. Mahieu, R. Zander, M. R. Gunson, R. J. Salawitch, A. Y. Chang, A. Goldman,

M. C. Abrams, M. M. Abbas, M. J. Newchurch, and F. W. Irion

Increase of stratospheric carbon tetrafluoride $\left(\mathrm{CF}_{4}\right)$ based on ATMOS observations from space (Paper 96GL00957) 2353

R. Zander, S. Solomon, E. Mahieu, A. Goldman, C. P. Rinsland, M. R. Gunson, M. C. Abrams, A. Y. Chang, R. J. Salawitch, H. A. Michelsen, M. J. Newchurch, and G. P. Stiller

The 1994 northern midlatitude budget of stratospheric chlorine derived from ATMOS/ATLAS 3 observations (Paper 96GL01792) 2357

R. Zander, E. Mahieu, M. R. Gunson, M. C. Abrams, A. Y. Chang, M. Abbas, C. Aellig, A. Engel, A. Goldman, F. W. Irion, N. Kämpfer, H. A. Michelsen, M. J. Newchurch, C. P. Rinsland, R. J. Salawitch, G. P. Stiller, and G. C. Toon

Stratospheric chlorine partitioning: Constraints from shuttle-borne measurements of [HCl], [ClNO 3$]$, and [ClO] (Paper 96GL00787) 2361

H. A. Michelsen, R. J. Salawitch, M. R. Gunson, C. Aellig, N. Kämpfer, M. M. Abbas, M. C. Abrams,

T. L. Brown, A. Y. Chang, A. Goldman, F. W. Irion, M. J. Newchurch, C. P. Rinsland, G. P. Stiller, and $R$. Zander

ATMOS/ATLAS 3 measurements of stratospheric chlorine and reactive nitrogen partitioning inside and outside the November 1994 Antarctic vortex (Paper 96GL01474) 2365

C. P. Rinsland, M. R. Gunson, R. J. Salawitch, H. A. Michelsen, R. Zander, M. J. Newchurch, M. M. Abbas, M. C. Abrams, G. L. Manney, A. Y. Chang, F. W. Irion, A. Goldman, and E. Mahieu

NO $y$ correlation with $\mathrm{N}_{2} \mathrm{O}$ and $\mathrm{CH}_{4}$ in the midlatitude stratosphere (Paper 96GL00870) 2369

Y. Kondo, U. Schmidt, T. Sugita, A. Engel, M. Koike, P. Aimedieu, M. R. Gunson, and J. Rodriguez

Stratospheric $\mathrm{NO}$ and $\mathrm{NO}_{2}$ abundances from ATMOS solar-occultation measurements

(Paper 96GL01196) 2373

M. J. Newchurch, M. Allen, M. R. Gunson, R. J. Salawitch, G. B. Collins, K. H. Huston, M. M. Abbas,

M. C. Abrams, A. Y. Chang, D. W. Fahey, R. S. Gao, F. W. Irion, M. Loewenstein, G. L. Manney,

H. A. Michelsen, J. R. Podolske, C. P. Rinsland, and R. Zander

Heavy ozone enrichments from ATMOS infrared solar spectra (Paper 96GL01695) 2377

F. W. Irion, M. R. Gunson, C. P. Rinsland, Y. L. Yung, M. C. Abrams, A. Y. Chang, and A. Goldman

Stratospheric observations of $\mathrm{CH}_{3} \mathrm{D}$ and HDO from ATMOS infrared solar spectra: Enrichments of deuterium in methane and implications for HD (Paper 96GL01402) 2381

F. W. Irion, E. J. Moyer, M. R. Gunson, C. P. Rinsland, Y. L. Yung, H. A. Michelsen, R. J. Salawitch,

A. Y. Chang, M. J. Newchurch, M. M. Abbas, M. C. Abrams, and R. Zander

ATMOS stratospheric deuterated water and implications for troposphere-stratosphere transport (Paper 96GL01489) 2385

Elisabeth J. Moyer, Fredrick W. Irion, Yuk L. Yung, and Michael R. Gunson.

A comparison of measurements from ATMOS and instruments aboard the ER 2 aircraft: Tracers of atmospheric transport (Paper 96GL01677) 2389

A. Y. Chang, R. J. Salawitch, H. A. Michelsen, M. R. Gunson, M. C Abrams, R. Zander, C. P. Rinsland, M. Loewenstein, J. R. Podolske, M. H. Proffitt, J. J. Margitan, D. W. Fahey, R. -S. Gao, K. K. Kelly,

J. W. Elkins, C. R. Webster, R. D. May, K. R. Chan, M. M. Abbas, A. Goldman, F. W. Irion, G. L. Manney,

M. J. Newchurch, and G. P. Stiller 
A comparison of measurements from ATMOS and instruments aboard the ER 2 aircraft: Halogenated gases (Paper 96GL01678) 2393

A. Y. Chang, R. J. Salawitch, H. A. Michelsen, M. R. Gunson, M. C. Abrams, R. Zander, C. P. Rinsland, J. W. Elkins, G. S. Dutton, C. M. Volk, C. R. Webster, R. D. May, D. W. Fahey, R.-S. Gao, M. Loewenstein, J. R. Podolske, R. M. Stimpfle, D. W. Kohn, M. H. Proffitt, J. J. Margitan, K. R. Chan, M. M. Abbas,

A. Goldman, F. W. Irion, G. L. Manney, M. J. Newchurch, and G. P. Stiller

ATMOS measurements of $\mathrm{H}_{2} \mathrm{O}+2 \mathrm{CH}_{4}$ and total reactive nitrogen in the November 1994 Antarctic stratosphere: Dehydration and denitrification in the vortex (Paper 96GL00048) 2397

C. P. Rinsland, M. R. Gunson, R. J. Salawitch, M. J. Newchurch, R. Zander, M. M. Abbas, M. C. Abrams,

G. L. Manney, H. A. Michelsen, A. Y. Chang, and A. Goldman

Seasonal variations of water vapor in the lower stratosphere inferred from ATMOS/ATLAS 3 measurements of $\mathrm{H}_{2} \mathrm{O}$ and $\mathrm{CH}_{4}$ (Paper 96GL01321) 2401

M. M. Abbas, H. A. Michelsen, M. R. Gunson, M. C. Abrams, M. J. Newchurch, R. J. Salawitch, A. Y. Chang

A. Goldman, F. W. Irion, G. L. Manney, E. J. Moyer, R. Nagaraju, C. P. Rinsland, G. P. Stiller, and R. Zander

The hydrogen budget of the stratosphere inferred from ATMOS measurements of $\mathrm{H}_{2} \mathrm{O}$ and $\mathrm{CH}_{4}$ (Paper 96GL01320) 2405

M. M. Abbas, M. R. Gunson, M. J. Newchurch, H. A. Michelsen, R. J. Salawitch, M. Allen, M. C. Abrams,

A. Y. Chang, A. Goldman, F. W. Irion, E. J. Moyer, R. Nagaraju, C. P. Rinsland, G. P. Stiller, and R. Zander

Stratospheric meteorological conditions from the November 3-12, 1994, ATMOS/ATLAS 3 measurements (Paper 96GL00774) 2409

G. L. Manney, R. Swinbank, and A. O'Neill

\section{ERRATA}

Cover figure titled "ATLAS-1 Solar Irradiance Spectra" has an incorrect label of MG $1 H \& K$ the label should be $\mathrm{Mg} \Pi h \& k$

The paper "The ATLAS Series of Shuttle Missions" by Jack A. Kaye and Timothy L. Miller (Geophys. Res. Lett., 23(17), 2285-2288),contained an incorrect Figure 2 caption. The correct caption appears below:

Figure 2. Plots showing two measures of solar activity for the time period July, 1991 to June, 1995. The upper curve (left ordinate) shows the SBUV/2 Mg II Index as determined from the NOAA 11 and NOAA 9 satellites (NOAA 9 data, which make up the last part of the this curve, are preliminary), while the lower curve (right ordinate) shows the $10.7 \mathrm{~cm}$ flux. Figure supplied by Rich Cebula of Hughes STX Corporation.

\section{Copyright 1996 by American Geophysical Union}




\title{
The ATLAS Series of Shuttle Missions
}

\author{
Jack A. Kaye \\ Office of Mission to Planet Earth, NASA Headquarters, Washington, DC \\ Timothy L. Miller \\ Earth System Science Division, NASA/Marshall Space Flight Center, Huntsville, Alabama
}

\begin{abstract}
The ATLAS space shuttle missions were conducted in March 1992, April 1993, and November 1994. The ATLAS payload and companion instruments made measurements of solar irradiance and middle atmospheric temperatures and trace gas concentrations. The solar irradiance measurements included total and spectrally resolved solar irradiance. The atmospheric measurements included microwave, infrared, and ultraviolet limb sounding, nadir ultraviolet backscatter, and solar occultation techniques. This paper introduces a special section in this issue of Geophysical Research Letters.
\end{abstract}

\section{Introduction}

The Atmospheric Laboratory for Applications and Science (ATLAS) was a series of three Spacelab missions flown in 1992, 1993, and 1994 with three major purposes: (i) determination of highly accurate and precise information about the trace chemical composition of the Earth's middle and upper atmosphere and incident solar radiation for comparison with information from other space-based instruments measuring these quantities; (2) measurement of a range of atmospheric and solar parameters such that very detailed scientific studies on atmospheric composition could be carried out for the brief duration of a flight of the space shuttle (8-11 days); and (3) provision of space-flight opportunities for instruments with objectives compatible to those of the ATLAS instruments so that comparisons of their measurements with those of the ATLAS instruments could be done as well as complementary science between two or more instruments. Originally envisioned as a series of 10 missions over an 11-year solar cycle, the ATLAS series was truncated after three missions due to a combination of funding constraints and limited availability of the space shuttle with a reduced flight rate and increased demands on the shuttle for other activities.

A major focus of the ATLAS series has been on the accuracy and precision of the measurements made. The simultaneous measurements of an identical parameter by different instruments allows to assess absolute accuracy, noise level, and efficiency of method of retrieval. This strategy is not unique, being similar to that of the Upper Atmosphere Research Satellite (UARS) [Reber, 1993]. Unlike instruments flown on free-flying satellites, however, instruments flown on the space shuttle are brought back to Earth, so that for each measurement both pre- and post-flight calibrations can be carried out, along with any on-board calibration activities conducted over the course of the shuttle missions. Combining this calibration regimen with the short-duration flights that limit onorbit instrument degradation should allow the ATLAS mea-

This paper is not subject to U.S. copyright. Published in 1996 by the American Geophysical Union

Paper number 96GL.02228 surements to be among the best characterized space-based measurements of atmospheric chemistry and solar radiation.

\section{Mission Descriptions}

The three ATLAS flights took place on March 24-April 2, 1992 (ATLAS-1), April 8-17, 1993 (ATLAS-2), and November 3-14, 1994 (ATLAS-3). Summaries of the individual ATLAS missions have already appeared [Torr, 1993; Miller et al., 1993; Miller et al., 1995]. The present overview article summarizes the three ATLAS missions, including the differences among them, and serves as an introduction of the 33 papers presenting results from the instruments which flew aboard these missions.

The ATLAS core instrument package consisted of six instruments, which are briefly described in Table 1. Two are for atmospheric chemistry measurements (ATMOS and MAS), two are for measurements of spectrally resolved solar radiation (SOLSPEC and SUSIM), and two are for measurements of total solar irradiance (ACRIM and SOLCON). With the exception of MAS, all of these instruments flew on the space shuttle prior to the ATLAS series-ACRIM, SOLCON, and SOLSPEC aboard the Spacelab-1 mission in 1983, SUSIM aboard the Spacelab2 mission in 1985 (as well as the earlier OSS-1 in 1982), and ATMOS aboard the Spacelab-3 mission in 1985. Flying along with the ATLAS payload on all three missions was the SSBUV instrument, which has now flown a total of eight times aboard the space shuttle. Other instruments have accompanied the ATLAS instruments as well, including several atmospheric science, space physics, and astronomy instruments which flew aboard ATLAS- 1 as part of a reflight of much of the Spacelab-1 package and a student-run solar instrument from the University of Colorado (ESCAPE) which flew aboard ATLAS-2 and ATLAS-3. Co-manifested payloads deployed and retrieved from the space shuttle which accompanied the ATLAS missions were the SPARTAN-201 solar science package [Fisher and Guhiathakurta, 1995] aboard ATLAS-2 and the German Shuttle Pallet Satellite (SPAS) platform which carried the CRISTA and MAHRSI atmospheric science instruments (see Table 1) aboard ATLAS-3 [Offermann and Conway, 1995].

A variety of "correlative measurement opportunities" were identified for the ATLAS instruments. Most notable among these is NASA's Upper Atmosphere Research Satellite (UARS), launched in September 1991. There is a great deal of complementarity between the UARS and ATLAS payloads, including each having two near-twin instruments (ACRIM and SUSIM), as well as instruments with very similar measurement objectives and experimental techniques (e.g., infrared absorption by occultation and microwave emission spectrometry). Significant attention has been paid to correlative measurement opportunities between the ATLAS and UARS payloads [Harrison et al., 1992; 1993; 1995], and ATLAS data have 
Table 1. ATLAS Instruments

\begin{tabular}{|c|c|c|}
\hline $\begin{array}{l}\text { Active Cavity Radiometer Irradiance } \\
\text { Monitor (ACRIM) }\end{array}$ & Total solar irradiance & Jet Propulsion Laboratory \\
\hline Solar Constant (SOLCON) & Total solar irradiance & Institut Royal Meteorologique Belgium \\
\hline Solar Spectrum (SOLSPEC) & Solar spectrum, IR through UV & CNRS France \\
\hline $\begin{array}{l}\text { Solar Ultraviolet Spectral Irradiance } \\
\text { Monitor (SUSIM) }\end{array}$ & Solar UV output & Naval Research Laboratory \\
\hline $\begin{array}{l}\text { Millimeter-Wave Atmospheric Sounder } \\
\text { (MAS) }\end{array}$ & $\begin{array}{l}\text { Middle atmosphere chemistry } \\
\text { (mm-wave emissions) }\end{array}$ & $\begin{array}{l}\text { Max Planck Institute of Aeronomy } \\
\text { (Germany) }\end{array}$ \\
\hline $\begin{array}{l}\text { Shuttle Solar Backscatter Ultraviolet } \\
\text { (SSBUV) }\end{array}$ & Stratospheric ozone (UV backscatter) & NASA/Goddard Space Flight Center \\
\hline $\begin{array}{l}\text { Cryogenic Infrared Spectrometers and } \\
\text { Telescopes for the Atmosphere } \\
\text { (CRISTA)* }\end{array}$ & $\begin{array}{l}\text { Middle atmosphere chemistry } \\
\text { (IR emissions) }\end{array}$ & University of Wuppertal (Germany) \\
\hline $\begin{array}{l}\text { Experiment of the Sun for } \\
\text { Complementing the ATLAS Payload } \\
\text { and for Education (ESCAPE)** }\end{array}$ & Solar far-UV output & University of Colorado \\
\hline
\end{tabular}

*CRISTA and MAHRSI flew with ATLAS-3 only.

**ESCAPE, a student experiment, flew with ATLAS-2 and ATLAS-3.

been used extensively in UARS validation [Grose and Gille, 1995; Woods et al., 1996; Gille et al., 1996]. SSBUV plays a critical role in the validation of the ozone and solar spectral irradiance measurements of the Solar Backscatter Ultraviolet/2 (SBUV/2) instruments aboard NOAA's polar-orbiting operational meteorological satellites [Hilsenrath et al., 1995]. Other correlative measurement opportunities included solar flux measurements on the European Retrieval Carrier (EURECA) and the Earth Radiaion Budget Satellite (ERBS), and ozone measurements from the Total Ozone Mapping Spectrometer (TOMS) instruments on Nimbus-7 and Meteor satellites.

The two total solar irradiance instruments in the ATLAS payload, SOLCON and ACRIM, are both pyrheliometers, measuring the amount of energy absorbed in a cavity exposed to sunlight. The total solar irradiance ("solar constant") was measured to within $0.1 \%$ accuracy and precision [Crommelynck et al., this issue]. In the context of a larger program with many measurements on several platforms over a long period of time, as well as instruments on long-duration, free-flying platforms, such as the UARS ACRIM instrument, this accuracy on periodic short missions can help establish our knowledge of possible variations in the total solar energy that drives Earth's climate system.

The two spectrally resolved solar irradiance instruments, SOLSPEC and SUSIM, covered different wavelength ranges, with SOLSPEC covering from the ultraviolet (UV) to near infrared wavelengths, while SUSIM examined only UV wavelengths but down to a short-wavelength cutoff of $110 \mathrm{~nm}$. SSBUV, whose primary purpose is to measure the vertical profile of stratospheric ozone, also made measurements of solar UV radiation [Cebula et al., this issue]. The ESCAPE instrument was designed to make measurements in the far UV, extending the ATLAS measurement range to wavelengths below the SUSIM short-wavelength cutoff.

A large number of atmospheric trace constituents and temperatures were measured using several methods (see Table 2).
The MAS instrument [Hartmann et al, this issue] measured altitude profiles of the emissions of atmospheric radiation in the millimeter wave range, specifically at frequencies corresponding to water vapor, ozone, chlorine monoxide, and molecular oxygen (used for temperature and pressure). ATMOS measured the absorption of infrared solar radiation by the Earth's atmosphere and from those data the concentrations of a very large number of trace species can be inferred as a function of altitude [Gunson et al., this issue]. SSBUV measured the scattering of solar UV radiation from the Earth's atmosphere, and used this information to obtain both total column ozone and ozone vertical profile distributions, as well as nitric oxide amounts in the mesosphere and lower thermosphere. CRISTA measured emissions of infrared radiation in three viewing directions, obtaining detailed three-dimensional datasets of various trace gases. MAHRSI made limb scanning measurements of the UV solar resonance fluorescence of $\mathrm{OH}$ and NO to obtain their vertical density profiles in the middle atmosphere (Conway et al., 1996).

All three ATLAS missions were carried out with an orbital inclination of 57 degrees, allowing the viewing of most of the Earth's surface. The orbit altitudes of ATLAS-1 and ATLAS-2 were both $296 \mathrm{~km}$, and the altitude of ATLAS-3 was slightly higher $(302 \mathrm{~km})$. Because of the emphasis on highly accurate and precise measurements, significant attention was placed on consideration of contamination issues. In addition to the cleanliness requirements on the individual instruments and Spacelab pallet, a special wipe-down of the shuttle payload bay was used prior to the ATLAS-2 and ATLAS-3 missions. The solar instruments did not operate until the platform had been in orbit for some 24 hours to allow for outgassing of any contaminants.

Due to different launch times and seasons, the viewing regions and times of the ATLAS instruments differed from one mission to the next. This was particularly true for ATMOS, as it uses the occultation technique, and the position of the occultations is very sensitive to launch time. For ATLAS-1, the 
Table 2. Atmospheric Constituents Measured by ATLAS-3 Approximate Altitude Range (km)

\begin{tabular}{|c|c|c|c|c|c|}
\hline Gas & ATMOS & MAS & SSBUV & MAHRSI & CRISTA \\
\hline $\mathrm{O}_{3}$ & $10-100$ & $20-90$ & $20-55$ & & $15-95$ \\
\hline No & $10-140$ & & $50-100$ & $45-145$ & $100-180$ \\
\hline $\mathrm{NO}_{2}$ & $15-50$ & & & & $15-40$ \\
\hline $\mathrm{N}_{2} \mathrm{O}$ & $10-60$ & & & & $15-45$ \\
\hline $\mathrm{N}_{2}^{2} \mathrm{O}_{5}$ & $20-40$ & & & & $20-40$ \\
\hline $\mathrm{H}_{2} \mathrm{O}$ & $10-90$ & $20-90$ & & & $15-80$ \\
\hline $\mathrm{HNO}_{3}$ & $15-40$ & & & & $15-40$ \\
\hline $\mathrm{CH}_{4}{ }^{3}$ & $10-70$ & & & & $15-70$ \\
\hline $\mathrm{CCl}_{3} \mathrm{~F}$ & $10-25$ & & & & $15-20$ \\
\hline $\mathrm{CCl}_{2} \mathrm{~F}_{2}$ & $10-30$ & & & & $15-30$ \\
\hline $\mathrm{ClONO}_{2}$ & $10-35$ & & & & $20-40$ \\
\hline $\mathrm{ClO}$ & & $30-45$ & & & \\
\hline $\mathrm{HCl}$ & $10-65$ & & & & $*$ \\
\hline $\mathrm{HF}$ & $10-60$ & & & & $40-65$ \\
\hline $\mathrm{OH}$ & & & & $40-90$ & \\
\hline $\mathrm{CO}$ & $10-60$ & & & & * \\
\hline $\begin{array}{l}\mathrm{CO}_{2} \\
\mathrm{O}(3 \mathrm{P})\end{array}$ & $10-120$ & & & & $\begin{array}{l}15-150 \\
80-180\end{array}$ \\
\hline
\end{tabular}

*Altitude range as yet undetermined.

In addition to the above, there are some 20 other constituents available in the spectra measured by ATMOS, and temperature and pressure were measured by ATMOS, MAS, and CRISTA.

occultations spanned a range of tropical and mid-latitude regions in both hemispheres, while for ATLAS-2, sunrise occultations were at high northern latitudes with sunset occultations at mid-high southern latitudes, and for ATLAS-3, sunrise occultations were at high southern latitudes with sunsets varying from the tropics to mid-latitudes in the northern hemisphere [Gunson et al., this issue]. The ATLAS-2 and ATLAS-3 missions took place during the northern and southern hemisphere spring seasons, respectively, during which the

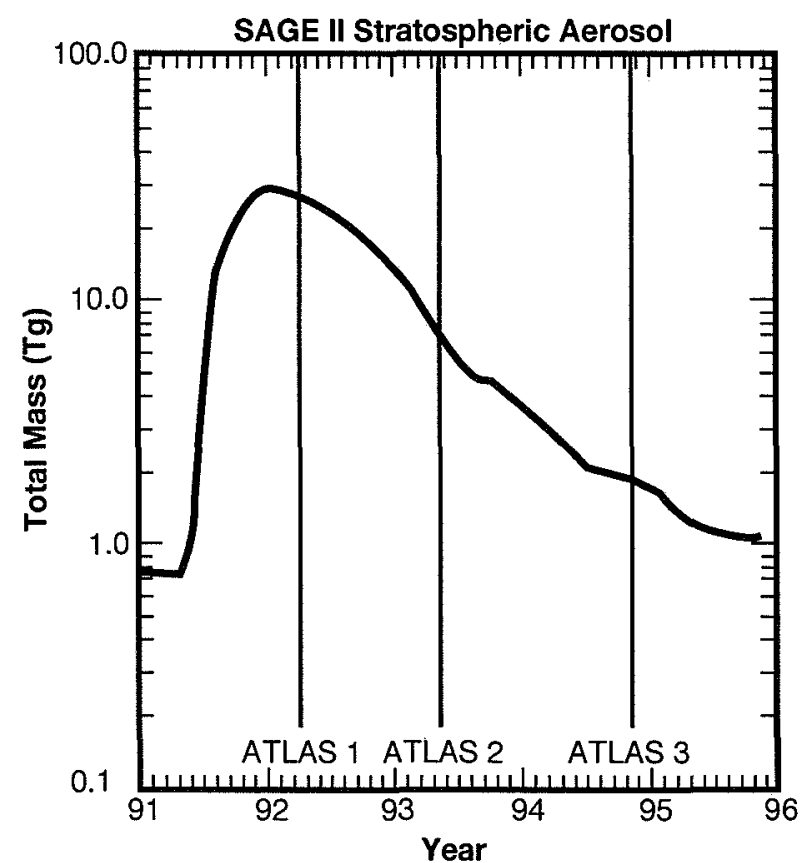

Figure 1. Plot showing estimated total mass of stratospheric aerosol as inferred from measurements made by the SAGE II instrument. Vertical bars are used to represent the dates of the ATLAS flights. Figure supplied by Larry Thomason of NASA's Langley Research Center. corresponding polar vortices persisted (displaced somewhat from the pole), allowing for observations both inside and outside. More details on the meteorological conditions of the ATLAS-3 mission are summarized in a paper by Manney et al. (this issue).

Since instrument pointing was provided by the space shuttle, atmospheric and solar observations were carried out sequentially. For most of the mission, the shuttle was in a cargo-bay-down attitude for atmospheric observations, inter-

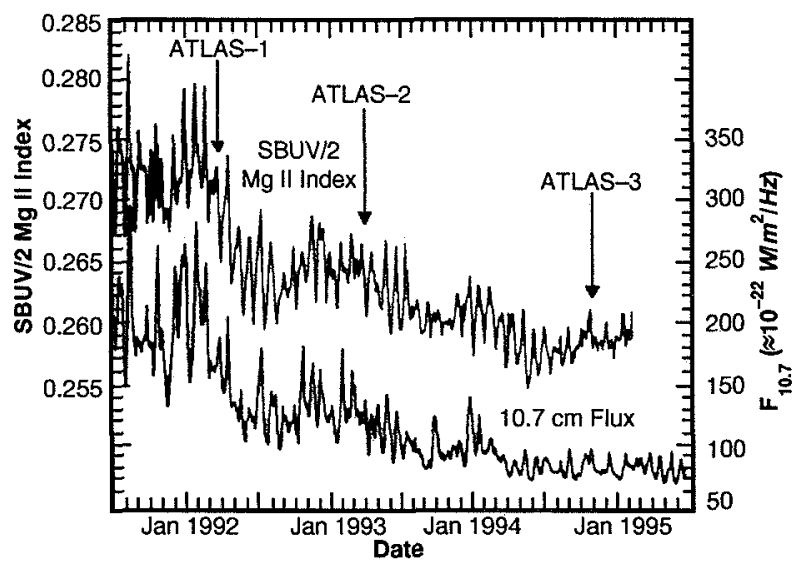

Figure 2. Plot showing globally averaged (65 degrees north to south) total ozone in Dobson Units (DU) for the period 1992 through 1994 as determined by the TOMS instrument. Arrows indicate the times of the ATLAS flights. The shaded area represents the climatology for the period 1979-1990, with the white line showing the mean of that period. The dashed line shows data from the Nimbus-7 TOMS instrument, which failed in May 1993, while the solid line shows data from the Meteor-3 TOMS, which failed in December 1994. Gaps in the latter curve reflect time periods where the orbit of the Meteor-3 satellite was inappropriate for quantitative determination of global total ozone. Figure supplied by Jim Gleason of NASA's Goddard Space Flight Center. 
rupted approximately every 2 days for roughly 12 hours of solar observations. During solar observations, the orbiter changed orientations every orbit between the cargo bay pointing towards the Sun (day side) to pointing toward deep space for cooling (night side). Scientific observations were suspended during the deployment and retrieval of the SPARTAN 201 (ATLAS-2) and CRISTA/SPAS (ATLAS-3) carriers. There were also several short periods of time during the latter mission when scientific observations by the ATLAS instruments were halted so that the shuttle attitude was changed to one dedicated to communications with the SPAS.

All instruments performed well during the ATLAS missions, with the exception of the MAS instrument, which failed after 1 day of operations during ATLAS-3. However, MAS was still able to obtain near-global measurements of $\mathrm{H}_{2} \mathrm{O}, \mathrm{O}_{3}, \mathrm{ClO}$, and temperature during that period [Hartmann et al., this issue].

The 2.5-year time interval between the first and last ATLAS missions saw several interesting and important changes in the atmosphere and solar forcing: (1) There was a significant decrease in the stratospheric aerosol burden (see Fig. 1) over this time period, since the ATLAS-1 mission occurred only 9 months after the Mt. Pinatubo eruption (June 1991) and only a few months after the peak in total stratospheric aerosol abundance as inferred from the Stratospheric Aerosol and Gas Experiment (SAGE). Given that there is an approximate 1-year lifetime for stratospheric aerosols, one sees that the stratospheric aerosol loading decayed by nearly a factor of 100 from the ATLAS-1 to ATLAS-3 mission. This decay allowed for significantly improved penetration of ATMOS occultations into the lower stratosphere and troposphere in ATLAS-3 as opposed to ATLAS-1 when most occultations could not observe the lower stratosphere (but interesting studies could be carried out on the aerosol optical properties [Rinsland et al., 1994]; (2) There was a marked decline in global total ozone in the 1992-1993 time period believed to be related to the Mt. Pinatubo eruption. This decline was just beginning at the time of the ATLAS-1 flight, was near its maximum at the time of the ATLAS- 2 flight, and was essentially recovered by the ATLAS-3 flight. The SSBUV instrument clearly showed the decrease in ozone from spring 1992 to spring 1993 [Hilsenrath et al., this issue] which has also been seen from other instruments; and (3) There was a marked decrease in solar activity from the ATLAS-1 to ATLAS-3 mission (see Fig. 2), as measured from a variety of solar indices, including the F10.7 flux and the Mg II Index determined from the SBUV/2 instrument. The ATLAS-1 and ATLAS-2 flights were during the declining phase of solar cycle 22, while ATLAS-3 was very close to solar minimum. It is also worth noting that the halogen burden in the stratosphere continued to increase over this time period [Gunson et al., this issue].

Acknowledgments. In addition to the principal investigator teams represented by the papers in this issue, the ATLAS missions would not have been possible without the efforts and expertise of the support teams. The Marshall Space Flight Center, under the direction of Mission Managers A. O'Neil (ATLAS-1), T. Vanhooser (ATLAS-2), and P. Hamby (ATLAS-3), managed payload integration and mission operations. Payload Operations Directors P. Nelson (ATLAS-1), L. Wooten (ATLAS-2), and D. Gunter (ATLAS-3) are especially acknowledged. Thanks are due to the Kennedy Space Center for payload integration and launch services, and to the Johnson Space Center for shuttle operations. D. Butler, E. Montoya, G. Esenwein, L. Caudill, and D. Jarrett of NASA Headquarters served in the capacity as program scientist, instrument manager, and program manager for the ATLAS series and made important contributions to the program. The payload partners from the Goddard Space Flight Center and from DARA (Germany) were gracious and cooperative in the many stages of planning and compromise that were necessary to conduct these joint science missions. Finally, many thanks are due to the orbiter and payload crews, who expertly worked with the payload in orbit and helped to communicate the ATLAS science goals to the public.

\section{References}

Cebula, R. P., et al., Observation of the solar irradiance in the 200-360 $\mathrm{nm}$ interval during the ATLAS-1 mission: A comparison between the SSBUV, SOLSPEC, and SUSIM measurements, Geophys. Res. Lett., this issue.

Conway, R. R., M. H. Stevens, J. G. Cardon, S. E. Zasadil, C. M. Brown, J. S. Morrill, and G. H. Mount, Satellite measurements of hydroxyl in the mesosphere, Geophys. Res. Lett., in press, 1996.

Crommelynck, D., et al., SOLCON solar constant observations from the ATLAS missions, Geophys. Res. Lett., this issue.

Fischer, R., and M. Guhathakurta, Physical properties of polar coronal rays and holes as observed with the Spartan 201-01 coronagraph, Astrophys J., 447, L139-L142, 1995.

Gille, J., S. Massie, and W. Mankin, Introduction to UARS Validation Special Issue, J. Geophys. Res., 101, 9539, 1996.

Grose, W. L., and J. Gille, Upper Atmosphere Research Satellite Validation Workshop III: Temperature and Constituents Validation, NASA Conf. Publ. 3317, NASA Langley Research Center, Hampton, VA, 1995.

Gunson, M. R., et al., The atmospheric trace molecule spectroscopy (ATMOS) experiment: Deployment on the ATLAS space shuttle missions, Geophys. Res. Lett., this issue.

Harrison, E. F., F. M. Denn, and G. G. Gibson, Correlative Measurement Opportunities between ATLAS-1 and UARS Experiments, NASA Tech. Memo. 107530, NASA Langley Research Center, Hamplon, VA, 1992.

Harrison, E. F., F. M. Denn, and G. G. Gibson, ATLAS-2 and UARS Correlative Measurement Opportunities During Space Shuttle Mission on April 8-17, 1993, NASA Tech. Memo. 109020, NASA Langley Research Center, Hampton, VA, 1993.

Harrison, E. F., F. M. Denn, and G. G. Gibson, ATLAS-3 Correlative Measurement Opportunities with UARS and Surface Observations, NASA Tech. Memo. 110159, NASA Langley Research Center, Hampton, VA, 1995.

Hartmann, G., et al., Measurements of $\mathrm{O}_{3}, \mathrm{H}_{2} \mathrm{O}$, and $\mathrm{ClO}$ in the middle atmosphere using the Millimeter-wave Atmospheric Sounder (MAS), this issue.

Hilsenrath, E., et al., Ozone depletion from 1992 to 1993 as observed from SSBUV on the ATLAS-1 and ATLAS-2 missions, this issue.

Hilsenrath, E., et al., Calibration of the NOAA 11 solar backscatter ultraviolet/2 (SBUV/2) ozone data set from 1989 to 1993 using inflight calibration data and SSBUV, J. Geophys. Res., 100, 1351-1366, 1995.

Manney, G. L., R. Swinbank, and A. O'Neill, Stratospheric meteorological conditions for the 3-12 Nov. 1994 ATMOS/ATLAS-3 measurements, this issue.

Miller, T. L., S. A. Smith, and J. A. Kaye, ATLAS space shuttle studies Earth's atmosphere and solar input, Eos, 75, 321-325, 1994.

Miller, T. L., S. A. Smith, and J. A. Kaye, Mission studies Earth's atmosphere and solar input, Eos, 76, 345-350, 1995.

Offermann, D., and R. R. Conway, Mission studies the composition of Earth's middle atmosphere, Eos, 76, 337-338, 1995.

Reber, C. A., The Upper Atmosphere Research Satellite (UARS), Geophys. Res. Lett., 20, 1215-1218, 1993.

Rinsland, C. P., et al., Mid-infrared extinction by sulfate aerosols from the Mt. Pinatubo eruption, J. Quant. Spectrosc. and Rad. Trans., 52, 241-252, 1994

Torr, M. R., The scientific objectives of the ATLAS-1 shuttle mission. Geophys. Res. Lett., 20, 487-490, 1993.

Woods, T. N., et al., Validation of the UARS solar UV irradiances: Comparison with the ATLAS-1 and -2 measurements, J. Geophys. Res., 101, 9541-9601, 1996.

J. A. Kaye, Office of Mission to Planet Earth, NASA Headquarters, Washington, DC 20546. (e:mail:jkay@mtpe.hq.nasa.gov)

T. L. Miller, Earth System Science Division, NASA/Marshall Space Flight Center, Huntsville, AL 35812. (e:mail: tim.miller@msfc.nasa.gov)

(Received June 28, 1996; accepted July 2, 1996.) 


\title{
Observations of the solar irradiance in the $200-350 \mathrm{~nm}$ interval during the ATLAS-1 mission: a comparison among three sets of measurements - SSBUV, SOLSPEC, and SUSIM
}

\author{
R. P. Cebula ${ }^{1}$, G. O. Thuillier ${ }^{2}$, M. E. VanHoosier ${ }^{3}$, E. Hilsenrath ${ }^{4}$, M. Herse ${ }^{2}$, G. E. \\ Brueckner $^{3}$, and P. C. Simon ${ }^{5}$
}

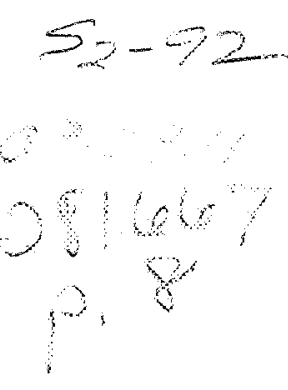

\begin{abstract}
The SOLSPEC, SSBUV, and SUSIM spectrometers simultaneously observed the solar spectral irradiance during the ATLAS-1 mission flown on board the Space Shuttle Atlantis in March 1992. The three instruments use different methods and means of absolute calibration and were each calibrated preflight and postflight. The three data sets are reported from 200 to $350 \mathrm{~nm}$ at $1.1 \mathrm{~nm}$ resolution. The method of comparing the three independent data sets is discussed. The importance of a common, precise wavelength scale is shown when comparing the data in wavelength regions of strong Fraunhofer lines. The agreement among the solar irradiance measurements is better than $\pm 5 \%$. The fact that the calibrations of the three instruments were based on three independent standards provides confidence that the absolute solar spectral irradiance in the range $200-350 \mathrm{~nm}$ is now known with an accuracy better than $\pm 5 \%$. The mean ATLAS-1 solar spectrum is compared with simultaneous solar observations from the UARS SOLSTICE and UARS SUSIM instruments. The two mean solar spectra agree to within $\pm 3 \%$.
\end{abstract}

\section{Introduction}

Solar spectral irradiance data and its changes, particularly in the middle and near ultraviolet (UV) are needed for photochemical studies and understanding the thermal structure, dynamics, and energy budget of the earth's atmosphere. As a result of molecular and aerosol scattering and, to a lesser extent, absorption by trace gases, approximately $50 \%$ of the extraterrestrial irradiance in the region between 300 and $400 \mathrm{~nm}$ reaches the Earth's surface. Shortward of $300 \mathrm{~nm}$ the solar irradiance is strongly absorbed by ozone and molecular. oxygen. Tracking solar irradiance changes at these wavelengths can therefore best be done above the Earth's atmosphere. The Upper Atmospheric Research Satellite (UARS) and the ATLAS Space Shuttle missions each carried multiple instruments to measure the extraterrestrial solar irradiance. The two spacecrafts' instruments complemented each other in that the UARS instruments were designed to monitor the sun over time, while the ATLAS instruments were designed to perform periodic checks of the UARS (and other satellite) instruments each time an ATLAS mission was conducted.

In this paper we compare simultaneous ATLAS-1 solar spectral irradiance observations from the Solar Spectrum (SOLSPEC), the

${ }^{1}$ Hughes STX Corporation, Greenbelt, MD 20770
${ }^{2}$ Service d'Aeronomie du CNRS, Verrieres le Buisson, France 91371
${ }^{3}$ Naval Research Laboratory, Washington, DC 20375-5352
${ }^{4}$ NASA/Goddard Space Flight Center, Greenbelt, MD 20771
${ }^{5}$ Institut d'Aeronomie Spatiale de Belgique, B1180 Bruxelles, Belgium

Copyright 1996 by the American Geophysical Union.

Paper number 96GL01109

0094-8534/96/96GL-01109\$05.00
Shuttle Solar Backscatter Ultraviolet (SSBUV), and the Solar Ultraviolet Spectral Irradiance Monitor (SUSIM) instruments obtained on 29 March 1992. The three ATLAS instruments' solar spectral irradiance measurements are first compared to one another and then the average of the ATLAS data is compared to simultaneous data taken by the UARS Solar Stellar Irradiance Comparison Experiment (SOLSTICE) and UARS SUSIM instruments [Rottman et al., 1993; Brueckner et al., 1993]. This paper complements the results given by Woods et al. [1996], where the UARS solar irradiance data are validated by direct comparison and by using SSBUV and SUSIM data from ATLAS-1 and -2 .

Observing the sun in the UV is complicated by instrument degradation caused by the damaging space environment and the difficulty in achieving an accurate absolute calibration. In earlier comparisons [Heath, 1980; Mount and Rottman, 1985; Nicolet, 1989; Lean, 1991], differences among the measurements were as large as $20 \%$ in the middle UV. It is now well known that both solar rotation and solar cycle variability are significantly lower, typically 7 $9 \%$ at $200 \mathrm{~nm}$, and, except for isolated absorption features, decrease with increasing wavelength [Lean, 1991]. The accuracy of solar irradiance measurements has significantly improved during the last two decades. We are now at the point at which the accuracies of the primary standards are no longer negligible with respect to other sources of measurement uncertainty. A unique aspect of the solar observations reported here is that the three spectrometers have different designs, are calibrated with different methods, and employ three different absolute standards. This comparison therefore can determine if the measured absolute irradiance data are consistent. If so, then the absolute value of solar spectral irradiance in the middle UV can be established with much improved confidence.

\section{SOLSPEC}

The Shuttle SOLSPEC instrument first flew in 1983 on the Spacelab-1 mission [Labs et al., 1987] and has since flown on the three ATLAS missions. A companion SOLSPEC experiment also flew on the European Space Agency's EURECA platform from August 1992 to May 1993. The instrument was described by Thuillier et al. [1981]. SOLSPEC observes from 200 to $3000 \mathrm{~nm}$ using three similar double monochromators. The UV spectrometer (200-350 nm) has $1.1 \mathrm{~nm}$ bandpass, steps in $0.4 \mathrm{~nm}$ increments, and its wavelength positioning, determined from metrology of the grating drive mechanism, is about $\pm 0.01 \mathrm{~nm}$. SOLSPEC incorporates diffusers in front of each entrance slit to minimize the impact of any pointing offset. The UV spectrometer uses a photomultiplier tube (PMT) as its detector. The experiment includes calibration lamps to monitor the responsivity and the spectral characteristics of the optics in space and on the ground. Two deuterium $\left(D_{2}\right)$ lamps were periodically activated to check the spectrometer's radiometric sensitivity. Seven emission lines from a Cu-He hollow cathode lamp were used to monitor the wavelength scale and the instrument slit function. For the 28 spectra used to construct the mean SOLSPEC 
spectrum, the stability of the wavelength scale, determined using the $280 \mathrm{~nm} \mathrm{Mg}$ II line profile, was found to be $\pm 0.02 \mathrm{~nm}$.

The Heidelberg Observatory blackbody is used as the irradiance source for the SOLSPEC radiometric calibration. The windowless blackbody is heated to approximately $3050 \mathrm{~K}$ and its temperature is measured periodically using a pyrometer with an accuracy of $\pm 6 \mathrm{~K}$, including the pyrometer calibration error. The blackbody's irradiance is calculated using Planck's law and the instrument's responsivity determined from the instrument counts recorded when observing the cavity under the same solid angle as is used to view the sun. The estimated $3 \sigma$ uncertainty of the SOLSPEC radiometric calibration decreases from $\pm 4.3 \%$ at $200 \mathrm{~nm}$ to $\pm 3.3 \%$ at $250 \mathrm{~nm}$ and $\pm 2.4 \% 350$ $\mathrm{nm}$. The SOLSPEC wavelength calibration was determined via a least squares regression of the observed positions of lines from argon, krypton, and neon laboratory sources and the internal $\mathrm{Cu}-\mathrm{He}$ lamp to a second order polynomial dispersion equation. The $2 \sigma$ uncertainty in the absolute wavelength calibration of SOLSPEC is $\pm 0.1 \mathrm{~nm}$.

Details of the SOLSPEC ATLAS-1 calibration procedures, data processing, and analysis are discussed by Thuillier et al. [Observation of the UV solar spectral irradiance between 200 and $360 \mathrm{~nm}$ during the ATLAS 1 mission by the SOLSPEC spectrometer, submitted to Solar Physics]. The mean SOLSPEC solar spectrum for March 29 is shown as the blue curve in Fig. 1. The standard deviation of the 28 spectral scan mean was $\pm 1 \%$. The final uncertainty in the derived SOLSPEC irradiance, including the accuracy of the radiometric calibration and precision of the solar measurements, ranges from $\pm 4.5 \%$ at $200 \mathrm{~nm}$ to $\pm 3.4 \%$ at $250 \mathrm{~nm}$ and $\pm 2.5 \%$ at $350 \mathrm{~nm}$.

\section{SSBUV}

SSBUV supports the long-term global stratospheric ozone and solar UV monitoring programs by providing repeated checks on the calibrations of UV ozone and solar monitoring instruments flying on US and international satellites. These include the Nimbus-7 SBUV and Total Ozone Mapping Spectrometer (TOMS) instruments, the Meteor TOMS instrument, and the SBUV/2 instruments on NOAA-9, -11 , and -14. SSBUV flew 8 times between October 1989 and January 1996; the ATLAS-1 mission was the fourth SSBUV flight. The instrument consists of a double holographic grating spectrometer with a PMT detector. The SSBUV wavelength range is 200 to 405

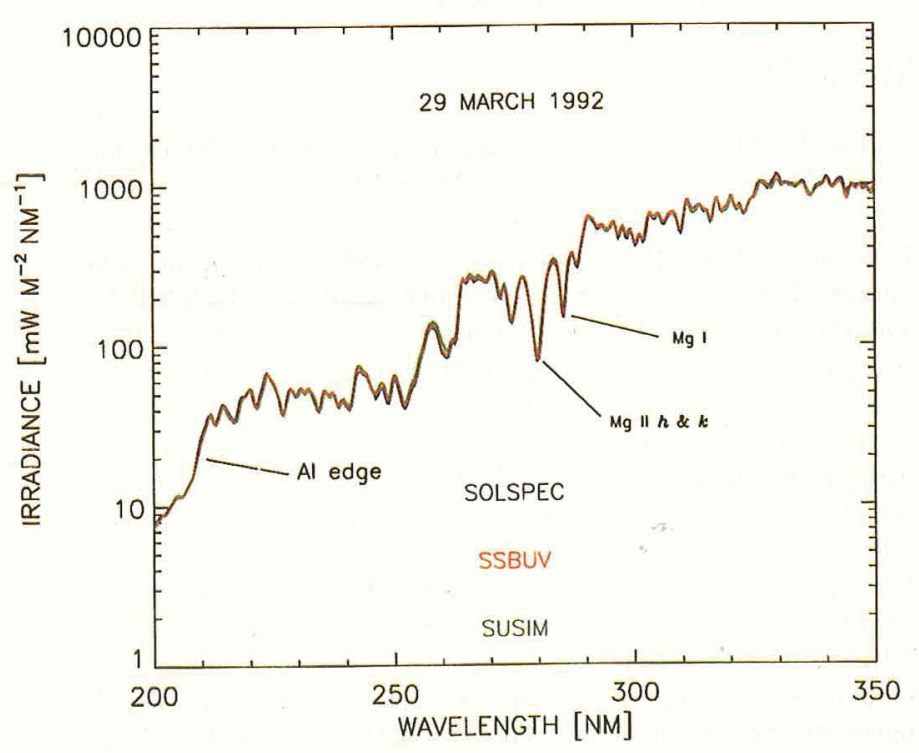

Figure 1. The average SOLSPEC (bule), SSBUV (red), and SUSIM (green) ATLAS-1 solar irradiance measured on 29 March 1992, each presented at $1.1 \mathrm{~nm}$ resolution. $\mathrm{nm}$ and the bandpass is $1.1 \mathrm{~nm}$. The instrument is identical to the SBUV/2 satellite instruments except that SSBUV uses a transmission rather than the reflection solar diffuser used on the satellite instruments. An onboard calibration system uses quartz halogen, $\mathrm{D}_{2}$, and mercury lamps to monitor the instrument radiometric sensitivity and wavelength registration. SSBUV solar irradiance observations from the first four missions were reported by Cebula et al. [1994].

The SSBUV calibration approach and results were described by Cebula et al. [1989] and Hilsenrath et al. [1991, 1993]. Multiple tungsten quartz halogen (FEL) secondary standard lamps, calibrated by the National Institute for Standards and Technology (NIST), are the primary standards used to calibrate SSBUV between 250 and $405 \mathrm{~nm}$. The FEL calibrations are traceable to a NIST gold point blackbody [Walker et al., 1987]. NIST-calibrated $\mathrm{D}_{2}$ arc lamps also provide calibration data in the $250-350 \mathrm{~nm}$ region and are used to extend the SSBUV calibration to $200 \mathrm{~nm}$. The $\mathrm{D}_{2}$ measurements are normalized to the FEL data in the spectral overlap region [Cebula et al., 1989].

The wavelength calibration of SSBUV as a function of the grating encoder step number is determined via a least squares regression of the observed position of lines from multiple laboratory sources. The estimated $2 \sigma$ uncertainty of this calibration is \pm 0.02 to $\pm 0.03 \mathrm{~nm}$. During each mission the stability of the SSBUV wavelength calibration is monitored using the onboard $\mathrm{Hg}$ lamp and 13 solar absorption features. Over the first 6 flights, long-term changes in the SSBUV wavelength calibration were less than $\pm 0.02 \mathrm{~nm}$ and thermally driven intraflight changes were $0.05 \mathrm{~nm}$ or less [Cebula et al., 1996]. The SSBUV solar data were corrected for these changes.

SSBUV observed the sun during 4 solar observation periods on 29 March 1992. Each solar observation consisted of 6 to 8 complete spectral scans of the sun. The arithmetic mean of all scans for each solar observation period was first computed. The arithmetic mean of the mean solar irradiance measured during the second, third, and fourth of the March 29 SSBUV solar observation periods, shown as the red curve in Fig. 1, was then computed. Data from the first solar observation period were excluded in order to ensure that the instrument had fully stabilized. The SSBUV solar data were corrected for a small amount of degradation which occurred during flight. The estimated $2 \sigma$ uncertainty in the SSBUV solar irradiance ranges from $\pm 2.4 \%$ near $350 \mathrm{~nm}$ to $\pm 6 \%$ near $200 \mathrm{~nm}$ [Woods et al., 1996].

\section{SUSIM}

The Shuttle SUSIM instrument [VanHoosier et al., 1988] consists of two identical double-dispersion scanning spectrometers which cover the wavelength range $110-420 \mathrm{~nm}$ with both $0.15 \mathrm{~nm}$ and $5.0 \mathrm{~nm}$ resolution, which is controlled by changeable slits. The instrument flew five times between March 1982 and November 1994 on OSS-1, Spacelab, and three ATLAS missions. The Shuttle instrument is the precursor and similar to the UARS SUSIM instrument flying on the UARS satellite [Brueckner et al., 1993]. In-flight sensitivity changes are tracked by using one of the two spectrometers only to view an onboard $\mathrm{D}_{2}$ calibration lamp in order to characterize changes in the filters and detectors. The second spectrometer is used to make solar measurements and to periodically make $\mathrm{D}_{2}$ lamp scans to compare with the calibration spectrometer to determine sensitivity changes. The $5 \mathrm{~nm}$ resolution is used for the highest accuracy using photodiode detectors, while the $0.15 \mathrm{~nm}$ resolution uses PMTs.

The calibration standard for the SUSIM is the NIST Synchrotron Ultraviolet Radiation Facility (SURF), which is a primary standard [Saloman et al., 1982]. The SURF beam irradiance is determined by synchrotron theory. During calibration SUSIM is oriented at $45^{\circ}$ and $135^{\circ}$ to the plane of the synchrotron; the average of the calibration factors for these two orientations removes almost all the effects of the polarization of the SURF beam. Longward of $250 \mathrm{~nm}$ the SUSIM calibration is validated using a NIST-calibrated FEL lamp. The two independent calibrations, SURF and FEL, agree to within $2 \%$. An aging correction to account for SUSIM inflight degradation ( $4 \%$ or 
less between 200 and $350 \mathrm{~nm}$ ) was based on a comparison of the preflight and postflight calibrations. The total 20 uncertainty in the SUSIM absolute calibration, including uncertainties in the SURF irradiance, polarization effects, and instrument degradation during the calibration, is estimated to be $\pm 4 \%$ [Woods et al, 1996].

The wavelength scale for the high resolution SUSIM spectrum was derived by a second order polynomial fit to 11 well defined solar spectral lines across the range of the grating encoder positions. These 11 wavelengths were determined by convolving high resolution spectra [Moe et al., 1976; Anderson and Hall, 1989; Kurucz et al., 1984] to the $0.15 \mathrm{~nm}$ resolution of the SUSIM instrument. Instrument power was cycled several times during ATLAS-1, which necessitated resetting the grating encoder and determining new wavelength coefficients. This made wavelength tracking difficult. The SUSIM wavelength calibration has an estimated $2 \sigma$ uncertainty of no worse than $\pm 0.4 \mathrm{~nm}$.

The SUSIM solar spectral irradiance used for this comparison, shown as the green curve in Fig. 1, consists of the mean of 2 spectra taken during a 3 orbit period on March 29. This spectrum was computed via a convolution of the observed $0.15 \mathrm{~nm}$ spectra with a $1.1 \mathrm{~nm}$ FWHM triangular slit function in order to match the resolution of other two instruments. The total $2 \sigma$ uncertainty in the mean SUSIM solar irradiance ranges from $\pm 5 \%$ to $\pm 8 \%$ from $200-350 \mathrm{~nm}$. The larger uncertainty applies only in those areas where the solar spectrum is steep and uncertainty in the wavelength registration of the calibration and solar data dominates.

\section{Wavelength scale impact}

Each experiment team applied a detailed wavelength calibration to their data. However, small differences in the wavelength assigned to each instrument's solar spectrum can have a dramatic effect when spectra are compared. Particular challenges occur near the numerous solar absorption lines, as well as regions where the spectrum makes large changes in magnitude, for example the Al edge near $208 \mathrm{~nm}$. Each instrument has a slightly different bandpass width and shape, further amplifying sensitivity to wavelength error; SOLSPEC has a gaussian shape while the SSBUV and SUSIM slit functions are nearly triangular. Among the techniques used by the experiment teams to establish accurate wavelength scales for their spectra are comparison to high resolution spectra [Anderson and Hall, 1989; Kurucz et al., 1984], which can be determined with higher wavelength accuracy. The high resolution data were first convolved with each of the ATLAS instruments' slit functions, then the resulting spectra were compared to the ATLAS instruments' spectra in $10 \mathrm{~nm}$ intervals over the entire spectral region from $200 \mathrm{~nm}$ to $350 \mathrm{~nm}$. For each instrument the comparison typically exhibited some scatter with respect to the high resolution data, but no errors greater than $\pm 0.1 \mathrm{~nm}$ were revealed, hence no adjustments were made. However, $\mathrm{a} \pm 0.1 \mathrm{~nm}$ wavelength shift between the spectra can generate differences of about $\pm 5 \%$, which is comparable to the structure observed in the comparisons presented later in this paper.

\section{Comparison of the three ATLAS-1 spectra}

In order to compare the data from the three ATLAS instruments, the unweighted average of the three spectra was first calculated, then the ratio of each instrument's spectrum to the mean ATLAS-1 spectrum was computed. These ratios are shown as the dotted lines in Fig. 2. The $1.1 \mathrm{~nm}$ instrument resolution ratios demonstrate the impact of small differences in wavelength scale and slit function discussed previously, particularly near strong solar absorption features. To minimize the impact of wavelength misregistration and small differences in slit function, each instrument's spectrum was also smoothed over $5 \mathrm{~nm}$ using a running mean. The arithmetic mean of the three instruments' degraded spectra was then constructed, and the ratio of each instrument's spectrum to this mean spectrum computed.
The result of this procedure is shown as the solid lines in Fig. 2. While these latter curves show generally good agreement within each instrument's calibration uncertainties, two types of features as a function of wavelength are observed. First, despite the integration over $5 \mathrm{~nm}$, the presence of strong Fraunhofer lines, such as the Mg II line at $280 \mathrm{~nm}$, remains noticeable. Deep lines can generate discrepancies which extend over several nanometers due to the running mean integration effect. Second, variations over tens of nanometers, particularly evident between 240 and $280 \mathrm{~nm}$, are likely due to radiometric calibration errors. Here the differences with respect to the ATLAS-1 mean spectrum are approximately $-5 \%$ for SOLSPEC and $+5 \%$ for SUSIM, with SSBUV lying roughly midway between the other two instruments. This implies an approximate $10 \%$ relative difference between SOLSPEC and SUSIM in this spectral region. Taking into account the uncertainty in the two instruments, it appears this difference is real and may be due to a discrepancy in their relative calibrations. At other wavelengths the agreement among the three instruments improves by approximately a factor of two over that seen between 240 and $280 \mathrm{~nm}$. The data presented in Fig. 2 demonstrate the difficulties in measuring the solar irradiance in the middle UV. While at first it might appear that the SSBUV data approximately replicate the mean ATLAS-1 spectrum, this is not the case. The SSBUV irradiances are approximately $2 \%$ greater than the mean ATLAS-1 irradiance in the region between 290 and $320 \mathrm{~nm}$ and are thus approximately $3 \%$ higher than the average of the SOLSPEC and SUSIM irradiances in this interval. Future comparisons of ATLAS-2 and ATLAS-3 data will help identify the source(s) of the differences observed in Fig. 2.

A unique feature of this comparison is that each of the three instruments traces its calibration to an independent calibration standard: the Heidelberg blackbody for SOLSPEC, FEL lamps for SSBUV, and SURF for SUSIM. Hence, the differences shown in Fig. 2 can arise from errors in correcting for instrument aging during the mission, from errors in transferring the absolute calibrations from the laboratory standards to the instruments, and from fundamental radiometric differences between the irradiance scales of the laboratory standards. The three ATLAS instruments participated in a special NIST-coordinated Round Robin [Woods et al, 1996], which compared calibration standards and laboratory procedures. Further, the comparisons presented herein represent the result of several

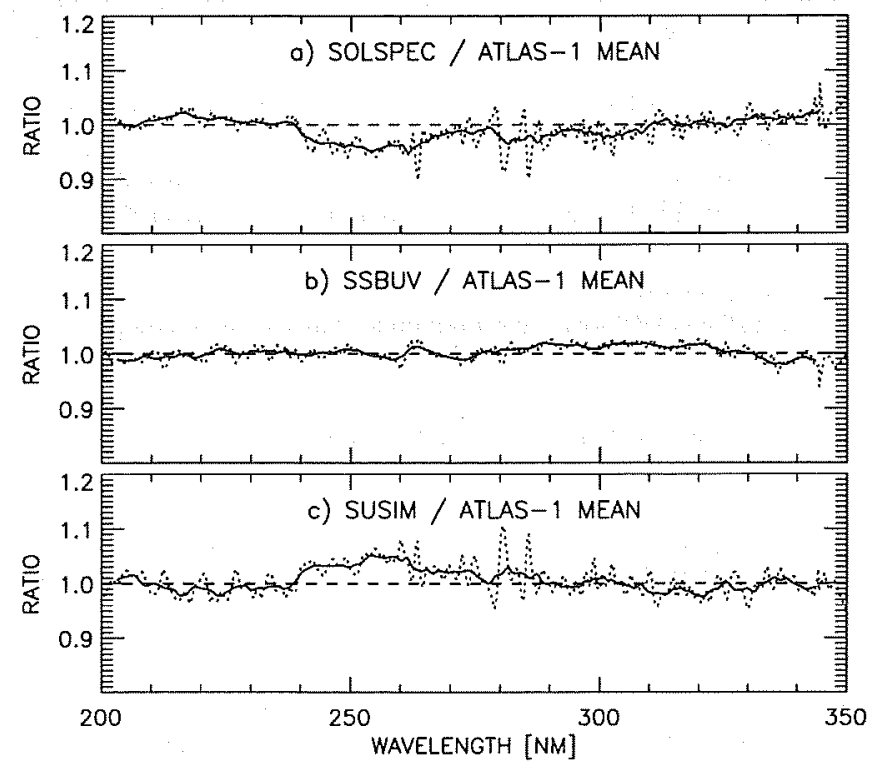

Figure 2. Ratio of the individual spectra to the mean ATLAS-1 spectrum: a) SOLSPEC/ATLAS-1; b) SSBUV/ATLAS-1; c) SUSIM/ATLAS-1. The comparisons are presented at both $1.1 \mathrm{~nm}$ (dotted lines) and $5 \mathrm{~nm}$ (solid lines) resolution. 


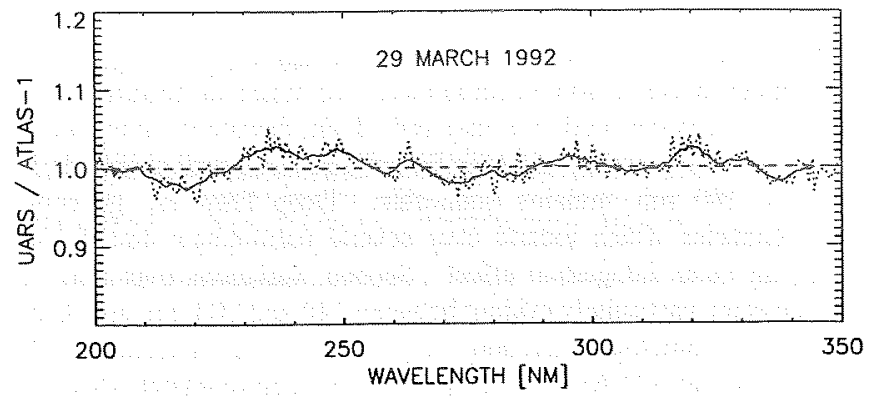

Figure 3. Ratio of the mean UARS spectrum [Woods et al., 1996] to the mean ATLAS-1 spectrum. The comparison is presented at both $1.1 \mathrm{~nm}$ (dotted line) and $5 \mathrm{~nm}$ (solid line) resolution.

iterations of preliminary versions of these data. As a result of both the Round Robin and the earlier comparisons, individual biases were revealed and subsequently explained. Yet, non-negligible differences remain. The comparison shown in Fig. 2 represents the best agreement to date among three simultaneous, yet independent middle UV solar spectral irradiance measurements. These results suggest that the middle UV solar spectral irradiance can now be measured by independent instruments to an absolute accuracy on the order of $\pm 5 \%$.

\section{Comparison of the mean ATLAS-1 and UARS spectra}

The UARS SOLSTICE and UARS SUSIM instruments also measured the solar spectral irradiance on 29 March 1992. A mean UARS spectrum and its validation using SSBUV and SUSIM on ATLAS-1 and -2, is presented by Woods et al. [1996]. The ratio between the mean UARS and the mean ATLAS-1 spectra is shown in Fig. 3 at both $1.1 \mathrm{~nm}$ and $5 \mathrm{~nm}$ resolution. As expected, the effect of the Fraunhofer lines is still present (although reduced in magnitude) in the $1.1 \mathrm{~nm}$ comparison. Considering the $5 \mathrm{~nm}$ resolution only, the discrepancies are now within $\pm 3 \%$, which is less than the uncertainty in any one instrument's measurement of the middle UV solar spectral irradiance. Between $200 \mathrm{~nm}$ and $350 \mathrm{~nm}$ the mean ratio of UARS solar irradiance to the ATLAS- 1 solar irradiance is $1.0014 \pm 0.0020$ $(2 \sigma)$ and no spectral bias is observed. Note that small wavelength errors and differences in slit function have little impact on the $5 \mathrm{~nm}$ comparison. This is expected since the mean spectra from the two missions, ATLAS-1 and UARS, tend to average out instrument-toinstrument differences in wavelength assignment and bandpass.

The comparison between the individual ATLAS-1 spectra shows agreement within the calibration uncertainties. Three instruments of different design, calibrated with different techniques using different standards, provided consistent results among themselves and with respect to the UARS solar measurements. The excellent comparisons among instruments on one space platform (ATLAS) and among instruments on another platform (UARS) indicate that the middle UV solar spectral irradiance has now been measured with an absolute accuracy better than $\pm 5 \%$.

Acknowledgements. We acknowledge the dedicated efforts of the SOLSPEC, SSBUV, and SUSIM experiment teams and the flight and ground crews of ATLAS-1 for their excellent support. We also tharik the UARS SOLSTICE and UARS SUSIM experiment teams for both the use of their data and many useful discussions. The SOLSPEC investigation was also supported by the Centre National d'Etudes Spatiales (France), the Departement de la Recherche Scientifique du Ministere de l'Education Nationale (Belgium), the
Bundesministerium fur Forschung und Technologie (Germany). The ATLAS SUSIM experiment is supported by NASA DRP H-27297B. R. P. Cebula is supported by NASA contract NAS5-31755 and NASA grant NASW-4864. The mean ATLAS-1 solar spectrum can be accessed via the World Wide Web at http://wwwsolar.nrl.navy.mil/susim.html.

\section{References}

Anderson, G.P., and L. A. Hall, Solar irradiance between 2000 and 3100 Angstroms with spectral band pass of 1.0 Angstroms, J. Geophys. Res. 54, 6435-6441,1989.

Brueckner, G.E, et al., The Solar Ultraviolet Spectral Irradiance Monitor (SUSIM) on board the Upper Atmospheric Research Satellite (UARS), $J$. Geophys. Res., 98, 10695-10711, 1993.

Cebula, R.P., et al., Calibration of the Shuttle Borne Solar Backscatter Ultraviolet Spectrometer, Proc. SPIE, 1109, 205-218, 1989.

Cebula, R.P., et al., The SSBUV experiment wavelength scale and stability: 1988 to 1994, Metrologia, in press, 1996.

Cebula, R.P., et al., Middle ultraviolet solar spectral irradiance measurements, 1985-1992, from the SBUV/2 and SSBUV instruments, in The Sun as Variable Star: Solar and Stellar Variations, edited by J.M. Pap et al., pp. 81-88, Cambridge University Press, Cambridge, England, 1994.

Hilsenrath, E., et al., Implications of Space Shuttle flight on the calibration of instruments observing atmospheric ozone and the solar irradiance, Metrologia, 28, 301-308, 1991.

Hilsenrath, E., et al, Calibration and radiometric stability of the Shuttle Solar Backscatter Ultraviolet (SSBUV) experiment, Metrologia, 30, 243-248, 1993.

Kurucz, R.L., et al., National Solar Observatory Atlas No. 1: Solar Flux Atlas from 296 to $1300 \mathrm{~nm}$, NSO, Sunspot, NM, 1984.

Labs, D., et al., Ultraviolet solar irradiance measurement from 200 to $358 \mathrm{~nm}$ during Spacelab 1 Mission., Solar Phys., 107, 203-219, 1987.

Lean, J., Variations in the Sun's radiative output, Rev. Geophys, 29, 839-868, 1991.

Moe, O.K., et al., $A$ spectral atlas of the sun between 1175 and 2100 angstroms, NRL Report 8056, NRL, Washington, DC, 1976.

Mount, G., and G. Rottman, Solar absolute spectral irradiance $118-300 \mathrm{~nm}$ : July 25 1983, J. Geophys. Res., 90, 13031-13036, 1985.

Nicolet, M., Solar spectral irradiances with their diversity between 120 and 900 nm, Planet. Space Sci., 37, 1249-1289, 1989.

Rottman, G.J.; et al., Solar Stellar Irradiance Comparison Experiment 1: 1. instrument design and operation, J. Geophys. Res., 98, 10667-10678, 1993.

Saloman, E.B., et al., Vacuum-ultraviolet and extreme ultraviolet radiometry using synchrotron radiation at the National Bureau of Standards, Opt. Eng., $21(5), 951,1982$.

Thuillier, G., et al., An instrument to measure the solar spectrum from 170 to $3200 \mathrm{~nm}$ on board SpaceLab, Solar Phys., 74, 531, 1981.

VanHoosier, M.E., et al., Absolute solar spectral irradiance $120 \mathrm{~nm}-410 \mathrm{~nm}$ (results from the Solar Ultraviolet Spectral Irradiance Monitor - SUSIM Experiment onboard Spacelab 2), Astrophys. Lett. Commun., 27, 163, 1988.

Walker, J.H., et al., Spectral Irradiance Calibrations, NBS Special Publication 250-20, 37 pp.,U.S. Department of Commerce/National Bureau of Standards, Gaithersburg, MD, 1987.

Woods, T.N., et al., Validation of the UARS solar ultraviolet irradiances: comparison with the ATLAS-1, -2 measurements, $J$. Geophys. Res., in press, 1996.

R. P. Cebula, Hughes STX Corporation, 7701 Greenbelt Road, Greenbelt, MD 20770 (email: cebula@ssbuv.gsfc.nasa.gov)

G.E. Brueckner and M. E. VanHoosier, Naval Research Laboratory, Washington, DC 20375

M. Herse and G. O. Thuillier, Service d'Aeronomie du CNRS, Verrieres le Buisson, France 91371

E. Hilsenrath, Code 916, NASA/GSFC, Greenbelt, MD 20771

P. Simon, Institut d'Aeronomie Spatiale de Belgique, B1180 Bruxelles, Belgium

(Received September 27, 1995; revised March 21, 1996; accepted April 4, 1996) 


\title{
SOLCON solar constant observations from the ATLAS missions
}

\author{
Dominique Crommelynck and Alain Fichot \\ Institut Royal Météorologique de Belgique \\ Vicente Domingo \\ Space Science Department, European Space Agency, The Netherlands \\ Robert Lee III \\ NASA Langley Research Center, Hampton, MD
}

\begin{abstract}
The solar constant observations obtained by the SOLCON/ATLAS experiment during the three successive missions are presented based on the Space Absolute Radiometric Reference (SARR) defined during the ATLAS-2 mission. The objectives of SOLCON, namely to obtain accurate measurements of the solar constant and to compare them with the observations obtained from free flyers in the hope of establishing a baseline and strategy for monitoring the solar constant at climate scale, have been achieved successfully with the three ATLAS missions. The long range objective of insuring the solar constant data continuity will, however, require that an alternative approach than that of the ATLAS program be found to fly and retrieve SOLCON.
\end{abstract}

\section{Introduction}

The following results were obtained by the Belgian experiment SOLCON flown with the ATLAS/NASA program. SOLCON is part of the core payload of the ATLAS program. Its scientific objective is to measure the solar constant during each of the solar observation periods of each ATLAS mission. The solar constant is defined as the total radiated flux from the sun, on a square meter area, at the mean sun to Earth distance. In the context of potential "global climate change", it is well recognized that the knowledge of the long term behavior of the solar constant is of basic importance.

To obtain this knowledge, it is necessary that measurements be performed continuously with the highest feasible absolute accuracy. However, solar irradiance continuous and drift free measurements can not be really guaranteed on a long term basis due to possible program budget problems and the susceptibility of the experiment to aging in space.

Therefore, the rational strategy to follow is regular, if not periodic, comparisons of the observations obtained from free flyers with those from retrieved absolute radiometers like SOLCON and SOVA 1, which carry the Space Absolute Radiometric Reference (SARR) heritage. This is what has been done with the ATLAS missions from the space shuttle.

\section{The SOLCON Instrument}

The mass of the SOLCON experiment is $9.962 \mathrm{~kg}$. Its power consumption is $13.6 \mathrm{~W}$ and its data rate is 60 bits per second.

Copyright 1996 by the American Geophysical Union.

Paper number 96GL01878

0094-8534/96/96GL-01878\$05.00
It is composed of two parts : the digital processing unit (DPU) providing the interface to the ATLAS pallet and the absolute radiometer with its electronics (see Figure 1).

SOLCON is a differential absolute radiometer with two channels. The incident radiative energy absorbed in a cavity is compared to electrical energy dissipated by the Joule effect, taking into account the nonequivalence of energies, as well as the geometric, thermal, optic and electrical characteristics of the instrument.

When the SOLCON radiometer is pointed toward the sun, one shutter of the cavity is opened. The heat balance system compensates for the added heat until the heat fluxes are again balanced between the open and closed cavities. The shutter is then closed, and power is adjusted to its original value automatically. The difference in the power required to maintain a heat balance between the two cavities during open and closed operations is a function of total solar radiation.

The SOLCON/DIARAD radiometer for the ATLAS missions is identical to the SOVA 1/DIARAD radiometer flown on the European Retrievable Carrier (EURECA). Both instruments are improved versions of the absolute radiometer flown on SPACELAB 1. Both SOLCON and SOVA 1 have been designed to provide the ultimate radiometric accuracy consistent with the state of the art metrological usage. The measurement philosophy is primarily based on :

a) the differential design of the radiometer

b) the independent characterization of each channel

c) the continuous electrical calibration interwaved with the solar measurements, with a high, accurate and stable voltage reference. d) the verification of the internal coherence of the measurements such as the accurate retrieval of the heaters resistance.

SOLCON operates efficiently, allowing temperature changes of up to $35^{\circ} \mathrm{C}$ with the uncertainty in the absolute accuracy of the solar constant measurement evaluated to be less than $\pm 0.1 \%$. The data resolution is 22 bits.

\section{The Results Obtained during the ATLAS Missions}

During the first ATLAS mission in March 1992, at the middle of the descending part of solar cycle 22 , the sun was quite active and a cluster of sunspots passed over the solar disk from one limb to the other. The data obtained on the two successive solar observation periods of days 85 and 89 show a difference of more than $1.5 \mathrm{Wm}^{-2}$, which is more than $0.1 \%$ variance.

The SOLCON left and right channel measurements of the differential absolute radiometer, characterized independently, showed a difference of $0.06 \%$ consistent with the level of uncertainty on the evaluated absolute accuracy of SOLCON. 


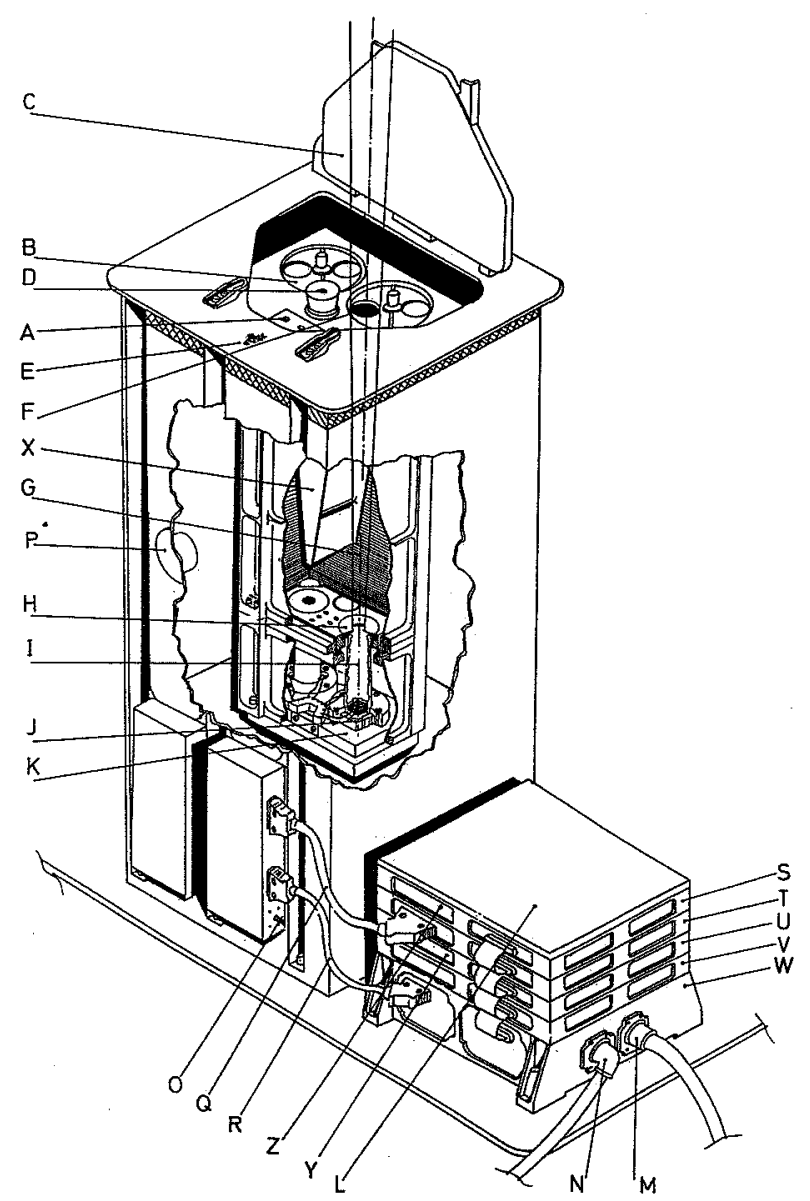

spacecrafts as well as the European Retrievable Carrier (EURECA) were carrying six other radiometers to measure the solar irradiance, representing the first time that such a space radiometric comparison has been performed. Compared to usual ground radiometric comparison conditions at high altitude sites like Table Mountain Observatory (TMO) or WRCD/Davos (CH), the solar source target was much more stable in the absence of circumsolar sky radiation and variable absorption of the atmosphere.

A mean solar constant was calculated at $1366.22 \mathrm{Wm}^{-2}$ and for each radiometric channel an "adjustment factor" (see Table 1) was determined, realizing de facto the definition of the SARR.

The Adjustment Factor (or multiplication factor) given for each instrument enables all the data to be reduced to the SARR. The resulting Solar constant value provides the state of the art accuracy.

During the third ATLAS mission in October 1994, the sun was again very quiet and the solar irradiance very stable. Over the

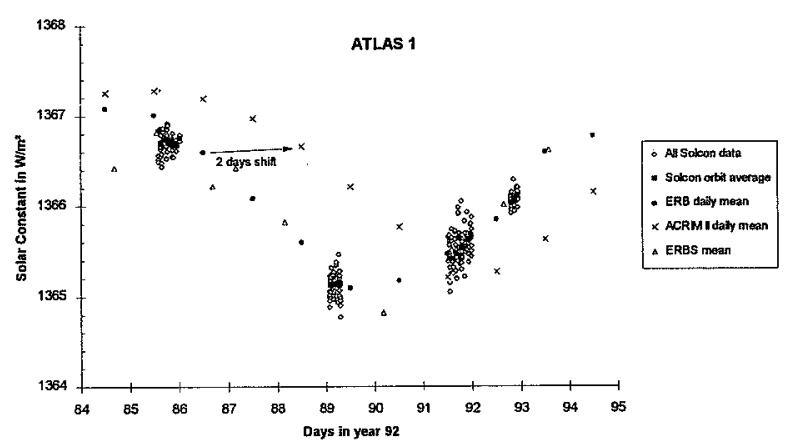

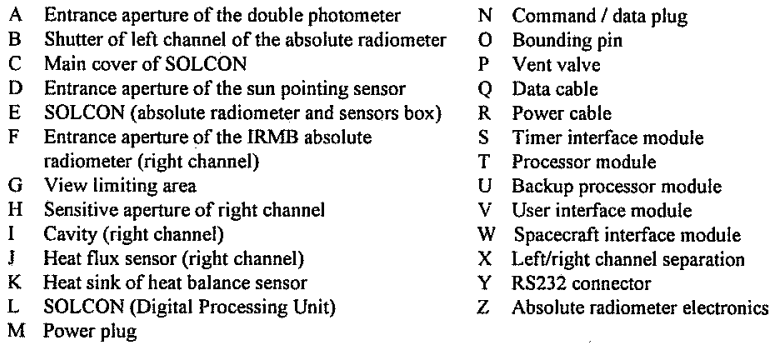

Figure 1. SOLCON radiometer and DPU

On days 91 and 92 , the irradiance values increased as the sunspots were reaching the solar limb. The situation encountered during this mission is very favorable for the detection of time stamp errors in databases. This is indeed illustrated with the ACRIM II daily mean observations, in which a two day shift is identified (see Figure 2) (Data can be acquired from the jpl.simdac anonymous ftp server, April 14th, 1995, file).

During the second ATLAS mission in April, 1993, the Sun was very quiet and the Solar irradiance very stable. Very few to no sunspots were observed.

Over the whole ATLAS-2 mission, the measured mean solar constant of each of the five solar observation periods did not change more than $0.3 \mathrm{Wm}^{-2}$. During the first mission, meanwhile, the dispersion during each orbit was of the order of $0.5 \mathrm{Wm}^{-2}$. This difference is mainly due to solar noise as the SOLCON instrument noise is about one order of magnitude smaller.

Of particular significance to the ATLAS-2 mission is the fact that simultaneously to ACR and SOLCON, the UARS and ERBS
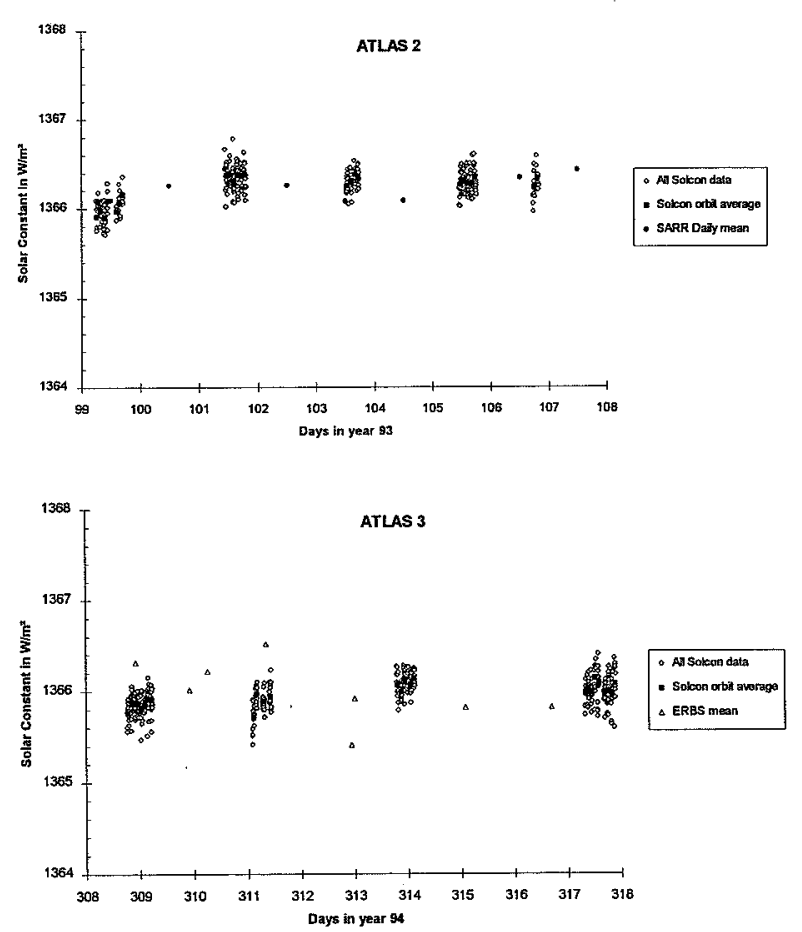

Figure 2. Compared results obtained by the SOLCON experiment during the three successive ATLAS missions. All the data shown are reduced to the SARR scale by application of the ad hoc adjustment coefficient to the data of ACRIM II, ERB, ERBS and the SOLCON left and right radiometric channels. A shift of two days is found in the ACRIM II database. 
Table 1. "Adjustment Factors" for the radiometers participating de facto in the space radiometric comparison realized during the ATLAS-2 mission

\begin{tabular}{lc}
\hline Experiments & $\begin{array}{c}\text { Adjustment factor to } \\
\text { MEAN }\end{array}$ \\
\hline SOVA1-L (EURECA/IRMB) & 1.000819 \\
ERBS (LARC) & 1.000453 \\
ACRIM II (UARS/JPL) & 1.000375 \\
SOVA2a (EURECA/PMOD) & 0.999691 \\
SOVA2b (EURECA/PMOD) & 0.999830 \\
SOLCON R (ATLAS/IRMB) & 0.999823 \\
SOVA1-R (EURECAJIRMB) & 0.999781 \\
SOLCON L (ATLAS/IRMB) & 0.999228 \\
\multicolumn{1}{c}{ Operational during ATLAS-2, identified as outlayers } \\
ACR502 (ATLAS/JPL) & 0.994893 \\
ACR504 (ATLAS/JPL) & 0.994423 \\
\hline NOte that for the eight radiometers, the dispersion is less than $+0.1 \%$
\end{tabular}

whole mission, the measured mean solar constant of each of the four solar periods did not change more than $0.4 \mathrm{Wm}^{-2}$.

Compared to the previous mission, the solar constant during ATLAS-3 was about $0.3 \mathrm{Wm}^{-2}$ lower than one would expect in the descending phase of activity of solar cycle 22 .

With the results of SOLCON during ATLAS-3, we could validate the actual Earth Radiation Budget Satellite/Solar Monitor (ERBS/SM) accuracy by using its SARR 1.000453 adjustment factor.

Indeed we find that some ERBS/SM values agree very well within $0.2 \mathrm{Wm}^{-2}$ but that some others are scattered by $\pm 0.6 \mathrm{Wm}^{-2}$. This is due probably either to the effect of solar noise undersampled or to some intrinsic ERBS/SM noise. The same verification will be done later on the ACRIM II and the ACR instruments flown on ATLAS when the data are made available.

During the ATLAS-3 mission, an unscheduled experiment with SOLCON pointing successively into the shuttle velocity vector and free space provided a unique measurement of the density of air around the shuttle.

With the assumption that the conversion factor of the kinetic energy of particles to heat is 0.4 , we found a density of $5.10^{-12} \mathrm{~kg}$ $\mathrm{m}^{-3}$ at $295 \mathrm{~km}$ altitude on 11/11/1994 at 18H GMT day time of the ATLAS-3 orbit in agreement with the current air density models (Hedin A.E., 1991).

This result shows that radiometers measuring the solar constant and aiming in the velocity vector will be affected by an error function of the air density. It demonstrates also that a SOLCON radiometer-like instrument could be designed specially for the purpose of air density measurements. This instrument would be useful for monitoring the flight conditions of the space station.

\section{Conclusions}

The objectives of experiment SOLCON have been fully accomplished during the three ATLAS missions.

Particularly noteworthy is the realization and the definition of the Space Absolute Radiometric Reference (SARR). Further experiments with retrievable instruments such as SOLCON and SOVA 1 , will maintain state of the art accuracy and long range continuity at climate-change time scale of the solar constant monitoring.

It is hoped, for example, that SOLCON will be able to fly over the $\mathrm{SOHO}$ satellite that carries solar constant instrumentation also designed by the SOLCON/ATLAS Principal Investigator and sharing the data acquisition system of the experiment VIRGO.

\section{References}

Crommelynck D., Fichot A., Lee III R.B. and Romero J. , First realisation of the space absolute radiometric reference (SARR) during the ATLAS 2 flight period, Advances in Space Research, Vol. 16, $n^{\circ} 8$, $\mathrm{pp}(8) 17-(8) 23,1995$.

Crommelynck D., Domingo V., Barkstrom B, Lee III R.B, Donaldson J. Telljohann U., Warren L. and Fichot A., Preliminary results of Solar Constant Observations with the SOLCON experiment on ATLAS 1, Advances in Space Research, Vol. 14, $\mathrm{n}^{\circ}$ 9, pp (9)253-(9)262, COSPAR, 1994

Crommelynck D., Domingo V., Fichot A. and Lee III R.B., Total Solar Irradiance Observations from the EURECA and ATLAS Experiments, The Sun as a Variable Star : Solar and Stellar Irradiance Variations, Proc. IAU Col. 143, Cambridge Univ. Press, pp 63-69, 1994.

Crommelynck D., Domingo V., Fichot A., Fröhlich C., Penelle B., Romero J. and Wherli Ch., Preliminary Results from the SOVA experiment on board the European Retrievable Carrier (EURECA) Metrologia, 30, pp 372-379, 1993.

Crommelynck D., Fundamentals of absolute pyrheliometry and objective characterisation, Earth Radiation Science Seminars, Lectures from a series of seminars held at Langley Research Center, Hampton, NASA Conference Publication 2239, pp 53-88, 1982.

Crommelynck D., Brusa R.W. and Domingo V., Results of the solar constant experiment onboard SPACELAB 1, Solar Physics 107, ISSN0038-0938, pages 1-9, 1986.

Heding A.E., Extension of the MSIS Thermosphere Model into the middle and lower atmosphere, J. Geophys. Res., 96, 1159-1172, 1991.

D. Crommelynck and A. Fichot, Royal Meteorological Institute of Belgium, Department Aerology, 3, avenue Circulaire, B -1180 Bruxelles. (e-mail:dcr@oma.be)

V. Domingo, European Space Agency, Space Science Department, ESTEC, SC, Keplerlaan 1, Postbus 299, 2200 Noordwijk AG, The Netherlands. (e-mail : vdomingo@vmprofs.estec.esa.nl)

R. B. Lee III, Langley Research Center/NASA, Mail stop 420, Hampton, VA 23665-5679, USA (e-mail : lee@rblee.larc.nasa.gov).

(Received October 12, 1995; revised May 17, 1996; accepted May 28, 1996.) 


\section{Page intentionally left blank}




\title{
Observations of the lunar geometric albedo during the ATLAS-3 mission
}

\author{
S. J. Janz \\ International Development and Energy Associates Inc., Beltsville, MD \\ E. Hilsenrath \\ Code 916, NASA Goddard Space Flight Center, Greenbelt, MD
}

\author{
R. P. Cebula and T. J. Kelly
}

Hughes STX Corporation, Greenbelt, MD

\begin{abstract}
During the ATLAS-3 mission on the Space Shuttle Atlantis (STS-66) in November of 1994, a unique opportunity arose that enabled the solar-viewing instruments to observe ultraviolet radiation from the Moon. During these observations the Moon was at a phase angle of $51.8^{\circ}$. Measurements by the Shuttle Solar Backscatter Ultraviolet (SSBUV) instrument indicate lunar geometric albedo values, after correction to full moon, of $4.4 \%-8.3 \%$ in the wavelength range from $250 \mathrm{~nm}$ to $400 \mathrm{~nm}$. These results are in good agreement with past measurements.
\end{abstract}

\section{Introduction}

Beginning with measurements of the lunar phase function, which describes the amount of reflected light as a function of lunar phase angle relative to the full Moon, at mean wavelengths of $550 \mathrm{~nm}$ [Russell, 1916] and $445 \mathrm{~nm}$ [Rougier, 1934; Rougier, 1937], significant work has been performed dealing with the radiometric properties of the Moon. During the 1950's, 1960's and early 1970's there were many ground-based and rocket measurements of the lunar phase function. A compilation of these results can be found in Lucke et al. [1976]. During this time several advances were made in the understanding of lunar reflective properties, including detailed ground-based measurements on the wavelength dependence of the phase function and geometric albedo [Lane et al., 1973], comparing lunar dust sample reflectivity measurements from Apollo 11, 12, and 14 to lunar reflectivity measured during Apollo 17 [Lucke et al., 1973; Lucke et al., 1976], and extending measurements into the far-ultraviolet [Lucke et al., 1976]. A discussion of the theoretical photometric function of the Moon can be found in Hapke $[1963 ; 1966]$ and comprehensive literature reviews on the photometry of the moon may be found elsewhere [Minnaert, 1961; Hapke, 1971].

The geometric albedo of the Moon is defined as the ratio, at a phase angle of zero, of the Moon's radiance to the incident solar irradiance, divided by the ratio one would measure if the Moon were a Lambertian reflector. The phase angle of the Moon is the angle between the Sun and the observer as seen from the Moon. The albedo is of interest in interpreting observations of other

\section{Copyright 1996 by the American Geophysical Union.}

Paper number 96GL01122

0094-8534/96/96GL-01122\$05.00
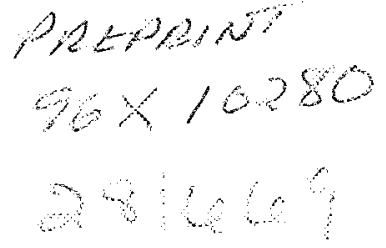

$+2$ planets and asteroids and can also be used directly for the calibration of orbiting satellites, as proposed for the Global Ozone Monitoring Experiment (GOME) [Vuorilehto et al., 1994]. The Earth Observing System (EOS) platform will also contain instruments which plan on using the Moon as a calibration target, including the Advanced Spaceborne Thermal Emission and Reflectance Radiometer (ASTER), and the Moderate Resolution Imaging Spectroradiometer (MODIS).

In this paper we present measurements of the lunar geometric albedo in the ultraviolet, taken at one phase angle and then corrected to zero phase, and compare these results with previous measurements. These measurements are unique in the fact that the solar irradiance used to calculate the geometric albedo of the Moon was measured during the orbit immediately following the lunar observations. They also provide additional information in the region below $300 \mathrm{~nm}$ where ground-based observations are not possible. Among the solar-viewing instruments participating in the third Atmospheric Laboratory for Applications and Science (ATLAS-3) mission, the Solar Constant (SOLCON) and Solar Spectrum (SOLSPEC) signal levels when viewing the Moon were beneath instrument noise levels. The Solar Ultraviolet Spectral Irradiance Monitor (SUSIM) instrument did see a statistically significant amount of lunar light [Andrews et al., 1995], however, the calibration of the instrument for this data is not yet complete.

\section{Observations and Data Reduction}

\section{The SSBUV Program}

The Shuttle Solar Backscatter Ultraviolet (SSBUV) experiment is part of a program for long term ozone monitoring of the earth's atmosphere [NOAA, 1989], and has as its primary goal the transfer of ground calibrations to orbiting satellites via coincident measurements of the Earth's ultraviolet albedo. These periodic checks provide benchmarks for determining in-flight instrument degradation of the optics and electronics of a series of SBUV/2 instruments on the NOAA polar orbiting satellites. SSBUV has also participated in the three ATLAS missions [Miller et al., 1994; see overview in this issue].

The SSBUV instrument has a spectral range of $160 \mathrm{~nm}$ to 405 $\mathrm{nm}(200 \mathrm{~nm}$ to $405 \mathrm{~nm}$ calibrated), with a $1.1 \mathrm{~nm}$ full width at half maximum (FWHM) triangular bandpass. During a typical mission the instrument will view either the Sun by deploying a quartz diffuser into the optical path, or the Earth. Details of the SSBUV program and calibration techniques are described 
elsewhere [Cebula et al., 1991; Hilsenrath et al., 1991; Hilsenrath et al., 1993; Janz et al., 1996].

The SSBUV instrument is designed to measure extended sources of uniform radiance. When viewing the Sun a quartz diffuser is deployed to minimize the effect of field-of-view (FOV) non-uniformities on the measurement. Due to the low signal levels expected, SSBUV could not view the Moon through the diffuser so data were collected in the Earth-view mode. The instrument was set to its discrete sample mode which scans a set of 12 wavelengths with an integration time of 1.25 seconds at each wavelength. Sixteen scans were collected during the time the shuttle was pointed at the Moon and the 1-sigma -standard deviation in this data set was about $10 \%$ at $250 \mathrm{~nm}$ and $2 \%$ at $400 \mathrm{~nm}$. The lunar observations were performed during the shadowed portion of orbit 157 of the ATLAS-3 mission, with data collected between 12:55 UT and 13:13 UT on November 13, 1994. The standard deviation in the shuttle pointing angle during this time was $0.06^{\circ}$, with a measured offset of the Moon with respect to the instrument's optical axis of $-0.7^{\circ}$ in the shuttle $\mathrm{xz}$ plane, and $-0.2^{\circ}$ in the $y z$ plane. Solar irradiance measurements were collected periodically during the flight, and measurements at the lunar observation wavelengths were performed during orbit 158 with 1-sigma standard deviations less than $0.5 \%$.

\section{Geometrical Correction of the Calibration}

It is useful to define the calibration constants for the two viewing modes of the SSBUV instrument. The equation describing the instrument sensitivity in the irradiance or solar-view mode is

$$
K(\lambda)=\frac{E(\lambda) G(\theta, \varphi)}{C(\lambda)}
$$

where $E(\lambda)$ is the irradiance of a standard source of spectral irradiance, $G(\theta, \phi)$ is a goniometric factor, and $C(\lambda)$ is the instrument response in counts on one of three gain ranges. The equivalent calibration constant for the radiance or Earth-view mode, assuming normal illumination of the target, is

$$
k(\lambda)=\frac{E(\lambda) B R D F(\lambda, \theta) F(\theta, \phi)}{c(\lambda)},
$$

where $F(\theta, \phi)$ is a correction factor for instrument vignetting and off-axis illumination of the viewed target, BRDF is the bidirectional reflectance distribution function of the illuminated target, and $c(\lambda)$ the response of the instrument in counts.

Since the calibration of SSBUV in the Earth-view mode consists of illuminating an extended source which fills the $11.5^{\circ}$ degree rectangular FOV, and the Moon is under-filling this view with an angular extent of $0.504^{\circ}$, we need to calculate the difference in throughput factors between these two geometries. The throughput at the position of an aperture stop in an optical system can be defined as

$$
\Gamma=a \Omega,
$$

where $\mathrm{a}$ is the area of the aperture (entrance slit in our case), and $\Omega$ is the solid angle subtended by the source. Using (3) and assuming there are no variations in the FOV response of the instrument, the calibration parameters are modified by the ratio of solid angles subtended by the two sources. This was calculated to be 670. However, the SSBUV FOV response is not constant over the full FOV and one must account for these variations.

Using the SSBUV instrument manufacturer's measurements of the response within the FOV [Ball Aerospace, 1984], an

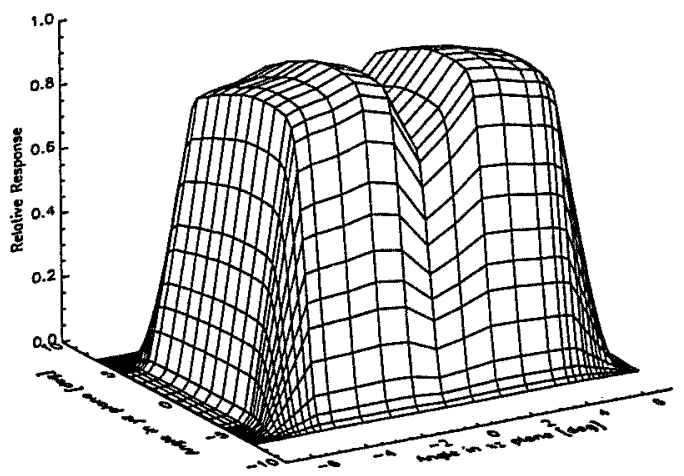

Figure 1. SSBUV measured FOV response at $1.1^{\circ}$ resolution. The $x$-axis represents the variation in FOV response in the plane defined by the optical axis of the instrument and the short dimension of the instrument aperture, The $y$-axis refers to the long dimension of the instrument aperture.

effective solid angle ratio was calculated by taking the solid angle of the Moon, weighted by the average FOV response over the Moon's angular extent, and dividing that quantity by a weighted full FOV average. Since there is a significant depression in the SSBUV FOV response at the center of the field, (see Figure 1), the calibration adjustment factor is reduced by about $17 \%$ to 555 .

The primary uncertainty in the calibration adjustment factor is due to the angular resolution of the FOV measurements, since the correction factor for scenes that subtend angles smaller than $1.1^{\circ}$ could be in error. Also, there may have been shifts in the FOV response over the ten year period since these measurements were made. For this reason an alternative calibration method was developed which does not rely on the extended source calibration described by Equation (2). This method uses the ratio of the instrument response to a source with small angular extent, with and without the instrument diffuser deployed. In this mode the instrument averages the source radiation over an area in the FOV which is similar to that illuminated by the Moon. Results from both calibration methods are compared in the next two sections.

\section{Geometric Albedo Calculation}

To calculate the geometric albedo, the ratio was taken between the digital counts in the lunar observations and digital counts in the solar observations, after correction for the appropriate gain ranges, and multiplied by the solid angle correction discussed in the previous section. The calculation of the geometric albedo from the ratio takes the form [Lucke, 1976]

$$
p(\lambda)=\frac{\left(\frac{S_{m}}{S_{s}}\right) K_{a}(\lambda)}{\left(\frac{I}{\pi}\right) F_{m}(\lambda)} \frac{\Gamma_{F O V}}{\Gamma_{m \times n}},
$$

where $S_{m}$ is the lunar count value, $S_{s}$ is the solar count value, $\mathrm{K}_{\mathrm{a}}(\lambda)$ is the instrument albedo calibration, which is the ratio of Equation (2) to Equation (1), the factor $1 / \pi$ is the Lambertian factor at $0^{\circ}$, and $F_{m}(\lambda)$ is the lunar phase correction used to convert the SSBUV measurement at a viewing angle of $51.8^{\circ}$ to what would be seen from a full Moon.

Figure 2 shows calculated values of the phase function for a phase angle of $51.8^{\circ}$ based on measurements by Lane and Irvine 


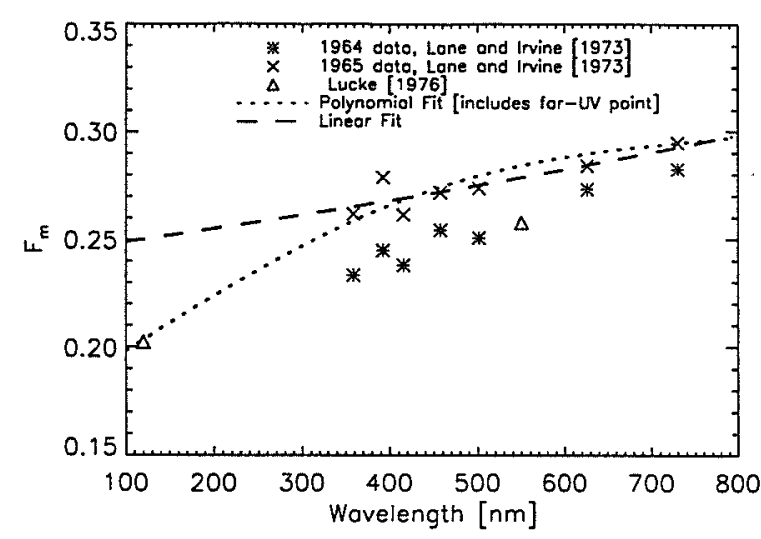

Figure 2. Phase function correction variation with wavelength, used to transform measurements at $51.8^{\circ}$ to full Moon.

[1973] and the calculations of Lucke et al. [1976]. The differences seen in the Lane and Irvine [1973] measurements between 1964 and 1965 are due primarily to the differences in lunar reflectivity between waxing and waning phases respectively. Since there are more bright features present in the waxing Moon, the reflectivity does not drop off as fast as it does during the waning portion. The data most appropriate for the SSBUV view angle are the 1965 measurements for a waxing Moon. A linear fit was used to interpolate these measurements to the SSBUV wavelengths. The theoretical wavelengths chosen to represent the visible and far-ultraviolet data points in the Lucke et al. [1976] calculations are somewhat arbitrary given the slowly varying nature of the single particle scattering function. Also, Lucke et al. [1976] argue that the far-ultraviolet point should be adjusted upward by approximately $10 \%$. For these reasons a linear fit, including only the measured points of Lane and Irvine [1973], is used to interpolate (and extrapolate) the phase function to the SSBUV wavelengths.

Using the alternative calibration approach the geometric albedo can be expressed as the ratio between lunar and solar

Table 1. SSBUV Measured Solar Irradiance, Lunar Radiance and Lunar Geometric Albedo $I_{s,} L_{R}, A$

\begin{tabular}{|c|c|c|c|c|c|}
\hline $\begin{array}{l}\lambda \\
(\mathrm{nm})\end{array}$ & $\begin{array}{l}\mathrm{I}_{\mathrm{s}} \\
\mathrm{x} 10^{-7} \\
\text { Watts } \mathrm{cm}^{-2} \\
\mathrm{~nm}^{-1}\end{array}$ & $\begin{array}{l}\mathrm{L}_{\mathrm{R}} \\
\mathrm{x} 10^{-7} \\
\text { Watts } \mathrm{cm}^{-2} \\
\mathrm{~nm}^{-1} \mathrm{ster}^{-1}\end{array}$ & $\begin{array}{l}\mathrm{L}_{\mathrm{R}}^{*} \\
\times 10^{-7} \\
\text { Watts } \mathrm{cm}^{-2} \\
\mathrm{~nm}^{-1} \mathrm{ster}^{-1}\end{array}$ & $\begin{array}{l}\text { A } \\
\%\end{array}$ & $\begin{array}{l}A^{*} \\
\%\end{array}$ \\
\hline 250.03 & 62.43 & 0.93 & 0.87 & 4.66 & 4.39 \\
\hline 258.03 & 130.74 & 1.92 & 1.88 & 4.62 & 4.52 \\
\hline 265.03 & 251.96 & 3.86 & 3.80 & 4.82 & 4.74 \\
\hline 276.97 & 265.54 & 4.33 & 4.20 & 5.13 & 4.97 \\
\hline 287.01 & 375.91 & 6.46 & 6.20 & 5.40 & 5.18 \\
\hline 299.99 & 467.44 & 8.85 & 8.40 & 5.95 & 5.65 \\
\hline 305.03 & 665.76 & 12.82 & 12.14 & 6.05 & 5.73 \\
\hline 320 & 809.77 & 16.46 & 15.59 & 6.39 & 6.05 \\
\hline 339.99 & 1072.40 & 22.67 & 22.38 & 6.64 & 6.56 \\
\hline 360.03 & 1155.18 & 28.39 & 26.37 & 7.72 & 7.17 \\
\hline 380.01 & 1137.36 & 31.06 & 28.05 & 8.58 & 7.75 \\
\hline 399.99 & 1713.25 & 50.84 & 45.38 & 9.32 & 8.32 \\
\hline
\end{tabular}

"Altemative calibration method irradiance, corrected for the angular size of the Moon and the distance between the Moon and the Shuttle. The albedo takes the form

$$
p(\lambda)=\frac{\left(\frac{S_{m}}{S_{s}}\right) K_{a}^{\prime}(\lambda) r^{2}}{F_{m}(\lambda)},
$$

where $r$ is the tangent of the angle subtended by the lunar radius at the position of the Shuttle, and $\mathrm{K}_{\mathrm{a}}^{\prime}(\lambda)$ is the modified albedo calibration constant. The modified albedo constant is the ratio of instrument counts viewing a source that has a similar angular extent compared with the Moon, with and without the instrument diffuser in the optical path. The two sources used were a quartz-tungsten-halogen (QTH) lamp and a deuterium lamp. The angular sizes of these sources was measured by scanning the source across the depression in the SSBUV instrument's FOV and calculating the resultant FWHM of the response. The result was $0.6^{\circ}$ for the deuterium lamp and $0.9^{\circ}$ for the QTH lamp. In calculating $\mathrm{K}_{\mathrm{a}}^{\prime}$ an average of six lamps (three QTH and three deuterium) was used with a standard deviation of $2.5 \%$. With Equations (1), (2), (4), and (5) we can calculate the solar irradiance, lunar radiance and lunar geometric albedo, using both calibration techniques. These values are given in Table 1 .

\section{Comparison with Previous Results}

A comparison of the SSBUV albedo values with previous measurements is presented in Figure 3. The dashed and dotted line in the figure is the fit that appears in Lucke [1976] to a variety of measurements including ground-based, rocket, and Apollo 17 measurements of the lunar albedo, and measurements of the reflectivity of lunar dust samples [Lucke, 1973].

The SSBUV values using both calibration methods agree with the linear fit given by Lucke [1976] to an average over this wavelength region of $10 \%$, with the alternative calibration approach yielding better agreement. The alternative calibration method yields values that are on average $2 \%$ higher than the reference fit. Previously reported values varied by as much as $50 \%$ relative to the reference fit. The FOV response method appears to incorrectly account for the wavelength dependence and overestimates the correction, especially toward longer wavelengths.

We believe the alternative calibration approach is superior for two reasons. First, because the angular size of the calibration

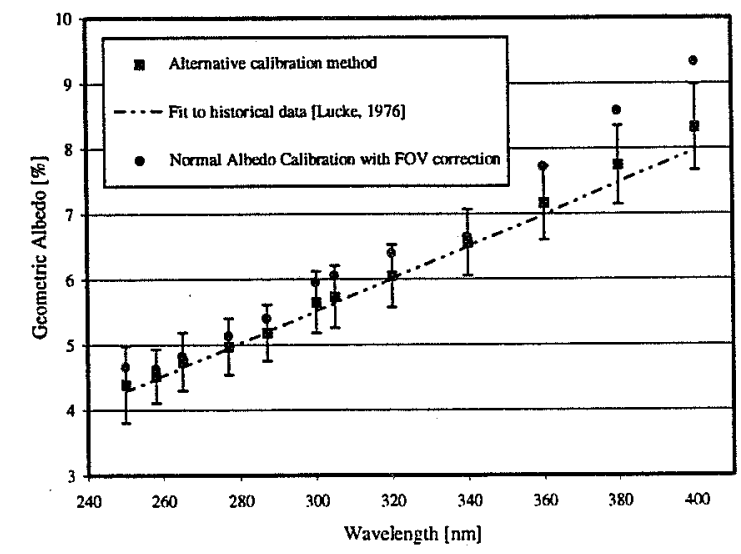

Figure 3. Geometric Albedo as measured by SSBUV using two calibration methods, compared with historical values. 
source is identical for the two instrument viewing modes during the calibration, no correction for the angular size of the Moon relative to an extended source is needed. The only correction required is to compensate for how the instrument averages the calibration source over variations in the FOV response relative to the Moon, at its position within the FOV. Using the FOV response measured with the QTH and deuterium lamps, this correction was calculated to be $21 \%$ due to the fact that the Moon was not viewed directly at the center of the FOV. Second, although the FOV response was measured at various wavelengths with little or no wavelength dependence observed, the resolution of this measurement could wash out such an effect for small angular sources. However, the alternative calibration method should correctly incorporate any wavelength dependence for sources of a similar size.

There are three main contributions to the uncertainties in the SSBUV measurements using the FOV calibration method. First, the correction due to the differing angular size of the Moon versus the calibrated view when the full instrument FOV. is illuminated. This error is estimated to be about $5 \%$ based on variations in the method used to integrate the function used for the FOV response (see Figure 1). The error could be larger if the FOV response of the instrument has changed since the time of the original measurements. Second, uncertainties in the exact position of the Moon within the FOV lead to an uncertainty in the adjustment of the calibration on the order of $5 \%$. Third, there is some uncertainty in the phase correction function, more so at the shorter wavelengths where we have extrapolated from previous measurements. If we use the standard deviation of the linear fit to the phase data in Figure 3 , this uncertainty is estimated to be $2 \%$ between $350 \mathrm{~nm}$ and $400 \mathrm{~nm}$ and possibly larger for wavelengths below $350 \mathrm{~nm}$ (The 1965 data points have an estimated uncertainty of about 5\% [Lane and Irvine, 1973]). We have eliminated the first uncertainty by using the alternative calibration approach. In addition the alternative approach removes any uncertainty in the measurement of the reflectivity (BRDF) of the extended source (see Equation (2)). The primary error sources remaining are due to the lunar position and phase correction. These are represented by the error bars in Figure 3 .

\section{Conclusions}

The lunar geometric albedo measured by the SSBUV instrument during the ATLAS-3 mission agrees to within $2 \%$ on average with the best fit to historical values. These measurements have provided additional data for the ultraviolet region between $250 \mathrm{~nm}$ and $350 \mathrm{~nm}$ with improved accuracy due to the contiguous measurement of the solar flux with the same instrument. We also presented calibrated lunar radiance measurements which should prove useful in estimating expected signal-to-noise levels for instruments that will use the Moon as a calibration source. On the most recent flight of SSBUV (STS-72) we were able to repeat these measurements at a phase angle near $90^{\circ}$ and at additional wavelengths. Results from this flight will be reported at a future time.

Acknowledgments. The authors wish to acknowledge all of the participants of the ATLAS-3 mission for the stimulating, collaborative atmosphere that was present during the shuttle flight, without which these lunar measurements would not have been possible. S. J. Janz is supported by NASA contract NAS5-31729 and R. P. Cebula and T. J. Kelly are supported by NASA contract NAS5-31755. R. P. Cebula is also supported by NASA grant NASW-4864.

\section{References}

Andrews, M. D., and M. E. VanHoosier, The ultraviolet albedo of the Moon as observed by the SUSIM instrument during the ATLAS-3 mission (abstract), EOS Trans. $A G U, 76,1995$.

Ball Aerospace, Specification compliance and calibration data book for SBUV/2 engineering flight unit, Tech. Rep. B6802-78, Ball Aerospace Division, Boulder, CO, 1984.

Cebula, R. P., E. Hilsenrath, and B. Guenther, Calibration of the Shuttle Borne Solar Backscatter Ultraviolet Spectrometer, Proc. Soc. Photo. Opt. Instrum. Eng., 1109, 205, 1989.

Hapke, B. W., A theoretical photometric function for the lunar surface, $J$. Geophys. Res., 68, 4571, 1963.

Hapke, B. W., An improved theoretical lunar photometric function, Astron. J., 71, 333, 1966.

Hapke, B. W., Physics and Astronomy of the Moon, edited by Z. Kopal Academic, New York, 155, 1971.

Hilsenrath, E., R. P. Cebula, R. Caffrey, and S. Hynes, Implications of space shuttle flight on the calibration of instruments observing atmospheric ozone and the solar irradiance, Metrologia, 28, 301, 1991.

hilsenrath, E., D. E. Williams, R. Caffrey, R. P. Cebula, and S. Hynes, Calibration and radiometric stability of the Shuttle Solar Backscatter Ultraviolet (SSBUV) experiment, Metrologia, 30, 243, 1993.

Janz, S. J., E. Hilsenrath, J. Butler, D. F. Heath, and R. P. Cebula, Uncertainties in radiance calibrations of backscatter ultraviolet (BUV) instruments, Metrologia, in press, 1996.

Lane, A. P., and W. M. Irvine, Monochromatic phase curves and albedos for the lunar disk, Astron. J., 78, 267, 1973.

Lucke, R. L., and R. C. Henry, Far ultraviolet reflectivity of lunar dust samples: Apollo 11, 12, and 14, Astron. J., 78, 263, 1973.

Lucke, R. L., R. C. Henry, and W. G. Fastie, The far-ultraviolet albedo of the moon, Astron. J., 81, 1162, 1976.

Miller, T. L., S. A. Smith, and J. A. Kaye, ATLAS space shuttle studies Earth's atmosphere and solar input, EOS Trans. $A G U, 75,321,1994$.

Minnaert, M., Planets and Satellites, edited by G. P. Kuiper and B. M. Middlehurst, U. of Chicago Press, p. 213, 1961.

NOAA, National plan for stratospheric monitoring and early detection of change, Rep. FCM-P17-1989, U. S. Dept. of Commerce, Washington, D.C., 1989 .

Rougier, G., Photometrie globale de la Lune, $L^{\prime}$ Astronomie, 48, 220-281, 1934.

Rougier, G., L'albedo des planetes et de leurs satellites, $L^{\prime}$ Astronomie, $51,165,1937$.

Russel, H. N., The stellar magnitudes of the Sun, Moon, and planets, Astrophys. J., 43, 103, 1916.

Vuorilehto, A., and S. Korpela, Study of the Sun and Moon as radiation calibration targets, Tech. Rep. SSF-ESA-SM-0002, ESA, 1994.

R. P. Cebula, Hughes STX corporation, Suite 400, 7701 Greenbelt road, Greenbelt, MD, 20770 (email:cebula@ @sbuv.gsfc.nasa.gov)

E. Hilsenrath, Code 916, NASA GSFC, Greenbelt, MD 20771. (email: hilsen@ssbuv.gsfc.nasa.gov)

S. J. Janz, International Development and Energy Associates Inc. Code 916, Blidg. 21, rm. 257A, NASA GSFC, Greenbelt, MD 20771. (email: janz@ssbuv.gsfc.nasa.gov)

T. J. Kelly, Hughes STX corporation, Code 916, Bldg. 21, rm. C218, NASA GSFC, Greenbelt, MD 20771. (email:kelly@ssbuv.gsfc.nasa.gov)

(Received October 12, 1995; revised March 8, 1996;

accepted March 28, 1996.) 


\title{
A comparison of ozone measurements made by the ATMOS, MAS, and SSBUV instruments during ATLAS 1,2, and 3
}

\author{
D. L. Kriebel, ${ }^{1}$ R. M. Bevilacqua, ${ }^{2}$ E. Hilsenrath, ${ }^{3}$ M. Gunson, ${ }^{4}$ G.K. Hartmann, ${ }^{5}$ M. \\ Abrams, ${ }^{4}$ M. Daehler, ${ }^{2}$ T. A. Pauls, ${ }^{2}$ M. Newchurch, ${ }^{6}$ C.P. Aellig, ${ }^{2}$ M.C. Bories ${ }^{7}$
}

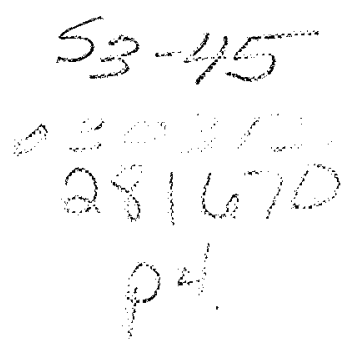

\begin{abstract}
Ozone profile measurements were made by three instruments, ATMOS, MAS, and SSBUV, using distinctly different observing techniques, as part of the ATLAS Space Shuttle missions in March 1992, April 1993, and November 1994. ATMOS makes solar-occultation observations of infrared spectra using a Fourier transform interferometer. MAS uses a limb-scanning antenna to measure emission spectra at millimeter wavelengths. SSBUV is a nadir-viewing instrument measuring the transmission of scattered solar ultraviolet radiation modified by ozone absorption. A sample of zonal-mean mixing ratio profiles indicates that these three ATLAS instruments generally agree to within $10 \%$, although a few potential biases have been noted. There are significant differences in the character of the agreement between ATLAS 1 and ATLAS 2 which will require further study.
\end{abstract}

\section{Introduction}

As part of NASA's Mission to Planet Earth program, the ATLAS remote sensing facility places particular emphasis on collecting data on constituents important in controlling the global ozone distribution. Ozone profile measurements have been conducted from the ground for nearly four decades, and from space for nearly twenty-five years with a variety of instruments and techniques. Because atmospheric ozone is a crucial environmental parameter, accurate measurements are required over the long term. Therefore, it is important that comparability between measurement techniques be well understood. Over the past decade, various satellite ozonemeasuring instruments have been intercompared with each other and with ground-based systems. In general, the agreement between datasets in the upper stratosphere is within about $5 \%$, but the variation becomes as large as $30 \%$ in the lower stratosphere (e.g. below $20 \mathrm{mb}$ ) (McPeters et al., 1994, Randel and $W u, 1995)$.

The NASA ATLAS series of spacelab shuttle missions includes the following atmospheric measurement instruments:

\footnotetext{
${ }^{1}$ Computational Physics, Inc., Fairfax, VA

2 Remote Sensing Division, Naval Research Laboratory, Washington, $D C$

${ }^{3}$ NASA Goddard Space Flight Center, Greenbelt, MD

4 Jet Propulsion Laboratory, California Institute of Technology, Pasadena, CA

${ }_{5}^{5}$ Max Planck Institute for Aeronomy, Lindau, Germany

6 Earth System Science Laboratory, University of Alabama at Huntsville, Huntsville, AL

${ }^{7}$ IDEA, Beltsville, MD
}

Copyright 1996 by the American Geophysical Union.

Paper number 96GL01024

0094-8534/96/96GL-01024\$05.00 the Atmospheric Trace Molecule Spectroscopy experiment (ATMOS), the Millimeter-wave Atmospheric Sounder (MAS), and the Shuttle Solar Backscatter Ultraviolet instrument (SSBUV). The observations made by these instruments have been designed to complement each other, with the advantage of sharing the same measurement. platform. The common thread among the three is the fact that all measure ozone. In this Letter we present a quantitative comparison of ozone measurements made by all three atmospheric instruments on board ATLAS 1,2, and 3. This is not a full validation study (such studies are in progress for each of the instruments). Rather, it represents an attempt to demonstrate the quality of the ATLAS database, and its adequacy for the scientific studies, based on ATLAS data, presented in this GRL special issue.

\section{Instruments and Coverage}

The ATMOS experiment employs a Fourier transform interferometer to measure high-resolution mid-infrared solarabsorption spectra of the atmosphere in the limb-viewing mode using solar occultation techniques [Farmer, 1987]. The vertical resolution of the ozone retrievals is $4-5 \mathrm{~km}$, and the estimated precision and accuracy are better than 4 and $10 \%$, respectively [Abrams et al., 1995].

MAS uses a 1-meter off-axis paraboloid antenna to scan the Earth's limb at millimeter wavelengths. The instrument is described in Croskey et al. [1992]. The vertical resolution of the ozone measurements is roughly $3 \mathrm{~km}$. The detailed error analysis for MAS ozone measurements is still in progress. Current estimates of the MAS uncertainties are as follows: Below $12 \mathrm{mb}$ : precision of about $7 \%$ and and accuracy of $14 \% ; 12$ to $0.2 \mathrm{mb}: 7 \%$ and $8 \%$ respectively. The details of the data analysis for MAS are described in Hartmann et al. [this issue].

The SSBUV is an SBUV/2 instrument modified for Shuttle flight [Hilsenrath et al., 1988]. Directional earth albedo is viewed from 250 to $340 \mathrm{~nm}$, and inverted to provide ozone profiles from about 55 to $20 \mathrm{~km}$ with a $7 \mathrm{~km}$ resolution [Bhartia et al., 1995]. The SSBUV precision is $1-2 \%$, and the accuracy ranges from $4 \%$ at $2 \mathrm{mb}$ to $10 \%$ at $30 \mathrm{mb}$ [Bhartia et al., 1995]. Flight-to-flight and absolute calibration errors have been discussed by Hilsenrath et al. [1991]. Stratospheric aerosols also scatter light, therefore, if not taken into account, they will also contribute an error to retrieved profiles. The ozone profile errors, resulting from stratospheric aerosol loading, are a function of aerosol optical depth and altitude, and solar zenith angle. In general, the ozone amounts are underestimated near $10 \mathrm{mb}$ and overestimated near $30 \mathrm{mb}$ by about $10 \%$ at $30^{\circ}$ solar zenith angle [Torres and Bhartia, 1995]. The contribution of this error is not taken into account in the SSBUV data presented here. 
Table 1. Latitudinal Coverage of ATMOS, MAS, and SSBUV during the ATLAS missions

\begin{tabular}{lccc} 
& ATLAS 1 & ATLAS 2 & ATLAS 3 \\
\hline ATMOS & $55^{\circ} \mathrm{S}-30^{\circ} \mathrm{N}$ & $50^{\circ} \mathrm{S}-10^{\circ} \mathrm{S} ;$ & $75^{\circ} \mathrm{S}-65^{\circ} \mathrm{S} ;$ \\
& & $65^{\circ} \mathrm{N}$ & $5^{\circ} \mathrm{N}-55^{\circ} \mathrm{N}$ \\
MAS & $40^{\circ} \mathrm{S}-70^{\circ} \mathrm{N}$ & $70^{\circ} \mathrm{S}-70^{\circ} \mathrm{N}$ & $40^{\circ} \mathrm{S}-70^{\circ} \mathrm{N}$ \\
SSBUV & $30^{\circ} \mathrm{S}-57^{\circ} \mathrm{N}$ & $57^{\circ} \mathrm{S}-57^{\circ} \mathrm{N}$ & $40^{\circ} \mathrm{S}-57^{\circ} \mathrm{N}$
\end{tabular}

Combining limb occultation, limb emission, and nadir measurements, for purposes of comparison, is complicated by the difference in spatial and temporal coverage of the instruments. ATMOS measures 32 profiles per day at specific locations determined by occultation requirements. MAS makes continuous measurements, barring other mission contingencies. The MAS data has been radiance-averaged over each mission into $5^{\circ}$ latitude bins. A zonal-mean ozone profile results from retrieval of these averaged spectra. SSBUV, being a nadir-viewing instrument, provides the greatest coverage, with roughly 420 profiles per day. These three data sets provide overlapping samples of the atmosphere from which we may attempt to ascertain the compatibility of the measurements. The latitudinal coverage of each of the instruments is indicated in Table 1. The coverage of both MAS and SSBUV is large enough to permit examination of a zonal cross-section of differences as a function of altitude/pressure and latitude. The nature of the ATMOS instrument is such that a more limited number of measurements were made. However, there were sufficient coincident data to make zonal mean comparisons at several latitudes. Therefore, two approaches are used here: zonal mean profiles within specific latitude bands are compared for all three instruments at $30^{\circ} \mathrm{S}$ (for ATLAS 1 and 2 ) and $50^{\circ} \mathrm{N}$ (for ATLAS 3), and zonal mean profiles as a function of latitude are compared for MAS and SSBUV. In all comparisons shown in this Letter, the means associated with a

ATLAS1 Ozone Zonal Mean Comparison, 30S

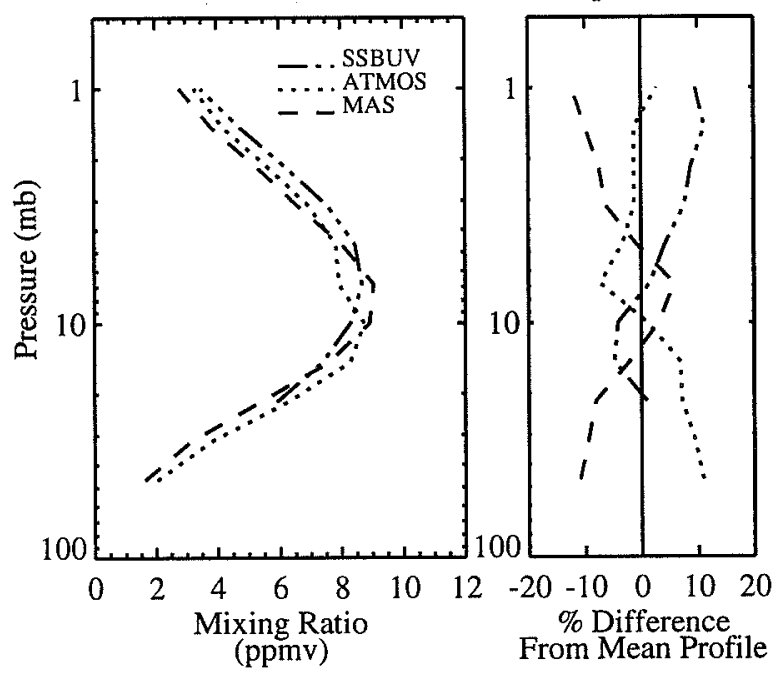

Figure 1a. Mean ozone profiles at $30 \mathrm{~S}$ latitude measured during the ATLAS 1 mission (March, 1992) by ATMOS, MAS, and SSBUV, and percentage differences from the average of the three profiles, with the same legend and pressure scale.
ATLAS2 Ozone Zonal Mean Comparison, 30S

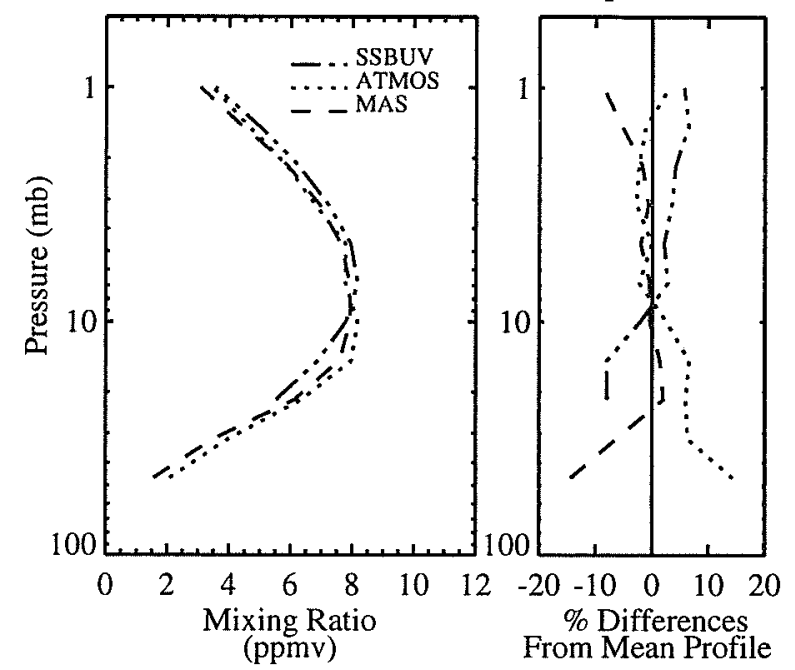

Figure 1b. Mean ozone profiles at $30 \mathrm{~S}$ latitude measured during the ATLAS 2 mission (April, 1993) by ATMOS, MAS, and SSBUV, and percentage differences from the average.

given latitude are calculated from measured profiles at locations within $\pm 5^{\circ}$ from the stated latitude.

\section{MAS / SSBUV / ATMOS Comparisons}

Figure 1a shows the ATLAS $130^{\circ} \mathrm{S}$ zonal-mean profiles over the entire mission for all three instruments, and the percentage difference between each zonal-mean profile and the average of the three. Because of large local time differences, and the diurnal variation of ozone, meaningful comparisons cannot be made above about $1 \mathrm{mb}$. Therefore, data at smaller pressures are not shown, although each dataset extends into this region. Below $20 \mathrm{mb}$, the vertical resolution

ATLAS 3 Ozone Zonal Mean Comparison, 50N

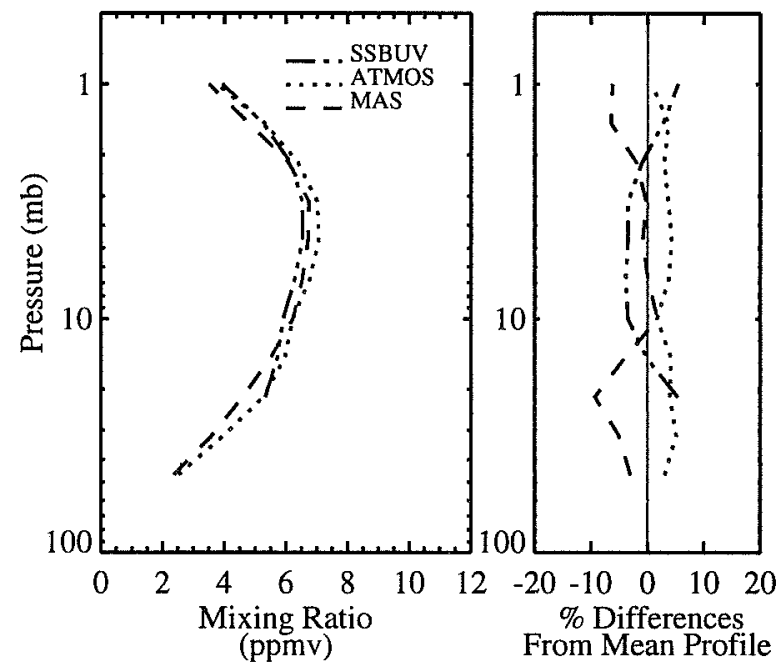

Figure 1c. Mean ozone profiles at $50 \mathrm{~N}$ latitude measured during the ATLAS 3 mission (November, 1994) by ATMOS, MAS, and SSBUV, and percentage differences from the average. 
of the SSBUV retrievals decreases rapidly; hence they are not included in the comparisons below this pressure.

In the upper stratosphere ( between about 2 to $4 \mathrm{mb}$ ), the MAS and ATMOS measurements are in fairly good agreement, within a few percent, and are both roughly $10-15 \%$ lower than SSBUV. Also, the SSBUV profile peaks at a lower pressure than the other two, which appear to peak at the same pressure. Near the ozone mixing ratio peak, the measurements from the three instruments disagree by up to $15 \%$. In the lower stratosphere, below $20 \mathrm{mb}$, MAS is about $20 \%$ lower than ATMOS. The reason for this discrepancy between MAS and ATMOS, which exists for all missions, is not clear, but comparisons with other datasets indicate that MAS is probably biased low below $20 \mathrm{mb}$ (and also near $1 \mathrm{mb}$ ).

Zonal means and percentage differences from ATLAS 2, also at $30^{\circ} \mathrm{S}$, are shown in Figure $1 \mathrm{~b}$. In general the comparisons are much better than for ATLAS 1, and all three profiles are in good agreement at the peak, with mixing ratios of about $8 \mathrm{ppmv}$. Once again, the SSBUV profile peaks at a lower pressure. In the upper stratosphere, from 2 to $10 \mathrm{mb}$, MAS and ATMOS are in excellent agreement, with less than $2 \%$ differences, while SSBUV is still somewhat higher, but within $5 \%$ of the mean. SSBUV appears to be biased low just below the peak, down to about $20 \mathrm{mb}$. MAS and ATMOS continue to agree well, within $5 \%$, from the peak down to 20 mb. Below $20 \mathrm{mb}$ MAS is again biased low relative to ATMOS, with differences reaching $20 \%$ at $50 \mathrm{mb}$.

It is noteworthy to compare the relative variations between ATLAS 1 and 2 indicated in the $30^{\circ} \mathrm{S}$ zonal average plots. At the mixing ratio peak, all three instruments observed lower ozone mixing ratios in ATLAS 2 than in ATLAS 1 by about 0.5 to $1.0 \mathrm{ppmv}$. We have also obtained data from the UARS Microwave Limb Sounder (MLS) instrument for the ATLAS1 and ATLAS 2 time periods from the Goddard Distributed Active Archive Center (DAAC) [Barath et al., 1993]. At the mixing ratio peak at $30^{\circ} \mathrm{S}$, MLS observed an ozone decrease between ATLAS 1 and 2 of 5-10\%, while the mean decrease observed by the ATLAS instruments is $7-8 \%$. In the lower stratosphere (below $20 \mathrm{mb}$ ), both the ATMOS and MAS retrieved mixing ratios are higher in ATLAS 2 than in ATLAS 1 , although the magnitude of this increase is greater in MAS than in ATMOS ( $15 \%$ compared to 5\%). MLS measurements indicate an increase of about $10 \%$, in good agreement with the ATLAS measurements. In the upper stratosphere centered on about $2 \mathrm{mb}$, higher mixing ratios are indicated in ATLAS 2 than in ATLAS 1. However, the magnitude of this increase varies significantly. In the MAS retrievals the increase was about $15 \%, 10 \%$ for ATMOS, and in SSBUV retrievals the increase is quite small $(<4 \%)$. The variation in the MLS data in this altitude region is also small ( $<5 \%)$, consistent with the SSBUV data.

ATLAS 3 zonal means and differences at $50^{\circ} \mathrm{N}$ are presented in Figure 1c. In the upper stratosphere there is agreement within about $10 \%$ from 1 to $2 \mathrm{mb}$. Down to $10 \mathrm{mb}$, ATMOS is about 5\% higher and SSBUV is about 5\% lower than MAS, which is roughly the average. Over the range of the peak, which is broad in all three cases because of the high latitude of the comparison, there are differences of $0.5 \mathrm{ppm}$.

As pointed out above, SSBUV and MAS can be compared over a much more extensive latitude range than that possible when comparing all three instruments. For this purpose, in figures $2 \mathrm{a}, \mathrm{b}$, and $\mathrm{c}$ we present contour plots of percentage zonal average differences between MAS and SSBUV for
ATLAS-1 Ozone: (MAS-SSBUV)/SSBUV \%

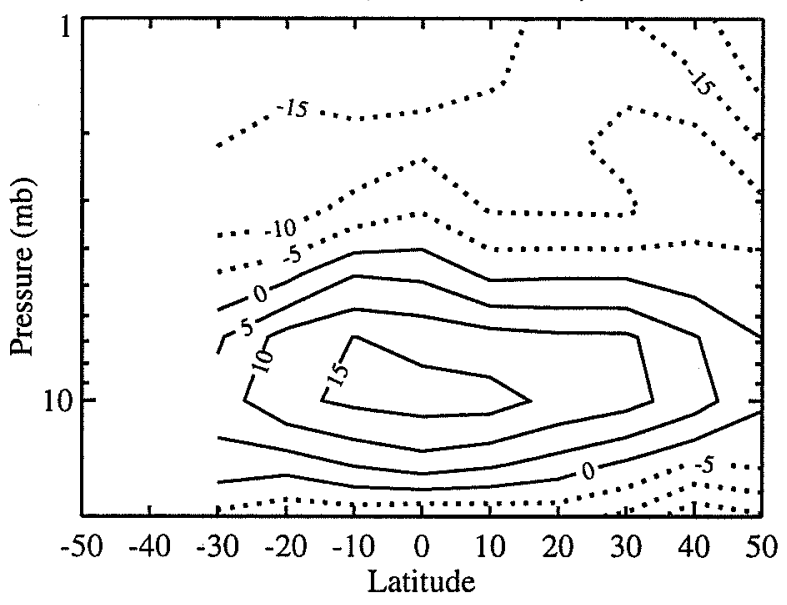

ATLAS-2 Ozone: (MAS-SSBUV)/SSBUV \%

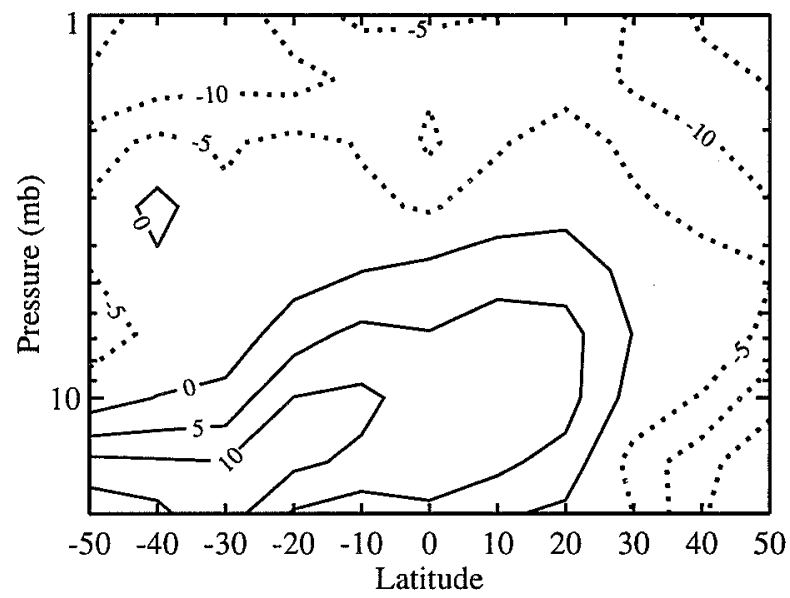

ATLAS-3 Ozone: (MAS-SSBUV)/SSBUV \%

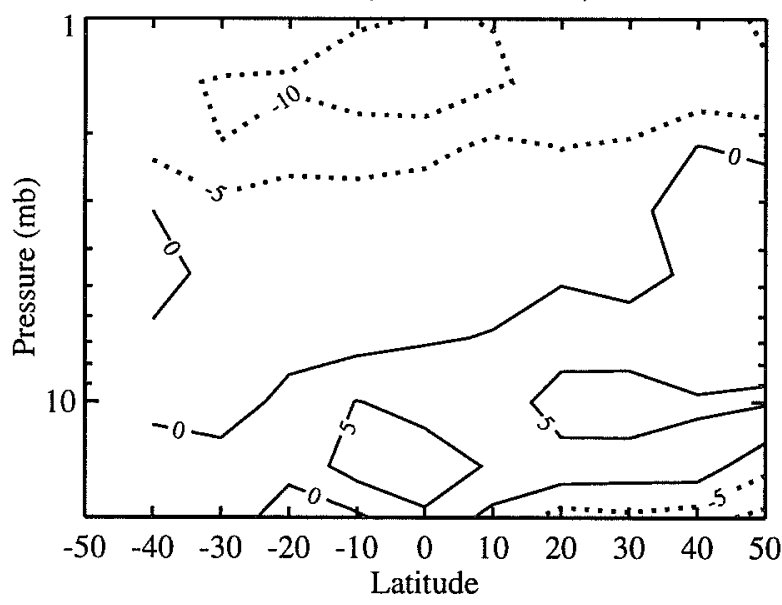

Figure 2. Contour plot of percentage differences in ozone measurements between MAS and SSBUV as a function of latitude and altitude for (a) ATLAS 1 , (b) ATLAS 2 , and (c) ATLAS 3.

ATLAS 1,2,and 3 respectively. Contours of the MAS mixing ratio values themselves, and the range of these measurements, may be found in the MAS overview Letter (Hartmann et al., this issue). In general, the contour plots show the same 
general characteristics as that indicated in the profile comparisons shown in Figure 1. The most striking feature is the significant improvement in the agreement between the two instruments in ATLAS 2 versus ATLAS 1, especially near 10 $\mathrm{mb}$ and again at $2 \mathrm{mb}$. The reason for this improvement is not completely understood currently, but in the lower stratosphere may be related to the significantly different stratospheric aerosol loading between the two missions. Because of Mt. Pinatubo, the aerosol loading over the equator was greater during ATLAS 1 than in ATLAS 2. The MAS retrievals should be largely immune to the aerosol loading environment, but, as mentioned previously, stratospheric aerosols could cause the SSBUV retrieved mixing ratios to be underestimated at $10 \mathrm{mb}$ by about $10 \%$. Therefore, stratospheric aerosols could be at least partially responsible for the large region of positive MAS-SSBUV differences centered on the equator at $10 \mathrm{mb}$. In ATLAS 2 and 3, the MAS and SSBUV retrievals agree to within $10 \%$, which is generally within the accuracy limits of the three instruments. However, even in ATLAS 2 and 3, there does appear to be a systematic positive bias in the lower stratosphere of order 510\% (MAS higher than SSBUV), and less than 5\% in the upper stratosphere.

\section{Conclusions}

In general, the comparisons between the ATLAS instruments are quite good, with the three agreeing to $\pm 10 \%$. This is generally within the combined measurement and retrieval errors. Thus, these limited comparisons have indicated that the measurements are suitable for the atmospheric studies appearing in this issue. Each of the three instruments employs a different observation technique and measures radiation in a different wavelength band, making these results particularly satisfying.

However, there are several systematic features of the comparisons which will require further study. First, the MAS ozone retrievals are consistently low below $20 \mathrm{mb}$, a discrepancy which may be due partly to differing instrumental sensitivities, but may also indicate a bias of up to $10 \%$. Second, the SSBUV retrievals appear to peak at lower pressures than the others, and to be biased low just below the peak down to $20 \mathrm{mb}$. Third, between ATLAS 1 and ATLAS 2 there seems to be a change in the nature of the agreement between the various measurements, namely, that MAS and ATMOS profiles were consistently lower than SSBUV in the mid- and upper stratosphere by about $10 \%$ during ATLAS 1 , but are in much better agreement during ATLAS 2 and 3 . In addition, at or below the peak of the ozone distribution, the MAS/SSBUV agreement is significantly better in ATLAS 2 and 3 compared to ATLAS 1, especially in the tropics. These changes may be due, in part, to differing aerosol environments during these missions.

\section{References}

Abrams, M.C., M.R. Gunson, A.Y. Chang, C.P. Rinsland, and R. Zander, Remote sensing of the earth's atmosphere from space with high resolution Fourier transform spectroscopy: Development and methodology of the data processing for the Atmospheric Tract Molecule Spectroscopy Experiment, Appl. Opt., in press, 1996.

Barath, F.T., et al., The Upper Atmosphere Research Satellite Microwave Limb Sounder instrument, J. Geophys. Res., 98, 10751-10762, 1993.

Bhartia, P.K., R.D. McPeters, C.L. Mateer, L.E. Flynn, and C. Wellemeyer, Algorithm for estimation of vertical profile from the backscatter ultraviolet (BUV) technique, J. Geophys. Res., under review, 1995.

Croskey, C. L., et al., The Millimeter-wave Atmospheric Sounder (MAS): a shuttle-based remote sensing experiment, IEEE Trans. Geosci. Remote Sensing, 40, 1090-1100,1992.

Farmer, C.B. High resolution infrared spectroscopy of the sun and the earth's atmosphere from space, Mikrochim. Acta (Wien), III, 189$214,1987$.

Hartmann, G.K., et al., Measurements of O3, $\mathrm{H} 2 \mathrm{O}$, and $\mathrm{ClO}$ in the middle atmosphere using the Millimeter-wave Atmospheric Sounder (MAS), this issue, 1996.

Filsenrath, E., D. Williams, and J. Frederick, Calibration of long-term data sets from operational satellites using the Space Shuttle, SPIE Proc., 924, 215-222, 1988.

Hilsenrath, E., R.P. Cebula, S.J. Hynes, and R.T. Caffrey, Implications of Space Shuttle flight on the calibration of instruments observing atmospheric ozone and the solar irradiance, Metrologia, 28, 301-308, 1991.

McPeters, R.D., T.Miles, L.E. Flynn, C.G. Wellemeyer, and J.M. Zawodny, Comparison of SBUV and SAGE II ozone profiles: implications for ozone trends, J. Geophys. Res., 99, 20513-20524, 1994.

Olivero, J.J., et al., Distinctive ozone structure in the high-latitude stratosphere: measurements by the Millimeter-wave Atmospheric Sounder, this issue, 1996.

Randel, W.J., and F. Wu, Climatology of stratospheric ozone based on SBUV and SBUV/2 data: 1978-1994, NCAR/TN-412+STR, 1995.

Torres, O., P.K. Bhartia, Effect of stratospheric aerosol on ozone profiles from BUV measurements, Geophys. Res. Lett., 22, 235$238,1995$.

D.L. Kriebel, Computational Physics, Inc., 2750 Prosperity Avenue, Suite 600, Fairfax, VA 22031.

R.M. Bevilacqua, M. Daehler, T.A. Pauls, and C.P. Aellig, Remote Sensing Division, Naval Research Laboratory, Washington DC 20375 E. Hilsenrath, NASA Goddard Space Flight Center, Greenbelt MD 2077

M. Gunson and M. Abrams, Jet Propulsion Laboratory, California Institute of Technology, Pasadena CA 91109

(Received September 22, 1995; revised February 16, 1996; accepted March 18, 1996) 


\title{
Ozone change from 1992 to 1993 as observed from SSBUV on the ATLAS-1 and ATLAS-2 missions
}

\author{
E. Hilsenrath ${ }^{1}$, P.A. Newman ${ }^{1}$, R.P. Cebula ${ }^{2}$, P.W. DeCamp ${ }^{2}$, T.J. Kelly ${ }^{2}$, L. Coy ${ }^{3}$

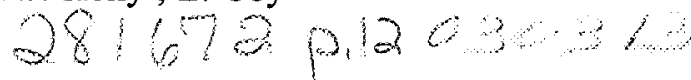

\begin{abstract}
The Shuttle SBUV (SSBUV) conducted its fourth and fifth flights in late March 1992 and early April 1993 along with the ATLAS-1 and ATLAS-2 Shuttle missions, respectively. The two successive SSBUV flights yielded ozone data nearly one year apart. An analysis of the meteorological conditions, namely temperature and winds, during the two flight periods indicate that the conditions in the stratosphere were very similar. The temperatures had significantly warmed from winter throughout most of the stratosphere and the circulation was approaching normal summertime conditions for both periods. SSBUV-4, flown in 1992, measured ozone from approximately $30 \mathrm{~S}$ to $60 \mathrm{~N}$ while SSBUV-5, flown in 1993, measured ozone from approximately $55 \mathrm{~S}$ to $60 \mathrm{~N}$. Zonal average column ozone amounts were derived from the two flights and compared to determine if a systematic change in ozone could be detected despite the fact that only a few days from each year were sampled. The comparison indicates that in the latitude range $30 \mathrm{~N}$ to $60 \mathrm{~N}$ total ozone was lower in 1993 than in 1992 by about $12 \%$. This change is larger than the observational errors and the expected interannual variations. This result verifies similar data taken from ground and satellites.
\end{abstract}

\section{Introduction}

Unprecedented ozone changes have been detected since 1991 over northern hemisphere mid-latitudes. Ozone depletion has been reported by Gleason et al. [1993], and Planet et al. [1994] from NOAA SBUV/2 (Solar Backscatter Ultraviolet/Mod2) and by Herman and Larko [1994] from NASA TOMS (Total Ozone Mapping Spectrometer) satellite data in the northern hemisphere after 1990. Similar findings have been reported from groundbased data by Hofmann et al. [1994], Bojkov et al. [1993], and more recently by Bojkov et al. [1995] who show ozone changes, in 1993, similar to the ATLAS data. The more recent decline of ozone values at mid to high latitudes have now been attributed to cold temperatures, increasing amounts of $\mathrm{ClO}$, and Pinatubo aerosols which spread to extra-tropical latitudes [WMO, 1994]. Ground, satellite, and now Shuttle-based data show that ozone reduction was more pronounced during the second winter after Pinatubo than during the first. Hofmann et al. [1994] concluded this decrease results from several factors, including unusually cold

temperatures, additional time for chemical processing, and aerosols reaching higher latitudes after the second year (winter 1992-1993). Additional causes could be attributed to steadily increasing

\footnotetext{
NASA Goddard Space Flight Center, Greenbelt MD

${ }^{2}$ Hughes STX Corporation, Greenbelt, MD

${ }^{3}$ General Science Corporation, Laurel MD
}

Copyright 1996 by the American Geophysical Union.

Paper number 96GL01120

0094-8534/96/96GL-01120\$05.00 amounts of ClO. The observed ozone changes are also consistent with a quasibiennial oscillation (QBO) effect as described by $Y$ ang and Tung [1994]. A solar cycle contribution is also possible since this period is during the steep descending period of solar cycle 22. However, for this period the changes in ozone are less than $1 \%$ for solar cycle effects.

Although satellites have given a fairly consistent picture of this depletion, the precise change has not been established since updates to the calibration of both SBUV/2 and TOMS data sets are planned using data from regular Shuttle SBUV (SSBUV) flights. For example, the SBUV/2 data reported by Planet et al. [1994] are still subject to calibration uncertainties [Hilsenrath et al., 1995]. Reprocessing of this data set from 1989 to 1995 is planned in 1996. The entire Nimbus TOMS data set (1979 thiough 1993) is also undergoing a major recalibration and will be available by mid 1996. Since the purpose of SSBUV is to provide a calibration transfer to SBUV/2 and to validate TOMS data, it was thought appropriate to use SSBUV data taken during the ATLAS flights to verify the conclusions derived from the satellite data sets that are now available. The first step in this verification is to demonstrate that the SSBUV measurements are sufficiently precise to detect year to year changes, particularly in the presence of aerosols, which can contaminate backscatter ultraviolet (BUV) radiances. The second step is to demonstrate that the observed changes do not result from the 1992 and 1993 samples being taken in different phases of the annual cycle.

\section{SSBUV Ozone Measurements}

The SSBUV has flown 8 times since 1989 where three of those flights were with ATLAS. The SSBUV is an SBUV/2 instrument modified for Shuttle flight. The primary goal is to provide calibration checks of BUV ozone instruments flying on NOAA operational and NASA research satellites [Hilsenrath et al., 1988]. SSBUV data have been used to update the calibration of the NOAA-11 SBUV/2 data set for the period 1989 to 1993 [Hilsenrath et al., 1995] and further refinements are planned. SSBUV observes the Earth's ultraviolet directional albedo to provide ozone profiles from about 20 to $55 \mathrm{~km}$ [Bhartia et al., 1995] as well as total column ozone [Klenk et al., 1982]. Ozone profiles from the ground to about $25 \mathrm{~km}$ are calculated from a climatology of low, mid, and high latitudes scaled by the total ozone amounts.

The accuracy of total ozone retrievals from SBUV instruments depends primarily on wavelength dependent calibration errors, because total ozone is derived from radiances taken in wavelength pairs, allowing wavelength independent calibration errors to cancel out [A hmad et al., 1994]. A $1 \%$ wavelength independent calibration error translates into less than a $0.3 \%$ error in total ozone. Wavelength dependent errors cause ozone errors ranging from $1-5 \%$, where the larger errors occur at higher solar zenith angles. SSBUV calibration and wavelength accuracy and precision have been discussed by Hilsenrath et al. [1991], Cebula 

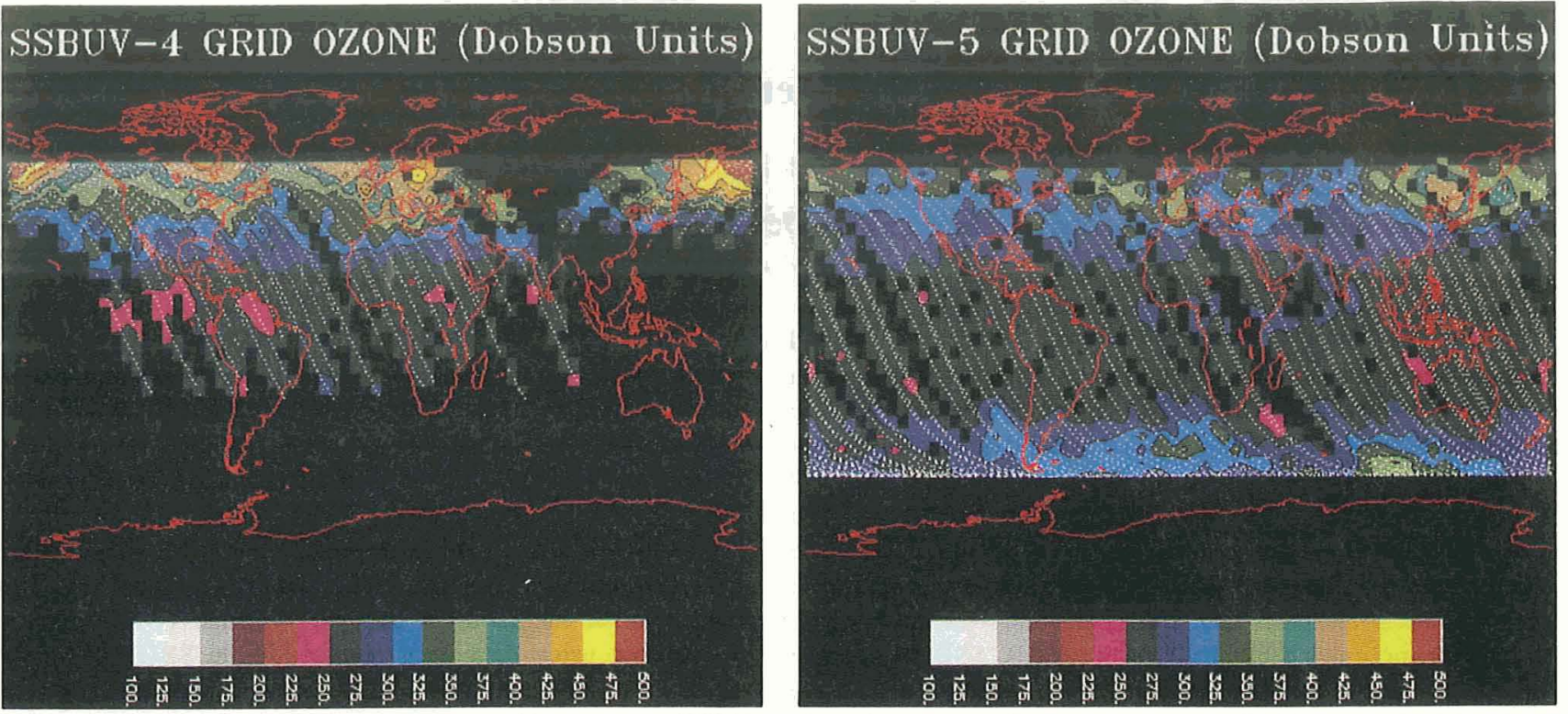

Figure 1. a) Total ozone map for SSBUV-4 on ATLAS-1 (1992). Column ozone amounts in Dobson Units are shown in color scale. b) Same as a) except for SSBUV-5 on ATLAS-2 (1993).

et al. [1995], and Janz et al. [1995] who show wavelength independent and dependent calibration errors to be less than $1 \%$ and of the order of $0.1 \%$, respectively from flight to flight. SSBUV total ozone values are therefore consistent to better than $1 \%$ from flight to flight. The accuracy of retrieved ozone profiles are more sensitive to absolute calibration errors. Bhartia et al. [1995] estimated profile errors from SBUV type measurements to range between $5-10 \%$ between 1 and $30 \mathrm{hPa}$.

Stratospheric aerosols modify ozone absorption of ultraviolet radiation through additional scattering, thereby contributing errors to the retrieved ozone profiles and column amounts [Torres and Bhartia, 1995]. The ozone profile error is a function of aerosol optical depth, aerosol altitude, and solar zenith angle. When observing through volcanic aerosols, ozone amounts are underestimated by $10 \%$ near $10 \mathrm{hPa}$ and overestimated by an equal percentage near $30 \mathrm{hPa}$ at a $30^{\circ}$ solar zenith angle. Radiative transfer calculations by Bhartia et al. [1993] show that except for very high solar zenith angles, errors in total ozone derived from aerosol contaminated BUV radiances range from -2 to $+2 \%$ depending on solar zenith angles. This error becomes larger at solar zenith angles greater than $75^{\circ}$. For ATLAS-1 the Pinatubo aerosols were never observed at angles larger than $70^{\circ}$ in latitudes greater than $50 \mathrm{~N}$, therefore total ozone errors remained below $2 \%$. For ATLAS-2 the aerosol spread to higher latitudes but their height and optical depth decreased, reducing errors in total ozone to less than $2 \%$ at mid to high latitudes.

\section{Discussion}

Ozone Change from the Spring of 1992 to Spring of 1993

SSBUV on ATLAS-1 collected ozone data during the period March 28-31, 1992 over a latitude range from $27 \mathrm{~S}$ to $57 \mathrm{~N}$. For ATLAS-2, ozone data were collected during the period April 9-13, 1993 from $57 \mathrm{~S}$ to $57 \mathrm{~N}$. These data were gridded and are illustrated in Figures 1a and 1b. To simplify the estimated change over the two measurement periods, zonal averages were computed from the two data sets and shown in Figure 2a. The ratio of the zonal averages between the two flights, 1993/1992, is shown in Figure $2 \mathrm{~b}$. There is a marked ozone decrease in northern hemisphere mid and high latitudes, exceeding $15 \%$ near $60 \mathrm{~N}$ (this latitude zone only contains data from $55-57^{\circ}$ since the inclination of the ATLAS- 2 orbits was $57^{\circ}$ ). The smaller ozone changes in tropical and subtropical latitudes which appear in Figure $2 \mathrm{~b}$ will be discussed later.

The SSBUV longitudinal coverage was not the same for the two data periods, as can be seen in Figure 1a and b, particularly over Siberia, a region which is usually covered by large ozone amounts in late winter. Exclusion of this local ozone high could bias the data such that the decrease in Figure $2 b$ is underestimated. In order to examine the effect of the Siberian high, a comparable region was excluded in the ATLAS-2 data and the comparison repeated. The comparison showed essentially the same latitude gradients seen in Figure 2a. A further screen, discussed in the next section, on the two data sets excluded air from the lower stratospheric polar vortex in the zonal averages.

In order to determine the vertical structure of the ozone change in the stratosphere, SSBUV measured ozone profiles were examined for this period. Zonally averaged height- latitude cross sections showed no significant change in ozone amounts at altitudes higher than $25 \mathrm{hPa}$. The absence of changes in the upper stratosphere, which would have to be large to contribute to the observed total ozone change, indicates that the ozone change seen in the two SSBUV flights primarily occurred below $25 \mathrm{~km}$. The specific altitude for the change can not be established since SBUV ozone profiles lack vertical resolution in this region. However, this observed change is consistent with the findings of Hofmann et al. [1994] who saw a $10-20 \%$ decrease in average profiles below $25 \mathrm{~km}$ from the spring of 1992 to the spring of 1993 over Boulder, Colorado and Wallops Island, Virginia.

Several investigations have shown that the 1992-1993 winter was highly unusual when compared to the last 10 or more years [Froidevaux et al. 1994, Manney et al. 1994a]. For this winter, the lower stratosphere Arctic vortex was stronger and more isolated than for previous winters. Furthermore, Gleason et al. [1993] showed that total ozone values were low before the onset of winter, suggesting fundamental circulation differences both 
SSBUV-4 \& SSBUV-5 TOTAL OZONE ZONAL MEANS
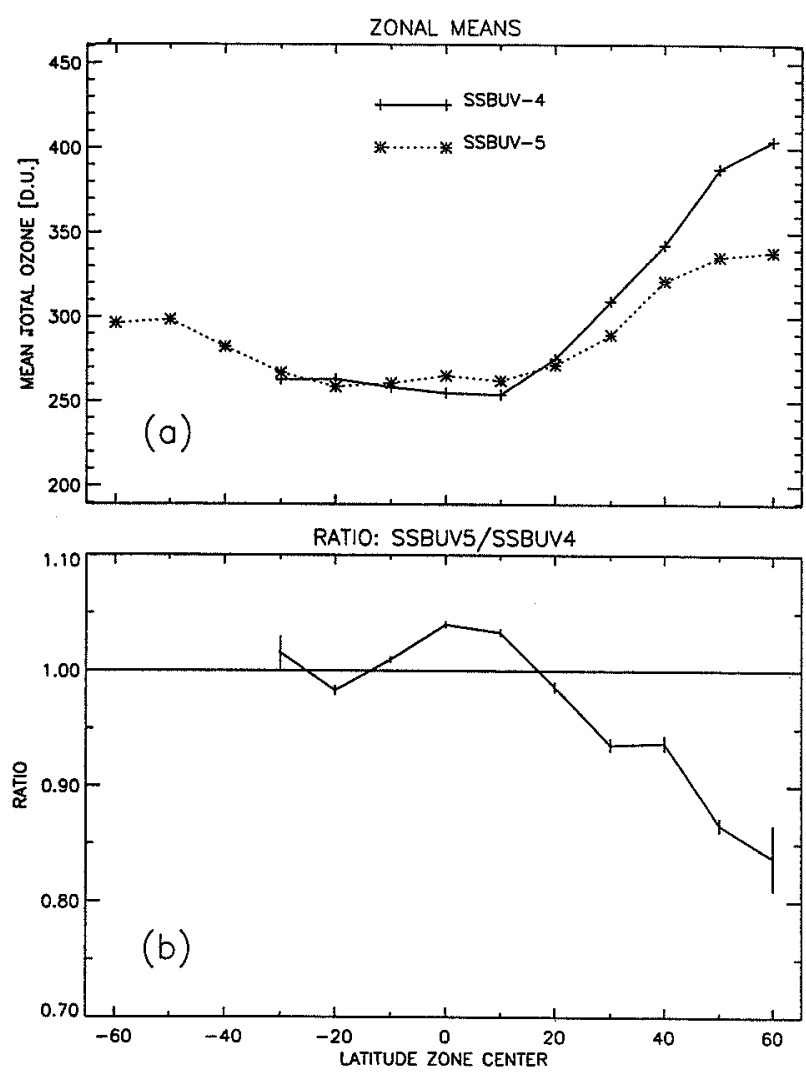

Figure 2. a) Total ozone zonal averages for SSBUV-4 (1992) and SSBUV-5 (1993) as a function of latitude. b) Zonal average ratio of SSBUV-5 and SSBUV-4. Data inside the polar vortex were excluded to remove aliasing (discussed in text).

prior to and during the winters of 1992 and 1993. This would delay and reduce the amount of ozone normally released from the polar night region from reaching mid latitudes. In addition, the colder temperatures would tend to form polar stratospheric clouds in the presence of Pinatubo aerosols, resulting in increased ozone depletion through heterogeneous chemistry. The ATLAS data show that the low ozone amounts persisted into the springtime even after the vortex broke down. Finally, some of the ozone change is attributable to the QBO since there was a complete turn around (easterly to westerly) in the $30 \mathrm{hPa}$ Singapore winds. Hollandsworth et al. [1995] have shown a 15 Dobson Unit variation in SBUV data (1979-1990) due to the QBO at mid and high latitudes during the spring months when the Singapore wind changes by 45 meters per second. This can account for most the change near $30 \mathrm{~N}$ but only about one third of the change at higher latitudes (Figure 2b).

\section{Stratospheric Conditions in the Spring of 1992 and 1993}

We have described how ozone changes from ATLAS-1 and ATLAS-2 do not result from observational errors due to inaccurate calibrations or by aerosol contaminated BUV radiances. The next step is to confirm that these changes do not result from sampling different phases of the annual cycle or differing meteorological conditions for the two years. Two meteorological parameters are analyzed to demonstrate that ATLAS data were most likely taken during the same phase of the annual cycle and in similar air masses. In general, for both ATLAS-1 and -2, the northern hemisphere was well on its way to a normal summertime circulation pattern. The mid-latitude temperatures had warmed since its coldest temperatures during the January-February period for both years throughout the mid to lower stratosphere. To illustrate the similarities of the two periods, the National Meteorological Center's $50 \mathrm{~N}$ zonal mean temperature, and the $60 \mathrm{~N}$ zonal mean zonal wind (both at $50 \mathrm{hPa}$ ) are shown in Figure 3a and $3 \mathrm{~b}$. Mid-latitude temperatures during the ATLAS-1 and ATLAS-2 periods were both near $218 \mathrm{~K}$. Zonal mean zonal wind reflects the decay from the strong mid-winter jet to the relatively weak jet in April. Both the ATLAS-1 and ATLAS-2 periods showed weakened but coherent vortices displaced off of the north pole into the Siberia region, with a larger vortex in 1992 than in 1993 [Manney et al. 1994b]. While these zonal mean zonal winds reflect similar wind speeds, about $10 \mathrm{~m} / \mathrm{s}$ in 1992 and just under $10 \mathrm{~m} / \mathrm{s}$ in 1993 , the polar vortex persisted with some SSBUV observations into this region. To remove aliasing in either the 1992 or 1993 data, ozone measurements inside the vortex (based on potential vorticity maps on the $400 \mathrm{~K}$ isentropic surface) were excluded from this analysis. The 1992 to 1993 comparison therefore only includes air outside the polar vortex.

Tropical and subtropical ozone changes seen in Figure $2 \mathrm{~b}$ are clearly related to the quasibiennial oscillation (QBO). Hilsenrath and Schlesinger [1981] and Oltmans and London [1982] demonstrated the phase, as a function of latitude, of the QBO total ozone relationship using satellite data. They showed the westerly phase of the QBO (as in 1993) is correlated with high tropical ozone and low subtropical ozone, while the easterly phase (as in 1992) has the opposite effect. This QBO - ozone relationship is driven by the secondary circulation associated with the QBO [Plumb and Bell, 1984] and is consistent with the ozone increase in the tropics between 1992 and 1993 and the corresponding decrease at subtropical latitudes. However, $Y$ ang and Tung [1994]

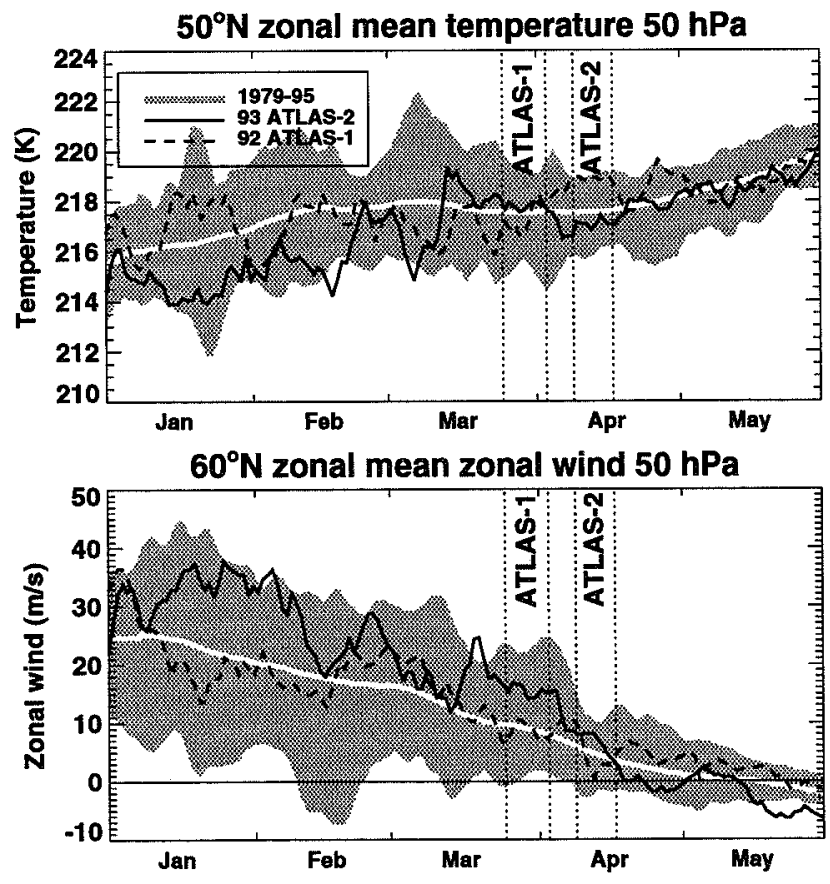

Figure 3. a) Zonal mean (50N) temperature at $50 \mathrm{hPa}$ for winterspring 1992 and 1993. b) Zonal mean $(60 \mathrm{~N})$ zonal wind at 50 $\mathrm{hPa}$ for winter spring 1992 and 1993. Shaded area is the range of values for the period 1979 to 1995. 
and Hollandsworth et al. [1995] have demonstrated that QBO induced ozone decreases for this period would only account for about one third of the change seen in the ATLAS data at high latitudes.

Despite the fact that ATLAS-1 and ATLAS-2 took only snapshots of the spring stratosphere in 1992 and 1993, a clear ozone decline over the two years was revealed at mid and high latitudes. The ozone change is well outside the errors of the SSBUV measurement, which are small, even when considering aerosol contaminated radiances. It also finally demonstrated that the zonal average decline is not due to sampling different times in the ozone annual cycle nor can the change be fully explained by the QBO. Therefore the large ozone decline observed from these two missions further verifies similar changes seen by other observations from space and ground stations over this period. Its likely cause is a combination of residual cold winter temperatures and the presence of active chlorine and Pinatubo aerosols.

Acknowledgements. The authors acknowledge the considerable efforts of the SSBUV engineering team and the STS-45 and STS- 56 Shuttle crews. R.P. Cebula, P.W. DeCamp and T.J. Kelly are supported by NASA contract NAS5-31755 and L. Coy is supported by NAS5-32332

\section{References}

Ahmad, Z., M.T. DeLand, R.P. Cebula, H. Weiss, C.G. Wellemeyer, W.G. Planet, J.H. Lienesch, H.D. Bowman, A.J. Miller, and R.M. Nagatani, Accuracy of total ozone retrieval from NOAA SBUV/2 Measurements: Impact of instrument performance, J. Geophy. Res., 99, 22975-22984, 1994.

Bhartia, P.K., J. Herman, and R.D. McPeters, Effect of Mount Pinatubo aerosols on total ozone measurements for backscatter ultraviolet (BUV) measurements, J. Geophys. Res., 98, 1854718544, 1993.

Bhartia, P.K., R.D. McPeters, C.L. Mateer, L.E. Flynn, and C. Wellemeyer, Algorithm for estimation of vertical profile from the backscatter Ultraviolet (BUV) technique, J. Geophys. Res., under review, 1996.

Bojkov, R.D., C.S. Zerefos, D.S. Balis, I.C. Ziomas, and A.F. Bais, Record low total ozone during winters of 1992 and 1993, Geophys. Res. Lett., 20, 1351-1354, 1993.

Bojkov, R.D., et al., Further decline during the northern hemisphere winter-spring of 1994-1995 and the new record low ozone over Siberia, Geophys. Res. Lett., 22, 2729-2732, 1996.

Cebula., R.P., E. Hilsenrath, and B. Guenther, Calibration of the Shuttle borne solar backscatter ultraviolet spectrometer, SPIE Proc., 1109, 205-218, 1989.

Cebula, R.P., E. Hilsenrath, P.W. DeCamp, K. Laamann, S. Janz, and $\mathrm{K}$. McCullough, The SSBUV experiment wavelength scale and stability: 1988 to 1994 , Metrologia, in press, 1996.

Froidevaux, L., J.W. Waters, W.G. Read, L.S. Elson, D.A Flower, and R.F. Jarnot, Global ozone observations from UARS MLS: An overview of zonal mean results, $J$. Atmos. Sci., 51, 2846-2866, 1994.

Gleason, J.F., et al., Record low global ozone in 1992, Science, 260, 523-526, 1993.

Herman, J.R., and D. Larko, Low ozone amounts during the 19921993 from the Nimbus TOMS and Meteor 3 Total Ozone Mapping Spectrometers, J. Geophys. Res., 99, 3483-3496, 1994.

Hilsenrath, E., D. Williams, and J. Frederick, Calibration of long term data sets from operational satellites using the space shuttle, SPIE Proc., 924, 215-222, 1988.

Hilsenrath, E., R.P. Cebula, S.J. Hynes, and R.T. Caffrey, Implications of space shuttle flight on the calibration of instruments observing atmospheric ozone and the solar Irradiance, Metrologia, 28, 301-304, 1991.

Hilsenrath, E., R.P. Cebula, M.T. DeLand, K. Laamann, S. Taylor, Wellemeyer, P.K. Bhartia, Calibration of the NOAA-11 SBUV/2 ozone data set from 1989 to 1993 using in-flight calibration data and SSBUV, J. Geophys. Res., 100, 1351-1356, 1995.

Hilsenrath, E., and B.S. Schlesinger, Total ozone seasonal and interannual variations derived from the 7 year Nimbus- 4 data set, J. Geophys. Res. 86, 12,087-12096, 1981.

Hofmann, D.J., S.J. Oltmans, W.D. Komyhr, J.M. Harris, J.A. Lathrop, A.O. Langford, T. Deshler, B.J. Johnson, A. Torres, and W.A. Matthews, Ozone loss in the lower stratosphere over the United States in 1992-1993: Evidence for heterogeneous chemistry on the Pinatubo aerosol, Geophys. Res. Lett., 21, 65$68,1994$.

Hofmann, et al., Recovery of stratospheric ozone over the United States in the winter of 1993-1994, Geophys. Res. Lett., 21, 1779-1782, 1994.

Hollandsworth, S.M., K.P. Bowman, and R. D. McPeters, Observational study of the quasibiennial oscillation in ozone, J. Geophys. Res., 100, 7347-7362, 1995.

Janz, S., E. Hilsenrath, J. Butler, D.F. Heath, and R.P. Cebula, Uncertainties in radiance calibrations of backscatter ultraviolet (BUV) instruments as determined from comparisons of BRDF measurements and integrating sphere calibrations, Metrologia, in press, 1996.

Klenk, K.F.,P.K. Bhartia, A.J. Fleig, V.G. Kaveshwar, R.D. McPeters, and P.M. Smith, Total ozone determination from backscatter ultraviolet (BUV) experiments, J. A ppl. Meteorol., 21, 1672-1684, 1982.

Manney, G.L., R.W. Zurek, M.E. Gelman, A.J. Miller, and R. Nagatani, The anomalous Arctic lower stratosphere polar vortex of 1992-1993, Geophys. Res. Lett., 21, 2405-2408, 1994a.

Manney, G., L. Froidevaux, J. Waters, R. Zurek, W. Read, L. Elson, J. Kumer, J. Mergenthaler, A. Roche, A. O'Neill, R. Harwood, I. MacKenzie, and R. Swinbank, Chemical depletion of lower stratospheric ozone in the 1992-1993 northern winter vortex, Nature, 370, 429-434, 1994b.

Oltmans, S.J., and J. London, The quasibiennial oscillation in atmospheric ozone, J. Geophys. Res., 87, 8981-8989, 1982.

Planet, W.G., et al., Northern hemisphere total ozone values from 1989 to 1993 determined with the NOAA-11 solar backscattered ultraviolet (SBUV/2) instrument, Geophys. Res. Lett., 21, 205- 208, 1994.

Plumb, R.A., and R.C. Bell, A model of the quasibiennial oscillation on an equatorial beta-plane, $Q . J . R$. Meteorol. Soc., 108, 335-352, 1982

Torres, O., and P.K. Bhartia, Effect of stratospheric aerosol on ozone profile from BUV measurements, Geophys. Res. Lett., 22, 235-238, 1995.

Tung, K.K., and H. Yang, Global QBO in circulation and ozone. Part I: Rexamination of observational evidence, J. Atmos. Sci., 51, 2699-2706, 1994

E. Hilsenrath and P. Newman, Code 916, Goddard Space Flight Center, Greenbelt, MD 20771, hilsenrath@ssbuv.gsfc,nasa.gov. R.P. Cebula, P.W. DeCamp, and T.J. Kelly, Hughes STX, 7701 Greenbelt Rd., Greenbelt, MD, 20771.

L. Coy, General Science Corp., 6100 Chevy Chase Dr., Laurel, MD, 20707.

(recieved September 20, 1995; revised February 16, 1996; accepted March 18, 1996.) 


\title{
Distinctive ozone structure in the high-latitude stratosphere: Measurements by the Millimeter-wave Atmospheric Sounder
}

\author{
J. J. Olivero, ${ }^{1}$ T. A. Pauls, ${ }^{2}$ R. M. Bevilacqua,${ }^{2}$ D. Kriebel,${ }^{3}$ M. Daehler, ${ }^{2}$ \\ M. L. Richards, ${ }^{4}$ N. Kämpfer, ${ }^{5}$ A. Berg, ${ }^{6}$ and C. Stodden ${ }^{1}$
}

\begin{abstract}
.
MAS (Millimeter-wave Atmospheric Sounder) observations from the shuttle ATLAS spacelab pallet have revealed some little known (and unexplained) structure in stratospheric ozone mixing ratio profiles at sub-polar latitudes, of both hemispheres. Qualitatively similar features are observed by UARS instruments. Another possibly related feature has been observed by groundbased remote sensing from the South Pole over an extended season. In all these cases, it seems likely that active photochemistry and highly structured horizontal and vertical transport play important roles. Some evidence of a similar feature is also present in a current 2-D photochemical model. This high latitude phenomenon is both an intriguing challenge for current 3-D models and potentially useful test for validating remote sensing experiments.
\end{abstract}

\section{Introduction}

The Millimeter-wave Atmospheric Sounder (MAS) is an international experiment which has flown on three Spacelab pallet missions - the ATLAS(ATmospheric Laboratory for Applications and Science) series - in 1992-1994, aboard the Space Shuttle [Croskey et al., 1992 and Hartmann et al., 1996]. The experiment consists of a large limb-viewing millimeter-wavelength total power radiometer-spectrometer system operating simultaneously in three frequency bands: 60,183 , and 204 $\mathrm{GHz}$; measuring the thermal microwave radiation from the Earth's limb emitted from rotational transitions of $\operatorname{oxygen}\left(\mathrm{O}_{2}\right)$, water vapor $\left(\mathrm{H}_{2} \mathrm{O}\right)$, ozone $\left(\mathrm{O}_{3}\right)$, and chlorine monoxide $(\mathrm{ClO})$, respectively. These observations yield mixing ratio profiles of ozone, chlorine monoxide,

\footnotetext{
${ }^{1}$ Embry-Riddle Aeronautical University, Daytona Beach, FL 32114

${ }^{2}$ Remote Sensing Division, Naval Research Laboratory, Washington, DC 20375-5351, USA.

${ }^{3}$ Computational Physics, Inc., Fairfax, VA.

${ }^{4}$ Max Planck Institute for Aeronomy, Lindau, Germany.

${ }^{5}$ University of Bern, Bern, Switzerland

${ }^{6}$ University of Bremen, Bremen, Germany
}

Copyright 1996 by the American Geophysical Union.

Paper number 96GL01044

0094-8534/96/96GL-01044\$05.00 water vapor, as well as temperature and pressure derived from three oxygen lines. Ozone measurements (with approximately $3 \mathrm{~km}$ vertical resolution) cover the altitude range from about $20 \mathrm{~km}$ to $80 \mathrm{~km}$ [Hartmann et al., 1996], and as high as $90 \mathrm{~km}$ (at night) [Bevilacqua et al., 1996].

The stratosphere at polar and sub-polar latitudes has received significant attention in the past decade. This is especially true for the winter through early spring periods when enormous reductions of ozone are observed [Farman et al., 1985; Solomon 1990]. Ozone observations by MAS occur in the late spring and late fall seasons. These observations reveal additional ozone structure at the same latitudes that is much less well known. The purpose of this Letter is to exhibit this phenomenon as it appears in the MAS data set and to offer a simple explanation for its existence.

\section{MAS Observations}

Depending upon the orientation of the Shuttle itself (at an altitude of $\sim 300 \mathrm{~km}$, and a $57^{\circ}$ inclination orbit), MAS can make measurements from about $70^{\circ}$ in one hemisphere to about $40^{\circ}$ in the other hemisphere. MAS observing periods have occurred as follows: ATLAS1, March 24 to April 2, 1992 (with coverage mostly between $40^{\circ} \mathrm{S}$ and $70^{\circ} \mathrm{N}$ ); ATLAS-2, April 8 to April $17,1993\left(70^{\circ} \mathrm{S}\right.$ to $\left.70^{\circ} \mathrm{N}\right)$; and ATLAS-3, November 3 and November $4,1994\left(40^{\circ} \mathrm{S}\right.$ to $\left.70^{\circ} \mathrm{N}\right)$. Thus MAS has observed the Arctic in two successive years in spring and once in fall, the Antarctic once in fall.

In Figures 1-3, we show examples of ozone vertical profiles from these ATLAS missions. The observations at high latitudes show unexplained structure in the 330 mbar levels. A notch in the zonal mean vertical ozone profiles is evident for $65^{\circ} \mathrm{N}$ and an even more pronounced notch appears at $4-10 \mathrm{mbar}$ at $65^{\circ} \mathrm{S}$ during ATLAS-1 (Figure 1a). A year later, during ATLAS2 (Figure 1b) a notch is still apparent at $65^{\circ} \mathrm{S}$, but is all but gone in the Arctic. In Figure 2, we show the zonal mean ozone profile at $60^{\circ} \mathrm{S}$ also for ATLAS2. Here the notch is quite well developed at the 10 mbar level. Also plotted in this figure is the corresponding zonal mean ozone profile for MLS (Millimeterwave Limb Sounder [Froidevaux et al., 1995]) on UARS (Upper Atmospheric Research Satellite). The agreement between the two measurements is quite satisfying and helps add credence to the MAS dataset. (A full 
MAS Zonal Mean Ozone Retrievals
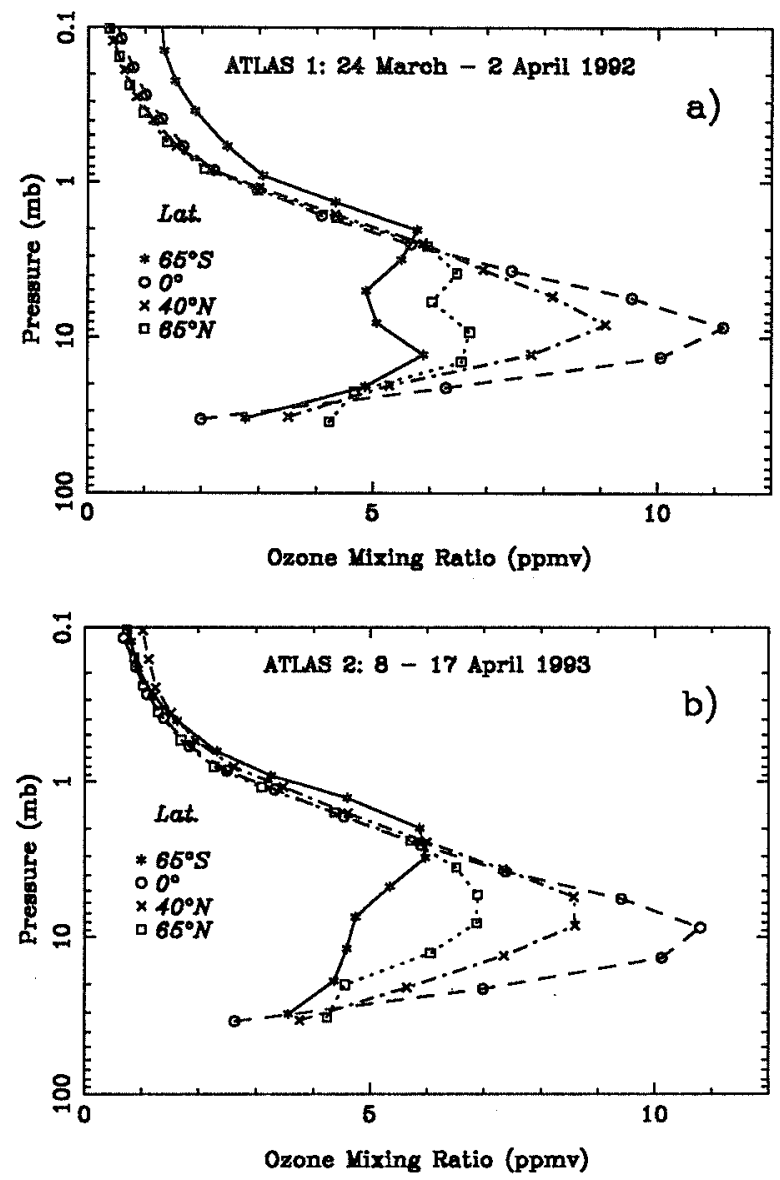

Figure 1. Zonal mean ozone retrievals for selected latitude bands: a) MAS/ATLAS-1, b) MAS/ATLAS-2.

MAS ozone validation study is currently underway, see Daehler et al., 1996]). Figure 3 shows ATLAS-3 observations in the Arctic. Here, at roughly $68^{\circ} \mathrm{N}$, we display ozone profiles centered on 5 longitudes, the notch structure is present in all, but longitudinal asymmetry is also apparent.

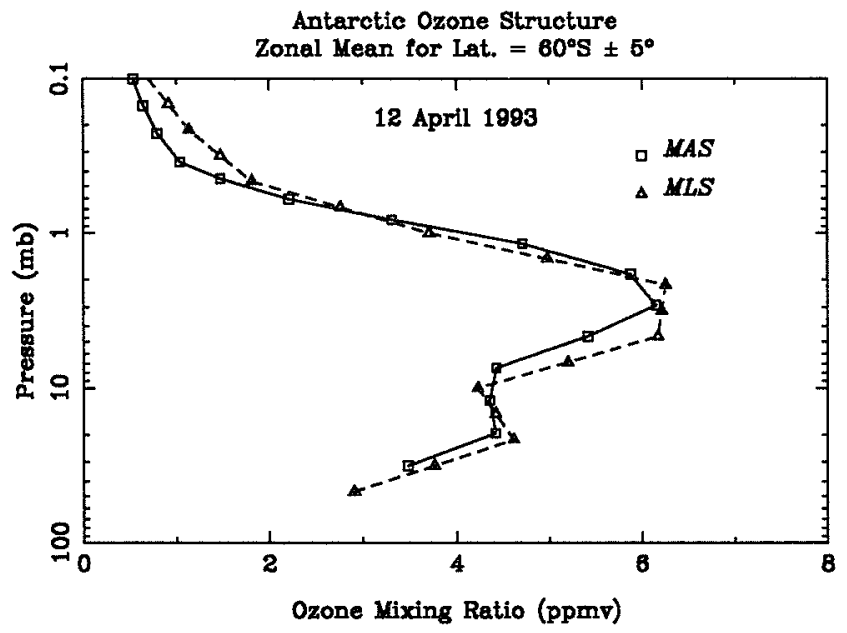

Figure 2. Comparison of zonal mean ozone retrievals from MAS/ATLAS-2 and MLS/UARS near $60^{\circ} \mathrm{S}$.

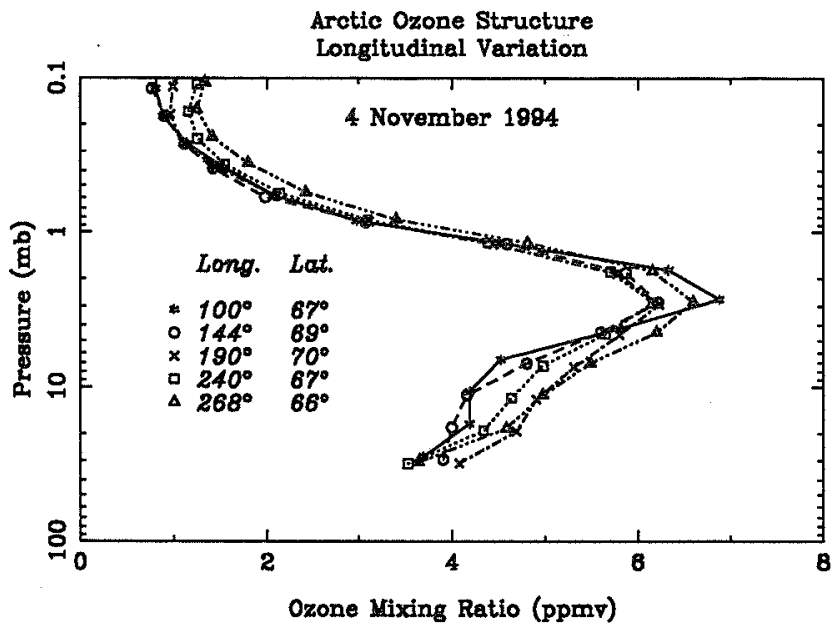

Figure 3. Ozone retrievals at specific longitudes near $68^{\circ} \mathrm{N}$ for MAS/ATLAS-3.

\section{Genesis of the Ozone Structure}

Strong summer depletion of high latitude, stratospheric ozone, resulting from enhanced photolytic production of $N O_{x}$, was modeled by Perliski et al., [1989], based upon suggestions from Johnston [1975]. Perliski et al. also demonstrated the plausibility of their 2-D photochemical model by comparing its predictions with an nine-year climatology of SBUV (Solar Backscatter Ultraviolet) measurements of ozone on constant pressure surfaces (as zonal and monthly means).

Recently Park and Russell [1994] reported confirming ozone measurements from HALOE (Halogen Occultation Experiment) on UARS. Their late summer observations show evidence of the low ozone predicted in Perliski et al. , however, the distribution is highly asymmetric in longitude. Park and Russell are also able to use HALOE solar occultation measurements of $\mathrm{HCl}$, $N O$, and $N_{2}$ to offer an explanation for this ozone asymmetry. They find that the region of lowest ozone is coincident with the highest relative abundances of $\mathrm{HCl}$, $\mathrm{NO}$, and $\mathrm{NO}_{2}$, which supports the photochemical loss hypothesis of Perliski et al. Moreover, Park and Russell examine the distribution of a passive tracer, $\mathrm{CH}_{4}$, to hypothesize that the higher ozone regions were created by a "polar intrusion" of mid-latitude air, air much richer in ozone. They find this asymmetric structure to be present for extended periods of time but subject to longitudinal displacements.

MAS observations of ozone below 3 mbar at latitudes poleward of about $60^{\circ}$ in local fall show the same low abundance signature first described by Perliski et al. for mid-summer. MAS, with $\sim 3 \mathrm{~km}$ vertical resolution, is able to examine this structure in some detail, (see Figs. 1 and 2). Now, in Perliski et al., the SBUV observations did not follow the ozone recovery at all stratospheric levels predicted by the model (see their Fig. 5), but instead the observations show a continued decrease through October in the Arctic. MAS ozone observations made during ATLAS-3 in early November 
1994 (Fig. 3) tend to support the SBUV trend. We see an even stronger structure (a notch) in the Antarctic both in March 1992 (ATLAS-1, Fig. 1a) and in April 1993 (ATLAS-2, Fig. 1b).

Recently Cheng et al. [1995] reported long term ground-based observations of stratospheric ozone from the South Pole station. This group used a millimeterwave spectrometer to measure thermal ozone emission from the atmosphere at $\sim 3$-day intervals for 11 months. These authors report a persistent double peak structure which is qualitatively similar to our notch; we emphasize the ozone minimum which is found at levels between about 5 and $10 \mathrm{mbar}$, while they draw attention to the lower ozone peak near 50-55 mbar. It is reasonable to expect some differences in the heights of these features because MAS observations are centered quite a distance from the pole $\left(20^{\circ}\right.$ to $30^{\circ}$ of latitude).

Cheng et al. attribute the minimum to continued photochemical loss and the second (or lower) peak to transport of air with higher ozone abundances into this region. Thus their hypothesis has some similarity to the transport role suggested by Park and Russell and supported by the HALOE observations. Meridional transport rates are expected to be both large and to have large vertical and meridional gradients at these altitudes in summer and fall at high southern latitudes, according to the GSFC 2-D stratospheric photochemistry model [C. Jackman, personal communication, 1995]. The model [Douglass et al. 1989 and Jackman et al. 1990 ] has a $10^{\circ}$ latitudinal resolution, extending from $85^{\circ} \mathrm{S}$ to $85^{\circ} \mathrm{N}$ and 46 levels equally spaced in log pressure, from the surface to $0.0024 \mathrm{mbar}$ (approximately 2 $\mathrm{km}$ intervals, 0 to $90 \mathrm{~km}$ ). The model time step is 1 day. A residual circulation is calculated using a 4-year zonal average of NMC temperatures. We have examined the current output from this model and find that the notch structure is well developed at $75^{\circ} \mathrm{S}$ for April, when it is at its seasonal maximum. It is also apparent at $75^{\circ} \mathrm{N}$ for November, but is only significant at $85^{\circ} \mathrm{N}$ in April. Model runs suggest that vertical structure in the meridional transport may play a dominant role in producing the notch. In this photochemical model, the notch structure becomes more striking as one approaches the pole, in both hemispheres near equinox. The model indicates that for latitudes poleward of about $65^{\circ}$ this notch structure reaches its maximum in fall, decreases throughout the winter, and is least pronounced in the spring.

\section{Summary}

Highly structured stratospheric ozone profiles are being observed at high latitudes in both hemispheres, by a number of space-based systems; we introduce here the measurements made by MAS on ATLAS 1, 2, and 3. Qualitatively similar observations have also been made at the South Pole by ground-based mm-wave spectroscopy. The question remains: are all of these observations part of a single phenomenon? A contemporary 2-D photochemical model appears to offer hope that a common framework for understanding all of these measurements is possible.

These and similar observations will likely serve as useful tests of 3-D photochemical models in the future. In addition, the notch structure provides a quite useful phenomenon for measurement comparisons. Even remote sensing systems with moderate resolution should be able to capture this vertical and horizontal structure.

Acknowledgments. We thank Dr. Charles Jackman of NASA-GSFC for his assistance with the 2-D model work. The MLS data used in this paper is courtesy of the EOS Distributed Active Archive Center at the NASA Goddard Space Flight Center.

\section{References}

Bevilacqua, R. M., et al., MAS Measurements of the Latitudinal Distribution of Water Vapor and Ozone in the Mesosphere and Lower Thermosphere, Geophys. Res. Lett., this issue, 1996.

Cheng, D., R. de Zafra, and C. Trimble, Millimeter-wave spectroscopic measurements over the South Pole, I: An 11-month cycle of stratospheric ozone observations during 1993-1994, submitted to J. Geophys. Res., 1995.

Croskey, C. L., et al., The Millimeter-Wave Atmospheric Sounder (MAS): A shuttle based remote sensing experiment, IEEE Trans. Microwave Theory and Techniques, 40, 1090-1100, 1992.

Daehler, M., et al., in preparation, 1996.

Douglass, A. R., C. H. Jackman, and R. S. Stolarski, Comparison of model results transporting the odd nitrogen family with results transporting the odd nitrogen species, J. Geophys. Res., 94, 9862-9872, 1989.

Farman, J. C., R. J. Murgatroyd, A. M. Silnickas, and B. A. Thrush, Ozone photochemistry in the antarctic stratosphere in summer. Q. J. R. Meteorol. Soc., 111, 1013$1028,1985$.

Froidevaux, L., et al., Validation of UARS MLS ozone measurements, J. Geophys. Res., in press, 1996.

Hartmann, G. K., et al., Measurements of $\mathrm{O}_{3}, \mathrm{H}_{2} \mathrm{O}$ and $\mathrm{ClO}$ in the Middle Atmosphere using the Millimeter-Wave Atmospheric Sounder (MAS), Geophys. Res. Lett., this issue, 1996.

Jackman, C. H., A. R. Douglass, R. B. Rood, R. D. McPeters, and P. E. Meade, Effect of solar proton events on the middle atmosphere during the past two solar cycles, J. Geophys. Res., 95, 7414-7428, 1990.

Johnston, H. S., Global ozone balance in the natural stratosphere. Rev. Geophys., 13, 637-649, 1975.

Park, J. H., J. M. Russell III, Summer polar chemistry observations in the stratosphere made by HALOE. J. Atmos. Sci., 51, 2903-2913, 1994.

Perliski, L., S. Solomon, and J. London, On the interpretation of seasonal variations of stratospheric ozone, Planet. Space. Sci., 37, 1527-1538, 1989.

Solomon, S., The mystery of the Antarctic ozone hole, Rev. Geophys., 26, 131-142, 1990.

J.J. Olivero, Embry-Riddle Aeronautical University, 600 S. Clyde Morris Blvd., Daytona Beach, FL 32114-3900. (oliveroj@db.erau.edu)

T.A. Pauls, Code 7213, Naval Research Laboratory, Washington, DC 20375-5351. (pauls@atlas.nrl.navy.mil)

(received September 22, 1995; revised February 16, 1996; accepted March 18, 1996.) 


\section{Page intentionally left blank}




\title{
Measurements of $\mathrm{O}_{3}, \mathrm{H}_{2} \mathrm{O}$ and $\mathrm{ClO}$ in the middle atmosphere using the millimeter-wave atmospheric sounder (MAS) ${ }^{\dagger}$
}

\author{
G. K. Hartmann, ${ }^{1}$ R. M. Bevilacqua,${ }^{2}$ P. R. Schwartz, ${ }^{2}$ N. Kämpfer, ${ }^{3}$ \\ K. F. Künzi, ${ }^{4}$ C. P. Aellig, ${ }^{2}$ A. Berg, ${ }^{4}$ W. Boogaerts,${ }^{1}$ B. J. Connor, ${ }^{5,10}$ \\ C. L. Croskey, ${ }^{6}$ M. Daehler, ${ }^{2}$ W. Degenhardt, ${ }^{1}$ H. D. Dicken, ${ }^{4}$ \\ D. Goldizen, ${ }^{6,14}$ D. Kriebel, ${ }^{7}$ J. Langen, ${ }^{4,11}$ A. Loidl, ${ }^{1}$ J. J. Olivero, ${ }^{8}$ \\ T. A. Pauls, ${ }^{2}$ S. E. Puliafito, ${ }^{9}$ M. L. Richards,${ }^{1}$ C. Rudin,,${ }^{3,12}$ J. J. Tsou, ${ }^{5,13}$ \\ W. B. Waltman, ${ }^{2}$ G. Umlauft, ${ }^{1}$ and R. Zwick ${ }^{1}$
}

\begin{abstract}
The Millimeter-Wave Atmospheric Sounder (MAS) is a shuttle-based limb-sounding instrument designed for global spectroscopic studies of $\mathrm{O}_{3}$, and constituents important in $\mathrm{O}_{3}$ photochemistry, in the middle atmosphere. It is part of the NASA's Atmospheric Laboratory for Applications and Science (ATLAS) spacelab shuttle mission. This paper presents an overview of the instrument, operation, and data analysis. In addition, as an example of the results, we present zonal average retrievals for $\mathrm{O}_{3}, \mathrm{H}_{2} \mathrm{O}$, and $\mathrm{ClO}$ obtained in ATLAS 1. The MAS $\mathrm{O}_{3}$ and $\mathrm{H}_{2} \mathrm{O}$ measurements are shown to agree well with simultaneous observations made with the UARS MLS instrument.
\end{abstract}

\section{Introduction}

Space-based mm-wave spectroscopy in the limb-scanning mode is a powerful technique for study of the middle atmosphere. The technique is successful because there is an abundance of line emissions from molecules

\footnotetext{
${ }^{1}$ Max Planck Institute for Aeronomy, Lindau, Germany.

${ }^{2}$ Remote Sensing Division, Naval Research Laboratory, Washington, DC.

${ }^{3}$ Institute for Applied Physics, University of Bern, Bern, Switzerland.

${ }^{4}$ Institute for Remote Sensing, University of Bremen, Bremen, Germany.

${ }^{5}$ NASA Langley Research Center, Hampton, VA.

${ }^{6}$ Communications and Space Studies Laboratory, The Pennsylvania State University, University Park, PA.

${ }^{7}$ Computational Physics, Inc., Fairfax, VA.

${ }^{8}$ Embry-Riddle Aeronautical University, Daytona Beach, FL.

${ }^{9}$ University of Mendoza, Mendoza, Argentina.

${ }^{10}$ Now at NIWA Lauder, New Zealand.

${ }^{11}$ Now at ESA-ESTEC, Noordwijk, The Netherlands.

${ }^{12}$ Now at IUB Ingenieur-Unternehmung AG, Bern, Switzerland.

${ }^{13}$ Now at GATS Inc., Hampton, VA.

${ }^{14}$ Now at Air Force Institute of Technology, Wright Patterson $\mathrm{AFB}, \mathrm{OH}$.
}

Copyright 1996 by the American Geophysical Union.

Paper number $96 \mathrm{GL} 01475$

0094-8534/96/96GL-01475\$05.00 radiating under well-known excitation conditions in a medium characterized by local thermodynamic equilibrim. Also, mm-wave spectrometers can achieve very high spectral resolution so that closely spaced or multiple lines can be resolved, and the effects of pressure broadening can be exploited to increase the information content of the limb scan spectroscopic measurements.

MAS is a shuttle-based, limb-scanning spectrometer which measures emissions from six mm-wave transitions of four molecular species: the rotational transitions of ozone $\left(\mathrm{O}_{3}\right)$ at $184 \mathrm{GHz}$ and water vapor $\left(\mathrm{H}_{2} \mathrm{O}\right)$ at 183 $\mathrm{GHz}$; the l-doubling multiplet of the chlorine monoxide $(\mathrm{ClO})$ radical at $204 \mathrm{GHz}$; and three magnetic dipole transitions of molecular oxygen $\left(\mathrm{O}_{2}\right)$ in the $55-65 \mathrm{GHz}$ complex. From these measurements we deduce the abundance profiles of $\mathrm{O}_{3}, \mathrm{H}_{2} \mathrm{O}$, and temperature (20 to $80 \mathrm{~km})$, and $C l O(20-45 \mathrm{~km})$. MAS is part of the NASA ATLAS series of spacelab shuttle missions, and has flown on ATLAS 1 (24 March - 2 April 1992), ATLAS 2 (8-17 April 1993), and ATLAS 3 (3-14 November 1994). In this Letter we present an overview of the instrument and its operation, data analysis, and, as an example of the results, we show zonal average measurements of $\mathrm{O}_{3}, \mathrm{H}_{2} \mathrm{O}$, and $\mathrm{ClO}$ obtained during ATLAS 1. We also compare the MAS ATLAS $1 \mathrm{O}_{3}$ and $\mathrm{H}_{2} \mathrm{O}$ measurements to those obtained simultaneously with the UARS MLS instrument [Barath et al., 1993]. More detailed MAS measurements obtained in all three missions can be found in Hartmann et al. [this issue], Olivero et al. [this issue], Kriebel et al. [this issue], Aellig et al., a,b, [this issue], and Bevilacqua et al. [this issue].

\section{Instrumentation and Observations}

A detailed account of the MAS instrument characteristics and measurement capabilities has been published by Croskey et al. [1992]. Briefly, the sensor system consists of a 1-m off-axis paraboloid antenna and three uncooled subharmonically pumped heterodyne radiometers at $60 \mathrm{GHz}, 184 \mathrm{GHz}$, and $204 \mathrm{GHz}$. These feed six separate multifilter spectrometers, each with a total bandwidth of $400 \mathrm{MHz}$ and a maximum resolution of 2 or $0.2 \mathrm{MHz}$. The sensor is mounted on the left side of the ATLAS pallet and scans the atmospheric limb perpendicular to the shuttle velocity vector. The 
nominal tangent altitude range is 10 to $120 \mathrm{~km}$. The MAS data sampling interval is $40 \mathrm{~ms}$, and each complete MAS measurement cycle, which includes an atmospheric measurement, a cold space calibration and a hot calibration, is $12.8 \mathrm{~s}$. The beamwidth of the antenna ranges from $0.30^{\circ}$ at $60 \mathrm{GHz}$ to $0.15^{\circ}$ at $204 \mathrm{GHz}$. For the $300 \mathrm{~km}$ ATLAS orbit, this provides a vertical cross section at the tangent point of $4.7 \mathrm{~km}$ to $9.5 \mathrm{~km}$.

For the ATLAS missions the shuttle orbital inclination was $57^{\circ}$. Atmospheric measurements were made in either the bay down/tail forward, or bay down/nose forward shuttle attitude. In the tail-forward orientation, MAS observes north of the orbital track and the measurement coverage is $73^{\circ} \mathrm{N}$ to $41^{\circ} \mathrm{S}$. In the noseforward orientation MAS observes to the south, giving a $41^{\circ} \mathrm{N}$ to $73^{\circ} \mathrm{S}$ measurement range. During ATLAS 1 the shuttle was deployed mainly in the tail-forward attitude so little data was obtained poleward of $41^{\circ} \mathrm{S}$. Furthermore, the ATLAS 1 launch time (1300) GMT was such that most of the southern hemisphere was on the night side of the orbit. In ATLAS 2 the deployment time in the two measurement orientations was roughly equal, giving a more uniform coverage between $73^{\circ} \mathrm{N}$ and $73^{\circ} \mathrm{S}$. In ATLAS 3 MAS failed after ten hours of observations. All measurements were obtained in the tail-forward attitude, giving no observations south of $41^{\circ} \mathrm{S}$.

\section{Data Acquisition and Analysis}

In order to determine measurement tangent altitudes we use the MAS $\mathrm{O}_{2}$ meaurements. Two of the three $\mathrm{O}_{2}$ lines measured by MAS have relatively small temperature sensitivity and can therefore be used to infer atmospheric pressure. The pressure determination is performed by matching the averaged measured brightness temperatures of these two lines to radiative transfer calculations in which the limb scan geometry, antenna beam pattern, and spectrometer filter response curves are modeled [Langen et al., 1994]. The pressures are converted to an approximate altitude scale using tem-

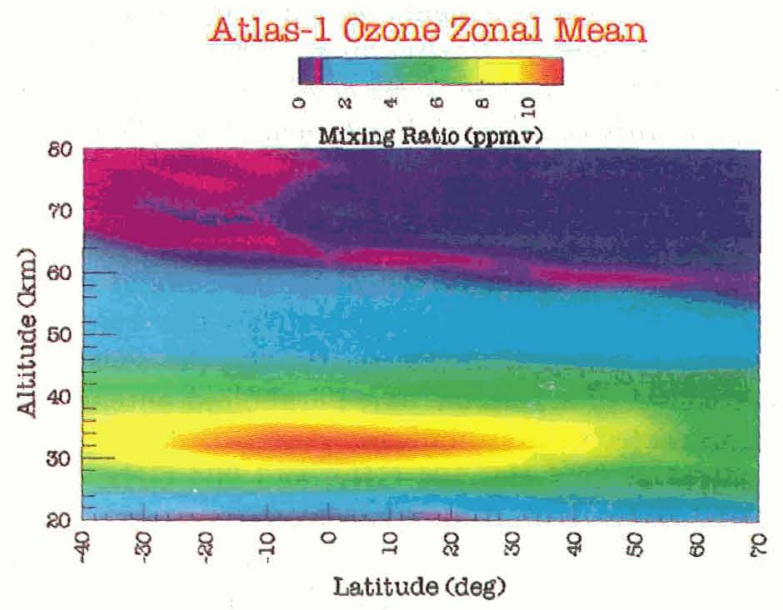

Figure 1a. Zonal mean $\mathrm{O}_{3}$ mixing ratio (in ppmv) measured by MAS during the ATLAS 1 mission.

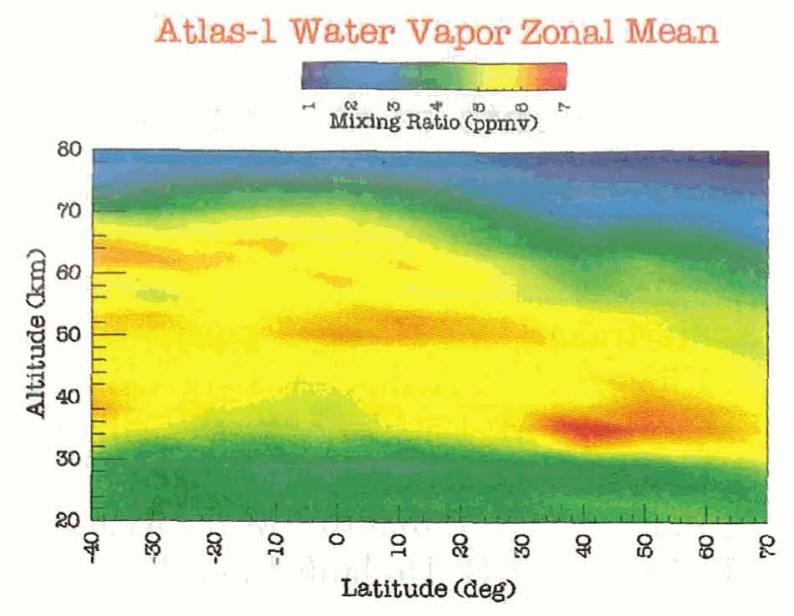

Figure 1b. Same as Figure 1a, but for $\mathrm{H}_{2} \mathrm{O}$.

perature/height profiles obtained from the National Meteorological Center.

Before mixing ratio retrieval, we use radiance averaging to increase the signal-to-noise ratio. Data are binned into height intervals of $3 \mathrm{~km}$ for $\mathrm{O}_{3}$ and $\mathrm{H}_{2} \mathrm{O}$ and $6 \mathrm{~km}$ for $\mathrm{ClO}$. In the $12.8 \mathrm{~s}$ measurement cycle, data from approximately seven $40 \mathrm{~ms}$ measurement intervals fall into each $3 \mathrm{~km}$ bin. The data are subsequently averaged either along the orbital track (for example, in groups of ten consecutive measurement cycles), or over geographical intervals (for example, latitude zones of $5^{\circ}$ or $10^{\circ}$ width, or regions near specific geographical locations). The averaging method is chosen appropriate to the diurnal or geographical dependence being investigated.

The fundamental data products from MAS are the mixing ratio profiles of $\mathrm{O}_{3}, \mathrm{H}_{2} \mathrm{O}$ and $\mathrm{ClO}$ as a function of position and altitude. These profiles are retrieved from the spectral data using the optimal estimation inversion technique [see e.g., Rodgers, 1976]. The procedure includes use of the pressure broadening of the $\mathrm{O}_{3}$ and $\mathrm{H}_{2} \mathrm{O}$ lines to infer atmospheric pressure and, thereby, determine the offset in elevation between the 60 $\mathrm{GHz}$ antenna beam pattern and the $184 \mathrm{GHz}$ pattern. This offset is approximately $0.09^{\circ}$. For details on the implementation of the optimal estimation technique to the retrieval of limb scan MAS spectral measurements see Aellig et al. [1993] and Puliafito et al. [1995].

\section{Sample Results and Comparisons}

As an example of the retrieval results, in Figure 1a we present a contour plot of the MAS ATLAS 1 zonal average $\mathrm{O}_{3}$ mixing ratios as a function of latitude and altitude. The retrievals were obtained by averaging the radiance data from the entire mission into 5 degree latitude bins. The total retrieval error is about $14 \%$ below $30 \mathrm{~km}$ (12 mb) decreasing to about $8 \%$ between 30 and $60 \mathrm{~km}(12-0.2 \mathrm{mb})$ and increasing to about $15 \%$ above this altitude. The contour plot shows features expected from $\mathrm{O}_{3}$ climatology [Keating and Young, 1985]. Peak mixing ratios of just over 10 ppmv occur in the trop- 
ics at about $35 \mathrm{~km}$ where the $\mathrm{O}_{3}$ layer is very narrow. At high latitudes the peak is broader with smaller amplitude. The increase in the $\mathrm{O}_{3}$ mixing ratio in the southern hemisphere mesosphere is the result of the $\mathrm{O}_{3}$ diurnal variation, because during ATLAS 1 the southern hemisphere was generally in the night portion of the shuttle orbit, and the northern hemisphere in the day portion. The mesospheric $\mathrm{O}_{3}$ diurnal variation observed by MAS during ATLAS 2 is discussed in Bevilacqua et al. [this issue].

The zonal average $\mathrm{H}_{2} \mathrm{O}$ distribution for ATLAS 1 is shown in Figure 1b. The data show the expected increase in the water vapor mixing ratio with altitude in the stratosphere, presumably resulting from methane oxidation, and the decrease in the mixing ratio beginning in the lower mesosphere, caused by photodissociation [Le Texier et al., 1988]. In the upper mesosphere, in both hemispheres, there is also a large meridional gradient directed toward the equator. These results are consistent with the mean meridional transport which, at equinox, is expected to be dominated by a 2-cell pattern with upward motion over the equator, poleward flow in the upper mesosphere, and downward motion over both poles [Holton and Schoeberl, 1988].

The MAS $\mathrm{O}_{3}$ measurements are compared to those made with the other atmospheric instruments on board ATLAS (ATMOS and SSBUV) in Kriebel et al. [this issue]. Also, detailed validation papers, in which the measurements are compared with the available data base, for both $\mathrm{O}_{3}$ and $\mathrm{H}_{2} \mathrm{O}$, are in progress. As an example of the results, in Figures $2 \mathrm{a}$ and $2 \mathrm{~b}$ we compare the MAS ATLAS 1 zonal average $\mathrm{O}_{3}$ and $\mathrm{H}_{2} \mathrm{O}$ measurements with those obtained simultaneously with the UARS MLS instrument [Barath et al., 1993]. The version 3 MLS data used in these comparisons were obtained from the NASA Goddard Distributed Active Archive Center (DAAC), and the MAS results have been interpolated to the standard UARS pressure grid.

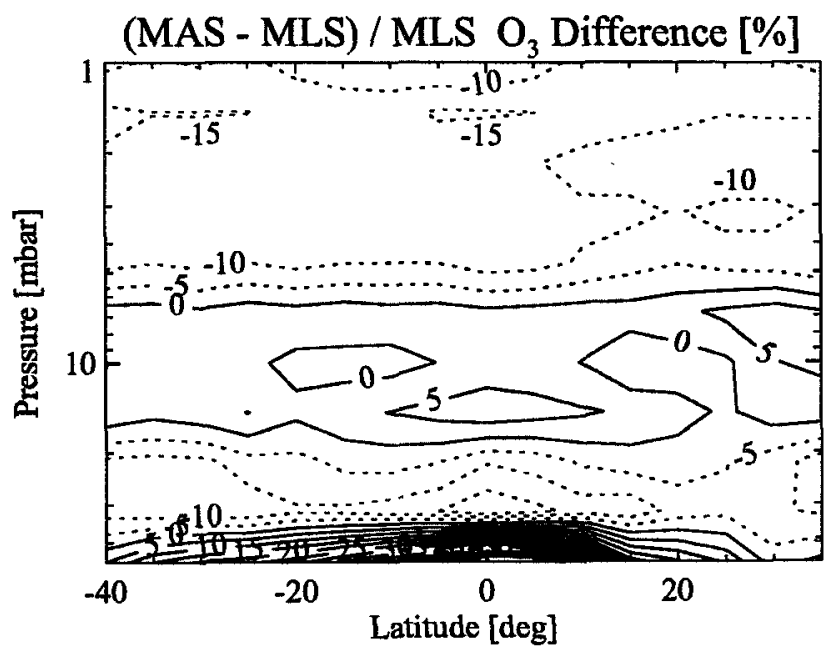

Figure 2a. Percentage differences of zonal mean MAS and $\mathrm{MLS}_{3}(184 \mathrm{GHz})$ mixing ratio retrievals obtained during the ATLAS 1 time-period.

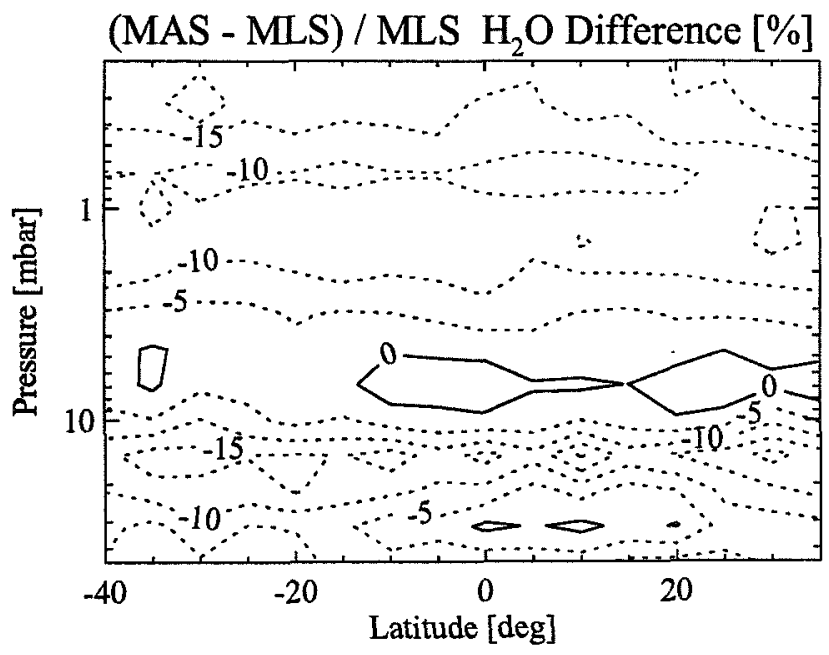

Figure 2b. Same as Figure 2a, but for $\mathrm{H}_{2} \mathrm{O}(183 \mathrm{GHz})$.

The $O_{3}$ comparisons were carried out only up to $1 \mathrm{mb}$ because at lower pressures the $\mathrm{O}_{3}$ diurnal variation becomes important, significantly complicating the comparison. The $\mathrm{H}_{2} \mathrm{O}$ comparisons are carried out to the $0.2 \mathrm{mb}$ pressure level given as the lower pressure limit for useful MLS $\mathrm{H}_{2} \mathrm{O}$ version 3 data [DAAC, 1994]. The MAS and MLS instruments are similar and observe the same spectral lines. Thus there is a possibility that they could share systematic errors, such as retrieval errors resulting from spectral line parameter uncertainties. However, in the $\mathrm{mm}$-wave region, these errors are expected to be small. Also, MLS is the only other currently operational instrument with the cability to make measurements over such an extended latitude range on shuttle mission time-scales. Unfortunately, MLS was in its southward looking mode during ATLAS 1, limiting the region of MLS/MAS overlap to $40^{\circ} \mathrm{S}$ to $30^{\circ} \mathrm{N}$.

The MAS/MLS $O_{3}$ comparisons shown in Figure 2a are generally favorable, with the measurements agreeing to within the expected errors down to at least 30 $\mathrm{mb}(\approx 23 \mathrm{~km})$. However, there are systematic features evident in the comparison which may be significant. In general, the MAS measurements appear to be somewhat low both below and above the $\mathrm{O}_{3}$ mixing ratio peak (with the discrepancy largest below $20 \mathrm{mb}$, where it is $>10 \%)$, and slightly low at the peak $(<5 \%)$. Comparison with other data sets has indicated that these systematic differences may be the result of an artifact in the MAS retrievals. The origin of this bias is currently under investigation. We have also examined nighttime MLS and MAS $O_{3}$ retrievals in order to compare the measurements in the mesosphere. Again we find agreement generally within $10 \%$, with the MAS retrievals tending to be higher than the MLS results. The comparison for ATLAS 2 is similar, but the agreement is better by a few percent.

Figure 2b is similar to Figure 2a, but for $\mathrm{H}_{2} \mathrm{O}$. The errors for the MAS $\mathrm{H}_{2} \mathrm{O}$ retrievals are generally similar to those for $\mathrm{O}_{3}$. In general, the MAS results are systematically lower than those obtained by MLS by 5 to 


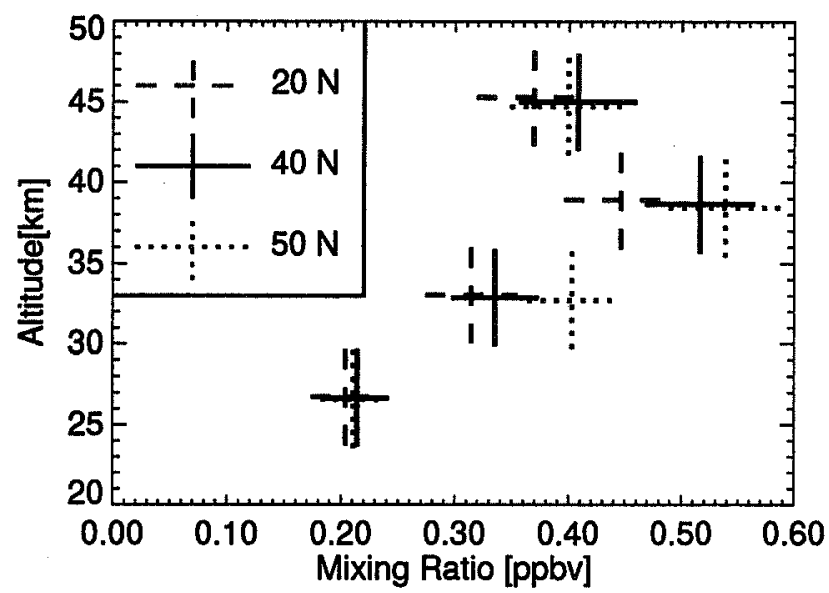

Figure 3. Daytime zonal mean $\mathrm{ClO}$ profiles measured by MAS during ATLAS 1 for $20^{\circ} \mathrm{N}, 40^{\circ} \mathrm{N}$, and $50^{\circ} \mathrm{N}$. Horizontal error bars represent the $1 \sigma$ mixing ratio retrieval precision. Vertical error bars indicate the vertical resolution.

15\%. However, the MLS team has found that comparisons with other data sets suggest that the MLS $\mathrm{H}_{2} \mathrm{O}$ retrievals may be biased high by approximately 10-30\% in the 46 to $0.2 \mathrm{mb}$ region [ $D A A C, 1994$ ]. Thus, the origin of the apparent bias between the MLS and MAS $\mathrm{H}_{2} \mathrm{O}$ data sets is not clear at this time.

Figure 3 shows retrieved $\mathrm{ClO}$ mixing ratio profiles for low- and mid-latitude bands obtained with MAS during ATLAS 1. The horizontal error bars represent the $1 \sigma$ mixing ratio retrieval precision. The results in Figure 3 indicate a slight latitudinal gradient near the peak of the $\mathrm{ClO}$ distribution, with mixing ratios increasing from low to mid latitudes. These results are qualitatively consistent with model calculations [e.g. Solomon and Garcia, 1984). Further details and comparisons of MAS ClO with MLS data can be found in Aellig et al. [this issue].

Acknowledgments. The authors wish to thank the Upper Atmosphere Research Satellite (UARS) Project (Code 916) and the Distributed Active Archive Center (Code 902.2) at the Goddard Space Flight Center, Greenbelt, MD 20771 for the production and distribution, respectively, of the MLS data. We thank NASA, ONR, the German BMFT and DARA, and the Swiss National Science Foundation for their generous support.

\section{References}

Aellig, C. P., N. Kaempfer, and R. M. Bevilacqua, Error analysis of $\mathrm{ClO}, \mathrm{O}_{3}$, and $\mathrm{H}_{2} \mathrm{O}$ abundance profiles retrieved from millimeter-wave limb sounding measurements, J. Geophys. Res., 98, 2,975-2,983, 1993.

Aellig, C. P., et al., Latitudinal distribution of upper stratospheric $\mathrm{ClO}$ as derived from space borne microwave spectroscopy, Geophys. Res. Lett., this issue, 1996a.

Aellig, C. P., et al., Space-borne $\mathrm{H}_{2} \mathrm{O}$ observations in the Arctic stratosphere and mesosphere in the spring of 1992, Geophys. Res. Lett., this issue, 1996b.
Barath, F.T., et al., The Upper Atmosphere Research Satellite Microwave Limb Sounder instrument, J. Geophys. Res., 98, 10,751-10762, 1993.

Bevilacqua, R. M., et al., MAS measurements of the latitudinal distribution of water vapor and ozone in the mesosphere and lower thermosphere, Geophys. Res. Lett., this issue, 1996.

Croskey, C. L., et al., The millimeter-wave atmospheric sounder (MAS): a shuttle based remote sensing experiment, IEEE Trans. Microwave Theory and Techniques, 40, 1,090-1,100, 1992.

DAAC, software document mls_quality_v3.doc.Z, $M L S$ product quality summary document, Goddard DAAC User Services Office, Code 902, NASA/Goddard Space Flight Center, Greenbelt, MD, 1994.

Holton, J. R., and M. R. Schoeberl, The role of gravity wave generated advection and diffusion in the transport of tracers in the mesosphere, J. Geophys. Res., 93, 11,07511,082, 1988.

Langen, J., A. Berg, H. D. Dicken, T. Wehr, K. Kuenzi, W. Degenhardt, N. Kaempfer, and R. Bevilacqua, Temperature and pressure profiles of the middle atmosphere retrieved from millimeter wave atmospheric sounder (MAS) onboard the space shuttle mission Atlas-1, International Geoscience and Atmospheric Remote Sensing Symposium, 940489, Pasadena, CA, 1994.

LeTexier, H., S. Solomon, and R. R. Garcia, The role of molecular hydrogen and methane oxidation in the water vapor budget of the stratosphere, Q.J.R. Meteorol. Soc., 114, 281-295, 1988.

Keating, G. M., and D. F. Young, Interim ozone reference models for the middle atmosphere, Handbook for MAP, 16, 205-229, University of Illinois, Urbana, 1985.

Kriebel, D. L, et al., A comparison of ozone measurements made by the ATMOS, MAS, and SSBUV instruments during ATLAS-1, 2, and 3, Geophys. Res. Lett., this issue, 1996.

Olivero, J. J. et al., Distinctive ozone structure in the highlatitude stratosphere: measurements by the millimeterwave Atmospheric Sounder, Geophys. Res. Lett., this issue, 1996.

Puliafito, S. E., R. M. Bevilacqua, J. J. Olivero, and W. Degenhardt, Retrieval error comparison for several inversion techniques used in limb-sounding $\mathbf{~ m m}$-wave spectroscopy, J. Geophys. Res., 100, 14,257-14,267, 1995.

Rodgers, C. D., Retrieval of atmospheric temperature and composition from remote measurements of thermal radiation, Rev. Geophys., 14, 609-624, 1976.

Solomon, S., and R. R. Garcia, On the distributions of long-lived tracers and chlorine species in the middle atmosphere, J. Geophys. Res., 89, 11,633-11,644, 1984.

†The MAS team would like to dedicate this paper to the memory of our friend and colleague Richard Zwick.

R. M. Bevilacqua, Remote Sensing Division, Naval

Research Laboratory, Washington, DC 20375-5351.

(e-mail: bevilacqu@map.nrl.navy.mil)

G. Hartmann, Max Planck Institute for Aeronomy, Postfach 20, D-37189 Katlenburg-Lindau, Germany.

(e-mail: ghartmann@linax1.mpae.gwdg.de)

N. Kämpfer, Institute for Applied Phyiscs, University of Bern, Sidlerstrasse 5, CH-3012 Bern, Switzerland. (e-mail: kaempfer@sun.iap.unibe.ch)

K. F. Künzi, Institute for Remote Sensing, University of Bremen/FB1, P. O. Box 330440, D-28334 Bremen; Germany. (e-mail: berg@physik.uni-bremen.de; kunzi@theo.physik.uni-bremen.de)

(received September 22, 1995; revised February 8, 1996; accepted April 25, 1996.) 


\title{
MAS measurements of the latitudinal distribution of water vapor and ozone in the mesosphere and lower thermosphere
}

\author{
R. M. Bevilacqua,${ }^{1}$ D. L. Kriebel,${ }^{2}$ T. A. Pauls, ${ }^{1}$ C. P. Aellig, ${ }^{1}$ D. E. Siskind,${ }^{1}$ \\ M. Daehler, ${ }^{1}$ J. J. Olivero, ${ }^{3}$ S. E. Puliafito, ${ }^{4}$ G. K. Hartmann,${ }^{5}$ N. Kämpfer, ${ }^{6}$ \\ A. Berg, ${ }^{7}$ and C. L. Croskey ${ }^{8}$
}

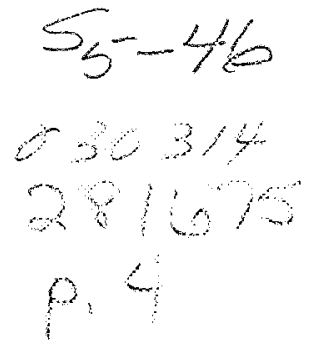

\begin{abstract}
We present measurements of the latitudinal variation of nighttime $\mathrm{O}_{3}$ and $\mathrm{H}_{2} \mathrm{O}$ in the mesosphere and (for $\mathrm{O}_{3}$ ) lower thermosphere obtained with the Millimeter-wave Atmospheric Sounder (MAS) instrument during the ATLAS 2 mission (8-15 April 1993). These are the first such measurements that have ever been reported. They indicate an $O_{3}$ mixing ratio minimum at mid-latitudes in the upper mesosphere, with maxima in the tropics and at high latitudes. The $\mathrm{H}_{2} \mathrm{O}$ retrievals indicate $\mathrm{H}_{2} \mathrm{O}$ mixing ratios decreasing toward the poles in both hemispheres in the upper mesosphere. We also present measurements of the diurnal variation of $\mathrm{O}_{3}$ at southern mid-latitudes, at higher vertical resolution than has ever been reported previously. The results are generally consistent with previous measurements and modeling studies.
\end{abstract}

\section{Introduction}

Measurements of mesospheric $\mathrm{O}_{3}$ and $\mathrm{H}_{2} \mathrm{O}$ have historically been of great interest because of the important roles these species play in the chemistry and energetics of the mesosphere [Allen et al., 1984] and, in the case of $\mathrm{H}_{2} \mathrm{O}$, as a tracer of atmospheric dynamics [Strobel et al., 1987]. Regarding $O_{3}$, there has been a long standing discrepancy between the models and the observations (the "ozone deficit" [e.g., Siskind et al., 1995] ). Several studies [e.g.,Allen and Delitsky, 1993] found that this deficit is largest in the upper mesosphere where the odd hydrogen catalytic cycle dominates $\mathrm{O}_{3}$ loss. Thus, simultaneous measurements of mesospheric

\footnotetext{
${ }^{1}$ Naval Research Laboratory, Washington, DC.

${ }^{2}$ Computational Physics Incorporated, Fairfax, VA.

${ }^{3}$ Embry-Riddle Aeronautical University, Daytona Beach, FL. ${ }^{4}$ University of Mendoza, Mendoza, Argentina.

${ }^{5}$ Max Planck Institute for Aeronomy, Lindau, Germany.

${ }^{6}$ Institute for Applied Physics, University of Bern, Bern, Switzerland.

${ }^{7}$ Department of Physics, University of Bremen, Bremen, Germany.

${ }^{8}$ Communications and Space Studies Laboratory, The Pennsylvania State University, University Park.
}

Copyright 1996 by the American Geophysical Union.

Paper number 96GL01119

0094-8534/96/96GL-01119\$05.00
$\mathrm{O}_{3}$ and $\mathrm{H}_{2} \mathrm{O}$ (the primary source of odd hydrogen) are important in studying this problem.

Previous global measurements of $\mathrm{O}_{3}$ in the middle to upper mesosphere have been limited to those obtained from the Solar Mesospheric Explorer (SME) [Thomas et al., 1984] in which $\mathrm{O}_{3}$ densities were inferred from measurements of $1.27 \mu \mathrm{m}$ radiation. This technique requires many assumptions to be made about the kinetics of the metastable $\mathrm{O}_{2}$ and, thus, may contain inherently large systematic uncertainties [Mlynczak and Olander, 1995]. It is also limited to daytime conditions. By contrast, millimeter-wave measurements are inherently of LTE emissions and can be made both day and night. Recently, Connor et al. [1994] published an extensive set of mesospheric $\mathrm{O}_{3}$ measurements. However, those data were ground-based and, thus, limited to a single location. Furthermore, they are of relatively low vertical resolution, and did not extend above $70 \mathrm{~km}$.

The shuttle-based Millimeter-wave Atmospheric Sounder (MAS) instrument (see e.g., Croskey et al. [1992]; Hartmann et al. [this issue]) is part of the NASA ATLAS series of spacelab missions. MAS observes the $\mathrm{O}_{3}$ line at $184 \mathrm{GHz}$, as well as the $\mathrm{H}_{2} \mathrm{O}$ line at $183 \mathrm{GHz}$ at high spectral resolution $(200 \mathrm{kHz})$, allowing simultaneous retrievals of these two constituents extending into the upper part of the middle atmosphere.

In this Letter we present MAS mesospheric and lower thermospheric $\mathrm{O}_{3}$ and $\mathrm{H}_{2} \mathrm{O}$ retrievals obtained during the ATLAS 2 mission (8-15 April 1993), which gave the largest nighttime latitudinal coverage of the three ATLAS missions. We concentrate on nighttime measurements because the large mesospheric enhancement of $\mathrm{O}_{3}$ at night allows retrievals to be made up to $92 \mathrm{~km}$ (see Figure 1a), whereas in the daytime the signal-tonoise ratio (SNR) becomes very small above $75 \mathrm{~km}$. We show the first near-global measurements of the nighttime latitudinal structure of $\mathrm{O}_{3}$ in the mesosphere. In addition, we show unique, high vertical resolution measurements of the diurnal variation of $\mathrm{O}_{3}$ in the mid and high latitude southern hemisphere.

\section{Data Analysis and Results}

For this study, "night" and "day" 12.8-sec atmospheric limb scans were selected according to the solar zenith angle at the tangent point. The "night" spectra included all data with the absolute value of the solar hour angle $>110^{\circ}$ (local time between 7:20 PM and 


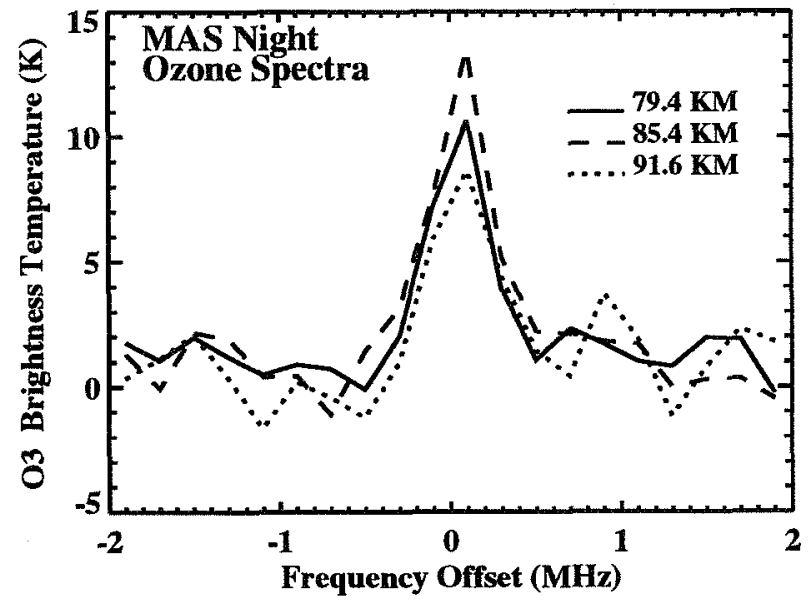

Figure 1a. Integrated MAS nighttime $O_{3}$ radiance spectra at $55^{\circ} \mathrm{S}$ latitude, for the indicated tangent altitudes, measured at $200 \mathrm{KHz}$ resolution.

4:40 AM); the "day" spectra required solar angle $<30^{\circ}$ (within 2 hours of local noon). This simple selection scheme is adequate because the ATLAS 2 mission was conducted very close to equinox. The data in each of these two groups were further binned into 10-degree latitude zones, and $3-\mathrm{km}$ altitude bins extending from 17 to $92 \mathrm{~km}$, compared to $17-80 \mathrm{~km}$ used in the operational retrievals. Within each bin, radiances were averaged to produce a spectrum with adequate SNR. Nighttime average spectra were produced from $65^{\circ} \mathrm{S}$ to $35^{\circ} \mathrm{N}$, but because of the ATLAS 2 orbit, daytime spectra with sufficient integration time were only obtained in the southern hemisphere (poleward of $25^{\circ} \mathrm{S}$ ). Except for the extended altitude range, the profile retrievals were performed as described in Hartmann et al. [this issue].

As an example of the diurnally binned spectra, in Figure la we show the MAS nighttime radiance spectra (expressed in units of brightness temperature) at $55^{\circ} \mathrm{S}$ for the indicated altitudes, and in Figure $1 \mathrm{~b}$ we show the similar results for $\mathrm{H}_{2} \mathrm{O}$. For both $\mathrm{H}_{2} \mathrm{O}$ and $\mathrm{O}_{3}$, the spectral line is only seen in the central channels; the

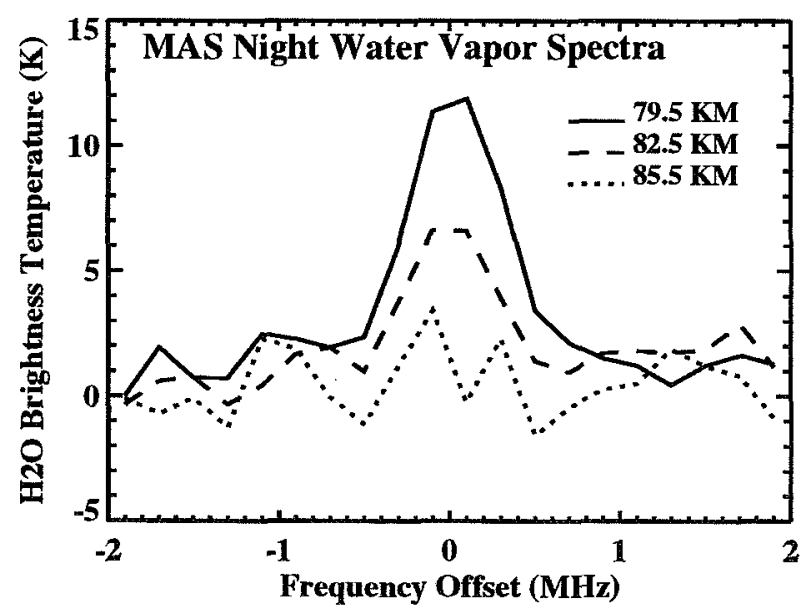

Figure 1b. Same as Fig. Ia, but for the $\mathrm{H}_{2} \mathrm{O}$ spectral line measured simultaneously with $\mathrm{O}_{3}$. variations in the other channels are indicative of the random component of the measurement error. The $\mathrm{O}_{3}$ line is clearly seen above the noise up to $92 \mathrm{~km}$, whereas the $\mathrm{H}_{2} \mathrm{O}$ line is not apparent above $86 \mathrm{~km}$, attesting to the different vertical distributions of those two species. In Figure 2 we show the $\mathrm{O}_{3}$ and $\mathrm{H}_{2} \mathrm{O}$ retrieval results for the $55^{\circ} \mathrm{S}$ case, along with the associated error bars. The error bars are the formal errors from the optimal estimation retrieval $(1 \sigma)$, and include the error resulting from the measurement noise and the smoothing error (see Marks and Rodgers, [1993]). The error bars are quite small below $70 \mathrm{~km}$, but increase very rapidly above this altitude to values exceeding $2 \mathrm{ppmv}$ (40\%) at $92 \mathrm{~km}$. The $\mathrm{O}_{3}$ profile shows a distinct minimum at $80 \mathrm{~km}$, and a rapid increase above this altitude. The secondary maximum (discussed in detail in Allen et al. [1984] ) is a significant retrieval feature, and is apparent in the integrated radiances (shown in Figure 1) as well. The $\mathrm{H}_{2} \mathrm{O}$ retrieval is shown only to $86 \mathrm{~km}$ because, above this altitude, the spectral line is not visible above the noise. An interesting feature of the retrieval is the region of reduced $\mathrm{H}_{2} \mathrm{O}$ falloff between 70 and $80 \mathrm{~km}$. This feature is barely significant considering the error bars. However, as shown below, it is persistent at midlatitudes.

\section{Ozone and Water Vapor Latitudinal Variations}

Nighttime retrievals, such as those shown in Figure 2 , were obtained in $10^{\circ}$ latitude bins from $65^{\circ} \mathrm{S}$ to $35^{\circ} \mathrm{N}$. In Figure 3a we show a contour plot of the nighttime $\mathrm{O}_{3}$ retrieval results in the form of mixing ratios as a function of latitude and altitude. The retrievals were carried out down to $17 \mathrm{~km}$; however, we have cut-off the plot at $50 \mathrm{~km}$ to emphasize mesospheric structure. The latitudinal variation of nighttime $\mathrm{O}_{3}$ over this altitude range has not been presented previously. Above about $68 \mathrm{~km}$, the data show mixing ratio minima at mid-latitudes in both hemispheres, and maxima at both the tropics and at high latitudes. The peak amplitude of this wave-

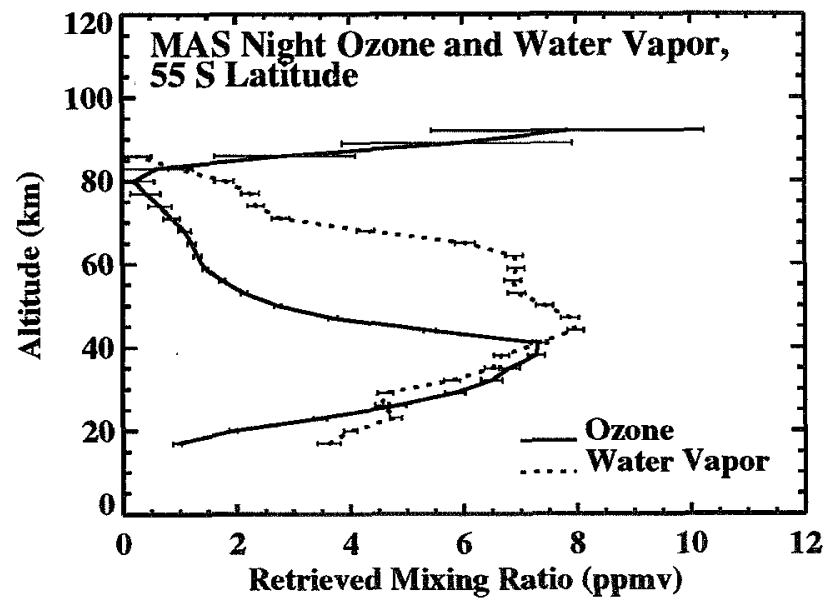

Figure 2. MAS $\mathrm{O}_{3}$ and $\mathrm{H}_{2} \mathrm{O}$ mixing ratio retrievals at $55^{\circ} \mathrm{S}$ latitude. The error bars are the $1 \sigma$ formal errors from the optimal estimation retrieval. 


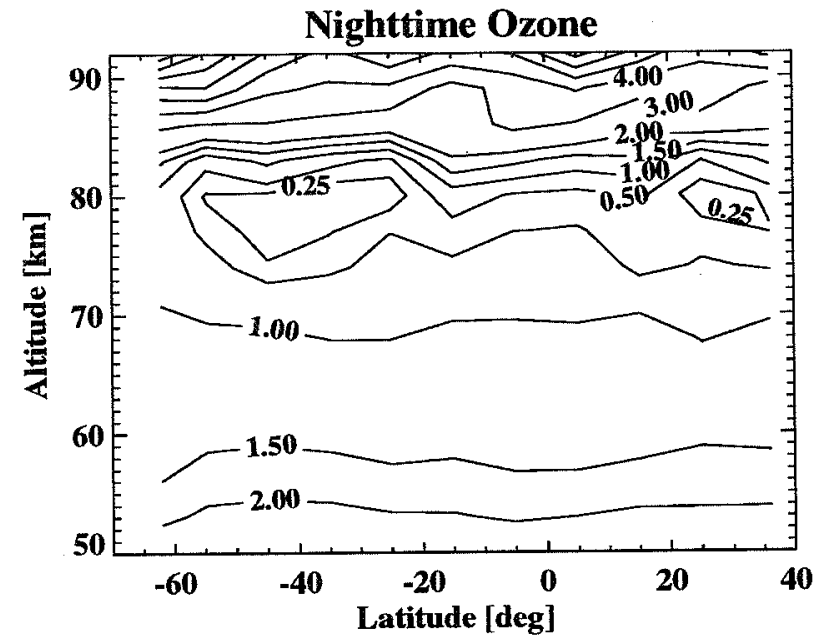

Figure 3a. Contour plot of MAS nighttime retrieved $\mathrm{O}_{3}$ mixing ratio (ppmv) as a function of latitude and altitude.

like structure is about a factor of 2 , occurring at $78 \mathrm{~km}$, and decreasing rapidly below this altitude. Below about $60 \mathrm{~km}$ the variation is of opposite phase, with maxima at mid-latitudes. However, the amplitude of this lower mesospheric feature is quite small (20\%) compared to the upper mesospheric variation. Above about $85 \mathrm{~km}$ the latitudinal variation is dominated by large mixing ratios at the high latitude southern hemisphere.

In Figure 3b, we show the $\mathrm{H}_{2} \mathrm{O}$ retrievals obtained simultaneously with the $\mathrm{O}_{3}$ retrievals discussed above. The contour plot shows a clear maximum in the upper mesosphere in the tropics, with mixing ratios falling off toward the poles in both hemispheres. Above 70 $\mathrm{km}$, the gradient is approximately a factor of 2 . Near the stratopause there is very little structure between the tropics and $35^{\circ} \mathrm{S}$. However, between $35^{\circ} \mathrm{S}$ and $65^{\circ} \mathrm{S}$ the gradient is reversed from that obtained in the upper mesosphere, with mixing ratios increasing toward the pole by less than $20 \%$. The latitudinal distribution of $\mathrm{H}_{2} \mathrm{O}$ shown in Figure $3 \mathrm{~b}$ is consistent with the upwelling over the tropics and subsidence over both poles

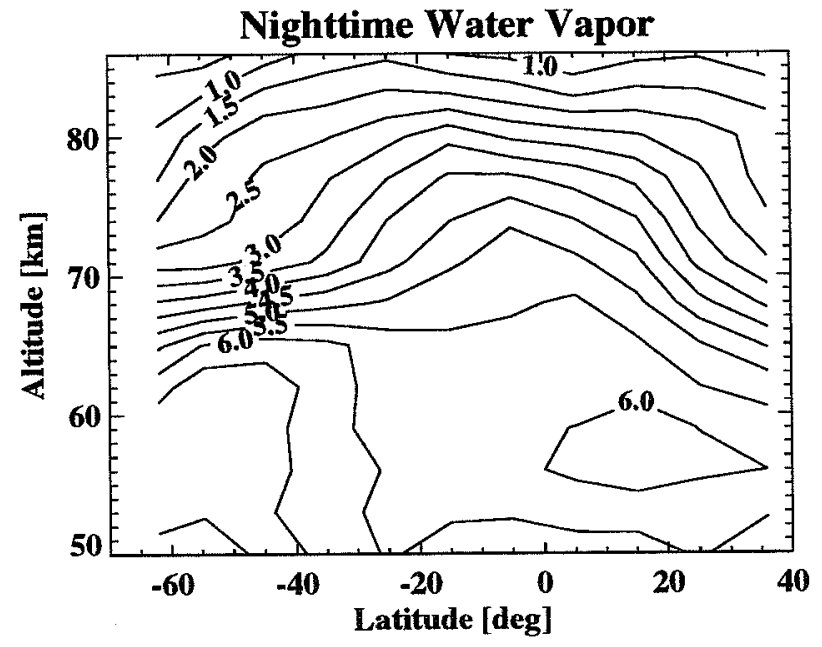

Figure 3b. Same as Figure $3 a$, but for $\mathrm{H}_{2} \mathrm{O}$. expected from the equinoctial mean circulation [Holton and Schoeberl, 1988]. However, although the retrievals extend only to $35^{\circ} \mathrm{N}$ latitude, there is some evidence of a hemispherical asymmetry in the upper mesosphere, with mixing ratios decreasing with latitude faster in the northern hemisphere than in the southern hemisphere. This is reasonable since the ATLAS 2 mission was conducted in April. Therefore, the atmosphere was still in the early stages of its reversal from northern hemisphere winter to southern hemisphere winter circulation.

\section{Ozone Dirunal Variation}

Satellite based observations, such as those presented in this study, are not ideal for studying diurnal variations, because day and night profiles at the same latitude are obtained, in general, on opposite sides of the earth. Therefore, longitudinal variations are convolved with temporal variations. Nevertheless, because of the high vertical resolution and large altitude range of the present measurements, it may still be instructive to examine night-day differences. In order to examine the $\mathrm{O}_{3}$ diurnal variation, we concentrate on southern midlatitudes because, in this region, the integration times in the day and night spectra are approximately the same. In Figure 4, we plot the average day and night $\mathrm{O}_{3}$ retrievals for the $55^{\circ} \mathrm{S}$ latitude bin. The approximately $10 \%$ difference in the profiles in the stratosphere (where no diurnal variation is expected) is indicative of the magnitude of longitudinal variations present in this comparison. However, above $50 \mathrm{~km}$ differences much larger than $10 \%$ become apparent, which are presumably the result of the $\mathrm{O}_{3}$ diurnal variation. In the mesosphere, the day profile decreases very rapidly to mixing ratios less than $0.5 \mathrm{ppmv}$ above $60 \mathrm{~km}$. However, in the night retrieval there is an enhancement of $\mathrm{O}_{3}$ in the midmesosphere. The different characteristics of the two profiles produce a maximum mesospheric mixing ratio difference of about $1.0 \mathrm{ppmv}$ above about $65 \mathrm{~km}$. These differences then begin to decrease up to the daytime detection limit of $75 \mathrm{~km}$. The nighttime enhancement

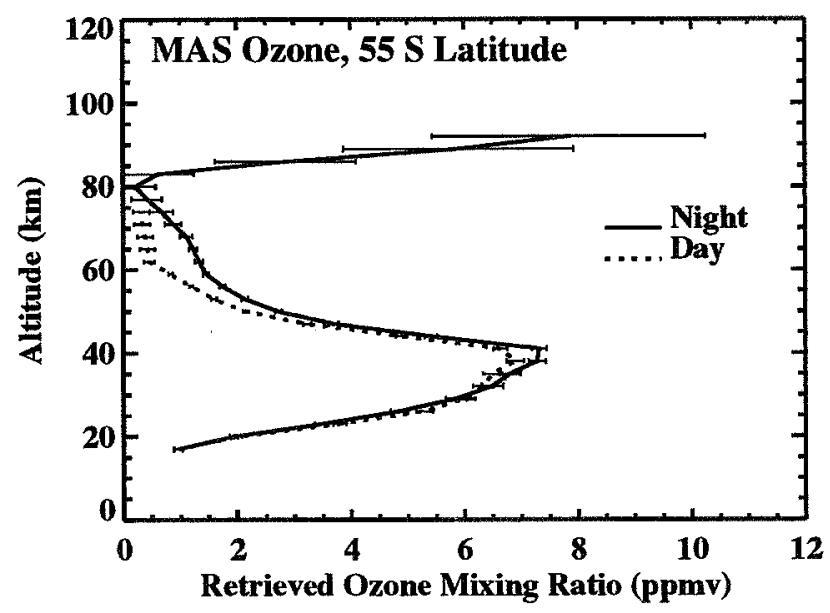

Figure 4. Comparison of the nighttime and daytime retrieved $\mathrm{O}_{3}$ mixing ratio profiles at $55^{\circ} \mathrm{S}$ latitude. 
of $\mathrm{O}_{3}$ in the mesosphere results from the conversion of atomic oxygen to $\mathrm{O}_{3}$ through the reaction $\mathrm{O}+\mathrm{O}_{2}+\mathrm{M}$ $\rightarrow O_{3}+M\left[\right.$ Allen et al., 1984]. Thus, the $O_{3}$ day-night difference is a good proxy measurement of mesospheric daytime atomic oxygen abundances. Therefore, the decrease in the magnitude of the diurnal variation of $\mathrm{O}_{3}$ from $65 \mathrm{~km}$ to $75 \mathrm{~km}$, observed in Figure 4, implies that atomic oxygen abundances are decreasing with altitude over this altitude range. This feature in the atomic oxygen profile is predicted in the model calculations of Allen et al. [1984], but, to our knowledge, has not previously been observed.

Connor et al. [1994] performed the most detailed study to date of the diurnal variation of $\mathrm{O}_{3}$, using ground-based measurements obtained at the JPL Observatory at Table Mountain ( $34^{\circ} \mathrm{N}$ latitude). Their results agree well with the measurements shown in Figure 4 up to about $65 \mathrm{~km}$. However, the decrease in the magnitude of the diurnal variation above that altitude, observed by MAS, is of much too narrow a vertical extent to be resolved in the low vertical resolution groundbased microwave measurements. The diurnal variation depicted in Figure 4 is also qualitatively consistent with the model results of Allen et al. [1984], including the decrease above $65 \mathrm{~km}$.

\section{Conclusions}

We have used MAS measurements, obtained during the ATLAS 2 mission, to present unique observations of the nighttime latitudinal structure of $\mathrm{O}_{3}$ up to $92 \mathrm{~km}$. In the upper mesosphere we find a significant mixing ratio minimum at mid-latitudes and maxima at both high latitudes and tropics. This latitudinal variation may be the result of the two competing effects which are thought to control $O_{3}$ abundances in this altitude region: solar zenith angle, which controls $\mathrm{O}_{3}$ production, and water vapor, which controls $O_{3}$ loss. The solar zenith angle effect tends to decrease $\mathrm{O}_{3}$ abundances with increasing latitude, whereas the $\mathrm{H}_{2} \mathrm{O}$ abundance (which is shown to decrease with latitude in this altitude region) tends to increase $\mathrm{O}_{3}$.

We also present unique high vertical resolution measurements of the diurnal variation of $\mathrm{O}_{3}$ at southern mid-latitudes. The results are qualitatively consistent with those obtained in the measurement study of Connor et al. [1994] and the modeling study of Allen et al. [1984]. A significant feature of these measurements is the region of decreasing $\mathrm{O}_{3}$ night-day differences with altitude from 65 to $75 \mathrm{~km}$, implying decreasing daytime atomic oxygen densities over this altitude range. In future work, the measurements presented in this Letter (both the latitudinal and diurnal variations) will be compared with detailed $\mathrm{O}_{3}$ photochemical model calculations obtained with the NRL 1- and 2-D models.

\section{References}

Allen, M., J.I. Lunine, and Y.L. Yung, The vertical distribution of ozone in the mesosphere and lower thermosphere, J. Geophys. Res., 89, 4841-4872, 1984.
Allen, M., and M.L. Delitsky, A test of odd oxygen photochemistry using Spacelab 3 atmospheric trace molecule spectroscopy observations, J. Geophys. Res., 96, 1288312891, 1991.

Connor, B.J., D.E. Siskind, J.J. Tsou, A. Parrish, and E.E. Remsberg, Ground-based microwave observations of ozone in the upper stratosphere and mesosphere, J. Geophys. Res., 99, 16757-16770, 1994.

Croskey, C.L., et al., The millimeter-wave atmospheric sounder (MAS): A shuttle-based remote sensing experiment, IEEE Trans. Microwave Theory and Techniques, 40, 1090-1100, 1992.

Hartmann, G.K., et al., Measurements of $\mathrm{O}_{3}, \mathrm{H}_{2} \mathrm{O}$ and $\mathrm{ClO}$ in the middle atmosphere using the millimeter-wave atmospheric sounder (MAS), Geophys. Res., Lett., this issue, 1996.

Holton, J.R., and M.R. Schoeberl, The role of gravity wave generated advection and diffusion in the transport of tracers in the mesosphere, J. Geophys. Res., 93, 11075-11082, 1988.

Marks, C.J., and C.D. Rodgers, A retrieval method for atmospheric composition from limb emission measurements, J. Geophys. Res., 98, 14939-14953, 1993.

Mlynczak, M.G., and D.S. Olander, On the utility of the molecular oxygen dayglow emissions as proxies for middle atmospheric ozone, Geophys. Res. Lett., 22, 1377-1380, 1995.

Siskind, D.E., B.J. Connor, R.S. Eckman, E.E. Remsberg, J.J. Tsou, and A. Parrish, An intercomparison of model ozone deficits in the upper stratosphere and mesosphere from two data sets, J. Geophys. Res., 100, 1995.

Strobel, D.F., M.E. Summers, R.M. Bevilacqua, M.T. DeLand, and M. Allen, Vertical constituent transport in the mesosphere, J. Geophys. Res., 92, 6691-6698, 1987.

Thomas, R.J., C.A. Barth, D.W. Rusch, and R.W. Sanders, Solar Mesosphere Explorer near-infrared measurements of $1.27 \mu \mathrm{m}$ radiances and the inference of mesospheric ozone, J. Geophys. Res., 89, 9569-9580, 1984.

C. P. Aellig, R. M. Bevilacqua, M. Daehler, T. A. Pauls, and D. E. Siskind, Remote Sensing Division, Naval Research Laboratory, Washington, DC 20375-5351. (e-mail: aellig@quate.nrl.navy.mil;

bevilacqu@map.nrl.navy.mil; daehler@rira.nrl.navy.mil; pauls@atlas.nrl.navy.mil; siskind@map.nrl.navy.mil)

A. Berg, Institute for Remote Sensing, University of Bremen/FB1, P. O. Box 330440, D-28334 Bremen,

Germany. (e-mail: berg@physik.uni-bremen.de)

C. L. Croskey, Communications and Space Studies

Laboratory, The Pemnsylvania State University, University

Park, PA 16802-2707. (e-mail: csc@ecl.psu.edu)

G. Hartmann, Max Planck Institute for Aeronomy,

Postfach 20, D-37189 Katlenburg-Lindau, Germany.

(e-mail: ghartmann@linax1.mpae.gwdg.de)

N. Kämpfer, Institute for Applied Phyiscs, University of Bern, Sidlerstrasse 5, CH-3012 Bern, Switzerland. (e-mail: kaempfer@sun.iap.unibe.ch)

D. L. Kriebel, Computational Physics Inc., 2750

Prosperity Ave., Suite 600, Fairfax, VA 22031. (e-mail: kriebel@kepler.cpi.com)

J. J. Olivero, Department of Physical Sciences,

Embry-Riddle Aeronautical University, 600 S. Clyde

Morris Boulevard, Daytona Beach, FL 32114. (e-mail: oliveroj@db.erau.edu)

S. E. Puliafito, University of Mendoza, 5500 Mendoza, Argentina. (e-mail: epuliafi@umdz.edu.ar)

(received September 22, 1995; revised February 16, 1996; accepted March 22, 1996.) 


\title{
Latitudinal distribution of upper stratospheric $\mathrm{ClO}$ as derived from space borne microwave spectroscopy
}

\author{
C.P. Aellig, ${ }^{1,2}$ N. Kämpfer, ${ }^{3}$ C. Rudin, ${ }^{4}$ R.M. Bevilacqua,${ }^{1}$ W. Degenhardt,${ }^{5}$ \\ P. Hartogh, ${ }^{5}$ C. Jarchow ${ }^{5}$ K. Künzi, ${ }^{6}$ J.J. Olivero, ${ }^{7}$ C. Croskey, ${ }^{8}$ \\ J.W. Waters, ${ }^{9}$ and H.A. Michelsen ${ }^{10}$
}

\begin{abstract}
Latitudinal distributions of upper stratospheric ClO measured by MAS during the three ATLAS missions are presented for northern hemisphere (NH) spring equinox in 1992, southern hemisphere (SH) early fall in 1993, and NH fall in 1994. The MAS ClO results are shown along with correlative MLS observations. The results of both instruments consistently show the same latitudinal features. The $\mathrm{ClO}$ maximum in the NH spring occurs at mid latitudes, whereas the latitudinal ClO maximum in both the NH and SH fall occurs at high latitudes. The volume mixing ratio maxima were significantly higher in the fall $(0.7-0.8 \mathrm{ppbv})$ than in spring $(0.5-0.6 \mathrm{ppbv})$. Qualitatively, these results are consistent with calculations of several 2-D models.
\end{abstract}

\section{Introduction}

Chlorine monoxide (ClO) is the predominant form of reactive chlorine in the stratosphere. Knowledge of the stratospheric $\mathrm{ClO}$ distribution, and an understanding of its chemistry, are of great interest because $\mathrm{ClO}$ is a key constituent in ozone $\left(\mathrm{O}_{3}\right)$ depletion. The large springtime loss of lower stratospheric $\mathrm{O}_{3}$ over Antarctica is largely driven by a chlorine catalytic cycle in which the formation of the $\mathrm{ClO}$ dimer $\left(\mathrm{Cl}_{2} \mathrm{O}_{2}\right)$ is the rate limiting step [Molina and Molina, 1987]. The ClO abundance is also important in controlling the $\mathrm{O}_{3}$ distribution in the upper stratosphere, where it participates in a catalytic cycle that destroys $\mathrm{O}_{3}$ very effectively [Molina and Rowland, 1974].

Because of interest in the ozone hole and polar $\mathrm{O}_{3}$ depletion in general, there has been a great deal published over the past few years about the distributions of enhanced $\mathrm{ClO}$ in the lower stratosphere during pho-

\footnotetext{
${ }^{1}$ Naval Research Laboratory, Washington, DC.

${ }^{2}$ George Mason University, Fairfax, VA.

${ }^{3}$ University of Berne, Switzerland.

${ }^{4}$ Formerly, University of Berne, Switzerland.

${ }^{5} \mathrm{Max}$ Planck Institute for Aeronomy, Lindau, Germany

${ }^{6}$ University of Bremen, Germany.

${ }^{7}$ Embry-Riddle Aeronautical University, Daytona Beach, FL.

${ }^{8}$ Pennsylvania State University, State College, PA.

'Jet Propulsion Laboratory, Pasadena, CA.

${ }^{10}$ Harvard University, Cambridge, MA.
}

Copyright 1996 by the American Geophysical Union.

Paper number 96GL01215

0094-8534/96/96GL-01215\$05.00 tochemically perturbed conditions. These studies have used ground-based [e.g. de Zafra et al., 1989], air-borne in-situ [e.g. Anderson et al., 1991], or space-based [e.g. Santee et al., 1995] measurements. However, less has been published [e.g. Crewell et al., 1995] on the latitudinal distribution of $\mathrm{ClO}$ in the upper stratosphere. Uncertainties regarding Cly partitioning [Michelsen et al., this issue] can be addressed by such upper stratospheric observational studies in conjunction with $\mathrm{HCl}$ and $\mathrm{CH}_{4}$ measurements [Solomon and Garcia, 1984].

In this paper we present measurements of the vertical and latitudinal distribution of $\mathrm{ClO}$ measured by the Millimeter-wave Atmospheric Sounder (MAS) [Croskey et al., 1992], with special emphasis on the ClO abundance in the upper stratosphere in the vicinity of the mixing ratio peak. MAS is part of the NASA Atmospheric Laboratory for Application and Science (ATLAS) spacelab shuttle mission. Stratospheric ClO measurements obtained from 24 March-2 April 1992 (ATLAS 1), from 8-17 April 1993 (ATLAS 2), and from 3-4 November 1994 (ATLAS 3) are presented along with correlative observations from the Microwave Limb Sounder (MLS).

\section{MAS ClO Observations and Analysis}

The signal strength of the upper stratospheric ClO emission line at $204 \mathrm{GHz}$ is very weak and provides double side band (DSB) brightness temperature amplitudes of the order of only $0.5 \mathrm{~K}$ for limb sounding observations. To reduce the effect of measurement noise on the retrieved $\mathrm{ClO}$ profiles, extensive radiance averaging of about $1000 \mathrm{~s}$ per retrieval were performed [Aellig et al., 1993]. ClO also exhibits a distinct diurnal variation, which is especially pronounced at pressures higher than about $5 \mathrm{mb}$ where virtually all $\mathrm{ClO}$ reacts with $\mathrm{NO}_{2}$ to form $\mathrm{ClONO}_{2}$ at night [Ko and Sze, 1984]. Therefore, only daytime $\mathrm{ClO}$ spectra with solar hour angles (SHA) between $-60^{\circ}$ and $+60^{\circ}$ were used for the ClO retrievals.

The measured radiances were first binned in latitude bands of ten degrees on a pressure grid between 80 and $1 \mathrm{mb}$, using 30 grid points vertically spaced at approximately $1 \mathrm{~km}$. (Pressure was provided by simultaneous measurements of $\mathrm{O}_{2}$ lines near $60 \mathrm{GHz}$ [Langen et al., 1994].) These spectra are affected by blending of lines from other species, which increases with pressure. In the ATLAS 1 and 2 measurements, there are also persistent instrumental baseline artifacts over the entire pressure range. (The long term baseline stability, how- 
ever, was for all missions generally less than $0.05 \mathrm{~K}$, which is approximately $10 \%$ of the DSB amplitude.) Therefore, in order to minimize retrieval error resulting from the atmospheric and instrumental baseline, in the ATLAS 1 and 2 retrievals, nighttime (11 p.m.- 4 a.m.) measurements (binned on the same pressure grid) have been subtracted from the daytime spectra over the entire pressure range. Prior to ATLAS 3, the $\mathrm{ClO}$ receiver was replaced, significantly reducing the instrumental baseline compared to that obtained in the two previous missions. Therefore, in the ATLAS 3 retrievals, the day-night spectral subtraction was performed only at pressures higher than $5 \mathrm{mb}$. The approach used in the ATLAS 3 retrievals is appropriate, and causes litthe retrieval bias because there is virtually no $\mathrm{ClO}$ at pressures higher than $5 \mathrm{mb}$ at night, according to theory [Ko and Sze, 1984]. Above $5 \mathrm{mb}$, however, nighttime $\mathrm{ClO}$ becomes increasingly abundant. Therefore, the day-night subtraction does produce a significant retrieval bias in the ATLAS 1 and 2 retrievals above $5 \mathrm{mb}$. This bias is constant with latitude for each mission because the same night spectra were used for all latitudes. ClO mixing ratios were retrieved from these averaged and differenced spectra on six layers, each of a thickness of about $6 \mathrm{~km}$, using an optimal estimation approach described in Aellig et al. [1993]. To account for the bias in the ATLAS 1 and 2 retrievals introduced by the difference of day and night spectra, estimated ClO night profiles were then added to the ATLAS 1 and 2 retrievals. Using correlative Atmospheric Trace Molecule Spectroscopy (ATMOS) experiment measurements [Gunson et al., this issue] as constraints, $\mathrm{ClO}$ night time estimates were derived from 1-dimensional photochemical model calculations.

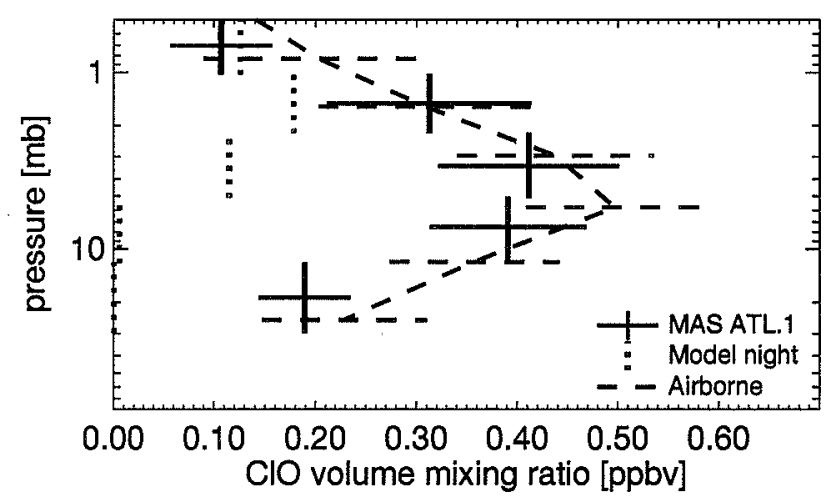

Figure 1. Comparison of MAS and correlative airborne $\mathrm{ClO}$ observations. The MAS measurements are zonal means between 25 and $35 \mathrm{~N}$, averaged between 24 March and 2 April 1992. The model night profile was already added to the MAS retrievals to obtain the values shown. The airborne measurements were obtained on a flight from the Azores (39 N) over Teneriffe (28 $\mathrm{N})$ to Lisbon $(39 \mathrm{~N})$ on 29 March 1992. The range of solar hour angle is $32^{\circ}$ to $42^{\circ}$ for MAS and $-30^{\circ}$ to $105^{\circ}$ for the aircraft observations. Discrepancies in the results due to unequal diurnal sampling are believed to be $10 \%$ or less between 20 and $1 \mathrm{mb}$.

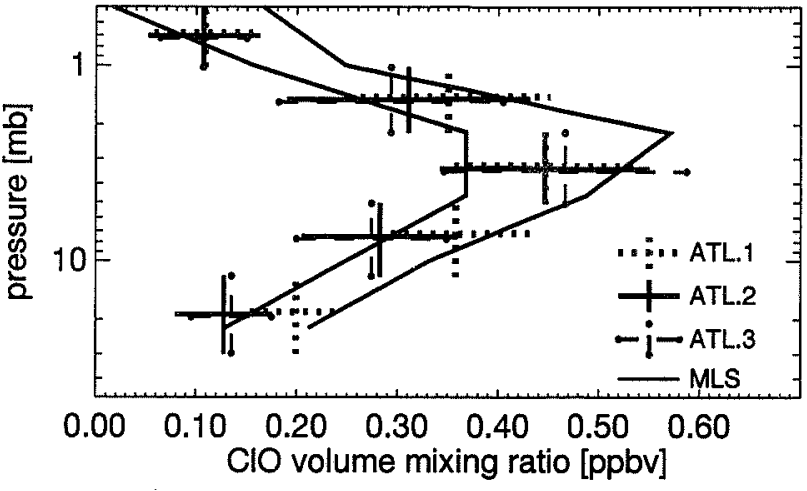

Figure 2. Comparison of MAS and MLS ClO version 3 (scaled by 0.92 , see text) measurements. The MAS measurements are zonal averages for ATLAS 1 (20-40 N), ATLAS 2 (20-40 S), and ATLAS 3 (25-35 N). MLS results represent the range of $\mathrm{ClO}$ monthly means averaged between 20 and $40 \mathrm{~N}$ [Waters et al., 1996].

\section{Validation of MAS Observations}

As a part of the MAS validation campaign, correlative airborne measurements of $\mathrm{ClO}$ at $649 \mathrm{GHz}$ were performed during ATLAS 1 by the Max Planck Institute for Aeronomy and by the University of Bremen. A description of the sub-mm instrument used and the results of earlier measurements can be found in Wehr et al. [1995]. A comparison of MAS and airborne ClO profiles obtained at similar latitudes is shown in Figure 1. The two profiles are in good agreement within the error bars $(1 \sigma)$, which represent the total retrieval errors. The estimated retrieval precision of $\mathrm{MAS} \mathrm{ClO}$ is of the order of 0.05 parts per billion by volume (ppbv) at the ClO vmr peak, which is significantly better than the total retrieval error.

Comparisons of MAS ClO with MLS ClO version 3 climatology were also performed. (An error in the data processing caused the MLS ClO version 3 retrievals to be too large by $8 \%$. Thus, these retrievals need to be multiplied by 0.92 to correct for this systematic error. This correction was made for all MLS ClO version 3 data shown in this Letter. The estimated MLS CIO version 3 bias uncertainties are $0.15-0.2 \mathrm{ppbv}(2 \sigma)$ and the estimated scaling error is $15-20 \%(2 \sigma)$. See Waters et al. [1996] for validation of the MLS ClO version 3 data.) As pointed out by Waters et al. [1996], zonal averages in the region between $20 \mathrm{~N}$ and $40 \mathrm{~N}$ are meaningful for comparisons because the seasonal variation is small in this latitude band. We therefore compared MAS data averaged on the same or on similar latitude bands with MLS monthly means. It can be seen in Figure 2 that the MAS ClO measurements for all missions are generally within the range of the MLS measurements.

\section{ClO Latitudinal Distributions}

Figure 3 is a contour plot of northern hemisphere $\mathrm{ClO}$ zonal mean volume mixing ratios (vmr) measured by MAS (a) along with MLS (b) results, both taken within 


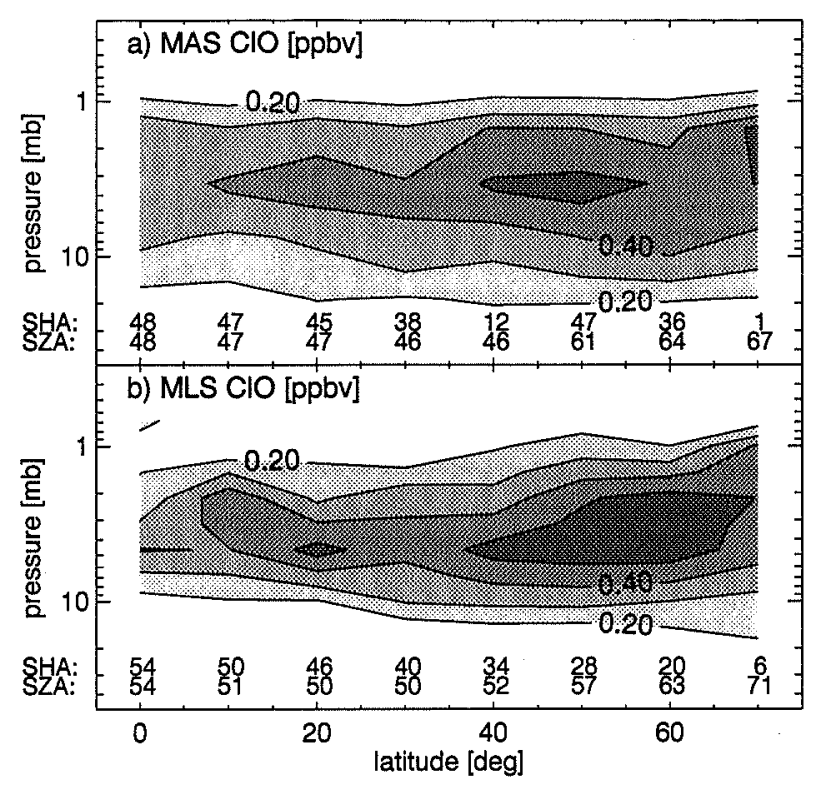

Figure 3. a) MAS ATLAS $1 \mathrm{ClO}$ distributions in ppbv measured between 24 March and 2 April 1992. b) MLS daytime $\mathrm{ClO}$ version 3 (scaled by 0.92 , see text) in ppbv taken between 16 and 19 March 1992. Only MLS data with DATA_QUALITY_UARS $=0.4$, DATA_QUALITY PI $=0.4$, and positive data quality numbers were used. Contour levels are $0.1 \mathrm{ppbv}$, which is about twice the retrieval precision $(1 \sigma)$ of the MAS and MLS results shown. Average solar hour angle (SHA) and solar zenith angle (SZA) in degrees are indicated as a function of latitude for both MAS and MLS.

ten days of spring equinox in 1992. MLS data shown in this and the following figures were obtained from the Distributed Active Archive Center (DAAC). Single daytime profiles (SHA: $-45^{\circ}$ to $+60^{\circ}$ ) were binned into $10^{\circ}$ latitude zones. SHA and SZA (solar zenith angle) for both MAS and MLS measurements are also indicated in Figure 3. The SHA shows that MAS and MLS measurements were taken in similar diurnal periods over the entire latitude region. Therefore, no substantial discrepancies due to unequal diurnal sampling are expected. In fact, diurnal model results have shown that the discrepancies in the $\mathrm{ClO}$ observations due to unequal diurnal sampling of MAS and MLS are $5 \%$ or less for all observation periods near the $\mathrm{ClO}$ peak at 2-4 mb. A latitudinal gradient of 0.1-0.2 parts per billion by volume (ppbv) in the $\mathrm{ClO}$ distribution between the tropics and northern mid latitudes is evident in both the MAS and MLS measurements in NH spring. MLS clearly observed a $\mathrm{ClO}$ vmr maximum at higher mid latitudes. MAS also observed a $\mathrm{ClO}$ vmr maximum at mid latitudes, and also high vmr at high latitudes. The difference in the peak ClO vmr measured by MAS and MLS is less than $0.1 \mathrm{ppbv}$, well within the combined error bars. In Figure 4, $\mathrm{ClO}$ zonal mean vmr measured by MAS and MLS in mid April 1993 between $70 \mathrm{~S}$ and the equator are displayed. These are fall results taken roughly three weeks after equinox. These southern fall observations show a distinct $\mathrm{ClO}$ vmr maximum at high

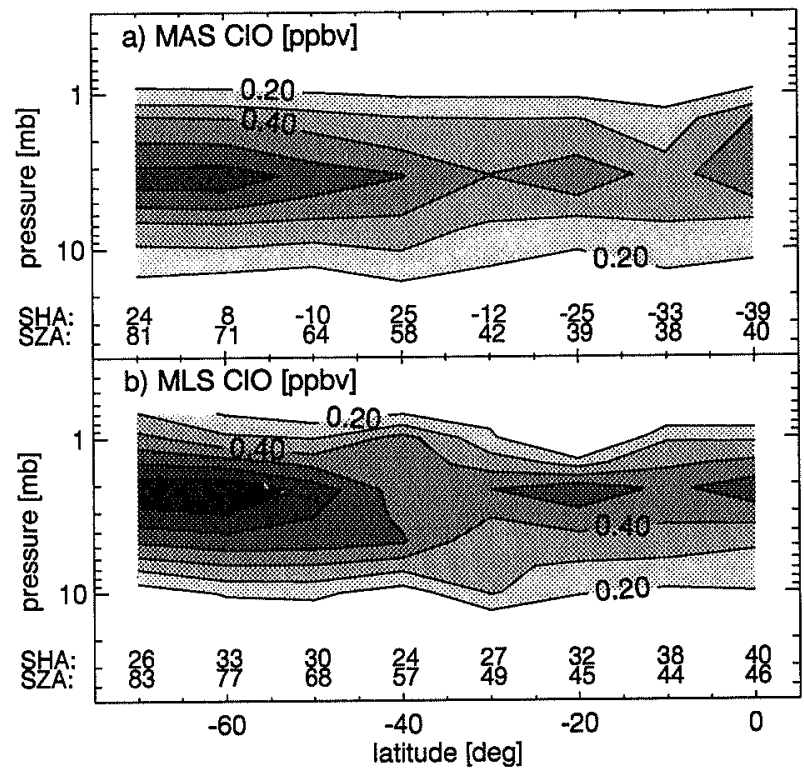

Figure 4. Similar to Figure 3, but for ATLAS 2 (13-15 April 1993). MLS measurements were taken between 10 and 22 April 1993.

latitudes, unlike the northern spring observations displayed in Figure 3. For both the MAS and the MLS measurements, the magnitude of the maximum is higher than that of the mid latitude maximum measured in the spring by about $0.1-0.3 \mathrm{ppbv}$. In fact, the latitudinal gradients observed with the two instruments are remarkably consistent. In both measurements, a distinct $\mathrm{ClO}$ gradient of $0.2-0.3 \mathrm{ppbv}$ occurs between mid and high latitudes, but no significant gradient is evident between low and mid latitudes. While the MAS and MLS latitudinal gradients and the approximate gradients of

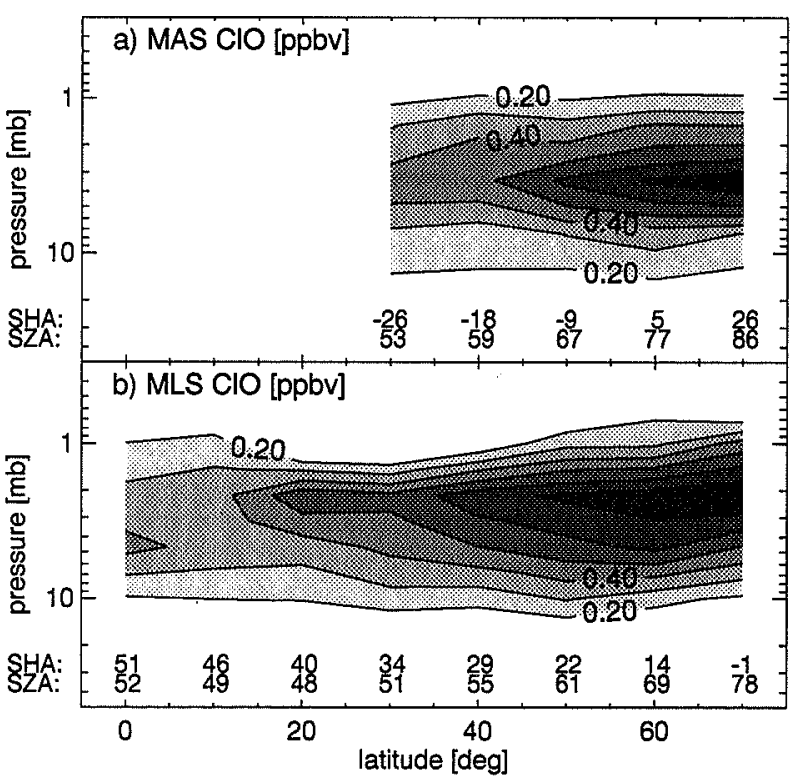

Figure 5. Similar to Figure 3 and 4, but for ATLAS 3 (3-4 November 1994). MLS measurements were taken between 12 and 23 October 1994. 
the peak mixing ratios are consistent, the MAS/MLS comparison indicates that the altitude of the mixing ratio peak measured by MAS is lower (3-4 mb) than measured by MLS (2-3 mb). Figure 5 displays the northern ClO latitudinal distribution measured in early November 1994 by MAS, and in mid October 1994 by MLS. These are also fall measurements taken 6 weeks (MAS) and 3-4 weeks (MLS) after equinox. The MAS measurements cover only the region between 30 and $70 \mathrm{~N}$, because of the short measurement period during ATLAS 3. These northern fall measurements show similar features to those observed in the southern hemisphere fall results displayed in Figure 4. Maximum $\mathrm{ClO}$ mixing ratios of $0.7-0.8 \mathrm{ppbv}$ occur at high latitudes. The altitude of the mixing ratio peak measured by MAS is somewhat lower than measured by MLS, similar to the southern fall results shown in Figure $4 a$ and $4 b$.

Model calculations of the latitudinal distribution of $\mathrm{ClO}$ in the stratosphere for solstice conditions have been presented and discussed by Solomon and Garcia [1984]. Their model results indicate a mid-latitude maximum in winter, and a high latitude maximum in summer. The increase of $\mathrm{ClO}$ from the equator to higher latitudes obtained in the model calculations for both seasons is explained mainly by the decrease of $\mathrm{CH}_{4}$ towards high latitudes. The reaction $\mathrm{CH}_{4}+\mathrm{Cl} \rightarrow \mathrm{HCl}+\mathrm{CH}_{3}$ converts reactive chlorine $\left(\mathrm{Cl}+\mathrm{ClO}=\mathrm{Cl}_{x}\right)$ into the inert $\mathrm{HCl}$. In the winter polar region, however, the reduced conversion of $\mathrm{Cl}_{x}$ into $\mathrm{HCl}$ is expected to be balanced by the slow production of $\mathrm{Cl}_{x}$ from all chlorine reservoir gases $\left(\mathrm{HCl}, \mathrm{ClONO}_{2}\right.$, and $\left.\mathrm{HOCl}\right)$, resulting from the lack of sunlight.

A compilation of 2-dimensional model calculations of the stratospheric ClO distribution was published by Jackman et al. [1989]. The March results of several models feature the ClO maxima at mid latitudes in the $\mathrm{NH}$ and at high latitudes in the $\mathrm{SH}$, similar to the March/April measurements in 1992 and 1993. The AER and GSFC2 model results in Jackman et al. in particular exhibit higher $\mathrm{ClO}$ distributions in the $\mathrm{SH}$ fall than in the NH spring, in agreement with the MAS and MLS measurements. For a more quantitative datamodel comparison, up-to-date stratospheric chlorine loading and chlorine chemistry need to be applied.

Acknowledgments. We thank M. Daehler, L. Froidevaux, and D. Kriebel for carefully reading the manuscript. We also wish to acknowledge two anonymous reviewers for their constructive reviews. The MLS data used in this letter is courtesy of the EOS DAAC at Goddard Space Flight Center. This work has been supported by the Swiss National Science Foundation under contract 20-36415.92, and by a postdoctoral fellowship to C.P. Aellig.

\section{References}

Aellig, C.P., N. Kämpfer, and R.M. Bevilacqua, Error analysis of $\mathrm{ClO}, \mathrm{O}_{3}$, and $\mathrm{H}_{2} \mathrm{O}$ abundance profiles retrieved from millimeter wave limb sounding measurements, $J$. Geophys. Res., 98, 2975-2983, 1993.
Anderson, J.G., D.W. Toohey, and W.H. Brune, Free radicals within the Antarctic vortex: The role of CFSs in the Antarctic ozone loss, Science, 251, 39-46, 1991.

Crewell, S., R. Fabian, K. Künzi, H. Nett, T. Wehr, W. Read, and J. Waters, Comparison of ClO measurements by airborne and spaceborne microwave radiometers in the arctic winter stratosphere 1993, Geophys. Res. Lett., 22, 1489-1492, 1995.

Croskey, C.L., et al., The Millimeter wave Atmospheric Sounder (MAS): A shuttle-based remote sensing experiment, IEEE Transactions on Microwave Theory and Techniques, 40, 1090-1100, 1992.

de Zafra, R.L., M. Jaramillo, J. Barett, L.K. Emmons, P.M. Solomon, and A. Parrish, New observations of a large concentration of $\mathrm{ClO}$ in the springtime lower stratosphere over Antarctica and its implications for ozone-depleting chemistry, J. Geophys. Res., 94, 11423-11428, 1989.

Gunson, M.R., et al., The Atmospheric Trace Molecule Spectroscopy (ATMOS) experiment: Deployment on the ATLAS space shuttle missions, Geophys. Res. Lett., this issue, 1996.

Jackman, C.H., R.K. Seals Jr., and M.J. Prather (Eds.), Two-Dimensional Intercomparison of Stratospheric Models, NASA Conf. publ., 3042, 608 pp., 1989.

Ko, M.K.W., and N.D. Sze, Diurnal variation of ClO: Implications for the stratospheric chemistries of $\mathrm{ClONO}_{2}$, HOCl, and HCl, J. Geophys. Res., 89, 11619-11632, 1984. Langen, J., A. Berg, H.D. Dicken, T. Wehr, W. Degenhardt, N. Kaempfer, and R. Bevilacqua, Pressure profiles of the middle atmosphere retrieved from the Millimeter-wave Atmospheric Sounder (MAS) onboard the space shuttle mission ATLAS-1, Proc. of International Geoscience and Remote Sensing Symposium (IGARSS), Pasadena, 1994.

Michelsen, H.A., et al., Stratospheric chlorine partitioning: Constraints from shuttle-borne measurements of [HCl], $\left[\mathrm{ClNO}_{3}\right]$, and [ClO], Geophys. Res. Lett., this issue, 1996.

Molina, L.T., and M.J. Molina, Production of $\mathrm{Cl}_{2} \mathrm{O}_{2}$ from the self-reaction of the ClO radical, J. Phys. Chem., 91, 433-436, 1987.

Molina, M.J., and F.S. Rowland, Stratospheric sink for chlorofluoromethanes: Chlorine atomcatalysed destruction of ozone, Nature, 249, 810-812, 1974.

Santee, M.L., W.G. Read, J.W. Waters, L. Froidevaux, G.L. Manney, D.A. Flower, R.F. Jarnot, R.S. Harwood, and G.E. Peckham, Interhemispheric differences in polar stratospheric $\mathrm{HNO}_{3}, \mathrm{H}_{2} \mathrm{O}, \mathrm{ClO}$, and $\mathrm{O}_{3}$, Science, 267, 849-852, 1995.

Solomon, S., and R.R. Garcia, On the distribution of longlived tracers and chlorine species in the middle atmosphere, J. Geophys. Res., 89, 11633-11644, 1984.

Waters, J.W., et al., Validation of UARS MLS ClO measurements, J. Geophys. Res., in press 1996.

Wehr, T., S. Crewell, K. Künzi, J. Langen, H. Nett, J. Urban, and $\mathrm{P}$. Hartogh, Remote sensing of $\mathrm{ClO}$ and $\mathrm{HCl}$ over northern Scandinavia in winter 1992 with an airborne submillimeter radiometer, J. Geophys. Res., 100, 20957-20968, 1995.

C.P. Aellig, R.M. Bevilacqua, code 7227, Naval Research Laboratory, Washington, DC 20375-5351. (e-mail: aellig@quate.nrl.navy.mil, bevilacqu@map.nrl.navy.mil)

N. Kämpfer, University of Berne, Sidlerstrasse 5, 3012

Berne, Switzerland. (e-mail: kaempfer@sun.iap.unibe.ch)

(received September 22, 1995; revised February 16, 1996; accepted March 18, 1996.) 


\title{
Space-borne $\mathrm{H}_{2} \mathrm{O}$ observations in the Arctic stratosphere and mesosphere in the spring of 1992
}

\author{
C.P. Aellig, ${ }^{1,2}$ J. Bacmeister, ${ }^{1}$ R.M. Bevilacqua,${ }^{1}$ M. Daehler, ${ }^{1}$ D. Kriebel, ${ }^{3}$ \\ T. Pauls, ${ }^{1}$ D. Siskind, ${ }^{1}$ N. Kämpfer,${ }^{4}$ J. Langen, ${ }^{5}$ G. Hartmann, ${ }^{6}$ A. Berg, ${ }^{7}$ \\ J.H. Park, ${ }^{8}$ and J.M. Russell III ${ }^{8}$
}

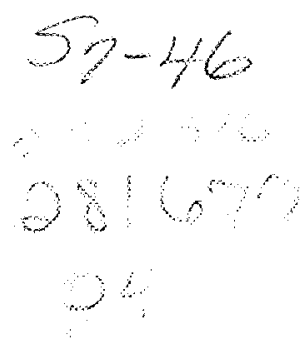

\begin{abstract}
We report on stratospheric and mesospheric water vapor $\left(\mathrm{H}_{2} \mathrm{O}\right)$ observations obtained by the Millimeter wave Atmospheric Sounder (MAS) in the Arctic spring of 1992 . In the lower stratosphere, the observations show enhanced $\mathrm{H}_{2} \mathrm{O}$ inside the vortex between $450 \mathrm{~K}$ and $625 \mathrm{~K}$, in agreement with other $\mathrm{H}_{2} \mathrm{O}$ observations. In the upper stratosphere and lower mesosphere, at potential temperatures between $1850 \mathrm{~K}$ and $2200 \mathrm{~K}$, we find regions of depressed $\mathrm{H}_{2} \mathrm{O}$ volume mixing ratio coincident with remnants of high potential vorticity. The depressed mesospheric $\mathrm{H}_{2} \mathrm{O}$, as well as the enhanced lower stratospheric $\mathrm{H}_{2} \mathrm{O}$, are consistent with wintertime descent. It also suggests effective containment of air up into the lower mesosphere.
\end{abstract}

\section{Introduction}

Several detailed studies have recently been published on the motion of air through the stratospheric polar vortex [e.g. Bacmeister et al., 1995; Fisher et al., 1993]. They describe the classical picture of poleward motion of winter mesospheric air and subsequent descent into the polar vortex. However, as discussed by Randel [1993], significant controversy remains regarding the degree of isolation of vortex air, and descent rates and patterns in the vortex. Several recent studies (as well as this Letter) deal specifically with the development of the stratospheric 1991/92 Arctic vortex [e.g. Rosenfield et al., 1994; Lahoz et al., 1994]. The dynamics of the high latitude winter and spring mesosphere has been less well studied. Previous observations, e.g. the HALOE NO results presented by Siskind and Russell [1996], have shown descent of upper atmospheric air into the stratosphere. However, to our knowledge, the signature of this descent within the mesosphere has not

\footnotetext{
${ }^{1}$ Naval Research Laboratory, Washington, DC.

${ }^{2}$ George Mason University, Fairfax, VA.

${ }^{3}$ Computational Physics, Inc., Fairfax, VA.

${ }^{4}$ University of Berne, Switzerland.

${ }^{5}$ European Space Agency, Nordwijk, Netherlands.

${ }^{6}$ Max Planck Institute for Aeronomy, Lindau, Germany.

${ }^{7}$ University of Bremen, Germany.

${ }^{8}$ NASA Langley Research Center, Hampton, VA.
}

Copyright 1996 by the American Geophysical Union.

Paper number 96GL01571

0094-8534/96/96GL-01571\$05.00 been reported. Furthermore, the containment properties of the winter mesosphere are not well understood.

The Millimeter-wave Atmospheric Sounder (MAS) experiment is part of the NASA ATLAS mission, and has flown on ATLAS $1(3 / 24 / 92-4 / 2 / 92)$, ATLAS 2 (4/8/93-4/17/93), and ATLAS 3 (11/3/94-11/4/94). One noteworthy feature of MAS is that it measures the $183 \mathrm{GHz} \mathrm{H}_{2} \mathrm{O}$ spectral line (as well as ozone at $184 \mathrm{GHz}$ ) at high spectral resolution $(200 \mathrm{kHz})$. These high spectral resolution measurements enable the $\mathrm{H}_{2} \mathrm{O}$ profiles to be retrieved up to $80 \mathrm{~km}$ [Bevilacqua et al., this issue]. The high altitude retrieval capability is significant because, in addition to its well documented usefulness as a tracer of vertical motions in the stratosphere, $\mathrm{H}_{2} \mathrm{O}$ has also been shown to be well suited for this purpose in the mesosphere [e.g. Bevilacqua et al., 1990]. In this Letter, we examine the MAS stratospheric and mesospheric $\mathrm{H}_{2} \mathrm{O}$ measurements made during the ATLAS 1 mission in the vicinity of the dissipating Arctic vortex.

\section{Dynamical State of the Arctic Vortex During ATLAS 1}

Computations of potential vorticity (pv) on levels of potential temperature $(\Theta)$ between 450 and $2200 \mathrm{~K}$ have been made for the days of our $\mathrm{H}_{2} \mathrm{O}$ observations using the Goddard Space Flight Center (GSFC) pv analysis routines [Newman et al., 1988]. These analyses show that the Arctic vortex was in advanced stages of dissipation at the end of March 1992. The vortex was intact, although weak, between about 500 and $800 \mathrm{~K}$ by March 25. The dissipation then rapidly progressed downward from the upper boundary, and was evident as low as the $700 \mathrm{~K}$ level on March 27. This dissipation from the upper stratosphere downward is typical of late winter/early spring conditions and has been reported by Manney et al. [1994]. Thus, during the ATLAS 1 mission, an intact, though weak, vortex was present only between approximately $500 \mathrm{~K}$ and $650 \mathrm{~K}$.

In order to illustrate the stratospheric meteorological situation during ATLAS 1 in more detail, in Figure 1 we present contours of pv on 27 March for the lower stratosphere (1a), the mid stratosphere (1b), and upper stratosphere/lower mesosphere (1c). At $600 \mathrm{~K}$, a coherent area of high pv can be seen over Siberia. At this level, the region of maximum winds $(40 \mathrm{~m} / \mathrm{s})$ is coincident with the region of steep pv gradients, which indicates that the vortex was still in an intact configuration. In the mid stratosphere and upper stratosphere/lower 

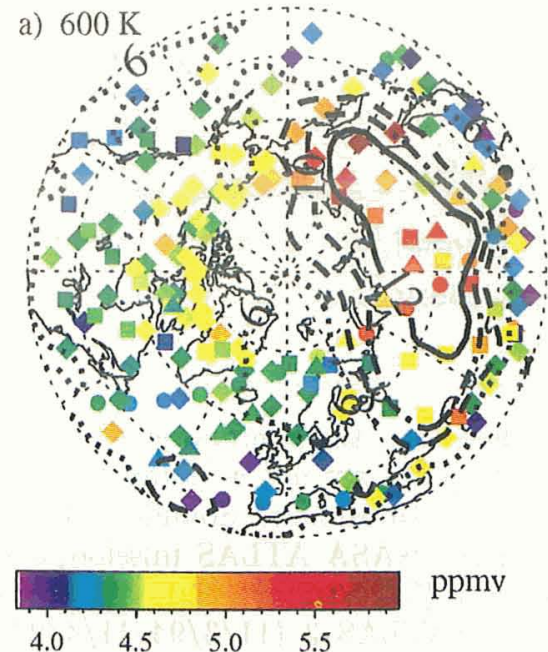
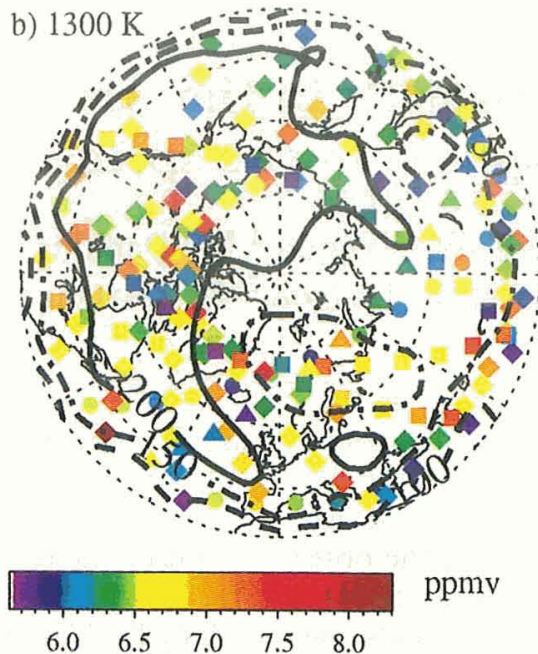
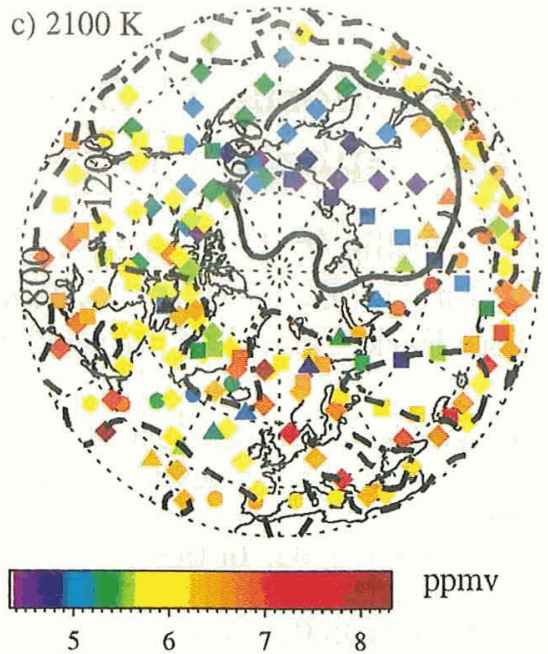

Figure 1. Potential vorticity $\left[10^{-5} \mathrm{Km}^{2} \mathrm{~kg}^{-1} \mathrm{~s}^{-1}\right]$ for 27 March 1992 as derived from the NMC analysis for the (a) $600 \mathrm{~K}$, (b) $1300 \mathrm{~K}$, and (c) $2100 \mathrm{~K} \Theta$-levels. The contour levels are 6, 8, 10, and 12 in (a); 100, 150, and 200 in (b); and 800,1200, and 1600 in (c). Maximum contour levels are in solid lines. The color symbols show MAS $\mathrm{H}_{2} \mathrm{O}$ volume mixing ratios on the respective level measured on March $25(\circ), 27(\diamond), 29(\square)$, and on April $1(\triangle)$.

mesosphere (Figures 1b/1c) there are regions of high pv, but pv gradients appear weak.

\section{MAS $\mathrm{H}_{2} \mathrm{O}$ Observations}

MAS is a limb-scanning passive microwave spectrometer that measures $\mathrm{H}_{2} \mathrm{O}(183 \mathrm{GHz})$, O3 $(184 \mathrm{GHz})$, $\mathrm{ClO}(204 \mathrm{GHz})$, as well as temperature and pressure (near $60 \mathrm{GHz}$ ). The MAS atmospheric limb-scan measurement time is $12.8 \mathrm{~s}$. However, in order to increase the signal-to-noise ratio in the mesospheric retrievals, in this study we have averaged the radiances obtained in ten successive MAS measurement cycles before performing the mixing ratio retrieval. Thus, each $\mathrm{H}_{2} \mathrm{O}$ mixing ratio profile represents 128 seconds of alongthe-orbital-track integration, which results in a spatial resolution of approximately $900 \mathrm{~km}$. Retrievals were obtained between 17 and $80 \mathrm{~km}$ at a $3 \mathrm{~km}$ vertical resolution. The $\mathrm{H}_{2} \mathrm{O}$ retrievals were then interpolated onto a vertical grid of $\Theta$, using National Meteorological Center (NMC) pressure and temperature data below $0.4 \mathrm{mb}$ and MSISE [Hedin, 1991] model results above $0.4 \mathrm{mb}$. The locations of the northern hemisphere (NH) mid and high latitude retrievals can be seen in Figure 1. MAS obtained a good sampling of measurements inside and outside of both the lower stratospheric vortex and the region of enhanced pv located in the upper stratosphere/lower mesosphere.

\section{Results and Discussion}

In Figure 2, examples of one tropical and two northern high latitude $\mathrm{H}_{2} \mathrm{O}$ profiles are shown. The tropical profile (a) is a zonal average, whereas (b) and (c) represent single high latitude retrievals obtained inside and outside the lower stratospheric vortex, respectively. The error bars represent the $1 \sigma$ standard deviation of the latitudinal average profile and, thus, are conservative estimates of the precision of an individual retrieval.
Profile (a) shows a $\mathrm{H}_{2} \mathrm{O}$ distribution that is typical of low latitudes. In the stratosphere, the volume mixing ratio increases with altitude, as a result of oxidation of methane $\left(\mathrm{CH}_{4}\right)$, and forms a broad maximum in the upper stratosphere/lower mesosphere. Above the peak, in the mesosphere, $\mathrm{H}_{2} \mathrm{O}$ decreases due to photodissociation. While basically similar to the tropical profile, the high latitude profiles show the following distinct features: (1) The lower to mid stratospheric (500-1500 K) $\mathrm{H}_{2} \mathrm{O}$ volume mixing ratio (vmr) is higher in the high latitude profiles than in the tropical average, (2) the upper stratospheric/mesospheric (2000-4000 K) $\mathrm{H}_{2} \mathrm{O} \mathrm{vmr}$ is lower in the high latitude profiles than in the tropical profiles, and (3) the peak in the $\mathrm{H}_{2} \mathrm{O}$ vmr vertical profile occurs at a lower altitude in high latitudes than in the tropics. These three main features may be explained by the mean middle atmospheric meridional circulation pattern consisting of flow toward the winter pole in the upper mesosphere, and descent into the winter stratosphere at high latitudes. In addition to

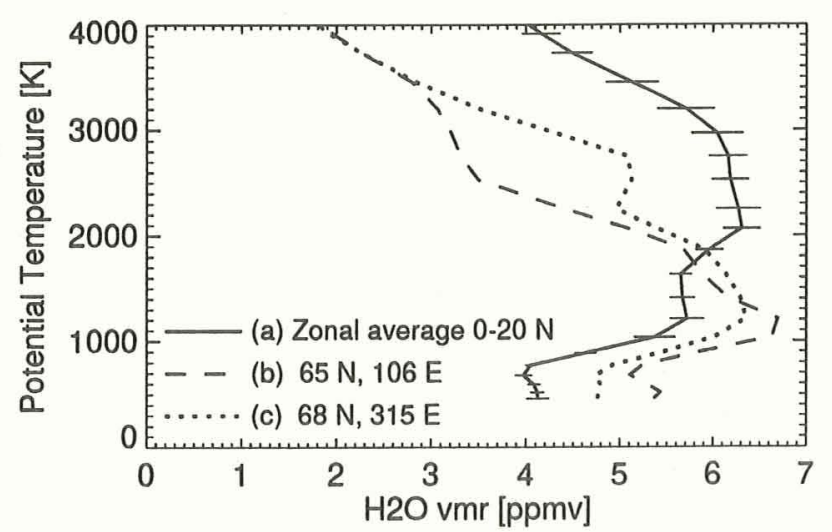

Figure 2. Average tropical $\mathrm{H}_{2} \mathrm{O}$ volume mixing ratio profile (a), and individual high latitude retrievals (b, c) measured by MAS. 

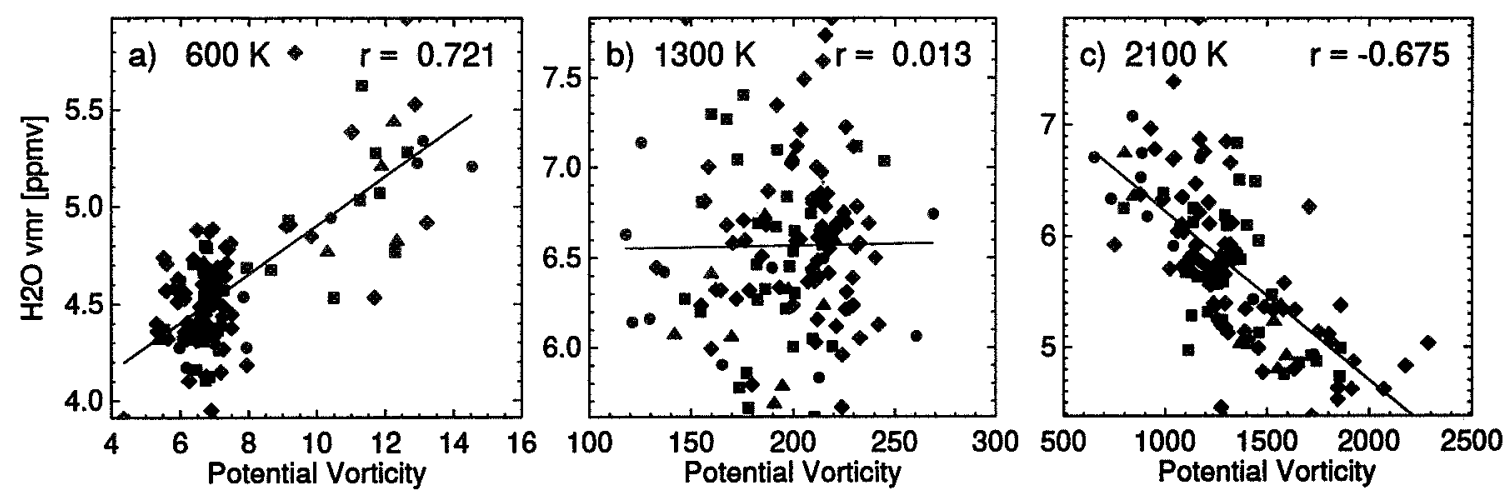

Figure 3. MAS $\mathrm{H}_{2} \mathrm{O}$ volume mixing ratio versus NMC potential vorticity $\left[10^{-5} \mathrm{Km}^{2} \mathrm{~kg}^{-1} \mathrm{~s}^{-1}\right]$ for (a) $600 \mathrm{~K}$, (b) $1300 \mathrm{~K}$, and (c) $2100 \mathrm{~K}$. Only observations made in the north of $45 \mathrm{~N}$ were used. The symbols are the same as in Figure 1. The regression line and correlation coefficient, $r$, are also indicated.

the general latitudinal features, similar differences, but less pronounced, between the high latitude vortex profile (b) and out of the vortex profile (c) are evident. In particular, profile (b) has higher $\mathrm{H}_{2} \mathrm{O}$ vmr over the vertical extent of the vortex $(500-650 \mathrm{~K})$ and also lower $\mathrm{H}_{2} \mathrm{O}$ vmr above $2000 \mathrm{~K}$, than profile (c).

Figure 1 displays polar projections of $\mathrm{H}_{2} \mathrm{O} \mathrm{vmr}$, in addition to the contours of pv, on the $600 \mathrm{~K}, 1300 \mathrm{~K}$, and $2100 \mathrm{~K} \Theta$-levels. Figure 1a shows a region of enhanced $\mathrm{H}_{2} \mathrm{O}$ in the lower stratosphere, coincident with the region of high pv. This enhancement of $\mathrm{H}_{2} \mathrm{O}$ in the lower stratospheric vortex was also seen in HALOE version $17 \mathrm{H}_{2} \mathrm{O}$ observations. In fact, the details of the region of high $\mathrm{H}_{2} \mathrm{O}$ deduced from the HALOE $\mathrm{H}_{2} \mathrm{O}$ observations are remarkably consistent with that indicated by the MAS $\mathrm{H}_{2} \mathrm{O}$ measurements at $600 \mathrm{~K}$. This enhancement is explained by descent during the winter, which transports moist air from the upper stratosphere down into the lower stratosphere. The vortex, which was still intact between 500 and $650 \mathrm{~K}$ during the time of our measurements, has preserved the high $\mathrm{H}_{2} \mathrm{O}$ inside the vortex.

In Figure $1 \mathrm{~b}$, the $\mathrm{H}_{2} \mathrm{O}$ distribution along with contours of $\mathrm{pv}$ can be seen at the $1300 \mathrm{~K}$ surface. No coherent pattern in the $\mathrm{H}_{2} \mathrm{O}$ distribution is evident. Measurements of low and high $\mathrm{H}_{2} \mathrm{O} \mathrm{vmr}$ are not apparently correlated to either geographic location or pv values.

Figure 1c shows the $\mathrm{H}_{2} \mathrm{O}$ distribution and pv contours at the $2100 \mathrm{~K}$ surface. As mentioned earlier, although no vortex is expected at these altitudes at this time of the year, there is a region of high pv centered over East Siberia. We find depressed $\mathrm{H}_{2} \mathrm{O}$ values in good congruence with this region of high pv. The relatively low $\mathrm{H}_{2} \mathrm{O}$ values may be indicative of descent at the $2100 \mathrm{~K}$ surface, because this level is above the $\mathrm{H}_{2} \mathrm{O}$ vmr peak at winter high latitudes (see Figure 2). Thus, the $\mathrm{H}_{2} \mathrm{O}$ observations provide evidence that the region of high pv at this level may be a vortex remnant.

In order to examine more closely the relationship between $\mathrm{H}_{2} \mathrm{O}$ vmr and pv, in Figure 3 we present scatter plots of $\mathrm{H}_{2} \mathrm{O} \mathrm{vmr}$ and pv for the same $\Theta$-surfaces used in Figure 1. In the plots we use the 115 MAS ATLAS 1 $\mathrm{H}_{2} \mathrm{O}$ results obtained poleward of $45 \mathrm{~N}$ latitude. Each MAS measurement point is correlated with the GSFC pv value obtained for the day and location of the measurement. Figure 3 shows quantitatively the correlation between $\mathrm{H}_{2} \mathrm{O}$ vmr and pv suggested in Figure 1 and 2 . At $600 \mathrm{~K}$, where the vortex was still intact, positive correlation of $\mathrm{H}_{2} \mathrm{O}$ and $\mathrm{pv}$ is evident, consistent with the suggestion of stratospheric descent and of containment of the vortex air. The correlation coefficient, $r$, for the regression analysis is 0.721 . For reference, in a random sample of 115 from an uncorrelated parent population, a value of $|r|$ exceeding 0.32 is significant on the $99.9 \%$ level. Thus, the present correlation is statistically significant.

At $1300 \mathrm{~K}$, the correlation between the two fields is virtually zero. However $1300 \mathrm{~K}$ is, roughly, near the broad $\mathrm{H}_{2} \mathrm{O}$ vmr peak (see Figure 2). Thus, in this region, $\mathrm{H}_{2} \mathrm{O}$ is not a particular good tracer of vertical motions. However, $\mathrm{CH}_{4}$ is an excellent tracer of vertical motion in the mid and upper stratosphere because of its steep and consistent vertical gradient. We have also performed a regression analysis between pv and HALOE $\mathrm{CH}_{4}$ measurements obtained during the ATLAS-1 time frame in the high latitude NH. We find a strong anticorrelation between pv and $\mathrm{CH}_{4}$ at all levels between 800 and $1500 \mathrm{~K}$. Since the $\mathrm{CH}_{4}$ mixing ratio decreases rapidly with altitude in the stratosphere, this negative correlation is indicative of wintertime descent

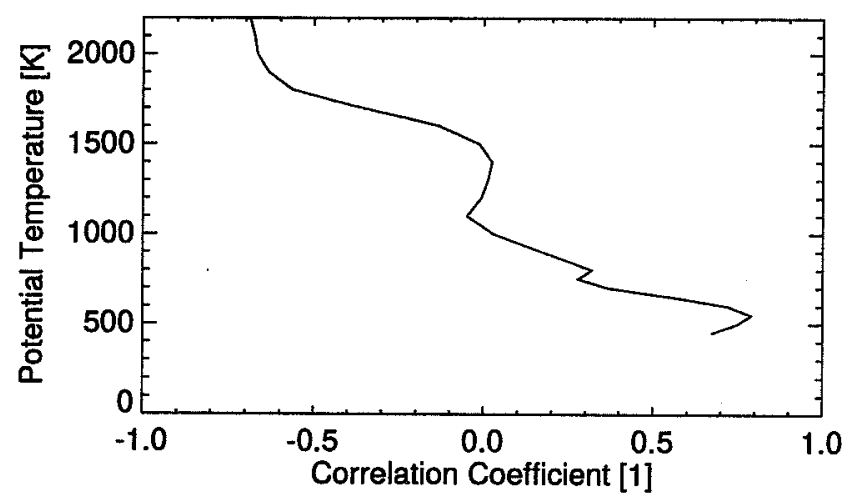

Figure 4. Correlation coefficient, $r$, of $\mathrm{H}_{2} \mathrm{O}$ volume mixing ratio and potential vorticity as a function of potential temperature, $\Theta$. 
in the vortex, which has not yet been completely dissipated by horizontal mixing after the vortex break up.

At $2100 \mathrm{~K}$, we find a pronounced $\mathrm{H}_{2} \mathrm{O} \mathrm{vmr} / \mathrm{pv}$ anticorrelation. Although no pv analysis is available above approximately $2200 \mathrm{~K}$, regions of depressed $\mathrm{H}_{2} \mathrm{O}$ are evident at least up to $3000 \mathrm{~K}$ (Figure 2). Again, we believe that this significant anticorrelation may be the residual effects of descent and containment of mesospheric air which occurred within the vortex during the winter. This is consistent with the vortex analyses by Bacmeister et al. [1995] and Fisher et al. [1993], which suggest mesospheric descent into the stratospheric polar vortex. Evidence of containment in the mid to high latitude winter mesosphere is also given in model studies. 2-D models [e.g. Garcia et al. 1992] incorporating planetary wave parametrization suggest low values of $\mathrm{K}_{y y}$ (meridional mixing coefficient) on the poleward flank of the mesospheric polar night jet. This finding implies a certain degree of isolation in the polar winter mesosphere and is consistent with our $\mathrm{pv} / \mathrm{H}_{2} \mathrm{O}$ correlation analysis.

In order to summarize the $\mathrm{H}_{2} \mathrm{O} / \mathrm{pv}$ regression analysis discussed above, in Figure 4, we plot $r$ as a function of $\Theta$. It shows strong correlation in the $450 \mathrm{~K}$ to $625 \mathrm{~K}$ region, strong anticorrelation above $1850 \mathrm{~K}$, and a transition region between the two regimes between $800 \mathrm{~K}$ and $1700 \mathrm{~K}$. As a check on the MAS observations, we have also performed a linear regression analysis between pv and the HALOE $\mathrm{H}_{2} \mathrm{O}$ data. The HALOE profile generally shows the same basic features as the MAS profile displayed in Figure 4, with positive $\mathrm{H}_{2} \mathrm{O} / \mathrm{pv}$ correlation in the lower stratosphere, no correlation in the mid stratosphere, and negative correlation in the upper stratosphere/lower mesosphere. Differences are evident, however, in the vertical extent of the region with high correlation, and in the degree of the correlation. In particular, the region of transition from positive to negative correlation occurs at a higher altitude in the HALOE data than in the MAS data. Since the transition is expected to correspond roughly to the altitude of the vmr peak, this may be consistent with the fact that (at high latitudes) there is a disagreement between HALOE and MAS $\mathrm{H}_{2} \mathrm{O}$ data in the altitude of the vmr peak, with the HALOE peak generally several $\mathrm{km}$ higher than that obtained in the MAS data.

\section{Conclusions}

The major finding of this paper is the evidence of descent in the upper stratosphere/lower mesosphere $(1850 \mathrm{~K}-2200 \mathrm{~K})$ that was observed in the MAS $\mathrm{H}_{2} \mathrm{O}$ measurements within a vortex remnant. This suggests that effective vortex containment may extend well into the mesosphere. Although the high altitude portion of the stratospheric vortex had dissipated in the weeks prior to the MAS observations presented in this Letter, the vortical descent signature had evidently not yet been completely dissipated by the horizontal mixing occurring subsequent to the vortex break up. Previous observations, e.g. the HALOE NO results presented by Siskind and Russell [1996], have shown descent of upper atmospheric air into the stratosphere. However, to our knowledge, the signature of descent within the mesosphere has not been reported.

Acknowledgments. The authors wish to acknowledge two anonymous reviewers for their constructive reviews. The HALOE data used in this letter is courtesy of the EOS Distributed Active Archive Center at GSFC. C.P. Aellig is supported by the Swiss National Science Foundation.

\section{References}

Bacmeister, J.T., M.R. Schoeberl, M.E. Summers, J.R. Rosenfield, and X. Zhu, Descent of long-lived trace gases in the winter polar vortex, J. Geophys. Res., 100, 1166911684, 1995.

Bevilacqua, R.M., D.F. Strobel, M.E., Summers, J.J. Olivero, and M. Allen, The seasonal variation of water vapor and ozone in the upper mesosphere: Implications for vertical transport and ozone photochemistry, J. Geophys. Res., 95, 883-893, 1990.

Bevilacqua, R.M., et al., MAS measurements of the latitudinal distribution of water vapor and ozone in the mesosphere and lower thermosphere, Geophys. Res. Lett., this issue, 1996.

Fisher, M., A. O'Neill, and R. Sutton, Rapid descent of mesospheric air into the stratospheric polar vortex, Geophys. Res. Lett., 20, 1267-1270, 1993.

Garcia, R.R., F. Stordal, S. Solomon, and J.T. Kiel, A new numerical model of the middle atmosphere, 1 , dynamics and transport of tropospheric source gases, J. Geophys. Res., 97, 12967-12991, 1992.

Hedin, A.E., Extension of the MSIS thermosphere model into the middle and lower atmosphere, J. Geophys. Res., 96, 1159-1172, 1991.

Lahoz, W.A., et al., Three-dimensional evolution of water vapor distributions in the northern hemisphere stratosphere as observed by the MLS, J. Atmos. Sci., 51, 29142930, 1994.

Manney, G.L., R.W. Zurek, A. O'Neill, R. Swinbank, On the motion of air through the stratospheric polar vortex, J. Atmos. Sci., 51, 2973-2994, 1994.

Newman, P.A., et al., Meteorological atlas of the southern hemisphere lower stratosphere for August and September 1987, NASA Technical Memorandum 4049, 131 pp., 1988.

Randel, W., Ideas flow on Antarctic vortex, Nature, 364, 105-106, 1993.

Rosenfield, J.E., P.A. Newman, and M.R. Schoeberl, Computations of diabatic descent in the stratospheric polar vortex, J. Geophys. Res., 99, 16677-16689, 1994.

Siskind, D.E., and J.M. Russell, Coupling between middle and upper atmospheric NO: Constraints from HALOE observations, Geophys. Res. Lett., 23, 137-140, 1996.

C.P. Aellig, R.M. Bevilacqua, code 7220, Naval Research Laboratory, Washington, DC 20375-5351. (e-mail: aellig@quate.nrl.navy.mil, bevilacqu@map.nrl.navy.mil)

J. Bacmeister, code 7641, Naval Research Laboratory, Washington, DC 20375-5351. (e-mail:

julio@ismap5.nrl.navy.mil)

(received September 22, 1995; revised April 19, 1996; accepted April 30, 1996.) 


\title{
Zeeman splitting of the 61 Gigahertz Oxygen $\left(\mathrm{O}_{2}\right)$ line in the mesosphere.
}

\author{
G. K. Hartmann, W. Degenhardt, M. L. Richards \\ Max-Planck-Institut für Aeronomie, Katlenburg-Lindau, Germany \\ H. J. Liebe, G. A. Hufford, M. G. Cotton
}

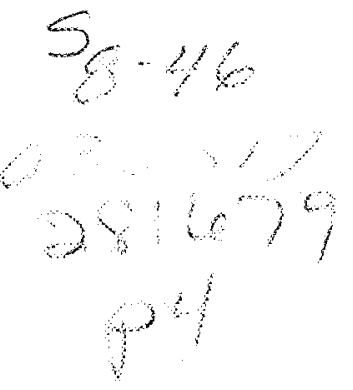

National Telecommunications and Information Administration, Institute for Telecommunication Sciences, Boulder, CO, USA

\author{
R. M. Bevilacqua \\ Naval Research Laboratory, Washington, DC, USA
}

\section{J. J. Olivero}

Dept. of Physical Science, Embry-Riddle Aeronautical University, Daytona Beach, FL, USA

\section{N. Kämpfer}

IAP University Bern, Bern, Switzerland

\author{
J. Langen \\ IUP, University Bremen, Bremen, Germany
}

\begin{abstract}
Zeeman splitting of $\mathrm{O}_{2}$ molecular states in the Earth's upper atmosphere leads to polarized emission spectra. A $61 \mathrm{GHz}$ radiometer operated as part of the Millimeter-wave Atmospheric Sounder (MAS), a core payload instrument of the NASA Space Shuttle ATLAS missions, observed such emissions. This instrument's high resolution spectrometer $(200 \mathrm{kHz})$ allows us to verify for the first time Zeeman effect model calculations for the upper atmosphere in some detail. The results suggest some interesting new aspects for the research of the upper atmosphere.
\end{abstract}

\section{Introduction}

J.W. Waters [1973] reported that Zeeman splitting of millimeter-wavelength emission of oxygen molecules must be taken into account for altitudes above $45 \mathrm{~km}$ in the terrestrial atmosphere, in modeling the radiative transfer of these molecules. Because of their inherent magnetic dipole moments, oxygen molecules interact with the Earth's magnetic field in the mesosphere and lower thermosphere leading to complex Zeeman refractivity patterns [Townes and Schawlow, 1955; Rosenkranz and Staelin, 1988; Hufford and Liebe, 1989]. Almost 20 years later, this assertion can be verified by the Millimeter-wave Atmospheric Sounder (MAS) ex-

Copyright 1996 by the American Geophysical Union.

Paper number 96GL01043

0094-8534/96/96GL-01043\$05.00 periment [Hartmann, 1988, Croskey et al, 1992], as a part of the shuttle ATLAS-1 (Atmospheric Laboratory for Applications and Science) mission. MAS was designed to measure important trace gases in the middle atmosphere, such as ozone, water vapor, and chlorine monoxide. It also uses the emissions from molecular oxygen to infer pressure and temperature. Here we show that MAS can also obtain new information in the upper atmosphere (above $80 \mathrm{~km}$ ).

\section{Method}

We use calculations of oxygen emission spectra from the Zeeman Propagation Model (ZPM) [Liebe and Hufford, 1989; Liebe et al, 1993] to compare with selected MAS brightness temperature observations of the $9^{+}$line in the $61.1506 \pm 0.002 \mathrm{GHz}$ frequency range. These spectra were obtained for heights between 70 and 115 $\mathrm{km}$. ZPM was run using the U.S. standard atmosphere [COESA, 1976] and the International Reference Geomagnetic Field from 1985 [Barraclough, 1985]. The temperature and pressure profiles used for the analyses, as well as the mixing ratios of the absorber molecule, $\mathrm{O}_{2}$ between 50 and $130 \mathrm{~km}$ altitude, were taken from the US Standard Atmosphere 1976.

\section{Results}

Figure 1 shows two 1 minute data samples of the $9^{+}$ $61 \mathrm{GHz} \mathrm{O}_{2}$ line, obtained during the ATLAS-1 mission on March 27th, 1992. 


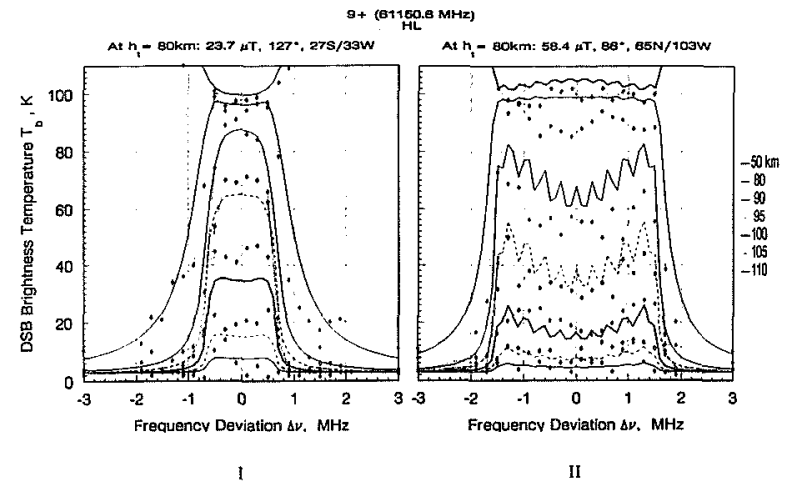

Figure 1. Mesospheric $\mathrm{O}_{2}$ Line Emission at Min. and Max. Magnetic Field Locations (ZPM vs. MAS data).

Part I was taken at a tangential height of $80 \mathrm{~km}$, at $27^{\circ} \mathrm{S}$ and $33^{\circ} \mathrm{W}$, where the Earth magnetic field strength was $23.7 \mu \mathrm{T}$ and the angle between the magnetic field and the direction of observation $127^{\circ}[\mathrm{Barr}$ aclough, 1985]. Part II was taken at $65^{\circ} \mathrm{N}$ and $103^{\circ} \mathrm{W}$ and $58.4 \mu \mathrm{T}$ and at a propagation angle of $86^{\circ}$, which is, therefore, approximately the perpendicular propagation condition. The lines show the ZPM model results for 50 $\mathrm{km}, 80 \mathrm{~km}, 90 \mathrm{~km}, 100 \mathrm{~km}, 105 \mathrm{~km}$, and $110 \mathrm{~km}$, and the diamonds are the actual MAS observations. ZPM data were calculated for a linear horizontal polarized (HL) observing antenna in steps of $10 \mathrm{kHz}$. We wish to stress the similarity in shape between calculated and observed spectra, and the contrast in width for the two cases.

The $9^{+}$line splits into $19\left(\sigma^{-}\right)$left hand circular components, $19\left(\sigma^{+}\right)$right hand circular components and

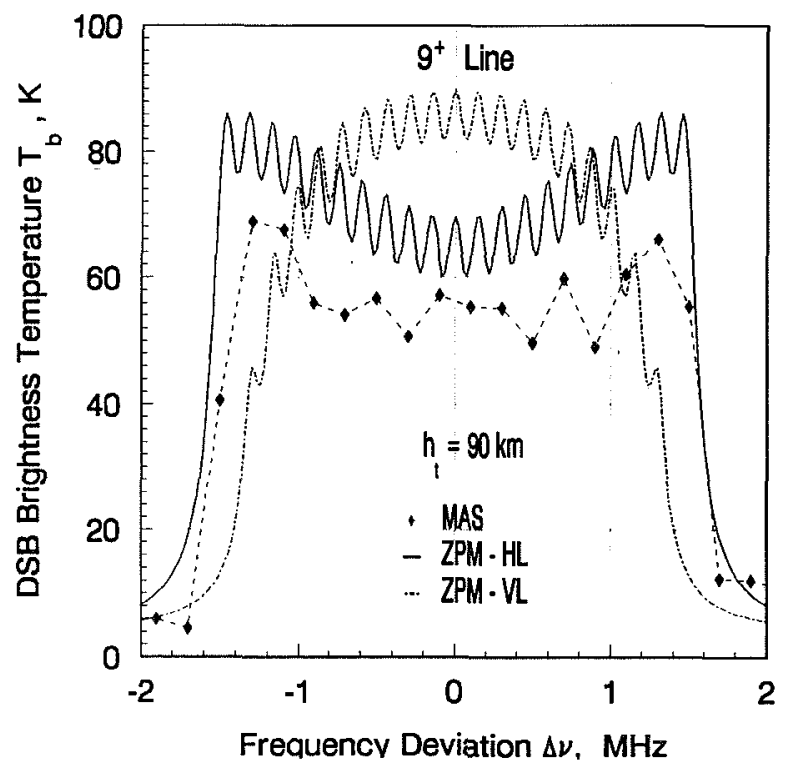

Figure 2. As in Fig. 1 part II except ZPM results for high resolution in both horizontal linear and vertical linear polarizations and MAS observations (black diamonds). $19(\pi)$ linear polarized components [Hufford and Liebe, 1989], where individual line amplitude and frequency offset from line center are determined by total angular and azimuthal momentum quantum numbers. Instead of one doppler and pressure broadened $\mathrm{O}_{2}$ line (Voigt line shape), we record a combination of 57 such lines, where the combination depends on the observing geometry. This scale of splitting cannot be resolved in the MAS Brightness Temperature spectra by the $200 \mathrm{kHz}$ MAS filters, and is not indicated in figure 1, but the resulting envelope of their combination can be detected with sufficient resolution. Characteristic of this envelope is a region symmetric about the $9^{+}$line center. There the form can be convex (part I) or concave (part II), but the width of the region remains constant over the range of tangent heights at a given location, and the width varies with the total magnetic field. This is apparently the effect of the $\sigma^{+}$and $\sigma^{-}$Zeemann components, for which the maximum amplitude is expected for maximum frequency offset, and theory predicts the width to be directly proportional to the total field. Thus, comparing the central portions of the spectra in the cases II and I of figure 1, these are in the expected ratio of $\sim 2.5$.

To further verify the observation of Zeeman splitting, additional ZPM model calculations were carried out for vertical linear polarization (VL). The results for the same case as in figure 1, part II, at tangential height of $90 \mathrm{~km}$ are shown in figure 2 , together with the HL polarization results and MAS data. Despite the fact that the 1 minute $200 \mathrm{KHz}$ data samples are quite noisy the VL results are seen to be significantly different. The concave shape of the envelope from the model calculations is also visible in the data. It can be concluded that during the observation period MAS measured a horizontally linear polarized emission signal (as expected).

For a more quantitative confirmation of the total magnetic field dependence, the data from four orbits

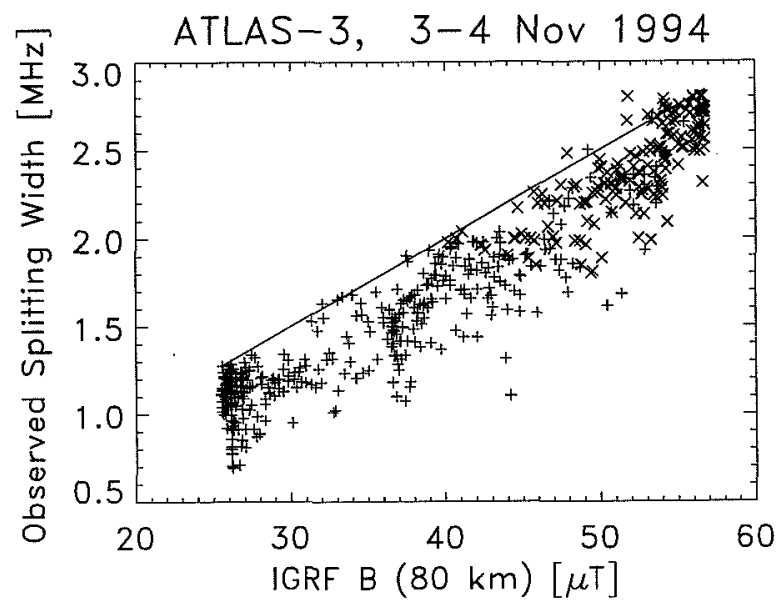

Figure 3. Width of the $9^{+}$lines as a function of the The solid line represents the theoretical width. 


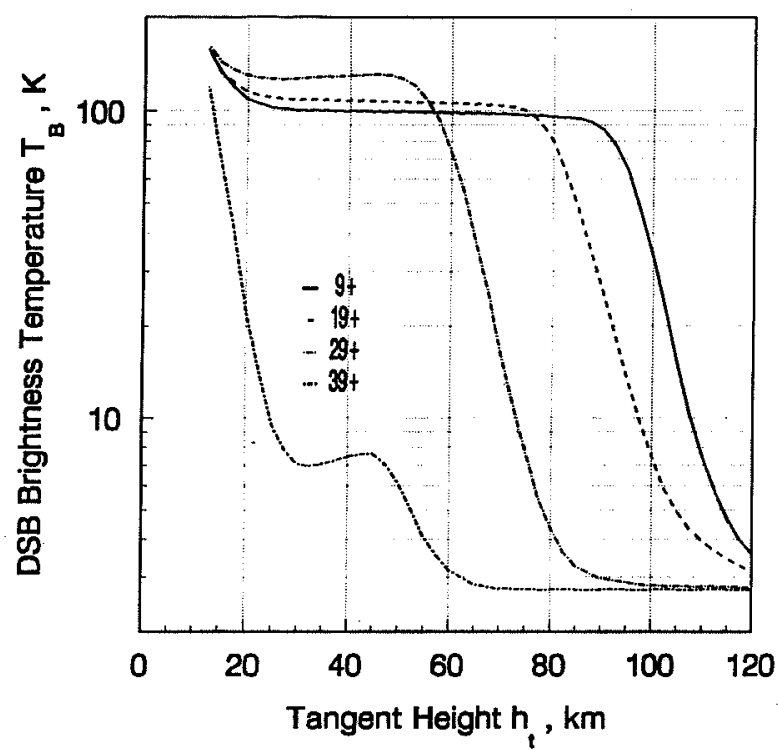

Figure 4. Brightness temperature for various $\mathrm{O}_{2}$ lines as a function of the tangent height calculated with the ZPM model.

during the more recent ATLAS-3 flight on Nov 4, 1994 were analyzed in more detail. Figure 3 shows the width variation with IGRF field for these data. Individual points represent 12.8 seconds of data for the tangent height interval 78.5 to $81.5 \mathrm{~km}$. From the theory it is expected that the width equals $0.05 \times B_{0}$ where $B_{0}$ is the total magnetic strength; these results can be summarized as widt $h \leq 0.05 \times B_{0}$. In addition to the spread due to noise there is a bias, for which one suggestion is that $\mathrm{O}_{2}$ line interference in overlapping regions reduces the signal [Rosenkranz and Staelin, 1988]. We exploit the concave shape of the envelope to estimate the width as the frequency separation of the strongest expected Zeeman components, which are also the furthest components either side of line center. Our procedure minimizes Doppler Broadening influence but tends to underestimate the width somewhat. Another suggestion for the bias is that the IGRF model overestimates the field strength at these altitudes. To test that hypothesis versus estimation bias, one must employ a detailed model such as ZPM.

As a further comment, simple limb viewing radiative transfer arguments suggest that the ambient temperature at about $80 \mathrm{~km}$ can be estimated directly using the $9^{+} \mathrm{O}_{2}$ line, divided by the sideband ratio of the receiver, which is about 0.5 . If one were to measure simultaneously the $\mathrm{I}^{-}$line $(118.7503 \mathrm{GHz})$, one could also estimate the ambient temperature at about $95 \mathrm{~km}$. Likewise, if one also measures the $25^{+}$line $(65.7648 \mathrm{GHz})$, one could have an estimate of the ambient temperature at about $60 \mathrm{~km}$. Figure 4 summarizes the idea, using the same ZPM results as in figure 1 .

The brightness temperature at the line center for the $39^{+}$line $(69.49 \mathrm{GHz})$ the $29^{+}$line $(66.83 \mathrm{GHz})$, the $19^{+}$ line $(\mathrm{GHz})$ and the $9+$ line $(61.15 \mathrm{GHz})$ are displayed as a function of the tangent height $h_{t}$. Figure 4 shows that the height sensitive portion of the $9^{+}$line lies above 85 $\mathrm{km}$ (for the $19^{+}$line above $75 \mathrm{~km}$ ). There the atmosphere is transparent. Below $80 \mathrm{~km}$ the atmosphere is opaque, and the transition point between opaque and transparent, the "knee" of the curves can be used to calculate the ambient temperature at that height. For a perfect double side band receiver this implies that one multiplies the observed brightness temperature by two. Data from the four ATLAS-3 orbits show clearly the knee in about $80 \mathrm{~km}$ altitude.

\section{Concluding Discussions}

We have made use of the relatively high frequency resolution of the MAS radiometer-spectrometer [Hartmann et al., 1985; Hartmann, 1988; Croskey et al., 1992; NASA, 1993] (the $200 \mathrm{kHz}$ filters are a factor of 10 higher in resolution than microwave spectrometers hitherto used in space applications) to verify Zeeman effect model calculations for the $\mathrm{O}_{2}$ molecule above 70 $\mathrm{km}$. This is the first time that such model calculations could be verified in some detail.

In addition, simple limb viewing radiative transfer arguments suggest that the ambient temperature at about $80 \mathrm{~km}$ can be estimated directly using the $9^{+} \mathrm{O}_{2}$ line. If one were to simultaneously measure the $\mathrm{I}^{-}$line, one could also estimate the ambient temperature at about $95 \mathrm{~km}$. Likewise, if one also measures the $25^{+}$line, one could have an estimate of the ambient temperature at about $60 \mathrm{~km}$. These three temperatures could be monitored continuously and globally from space. The geographical resolution would improve with lower noise radiometers, which now can be made by using modern semiconductor technology.

Acknowledgments. The MAS-PI Dr. G. K. Hartmann and the Co-PIs Dr. N. Kämpfer, Dr. P. R. Schwartz, Prof. Dr. K. F. Künzi and the project manager Dr. G. Schneppe, Deutsche Agentur für Raumfahrtangelegenheiten (DARA), and the other MAS Co-investigators like to thank the U.S. Space Agency NASA, the German Space Agency DARA, all involved contractors - coordinated by Dornier GmbH - and all contributing research institutions for their support. Dr. H. J. Liebe, Dr. G. A. Hufford, and M. G. Cotton like to thank NTIA/ITS for supporting this work.

\section{References}

Barraclough, D. R., International Geomagnetic Reference Field, Pure and Applied Geophysics, 123, 641-645, 1985.

COESA, U.S., Committee on Extension to the Standard Atmosphere, NOAA-S/T 76-1562, Gov. Printing office, Washington, D.C., Sup. Docs. Stock No. 003-017-00232$0,1976$.

Croskey, C. L., N. Kämpfer, R. M. Bevilacqua, G. K. Hartmann, K. F. Künzi, P. R. Schwartz, J. J. Olivero, S. E. Puliafito, C. Aellig, G. Umlauft, W. B. Waltman, and W. Degenhardt, The Millimeter-wave Atmo- 
spheric Sounder (MAS): A Shuttle-based remote sensing experiment, IEEE Transactions on Microwave Theory and Techniques, 40, 6, 1090-1100, 1992.

Hartmann, G. K., K. F. Künzi, and R. P. Schwartz, Millimeterwellen-Atmosphären-Sondierer (MAS) für den Einsatz auf Space Shuttle (STS), Mikrowellen Magazin, 11, 3, 254-267, 1985.

Hartmann, G. K., Millimeter-Wave Atmospheric Sounder (MAS), In: Atmospheric Laboratory for Application and Science (ATLAS) Mission 1, Ed. P. D. Craven, and M. R. Torr, NASA Technical Memorandum 4101, NASA Scientific and Technical Information Division, I-27 - I-31, 1988.

Hufford, G. A., and H. J. Liebe, Millimeter-Wave Propagation in the Mesosphere, NTIA Report, 89-249, 1989.

Liebe, H. J., and G. A. Hufford, Modeling Millimeter-wave propagation effects in the Atmosphere, AGARD CP-454, 18, Copenhagen, Denmark, 1989.

Liebe, H. J., G. A. Hufford, and M. G. Cotton, Propagation modeling of moist air and suspended water/ice particles at frequencies below $1000 \mathrm{GHz}, A G A R D C P-542,3, \mathrm{Mal}-$ lorca, Spain, 1993.

NASA Marshall Space Flight Center (MSFC), ATLAS 1: Encountering Planet Earth, Payload Projects Office at NASA, Huntsville, Alabama, USA, 1993.

Rosenkranz, P. W., and D. H. Staelin, Polarized thermal microwave emission from oxygen in the mesosphere, Radio Sci., 23, 721-729, 1988.
Townes, C. H., and A. L. Schawlow, Microwave Spectroscopy, Mc Graw-Hill Company, 174-184, 1955.

Waters, J. W., Ground-based Measurement of Millimeterwavelength Emission by Upper Stratospheric $\mathrm{O}_{2}$, Nature, 242, 506-508, 1973.

G. K. Hartmann, W. Degenhardt, M. L. Richards Max-Planck-Institut für Aeronomie, PO Box 20, D-37189 Katlenburg-Lindau, Germany

H. J. Liebe, G. A. Hufford, M. G. Cotton, National Telecommunications and Information Administration, Institute for Telecommunication Sciences, 325 Broadway, Boulder, CO 80303, USA

R. M. Bevilacqua, Naval Research Laboratory, Code 4213 BE, Washington, DC 20375-5000, USA

J. J. Olivero, Dept. of Physical Science, Rm. A117, Embry-Riddle Aeronautical University, $600 \mathrm{~S}$. Clyde Morris Boulevard, Daytona Beach, FL 32114, USA

N. Kämpfer, IAP University Bern, Sidlerstr. 5, 3012 Bern, Switzerland

J. Langen, University Bremen, IUP, Postfach 3304 40, 28334 Bremen, Germany

(received September 27, 1995; revised February 16, 1996; accepted March 18, 1996.) 


\title{
The Atmospheric Trace Molecule Spectroscopy (ATMOS) experiment: Deployment on the ATLAS Space Shuttle missions
}

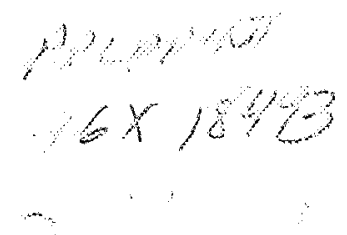

\author{
M. R. Gunson, ${ }^{1}$ M. M. Abbas, ${ }^{2}$ M. C. Abrams, ${ }^{3}$ M. Allen, ${ }^{1,4}$ L. R. Brown, ${ }^{1}$ \\ T. L. Brown, ${ }^{1}$ A. Y. Chang, ${ }^{1}$ A. Goldman, ${ }^{5}$ F. W. Irion, ${ }^{4}$ L. L. Lowes, ${ }^{1}$ E. Mahieu, ${ }^{6}$ \\ G. L. Manney, ${ }^{1}$ H. A. Michelsen, ${ }^{7}$ M. J. Newchurch, ${ }^{8}$ C. P. Rinsland, ${ }^{9}$ \\ R. J. Salawitch, ${ }^{1}$ G. P. Stiller, ${ }^{10}$ G. C. Toon, ${ }^{1}$ Y. L. Yung, ${ }^{4}$ and R. Zander ${ }^{6}$
}

\begin{abstract}
The ATMOS Fourier transform spectrometer was flown for a fourth time on the Space Shuttle as part of the ATLAS-3 instrument payload in November 1994. More than 190 sunrise and sunset occultation events provided measurements of more than 30 atmospheric trace gases at latitudes $3-49^{\circ} \mathrm{N}$ and $65-72^{\circ} \mathrm{S}$, including observations both inside and outside the Antarctic polar vortex. The instrument configuration, data retrieval methodology, and mission background are described to place in context analyses of ATMOS data presented in this issue.
\end{abstract}

\section{ATMOS Instrument}

The Atmospheric Trace MOlecule Spectroscopy (ATMOS) project was initiated in the 1970 s, when the central scientific objectives were to extend our base knowledge of the infrared spectral response of the stratosphere, measure nearly simultaneously the vertical profiles of many atmospheric constituents on a global scale, determine seasonal and annual variations in atmospheric composition, and quantify the catalytic cycles and other processes controlling ozone depletion. This goal led to the ATMOS instrument, a Fourier transform spectrometer first flown as part of the Spacelab 3 (SL-3) Space Shuttle payload in 1985. The current instrument remains essentially as described by Farmer [1987].

ATMOS was designed for operation in solar occultation mode, acquiring high-resolution, infrared solar absorption spectra of the Earth limb during orbital sunrises and sunsets. In a typical 4 minute observation period, close to 100 spectra are obtained with an apodized resolution of $\sim 0.01 \mathrm{~cm}^{-1}$. In low Earth orbit $(\sim 300 \mathrm{~km})$ from the Space Shuttle, the height spacing between successive ATMOS spectra varies between 4 $\mathrm{km}$ to less than $1 \mathrm{~km}$ at tangent heights in the troposphere, with typical values of $\sim 2 \mathrm{~km}$ at tangent heights through the

\footnotetext{
1Jet Propulsion Laboratory, California Institute of Technology, Pasadena, CA.

${ }^{2}$ NASA Marshall Space Flight Center, Huntsville, AL.

${ }^{3}$ SAIC, NASA Langley Research Center, Hampton, VA.

${ }^{4}$ California Institute of Technology, Pasadena, CA.

${ }^{5}$ Department of Physics, University of Denver, Denver, CO.

${ }^{6}$ Institute of Astrophysics, Univ. of Liège, Liège-Cointe, Belgium.

${ }^{7}$ Harvard University, Cambridge, MA.

${ }^{8}$ University of Alabama in Huntsville, Huntsville, AL.

${ }^{9}$ NASA Langley Research Center, Hampton, VA.

${ }^{10} \mathrm{IMK}$, Forschungszentrum Karlsruhe, Karlsruhe, Germany
}

Copyright 1996 by the American Geophysical Union.

Paper number 96GL01569

0094-8534/96/96GL-01569\$05.00 stratosphere. The projection of the instrument field-of-view $(1-2.8 \mathrm{mrad})$ at the tangent point limits the effective vertical resolution in retrieved profiles to $\sim 4 \mathrm{~km}$. The signal-to-noise ratios of the resulting spectra are in the range 250 to $100: 1$, depending on the optical bandpass filter used to select spectral regions between $600-4750 \mathrm{~cm}^{-1}$ (16.6 - 2.1 microns). Up to eight different optical filters were carried on each flight, with some changed between flights to extend the multiple species measurement within individual observations, while preserving the signal-to-noise ratio of the resulting spectra. Abrams et al. [this issue (a)] discuss the estimated precision reported with the ATMOS vertical profiles and compare these with the standard deviation of the mean of several profiles obtained at low latitudes. The altitude range over which ATMOS profiles are reported, together with a general guide to the precisions achieved for each gas are shown in Figure 1. The upper altitudes coincide with the first appearance of absorption features of a particular gas that vary slightly between successive occultations. The lower altitude limit is determined by a combination of factors: rapid increase in opacity through the troposphere, aerosols and cloud tops obscuring the sun, lack of accuracy in assigning tangent heights and pressures to individual spectra, and the difficulty in retrieving stratospheric constituent volume mixing ratios (vmrs) below their peak concentrations. Issues limiting the lower altitude range will be addressed in future analyses.

The data processing and inversion methods employed with the ATMOS data follow those described by Norton and Rinsland [1991]. As some changes have been incorporated to enhance routine data processing and to distinguish between this current dataset and previous published analyses, the ATMOS data utilized in the papers in this issue have been ascribed to 'Version 2'. In brief, after transformation of the interferograms to power spectra, an average exoatmospheric spectrum is formed for each occultation against which ratios are made with successive atmospheric spectra. The resulting atmospheric transmission spectra are essentially free of solar and instrumental spectral features. The inversion of these transmission spectra follows a general sequence: assignment of a first-guess tangent height based on spacecraft ephemeris data, refinement of the tangent height (or more correctly, tangent pressure) from spectral fitting to the observed $\mathrm{CO}_{2}$ absorption bands [Abrams et al., 1996], and retrieval of atmospheric temperature-pressure profiles [Stiller et al., 1995], followed by a final tangent pressure assignment. Retrievals of vmr profiles for individual species use spectral intervals that are chosen based on the shape and strength of target gas absorption, the insensitivity of these features to errors in temperature, and their relative freedom from interfering absorption from other constituents [e.g., Gunson 
et al., 1990]. The ATMOS spectral linelist and associated spectroscopic uncertainties (leading to systematic uncertainties in the vmrs) are discussed in Brown et al. [1996].

\section{ATMOS and ATLAS}

The results from the 1985 SL-3 deployment have been extensively reported in the literature, and have demonstrated the potential for high-resolution, infrared solar occultation observations to measure the vertical profiles of more than thirty atmospheric trace gases. Included in this dataset were measurements of vertical profiles of trace gases in the stratosphere not measured previously [e.g., $\mathrm{N}_{2} \mathrm{O}_{5}$, Toon et al., 1986; $\mathrm{ClONO}_{2}$, Zander et al., 1986; $\mathrm{HO}_{2} \mathrm{NO}_{2}$, Rinsland et al., $1986 \mathrm{a} ; \mathrm{CH}_{3} \mathrm{Cl}$, Park et al., 1986; $\mathrm{COF}_{2}$, Rinsland et al., $1986 \mathrm{~b}, \mathrm{SF}_{6}$, Rinsland et al., 1990], which, combined with the measured vertical profiles of other trace constituents, provided opportunities to critically evaluate aspects of our understanding of the photochemistry of the upper stratosphere [e.g., McElroy and Salawitch, 1989; Allen and Delitsky, 1990; Natarajan and Callis, 1991]. The absorption features of several gases were discernible well above heights in the stratosphere, providing information for analyses on the composition of the mesosphere and thermosphere [Kumar et al., 1995; Lopez-Puertas et al., 1992].

The operational success of ATMOS was realized over the three flights of the 1992-1994 ATLAS series (AT-1, AT-2, and AT-3). Together, these missions provided observations of vertical profiles of atmospheric constituents throughout the tropics and mid-latitudes, in both hemispheres and over two seasons (Figure 2). In addition, ATMOS obtained measurements inside and outside the Arctic polar vortex during AT-2, and sampled inside and outside the Antarctic vortex in AT-3.

The early November mission of AT-3 provided the first opportunity to obtain space-based measurements on the chemical and dynamical state of the Antarctic vortex with the breadth of species and measurement precision provided by
ATMOS, comparing conditions inside and outside the region of maximum ozone loss that had occurred in the preceding months. Manney et al. [this issue] discuss meteorological conditions relevant to ATMOS observations during AT-3. Rinsland et al. [this issue (a,b)] have used the ability of ATMOS to measure the components of the family of reactive nitrogen species as well as $\mathrm{H}_{2} \mathrm{O}$ and $\mathrm{CH}_{4}$, to show that the level of denitrification within the vortex through formation and sedimentation of polar stratospheric clouds (PSCs) occurs at a slightly higher altitude of $\sim 20 \mathrm{~km}$ than the maximum level of dehydration (as measured by $\mathrm{H}_{2} \mathrm{O}+$ $2 \times \mathrm{CH}_{4}$ ), which appears at $\sim 18 \mathrm{~km}$ altitudes. Near $25 \mathrm{~km}$, the long-lived tracers $\mathrm{N}_{2} \mathrm{O}$ and $\mathrm{CH}_{4}$ are measured with vmrs equivalent to those found at $\sim 50 \mathrm{~km}$ [Abrams et al., this issue (b)]. This air brings higher levels of inorganic chlorine $(\mathrm{HCl}$ $+\mathrm{ClONO}_{2}+\mathrm{ClO}$ ), which, by the time of the AT- 3 mission, has been converted mainly to $\mathrm{HCl}$ under the low ozone levels in the vortex, which lead also to higher vmrs of NO [Rinsland et al., this issue (a)]. In contrast, measurements obtained by ATMOS during AT-2 through the Arctic vortex in April 1993 show similar descent rates but, because of the shorter descent period, less total descent [Abrams et al., this issue (c)].

The conditions within the polar vortices can be viewed as perturbed relative to the typical conditions encountered at mid-latitudes or within the tropics. However, detailed model studies of mid-latitude ATMOS observations of chlorine sink and reservoir species reveal a deficit in the computed levels of $\mathrm{HCl}$ that can be reconciled if an additional source of stratospheric $\mathrm{HCl}$ production, such as $\mathrm{ClO}+\mathrm{OH} \rightarrow \mathrm{HCl}+\mathrm{O}_{2}$, is allowed [Michelsen et al., this issue]. The internal consistency of the ATMOS halogen measurements has been evaluated previously by Zander et al. [1992] by comparing the altitude variation of the total chlorine-atom content from all measured species, which includes not only the major sink and reservoirs, but also most of the major source gases. This analysis is extended using the AT-3 measurements from ATMOS [Zander et al., this issue (a)] and MAS (Millimeter

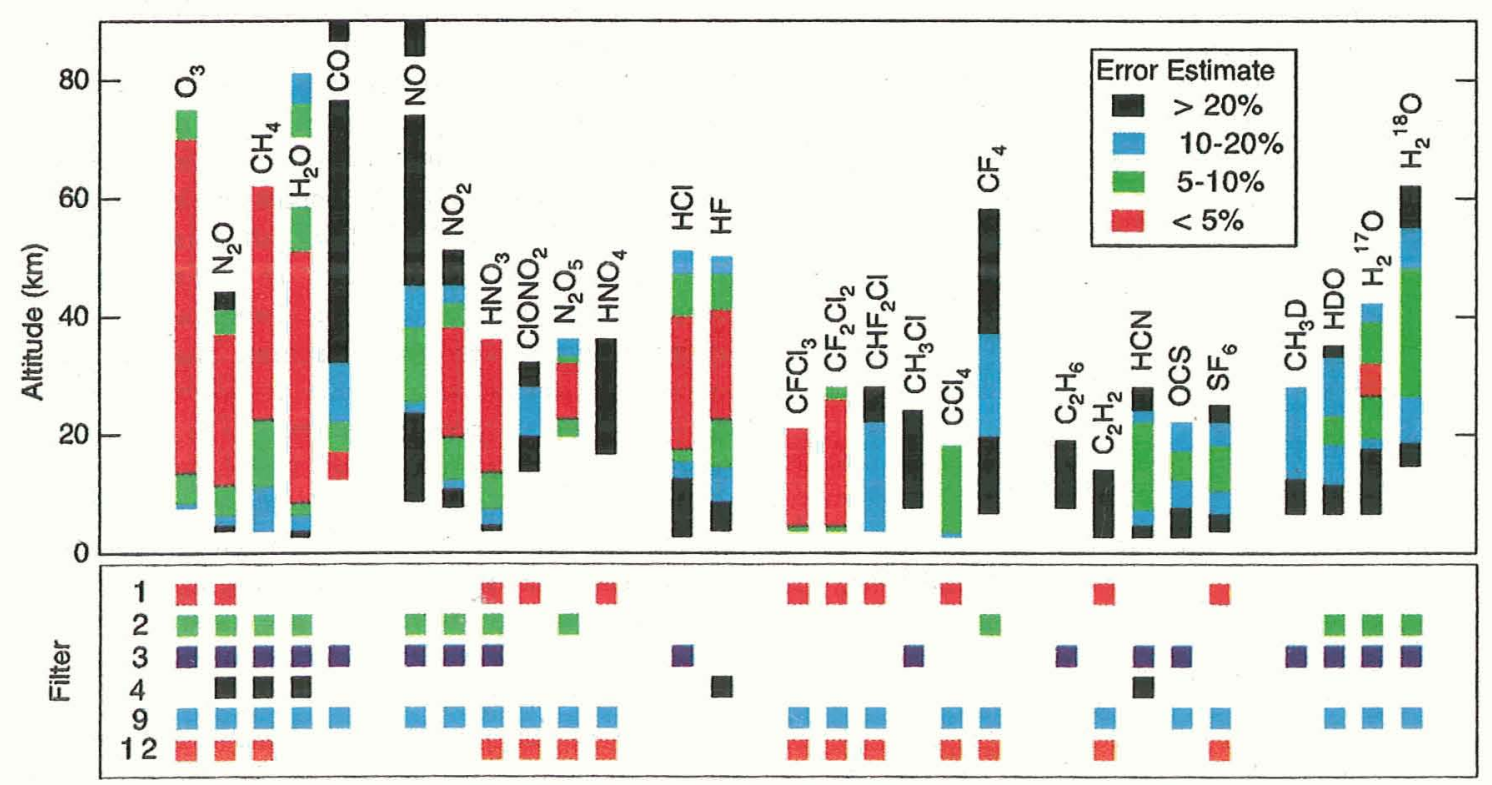

Figure 1. Vertical range of trace gas measurement in ATMOS 'Version 2' data. Shadings correspond to estimated $1 \sigma$ precision error in retrieved volume mixing ratio The lower panel maps gases to the ATMOS optical bandpass filters in which they are measured. The spectral bandpasses (in $\mathrm{cm}^{-1}$ ) correspond to: filter 1, 600-1180; filter 2, 1100-2000; filter 3, 1580-3340; filter 4, 3150-4800; filter 9, 600-2450; filter 12, 600-1400. 

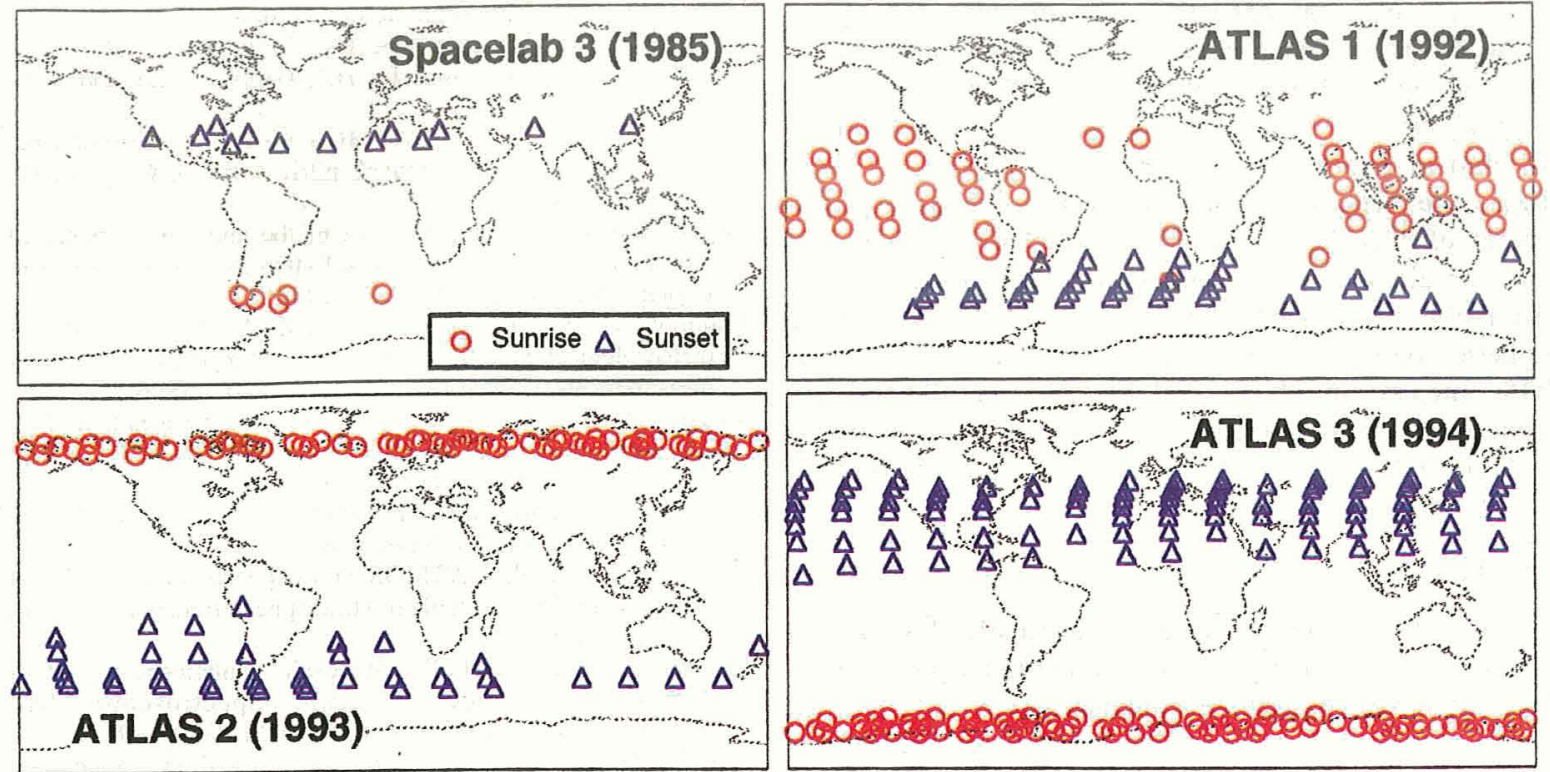

Figure 2. Sunrise (circles) and sunset (triangles) occultations observed by ATMOS on Space Shuttle for (a) Spacelab 3 , 29 April-May 7, 1985; (b) ATLAS-1, 24 March - 3 April 1992; (c) ATLAS-2, 8 - 16 April 1993; and (d) ATLAS-3, 3 - 14 Nov 1994.

Atmospheric Sounder) which measures ClO [Aellig et al., this issue], and the total stratospheric chlorine-loading compared with values measured by ATMOS on all four shuttle flights. This shows that the measured trends in stratospheric inorganic chlorine and fluorine are consistent with the growth in halocarbon source gases, such as chlorofluorocarbons. Following the approach discussed in Gunson et al. [1994], the measured upper stratospheric $\mathrm{HCl}$ and $\mathrm{HF}$ from all four shuttle flights are shown in Figure 3. The roughly 9.5 year interval between the first and latest deployment is sufficiently long to allow significant trends to be discernible not only in total chlorine and fluorine loading, but also in many individual species [Rinsland et al., this issue (c); Zander et al., this issue (b)].

The broad range of species measured at the precision and accuracy achieved by ATMOS over a long time base of observations provides correlative measurements with satellite instruments such as those on the Upper Atmospheric Research Satellite [see special issue, J. Geophys. Res., 101, No. D6, 1996]. Relating broad but global space-based observations to the detailed measurements made by in situ sensors carried on research aircraft has not always been possible. Chang et al. [this issue, $(\mathrm{a}, \mathrm{b})$ ] explore inter-comparisons of ATMOS measurements at northern mid-latitudes with those made by instruments on the ER-2 deployed in the same region. These comparisons reveal good agreement for many species to within a few percent, and certainly within the estimated precision of either data set, confirming the potential to utilize observations from such disparate sources to study atmospheric processes. However larger systematic differences were evident in the measurements of $\mathrm{CO}$ [Chang et al., this issue (a)], $\mathrm{CCl}_{4}$, and $\mathrm{HCl}$ [Chang et al., this issue (b)]. The good agreement for measurements of $\mathrm{NO}_{\mathrm{y}}$ (in correlations against $\mathrm{N}_{2} \mathrm{O}$ ) corroborates the accuracy of the in situ measurements obtained with a gold catalyst total- $\mathrm{NO}_{\mathrm{y}}$ sensor. Newchurch et al. [this issue] describe the sensitivities and results of accounting for the rapid diurnal variation of $\mathrm{NO}$ and $\mathrm{NO}_{2}$ along the line-of-sight through the terminator. The excellent agreement of the diurnally corrected NO from
ATMOS with in situ measurements on the ER-2 provides evidence that these space-borne measurements can be used to quantify $\mathrm{NO}_{\mathrm{x}}$ in the lower stratosphere.

Co-located measurements of water and methane by ATMOS have revealed further evidence of the seasonal variation in the vmr of water entering the stratosphere [Abbas et al., this issue, (a)]. Also, the sum $\mathrm{H}_{2} \mathrm{O}+2 \times \mathrm{CH}_{4}$ has been shown to be relatively constant in the lower stratosphere but exhibits a maximum between 35 and $65 \mathrm{~km}$, consistent with oxidation
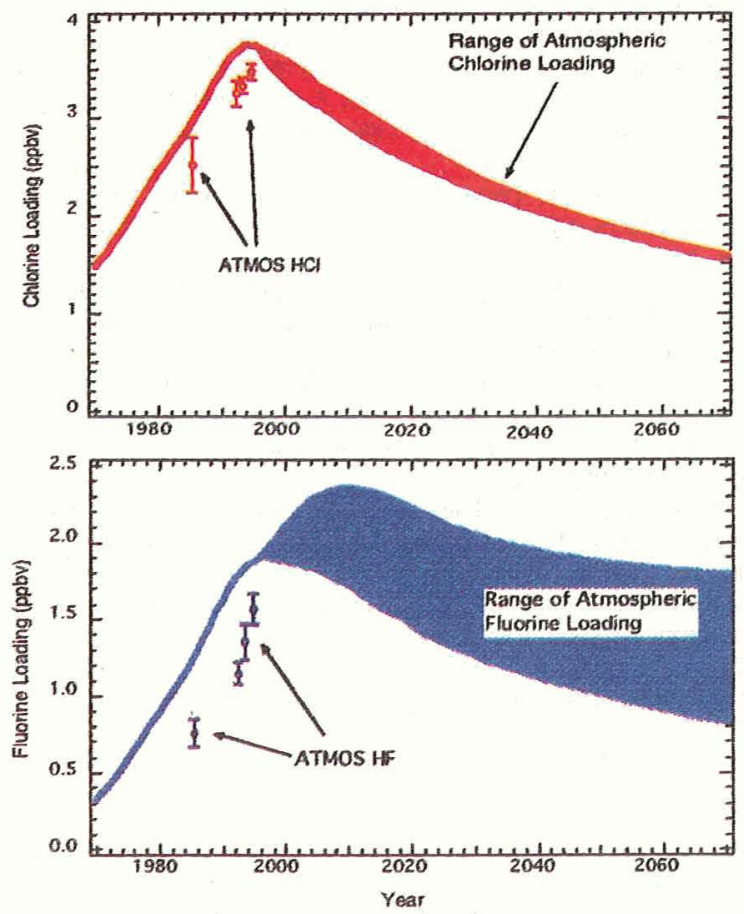

Figure 3. Modeled changes in total tropospheric chlorine $\left(\mathrm{Cl}_{\mathrm{y}}\right)$ and fluorine $\left(\mathrm{F}_{\mathrm{y}}\right)$ as described by Gunson et al. [1994] (Fig. 3), along with ATMOS data for upper stratospheric $\mathrm{HCl}$ and HF from 1985, 1992, 1993, and 1994. 
of $\mathrm{H}_{2}$ as a net source of water [Abbas et al., this issue, (b)]. The ATMOS spectral range and resolution allow analyses of several isotopomers of ozone, water, and methane, and offers the potential for new insight into atmospheric photochemical and dynamical processes. Irion et al. [this issue, (a)] investigate the vertical structure of heavy ozone and find enrichment of ${ }^{16} \mathrm{O}^{16} \mathrm{O}^{18} \mathrm{O}$ and ${ }^{16} \mathrm{O}^{18} \mathrm{O}^{16} \mathrm{O}$ to be relatively constant with altitude and season. Irion et al. [this issue, (b)] quantitatively investigate the oxidation of $\mathrm{CH}_{3} \mathrm{D}$ as a stratospheric source of HDO. Moyer et al. [this issue] use ATMOS measurements of deuterium depletion in stratospheric water as evidence that much of troposphericstratospheric exchange is associated with rapid convective events in the troposphere.

\section{ATMOS Data}

The ATMOS 'Version 2' data are available through an anonymous ftp-site at ftp://remus.jpl.nasa.gov/pub/atmos/ version2, or through http://remus.jpl.nasa.gov.

Acknowledgments. This work was carried out at the Jet Propulsion Laboratory, California Institute of Technology, under contract to the National Aeronautics and Space Administration. The authors wish to thank the many people who have contributed their time and effort to the success of ATMOS including Reinhard Beer, Barney Farmer, Julie Foster, Greg Goodson, Gindi Lynch, Bert McKenna, Fred O'Callaghan, Odell Raper, Rudy Schindler, Bhaswar Sen to name but a few.

\section{References}

Abbas, M. M., et al., Seasonal variations of water vapor in the lower stratosphere inferred from ATMOS/ATLAS-3 measurements of $\mathrm{H}_{2} \mathrm{O}$ and $\mathrm{CH}_{4}$, Geophys. Res. Lett., this issue, 1996a.

Abbas, M. M., et al., The hydrogen budget of the stratosphere inferred from ATMOS measurements of $\mathrm{H}_{2} \mathrm{O}$ and $\mathrm{CH}_{4}$, Geophys. Res. Lett, this issue, $1996 \mathrm{~b}$

Abrams, M. C., et al., Pressure sounding of the middle atmosphere from ATMOS solar occultation measurements of atmospheric $\mathrm{CO}_{2}$ absorption lines, Appl. Opt., in press, 1996.

Abrains, M. C., et al., On the assessment and uncertainty of atmospheric trace gas burden measurements with high resolution infrared solar occultation spectra from space, Geophys. Res. Lett., this issue, 1996a.

Abrams, M. C., et al., ATMOS/ATLAS-3 observations of descent in the Antarctic vortex in November 1994, Geophys. Res. Lett., this issue, $1996 \mathrm{~b}$

Abrams, M. C., et al., Trace gas transport in the Arctic vortex inferred from ATMOS ATLAS-2 observations during April 1993, Geophys. Res. Lett., this issue, 1996c.

Aellig, C. P., et al., Latitudinal distribution of upper stratospheric $\mathrm{ClO}$ as derived from space borne microwave spectroscopy, Geophys. Res. Lett., this issue, 1996.

Allen, M., and M. L. Delitsky, Stratospheric NO, $\mathrm{NO}_{2}$, and $\mathrm{N}_{2} \mathrm{O}_{5}$ : A comparison of model results with Spacelab 3 Atmospheric Trace Molecule Spectroscopy (ATMOS) measurements, J. Geophys. Res., 95, 14077-14082, 1990.

Brown, L. R., et al., The 1995 Atmospheric Trace Molecule Spectroscopy (ATMOS) linelist, Appl. Opt., in press, 1996.

Chang, A. Y., et al., A comparison of measurements from ATMOS and instruments aboard the ER-2 aircraft: Tracers of atmospheric transport, Geophys. Res. Lett., this issue, 1996a.

Chang, A. Y., et al., A comparison of measurements from ATMOS and instruments aboard the ER-2 aircraft: Halogenated gases, Geophys. Res. Lett., this issue, $1996 \mathrm{~b}$.

Farmer, C. B., High resolution infrared spectroscopy of the Sun and the Earth's atmosphere from space, Mikrochim. Acta (Wien), III, 189. $214,1987$.

Gunson, M. R., et al., Measurements of $\mathrm{CH}_{4}, \mathrm{~N}_{2} \mathrm{O}, \mathrm{CO}, \mathrm{H}_{2} \mathrm{O}$, and $\mathrm{O}_{3}$ in the middle atmosphere by the ATMOS experiment on Spacelab 3, $J$. Geophys. Res., 95, 13867-13882, 1990

Gunson, M. R., et al., Increase in levels of stratospheric chlorine and fluorine loading between 1985 and 1992, Geophys. Res. Lett., 21, 2223-2226, 1994.

Irion, F. W., et al., Heavy ozone enrichments from ATMOS infrared solar spectra, Geophys. Res. Lett., this issue, 1996a.
Irion, F. W., et al., Stratospheric observations of $\mathrm{CH}_{3} \mathrm{D}$ and $\mathrm{HDO}$ from ATMOS infrared solar spectra: Enrichments of deuterium in methane and implications for HD, Geophys. Res. Lett., this issue, 1996b.

Kumar, C. K., et al., ATMOS/ATLAS-1 measurements of thermospheric and mesospheric nitric oxide, J. Geophys. Res., 100 , 16839-16846, 1995.

Lopez-Puertas, M., et al., Analysis of the upper atmosphere $\mathrm{CO}_{2}\left(\mathrm{v}_{2}\right)$ vibrational temperatures from ATMOS/Spacelab 3 observations, $J$. Geophys. Res., 97, 20469-20478, 1992.

Manney, G. L., R. Swinbank, and A. O'Neill, Stratospheric meteorological conditions for the 3-12 Nov 1994 ATMOS/ATLAS-3 measurements, Geophys. Res. Lett., this issue, 1996.

McElroy, M. B., and R. J. Salawitch, Changing composition of the global stratosphere, Science, 243, 763-770, 1989.

Michelsen, H. A., et al., Stratospheric chlorine partitioning: Constraints from shuttle-borne measurements of $[\mathrm{HCl}],\left[\mathrm{ClNO}_{3}\right]$, and $[\mathrm{ClO}]$, Geophys. Res. Lett., this issue, 1996.

Moyer, E. J., et al., ATMOS stratospheric deuterated water and implications for troposphere-stratosphere transport, Geophys. Res. Lett., this issue, 1996.

Natarajan, M., and L. B. Callis, Stratospheric photochemical studies with Atmospheric Trace Molecule Spectroscopy (ATMOS) measurements, J. Geophys. Res., 96, 9361-9370, 1991.

Newchurch, M. J., et al., Stratospheric $\mathrm{NO}$ and $\mathrm{NO}_{2}$ abundances from ATMOS solar-occultation measurements, Geophys. Res. Lett., this issue, 1996.

Norton, R. H., and C. P. Rinsland, ATMOS data processing and science analysis methods, Appl. Opt., 30, 389-400, 1991.

Park, J. H., et al., Spectroscopic detection of $\mathrm{CH}_{3} \mathrm{Cl}$ in the upper troposphere and lower stratosphere, Geophys. Res. Lett., 13, 765$768,1986$.

Rinsland, C. P., et al., Evidence for the presence of the $802.7 \mathrm{~cm}^{-1}$ band $Q$ branch of $\mathrm{HO}_{2} \mathrm{NO}_{2}$ in high resolution solar absorption spectra of the stratosphere, Geophys. Res. Lett., 13, 761-764, 1986a.

Rinsland, C. P., et al., Detection of carbonyl fluoride in the stratosphere, Geophys. Res. Lett., 13, 769-772, $1986 \mathrm{~b}$.

Rinsland, C. P., L. R. Brown, and C. B. Farmer, Infrared spectroscopic detection of sulfur hexafluoride $\left(\mathrm{SF}_{6}\right)$ in the lower stratosphere and upper troposphere, J. Geophys. Res., 95, 5577 - 5585, 1990.

Rinsland, C. P., et al., ATMOS/ATLAS-3 measurements of stratospheric chlorine and reactive nitrogen partitioning inside and outside the November 1994 Antarctic vortex, Geophys. Res. Lett., this issue, 1996a.

Rinsland, C. P., et al., ATMOS measurements of $\mathrm{H}_{2} \mathrm{O}+2 \mathrm{CH}_{4}$ and total reactive nitrogen in the November 1994 Antarctic stratosphere: Dehydration and denitrification in the vortex, Geophys. Res. Lett. this issue, $1996 \mathrm{~b}$.

Rinsland, C. P., et al., Trends of OCS, $\mathrm{HCN}, \mathrm{SF}_{6}, \mathrm{CHClF}_{2}$ (HCFC-22) in the lower stratosphere from 1985 and 1994 Atmospheric Trace Molecule Spectroscopy experiment measurements near $30 \mathrm{~N}$ latitude, Geophys. Res. Lett., this issue, 1996c.

Stiller, G. P., et al., Stratospheric and mesospheric pressure-temperature profiles from the rotational analysis of $\mathrm{CO}_{2}$ Lines of ATMOS/ATLAS-1 Observations, J. Geophys. Res., 100, 3107-3117, 1995.

Toon, G. C., C. B. Farmer, and R. H. Norton, Detection of stratospheric $\mathrm{N}_{2} \mathrm{O}_{5}$ by infrared remote sounding, Nature, 319, 570-571, 1986.

Zander, R., et al., Observation of Several Chlorine Nitrate $\left(\mathrm{ClONO}_{2}\right)$ Bands in Stratospheric Infrared Spectra, Geophys. Res. Lett., I3, $757-760,1986$

Zander, R., et al., The 1985 Chlorine and fluorine inventories in the stratosphere based on ATMOS observations at 30 North latitude, $J$. Atm. Chem., 15, 171-186, 1992.

Zander, R., et al., The 1994 northern midlatitude budget of stratospheric chlorine derived from ATMOS/ATLAS-3 observations, Geophys. Res. Lett., this issue, 1996a.

Zander, R., et al., Increase of stratospheric carbon tetrafluoride $\left(\mathrm{CF}_{4}\right)$ based on ATMOS observations from space, Geophys. Res. Lett., this issue, 1996b.

M. R. Gunson, Jet Propulsion Laboratory, 4800 Oak Grove Drive, Mail-Stop 183-301, Pasadena, CA 91109 (email: Michael.R.Gunson@ jpl.nasa.gov)

(Received April 3, 1996; revised April 25, 1996; accepted May 2, 1996.) 


\title{
On the assessment and uncertainty of atmospheric trace gas burden measurements with high resolution infrared solar occultation spectra from space by the ATMOS experiment
}

\author{
M. C. Abrams ${ }^{1}$, A. Y. Chang 2 , M. R. Gunson' 2 , M. M. Abbas ${ }^{3}$, A. Goldman 4 , F. W. \\ Irion $^{5,2}$, H. A. Michelsen ${ }^{6}$, M. J. Newchurch ${ }^{7}$, C. P. Rinsland ${ }^{8}$, G. P. Stiller', and R. \\ Zander $^{10}$
}

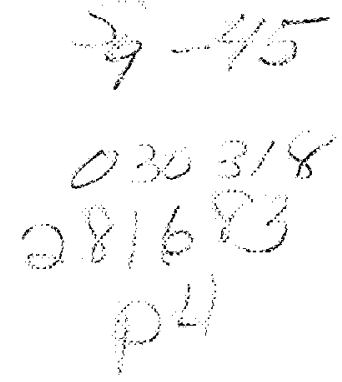

\begin{abstract}
The Atmospheric Trace Molecule Spectroscopy (ATMOS) instrument is a high resolution Fourier transform spectrometer that measures atmospheric composition from low Earth orbit with infrared solar occultation sounding in the limb geometry. Following an initial flight in 1985, ATMOS participated in the Atmospheric Laboratory for Applications and Science (ATLAS) 1, 2, and 3 Space Shuttle missions in 1992, 1993, and 1994 yielding a total of 440 occultation measurements over a nine year period. The suite of more than thirty atmospheric trace gases profiled includes $\mathrm{CO}_{2}, \mathrm{O}_{3}, \mathrm{~N}_{2} \mathrm{O}, \mathrm{CH}_{4}$, $\mathrm{H}_{2} \mathrm{O}$, NO, $\mathrm{NO}_{2}, \mathrm{HNO}_{3}, \mathrm{HCl}, \mathrm{HF}, \mathrm{ClONO}_{2}, \mathrm{CCl}_{3} \mathrm{~F}, \mathrm{CCl}_{2} \mathrm{~F}_{2}$, $\mathrm{CHF}_{2} \mathrm{Cl}$, and $\mathrm{N}_{2} \mathrm{O}_{5}$. The analysis method has been revised throughout the mission years culminating in the 'version 2' data set. The spectroscopic error analysis is described in the context of supporting the precision estimates reported with the profiles; in addition, systematic uncertainties assessed from the quality of the spectroscopic database are described and tabulated for comparisons with other experiments.
\end{abstract}

\section{Introduction}

Remote sensing measurements of the infrared telluric spectrum are a powerful means of studying chemical and dynamical processes in the atmosphere. The role of the ATMOS experiment as part of the shuttle-borne ATLAS 1-3 missions and as a component of the NASA Mission to Planet Earth has been widely discussed. Among the current family of space based remote sensing experiments ATMOS is unique in several aspects. The recoverability of the instrument and the self-calibrating nature of solar occultation limb sounding provide confidence in the reproducibility of the measurements. The instrument provides spectra of high photometric and spectral quality using a Fourier transform spectrometer at a sufficiently rapid rate (once per 2.2 seconds) to profile the limb of the Earth's atmosphere with high

\footnotetext{
${ }_{2}^{1}$ SAIC-NASA Langley Research Center

${ }_{3}$ Jet Propulsion Laboratory

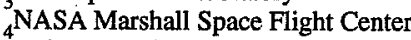

${ }_{5}^{4}$ University of Denver

${ }_{6}$ California Institute of Technology

${ }_{7}^{6}$ Harvard University

${ }_{8}$ University of Alabama at Huntsville

9 NASA Langley Research Center

${ }_{10}$ Forschungszentrum Karisruhe

Institute of Astrophysics, University of Liege
}

Copyright 1996 by the American Geophysical Union.

Paper number 96GL01794

0094-8534/96/96GL-01794\$05.00 vertical resolution $(2-3 \mathrm{~km})$. The high spectral resolution combined with broadband frequency coverage allows an assessment of constituent burdens from many spectral features. In the present letter, the method of analysis is discussed and assessed from the consistency of zonal averages of observations at tropical latitudes $(0-15 N)$ relative to the reported measurement precisions.

The ATMOS level-2 data processing retrieves vertical profiles of atmospheric composition from portions of the infrared (625$4800 \mathrm{~cm}^{-1}$, or 2.1-16 microns) absorption spectra. Each occultation uses a broad-band optical filter to improve the signal to noise ratio in the spectra (four of the primary filters have the following bandpasses: $\mathrm{f} \# 12: 625-1450 \mathrm{~cm}^{-1}$, $\mathrm{f} \# 3: 1580-3450 \mathrm{~cm}^{-1}$, f\#4: $3100-4800 \mathrm{~cm}^{-1}$, f\#9: $625-2450 \mathrm{~cm}^{-1}$ ). Atmospheric transmission spectra are 'self-calibrated' by ratioing each observed spectrum to an average of exo-atmospheric solar spectra obtained at tangent altitudes above $165 \mathrm{~km}$. In the ATMOS experiment, the high spectral resolution $\left(0.01 \mathrm{~cm}^{-1}\right.$ unapodized) permits the retrieval of atmospheric composition using narrow spectral windows or microwindows. For the minor gases $\left(\mathrm{O}_{3}\right.$, $\mathrm{CH}_{4}, \mathrm{H}_{2} \mathrm{O}, \mathrm{N}_{2} \mathrm{O}$, and $\mathrm{CO}$ ) it is possible to select many microwindows (with widths between 0.2 and $0.6 \mathrm{~cm}^{-1}$ ) that contain single lines with well defined continua and average the retrieved results; conversely, with many of the heavier gases $\left(\mathrm{CCl}_{3} \mathrm{~F}, \mathrm{CCl}_{2} \mathrm{~F}_{2}, \mathrm{~N}_{2} \mathrm{O}_{5}, \mathrm{CCl}_{4}\right.$, and $\mathrm{SF}_{6}$ for example) wider windows $\left(1.0-50 \mathrm{~cm}^{-1}\right)$ must be used and may require the pre-retrieval of interfering absorption features. The retrieval algorithm simulates the transmission spectrum within a microwindow and uses a least squares comparison between the observed and calculated spectra to adjust the assumed concentration of a gas in the model atmosphere until the spectral residual (observed minus calculated) is minimized. The selection, evaluation, and combination of microwindows remains a critical task in the definition of a retrieval strategy; broadband high resolution spectra offer a wide choice of spectral features suitable for retrievals at different altitude levels. However, obtaining consistent and statistically meaningful results requires careful analysis. Elements of the retrieval process have been presented by Norton and Rinsland [1990], Abrams et al., [1996a], and Abrams et al., [1996b], and rely upon a comprehensive database of spectroscopic parameters described by Brown et al., [1995]. A detailed recitation of the methodology is beyond the scope of this presentation, instead, a summary of the critical features of the processing methodology and a discussion of the error budget will be presented.

\section{ATMOS science analysis method}

The ATMOS retrieval method is a three step process of measuring temperature as a function of pressure, determining the viewing geometry, and assessing the burden of atmospheric 
constituents. Temperature is estimated from measurements of the slant column of $\mathrm{CO}_{2}$ using many spectral features with widely varying lower state energies (and hence range from temperature insensitive to highly temperature sensitive transitions) with a log regression technique that simultaneously estimates the temperature and pressure for each spectrum [Stiller et al., 1995] The retrieval provides profiles throughout the stratosphere and mesosphere with uncertainties of $\sim 3-4 \mathrm{~K}$. The viewing geometry is determined from the measurement of the slant column $\mathrm{CO}_{2}$ with an adjustment of the zenith angle until the calculated column matches the observed column [Abrams et al., 1996a]. Once the tangent pressure is assigned, the retrieval of constituent concentrations is in principle straightforward [Norton and Rinsland, 1991; Abrams et al., 1996b]. The propagation of temperature uncertainties into trace gas profiles is minimized by the selection, where ever possible, of microwindows that contain molecular transitions that are minimally temperature sensitive (as defined by the lower state energy of the transition).

The retrieval of atmospheric trace gas concentrations is performed with an onion-peeling algorithm that models the atmosphere with 150 one- $\mathrm{km}$ thick homogeneous layers. Spectroscopically derived tangent pressures and a consistent temperature-pressure model are assumed and used with the ATMOS linelist compilation [Brown et al., 1995] to calculate atmospheric transmittance $T_{c}$ along a slant path at the frequency $\sigma_{i}$

$$
\mathrm{T}_{\mathrm{c}}\left(\sigma_{\mathrm{i}}, \mathrm{z}\right)=\exp \left[-\sum_{\mathrm{j}} \Sigma_{\mathrm{k}} \kappa_{\mathrm{ijk}} \mathrm{n}_{\mathrm{k}} \mathrm{x}_{\mathrm{k}} \mathrm{v}_{\mathrm{jk}} \mathrm{g}_{\mathrm{k}}\right]
$$

where $z$ is the tangent height, $\kappa_{i j \mathrm{k}}$ is the absorption coefficient for the ith spectral point due to the $\mathrm{jth}$ gas in the kth layer, $\mathrm{n}_{\mathrm{k}}$ is the number density of air in the kth layer, $v_{j k}$ is the volume mixing ratio of the $j$ th gas in the $k$ th layer, $x_{k}$ is a multiplicative scale factor for the volume mixing ratio of the target gas ( $x_{k}=1$ unless $j=$ the target gas index), and $g_{k}$ is the geometrical slant path through the kth layer. For high resolution spectra, such as those produced by the ATMOS interferometer, single gas retrievals are sufficient and hence there is no subscript $\mathrm{j}$ for the scale factor $\mathrm{x}$. For certain gases, sequential retrievals of interfering gases are necessary before the retrieval of the target gas, but this simplified approach allows for rapid retrievals without compromising the quality of the retrievals. The retrieval algorithm computes a residual spectrum $\mathrm{r}\left(\sigma_{\mathrm{i}}\right)$ of the form

$$
\mathrm{r}\left(\sigma_{\mathrm{i}}, \mathrm{z}\right)=\mathrm{T}_{\mathrm{m}}\left(\sigma_{\mathrm{i}}, \mathrm{z}\right)-\mathrm{T}_{\mathrm{c}}\left(\sigma_{\mathrm{i}}, \mathrm{z}\right)
$$

(where $T_{m}\left(\sigma_{i}, z\right)$ is the measured spectrum) which is minimized, under a least squares criterion when

$$
\sum_{\mathrm{i}}\left[\partial \mathrm{T}_{\mathrm{c}}\left(\sigma_{\mathrm{i}}, \mathrm{z}\right) / \partial \mathrm{x}\right] \mathrm{r}\left(\sigma_{\mathrm{i}}, \mathrm{z}\right)=0
$$

to scale the a priori profile, through the factor $\mathrm{x}$, in a sequentia process to fit each spectrum. The partial derivative of the transmission with respect to scale factor $\mathrm{x}$ acts as a weighting function (The subscript $\mathrm{k}$ is dropped in Eq. 3, since in an onionpeeling algorithm, the scale factor $\mathrm{x}$ is adjusted for all layers between the tangent layer encompassing the current spectrum and the layer encompassing the previous (higher) spectrum.). The assumed profiles are based on the atmosphere obtained from the SPACELAB-3 mission, and the retrieval process consists of three iterations with vertical smoothing between each iteration to reflect the smearing of the field of view of the instrument during the measurement period. The numerical behavior of this algorithm has been evaluated and quantified [Abrams et al., 1996b] in terms of stability, convergence, independence from the a priori profile, and validated against simultaneous multigas retrieval algorithms. The method, despite its simplifications (single gas, smoothing, etc.), has survived extensive algorithm comparisons without compromising speed or accuracy and the vertical profiles have been widely utilized in the Upper Atmosphere Research Satellite validation program [Grose and Gille, 1995].
Since the final profile is reported at higher resolution than the measurements, the volume mixing ratios and uncertainties at different altitudes are correlated, the smoothing used in the retrieval process removes features with vertical scales finer than the $2-3 \mathrm{~km}$ field of view of the instrument before the retrieved profile (on the $1.0 \mathrm{~km}$ ATMOS altitude grid) is interpolated onto a pressure grid with 12 levels per decade of pressure. Zonal averages are computed for each altitude/pressure level independently.

The uncertainty in the gas burden is related to the spectroscopic residual in the following fashion

$$
\varepsilon=v_{\text {init }}\left\{\left[\sum_{\mathrm{i}} \mathrm{r}^{2}\left(\sigma_{\mathrm{i}}, \mathrm{z}\right)\right] /\left[\sum_{\mathrm{i}}\left(\partial \Gamma_{\mathrm{c}} \partial \mathrm{x}\right)^{2}\right]\right\}^{(1 / 2)}
$$

where $v_{\text {init }}$ is the assumed volume mixing ratio profile and $\mathrm{N}$ is one-half of the number of inflection points in $\partial T_{c} \partial x$ (effectively the number of target gas lines within the microwindow). The immediate advantage of (4) is that it readily lends itself to the statistical combination of errors arising from individual microwindows and tends to be conservative rather than optimistic. Two additional error terms are root-sum-squared into the total measurement precision, a continuum error and a propagation error resulting from mis-estimation of the burden in previous (higher altitude) layers. These terms are usually small compared to the residual error. Typically, several microwindows are utilized in the retrieval process, with the reported profile $v_{m}$ being the weighted average over the ' $n$ ' microwindows

$$
\mathrm{v}_{\text {mean }}=\left(\sum_{\mathrm{n}} \mathrm{v}_{\mathrm{n}} / \varepsilon_{\mathrm{n}}{ }^{2}\right) /\left(\sum_{\mathrm{n}} 1 / \varepsilon_{\mathrm{n}}{ }^{2}\right)
$$

where $v_{n}$ is the vmr retrieved for the nth microwindow. An estimate of the precision of the measurement is determined two ways. The first is simply the standard error of the mean $(1 \sigma)$

$$
\varepsilon_{\mathrm{se}}{ }^{2}=1 /\left[\sum_{\mathrm{n}} 1 / \varepsilon_{\mathrm{n}}^{2}\right]
$$

as a measure of the precision of the results. The second is the error on the estimation of the mean $\varepsilon_{\text {eem }}$ obtained from the standard deviation

$$
\varepsilon_{\mathrm{ecm}}{ }^{2}=\varepsilon_{\mathrm{se}}{ }^{2} * \sum_{\mathrm{n}}\left[\mathrm{v}_{\mathrm{n}}{ }^{2} / \varepsilon_{\mathrm{n}}{ }^{2}-\mathrm{v}_{\text {mean }}{ }^{2} / \varepsilon_{\mathrm{n}}{ }^{2} y(\mathrm{M}-1)\right. \text {. }
$$

Normally M-1 is the number of degrees of freedom, but in cases where the individual error estimates $\varepsilon_{\mathrm{n}}$ can be significantly different (as will occur when results from wide and narrow spectral windows are combined together) an alternate definition of $\mathrm{M}$ :

$$
M=\left[\Sigma_{n}\left(1 / \varepsilon_{n}\right)\right]^{2} /\left[\Sigma_{n} 1 / \varepsilon_{n}^{2}\right] .
$$

yields a more conservative (larger) error estimate. This effective $M$ is smaller than the actual number of microwindows except in the case where the weights are the same. The reported precision is taken as the larger of the two estimates. In many cases Eqs. 6 and 7 give quite similar results, which suggests that the line parameters used for each microwindow are not significantly biased relative to the spectroscopic precision (since inconsistencies in spectroscopic parameters would cause the standard deviation and the standard error to differ). The similarity of the standard error (based only on residuals) and the standard deviation (which assesses the weighted variance about the mean) should convey a degree of confidence that the reported uncertainties are reasonably conservative and plausibly reflect the quality of the measurements.

\section{Processing Methodology}

There have been three major versions of the ATMOS data, reflecting the increasing sophistication of the retrieval methodology. The three versions are distinguishable by the final processing date: preliminary results (version 0 ) were processed prior to 1 October 1994, version 1 data was processed between 1 October 1994 and 14 December 1994 and was widely used in evaluations of measurements made from the Upper Atmosphere Research Satellite (UARS), and version 2 data was processed between 15 December 1994 and 15 April 1995 and is presented in 


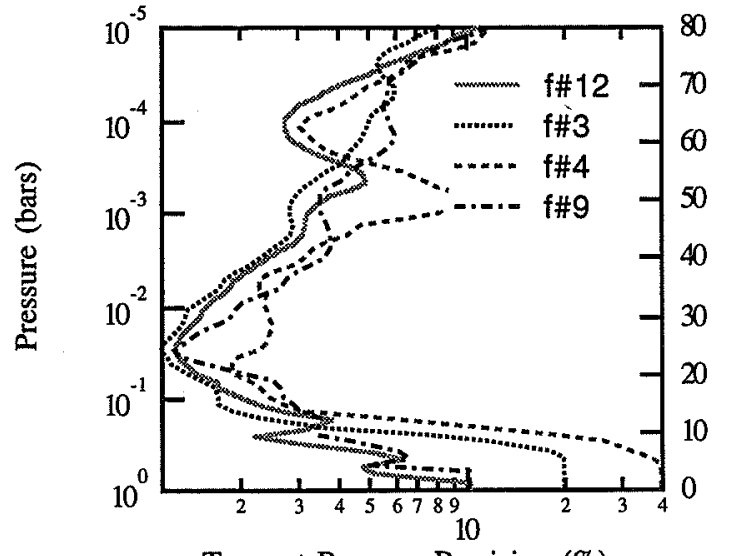

Tangent Pressure Precision (\%)

Figure 1. Tangent pressure precisions (viewing geometry).

the accompanying letters in this issue. All current ATMOS data has been processed with the version 2 methodology.

In early 1995, the ATMOS data set was revised to address certain limitation of the version 1 data set; specifically, inconsistencies in the derivation of pressures with the simultaneous pressure-temperature algorithm led to the conclusion that the version 1 constituent profiles could be locally biased by as much as $8-15 \%$. Three new elements were added to the process: (a) a final pressure sounding was performed using the version 1 temperature profile and the assumed mean stratospheric $\mathrm{CO}_{2}$ volume mixing ratio profile of 350 parts per million by

Table 1. ATMOS Version 2 Data Precision and Accuracy

\begin{tabular}{|c|c|c|c|c|}
\hline $\begin{array}{c}\text { Species } \\
\text { Range } \\
(\mathrm{km})\end{array}$ & $\begin{array}{c}\text { Altitude } \\
\text { Range } \\
(\mathrm{km})\end{array}$ & $\begin{array}{c}\text { Est. } \\
\text { Prec. } \\
(\%) \\
\end{array}$ & $\begin{array}{c}\text { Zonal } \\
\text { Std. Dev. } \\
(\%)\end{array}$ & $\begin{array}{l}\text { Est. } \\
\text { Acc. } \\
(\%) \\
\end{array}$ \\
\hline $\mathrm{O}_{3}$ & $10-77$ & $2-5$ & $2-8$ & 6 \\
\hline $\mathrm{N}_{2} \mathrm{O}$ & $10-53$ & $3-5$ & $2-10$ & 5 \\
\hline $\mathrm{CH}_{4}$ & $10-65$ & $3-5$ & $3-14$ & 5 \\
\hline $\mathrm{HNO}_{3}$ & $15-40$ & $3-6$ & $3-10$ & 16 \\
\hline $\mathrm{H}_{2} \mathrm{O}$ & $10-83$ & $5-7$ & $2-8$ & 6 \\
\hline $\mathrm{HCl}$ & $13-53$ & $5-8$ & $1-5$ & 5 \\
\hline $\mathrm{HF}$ & $12-55$ & $5-10$ & $1-10$ & 5 \\
\hline NO & $18-100$ & $10-20$ & $1-10$ & 5 \\
\hline $\mathrm{NO}_{2}$ & $15-48$ & $4-10$ & $1-10$ & 6 \\
\hline $\mathrm{CCl}_{3} \mathrm{~F}$ & $10-29$ & $3-5$ & 4-15 & 11 \\
\hline $\mathrm{CCl}_{2} \mathrm{~F}_{2}$ & $10-31$ & $3-5$ & $2-10$ & 9 \\
\hline $\mathrm{CHClF}_{2}$ & $10-31$ & $12-25$ & $3-10$ & 11 \\
\hline $\mathrm{ClONO}_{2}$ & $16-39$ & 40 & $3-20$ & 20 \\
\hline $\mathrm{N}_{2} \mathrm{O}_{5}$ (ss) & $21-40$ & $16-25$ & $8-35$ & 16 \\
\hline $\mathrm{N}_{2} \mathrm{O}_{5}(\mathrm{sr})$ & & $3-5$ & $12-20$ & 16 \\
\hline $\mathrm{SF}_{6}$ & $10-27$ & $7-20$ & $2-15$ & 11 \\
\hline OCS & $10-24$ & $15-20$ & $2-7$ & 9 \\
\hline $\mathrm{HCN}$ & $10-29$ & $7-18$ & $2-10$ & 6 \\
\hline HDO & $10-40$ & $20-30$ & $3-15$ & 7 \\
\hline $\mathrm{H}_{2}{ }^{17} \mathrm{O}$ & $10-49$ & $12-20$ & $5-15$ & 7 \\
\hline $\mathrm{H}_{2}{ }^{18} \mathrm{O}$ & $18-65$ & $13-25$ & $3-12$ & 7 \\
\hline $\mathrm{CH}_{3} \mathrm{D}$ & $10-35$ & $16-23$ & $3-7$ & 7 \\
\hline $\mathrm{CO}$ & $15-100$ & $5-20$ & $3-15$ & 5 \\
\hline $\mathrm{HNO}_{4}$ & $16-40$ & $30-60$ & $10-30$ & 20 \\
\hline $\mathrm{CCl}_{4}$ & $10-27$ & $7-15$ & $4-15$ & 20 \\
\hline $\mathrm{C}_{2} \mathrm{H}_{2}$ & $10-24$ & $>50$ & $>50$ & 7 \\
\hline $\mathrm{C}_{2} \mathrm{H}_{6}$ & $10-24$ & 25 & 25 & 11 \\
\hline $\mathrm{CH}_{3} \mathrm{Cl}$ & $10-27$ & $>50$ & $5-20$ & 11 \\
\hline $\mathrm{CF}_{4}$ & $10-58$ & $20-40$ & $2-7$ & 11 \\
\hline
\end{tabular}

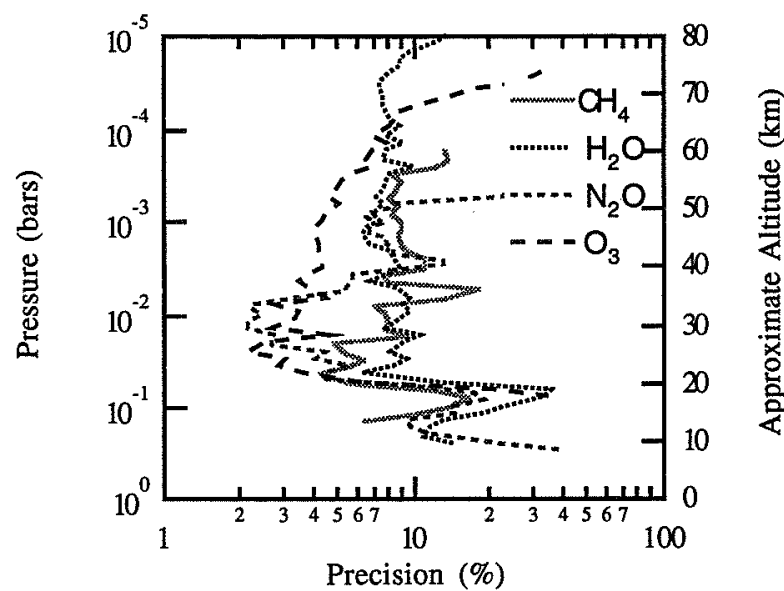

Figure 2. Precisions (root sum square of retrieval precision and tangent pressure precision) for the minor gases.

volume (ppmv) in 1994 to minimize the uncertainties in the viewing geometry determination, (b) improved low altitude tangent pressure determination (through the definition of new microwindows), and (c) the spectroscopic precision was redefined to be more consistent with the random errors in the retrieval process (Eq. 4 ). The precision of the tangent pressure soundings as functions of pressure are illustrated in Figure 1; the variation between the optical filters reflects differences in the spectroscopy and number of microwindows at each pressure level available for each of the optical filters. The fractional error for the pressure sounding ranges between $0.5 \%$ and $9 \%$, with the best results typically in the pressure range between 100 and 1 mbars (approximately 18 to $48 \mathrm{~km}$ altitude). Given a $7 \mathrm{~km}$ scale height, such an uncertainty corresponds to a relative pointing uncertainty of 35-630 meters (70 meters/percent uncertainty) which is modest with respect to the absolute pointing knowledge which is typically not better than $1 \mathrm{~km}$ (14\% in pressure). Below 100 mbars the uncertainty increases, but is still quite acceptable at 300 mbars (approximately $8 \mathrm{~km}$ ); similarly, in several filters there is a region around $50 \mathrm{~km}$ where the number of usable features is insufficient to maintain consistent precision.

\section{Error Budget}

The version 2 data set has been released to the general science community and utilized in the accompanying letters. Single profile precision ranges are given in Table 1 for each of the gases

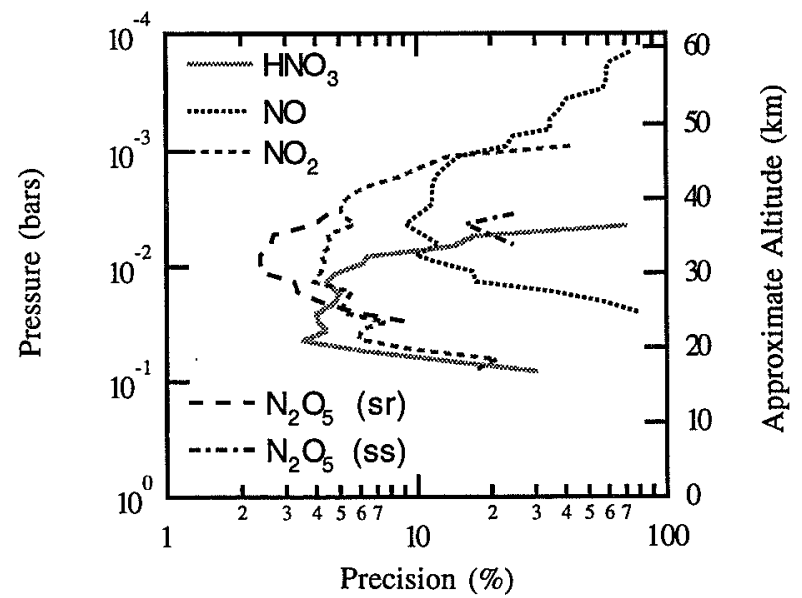

Figure 3. Precisions for odd nitrogen species. Notice the dramatic difference in dinitrogen pentoxide precisions depending on the time of day. 


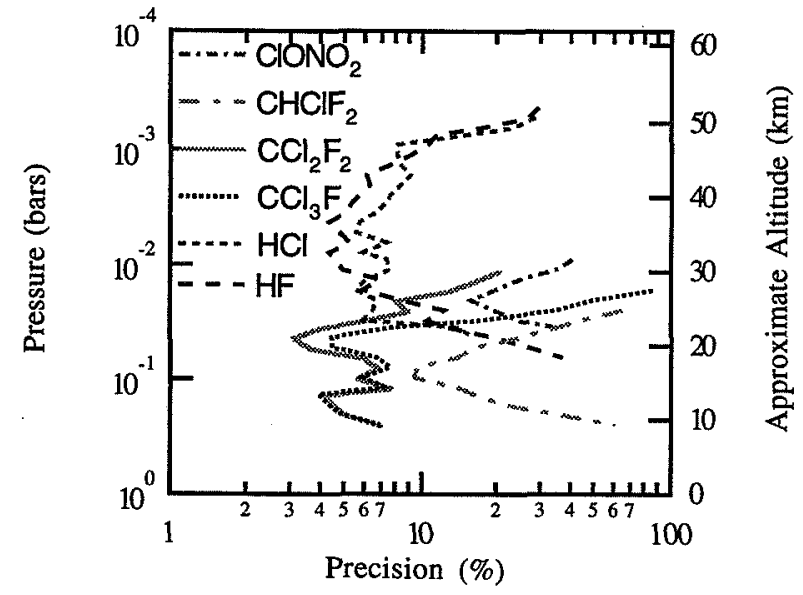

Figure 4. Precisions for chlorine- and fluorine-bearing species including the source gases $\left(\mathrm{CCl}_{3} \mathrm{~F}, \mathrm{CCl}_{2} \mathrm{~F}_{2}\right.$, and $\left.\mathrm{CHClF}_{2}\right)$ and reservoir species $\mathrm{HCl}$ and $\mathrm{HF}$.

routinely profiled from the ATMOS spectral database. For the minor and trace gases with well isolated spectral features $\left(\mathrm{CO}_{2}\right.$, $\mathrm{O}_{3}, \mathrm{~N}_{2} \mathrm{O}, \mathrm{CH}_{4}, \mathrm{H}_{2} \mathrm{O}, \mathrm{HCl}$, and $\mathrm{HF}$ ) where many microwindows may be combined the precision is typically less than $10 \%$. Retrievals of profiles of the chlorofluorocarbons $\left(\mathrm{CCl}_{3} \mathrm{~F}\right.$ and $\mathrm{CCl}_{2} \mathrm{~F}_{2}$ ) with broadband spectral features that are minimally overlapped by features of other gases yield small precision estimates due to the large amount of spectral information. At the other extreme are species such as $\mathrm{HNO}_{3}, \mathrm{~N}_{2} \mathrm{O}_{5}, \mathrm{CHCI}_{2}$, and $\mathrm{ClONO}_{2}$ which require pre-retrieval of 1-6 interfering species. In such cases, the residuals are dominated by interferences and lead to large precision estimates. As illustrated in Figs. 2-4, if the profiles are sharply peaked then the error estimates will tend to $100 \%$ at the top and bottom of the profiles, indicating the full range of measurable signal. $\mathrm{N}_{2} \mathrm{O}_{5}$ is a special case, since the diurnal variation is sufficiently large that sunset retrievals typically have an uncertainty of $16-25 \%$, while the sunrise measurements, sampling a much larger amount of gas along the line of sight, have smaller uncertainties (note that, the only tropical sunrise data obtained by ATMOS was during the 1992 ATLAS-1 mission).

The tangent pressure precision is included in quadrature with the retrieval precision (Eq. 4) in the precision estimates for each constituent (temperature errors are sufficiently small and may be neglected). Approximate altitude ranges given in Table 1 (column 2) correspond to altitude limits between which measurements with reasonable precision estimates (column 3) are obtained. Accuracy ranges, based on the combined uncertainties in spectroscopic parameters [Brown et al., 1995] of the retrieved constituent and the $\mathrm{CO}_{2}$ transitions used for pressure sounding, are given in Table 1 (column 5) to indicate the systematic uncertainty for comparison with other measurements and model calculations.

Both long-lived and reactive trace gases are expected to have minimal spatial variability in the tropics and consequently, the zonal average standard deviation of measurements at tropical latitudes may be used to assess the precision of the measurements relative to the expected natural variability of $5-8 \%$ at most altitudes between 15 and $50 \mathrm{~km}$ (column 4). For the trace gases, the standard deviation is typically comparable to the precision, indicating that the precision estimate and the measurement variance are in reasonable agreement. The standard deviations for the minor and source gases are sufficiently small that comparisons with other experiments should allow an assessment of the accuracy of the measurements. When the zonal standard deviation is smaller that the single profile precision there is the potential that a fraction of the precision estimate from the spectroscopic residual is indeed systematic in origin. For example, with $\mathrm{ClONO}_{2}$ the zonal standard deviation is less than the reported precision: the scatter among the different measurements was small because the residuals were not due to random noise but a systematic error in the calculated spectra which does not vary from fit to fit; conversely, the precision is an overestimate resulting from the inadequacy of the pre-retrieval of interfering spectral lines.

\section{Conclusions}

The methodology and accuracies of the ATMOS data processing have been summarized and evaluated. The reported error budget for version 2 data is presented and evaluated, with the intent of describing the quality and limitations of the database. Vertical error profiles highlight the range over which retrievals produce reasonable results, and the regions where the uncertainty in the measurement process dominates the result. A summary error budget provides a simple set of guidelines to the relative quality of ATMOS measurements trace gas burdens, which should permit users of the ATMOS data to focus on measured features of the atmosphere rather than features of the ATMOS retrieval process. The accuracy of the spectroscopic database provides a similar guideline for comparing ATMOS profiles with the results from other experiments.

Acknowledgments. Research at the Jet Propulsion Laboratory (JPL) California Institute of Technology, is performed under contract to the National Aeronautics and Space Administration (NASA). Research at the University of Liège was partially supported by the Belgian Global Change Program through the SSTC, Brussels. The authors greatly appreciate the careful scientific and editorial comments of several colleagues, especially those of $R$. J. Salawitch.

\section{References}

Abrams, M. C., et al., Pressure sounding of the middle atmosphere from ATMOS solar occultation measurements of atmospheric $\mathrm{CO}_{2}$ absorption lines, Appl. Opt., in press, 1996a.

Abrams, M. C., et al., Remote sensing of the Earth's atmosphere from space with high resolution Fourier transform spectroscopy: development and methodology of data processing for the Atmospheric Trace Molecule Spectroscopy experiment, submitted, Appl. Opt., $1996 \mathrm{~b}$.

Brown, L. R. et al.., The 1995 Atmospheric Trace Molecule Spectroscopy (ATMOS ) Linelist, submitted, Appl. Opt., 1995.

Grose, W. and J. Gille, eds., Upper Atmosphere Research Satellite Validation Workshop Report: Temperature and Constituents, I and II, NASA Ref. Pub., 1995.

Norton, R. H., and C. P. Rinsland, ATMOS data processing and science analysis methods, Appl. Opt., 30, 389-400, 1991.

Stiller, G. P., et al., Stratospheric and mesospheric pressure-temperature profiles from the rotational analysis of $\mathrm{CO}_{2}$ lines of the ATMOS/ATLAS-1 observations, J. Geophys. Res., 100, 3,107-3,117, 1995

M. C. Abrams, NASA LaRC, MS-475, Hampton, VA, 23681 , (m.c.abrams@larc.nasa.gov)

A. Y. Chang; M. R. Gunson, Jet Propulsion Laboratory

M. M. Abbas, NASA Marshall Space Flight Center

A. Goldman, University of Denver

F. W. Irion, California Institute of Technology

H. A. Michelsen, Harvard University

M. J. Newchurch, University of Alabama at Huntsville

C. P. Rinsland, NASA Langley Research Center

G. P. Stiller, Forschungszentrum Karlsruhe

R. Zander, Institute of Astrophysics, University of Liege

(Received November 27, 1995; revised March 4, 1996 ;

accepted April 25, 1996.) 


\title{
Trace gas transport in the Arctic vortex inferred from ATMOS ATLAS-2 observations during April 1993
}

\author{
M. C. Abrams, ${ }^{1}$ G. L. Manney, ${ }^{2}$ M. R. Gunson, ${ }^{2}$ M. M. Abbas, ${ }^{3}$ A. Y. Chang, ${ }^{2}$ \\ A. Goldman, ${ }^{4}$ F. W. Irion, ${ }^{5,2}$ H. A. Michelsen, ${ }^{6}$ M. J. Newchurch, ${ }^{7}$ C. P. Rinsland, ${ }^{8}$ \\ R. J. Salawitch, ${ }^{2}$ G. P. Stiller, ${ }^{9}$ and R. Zander ${ }^{10}$
}

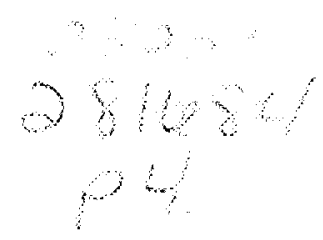

\begin{abstract}
Measurements of the long-lived tracers $\mathrm{CH}_{4}, \mathrm{~N}_{2} \mathrm{O}$, and HF from the Atmospheric Trace Molecule Spectroscopy (ATMOS) instrument during the Atmospheric Laboratory for Science and Applications-2 (ATLAS-2) Space Shuttle mission in April 1993 are used to infer average winter descent rates ranging from $0.8 \mathrm{~km} / \mathrm{month}$ at $20 \mathrm{~km}$ to $3.2 \mathrm{~km} /$ month at $40 \mathrm{~km}$ in the Arctic polar vortex during the 1992-93 winter. Descent rates in the mid-stratosphere are similar to those deduced for the Antarctic vortex using ATMOS/ATLAS-3 measurements in November 1994, but the shorter time period of descent in the Arctic leads to smaller total distances of descent. Strong horizontal gradients observed along the vortex edge indicate that the Arctic vortex remains a significant barrier to transport at least until mid-April in the lower to middle stratosphere.
\end{abstract}

\section{Introduction}

Recent theoretical studies have addressed the transport of air through the polar vortices and the evidence for and against the mixing of air confined within the vortex with extra-vortex air. Manney et al. [1994a] simulated the three-dimensional motion of air through the polar vortices using horizontal winds from the United Kingdom Meteorological Office (UKMO) data assimilation system [Swinbank and O'Neill, 1994] and vertical velocities from a radiation calculation. Rosenfield et al. [1994] combined a radiation model with National Meteorological Center (NMC) temperature observations to compute heating rates and descent rates for long-lived tracers in the Northern hemisphere and Southern hemisphere winter vortices. Schoeberl et al. [1995] obtained descent rates within the lower stratosphere in the southern hemisphere (SH) from HALOE observations, and Manney et al. [1995] calculated descent rates in the lower stratosphere in both the northern hemisphere (NH) and SH.

The third flight of the ATMOS instrument as part of the ATLAS-2 Shuttle mission captured a unique view of the late winter state of the Arctic stratosphere in April 1993 by recording

\footnotetext{
${ }_{2}^{1}$ SAIC-NASA Langley Research Center

2 Jet Propulsion Laboratory

${ }^{3}$ NASA Marshall Space Flight Center

${ }_{5}^{4}$ University of Denver

${ }^{5}$ California Institute of Technology

${ }_{7}^{6}$ Harvard University

${ }_{8}^{7}$ University of Alabama at Huntsville

${ }^{8}$ NASA Langley Research Center

${ }^{9}$ Forschungszentrum Karlsruhe

${ }^{10}$ Institute of Astrophysics, University of Liege
}

Copyright 1996 by the American Geophysical Union.

Paper number 96GL00705

0094-8534/96/96GL-00705\$05.00 solar occultation measurements inside and outside the polar vortex. The observed distributions of the long-lived tracers $\mathrm{N}_{2} \mathrm{O}$, $\mathrm{CH}_{4}$ and $\mathrm{HF}$ give information on transport effects that have occurred during the winter, and provide a dynamical context for analyses of the chemistry of reactive nitrogen and chlorine species as the polar vortex erodes in spring. Approximately 50 sunrise observations were concentrated in a narrow band of latitudes from $60-65^{\circ} \mathrm{N}$, with the remaining sunset observations latitudes between the Equator and $50^{\circ} \mathrm{S}$. The ATLAS-3 mission, flown eighteen months later in November 1994, provided measurements at high Northern midlatitudes, from which the seasonal variation in the upper stratospheric trace gases can be addressed. The results presented in this Letter focus on estimation of descent rates from profiles of $\mathrm{N}_{2} \mathrm{O}, \mathrm{CH}_{4}$, and HF measured inside and outside the Arctic polar vortex during late winter and complement an analysis of similar measurements presented in an accompanying Letter [Abrams et al., 1996] of descent within the Antarctic polar vortex.

\section{Mean tracer profiles and descent rates}

Figure 1 shows a cross-section of $\mathrm{N}_{2} \mathrm{O}$ as a function of longitude and potential temperature $(\theta)$, constructed from data taken throughout the nine day ATLAS-2 mission. Two regions of very strong gradients in $\mathrm{N}_{2} \mathrm{O}$ are seen between $50^{\circ}$ and $150^{\circ} \mathrm{E}$ longitude, at levels up to about $900 \mathrm{~K}$ (approx. $30 \mathrm{~km}$ ), indicating that vortex and extra-vortex air remain largely unmixed in the lower and middle stratosphere as late as mid-April 1993. As was the case in the SH [Abrams et al., 1996], contours of scaled potential vorticity (sPV) [Manney et al., 1994], overlaid in Figure 1, show large gradients coincident with the steep $\mathrm{N}_{2} \mathrm{O}$ gradients, although the SPV gradients extend up to about $1200 \mathrm{~K}$. The existence of strong $\mathrm{N}_{2} \mathrm{O}$ gradients along the vortex edge in the middle and lower stratosphere supports the theoretical calculations of Manney et al. [1994], who showed confinement of air in the lower to middle stratospheric vortex in early April 1993. Similar features are apparent in cross-sections of ATMOS measurements of $\mathrm{CH}_{4}$ and HF (not shown).

As in Abrams et al. [1996], we construct average vortex and extra-vortex profiles of $\mathrm{N}_{2} \mathrm{O}, \mathrm{CH}_{4}$, and $\mathrm{HF}$ on the basis of both tracer mixing ratios and sPV values on the $655 \mathrm{~K}$ isentropic surface. Profiles with $\mathrm{N}_{2} \mathrm{O}$ and $\mathrm{CH}_{4}$ less (greater) than $30 \mathrm{ppbV}$ and $0.6 \mathrm{ppmV}$, respectively, and sPV greater (less) than $1.6(1.2)$ are considered to be inside (outside) the vortex. Figures $2-4$ show the mean vortex and extra-vortex profiles of $\mathrm{N}_{2} \mathrm{O}, \mathrm{CH}_{4}$, and $\mathrm{HF}$ determined in this manner. As was apparent in Figure 1, there is a strong distinction between vortex (low $\mathrm{N}_{2} \mathrm{O}$, low $\mathrm{CH}_{4}$, high $\mathrm{HF}$ ) and extra-vortex (high $\mathrm{N}_{2} \mathrm{O}$, high $\mathrm{CH}_{4}$, low $\mathrm{HF}$ ) profiles at levels below about $35-40 \mathrm{~km}$. Below $20 \mathrm{~km}$, vortex and extra-vortex profiles begin to converge, suggesting that there may be a larger degree of horizontal mixing below this level. 

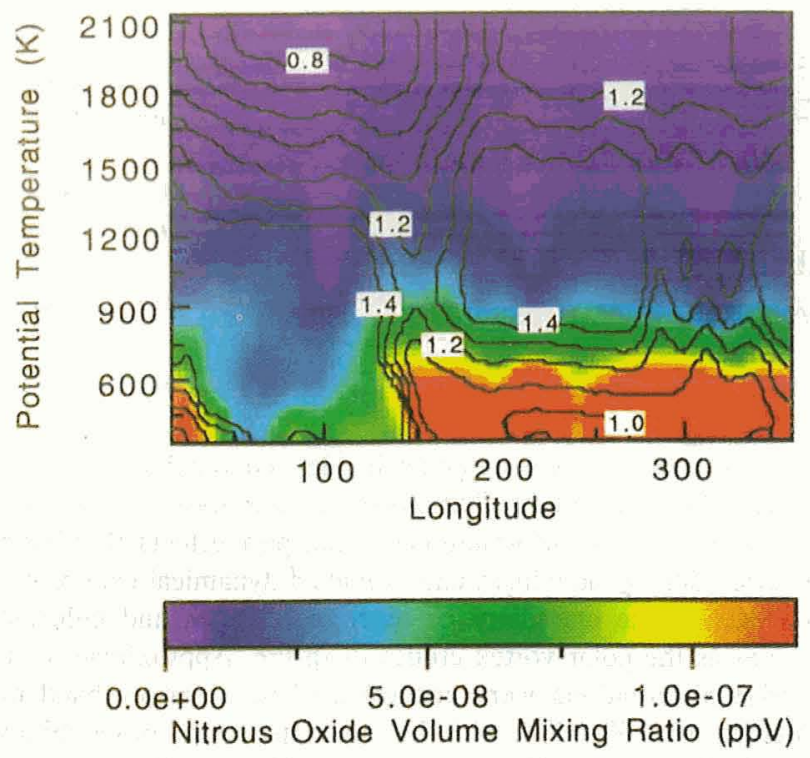

Figure 1. Potential temperature-longitude cross-section at $62^{\circ} \mathrm{N}$ of $\mathrm{N}_{2} \mathrm{O}$ (color) and sPV (contours). The region of the vortex is clearly defined by the steep gradients in potential vorticity between 1.2 and 1.6 in the lower stratosphere between $40^{\circ}$ and $150^{\circ}$ longitude.

Profiles of tracers obtained in the ATLAS-3 mission at Northern mid-latitudes $\left(46^{\circ} \mathrm{N}\right)$ in late fall (3-12 November, 1994), shown in Figures 2-4, provide a measure of seasonal variation in the mid-latitude upper stratosphere. These observations suggest some seasonal variation above about $35 \mathrm{~km}$. Between 25 and $35 \mathrm{~km}$, the vortex profiles of $\mathrm{N}_{2} \mathrm{O}$ and $\mathrm{CH}_{4}$ show a secondary maximum and HF a secondary minimum; above this, they closely resemble the extra-vortex profiles. This is evidence that there is no longer any significant barrier to horizontal transport in the upper stratosphere, since vortex and extra-vortex air are no longer distinguishable. However, despite the large amount of dynamical activity that has occurred throughout the winter [e.g., Manney

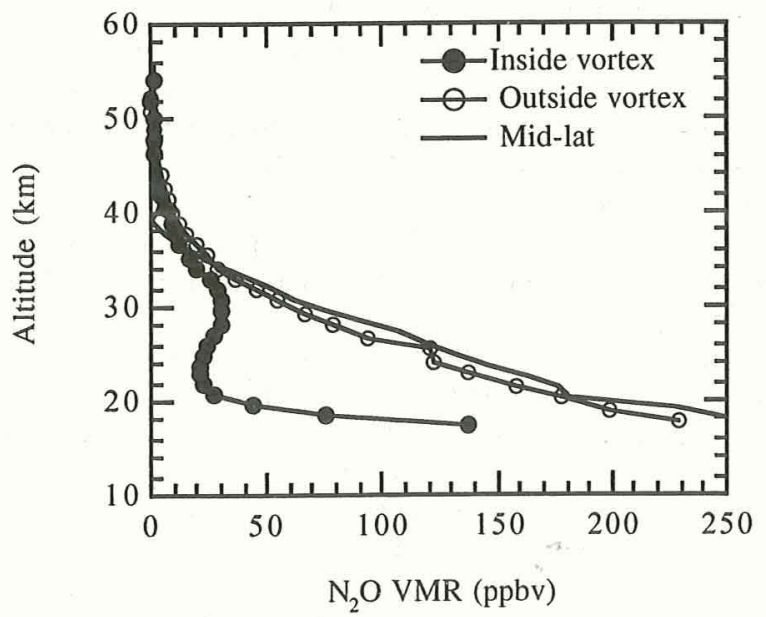

Figure 2. Mean $\mathrm{N}_{2} \mathrm{O}$ volume mixing ratio profiles for "inside" and "outside" the Antarctic polar vortex and a midlatitude zonal mean profile $\left(46^{\circ} \mathrm{N}\right)$ obtained during the ATLAS-3 mission (11/94) which indicates the minimal seasonal variation outside the vortex at midlatitudes. Descent rates will be inferred from the vertical separation between the mean late winter vortex and extravortex tracer profiles.

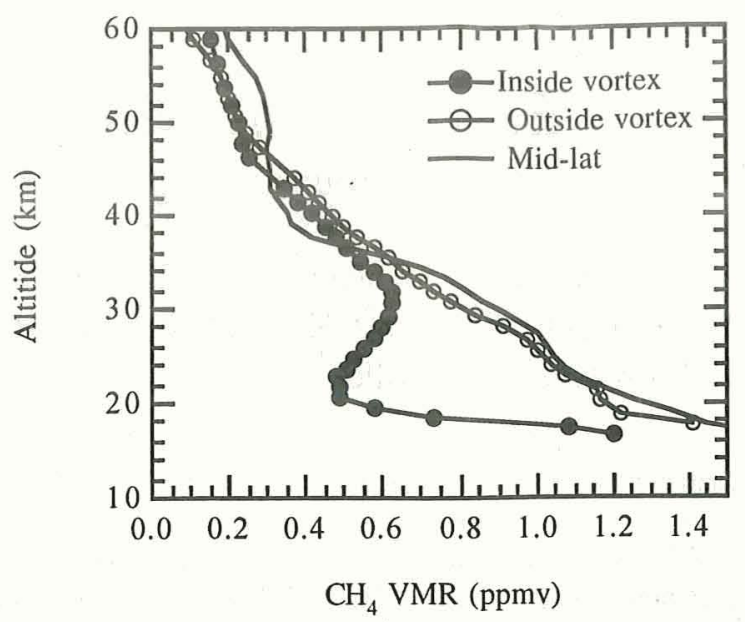

Figure 3. Mean $\mathrm{CH}_{4}$ volume mixing ratio profiles.

et al., 1994], at levels below about $30 \mathrm{~km}$ there is a distinct separation between the spring vortex and extra-vortex tracer mixing ratios. At these levels, a signature of relatively unmixed descent in the vortex is apparent. Mixing ratios in the lower stratospheric vortex (e.g., $20 \mathrm{~km}$ ) are typical of middle to upper stratosphere extra-vortex values (e.g., $35-40 \mathrm{~km}$ ). The lack of evidence of mesospheric air in the Arctic vortex, as was seen in the Antarctic during November 1994 [Abrams et al., 1996], is probably due primarily to the shorter period of descent and the greater degree of horizontal mixing in the Arctic.

Descent rates are inferred from the tracer measurements by estimating the vertical separation between the late winter vortex and extra-vortex tracer profiles for similar tracer mixing ratios, as discussed in greater detail by Abrams et al. [1996]. The estimates of descent rates depend upon an assumption of unmixed descent (that is, the only thing that alters the profiles is vertical motion); while this assumption has been shown to be less appropriate in the $\mathrm{NH}$ than in the $\mathrm{SH}$, the strong tracer gradients along the vortex edge below about $30 \mathrm{~km}$ suggest that it is a valid assumption at these levels. Differences between the extra-vortex spring profile and the winter midlatitude profile suggest some seasonal differences in the midlatitude upper stratosphere. In addition, the "vortex" profile is similar to the "extra-vortex" profile at these levels, since the vortex has broken down, and mixing occurred, as discussed above. Thus, any signature of unmixed descent at levels above about $35 \mathrm{~km}$ has been obscured by mixing.

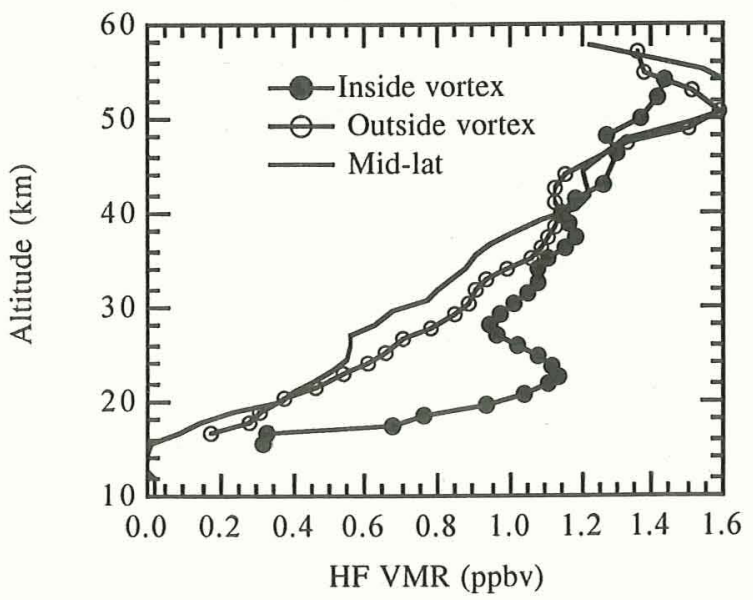

Figure 4. Mean HF volume mixing ratio profiles. 
Table 1. ATMOS/ATLAS-2 Net Arctic Descent Rates

\begin{tabular}{cccccc}
\hline $\begin{array}{c}\text { St. Alt. } \\
(\mathrm{km})\end{array}$ & $\begin{array}{c}\text { St. } \theta \\
(\mathrm{K})\end{array}$ & $\begin{array}{c}\text { Delta } \\
(\mathrm{km})\end{array}$ & $\begin{array}{c}\text { Rate } \\
(\mathrm{km} / \mathrm{mo})\end{array}$ & $\begin{array}{c}\text { St. Date } \\
(\mathrm{dd} / \mathrm{mm})\end{array}$ & $\begin{array}{c}\text { Adj. Rate } \\
(\mathrm{km} / \mathrm{mo})\end{array}$ \\
\hline 50 & 1700 & --- & --- & 1 Oct & --- \\
42 & 1300 & --- & --- & 1 Oct & -- \\
34 & 960 & 13.8 & 2.6 & 15 Oct & 2.4 \\
30 & 840 & 11.1 & 2.2 & 20 Oct & 1.9 \\
24 & 655 & 6.4 & 1.2 & 1 Nov & 1.2 \\
20 & 520 & 3.7 & 0.7 & 10 Nov & 0.74 \\
17 & 465 & --- & --- & 12 Nov & --- \\
15 & 25 & --- & --- & $*$ & --- \\
\hline
\end{tabular}

*at this level the starting date is uncertain, but much later than for the other levels.

Rosenfield et al. [1994] assumed a starting date of 1 November for $\mathrm{NH}$ descent rate calculations, and a 4.6 month period of descent ending on $21 \mathrm{March}$. As discussed by Abrams et al. [1996], the most appropriate starting date for calculations of unmixed descent is a function of altitude, and depends on when a barrier to transport has formed at the appropriate level. Table 1 shows two descent rates: the first calculated assuming a 1 November starting date (Rate), and the second assuming an adjusted starting date based on examination of sPV gradients (Adj. Rate, assuming the starting date (St. Date) given in the table), as described by Abrams et al. [1996]. Figure 5 shows descent rates calculated using the adjusted starting dates and observations of $\mathrm{N}_{2} \mathrm{O}, \mathrm{CH}_{4}$, and $\mathrm{HF}$. These are compared with several previous theoretical results. The results for each of the three tracers measured by ATMOS are consistent within the measurement precision. The results agree favorably with theoretical descent rates for the NH obtained by Manney et al. [1994] and Rosenfield et al. [1994] in the middle stratosphere between 30 and $35 \mathrm{~km}$, but below $30 \mathrm{~km}$ the theoretical results are $60 \%$ larger than the measurements at $25 \mathrm{~km}$ and $100 \%$ larger than the measurements at $20 \mathrm{~km}$. Recalling that the tracer profiles suggested more mixing near $20 \mathrm{~km}$, the calculations from ATMOS measurements may underestimate descent at low altitudes because the profiles are significantly affected by horizontal transport. In the upper stratosphere, there is no evidence of large scale descent of air, as suggested by the theoretical calculations [Rosenfield et al., 1994; Manney et al., 1994], or of air originating above the stratopause, as was apparent in the Antarctic [Abrams et al., 1996]. However, during the ATLAS-2 mission, horizontal mixing is expected to dilute the vortex air sufficiently at these altitudes so as to obscure any evidence of vertical motion. The absence of extremely low $\mathrm{N}_{2} \mathrm{O}$ and $\mathrm{CH}_{4}$ mixing ratios such as those observed in the Antarctic lower stratosphere may be explained by the shorter duration of the period of descent, and the mixing that has already occurred in the Arctic upper stratosphere.

Previous analyses of descent in the Arctic used one of three classes of long-lived tracer measurements: airborne column or in situ measurement which provide spatially distributed results without vertical information, or balloon-borne measurements which yield information about the vertical distribution of tracers, but only at a specific location. Mankin et al. [1990], Toon et al. [1992], and Traub et al. [1994] inferred 5-6 km of subsidence within the polar vortex from total column measurements obtained onboard the NASA DC-8 aircraft. Similarly, Lowenstein et al. [1990] inferred a subsidence of 4-6 km above $17 \mathrm{~km}$ based on measurements from the NASA ER-2 aircraft. Balloon measurements between 1987 and 1990 at $68^{\circ} \mathrm{N}$ suggested a vertical subsidence of about $7 \mathrm{~km}$ over the period between November and February [Schmidt et al., 1991]. To the degree possible without detailed vertical information inside and outside the polar vortex, these measurements are consistent with the measurements from the ATLAS-2 mission as well as model calculations. Descent rates have been inferred in several more recent experiments; Bauer et al. [1994] obtained vertical descent rates of $2.0 \mathrm{~km} / \mathrm{month}$ at $29.8 \mathrm{~km}$, which agrees adequately with the present measurements but also found rates of $3.1 \mathrm{~km} /$ month around $26 \mathrm{~km}$, which is nearly a factor of two larger than ATMOS measurements and theoretical estimates. Schoeberl et al. [1992] and Traub et al. [1995] obtained descent rates of 1.29 and $1.30 \mathrm{~km} /$ month in the lower stratosphere at 15.5 and $18 \mathrm{~km}$, respectively. The disagreement between the ATMOS/ATLAS-2 measurements and the theoretical results (supported by the measurements of Traub et al. [1995] and Schoeberl et al. [1992]) may result from differences between the years of the observations (1992 and 1990, respectively), the lack of vertical resolution, different assumptions about the descent period, or from the ATMOS observations underestimating descent once horizontal mixing becomes significant. The inclusion of measurements at the "edge" of the polar vortex which are likely to be most affected by mixing would lead to an underestimation of the descent rates, however, the statistical weight of such measurements was assessed and determined to be small relative to the measurement variance. The extra-vortex seasonal variation, illustrated in Figures $2-4$ is no more that $2 \mathrm{~km}$ at most altitudes and would results in a modest increase in the descent rates. Similarly, the estimation of "net" descent rates neglects the detailed motion of the air during the winter and consequently may overestimate the descent period. Manney et al. [1995] use results from CLAES to deduce descents rates of $\sim 1.4$ and $1.6 \mathrm{~km} / \mathrm{month}$ during the 1992-93 early and late winter, respectively, at about $21 \mathrm{~km}$, falling near the theoretical values, but between those and the estimates from ATMOS observations. In comparison with the analysis of descent from ATMOS observations within the Antarctic polar vortex [Abrams et al., 1996], the average descent rates in the Arctic are similar to those calculated for the Antarctic. However, because of the shorter period of descent in the Arctic, the magnitude of the total descent in the Arctic is much less.

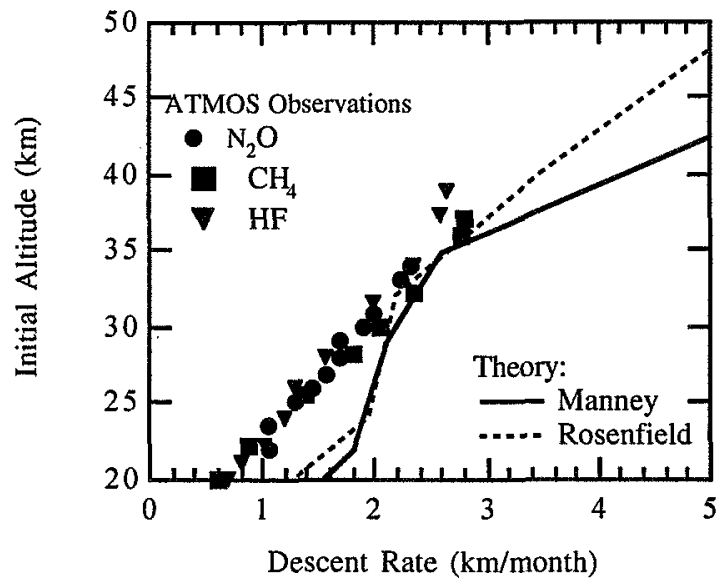

Figure 5. Descent rates obtained from ATMOS trace gas measurements compared with theoretical predictions. 


\section{Conclusions}

Trace gas measurements made by the ATMOS instrument as part of the ATLAS-2 and 3 Space Shuttle missions are used to infer descent rates in the Arctic polar vortex for the 1992-93 winter. The results compare favorably with the theoretical calculations of Manney et al. [1994a] and Rosenfield et al. [1994] in the mid-stratosphere and provide more information about the vertical extent of air motion and descent rates than previously available. Comparison to a similar analysis of ATMOS measurements in the Antarctic vortex shows similar descent rates in both hemispheres, but much deeper total descent in the Antarctic due to the longer period over which descent occurs. The ATMOS observations of $\mathrm{N}_{2} \mathrm{O}, \mathrm{CH}_{4}$, and $\mathrm{HF}$ in the Arctic indicate that, despite the erosion of the vortex that has already occurred in the upper stratosphere, a clear separation between vortex and extra-vortex air persists until mid-April 1993 in the middle and lower stratosphere. Future work will apply these descent rates to reactive species with the intention of characterizing the dynamical component present in ATMOS measurements of $\mathrm{O}_{3}, \mathrm{HCl}$, and $\mathrm{CO}$.

Acknowledgments. Research at the Jet Propulsion Laboratory (JPL), California Institute of Technology, is performed under contract to the National Aeronautics and Space Administration (NASA). Research at the University of Liège was partially supported by the Belgian Global Change Program through the SSTC, Brussels.

\section{References}

Abrams, M. C., et al., ATMOS/ATLAS-3 Observations of trace gas transport in the Antarctic vortex in November 1994, Geophys. Res. Lett., this issue, 1996.

Bauer, R. A., et al., Monitoring the vertical structure of the Arctic polar vortex over northern Scandinavia during EASOE: regular $\mathrm{N}_{2} \mathrm{O}$ profile observations, Geophys. Res. Lett., 21, 1211-1214, 1994.

Lowenstein, M., J. R. Podolske, K. R. Chan, and S. E. Strahan, $\mathrm{N}_{2} \mathrm{O}$ as a dynamical tracer in the Arctic vortex, Geophys. Res. Lett., 17, 477-480, 1990.

Mankin, W. G., M. T. Coffey, A. Goldman, M. R. Schoeberl, L. R. Lait, and P. A. Newman, Airborne measurements of stratospheric constituents over the Arctic in the winter of 1989, Geophys. Res. Lett., $17,473-476,1990$.
Manney, G. L., R. W. Zurek, A. O'Neill, and R. Swinbank, On the motion of air through the stratospheric polar vortex, J. Atmos. Sci., 51, 2973-2994, 1994a.

Manney, G. L., et al., Langrangian transport calculations using UARS data. Part I: Passive tracers, J. Atmos. Sci., 52, 3049-3068, 1995.

Rosenfield, J. E., P. A. Newman, and M. R. Schoeberl, Computations of diabatic descent in the stratospheric polar vortex, J. Geophys. Res., 99, $16677-16689,1994$.

Schmidt, U., R. Bauer, A. Khedim, E. Klein, G. Kulessa, and C. Schiller, Profile observations of long-lived trace gases in the Arctic vortex, Geophys. Res. Lett., 18, 767-770, 1991.

Schoeberl, M. R., L. R. Lait, P. A. Newman, and J. E. Rosenfield, The structure of the polar vortex, J. Geophys. Res., 97, 7859-7882, 1992.

Schoeberl, M. R., L. Mingzhao, and J. E. Rosenfield, An analysis of the Antarctic Halogen Occultation Experiment trace gas observations, J. Geophys. Res., 100, 5159-5172, 1995.

Swinbank, R., and A. O'Neill, A stratosphere-troposphere data assimilation system, Mon. Weather, Rev., 122, 686-702, 1994.

Toon, G. C., C. B. Farmer, P. W. Schaper, L. L. Lowes, R. H. Norton, M. R. Schoeberl, L. R. Lait, and P. A. Newman, Evidence for subsidence in the 1989 Arctic winter stratosphere from airborne infrared composition measurements, J. Geophys. Res., 97, 7963-7970, 1992.

Traub, W. A., K. W. Jucks, D. G. Johnson, and K. V. Chance, Chemical change in the Arctic vortex during AASE II, Geophys. Res. Lett., 21, 2595-2598, 1994.

Traub, W. A., K. W. Jucks, D. G. Johnson, and K. V. Chance, Subsidence of the Arctic stratosphere determined from thermal emission of hydrogen fluoride, J. Geophys. Res., 100, 11261-11267, 1995.

M. C. Abrams, SAIC-NASA LaRC, MS-475, Hampton, VA, 23681, (m.c.abrams@larc.nasa.gov)

A. Y. Chang, M. R. Gunson, G. L. Manney, and R. J. Salawitch, Jet Propulsion Laboratory

M. M. Abbas, NASA Marshall Space Flight Center

A. Goldman, University of Denver

F. W. Irion, California Institute of Technology

H. A. Michelsen, Harvard University

M. J. Newchurch, University of Alabama at Huntsville

C. P. Rinsland, NASA Langley Research Center

G. P. Stiller, Forschungszentrum Karlsruhe

R. Zander, Institute of Astrophysics, University of Liege

(Received September 20, 1995; revised November 21, 1995; accepted December 6, 1995.) 


\title{
ATMOS/ATLAS-3 observations of long-lived tracers and descent in the Antarctic vortex in November 1994
}

\author{
M. C. Abrams, ${ }^{1}$ G. L. Manney, ${ }^{2}$ M. R. Gunson, ${ }^{2}$ M. M. Abbas, ${ }^{3}$ A. Y. Chang, ${ }^{2}$ \\ A. Goldman, ${ }^{4}$ F. W. Irion, ${ }^{5,2}$ H. A. Michelsen, ${ }_{9}^{6}$ M. J. Newchurch, ${ }^{7}$ C. P. Rinsland, ${ }^{8}$ \\ R. J. Salawitch, ${ }^{2}$ G. P. Stiller, ${ }^{9}$ and R. Zander ${ }^{10}$
}

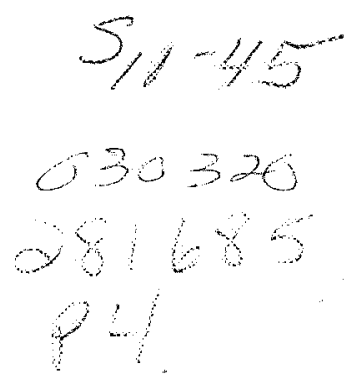

Abstract. Observations of the long-lived tracers $\mathrm{N}_{2} \mathrm{O}, \mathrm{CH}_{4}$ and HF obtained by the Atmospheric Trace Molecule Spectroscopy (ATMOS) instrument in early November 1994 are used to estimate average descent rates during winter in the Antarctic polar vortex of 0.5 to $1.5 \mathrm{~km} /$ month in the lower stratosphere, and 2.5 to $3.5 \mathrm{~km} /$ month in the middle and upper stratosphere. Descent rates inferred from ATMOS tracer observations agree well with theoretical estimates obtained using radiative heating calculations. Air of mesospheric origin $\left(\mathrm{N}_{2} \mathrm{O}<5 \mathrm{ppbV}\right)$ was observed at altitudes above about $25 \mathrm{~km}$ within the vortex. Strong horizontal gradients of tracer mixing ratios, the presence of mesospheric air in the vortex in early spring, and the variation with altitude of inferred descent rates indicate that the Antarctic vortex is highly isolated from midlatitudes throughout the winter from approximately $20 \mathrm{~km}$ to the stratopause. The 1994 Antarctic vortex remained well isolated between 20 and $30 \mathrm{~km}$ through at least mid-November.

\section{Introduction}

The transport of long-lived tracers remains a focus of considerable interest, especially with the results from the Upper Atmosphere Research Satellite (UARS). Schoeberl et al. [1995] reexamined the issue with Halogen Occultation Experiment (HALOE) data (version 16) with a carefully developed identification scheme to classify profiles as inside or outside the vortex. Late winter descent rates of $1.5-1.8 \mathrm{~km} / \mathrm{month}$ in the lower stratosphere were obtained which are consistent with theoretical analyses of meteorological data [Rosenfield et al., 1994; Manney et al., 1994].

The ATMOS instrument is a Shuttle-borne high resolution Fourier transform spectrometer that obtains vertical profiles of atmospheric composition from solar occultation measurements of infrared atmospheric transmission [Farmer, 1987]. Observations that sample inside, outside, and across the edge of the polar vortex were made at high Southern latitudes $\left(64-66^{\circ}\right)$ during the

\footnotetext{
${ }_{2}$ SAIC-NASA Langley Research Center

${ }_{3}$ Jet Propulsion Laboratory

${ }_{4}^{3}$ NASA Marshall Space Flight Center

${ }_{5}^{4}$ University of Denver

${ }_{6}$ California Institute of Technology

${ }_{7}^{6}$ Harvard University

${ }_{8}$ University of Alabama at Huntsville

${ }_{9}^{8}$ NASA Langley Research Center

Forschungszentrum Karlsruhe

Institute of Astrophysics, University of Liege
}

Copyright 1996 by the American Geophysical Union.

Paper number 96GL00704

0094-8534/96/96GL-00704\$05.00
ATLAS-3 mission (3 to 12 November, 1994). In the present Letter, measurements of three long-lived tracers, $\mathrm{N}_{2} \mathrm{O}, \mathrm{CH}_{4}$, and HF, are combined with potential vorticity (PV) from the United Kingdom Meteorological Office (UKMO) analyses to infer net winter descent rates. The results complement a similar analysis of descent rates within the NH polar vortex during the 1992-93 winter [Abrams et al., 1995].

\section{ATMOS and UKMO data analysis}

$\mathrm{N}_{2} \mathrm{O}, \mathrm{CH}_{4}$, and $\mathrm{HF}$ are among more than 30 gaseous constituents that can be profiled from ATMOS spectra. The 1994 measurements provide a snapshot of the Antarctica vortex in early spring that shows the net effects of wintertime transport on the tracer distributions. The tracer measurements are interpolated to isentropic (constant potential temperature, $\theta$ ) surfaces, upon which passive tracers and PV are conserved in the absence of diabatic and frictional effects. Figures 1 and 2 show the observed distributions of $\mathrm{N}_{2} \mathrm{O}$ and $\mathrm{CH}_{4}$ at $65^{\circ} \mathrm{S}$ as functions of $\theta$ and longitude. Manney et al. [1996] describe the degree to which the position of the vortex changes in relation to ATMOS measurements during this period: although its shape varies considerably, it is shifted off the pole in the same direction throughout the mission, so measurements over the 10 days give a reasonable estimate of the longitudinal structure of the tracer fields. The strong correlation between the behavior of $\mathrm{N}_{2} \mathrm{O}$ and $\mathrm{CH}_{4}$, which are fully independent measurements except for the common methodology used in the determination of pressure and temperature, demonstrates the consistency and accuracy of the ATMOS measurements. $\mathrm{N}_{2} \mathrm{O}$ mixing ratios of about $5 \mathrm{ppbV}$ and $\mathrm{CH}_{4}$ mixing ratios of about $0.3 \mathrm{ppmv}$ are seen within the vortex down to near $600 \mathrm{~K}$ (about $25 \mathrm{~km}$ ). These mixing ratios are typical of air with mesospheric origins.

Rossby-Ertel PV, obtained from the UKMO data assimilation system [Swinbank and O'Neill, 1994], can be used to characterize the location and extent of the polar vortex. PV is scaled [Manney et al., 1994] in "vorticity units" to provide a modest range of "sPV" values on surfaces of constant $\theta$ throughout the stratosphere. Strong horizontal SPV gradients coincide with the core of the polar night jet and represent a barrier to horizontal transport, facilitating the identification of the polar vortex edge. Contours of $\mathrm{SPV}$ are overlaid in Figures 1 and 2.

The vortex edge is clearly indicated in Figure 1 by very large gradients in $\mathrm{N}_{2} \mathrm{O}$ and $\mathrm{CH}_{4}$ at levels between about 420 and $1300 \mathrm{~K}$. Up to about $1000 \mathrm{~K}$, the strong tracer gradients are coincident with large horizontal gradients in sPV. Theoretical studies have shown that coherent fragments of air with vortexlike tracer values may remain well after the final warming, and after the signature of the vortex in PV has disappeared [e.g., Hess, 1991]. The $\mathrm{N}_{2} \mathrm{O}$ and $\mathrm{CH}_{4}$ in Figure 1 appear well-mixed below about $400 \mathrm{~K}$. Although there are strong sPV gradients below this 

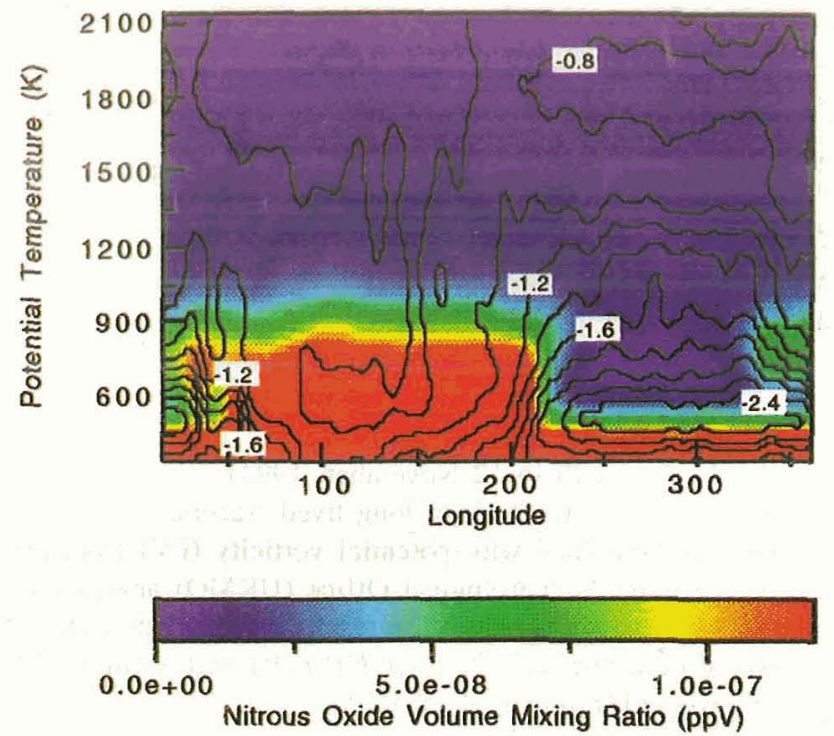

Figure 1. Potential temperature/longitude cross-section at $65^{\circ} \mathrm{S}$ of $\mathrm{N}_{2} \mathrm{O}$ (color) and sPV (contours). The vortex region is clearly defined by the steep gradients in potential vorticity between 1.2 and $-1.6 \times 10^{-4} \mathrm{sec}^{-1}$ in the lower stratosphere between $\sim 200^{\circ}$ and $350^{\circ} \mathrm{E}$.

level, they do not form until late winter [Manney et al., 1994], and are not continuous around the circumference of the vortex [Manney et al., 1996]. Thus, there is not expected to be as strong a barrier to transport at levels below $400 \mathrm{~K}$. These strong $\mathrm{N}_{2} \mathrm{O}$ and $\mathrm{CH}_{4}$ gradients clearly demonstrate the extremely strong barrier to horizontal transport at the edge of the Antarctic polar vortex between 400 and $1100 \mathrm{~K}$, and that this barrier remained intact in the lower stratosphere until at least mid-November 1994.

A vortex/extra-vortex classification scheme was developed which requires $\mathrm{N}_{2} \mathrm{O}, \mathrm{CH}_{4}$, and $\mathrm{sPV}$ values on the $655 \mathrm{~K}$ isentropic surface all to be characteristic of the vortex (mixing
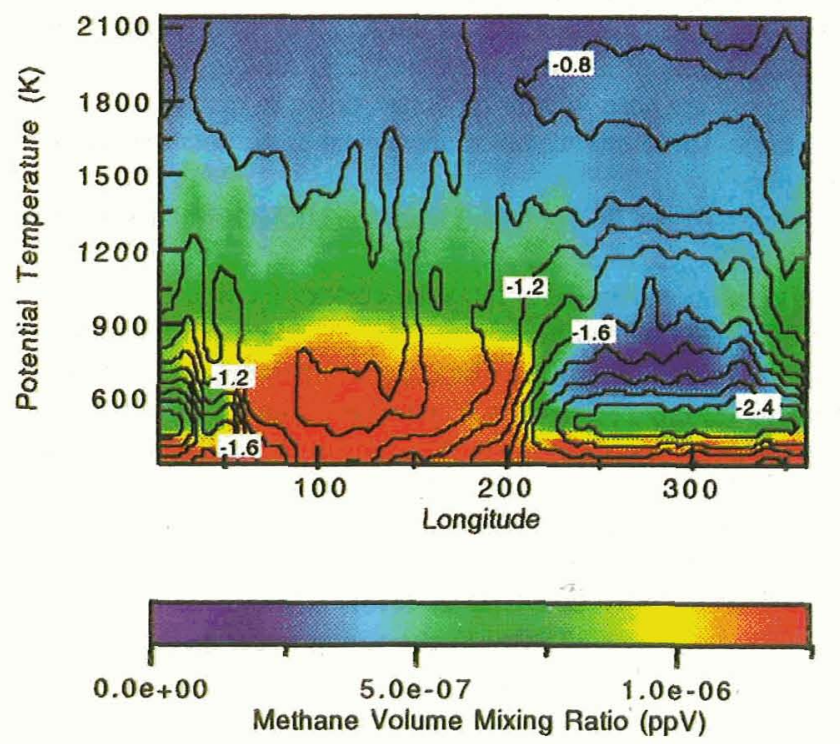

Figure 2. Potential temperature/longitude cross-section at $65^{\circ} \mathrm{S}$ of $\mathrm{CH}_{4}$ (color) and sPV (contours). Note that the $\mathrm{CH}_{4}$ and $\mathrm{N}_{2} \mathrm{O}$ maps do not display a gradient below $450 \mathrm{~K}$, whereas there is a significant gradient in SPV.

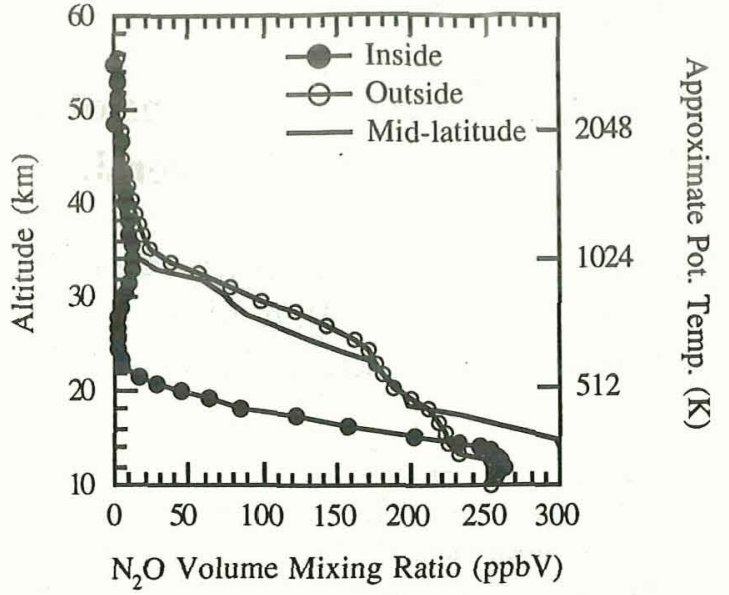

Figure 3. Mean $\mathrm{N}_{2} \mathrm{O}$ volume mixing ratio profiles.

ratios of less than $30 \mathrm{ppbv}$ for $\mathrm{N}_{2} \mathrm{O}$ and 0.60 ppmv for $\mathrm{CH}_{4}$ ). Figures 3-5 show mean vortex and extra vortex profiles of $\mathrm{N}_{2} \mathrm{O}$, $\mathrm{CH}_{4}$, and $\mathrm{HF}$. As was also apparent in Figure 1, there is an obvious distinction between vortex and extra-vortex air between $\sim 18$ and $35 \mathrm{~km}$.

\section{Descent rates inferred from tracer profiles}

Mean vortex and extra-vortex volume mixing ratio profiles of long-lived tracers measured in November 1994 (early spring) are compared with early winter profiles from the ATLAS-2 mission (April 1993) in Figures 3-5. The ATMOS measurements suggest minimal seasonal variation in the upper stratospheric profiles of long-lived tracers at midlatitudes $\left(45-60^{\circ} \mathrm{S}\right)$ and that descent rates within the polar vortex can be assessed relative to either extravortex air that has not been within the polar vortex or to spring air prior to the onset of winter-time descent. In the subsequent analysis, the former approach is used: comparison of vortex and extra-vortex profiles obtained during the same season (spring) and latitude, with corroboration from the opposite season extravortex profiles. An eight month period of descent (starting March 1) has been assumed in theoretical studies [Fisher et al., 1993 and Rosenfield et al., 1994]. However, the ATMOS measurements from the ATLAS-2 mission indicate that modest descent has occurred at altitudes above $35 \mathrm{~km}$ in early April, but not for lower

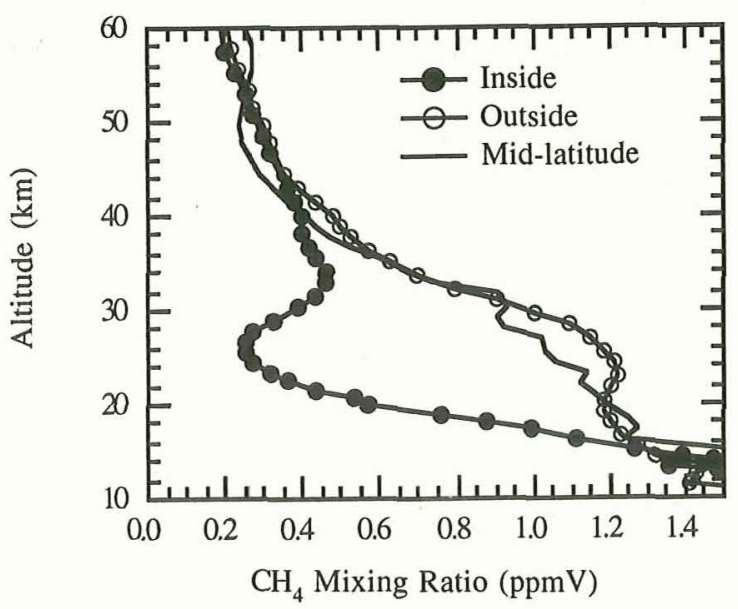

Figure 4. Mean $\mathrm{CH}_{4}$ volume mixing ratio profiles. 


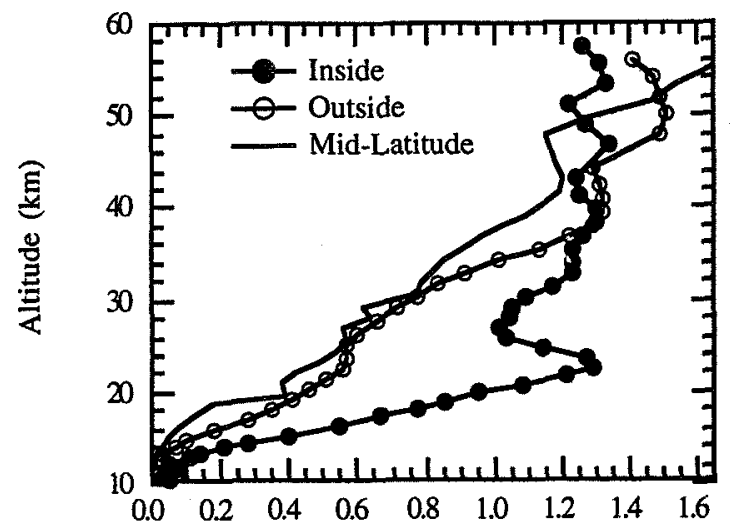

HF Volume Mixing Ratio (ppbV)

Figure 5. Mean HF volume mixing ratio profiles.

altitudes; hence defining a single starting date for all altitudes is incorrect. Since descent in much of the high-latitude stratosphere begins shortly after the summer solstice [Kiehl and Solomon, 1986], well before the vortex forms, the relevant starting time for examination of unmixed descent is the date after which the polar vortex has developed to the extent of providing a significant barrier to transport. Before this time, any signature of descent would be lost due to unrestrained horizontal mixing. To estimate this date, we examined the time evolution of SPV gradients as a function of sPV, during the SH fall of 1994, at levels throughout the stratosphere and located the first date on which sPV gradients associated with the polar vortex were significantly stronger than in surrounding regions. This leads to starting dates, given in Table 1, ranging from 25 March 1994 in the upper stratosphere to 12 May 1994 in the lower stratosphere.

Mean descent rates are determined from spring vortex and extra-vortex profiles of tracers assuming unmixed vertical descent within the vortex (i.e., vertical motion is the only factor that alters the profiles of long-lived tracers.), and that extra-vortex profiles are representative of fall conditions. For each of the three longlived tracers, the descent rate (Adj. Rate, in the table) has been inferred by dividing the vertical distance between the inside and outside profiles at similar gas amounts ( $\Delta z$ in Table 1$)$ by the time period of descent, based on starting dates derived from the evolution of sPV (St. Date in Table 1). Descent rates assuming an eight month period of descent are also given in Table 1 (Rate). Descent rates for each of the tracers, given in Figure 6, are in good agreement given the precisions of the measurements. The

Table 1. ATMOS/ATLAS-3 Antarctic Net Descent Rates

\begin{tabular}{cccccc}
\hline $\begin{array}{c}\text { St. Alt. } \\
(\mathrm{km})\end{array}$ & $\begin{array}{c}\text { St. } \theta \\
(\mathbf{K})\end{array}$ & $\begin{array}{c}\Delta \mathbf{z} \\
(\mathbf{k m})\end{array}$ & $\begin{array}{c}\text { Rate } \\
(\mathbf{k m} / \mathrm{mo})\end{array}$ & $\begin{array}{c}\text { St. Date } \\
(\mathrm{dd} / \mathrm{mm})\end{array}$ & $\begin{array}{c}\text { Adj. Rate } \\
(\mathrm{km} / \mathrm{mo})\end{array}$ \\
\hline 50 & 1700 & 26 & 3.25 & $25 \mathrm{Mar}$ & 3.6 \\
42 & 1300 & 20 & 2.5 & $25 \mathrm{Mar}$ & 2.7 \\
34 & 960 & 14.4 & 1.8 & $1 \mathrm{Apr}$ & 2.1 \\
30 & 840 & 12.0 & 1.5 & $5 \mathrm{Apr}$ & 1.8 \\
24 & 655 & 8.4 & 1.05 & $15 \mathrm{Apr}$ & 1.3 \\
20 & 520 & 4.4 & 0.55 & $8 \mathrm{May}$ & 0.8 \\
17 & 465 & 2.4 & 0.3 & $12 \mathrm{May}$ & 0.4 \\
15 & 425 & 1.4 & 0.18 & $*$ & --- \\
\hline
\end{tabular}

*at $425 \mathrm{~K}$ the starting date is uncertain, but much later.

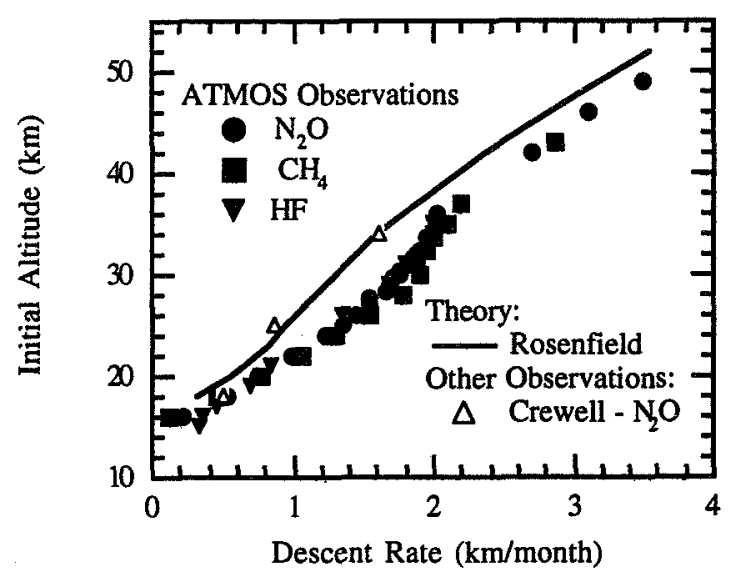

Figure 6. Adjusted descent rates obtained from ATMOS trace gas measurements compared with previous observations and theoretical calculations. Table 1 enumerates the starting dates used to adjust the descent rates. For comparison the rate of $1 \mathrm{~km} /$ month at $24 \mathrm{~km}$ corresponds to $32.9 \mathrm{~m} /$ day, or $0.039 \mathrm{~cm} / \mathrm{sec}$, assuming $365.25 / 12=30.44$ days $/$ month.

unweighted standard deviations are between 10 and $25 \%$, and the precision of the vertical registration ranges between $0.05 \mathrm{~km}$ (at $20 \mathrm{~km}$ ) and $0.4 \mathrm{~km}$ (at $50 \mathrm{~km}$ ) are in relatively good agreement with the scatter between the rates inferred from each of the three gases. Air initially at $20 \mathrm{~km}$ descended $0.6 \mathrm{~km} / \mathrm{month}$, while air initially at $30 \mathrm{~km}$ descended $1.5 \mathrm{~km} /$ month. Air in the lower mesosphere at initial heights above $50 \mathrm{~km}$ descended $3.3-3.5 \mathrm{~km} /$ month to levels around $600 \mathrm{~K}$ (approximately $20-25 \mathrm{hPa}$ ). The curvature of the descent rate profiles inferred from ATMOS measurements of $\mathrm{N}_{2} \mathrm{O}, \mathrm{CH}_{4}$, and $\mathrm{HF}$ provides additional corroborative evidence for a highly isolated vortex throughout the winter, during periods of rapid loss of $\mathrm{O}_{3}, \mathrm{H}_{2} \mathrm{O}$, and nitrogen oxides within the vortex. In 1994, the vortex remained strong and isolated between about 20 and $30 \mathrm{~km}$ until at least mid November. Therefore, at these levels, polar processes are not expected to have any strong direct impact on mid-latitude distributions of $\mathrm{O}_{3}, \mathrm{H}_{2} \mathrm{O}$, and nitrogen oxides until later in the spring.

Rosenfield et al. [1994] calculated diabatic descent rates using NMC temperatures during the $1992 \mathrm{SH}$ winter, and found average winter (March-October) descent rates of $0.4-0.9 \mathrm{~km} / \mathrm{month}$ for air starting in the lower stratosphere, and 3.3-3.6 km/month for air started at $52 \mathrm{~km}$. This profile is shown in Figure 6 . Rosenfield noted the paucity of information on descent rates inferred from tracer profiles in comparison with column measurements indicating 6-8 km of subsidence above $12 \mathrm{~km}$ [Toon et al., 1989]. Ground-based measurements of $\mathrm{N}_{2} \mathrm{O}$ [Crewell et al., 1995] at the South pole were used to derive early winter, late winter, and net winter descent rates. The net winter descent rates of Crewell et al. [1995] compare favorably with the present results. Analysis of UARS/HALOE satellite measurements [Schoeberl et al., 1995] yielded descent rates of $1.5-1.8 \mathrm{~km} /$ month for the lower stratosphere (altitude below $25 \mathrm{~km}$ ), during September and October and a net winter descent rate of $1.5 \mathrm{~km} /$ month. These descent rates agree closely with the present measurements and the theoretical calculations of Rosenfield et al. [1994].

The estimates from ATMOS presented here constitute minimum estimate of descent rates, since horizontal mixing will dilute the signature of unmixed descent in the vortex. This is expected 
to influence our results most at the highest and lowest levels, where there is evidence of significant horizontal mixing. Similarly, seasonal variation in the extra-vortex tracer levels, or mixing gradients at the vortex edge, and averaging over the entire season prior to the measurement period would compound the under-estimation of the descent rates. The descent rates for late winter [Schoeberl et al., 1995 and Crewell et al., 1995] are 2-3 times larger than the net rates. Given the differences between the measurement years (Toon-1987; Crewell-1993; Schoeberl-1992, and 1994 in the present work), periods, and durations, the results are remarkably consistent. The ATMOS measurements extend the altitude range of descent measurements up to the lower mesosphere and provide definitive evidence of transport of mesospheric air to levels near $25 \mathrm{~km}$.

\section{Conclusions}

Colocated measurements of the long-lived tracers $\mathrm{N}_{2} \mathrm{O}, \mathrm{CH}_{4}$ and $\mathrm{HF}$ during the ATMOS/ATLAS-3 mission provide conclusive evidence for descent within the vortex of air originating in the lower mesosphere to about $25 \mathrm{~km}$ over the course of the winter. Descent rates of $0.5-1.5 \mathrm{~km} /$ month in the lower stratosphere (below $~ 30 \mathrm{~km}, 850 \mathrm{~K}$, or $10 \mathrm{hPa}$ ), and $2.5-3.5 \mathrm{~km} /$ month in the upper stratosphere and lower mesosphere are inferred from the tracer measurements. The consistency of the descent rates inferred from profiles of three long-lived tracers attests to the precision of the measurements and the robustness of the overall conclusions. Our results are consistent with previous theoretical and observational estimates of descent, but extend the vertical range of descent estimate from observations. Strong horizontal gradients in $\mathrm{N}_{2} \mathrm{O}, \mathrm{CH}_{4}$, and HF demonstrate the vortex was highly isolated from mid-latitude air, between 20 and $30 \mathrm{~km}$, until midNovember.

Acknowledgments. We thank the UKMO for providing meteorological data. Research at the Jet Propulsion Laboratory (JPL), California Institute of Technology, is performed under contract to the National Aeronautics and Space Administration (NASA). Research at the University of Liège was partially supported by the Belgian Global Change Program through the SSTC, Brussels.

\section{References}

Abrams, M. C. et al., Trace gas transport in the Arctic vortex inferred from ATMOS ATLAS-2 observations during April 1993, Geophys. Res. Lett., this issue, 1996.

Crewell, S., D. Cheng, R. L. de Zafra, and C. Trimble, Millimeter-wave spectroscopic measurements over the South Pole, I: a study of stratospheric dynamics using $\mathrm{N}_{2} \mathrm{O}$ observations, J. Geophys. Res., in press, 1995 .

Farmer, C. B., High resolution infrared spectroscopy of the Sun and the Earth's atmosphere from space, Mikrochim. Acta (Wien), III, 189-214, 1987.

Fisher, J., A. O'Neill, and R. Sutton, Rapid descent of mesospheric air in the stratospheric polar vortex, Geophys. Res. Lett., 20, 1267-1270, 1993.

Hess, P. G., Mixing processes following the final stratospheric warming. J. Atmos. Sci., 48, 1625-1641, 1991.

Kiehl, J. T. and S. Solomon, On the radiative balance of the stratosphere, J. Atoms. Sci., 43, 1525-1534, 1986.

Manney, G. L., R. W. Zurek, A. O'Neill, and R. Swinbank, On the motion of air through the stratospheric polar vortex, J. Atmos. Sci., 51, 2973-2994, 1994.

Manney, G. L., R. Swinbank, and A. O'Neill, Stratospheric meteorological conditions for the 3-12 Nov. 1994 ATMOS/ATLAS-3 measurements, Geophys. Res. Lett., this issue, 1996.

Rosenfield, J. E., P. A. Newman, and M. R. Schoeberl, Computations of diabatic descent in the stratospheric polar vortex, J. Geophys. Res., 99, 16677-16689, 1994.

Schoeberl, M. R., L. Mingzhao, and J. E. Rosenfield, An analysis of the Antarctic Halogen Occultation Experiment trace gas observations, J. Geophys. Res., 100, 5159-5172, 1995.

Swinbank, R., and A. O'Neill, A stratosphere-troposphere data assimilation system, Mon. Weather, Rev., 122, 686-702, 1994.

Toon, G. C., C. B. Farmer, L. L. Lowes, P. W. Schaper, J.-F. Blavier, and R. H. Norton, Infrared aircraft measurements of stratospheric composition over Antarctica during September 1987, J. Geophys. Res., 94, 16571-16596, 1989.

M. C. Abrams, SAIC-NASA LaRC, MS-475, Hampton, VA, 23681, (m.c.abrams@larc.nasa.gov)

(Received September 20, 1995; revised November 21, 1995; accepted December 6, 1995.) 


\title{
Trends of OCS, HCN, $\mathrm{SF}_{6}, \mathrm{CHClF}_{2}$ (HCFC-22) in the lower stratosphere from 1985 and 1994 Atmospheric Trace Molecule Spectroscopy experiment measurements near $30^{\circ} \mathrm{N}$ latitude
}

\author{
C. P. Rinsland, ${ }^{1}$ E. Mahieu, ${ }^{2}$ R. Zander, ${ }^{2}$ M. R. Gunson, ${ }^{3}$ R. J. Salawitch, ${ }^{3}$ A. Y. Chang, ${ }^{3}$ \\ A. Goldman, ${ }^{4}$ M. C. Abrams, ${ }^{5}$ M. M. Abbas, ${ }^{6}$ M. J. Newchurch, ${ }^{7}$ and F. W. Irion ${ }^{8}$
}

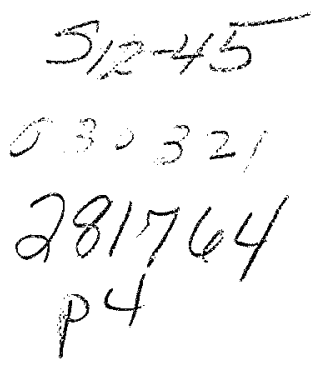

\begin{abstract}
Volume mixing ratio (VMR) profiles of OCS, $\mathrm{HCN}$, $\mathrm{SF}_{6}$, and $\mathrm{CHClF}_{2}$ (HCFC-22) have been measured near $30^{\circ} \mathrm{N}$ latitude by the Atmospheric Trace Molecule Spectroscopy Fourier transform spectrometer during shuttle flights on 29 April6 May 1985 and 3-2 November 1994. The change in the concentration of each molecule in the lower stratosphere has been derived for this $91 / 2$-year period by comparing measurements between potential temperatures of 395 to $800 \mathrm{~K}(\sim 17$ to $30 \mathrm{~km}$ altitude) relative to simultaneously measured values of the longlived tracer $\mathrm{N}_{2} \mathrm{O}$. Exponential rates of increase inferred for 1985-to-1994 from these comparisons are $(0.1 \pm 0.4) \% \mathrm{yr}^{-1}$ for OCS, $(1.0 \pm 1.0) \% \mathrm{yr}^{-1}$ for $\mathrm{HCN},(8.0 \pm 0.7) \% \mathrm{yr}^{-1}$ for $\mathrm{SF}_{6}$, and $(8.0 \pm 1.0) \% \mathrm{yr}^{-1}$ for $\mathrm{CHClF}_{2}$ (HCFC-22), 1 sigma. The lack of an appreciable trend for OCS suggests the background (i.e. nonvolcanic) source of stratospheric aerosol was the same during the two periods. These results are compared with trends reported in the literature.
\end{abstract}

\section{Introduction}

The Atmospheric Trace Molecule Spectroscopy (ATMOS) instrument is a $0.01-\mathrm{cm}^{-1}$ resolution Fourier transform spectrometer (FTS) designed to record broadband, infrared solar occultation spectra of the middle atmosphere from low Earth orbit [Farmer, 1987]. To date, the ATMOS FTS has flown 4 times on board the shuttle as part of the Spacelab 3 (29 April-6 May 1985), ATLAS (Atmospheric Laboratory for Applications and Science) 1 (24 March-2 April 1992), ATLAS 2 (8-17 April 1993), and ATLAS 3 (3-12 November 1994) missions.

An important geographic measurement overlap occurred at $28^{\circ} \mathrm{N}-33^{\circ} \mathrm{N}$ latitude where occultations were recorded at sunset during the Spacelab 3 and ATLAS 3 flights, respectively. The middle atmosphere profiles of more than 2 dozen molecules

ved from these measurements provide a unique record of

nges in stratospheric composition at northern lower midlautudes over the $91 / 2$ years separating these 2 ATMOS missions [i.e., Zander et al., 1996a,b].

\footnotetext{
${ }^{1}$ NASA Langley Research Center, Hampton, VA.

Institute of Astrophysics, University of Liège, 4000 Liège-Cointe, Belgium.

${ }^{3}$ Jet Propulsion Laboratory, California Institute of Technology, Pasadena.

${ }^{4}$ University of Denver, Denver, CO.

${ }^{5}$ Science Applications International Corporation, Hampton, VA.

${ }^{6}$ NASA Marshall Space Flight Center, Huntsville, AL

${ }^{7}$ University of Alabama, Huntsville.

${ }^{8}$ California Institute of Technology, Pasadena.
}

Copyright 1996 by the American Geophysical Union.

Paper number 96GL01234

0094-8534/96/96GL-01234\$05.00
The present Letter uses the Spacelab 3 and ATLAS 3 measurements in this common latitudinal band to assess changes in the volume mixing ratios (VMRs) of 4 important trace molecules: $\mathrm{OCS}, \mathrm{HCN}, \mathrm{SF}_{6}$, and $\mathrm{CHClF}_{2}$ (HCFC-22). For each molecule, the measured VMRs in the lower stratosphere are referenced to simultaneous values for the conserved, long-lived tracer $\mathrm{N}_{2} \mathrm{O}$ to remove the contribution of atmospheric dynamics to the measured variations of the target molecules. The 1985-1994 trends inferred from the VMR differences have been compared with values reported in the literature for the same time period.

\section{Spectroscopic Analysis}

Pressure-temperature and constituent profiles were derived from the ATMOS spectra with the Occultation Display Spectra (ODS) onion-peeling retrieval algorithm [Norton and Rinsland, 1991] and the 1995 ATMOS line parameters compilation [Brown et al., 1996]. Except for HCN, the spectroscopic parameters for the target molecules are significantly different than those used for the previous version of the ATMOS database [Brown et al., 1987]. The $\mathrm{CO}_{2}$ profile is a fundamental assumption in both the pressure-temperature and subsequent VMR retrievals. The $\mathrm{CO}_{2}$ VMRs assumed for the Spacelab 3 mission decrease from $3.40 \times 10^{-4}$ at the surface to $3.33 \times 10^{-4}$ in the middle and upper stratosphere. Corresponding values for the ATLAS 3 mission are $3.64 \times 10^{-4}$ and $3.51 \times 10^{-4}$, respectively. A synopsis of the procedure used to produce the version 2 VMR profiles reported in this paper and estimates of their precisions and accuracies have been provided by Abrams et al. [1996]. Data from all ATMOS missions, including Spacelab 3, were reprocessed.

For OCS and HCN, narrow intervals ("microwindows") containing isolated, unblended target spectral features were fitted by line-by-line methods over prespecified ranges in tangent altitude. A weighted mean and weighted standard deviation of the individual profiles were then calculated for each occultation and interpolated to a potential temperature $(\theta)$ grid. Six OCS microwindows in the $v_{3}$ band provided coverage between altitudes of 5 and $25 \mathrm{~km}$. Ten microwindows in the $v_{3}$ band were used to measure HCN between altitudes of 6 and $30 \mathrm{~km}$. The profiles for $\mathrm{SF}_{6}$ and $\mathrm{CHClF}_{2}$ were derived from unresolved $\mathrm{Q}$ branches on the basis of temperature- and pressure-dependent absorption coefficients from laboratory spectra. A single microwindow was fitted for each molecule. For $\mathrm{SF}_{6}$, the microwindow spanned 945.0 to $952.0 \mathrm{~cm}^{-1}$, covering the intense $v_{3}$ band $\mathrm{Q}$ branch between altitudes of 13 to $28 \mathrm{~km}$. For $\mathrm{CHClF}_{2}$, a $0.5-\mathrm{cm}^{-1}$ wide microwindow centered at $829.05 \mathrm{~cm}^{-1}$ was used to measure the $2 v_{6} \mathrm{Q}$ branch between 13 and $33 \mathrm{~km}$. Examples of the target features in lower stratospheric ATMOS solar occultation spectra have been presented previously [Zander et al., 1987; 1988; Rinsland et al., 1993]

Profiles of $\left[\mathrm{N}_{2} \mathrm{O}\right.$ ] (where the brackets are used to denote the species VMR) were obtained by combining measurements from 
microwindows in several bands, depending on the filter selected for the occultation. Ground-based spectroscopic measurements indicate a $\mathrm{N}_{2} \mathrm{O}$ total column exponential growth rate of $(0.36 \pm$ $0.06) \% \mathrm{yr}^{-1}$ between 1984 and 1992 [Zander et al., 1994a]. Assuming this rate, we detrend the $\mathrm{N}_{2} \mathrm{O}$ measurements by defining $\left[\mathrm{N}_{2} \mathrm{O} *\right]=1.035 *\left[\mathrm{~N}_{2} \mathrm{O}\right]$ for Spacelab 3 and $\left[\mathrm{N}_{2} \mathrm{O} *\right]=\left[\mathrm{N}_{2} \mathrm{O}\right]$ for ATLAS 3 data. [HCN] and [OCS] with relative uncertainties greater than $20 \%$ and $\left[\mathrm{SF}_{6}\right]$ and $\left[\mathrm{CHClF}_{2}\right]$ with relative uncertainties above $35 \%$ have been excluded from our analysis along with $\left[\mathrm{N}_{2} \mathrm{O}^{*}\right]$ above 330 ppbv.

\section{Results}

\section{Carbonyl Sulfide (OCS)}

Figure 1 presents a plot of simultaneous OCS and $\mathrm{N}_{2} \mathrm{O}^{*}$ VMRs from 4 Spacelab 3 and 6 ATLAS 3 occultations. Solid and dashed lines show the corresponding OCS VMRs derived by averaging the measurements in intervals of 0.05 in $\log _{10}\left(\mathrm{~N}_{2} \mathrm{O}^{*}\right)$. The 1994-to-1985 OCS VMR ratio was calculated for each bin. The average and standard deviation of these ratios are $1.01 \pm$ $0.04,1$ sigma. Assuming an exponential model for the OCS VMR increase with time, the corresponding trend is $(0.1 \pm$ $0.4) \% \mathrm{yr}^{-1}$. Hence, the measurements indicate no significant change in the OCS lower stratospheric VMR over this time period.

The inference of a negligible long-term OCS trend is consistent with the results obtained from high resolution groundbased IR measurements [Rinsland et al., 1992]. Total columns retrieved from spectra recorded at the National Solar Observatory (NSO, $31.9^{\circ} \mathrm{N}$ latitude, $111.6^{\circ} \mathrm{W}$ longitude) between May 1977 and March 1991 showed a trend of $(0.1 \pm 0.2) \% \mathrm{yr}^{-1}, 2$ sigma, while similar measurements at the International Station of the Jungfraujoch (ISSI, $46.5^{\circ} \mathrm{N}$ latitude, $8.0^{\circ} \mathrm{E}$ longitude) between October 1984 and April 1991 yielded a trend of $(-0.1 \pm$ $0.5) \% \mathrm{yr}^{-1}, 2$ sigma.

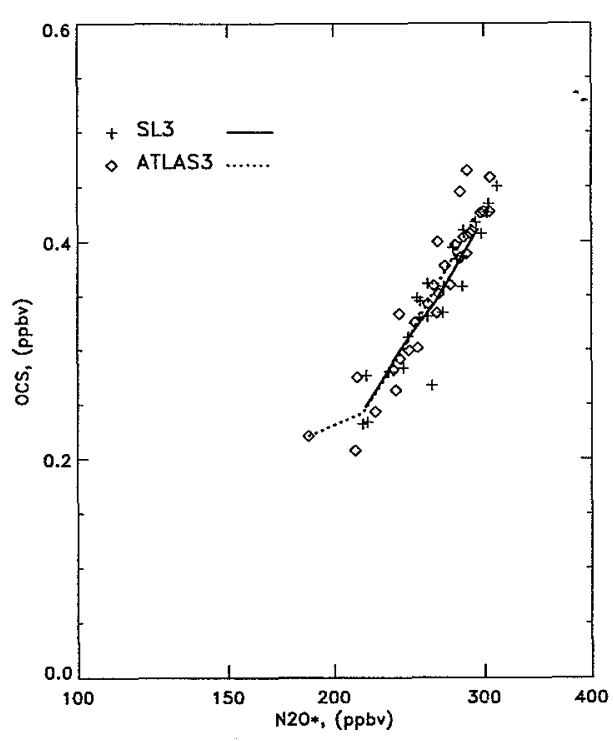

Figure 1. ATMOS Spacelab 3 and ATLAS 3 [OCS] versus $\left[\mathrm{N}_{2} \mathrm{O}^{*}\right]$ (in ppbv, $10^{-9}$ ) for measurements between $26^{\circ} \mathrm{N}$ and $33^{2} \mathrm{~N}$ latitude and $\mathrm{T}$ between 395 and $800 \mathrm{~K}$ (altitudes of $\sim 17$ to $30 \mathrm{~km}$ ). Solid and dashed lines show [OCS] averages as a function of $\left[\mathrm{N}_{2} \mathrm{O}^{*}\right]$ derived from the 1985 and 1994 measurements, respectively. See text for details.

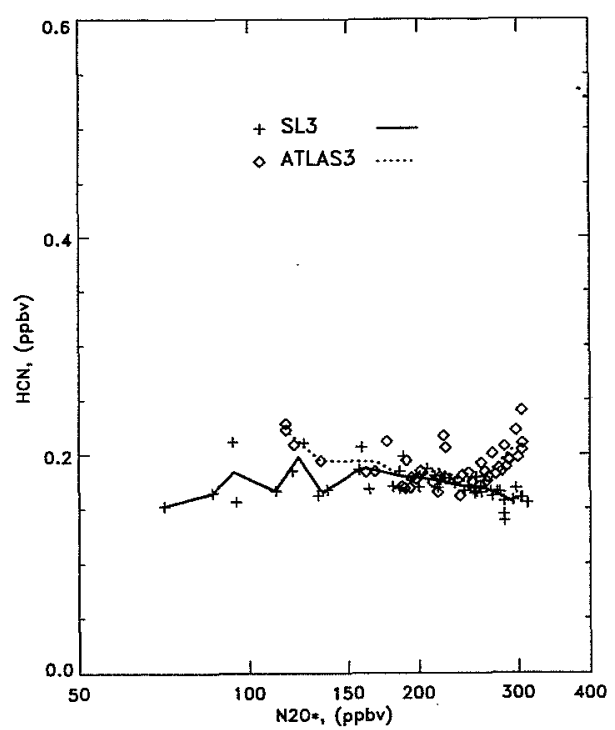

Figure 2. Same as Figure 1 except for $\mathrm{HCN}$.

The primary source of stratospheric sulfate aerosols under background (nonvolcanic) conditions is thought to be OCS. Budget studies suggest that the main sources of OCS are natural with biomass burning, the largest anthropogenic source, contributing about $10 \%$ to the total [Khalil and Rasmussen, 1984.] The eruption of Mount Pinatubo $\left(15.14^{\circ} \mathrm{N}, 120.35^{\circ} \mathrm{E}\right)$ in the Philippines on June 15, 1991, produced the largest volcanic input to the stratosphere ever observed by satellite instruments [McCormick, 1992], but as shown here, apparently no significant long-term change in stratospheric OCS loading resulted since the vast majority of the sulfur injected in the stratosphere was in the form of $\mathrm{SO}_{2}$. An increase in OCS concentrations could cause important climate changes [Turco et al., 1980] and increase stratospheric sulfate aerosol mass, altering $\mathrm{O}_{3}$ concentrations through heterogeneous chemical reactions [Hofmann, 1990]. Our measurements suggest little change in the background stratospheric aerosol mass between the two periods of observation.

\section{Hydrogen Cyanide (HCN)}

Figure 2 presents measurements of lower stratospheric [HCN] versus $\left[\mathrm{N}_{2} \mathrm{O}^{*}\right]$ from 6 Spacelab 3 and 4 ATLAS 3 occultations. The format is the same as in Figure 1. Based on the mean and standard deviation calculated from the averages shown by the solid and dashed lines, the average 1994-to--1985 HCN VMR ratio is $1.10 \pm 0.10,1$ sigma. This value corresponds to an exponential increase rate of $(1.0 \pm 1.0) \% \mathrm{yr}^{-1}$. Hence, there is marginal evidence for an increase in HCN VMRs in the lower stratosphere over the 91/2 year-period separating the Spacelab 3 and ATLAS 3 missions.

To our knowledge, the only previous investigation of the HCN long-term trend was reported by Mahieu et al. [1995]. In that study, high resolution ground-based IR measurements obtained at NSO between June 1984 and June 1993 and at ISSJ between May 1978 and July 1992 were analyzed to derive total vertical column abundances that indicated HCN long-term column trends of $(-0.30 \pm 0.50) \% \mathrm{yr}^{-1}$ above NSO and $(0.99 \pm 0.51) \% \mathrm{yr}^{-1}$ above ISSJ. Hence, the present results are in better agreement with the ISSJ measurements [Mahieu et al., 1995], which suggest a small, long-term increase in $\mathrm{HCN}$ abundance. 


\section{Sulfur hexafluoride $\left(\mathrm{SF}_{6}\right)$}

The lower stratospheric measurements of $\left[\mathrm{SF}_{6}\right]$ versus $\left[\mathrm{N}_{2} \mathrm{O}\right]$ near $30^{\circ} \mathrm{N}$ latitude from 1 Spacelab 3 and 6 ATLAS 3 occultations are presented in Figure 3. A substantial VMR increase over the $91 / 2$ years separating the 2 missions is readily apparent. Based on the measurements, the average 1994-to-1985 $\mathrm{SF}_{6}$ VMR ratio in the lower stratosphere is $2.14 \pm 0.14,1$ sigma, which corresponds to an exponential growth rate of $(8.0 \pm$ $0.7) \% \mathrm{yr}^{-1}$.

The present result is in agreement with 4 recent determinations of the $\mathrm{SF}_{6}$ long-term trend, all based on high resolution infrared solar absorption spectra. Rinsland et al. [1990] deduced an exponential growth rate of $(7.4 \pm 1.9) \% \mathrm{yr}^{-1}$ from comparison of solar occultation measurements recorded near $30^{\circ} \mathrm{N}$ latitude between March 1981 and June 1988 in the $12-18 \mathrm{~km}$ altitude region. Zander et al. [1991] reported $\mathrm{SF}_{6}$ total column exponential increase rates of $(6.6 \pm 7.2) \% \mathrm{yr}^{-1}, 2$ sigma, above NSO between March 1981 and June 1990 and $(6.9 \pm 2.8) \% \mathrm{yr}^{-1}$, 2 sigma, above ISSJ between June 1986 and June 1990. The larger uncertainty in the NSO measurement was the result of stronger $\mathrm{H}_{2} \mathrm{O}$ and $\mathrm{CO}_{2}$ interferences overlapping the target $\mathrm{SF}_{6} \mathrm{v}_{3}$ band Q branch (the NSO FTS is at an altitude of $2095 \mathrm{~m}$ as compared to $3580 \mathrm{~m}$ for the ISSJ FTS). An exponential growth rate of $(8.7 \pm 2.2) \% \mathrm{yr}^{-1}, 2$ sigma, was inferred by comparing University of Denver $\left(32^{\circ} \mathrm{N}\right)$, ATMOS Spacelab $3\left(31^{\circ} \mathrm{N}\right)$, and ATMOS/ATLAS $1\left(28^{\circ} \mathrm{S}, 54^{\circ} \mathrm{S}\right)$ measurements in the $12-18 \mathrm{~km}$ altitude region after applying a small correction for the $\mathrm{SF}_{6}$ interhemispheric gradient based on model calculations [Rinsland et al., 1993].

Recently, $\mathrm{SF}_{6}$ measurements with a gas chromatograph have been obtained from a monitoring site in rural eastern North Carolina [Hurst et al., 1995]. Preliminary results suggest an increase of $(0.151 \pm 0.021) \times 10^{-12}$ in baseline $\mathrm{SF}_{6}$ mixing ratios during 1995, corresponding to a rate of $(4.9 \pm 0.7) \% \mathrm{yr}^{-1}, 1$ sigma (D. F. Hurst, unpublished data, 1996). This growth rate is lower than indicated by the published IR measurements.

Although the atmospheric concentration of $\mathrm{SF}_{6}$ is increasing rapidly and the gas is likely to have a very long lifetime [Ko et al., 1993; Ravishankara et al., 1993], its abundance is very

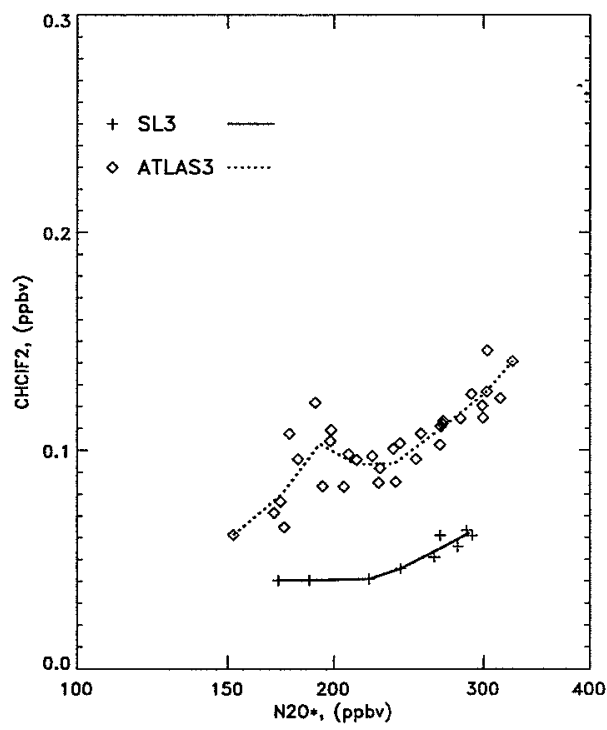

Figure 3. Same as Figure 1 except for $\mathrm{SF}_{6}$.

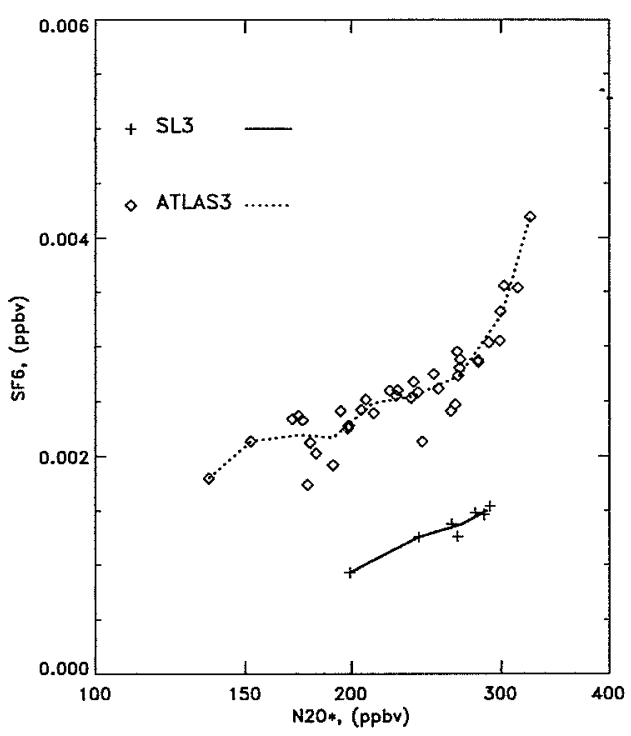

Figure 4. Same as Figure 1 except for $\mathrm{CHClF}_{2}(\mathrm{HCFC}-22)$.

low. The projected greenhouse warming potential of $\mathrm{SF}_{6}$ on a per molecule basis is high, but the net warming due to $\mathrm{SF}_{6}$ will remain negligible with respect to that of $\mathrm{CO}_{2}$, at least through 2010 [Ko et al., 1993].

\section{$\mathrm{CHClF}_{2}$ (HCFC-22)}

The comparison in Figure 4, derived from 3 Spacelab 3 and 4 ATLAS 3 occultations, shows the substantial growth of hydrochlorofluorocarbon (HCFC)-22 that has occurred in the lower stratosphere in response to the increasing use of this molecule as a substitute for the CFCs, particularly $\mathrm{CCl}_{2} \mathrm{~F}_{2}$ (CFC-12) and CClF (CFC-11) [AFEAS, 1995]. The average 1994-to-1985 HCFC-22 lower stratospheric VMR ratio derived from the ATMOS measurements is $2.14 \pm 0.21$, 1 sigma, which corresponds to an exponential HCFC-22 growth rate of $(8.0 \pm$ $1.0) \% \mathrm{yr}^{-1}$.

The present result is consistent with recent IR and in situ HCFC-22 trend determinations. Zander et al. [1994] reported total column exponential increase rates of $(7.0 \pm 0.23) \% \mathrm{yr}^{-1}$ above NSO from December 1980 to April 1992 and (7.0 \pm $0.35) \% \mathrm{yr}^{-1}$ above ISSJ from June 1986 to November 1992 . Irion et al. [1994] inferred an exponential total column growth rate of $(6.7 \pm 0.5) \% \mathrm{yr}^{-1}$ on the basis of IR solar spectra recorded from the Table Mountain Facility, Wrightwood, California $\left(34.4^{\circ} \mathrm{N}\right)$, between October 1985 and July 1990 . A slightly higher growth rate of $(7.3 \pm 0.3) \% \mathrm{yr}^{-1}$ was deduced by Montzka et al. [1993] from surface ambient air sampling measurements from mid-1987 through 1992. Solar occultation IR measurements near $30^{\circ} \mathrm{N}$ latitude between March 1981 and June 1988 also yielded a higher exponential growth rate, $(9.4 \pm 1.3) \% \mathrm{yr}^{-1}$ in the $12-18 \mathrm{~km}$ altitude region [Rinsland et al., 1990].

Empirically-based determinations of global ozone depletion potentials for HCFC-22 are up to a factor of 2 larger than values from some gas phase models [Solomon et al., 1992]. The similarity of the observed growth rates of HCFC-22 and $\mathrm{SF}_{6}$ and the similarity of the slopes of these gases relative to $\mathrm{N}_{2} \mathrm{O}$ suggest that the stratospheric lifetime of HCFC-22 is much longer than the published estimate of 210 years [NASA, 1994]. This result, coupled with the rapid growth of atmospheric HCFC-22 
concentrations, suggests that this molecule will continue to be an important contributor to ozone depletion in the stratosphere.

\section{Conclusions}

Trends in the volume mixing ratios of OCS, $\mathrm{HCN}, \mathrm{SF}_{6}$, and $\mathrm{CHClF}_{2}(\mathrm{HCFC}-22)$ in the lower stratosphere have been derived from ATMOS measurements obtained near $30^{\circ} \mathrm{N}$ latitude during shuttle flights on 29 April-6 May 1985 and 3-12 November 1994. Among the most significant results are (i) no significant increase in OCS was observed following the massive Mt. Pinatubo volcanic eruption; (ii) a marginally significant $\mathrm{HCN}$ increase was measured over this $91 / 2$-year span; and (iii) both $\mathrm{SF}_{6}$ and $\mathrm{CHClF}_{2}$ are accumulating rapidly in the lower stratosphere with rates of increase generally consistent with previously published measurements for the same time period.

Acknowledgments. Research at the Jet Propulsion Laboratory (JPL), California Institute of Technology is performed under contract with the National Aeronautics and Space Administration (NASA). R. Z. and E. M. are supported by Belgian funds supplied by S.S.T.C., Brussels. The authors thank the ATMOS data processing team at JPL and L. Chiou of Science Applications International Corporation (SAIC), Hampton, Virginia, for their help.

\section{References}

Abrams, M. C., et al., On the assessment of atmospheric trace gas burdens with high resolution infrared solar occultation measurements from space, Geophys. Res. Lett., this issue, 1996.

AFEAS, Alternative Fluorocarbons Environmental Acceptability Study, Production, sales and atmospheric release of fluorocarbons through 1993, Washington, D. C., 1995.

Brown, L. R., et al., Molecular line parameters for the atmospheric trace molecule spectroscopy experiment, Appl. Opt., 26, 5154-5182, 1987.

Brown, L. R., et al., The 1995 Atmospheric Trace Molecule Spectroscopy (ATMOS) linelist, Appl. Opt., in press, 1996.

Farmer, C. B., High resolution infrared spectroscopy of the Sun and the Earth's atmosphere from space, Mikrochim. Acta (Wien), III, 189-214, 1987.

Hofmann, D. J., Increase in the stratospheric sulfuric acid aerosol mass in the past 10 years, Science, 248, 996-1000, 1990.

Hurst, D. F., et al., Observations of sulfur hexafluoride from a very tall tower in the southeastern united states (abstract), Eos Trans. AGU, paper A11E-3, 1995.

Irion, $F$. W., et al., Increase in atmospheric $\mathrm{CHF}_{2} \mathrm{Cl}$ (HCFC-22) over southern california from 1985 to 1990 , Geophys. Res. Lett., 21, 1723-1726, 1994.

Khalil, M. A. K., and R. A. Rasmussen, Global sources, lifetimes and mass balances of carbonyl sulfide (OCS) and carbonyl disulfide ( $\left.\mathrm{CS}_{2}\right)$ in the earth's atmosphere, Atmos. Environ., 18, 1805-1813, 1984.

Ko, M. K. W., et al., Atmospheric sulfur hexafluoride: Sources, sinks, and greenhouse warming, J. Geophys. Res., 98, 10,499-10,507, 1993.

Mahieu, E., et al., Vertical column abundances of HCN deduced from ground-based infrared solar spectra; Long-term trend and variability, J. Atmos. Chem, 20, 299-310, 1995.

McCormick, M. P., Initial assessment of the stratospheric and climatic impact of the 1991 Mount Pinatubo eruption: Prologue, Geophys. Res. Lett., 19, 149, 1992.
Montzka, S. A., et al., Global tropospheric distribution and calibration scale of HCFC-22, Geophys. Res. Lett., 20, 703-706, 1993.

NASA Reference Publ. 1339, Table 5.9, 1994.

Norton, R. H., and C. P. Rinsland, ATMOS data processing and science analysis methods, Appl. Opt., 30, 389-400, 1991.

Ravishankara, A. R., et al., Atmospheric lifetimes of long-lived halogenated species, Science, 259, 194-199, 1993.

Rinsland, C. P., et al., Long-term trends in the concentrations of SF, $\mathrm{CHClF}_{2}$, and $\mathrm{COF}_{2}$ in the lower stratosphere from analysis of highresolution infrared solar occultation spectra, J. Geophys. Res., 95, $16,477-16,490,1990$.

Rinsland, C. P., et al., Ground-based infrared measurements of carbonyl sulfide total column abundances: Long-term trends and variability, J. Geophys. Res., 97, 5995-6002, 1992.

Rinsland, C. P., et al., ATMOS/ATLAS 1 Measurements of sulfur hexafluoride $\left(\mathrm{SF}_{6}\right)$ in the lower stratosphere and upper troposphere, J. Geophys. Res., 98, 20,491-20,494, 1993.

Solomon, S., et al., On the evaluation of ozone depletion potentials, J. Geophys. Res., 97, 825-42, 1992.

Turco, R. P., et al., OCS, stratospheric aerosols, and climate, Nature, 283, 283-286, 1980.

Zander, R., et al., Concentrations of carbonyl sulfide (OCS) and hydrogen cyanide (HCN) in the free upper troposphere and lower stratosphere deduced from ATMOS/Spacelab 3 infrared solar occultation spectra, J. Geophys. Res., 93, 1669-1678, 1988.

Zander, R., C. P. Rinsland, and P. Demoulin, Infrared spectroscopic measurements of the vertical column abundance of sulfur hexafluoride, $\mathrm{SF}_{6}$, from the ground, J. Geophys. Res., 96, 15,447$15,454,1991$.

Zander, R., et al., Secular trend and seasonal variability of the column abundance of $\mathrm{N}_{2} \mathrm{O}$ above the Jungfraujoch station determined from IR solar spectra, J. Geophys. Res., 99, 16,745-16,756, 1994a.

Zander, R. et al., Secular evolution of the vertical column abundances of $\mathrm{CHClF}_{2}$ (HCFC-22) in the Earth's atmosphere inferred from groundbased IR solar observations at the Jungfraujoch and at Kitt Peak, and comparison with model calculations, J. Atmos. Chem., 18, 129-148, $1994 \mathrm{~b}$.

Zander, R., et al., The 1994 northern midlatitude budget of stratospheric chlorine derived from ATMOS/ATLAS -3 observations, Geophys. Res. Lett., this issue, 1996a.

Zander, R., et al., Increase of stratospheric carbon tetrafluoride $\left(\mathrm{CF}_{4}\right)$ based on ATMOS observations from space, Geophys. Res. Lett., this issue, 1996b.

C. P. Rinsland, NASA LaRC, Mail Stop 401A, Hampton, VA 23681-0001.

E. Mahieu and R. Zander, Institute of Astrophysics, University of Liège, 4000 Liège-Cointe, Belgium.

A. C. Chang, M. R. Gunson, and R. J. Salawitch, JPL, Mail Stop 183-301, 4800 Oak Grove Drive, Pasadena, CA 91109.

A. Goldman, Department of Physics, University of Denver, Denver, CO 80208.

M. C. Abrams, NASA LaRC, Mail Stop 475, Hampton, VA 23681-0001.

M. M. Abbas, SSL, NMSFC, Huntsville, AL 35889.

M. J. Newchurch, University of Alabama at Huntsville, Huntsville, AL 35899.

F. W. Irion, Department of Chemical Engineering, Caltech, Pasadena, CA 91125.

(Received November 27, 1995; revised March 26, 1996; accepted April 5, 1996.) 


\title{
Increase of stratospheric carbon tetrafluoride $\left(\mathrm{CF}_{4}\right)$ based on ATMOS observations from space
}

\author{
R. Zander, ${ }^{1}$ S. Solomon, ${ }^{2}$ E. Mahieu, ${ }^{1}$ A. Goldman, ${ }^{3}$ C .P. Rinsland, ${ }^{4}$ \\ M. R. Gunson, ${ }^{5}$ M. C. Abrams, ${ }^{6}$ A. Y. Chang, ${ }^{5}$ R. J. Salawitch, ${ }^{5}$ \\ H. A. Michelsen, ${ }^{7}$ M. J. Newchurch, ${ }^{8}$ and G. P. Stiller ${ }^{9}$
}

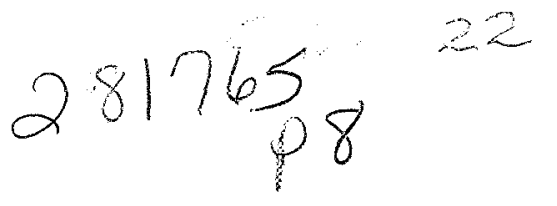

\begin{abstract}
Stratospheric volume mixing ratio profiles of carbon tetrafluoride, $\mathrm{CF}_{4}$, obtained with the Atmospheric Trace Molecule Spectroscopy (ATMOS) instrument during the ATLAS (Atmospheric Laboratory for Applications and Science) -3 mission of 1994 are reported. Overall the profiles are nearly constant over the altitude range 20 to $50 \mathrm{~km}$, indicative of the very long lifetime of $\mathrm{CF}_{4}$ in the atmosphere. In comparison to the stratospheric values of $\mathrm{CF}_{4}$ inferred from the ATMOS/Spacelab 3 mission of 1985 , the 1994 concentrations are consistent with an exponential increase of (1.6 \pm $0.6) \% / y r$. This increase is discussed with regard to previous results and likely sources of $\mathrm{CF}_{4}$ at the ground. Further, it is shown that simultaneous measurements of $\mathrm{N}_{2} \mathrm{O}$ and $\mathrm{CF}_{4}$ provide a means of constraining the lower limit of the atmospheric lifetime of $\mathrm{CF}_{4}$ at least 2,300 years, two sigma.
\end{abstract}

\section{Introduction}

Atmospheric carbon tetrafluoride $\left(\mathrm{CF}_{4}\right.$, also referred to as tetrafluoromethane, perfluoromethane, $\mathrm{FC}-14$ ) is thought to have both natural and anthropogenic sources at the ground [i.e., Cicerone, 1979; Penkett et al., 1981; Fabian et al., 1987] but their identification and relative strengths have not been well established so far. Being a fully fluorinated organic compound, $\mathrm{CF}_{4}$ is an inert tracer and has a very long lifetime recently estimated to lie between 25,000 and 50,000 years [Ravishankara et al., 1993]. Thus, it is expected to be uniformly mixed both geographically and vertically around the globe and to have a large "global warming potential" (GWP) due to its absorption characteristics in the infrared (IR) [Roehl et al., 1995]. The GWP is a measure of the potential globally averaged warming effect caused by the emission of a set amount (e.g., $1 \mathrm{~kg}$ ) of a trace gas. The total warming depends not only on the GWP but also on the total emissions; while $\mathrm{CF}_{4}$ has a large GWP, its current global emission and its corresponding impact on the climat system remain small (see IPCC, 1995).

Measurements of tropospheric $\mathrm{CF}_{4}$ mixing ratios by Gassmann [1974] over Europe and by Rasmussen et al.

\footnotetext{
"Institute of Astrophysics-University of Liège, Liège, Belgium 'NOAA Aeronomy Laboratory, Boulder, CO, USA

${ }^{3}$ University of Denver - Physics Department, Denver, CO, USA “ASD - NASA Langley Research Center, Hampton, VA, USA SJet Propulsion Laboratory, CalTec, Pasadena, CA, USA

'SAIC - NASA Langley Research Center, Hampton, VA, USA

${ }^{7}$ Harvard University, Cambridge, MA, USA

'University of Alabama at Huntsville, AL, USA

'IMK-Forschungszentrum Karlsruhe, Karlsruhe, Germany
}

Copyright 1996 by the American Geophysical Union.

Paper number 96GL00957

0094-8534/96/96GL-00957\$05.00
[1979] and Penkett et al. [1981] in both hemispheres, indicated levels of tropospheric background concentrations in the $(67 \pm 10)$ pptv (parts per trillion by volume, or $\times 10^{-12}$ by volume) range, nearly the same worldwide. Stratospheric values have been measured locally by Goldman et al. [1979], i.e., $75 \mathrm{pptv}$ at $25 \mathrm{~km}$ altitude, and over extended altitude ranges by Fabian and Goemer [1984] and by Fabion et al. [1987]. IR-remote measurements of the concentration versus altitude of $\mathrm{CF}_{4}$ throughout the stratosphere and upper troposphere were reported by Zander et al. [1987, 1992], based on observations with the ATMOS (Atmospheric Trace Molecule Spectroscopy) instrument during the Spacelab-3 mission of 1985 ; they suggested that $\mathrm{CF}_{4}$ has a nearly constant VMR ratio up to at least $50 \mathrm{~km}$.

From the analysis of air samples collected at the South Pole in 1978-79 and 1984, Khalil and Rasmussen [1985] concluded that $\mathrm{CF}_{4}$ accumulates in the Earth's atmosphere at a rate of about $2 \%$ or $1.3 \mathrm{pptv} / \mathrm{yr}$, which corresponds to a global emission of $1.8 \times 10^{10} \mathrm{gms} / \mathrm{yr}$; they suggested that the most likely anthropogenic source of $\mathrm{CF}_{4}$ may be from releases associated with electrolytic aluminum and steel reduction processes (see also Penkett et al., 1981).

In this Letter, we report volume mixing ratio profiles of $\mathrm{CF}_{4}$ retrieved from IR solar occultation observations with the ATMOS instrument during the 1994 ATLAS-3 mission and compare these with revised $\mathrm{CF}_{4}$ profiles derived from the ATMOS/Spacelab-3 flight of 1985 . We also report an estimate of the lower limit of the lifetime of $\mathrm{CF}_{4}$ in the atmosphere, based on $\mathrm{N}_{2} \mathrm{O}$ concentrations measured simultaneously.

\section{Data Base and Analysis}

The bulk of the results reported here have been derived from $0.01 \mathrm{~cm}^{-1}$ resolution infrared solar spectra obtained in the occultation mode with the ATMOS fast Fourier transform spectrometer [Farmer et al., 1987; Gunson et al., 1996] in low Earth orbit during the ATLAS-3 mission of November 312, 1994.

The spectral region analyzed for $\mathrm{CF}_{4}$ VMR profile retrievals extends from 1282.2 to $1285.2 \mathrm{~cm}^{-1}$, and includes the $\mathrm{Q}$ branch as well as a number of unresolved $\mathrm{R}$ branch manifolds of the very strong $v_{3}$ band of the target molecule. Target and major interfering absorptions by $\mathrm{CH}_{4}$ and $\mathrm{N}_{2} \mathrm{O}$ (also $\mathrm{CO}_{2}, \mathrm{H}_{2} \mathrm{O}, \mathrm{HNO}_{3}$ and $\mathrm{ClONO}_{2}$ below $30 \mathrm{~km}$ ) are accounted for in an iteration fitting sequence using the spectroscopic parameters maintained in the ATMOS line parameters compilation described by Brown et al. [1996]. In particular, the $\mathrm{CF}_{4}$ VMRs reported here were derived with a realistic set of spectroscopic parameters used previously [Brown et al., 1987; Zander et al., 1987] but scaled by a factor of 1.4 to account for new band strength estimates made by one of us (A.G.) using the $\mathrm{CF}_{4} \mathrm{~V}_{3}$ cross-sections reported 
by McDaniel et al. [1991]. Forward calculations and nonlinear least-squares spectral fittings were made with the ODS (Occultation Display Spectra) algorithm described by Norton and Rinsland [1991]. Details about data processing, retrieval procedure and ancillary input parameters are discussed by Gunson et al. [1996].

All sunset occultations that occurred between 3 and $49^{\circ} \mathrm{N}$ and encompassed the $v_{3}$ band of $\mathrm{CF}_{4}$ have been analyzed consistently as part of the ATMOS - "Version 2" data set; they comprise 17 and 30 events observed with filters Nr. $9(600$, $\left.2450 \mathrm{~cm}^{-1}\right)$ and Nr. $12\left(600-1400 \mathrm{~cm}^{-1}\right)$, respectively. This represents the largest single set of measurements which broadly overlaps the latitudes $\left(25\right.$ to $32^{\circ} \mathrm{N}$ ) of the 1985 ATMOS observations [Zander et al., 1987]. Averages corresponding to subsets of spectra binned over specific latitude and altitude ranges were also analyzed for consistency of the results. Such averages improve signal-to-noise ratios of the resulting spectra, thus extending the range of detectability of the weak $\mathrm{CF}_{4}$ features to higher altitudes. Typical examples of sample spectra covering the interval fitted for $\mathrm{CF}_{4}$ retrievals have been reported in Figs. 17 and 18 of Zander et al. [1987], showing the weakness of the target absorption features in the upper stratosphere and the extent of interfering absorptions below $30 \mathrm{~km}$.

In addition to the northern sunset occultations mentioned before, the present analysis also evaluated $\mathrm{CF}_{4} \mathrm{VMR}$ profiles from the 14 filter Nr. 9 and 27 filter Nr. 12 sunrise occultations that occurred between 65 and $72^{\circ}$ southern latitudes, both inside and outside the Antarctic vortex. $\mathrm{CF}_{4}$ VMRs derived from ATMOS observations during the ATLAS-1 (03/24 to 04/02/92) and ATLAS-2 (04/08 to 16 , 1993) missions are also reported for consistency.

\section{Results and Discussion}

Figure 1 reproduces VMR profiles of $\mathrm{CF}_{4}$ derived from the ATMOS/ATLAS-3 occultations observed at northern latitudes, over the pressure range from about 1 to $50 \mathrm{mb} \sim \sim 50$ to $20 \mathrm{~km}$ altitude). The increased scatter of the VMRs above $40 \mathrm{~km}$ is the result of the weakening of the target absorption features at high altitudes, while the curvature near the bottom of the profiles reflects a systematic effect resulting from the "Version-2" retrieval process and strong interfering absorption features. Local excursions of the data around the means are likely to result from propagation of errors associated with the onion-peeling retrieval method adopted here. One-sigma random errors with which individual profiles can be retrieved are discussed and documented by Gunson et al. [1996] and Abrams et al. [1996]. Except where mentioned specifically, and because of the large number of occultations involved in this analysis, the standard deviations are representative of the precision assigned to the reported VMR profiles.

A characteristic feature that emerges from the $\mathrm{CF}_{4}$ profiles displayed in Fig. 1 is their near-constant VMR in the stratosphere. The thick continuous and dotted vertical lines represent the average VMRs corresponding to the arithmetic means of the individual sunset profiles binned according to the ATMOS filter Nr. 9 and filter Nr. 12, respectively. These as well as similar averages derived from the ATLAS-3 sunrise occultations lie within the 71 to 76 pptv range (the filter Nr. 9 results which extend only over the 20 to $40 \mathrm{~km}$ altitude range for quality reasons, are slightly larger $(\sim 2 \%)$ than those obtained from filter Nr. 12) with their mean of $73.5 \mathrm{pptv}$ having a standard deviation of $2.7 \mathrm{pptv}$ and a precision of $3 \mathrm{pptv}$. This

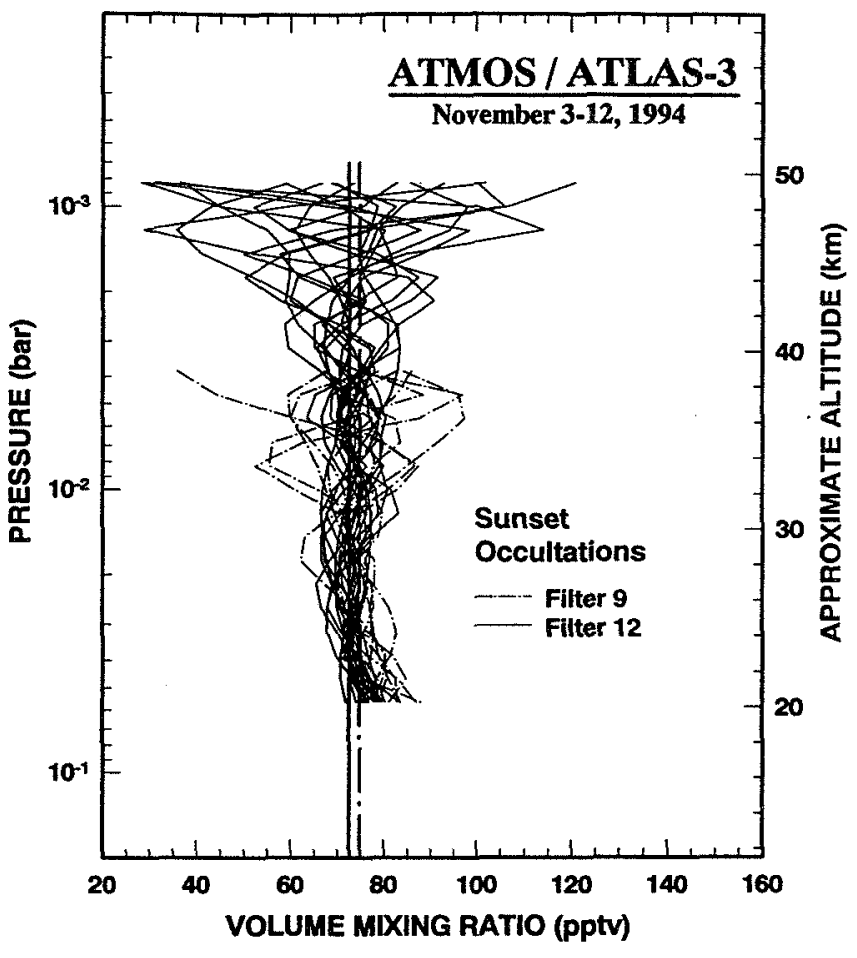

Figure 1. Series of $\mathrm{CF}_{4}$ VMR profiles retrieved from sunset occultations that occurred between 3 and $49^{\circ} \mathrm{N}$ during the ATMOS/ATLAS-3 mission of Nov. 3 to 12, 1994. The thick straight lines correspond to the mean constant VMRs obtained from all profiles with filter \# 9 (dotted line) and filter \# 12 (continuous line).

mean November 1994 VMR which applies at quite different latitudes around the Earth, is $17 \%$ larger than the April-May 1985 mean $\mathrm{CF}_{4} \mathrm{VMR}$ of $(63 \pm 4)$ pptv retrieved from the 3 sunset occultations observed by ATMOS near $30^{\circ} \mathrm{N}$ during the April-May 1985 Spacelab-3 mission. The same analytical procedure was adopted for both 1985 and 1994 retrievals, in order to eliminate any biasing problem among all results reported in this Letter. The mean $\mathrm{CF}_{4}$ volume mixing ratios retrieved here are summarized in Table 1.

The relative constancy of the VMR profiles versus altitude and latitude is indicative of the long lifetime of $\mathrm{CF}_{4}$ in the atmosphere. This has already been suggested in earlier investigations [i.e., Cicerone, 1979] and was confirmed more recently by Ravishankara et al. [1993] and Morris et al. [1995] who re-evaluated all likely destruction mechanisms of $\mathrm{CF}_{4}$ in the Earth's atmosphere and concluded that its lifetime may be as long as 25,000 to 50,000 years.

While constant VMR profiles (which would correspond to an infinite lifetime) were assumed in the above $\mathrm{CF}_{4}$ trend evaluation, a more detailed binning versus height (at pressure levels consistent with the UARS pressure grid) reveals a weak decrease of the $\mathrm{CF}_{4}$ VMRs with increasing altitude. This decrease derived from the large set of ATMOS/ATLAS-3 observations is statistically significant and can be used as an observational constraint on the lower limit of the atmospheric lifetime of $\mathrm{CF}_{4}$.

A sound approach for its evaluation was based on correlations between simultaneous measurements of $\mathrm{CF}_{4}$ and $\mathrm{N}_{2} \mathrm{O}$, both being long-lived gases for which the steady state ratio of gradients can be assumed to be proportional to their lifetimes [Plumb and Ko, 1992]. Figure 2 shows the 
Table 1. Summary of mean retrieved $\mathrm{CF}_{4} \mathrm{VMRS}$ over indicated pressure range

\begin{tabular}{lccccc}
\hline \multirow{2}{*}{ Mission } & \multirow{2}{*}{ Nr. of } & Filter & Pressure & \multicolumn{2}{c}{ Retr. CF 4 pptv } \\
& occulations & Nr. & range, mb & Mean & St.Dev. \\
\hline \multirow{2}{*}{ SL-3 } & IxSR & 2 & $70-2$ & 55.5 & 7.8 \\
& $3 \times S S$ & 2 & $70-2$ & 63.0 & 4.0 \\
& & & & & \\
AT-1 & $14 \times S R$ & 2,9 & $26-2$ & 71.8 & 1.5 \\
& $13 \times S S$ & 2,9 & $70-2$ & 71.3 & 2.8 \\
\multirow{2}{*}{ AT-2 } & $19 \times S R$ & 2,12 & $70-2$ & 70.0 & 3.9 \\
& $13 \times S S$ & $2,9,12$ & $70-2$ & 68.7 & 8.0 \\
\multirow{2}{*}{ AT-3 } & $41 \times S R$ & 9,12 & $70-2$ & 74.0 & 4.5 \\
& $47 \times S S$ & 9,12 & $70-2$ & 73.5 & 2.7 \\
\hline
\end{tabular}

correlation plot derived from all sunset occultations binned in Fig. 1. The data used were restricted to the range of $\mathrm{N}_{2} \mathrm{O}$ VMR values between $3 \times 10^{-8}$ and $2.5 \times 10^{-7} \mathrm{ppv}$ in order to avoid the extremes of the profiles where lack of sensitivity (above $45 \mathrm{~km}$ ) and uncertainty in the automatic retrieval procedure (near and below $20 \mathrm{~km}$ ) may degrade or bias the results. The slope of the straight line fitted to all data points in Fig. 2 is equal to $1.8944 \times 10^{-5}$; assuming that $\mathrm{N}_{2} \mathrm{O}$ has a lifetime of 120 years [WMO-Report $N r .37,1995$ ], this slope translates into a "first order" lifetime of $\mathrm{CF}_{4}$ ranging from 1,100 to 3,800 years, two sigma. A similar evaluation performed by one of us (S.S., private report) based on ATMOS/Spacelab 3 measurements of $\mathrm{CF}_{4}$ [Zander et al., 1992] and $\mathrm{N}_{2} \mathrm{O}$ [Gunson et al., 1990] led to a range of $\mathrm{CF}_{4}$ lifetimes between 1,500 and 5,800 years.

However, a more realistic analysis must account for nonsteady state effects due to (i) the $\mathrm{N}_{2} \mathrm{O}$ increase at a rate of 0.3 $\% / y r$ [WMO-Report Nr. 25, 1992; Zander et al., 1994], (ii) the $\mathrm{CF}_{4}$ increase at a rate of $1.6 \% / y r$ derived above and (iii) a troposphere-stratosphere mixing time of 4 years. One then finds a more representative $\mathrm{CF}_{4}$ lifetime lying between 2,300 years and infinity, 2 sigma. We believe that the 2,300 years minimum lifetime of $\mathrm{CF}_{4}$ in the atmosphere is a useful constraint for assessing that molecule's impact on our environment. Similar long lifetimes for $\mathrm{CF}_{4}$ have been suggested by von Clarmann et al. [1995] and based on MarkIV-balloon measurements $[B$. Sen, private communication, 1995]. It should be noted that the $\mathrm{CF}_{4}$ lifetime evaluation is insensitive to any systematic bias of the spectroscopic parameters used in the retrieval process, provided that such a bias applies similarly throughout the altitude range involved.

Concern may be raised about the above procedure's validity. Its applicability was assessed for various long-lived gases such as $\mathrm{CCl}_{2} \mathrm{~F}_{2}, \mathrm{CCl}_{3} \mathrm{~F}, \mathrm{SF}_{6}, \ldots$, measured simultaneously with $\mathrm{N}_{2} \mathrm{O}$ during the ATMOS missions. For example, the application to $\mathrm{CCl}_{2} \mathrm{~F}_{2}$ performed for 3 latitudinal zones, i.e., midlatitudes $\left(49-35^{\circ} \mathrm{N}\right)$, subtropics $\left(35-20^{\circ} \mathrm{N}\right)$ and tropics $\left(20-3^{\circ} \mathrm{N}\right)$, all encompassed by sunset occultations during the ATLAS-3 mission, returned lifetimes of $\mathrm{CCl}_{2} \mathrm{~F}_{2}$ for these zones equal to 121,104 and 94 years, respectively. These values are in excellent agreement with the mean global range of 102 to 111 years reported in WMO-Nr. 18 [1989] and WMO-Nr. 37 [1995] and reflect the latitudinal dependence of the lifetime, in particular its shorter value in the tropics where upward transport favours faster intrusion to altitudes where photodissociation proceeds.

How do the present results compare with other findings about atmospheric $\mathrm{CF}_{4}$ ?

The atmospheric lifetime derived here points to a minimum value of 2,300 years, ranging up to many thousands of years. The $(1.6 \pm 0.6) \% / y r$ rate of $\mathrm{CF}_{4}$ increase throughout the stratosphere derived for the period separating the ATMOS mission of 1985 and 1994 is commensurate with (i) the 2 $\% / y r$ increase derived by Khalil and Rasmussen [1985] for the 65 pptv tropospheric background loading of $\mathrm{CF}_{4}$ at the south pole between 1978 and 1984, and (ii) the $2.5 \% / y r$ increase

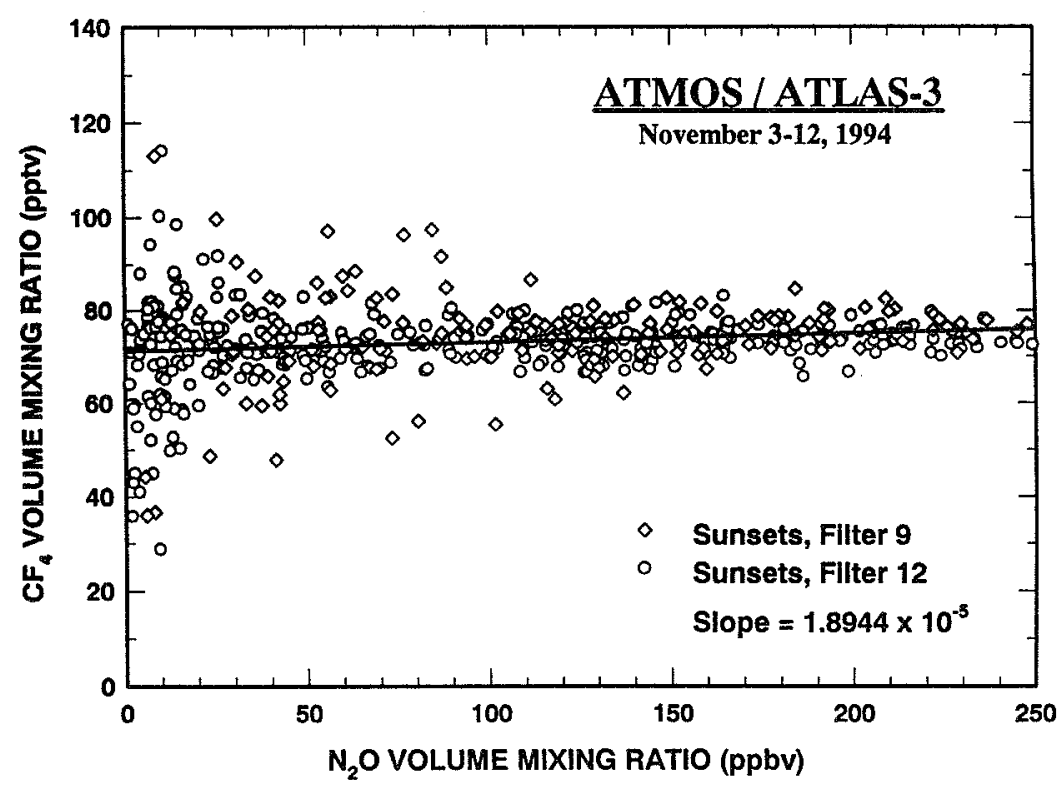

Figure 2. Correlation diagram of simultaneous $\mathrm{CF}_{4}$ and $\mathrm{N}_{2} \mathrm{O}$ VMR measurements for the sunset occultations reproduced in Fig. 1. The slope of the continuous straight line fitted to all data points has been used to evaluate the $\mathrm{CF}_{4}$ lifetime throughout the stratosphere (for details, see text). 
inferred from the $\mathrm{CF}_{4}$ balloon VMR profiles reported by Fabian et al. [1987] when considering the 10\% decrease observed between 10 and $35 \mathrm{~km}$ altitude and invoking a mixing time of 4 years. Overall, these trends are in good agreement (average of $2.0 \pm 0.5 \% / y r$ ) when considering the respective uncertainties in the various measurement methods involved and indicate that the global $\mathrm{CF}_{4}$ loading has increase at a rather constant rate during at least the last two decades. Based on the ATMOS findings reported here, the global burden of $\mathrm{CF}_{4}$ in the Earth's atmosphere was equal to $1.15 \times 10^{6}$ Tons in 1994 , increasing by about $1.8 \times 10^{4}$ Tons/yr.

\section{Conclusions}

Over the 9.5 year interval separating the ATMOS missions Spacelab-3 and ATLAS-3, the $\mathrm{CF}_{4}$ increase reported here is consistent with an exponential rate of $(1.6 \pm 0.6) \% / y r$, indicating that anthropogenic sources of $\mathrm{CF}_{4}$ have made significant contributions to the present global loading. This is an important outcome, since the extremely long lifetime (minimum of 2,300 years) for carbon tetrafluoride in the atmosphere, leads to a GWP which is among the largest reported thus far $[I P C C, 1995]$. The upcoming ATMOS data processing will incorporate improvements in the $\mathrm{CF}_{4}$ spectroscopic data base [Brown et al., 1996; A. Goldman and $P$. Varanasi, private communications] and is expected to reduce the absolute uncertainties of the findings presented here. The need for spectroscopic parameters about "new", non traditional molecules whose interest has emerged during the last decade (this is certainly the case for $\mathrm{CF}_{4}$ ) is stressed here; it should be performed in the laboratory under experimental spectral resolution conditions achieved with current state-ofthe-art field instruments and span temperature and pressure ranges encountered throughout the entire lower and middle atmosphere.

Acknowledgements This work was carried out within the overall ATMOS activities conducted at the Jet Propulsion Laboratory (JPL), California Institute of Technology, under contract with the National Aeronautics and Space Administration (NASA). The Liège involvement was partly supported by funds from the Belgian "Global Change" program coordinated by the S.S.T.C., Brussels.

\section{References}

Abrams, M. C., et al., A summary assessment of the precision and accuracy of ATMOS measurements of atmospheric composition during the ATLAS Shuttle missions, in preparation, 1996.

Brown, L. R., et al., Molecular line parameters for the atmospheric trace molecule spectroscopy experiment, Appl. Opt., 26, 5154$5182,1987$.

Brown, L. R., et al., The 1995 Atmospheric Trace Molecule Spectroscopy (ATMOS) linelist, submitted to Appl. Opt., 1996.

Cicerone, R., Atmospheric carbon tetrafluoride: a nearly inert gas, Science, 206, 59-61, 1979.

Fabian, P., and D. Goemer, The vertical distribution of halocarbons in the stratosphere, Fresenius Z. Anal. Chem., 319, 890-897, 1984.

Fabian, $\mathrm{P}$., et al., $\mathrm{CF}_{4}$ and $\mathrm{C}_{2} \mathrm{~F}_{6}$ in the atmosphere, J. Geophys. Res., 92, 9831-9835, 1987.

Farmer, C. B., et al., Final report on the first flight of the ATMOS instrument during the Spacelab 3 mission, 29 April through 6 May, 1985; JPL publication 87-32, Jet Propulsion Laboratory, California Institute of Technology, Pasadena, California, USA, 1987.

Gassmann, M., Freon 14 in the "ultra clean" krypton and in the atmosphere, Naturwissenschaften, 61, 127, 1974.

Goldman, A., et al., Identification of the $v_{3}$ vibration-rotation band of $\mathrm{CF}_{4}$ in balloon-borne infrared solar spectra, Geophys. Res. Lett., 6 , 609-612, 1979.
Gunson, M. R., et al., Measurements of $\mathrm{CH}_{4}, \mathrm{~N}_{2} \mathrm{O}, \mathrm{CO}, \mathrm{H}_{2} \mathrm{O}$, and $\mathrm{O}_{3}$ in the middle atmosphere by the ATMOS experiment on Spacelab 3, J. Geophys. Res., 95, 13867-13882, 1990.

Gunson, M. R. et al., The Atmospheric Trace Molecule Spectroscopy (ATMOS) experiment deployment on the ATLAS-3 Space Shuttle Mission, Geophys. Res. Lett., this issue, 1996.

IPCC (Intergovernmental Panel on Climate Change) Climate Change: 1994, J.T. Houghton et al., eds., Cambridge University Press, Cambridge, U.K., 1995.

Khalil, M.A.K, and R.A. Rasmussen, Atmospheric carbontetrafluoride $\left(\mathrm{CF}_{4}\right)$ : sources and trends, Geophys. Res. Lett., 10, 671-672, 1985.

McDaniel, A. H., et al., The temperature dependent absorption cross sections for the chlorofluorocarbons: CFC-11, CFC-12, CFC-13, CFC-14, CFC-22, CFC-113, CFC-114 and CFC-115, J. Atmos. Chem., 12, 211-227, 1991.

Morris, R. A., et al., Effect of electron and ion reactions on atmospheric lifetimes of fully fluorinated compounds, J. Geophys. Res., 100, 1287-1294, 1995.

Norton, R. H., and C. P. Rinsland, ATMOS data processing and science analysis methods, Appl. Opt., 30, 389-400, 1991.

Penkett, S. A., et al., Atmospheric measurements of $\mathrm{CF}_{4}$ and other fluorocarbons containing $\mathrm{CF}_{3}$ grouping, J. Geophys. Res., 86, 5172-5178, 1981.

Plumb, R. A., and M. K. W. Ko, Interrelationship between mixing ratios of long lived stratospheric constituents, J. Geophys. Res., 97, 145-156, 1992.

Rasmussen, R. A., et al., Measurements of carbontetrafluoride in the atmosphere, Nature, 277, 549-550, 1979.

Ravishankara, A. R., et al., Atmospheric lifetimes of long-lived halogenated species, Science, 259, 194-199, 1993.

Roehl, C. M., et al., Infrared band intensities and global warming potentials, Geophys. Res. Lett., 22, 815-818, 1995.

von Clarmann, T., et al., Determination of the stratospheric organic chlorine budget in the spring arctic vortex from MIPAS B limb emission spectra and air sampling experiments, J. Geophys. Res., $100,13,979-13,997,1995$.

WMO-Report Nr. 18, Report of the International Ozone Trends Panel 1988, World Meteorological Organzation, Vol. II, World Meteorological Organization, P.O. Box 5, Geneva 20, CH-1211, Switzerland, 1989.

WMO-Report Nr. 25, Scientific Assessment of Ozone Depletion: 1991, World Meteorological Organization, P.O. Box 2300, Geneva 20, CH-1211, Switzerland, 1992.

WMO-Report Nr. 37, Scientific Assessment of Ozone Depletion: 1994, World Meteorological Organization, P.O. Box 2300, Geneva 2, CH-1211, Switzerland, 1995.

Zander, R., et al., Infrared spectroscopic measurements of halogenated source gases in the stratosphere with the ATMOS instrument, $J$. Geophys. Res., 92, 9836-9850, 1987.

Zander, R., et al., The 1985 chlorine and fluorine inventories in the stratosphere based on ATMOS observations at $30^{\circ}$ North latitude, J. Atmos. Chem., 15, 171-186, 1992.

Zander, R., et al., Secular trend and seasonal variability of the column abundance of $\mathrm{N}_{2} \mathrm{O}$ above the Jungfraujoch station determined from IR solar spectra, J. Geoplyss. Res., 99, 16,745-16,756, 1994.

M. C. Abrams, NASA-LaRC, Mail Stop 475, VA 23681-0001

A. Y. Chang, M. R. Gunson, and R. J. Salawitch, JPL, 4800 Oak Grove Drive, Pasadena, CA 91109

A. Goldman, Dep. of Physics, University of Denver, CO 80208

H. A. Michelsen, Dep. of Earth and Planetary Sciences, Harvard University, Cambridge, MA 02138

E. Mahieu, and R. Zander, Institute of Astrophysics, University of Liège, 4000 Liège, Belgium

M. J. Newchurch, University of Alabama at Huntsville, Huntsville AL 35899

C. P. Rinsland, NASA-LaRC, Mail Stop 401A, Hampton, VA 23681-0001

S. Solomon, NOAA, Aeronomy Laboratory, P.O.Box 3000, Boulder, $\mathrm{CO} 80307$

G. P. Stiller, IMK-Forschungszentrum, Karlsruhe, Germany.

(Received September 20, 1995; revised March 8, 1996;

accepted March 18, 1996) 


\title{
The 1994 northern midlatitude budget of stratospheric chlorine derived from ATMOS/ATLAS-3 observations
}

\author{
R. Zander, ${ }^{1}$ E. Mahieu, ${ }^{1}$ M. R. Gunson, ${ }^{2}$ M. C. Abrams, ${ }^{3}$ A. Y. Chang, ${ }^{2}$ \\ M. Abbas, ${ }^{4}$ C. Aellig, ${ }^{5}$ A. Engel,${ }^{6}$ A. Goldman, ${ }^{7}$ F. W. Irion, ${ }^{8}$ N. Kämpfer, ${ }^{9}$ \\ H. A. Michelsen, ${ }^{10}$ M. J. Newchurch, ${ }^{11}$ C. P. Rinsland, ${ }^{12}$ R. J. Salawitch, ${ }^{2}$ \\ G. P. Stiller, ${ }^{13}$ and G. C. Toon ${ }^{2}$
}

\begin{abstract}
Volume mixing ratio (VMR) profiles of the chlorine-bearing gases $\mathrm{HCl}, \mathrm{ClONO}_{2}, \mathrm{CCl}_{3} \mathrm{~F}, \mathrm{CCl}_{2} \mathrm{~F}_{2}$, $\mathrm{CHClF}_{2}, \mathrm{CCl}_{4}$, and $\mathrm{CH}_{3} \mathrm{Cl}$ have been measured between 3 and $49^{\circ}$ northern- and 65 to $72^{\circ}$ southern latitudes with the Atmospheric Trace MOlecule Spectroscopy (ATMOS) instrument during the ATmospheric Laboratory for Applications and Science (ATLAS)-3 shuttle mission of 3 to 12 November 1994. A subset of these profiles obtained between 20 and $49^{\circ} \mathrm{N}$ at sunset, combined with ClO profiles measured by the Millimeter-wave Atmospheric Sounder (MAS) also from aboard ATLAS-3, measurements by balloon for $\mathrm{HOCl}, \mathrm{CH}_{3} \mathrm{CCl}_{3}$ and $\mathrm{C}_{2} \mathrm{Cl}_{3} \mathrm{~F}_{3}$, and model calculations for $\mathrm{COClF}$ indicates that the mean burden of chlorine, $\mathrm{Cl}_{\mathrm{TOT}}$, was equal to $(3.53 \pm 0.10)$ ppbv (parts per billion by volume), 1 sigma, throughout the stratosphere at the time of the ATLAS 3 mission. This is some $37 \%$ larger than the mean $2.58 \mathrm{ppbv}$ value measured by ATMOS within the same latitude zone during the Spacelab 3 flight of 29 April to 6 May 1985, consitent with an exponential growth rate of the chlorine loading in the stratosphere equal to $3.3 \% / y r$ or a linear increase of $0.10 \mathrm{ppbv} / \mathrm{yr}$ over the Spring-1985 to Fall-1994 time period. These findings are in agreement with both the burden and increase of the main anthropogenic Cl-bearing source gases at the surface during the $1980 \mathrm{~s}$, confirming that the stratospheric chlorine loading is primarily of anthropogenic origin.
\end{abstract}

\section{Introduction}

The ATMOS experiment is a multi-mission, shuttle-based project designed for regular, detailed remote soundings of the Earth's middle atmosphere [Farmer, 1987; Gunson et al., 1996].

\footnotetext{
Institute of Astrophysics, University of Liège, Belgium

${ }^{2} J e t$ Propulsion Laboratory, California Institute of Technology, CA

${ }^{3}$ Science Applications International Corporation, Hampton, VA

${ }^{4}$ NASA Marshall Space Flight-Center, AL

${ }^{5}$ Naval Research Laboratory, Washington, DC

${ }^{6}$ Forschungszentrum, Jülich, Germany

${ }^{7}$ University of Denver, Denver, CO

${ }^{8}$ California Institute of Technology, CA

${ }^{9}$ University of Bern, Switzerland

${ }^{10}$ Harvard University, Cambridge, MA

${ }^{11}$ University of Alabama, Huntsville, AL

12 NASA Langley Research Center, Hampton, VA

${ }^{13}$ IMK-Forschungszentrum Karlsruhe, Germany
}

Copyright 1996 by the American Geophysical Union.

Paper number 96GL01792

0094-8534/96/96GL-01792\$05.00
During its first mission of 1985 , ATMOS demonstrated the capability of establishing the budget of and partitioning among the nitrogen, chlorine and fluorine "families" [Russell et al., 1988; Zander et al., 1992] through infrared remote sensing from space. Subsequently, ATMOS was selected as a core instrument of the ATLAS program, performing additional missions in 1992, 1993 and 1994 [Gunson et al., 1996].

In this Letter, we report VMR profiles for the 7 most important $\mathrm{Cl}$-bearing gases derived from a subset of ATMOS/ATLAS-3 sunset occultations observed at northern latitudes comparable to those covered during ATMOS/ Spacelab-3 in 1985. By complementing these with profiles of 4 additional, non-negligible chlorinated species measured by other experiments, and the COClF contribution from model calculations, we evaluate the 1994 stratospheric $\mathrm{Cl}$ budget and derive its rate of change between 1985 and 1994.

\section{Data base and analysis}

The main absorption features used for VMR retrievals of the chlorine-bearing target molecules observed by ATMOS are primarily those reported in Table 1 of Zander et al. [1992]. This involves analyzing the ATMOS observations with the filters \# $12\left(600-1400 \mathrm{~cm}^{-1}\right), \# 9\left(600-2450 \mathrm{~cm}^{-1}\right)$ and $\# 3$ $\left(1580-3420 \mathrm{~cm}^{-1}\right)$ which were used alternately during the ATLAS-3 mission in predetermined sequences during 68 sunset occultations that occurred between 20 and $49^{\circ} \mathrm{N}$. In order to appraise the latitudinal dependence of the VMR profiles, we subdivided this range into "midlatitude" (49 to $35^{\circ} \mathrm{N}$ ) and "subtropical" ( 35 to $20^{\circ} \mathrm{N}$ ) zones. The molecules $\mathrm{HCl}$ and $\mathrm{CH}_{3} \mathrm{Cl}$ are studied in filter 3 occultations, while all other ATMOS-retrieved gases are from Filter 9 and 12 events; to eliminate any biasing arising from the sampling of the ensembles of the filter 3 or the filter 9 and 12 occultations, we used $\mathrm{N}_{2} \mathrm{O}$ as a dynamical tracer to register $\mathrm{HCl}$ and $\mathrm{CH}_{3} \mathrm{Cl}$ profiles on the same vertical scale as the other gases.

The VMR profiles were retrieved with the ODS (Occultation Display Spectra) onion-peeling retrieval algorithm developed at JPL and described by Norton and Rinsland [1991]. The input data to the code and parameters necessary to retrieve consistent composition of the atmosphere are discussed by Gunson et al. [1996].

The adoption of temperature-dependent absorption crosssections for a number of heavy molecules such as $\mathrm{CCl}_{2} \mathrm{~F}_{2}$, $\mathrm{CCl}_{3} \mathrm{~F}, \mathrm{CHClF}_{2}$ and $\mathrm{ClONO}_{2}$, instead of more subjective parameters used before [Zander et al., 1987; Brown et al., 1987] greatly improved the absolute quality of related ATMOS retrievals used here [Brown et al., 1996]. 


\section{Results}

The mean $\mathrm{HCl}$ VMR value obtained by averaging all individual measurements made during the ATLAS-3 mission near and above $50 \mathrm{~km}$ altitude, and over the 3 to $49^{\circ} \mathrm{N}$ latitude zone was found equal to $(3.52 \pm 0.10)$ ppbv. Sub-binnings performed over the northern midlatitude and subtropical zones identified here indicate no statistically significant difference with respect to that mean, thus confirming that $\mathrm{HCl}$ is well mixed at the hemispheric level near and above the stratopause. Previous ATMOS missions also demonstrated that the VMR measurement of $\mathrm{HCl}$ in the vicinity of the stratopause is a good surrogate for total stratospheric chlorine loading [Zander et al., 1992; Gunson et al., 1994]. Therefore, the value of $(3.52 \pm 0.10) \mathrm{ppbv}$ for $\mathrm{HCl}$ near $50 \mathrm{~km}$ should represent the mean stratospheric loading of chlorine for the Fall-1994 time.

To corroborate this, we evaluated the burden of chlorine, $\mathrm{Cl}_{\mathrm{TOT}}$, at various pressure levels by summing measured VMRs of the largest possible set of Cl-bearing gases (sources, sinks and reservoirs combined) weighted by the number of $\mathrm{Cl}$ atoms bound in each of them. For the ATLAS-3 time period, we performed the following summation:

$$
\begin{aligned}
\mathrm{Cl}_{\mathrm{TOT}} & =[\mathrm{HCl}]+\left[\mathrm{ClONO}_{2}\right]+4 \times\left[\mathrm{CCl}_{4}\right]+3 \times\left[\mathrm{CCl}_{3} \mathrm{~F}\right] \\
& +2 \times\left[\mathrm{CCl}_{2} \mathrm{~F}_{2}\right]+\left[\mathrm{CHClF}_{2}\right]+\left[\mathrm{CH}_{3} \mathrm{Cl}\right]+\{\mathrm{ClO}\} \\
& +3 \times\left\{\mathrm{CH}_{3} \mathrm{CCl}_{3}\right\}+3\left\{\mathrm{C}_{2} \mathrm{Cl}_{3} \mathrm{~F}_{3}\right\}+\{\mathrm{HOCl}\}+\{\{\mathrm{COClF}\}\}
\end{aligned}
$$

where the brackets indicate the VMRs of the seven important chlorinated constituents measured by ATMOS, and the 4 braces referring to VMRs of additional, non-negligible chlorine contributors not retrieved by ATMOS but by other experiments. In particular, the $\mathrm{ClO}$ data were derived from the MAS (Millimeter-wave Atmospheric Sounder) experiment [Croskey et al., 1992] which was also part of the ATLAS-3 payload; details about the $\mathrm{ClO}$ data analysis have been

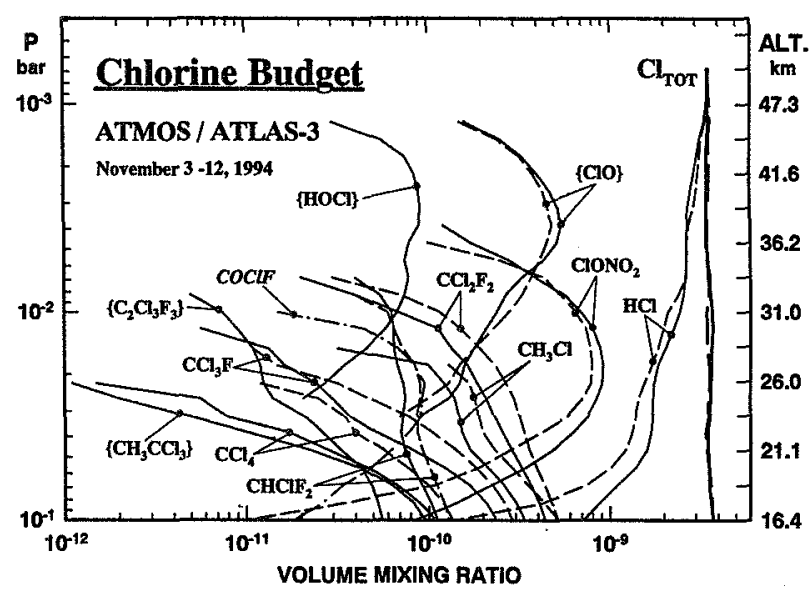

Figure 1. Graphical representation of the individual volume mixing ratio profiles included in the evaluation of the 1994 stratospheric Cl-loading for the northern midlatitude (35 to $49^{\circ} \mathrm{N}$; thin full lines) and subtropical $\left(20\right.$ to $35^{\circ} \mathrm{N}$; thin dashed lines) zones. The thicker full and dashed lines display the corresponding $\mathrm{Cl}$ budgets throughout the stratosphere, sources, sinks and reservoirs combined. While all subtropical profiles experience uplifting, opposite relative contributions to $\mathrm{Cl}_{\text {TOT }}$ are noticeable between sources on one hand, and sinks and reservois on the other hand. For details, see text.
Table 1. Numerical values (in ppbv) for $\mathrm{Cl}_{\mathrm{TOT}}$ and $\mathrm{CCl}_{\mathrm{y}}$ at northern mid- and subtropical latitudes in November 1994

\begin{tabular}{rccccc}
\hline & \multicolumn{3}{c}{$\begin{array}{c}\text { P, } \\
\text { mbar }\end{array}$} & Approx. & \multicolumn{2}{c}{ Midlatitudes } & \multicolumn{2}{c}{ Subtropics } \\
& Alt., $\mathrm{km}$ & $\mathrm{Cl}_{\text {TOT }}$ & $\mathrm{CCl}_{\mathrm{y}}$ & $\mathrm{Cl}_{\text {TOT }}$ & $\mathrm{CCl}_{\mathrm{y}}$ \\
\hline 0.68 & 50.2 & 3.52 & & 3.50 & \\
1.00 & 47.3 & 3.49 & & 3.52 & \\
1.47 & 44.4 & 3.47 & & 3.51 & \\
2.15 & 41.6 & 3.46 & & 3.55 & \\
3.16 & 38.9 & 3.45 & & 3.48 & \\
4.64 & 36.2 & 3.43 & 0.02 & 3.39 & \\
6.81 & 33.6 & 3.49 & 0.08 & 3.58 & 0.06 \\
10.00 & 31.0 & 3.65 & 0.22 & 3.41 & 0.31 \\
14.68 & 28.5 & 3.64 & 0.44 & 3.43 & 0.53 \\
21.54 & 26.0 & 3.62 & 0.69 & 3.57 & 0.98 \\
31.62 & 23.6 & 3.55 & 0.90 & 3.50 & 1.37 \\
46.42 & 21.2 & 3.49 & 1.34 & 3.43 & 1.86 \\
68.13 & 18.8 & 3.55 & 2.19 & 3.62 & 2.74 \\
100.00 & 16.4 & $\underline{3.74}$ & 2.92 & $\underline{3.72}$ & 3.56 \\
& Mean : & $3.54 \pm .09$ & & $3.52 \pm .09$ & \\
\hline
\end{tabular}

reported by Aellig et al. [1996]. The VMR profiles for $\mathrm{CH}_{3} \mathrm{CCl}_{3}$ and $\mathrm{C}_{2} \mathrm{Cl}_{3} \mathrm{~F}_{3}$ were derived from in situ measurements during a balloon flight performed by the Jülich group (A. Engel, private communication) in Aire-sur-l'Adour, France, on 7 October 1994, i.e., about one month prior to the ATLAS-3 mission. The HOCl profile was obtained from the analysis of sunset spectra recorded with the Mark IV FTIR during a balloon flight in September 1993 near $32^{\circ} \mathrm{N}$ (G.C. Toon, private communication), scaled by a $3 \%$ increase per year to represent the $\mathrm{HOCl}$ loading at the time of the ATLAS-3 mission. The double braced COCIF VMRs were extrapolated from model calculations by Kaye et al., [1991], assuming a 3\%/yr increase between their 1990 evaluations and 1994.

Figure 1 displays the profiles of the individual gases included in the present evaluation for both the midlatitude (thin full lines) and the subtropical (thin dotted lines) zones, as well as the corresponding total $\mathrm{Cl}$ profiles labeled $\mathrm{Cl}_{\mathrm{TOT}}$ displayed as thicker lines. For $\mathrm{CH}_{3} \mathrm{CCl}_{3}, \mathrm{C}_{2} \mathrm{Cl}_{3} \mathrm{~F}_{3}, \mathrm{HOCl}$ and $\mathrm{COClF}$, the same profiles were used in both binnings, because of unavailable measurements for the two latitudinal zones.

Table 1 provides numerical values for $\mathrm{Cl}_{\mathrm{TOT}}$ and $\mathrm{CCl}_{\mathrm{y}}$, for the two northern midlatitude and subtropical zones; the pressure levels were selected among standard values adopted by various experiments (including UARS) for date archiving.

The VMRs of the top and bottom pressure levels of the midlatitude $\mathrm{Cl}_{\text {тот }}$ profile (thick continuous line in Fig. 1) are equal, respectively, to 3.52 and $3.74 \mathrm{ppbv}$, with a corresponding mean VMR and standard deviation over the entire altitude span equal to $(3.54 \pm 0.09) \mathrm{ppbv}$. The extremes are indicative of a $6.3 \%$ decrease of $\mathrm{Cl}_{\text {TOT }}$ which can be explained by the time delay for air to mix between 100 and 0.68 mbar $(\sim 16.5$ to $50 \mathrm{~km})$ levels.

The $\mathrm{Cl}_{\text {Tor }}$ profile for the subtropical latitudinal zone (thick dotted line in Fig. 1) also indicates a decrease of $6.3 \%$ between bottom (3.72 ppbv) and top (3.50 ppbv) VMRs, with a corresponding mean mixing ratio and standard deviation equal to $(3.52 \pm 0.09)$ ppbv.

Figure 2 with its linear abscissa scale shows some details of the profiles of the key components which make up the $\mathrm{Cl}$ inventory for the northern midlatitude case $\left(35\right.$ to $\left.49^{\circ}\right)$. In particular, we have reproduced the $\mathrm{CCl}_{y}$ curve corresponding 


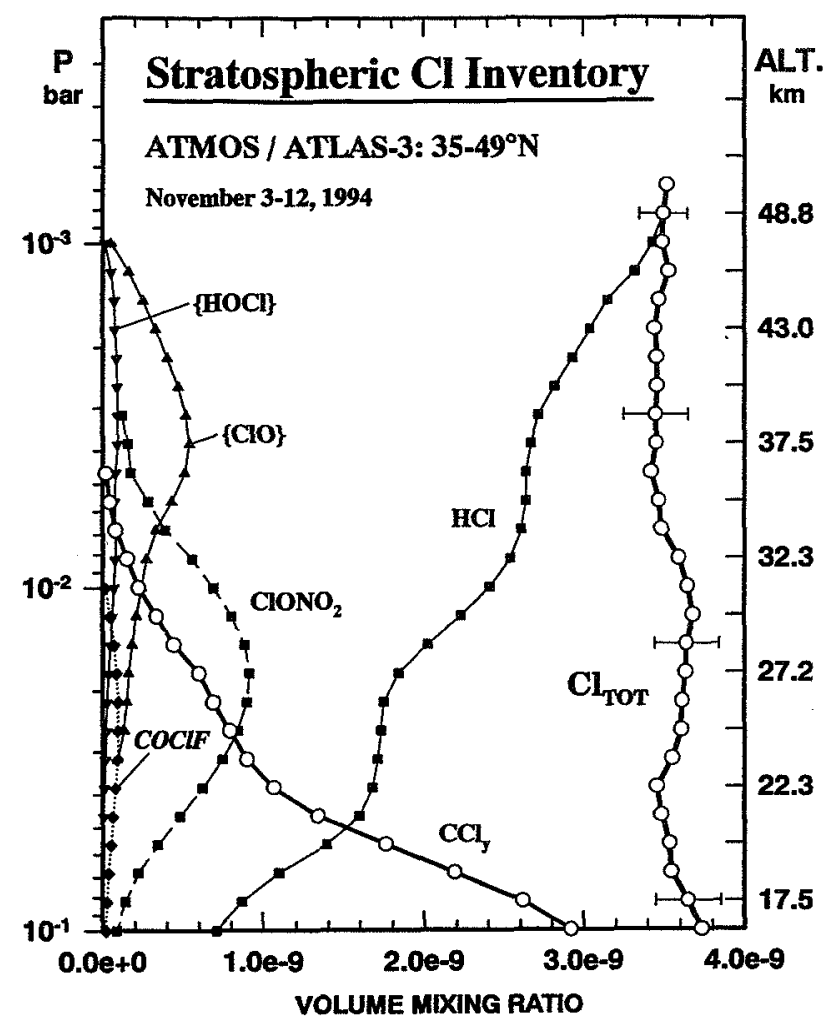

Figure 2. A linear-scale presentation of the key components entering in the total $\mathrm{Cl}$-loading evaluation throughout the stratosphere at northern midlatitudes. The $\mathrm{CCl}_{\mathrm{y}}$ curve refers to the total chlorine bound in all organic source gases listed in the text. $\mathrm{Cl}_{\text {TOr }}$ represents the summation over $\mathrm{CCl}_{\mathrm{y}}, \mathrm{HCl}$, $\mathrm{ClONO}_{2}, \mathrm{ClO}, \mathrm{HOCl}$ and $\mathrm{COClF}$.

to the sum of $\mathrm{Cl}$ atoms bound in all source gases considered here. By further summing over $\mathrm{CCl}_{\mathrm{y}}$ and the inorganic compounds $\mathrm{HCl}, \mathrm{ClONO}_{2}, \mathrm{ClO}, \mathrm{HOCl}$ and $\mathrm{COClF}$ also reproduced in Fig. 2, one obtains the thicker continuous curve which corresponds to the total chlorine loading $\mathrm{Cl}_{\text {TOT. }}$. Within the estimated uncertainty ranging from $4 \%$ at the top to $6 \%$ at the bottom of the $\mathrm{Cl}_{\text {TOT }}$ profile, the conservation of chlorine is properly demonstrated throughout the stratosphere. Within that uncertainty, however, we cannot rule out some missing second order contributions from either reservoirs or sources not accounted for here. Some uncertainty in the spectroscopic parameters used to derive the profiles of the heavier molecules [see Brown et al., 1996 and Gunson et al., 1996] could also reflect in the lower altitude range.

\section{Discussion and conclusions}

The measurement of the budget of chlorine in the middle atmosphere involve a large number of $\mathrm{Cl}$-bearing gases which cannot be studied by any one technique alone, and related investigations during the last years have sought approaches for its evaluation, based on a more limited number of measurements. These have generally invoked the work of Plumb and Ko [1992] who demonstrated that there exist compact correlations between the mixing ratios of long-lived atmospheric constituents, thus providing means of deriving the distribution versus altitude for some species, solely based on concentrations measured for others. The procedure has been applied in many cases [e.g., Schmidt et al., 1994; Woodbridge et al., 1995]. Among these, the application by Schmidt et al. [1994] is of relevance here; using $\mathrm{CCl}_{2} \mathrm{~F}_{2}$ as the "reference" gas, they derived the function

$$
\left[\mathrm{CCl}_{y}\right]=0.045 \times\left[\mathrm{CCl}_{2} \mathrm{~F}_{2}\right]^{1.82}+67.35
$$

in which the brackets refer to the VMRs of the related compounds, expressed in units of pptv ( $\times 10^{-12} \mathrm{ppv}$ ). Application of that formulation to the midlatitude mean $\mathrm{CCl}_{2} \mathrm{~F}_{2}$ profile derived in 1994 by ATMOS produces results which match very well the $\mathrm{CCl}_{\mathrm{y}}$ profiles obtained in this work, when accounting for the fact that Schmidt et al. [1994] sample species amounting to $95 \%$ of the total organic chlorine.

Model evaluations of the chlorine partitioning in the stratosphere as reported here for the ATLAS-3 mission are dealt with and discussed by Michelsen et al. [1996].

From the various independent results derived here, it can be concluded that the overall chlorine loading at the top of the stratosphere over the 20 to $49^{\circ} \mathrm{N}$ latitudinal zone was equal to $3.51 \mathrm{ppbv}$ at the time of the November 1994 ATLAS-3 mission, with a 1-sigma uncertainty of $0.15 \mathrm{ppbv}$ or $4 \%$; this uncertainty represents an estimated accuracy assessed as in previous investigations [Zander et al., 1990; Gunson et al., 1990]. Throughout the stratosphere, the $\mathrm{Cl}_{\text {TOT }}$ VMR profiles combining the important sources, sinks and reservoirs display consistent values which suggest only second order local deficit and a mean slope reflecting the time for air to mix from the lower to the upper stratosphere.

When compared to the mean stratospheric chlorine VMR of $2.58 \pm 0.10 \mathrm{ppbv}$ derived from the 1985 ATMOS/Spacelab3 mission [Zander et al., 1992], the present mean stratospheric chlorine loading of $3.53 \mathrm{ppbv}$ (see Table 1) corresponds to a $37 \%$ increase over the 9.5 years separating the two missions, i.e., an exponential rate of $3.3 \% / \mathrm{yr}$ or a linear change of $0.10 \mathrm{ppbv} / \mathrm{yr}$. The mean stratospheric $\mathrm{Cl}$ VMR derived from the ATMOS/Spacelab-3 mission was shown to be in excellent agreement with the corresponding $1980 \mathrm{Cl}$ inventory in the lower troposphere [Zander et al., 1992], indicative of a mixing time of about 5 years for free tropospheric air to be transported and mixed throughout the stratosphere. The upper stratospheric $\mathrm{Cl}$ loading derived here (3.51 ppbv) for the time of the ATLAS-3 mission of November 1994 mirrors precisely the tropospheric $\mathrm{Cl}$ content that prevailed in 1989-90 [WMO Reports No. 25, 1992 and NASA Pub. 1339, 1994], thus confirming the $4-5$ year lag time between tropospheric and stratospheric chlorine loading identity. In addition, the rate of change of $3.3 \% / y$ deduced here agrees very well with the increase of the organic chlorine burden in the lower troposphere (i.e., $0.104 \mathrm{ppbv}$ for a total of $3.18 \mathrm{ppbv}$ in 1986 and $0.109 \mathrm{ppbv}$ for a total loading of 3.46 ppbv in 1989; see WMO Reports No. 18, 1989 and No. 25, 1992).

As a consequence of the excellent agreement between the stratospheric chlorine loadings and rates of change derived from the ATMOS missions of 1985 and 1994 and the surface measurements of Cl-bearing gases some 4-5 years earlier, there remains no doubt that the bulk of stratospheric chlorine and its evolution are primarily associated with the release at the ground of chlorinated source gases produced industrially. As concluded by Gunson et al. [1994], this implies that natural sources of chlorine, in particular $\mathrm{CH}_{3} \mathrm{Cl}$ and perhaps 
$\mathrm{CCl}_{4}$, and volcanic activity, have contributed negligibly to the changes in the burden of stratopheric $\mathrm{Cl}$ over the last decade or so.

The 1987 Montreal Protocol and its London and Copenhagen Amendments are intended to reduce the future levels of atmospheric chlorine by regulating the production and release in the atmosphere of the most important anthropogenic, Cl-bearing source gases at the ground, primarily the chlorofluorocarbons (CFCs). The successive ATMOS missions have provided, thus far, the most consistent and complete set of measurements needed to "watch" the timely chlorine loading and to model its impact on ozone depletion throughout the entire stratosphere. Similar measurements will become critical in the near future when the stratospheric loading is expected to start decreasing, to test the implementation of the Protocol and the validity of the model predictions.

Acknowledgements This work was carried out at the Jet Propulsion Laboratory (JPL), California Institute of Technology, under contract with the National Aeronautics and Space Administration (NASA). The University of Liège involvement in the ATMOS project was partly supported within the frame of the Belgian 'Global Change' program coordinated by the S.S.T.C., Brussels.

\section{References}

Aellig, C. P., et al., Latitudinal Distribution of upper stratospheric ClO as derived from space borne microwave spectroscopy, this issue, 1996.

Brown, L. R., et al., Molecular line parameters for the atmospheric trace molecule spectroscopy (ATMOS) experiment, Appl. Opt., 26, 5154-5182, 1987.

Brown, L. R., et al., The 1995 Atmospheric Trace Molecule Spectroscopy (ATMOS) linelist, Appl. Opt., in press, 1996.

Croskey, C. L., et al., The Millimeter Wave Atmospheric Sounder (MAS): a Shuttle-based remote sensing experiment, IEEE Transactions on Geoscience and Remote Sensing, 40, 1090-1100, 1992.

Farmer, C. B., High resolution infrared spectroscopy of the sun and the earths atmosphere from space, Microchim. Acta/Wien/III, 189$214,1987$.

Gunson, M. R., et al., Measurements of $\mathrm{CH}_{4}, \mathrm{~N}_{2} \mathrm{O}, \mathrm{CO}, \mathrm{H}_{2} \mathrm{O}$, and $\mathrm{O}_{3}$ in the middle atmosphere by the Atmospheric Trace Molecule Spectroscopy experiment on Spacelab 3, J. Geophys. Res.,95, 13867-13882, 1990.

Gunson, M. R., et al., Increase in levels of stratospheric chlorine and fluorine loading between 1985 and 1992, Geophys. Res. Lett., 21, 2223-2226, 1994.

Gunson, M. R., et al., The Atmospheric Trace Molecule Spectroscopy (ATMOS) experiment deployment on the ATLAS-3 Space Shuttle Mission, Geophys. Res. Lett., this issue, 1996.

Kaye, J. A., et al., Two-dimensional model calculations of fluorinecontaining reservoir species in the stratosphere, $J$. Geophys. Res., $96,12,865-12,881,1991$

Michelsen, H. A., et al., Chlorine partitioning in the stratosphere: evidence from ATMOS measurements for an additional source of $\mathrm{HCl}$, Geophys. Res. Lett., this issue, 1996.
NASA Reference Publication 1339, Report on Concentrations, Lifetimes and Trends of CFCs, Halons and Related Species, J.A. Kaye, S.A Penkett and F.M. Ormond, eds., NASA Office of Missions to Planet Earth, Science Div., Washington, D.C., January 1994.

Norton, R. H., and C. P. Rinsland, ATMOS data processing and science analysis methods, Appl. Opt., 30, 389-400, 1991.

Plumb, R. A., and M. K. W. Ko, Interrelationships between mixing ratios of long-lived constituents, J. Geophys. Res., 95, 10145$10156,1992$.

Russell III, J. M., et al., Measurements of odd nitrogen compounds in the stratosphere by the ATMOS experiment on Spacelab 3, $J$. Geophys. Res., 93, 1718-1736, 1988.

Schmidt, U., et al., The variation of available chlorine, $\mathrm{Cl}_{y}$, in the Arctic polar vortex during EASOE, Geophys. Res. Lett., 21, 1215 $1218,1994$.

WMO-Report No. 18, Report of the International Ozone trends Panel-1988, World Meteorological Organization, P.O. Box 5, CH 1211, Geneva 20, Switzerland, 1989.

WMO-Report No. 25, Scientific Assessment of Ozone Depletion: 1991, World Meteorological Organization, P.O. Box 2300 Geneva 2, CH 1211, Switzerland, 1992.

Woodbridge, E. L., et al., Estimates of total organic and inorganic chlorine in the lower stratosphere from in situ and flask measurements during AASE II, J. Geophys. Res., 100, 3057-2064, 1995.

Zander, R., et al., Stratospheric $\mathrm{ClONO}_{2}, \mathrm{HCl}$ and $\mathrm{HF}$ concentration profiles derived from ATMOS/Spacelab 3 observations - An update, J. Geophys. Res., 95, 20519-20525, 1990.

Zander, R., et al., Infrared spectroscopic measurements of halogenated source gases in the stratosphere with the ATMOS instrument, $J$. Geophys. Res., 92, 9836-9850, 1987.

Zander, R., et al., The 1985 chlorine and fluorine inventories in the stratosphere based on ATMOS observations at $30^{\circ}$ North latitude, J. Atmos. Chem., 15, 171-186, 1992.

M. M Abbas, ESSL, NASA-MSFC, Huntsville, AL 35889

M. C. Abrams, NASA-LaRC, Mail Stop 475, VA 23681-0001

C. Aellig, Naval Research Laboratory, Code 7227, Washington, DC 20375

A. Y. Chang, M. R. Gunson, R. J. Salawitch, and G. C. Toon, JPL, 4800 Oak Grove Drive, Pasadena, CA 91109

A. Engel, Jülich Forschungszentrum, ICG3, D-5170 Jülich, Germany

A. Goldman, Dep. of Physics, Univ. of Denver, CO 80208

F. W. Irion, Dep. of Chemical Engineering, Caltech, Pasadena, CA 91125

N. Kämpfer, Institute of Applied Physics, Univ.of Bern, Bern, Switzerland

H. A. Michelsen, Dep. of Earth and Planetary Sciences, Harvard Univ., Cambridge, MA 02138

E. Mahieu, and R. Zander, Institute of Astrophysics, Univ. of Liège, 4000 Liège, Belgium

M. J. Newchurch, Univ.of Alabama at Huntsville, Huntsville AL 35899

C. P. Rinsland, NASA-LaRC, Mail Stop 401A, Hampton, VA 23681-0001

G. P. Stiller, IMK-Forschungszentrum, Karlsruhe, Germany.

(Received September 20, 1995; revised March 8, 1996; accepted May 2, 1996.) 


\section{Stratospheric chlorine partitioning: Constraints from shuttle-borne measurements of $[\mathrm{HCl}]$, $\left[\mathrm{ClNO}_{3}\right]$, and [ClO]}

H. A. Michelsen', R. J. Salawitch ${ }^{2}$, M. R. Gunson ${ }^{2}$, C. Aellig ${ }^{3}$, N. Kämpfer ${ }^{4}$ M. M. Abbas, , M. C. Abrams ${ }^{6}$, T. L. Brown', A. Y. Chang ${ }^{2}$, A. Goldman', F. W. Irion', M. J. Newchurch ${ }^{8}$, C. P. Rinsland ${ }^{6}$, G. P. Stiller', and R. Zander ${ }^{10}$

Abstract. Measured stratospheric mixing ratios of $\mathrm{HCl}$, $\mathrm{CINO}_{3}$, and $\mathrm{ClO}$ from ATMOS and MAS are poorly reproduced by models using recommended kinetic parameters. This discrepancy is not resolved by new rates for the reactions $\mathrm{Cl}+\mathrm{CH}_{4}$ and $\mathrm{OH}+\mathrm{HCl}$ derived from weighted fits to laboratory measurements. A deficit in modeled $[\mathrm{HCl}]$ and corresponding overprediction of $\left[\mathrm{ClNO}_{3}\right]$ and $[\mathrm{ClO}]$, which increases with altitude, suggests that production of $\mathrm{HCl}$ between 20 and 50 $\mathrm{km}$ is much faster than predicted from recommended rates.

\section{Introduction}

Identifying and determining the rates of reactions involved in partitioning inorganic chlorine is critical to understanding the processes that regulate stratospheric ozone. Collections of recommended rates published by NASA [DeMore et al., 1994] and NIST [Atkinson et al., 1992], referred to below as NASA94 and NIST92, have been invaluable for consolidation and review of the vast amount of kinetic information for hundreds of reactions. Nevertheless, models incorporating recommended reaction rates and absorption cross sections and allowing for production of $\mathrm{HCl}$ only via reactions of $\mathrm{Cl}$ with hydrocarbons and $\mathrm{HO}_{2}$ underpredict abundances of $\mathrm{HCl}$ and overpredict those of $\mathrm{ClNO}_{3}, \mathrm{ClO}$, and $\mathrm{HOCl}$ at altitudes above $\sim 24 \mathrm{~km}$ [e.g., McElroy and Salawitch, 1989; Allen and Delitsky, 1991; Natarajan and Callis, 1991; Stachnik et al., 1992]. This discrepancy leads to an overprediction of ozone loss rates by an estimated $5-30 \%$ near $40 \mathrm{~km}$ [Brasseur et al., 1985; Natarajan and Callis, 1991].

Previous suggestions for resolving the disagreement between theory and observation involve increasing the production of $\mathrm{HCl}$ either by enhancing the rate of $\mathrm{Cl}+\mathrm{HO}_{2} \rightarrow \mathrm{HCl}+\mathrm{O}_{2}[$ Allen and Delitsky, 1991] or by including a channel for $\mathrm{HCl}$ formation from the reaction $\mathrm{ClO}+\mathrm{OH}$ [McElroy and Salawitch, 1989; Natarajan and Callis, 1991; Stachnik et al., 1992].

\footnotetext{
${ }^{1}$ Div. of Applicd Sciences, Harvard Univ., Cambridge, MA

2 Jet Propulsion Lab., Calif. Inst. of Technology, Pasadena, CA

${ }^{3}$ Naval Research Lab, Washington, DC

${ }^{4}$ Inst. of Applied Physics, Univ. of Bem, Switzerland

5 NASA Marshall Space Flight Center, Huntsville, AL

6 NASA Langley Research Center, Hampton, VA;

7 Dept. of Physics, Univ. of Denver, Denver, CO

${ }^{8}$ Earth Systems Science Laboratory, Univ, of Alabama, Huntsville

${ }^{9}$ Forschungszentrum Karlsruhe, Univ. Karlsruhe, Karlsruhe, Germany

${ }^{10}$ Inst. of Astrophysics, Univ. of Liege, Liege-Ougree, Belgium

Copyright 1996 by the American Geophysical Union.

Paper number 96GL00787

0094-8534/96/96GL-00787\$05.00
}

The extensive array of species measured by the Atmospheric Trace Molecule Spectroscopy (ATMOS) instrument offers an opportunity to test photochemical models over a wide range of altitudes $(\sim 12-50 \mathrm{~km})$ and latitudes $\left(\sim 70^{\circ} \mathrm{S}-70^{\circ} \mathrm{N}\right)$. ATMOS has measured the volume mixing ratios of (1) $\mathrm{HCl}$ and $\mathrm{ClNO}_{3}$, which comprise most of inorganic chlorine (Cly) under conditions unperturbed by polar stratospheric clouds (PSCs), (2) species that influence the partitioning of Cly directly, e.g., $\mathrm{O}_{3}, \mathrm{CH}_{4}, \mathrm{C}_{2} \mathrm{H}_{6}, \mathrm{NO}$, and $\mathrm{NO}_{2}$, and (3) additional species that control $\mathrm{OH}$ and $\mathrm{HO}_{2}$ abundances and thus affect $\mathrm{Cly}$ partitioning indirectly, e.g., $\mathrm{H}_{2} \mathrm{O}, \mathrm{CO}$, and $\mathrm{HNO}_{3}$. These observations are complemented by correlative measurements of $\mathrm{ClO}$ made by the Millimeter-wave Atmospheric Sounder (MAS) [Croskey et al., 1992], which provide significant leverage in testing photochemical models at altitudes near 40 $\mathrm{km}$, where $\mathrm{ClNO}_{3}$ is not a major component of Cly.

We have studied the partitioning of Cly between 18 and 50 $\mathrm{km}$ under conditions sampled by ATMOS during ATLAS-2 (814 April 1993) and by ATMOS and MAS during ATLAS-3 (412 Nov 1994) and present here an analysis of data from the northern hemisphere during fall at sunset (tropics and midlatitudes) and spring at sunrise (high latitudes). We also present a review of laboratory studies of the kinetics of the primary reactions believed to control production and loss of $\mathrm{HCl}$. We conclude that (1) modifications to recommended rates for $\mathrm{Cl}+\mathrm{CH}_{4}$ and $\mathrm{OH}+\mathrm{HCl}$, based on published laboratory measurements, are insufficient to reconcile the differences between modeled and measured partitioning of inorganic chlorine, and (2) a mechanism leading to enhanced formation of $\mathrm{HCl}$, such as $\mathrm{ClO}+\mathrm{OH} \rightarrow \mathrm{HCl}+\mathrm{O}_{2}$, is required to resolve these differences.

\section{Stratospheric measurements}

Measurements of $\left[\mathrm{ClNO}_{3}\right]$ and $[\mathrm{HCl}]$ made by ATMOS and $[\mathrm{ClO}]$ by MAS are shown by the symbols in Fig. 1 ( $\mathrm{C}$ denotes volume mixing ratio). Each ATMOS vertical profile is an average of Version 2 retrievals from 3 to 6 occultations [Abrams et al., this issue]. Profiles were selected by (1) the geographic location $\left(65-69^{\circ} \mathrm{N}, 161-298^{\circ} \mathrm{E}\right.$ for the extra-vortex high latitude case, $40-48^{\circ} \mathrm{N}, 39^{\circ} \mathrm{W}-27^{\circ} \mathrm{E}$ for the midlatitude case, and $3-13^{\circ} \mathrm{N}, 187-343^{\circ} \mathrm{E}$ for the tropical case) and (2) the requirement that $\left[\mathrm{O}_{3}\right]$ not differ by more than $\sim 10 \%$ from the mean for each occultation used. The error bars were derived from the standard deviation of a weighted average of the measurements, added in quadrature to the systematic error associated with uncertainties in the pressure-temperature retrieval and band strength for each constituent [Abrams et al., this issue]. The data were weighted by the inverse square of the random error generated in the retrieval process. The purple triangles represent the sum of measured $\left[\mathrm{ClNO}_{3}\right],[\mathrm{HCl}]$, and $[\mathrm{ClO}]$, which agrees to within $10 \%$ with the value of [Cly] 
derived from measurements of organic source gases [Woodbridge et al., 1995; Zander et al., this issue].

$\mathrm{HCl}$ comprises most of $\mathrm{Cly}$ at all altitudes and latitudes (see Fig. 1). The ratio $\left[\mathrm{ClNO}_{3}\right] /[\mathrm{HCl}]$ has a maximum of $\sim 0.5$ at 26 $\mathrm{km}$. Higher values for this ratio were observed by ATMOS during ATLAS-2 for air believed to be recovering from PSC processing. The magnitude of this ratio and its near invariance with latitude above $\sim 20 \mathrm{~km}$ is consistent with UARS observations [Dessler et al., 1995]. These observations also agree with in situ measurements of [HCl] and organic source gases at $20 \mathrm{~km}$ [Chang et al., this issue].

\section{Laboratory measurements}

$\mathrm{OH}+\mathrm{HCl} \rightarrow \mathrm{Cl}+\mathrm{H}_{2} \mathrm{O}$. This reaction is the major sink for $\mathrm{HCl}$ under conditions unperturbed by PSCs. Measured and recommended rates for this reaction are plotted in Fig. 2a. The NASA94 rate is based on an unweighted fit to all data shown, except those represented by red symbols. The NIST92 rate is from a weighted fit to the same data.

Recent measurements of this rate at temperatures $\mathrm{T}<220 \mathrm{~K}$ have demonstrated strong non-Arrhenius behavior (red circles outlined in black) [Sharkey and Smith, 1993]. These measurements are the first to be made at $\mathrm{T}<240 \mathrm{~K}$ and demonstrate faster rates than either recommended value, although rates from this study are in agreement with recommendations at higher temperatures. At stratospheric temperatures these measurements are within the $\sim 40 \% 1 \sigma$ uncertainty of NASA94. We use a value of $5.38 \pm 0.06 \times 10^{-13}$ $\mathrm{cm}^{3} \mathrm{~s}^{-1}$ for this rate below (and the NASA94 rate above) $222 \mathrm{~K}$.

$\mathrm{Cl}+\mathrm{CH}_{4} \rightarrow \mathrm{HCl}+\mathrm{CH}_{3}$. The reaction of $\mathrm{Cl}$ with $\mathrm{CH}_{4}$ is thought to be the major source of $\mathrm{HCl}$ throughout the stratosphere. NASA94 notes that for $\mathrm{T}<240 \mathrm{~K}$ rates measured for this reaction using the discharge flow technique tend to be slower than those measured by flash photolysis. Competitive chlorination rate measurements (open symbols, Fig. 2b) tend to be lower than all other values. Ravishankara and Wine [1980] tentatively attributed these differences to nonequilibrated spin states of $\mathrm{Cl}$ affecting the results of the discharge flow and competitive chlorination experiments.

The Arrhenius parameters from NASA94 were derived from an unweighted fit to all data for $200 \mathrm{~K}<\mathrm{T}<300 \mathrm{~K}$. For $\mathrm{T}<240 \mathrm{~K}$ this rate is slower than the slowest absolute measurement but faster than the competitive chlorination measurements. The NIST92 recommendation is from a fit to data obtained at $\mathrm{T}<300 \mathrm{~K}$ and is slightly higher than that of NASA94.

We performed a least squares fit weighted by the $1 \sigma$ uncertainty for each observation. We included the competitive chlorination measurements using NASA94 rates and uncertainties for the reference reactions. Our fit to measurements obtained at $\mathrm{T} \leq 300 \mathrm{~K}$ yielded the expression $5.57 \pm 0.11 \times 10^{-12} e^{(-1203 \pm 5 / \mathrm{T})} \mathrm{cm}^{3} \mathrm{~s}^{-1}$. At $215 \mathrm{~K}$ this rate is $27 \%$ faster than (but within $1 \sigma$ of) that of NASA94 and $15 \%$ faster than that of NIST92.

$\mathrm{ClO}+\mathrm{OH} \rightarrow \mathrm{HCl}+\mathrm{O}_{2}$. The major product channel for the reaction of $\mathrm{ClO}$ with $\mathrm{OH}$ is formation of $\mathrm{Cl}$ and $\mathrm{HO}_{2}$. Laboratory measurements indicate a minor channel producing $\mathrm{HCl}$ and $\mathrm{O}_{2}$ might occur with a branching ratio between $0.02 \pm 0.12$ [Poulet et al., 1986] and $0.14 \pm 0.14(2 \sigma)$ [Burrows et al., 1984; Hills and Howard, 1984; Leu and Lin, 1979]. All results are consistent with a branching ratio of zero [NASA94]. Each observation is also consistent with a branching ratio of 0.14 , and only Burrows et al. reported a value for $\mathrm{T}<293 \mathrm{~K}$, i.e., $0.15 \pm 0.14$ between 243 and $298 \mathrm{~K}$. Hills and Howard pointed out that production of $\mathrm{HCl}$ from this reaction would have to occur via a four-centered transition state, which, although unusual, has been observed for $\mathrm{ClO}+\mathrm{ClO} \rightarrow \mathrm{Cl}_{2}+\mathrm{O}_{2}$.

\section{Comparison of models and observations}

The diurnally varying concentrations of 35 radical and reservoir species were calculated assuming a balance between production and loss for each species over a $24 \mathrm{hr}$ period. The model was constrained by profiles of temperature, $\left[\mathrm{O}_{3}\right],\left[\mathrm{H}_{2} \mathrm{O}\right]$, $\left[\mathrm{CH}_{4}\right],\left[\mathrm{C}_{2} \mathrm{H}_{6}\right]$, and [CO] measured by ATMOS and aerosol surface area from SAGE II [G.K. Yue and L. W. Thomason, private communication]. The calculated sum of $\left[\mathrm{ClNO}_{3}\right]$, $[\mathrm{HCl}]$, and $[\mathrm{ClO}]$, appropriate for the local time for each measurement, was constrained to reproduce the measured value, as was the predicted sum of $\left[\mathrm{ClNO}_{3}\right],\left[\mathrm{HNO}_{3}\right],[\mathrm{NO}],\left[\mathrm{NO}_{2}\right]$, $2\left[\mathrm{~N}_{2} \mathrm{O}_{5}\right]$, and $\left[\mathrm{HNO}_{4}\right]$, i.e., [NOy] [McElroy and Salawitch, 1989]. We used kinetic parameters from NASA94, except as noted below, and have included the heterogeneous hydrolysis of $\mathrm{BrNO}_{3}$ with a reaction probability of 0.5 in all simulations [Hanson and Ravishankara, 1995].

Figure 1 shows a comparison of observed mixing ratios of $\mathrm{HCl}, \mathrm{ClNO}_{3}$, and $\mathrm{ClO}$ with theoretical values corresponding to the local time at which the measurements were made: sunset in the midlatitudes and tropics and sunrise at high latitudes for $\mathrm{HCl}$ and $\mathrm{ClNO}_{3}$, and local times of 9:30 am in the tropics and 11:00 am in the midlatitudes for ClO. The "JPL94 model", which strictly uses rates and cross sections from NASA94 with $\mathrm{ClO}+\mathrm{OH} \Theta \mathrm{HCl}+\mathrm{O}_{2}(\Theta \equiv$ "does not produce") (blue dashed lines, right panels, and dashed lines, left panels), leads to overprediction of $\left[\mathrm{ClNO}_{3}\right]$ and $[\mathrm{ClO}]$, underprediction of $[\mathrm{HCl}]$, and overprediction of the ratios $\left[\mathrm{ClNO}_{3}\right] /[\mathrm{HCl}]$ and $[\mathrm{ClO}] /[\mathrm{HCl}]$ by up to a factor of three. For the high latitude case, where [ClO] measurements are not available, the [Cly] required to match observed $[\mathrm{HCl}]$ is unrealistically large $(>4 \mathrm{ppbv})$ above $40 \mathrm{~km}$ (dashed purple lines, top left panel). The disagreement between the JPL9 4 model and the measurements is not significant at $20 \mathrm{~km}$ but increases at higher altitudes.

We have examined the sensitivity of calculated abundances of $\mathrm{HCl}, \mathrm{ClNO}_{3}$, and $\mathrm{ClO}$ to changes in rates for $\mathrm{Cl}+\mathrm{CH}_{4}$, $\mathrm{OH}+\mathrm{HCl}$, and $\mathrm{ClO}+\mathrm{OH} \rightarrow \mathrm{HCl}+\mathrm{O}_{2}$, based on our analysis of laboratory data. The purple dotted lines (right panels) show model results using the rate for $\mathrm{Cl}+\mathrm{CH}_{4}$ from our fit to the laboratory data. Increasing this rate by $\sim 30 \%$ below $30 \mathrm{~km}$ decreases the ratio $\left[\mathrm{CINO}_{3}\right] /[\mathrm{HCl}]$ by $15-20 \%$, reflecting the increase in production of $\mathrm{HCl}$ and bringing the calculations into better agreement with the observations. Above $30 \mathrm{~km}$, however, the discrepancy is not resolved, particularly for $[\mathrm{ClO}] /[\mathrm{HCl}]$, since sensitivity to this rate decreases rapidly with altitude as $\mathrm{T}$ increases, $\left[\mathrm{CH}_{4}\right]$ decreases, and the rate of $\mathrm{HCl}$ production is influenced strongly by $\mathrm{Cl}+\mathrm{HO}_{2}$.

Using the faster rate for $\mathrm{OH}+\mathrm{HCl}$ below $222 \mathrm{~K}$ increases the loss rate of $\mathrm{HCl}$ and the ratio $\left[\mathrm{ClNO}_{3}\right] /[\mathrm{HCl}]$ by $<10 \%$ (orange dot-dashed lines), increasing the discrepancy between theory and observation slightly at all altitudes. Using quantum yields of $\mathrm{O}\left({ }^{1} \mathrm{D}\right)$ from photolysis of $\mathrm{O}_{3}$ (green dashed lines) from Michelsen et al. [1994] leads to enhanced O( $\left.{ }^{1} \mathrm{D}\right)$ below $30 \mathrm{~km}$, which increases the discrepancy by $15-20 \%$.

Allowing for the production of $\mathrm{HCl}$ from the reaction of $\mathrm{ClO}$ with $\mathrm{OH}$ has a large effect on calculated profiles of Cly 


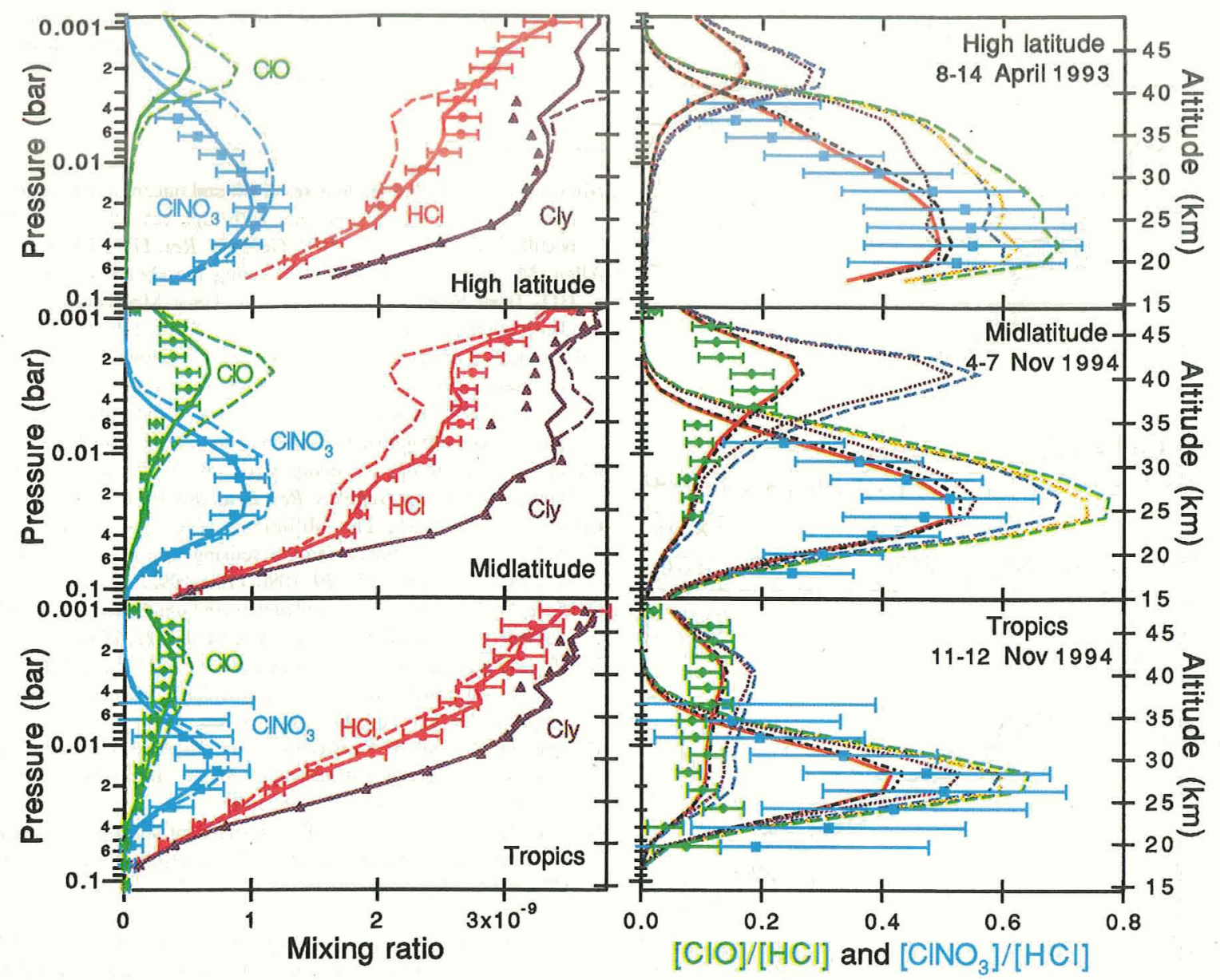

Figure 1. Comparison of modeled and measured $\left[\mathrm{ClNO}_{3}\right],[\mathrm{HCl}]$, and $[\mathrm{ClO}]$. Model results are represented by lines; measurements, by symbols. The vertical axis is pressure; approximate altitude is also shown. Model cases displayed and described in the text used the following rates: JPL94 model used recommended rates and $\mathrm{ClO}+\mathrm{OH} \Theta \mathrm{HCl}+\mathrm{O}_{2}(\mathrm{dashed}$ blue lines in right panels and dashed lines in left panels); JPL94 model with revised $\mathrm{Cl}+\mathrm{CH}_{4}$ rate (dotted purple); JPL94 model with revised $\mathrm{OH}+\mathrm{HCl}$ rate (dot-dashed orange); JPL94 model with Michelsen et al. $\mathrm{O}\left({ }^{1} \mathrm{D}\right)$ quantum yield (dashed green); JPL94 model with $\mathrm{ClO}+\mathrm{OH} \rightarrow \mathrm{HCl}+\mathrm{O}_{2}(7 \%)$ (dot-dashed black); Model C: revised $\mathrm{Cl}+\mathrm{CH}_{4}$ and $\mathrm{OH}+\mathrm{HCl}$ rates, $\mathrm{O}\left({ }^{1} \mathrm{D}\right)$ quantum yield, and $\mathrm{ClO}+\mathrm{OH} \rightarrow \mathrm{HCl}+\mathrm{O}_{2}(7 \%)$ (solid lines in right and left panels). Top panels show comparisons for high latitudes $\left(65-69^{\circ} \mathrm{N}\right)$ at sunrise, middle panels, for midlatitudes $\left(40-48^{\circ} \mathrm{N}\right)$ at sunset, and bottom panels, for tropics $\left(3-13^{\circ} \mathrm{N}\right)$ at sunset.

species. Assuming a yield of $7 \%$ results in agreement between measured and modeled profiles of $[\mathrm{HCl}],\left[\mathrm{ClNO}_{3}\right]$, and [ClO] within experimental uncertainty at nearly all altitudes and latitudes (black dot-dashed lines). This result is reproduced to within a few percent by Model C, which incorporates $\mathrm{O}\left({ }^{1} \mathrm{D}\right)$ quantum yields from Michelsen et al., our rates for $\mathrm{Cl}+\mathrm{CH}_{4}$ and $\mathrm{OH}+\mathrm{HCl}$, and a yield of $7 \%$ for production of $\mathrm{HCl}$ from $\mathrm{ClO}+\mathrm{OH}$ (solid red lines, right panels and solid lines, left panels). The effect of the new rate for $\mathrm{Cl}+\mathrm{CH}_{4}$ is nearly canceled by effects of the Michelsen et al. $\mathrm{O}\left({ }^{1} \mathrm{D}\right)$ quantum yield and the new rate for $\mathrm{OH}+\mathrm{HCl}$. These results are consistent with those for 5-11 Nov 1994 (sunrise) at southern high latitudes [Rinsland et al., this issue].

Although space limitations preclude listing all reaction rates investigated to resolve the discrepancy, we note that resolution is achieved if (1) $\mathrm{Cl}+\mathrm{HO}_{2}$ is increased by a factor of 6 or (2) $\mathrm{Cl}+\mathrm{O}_{3}$ is decreased by $30 \%$ below and $60 \%$ above 30 $\mathrm{km}$. Good agreement below $30 \mathrm{~km}$, with little effect above, is given by (1) an increase in $\mathrm{NO}_{2}+\mathrm{hv}$ by $50 \%$, (2) a decrease in $\mathrm{OH}+\mathrm{HCl}$ by $50 \%$, or (3) a yield of $3 \%$ for $\mathrm{ClO}+\mathrm{HO}_{2} \rightarrow \mathrm{HCl}+\mathrm{O}_{3}$.
These modifications are not supported by current laboratory measurements [NASA94]. Uncertainties in measured $\left[\mathrm{O}_{3}\right]$, estimated to be $\sim 6 \%$, cannot account for the discrepancy, which would require a $50 \%$ lower value above $30 \mathrm{~km}$.

\section{Conclusions}

Comparison of calculated and observed mixing ratios of $\mathrm{HCl}, \mathrm{ClNO}_{3}$, and $\mathrm{ClO}$ leads us to conclude that (1) there must be a mechanism leading to significantly enhanced photochemical production of $\mathrm{HCl}$ in the stratosphere, (2) the contribution from this source must increase with altitude up to $\sim 40 \mathrm{~km}$, and (3) the current set of recommended kinetic parameters and associated uncertainties cannot fully account for the observed errors in calculated $\mathrm{Cly}$ partitioning. Although we have presented $\mathrm{ClO}+\mathrm{OH} \rightarrow \mathrm{HCl}+\mathrm{O}_{2}$ as a candidate, contributions from an unknown source are possible. Including such a channel for repartitioning Cly will lower predicted ozone loss rates, especially in the upper stratosphere. 


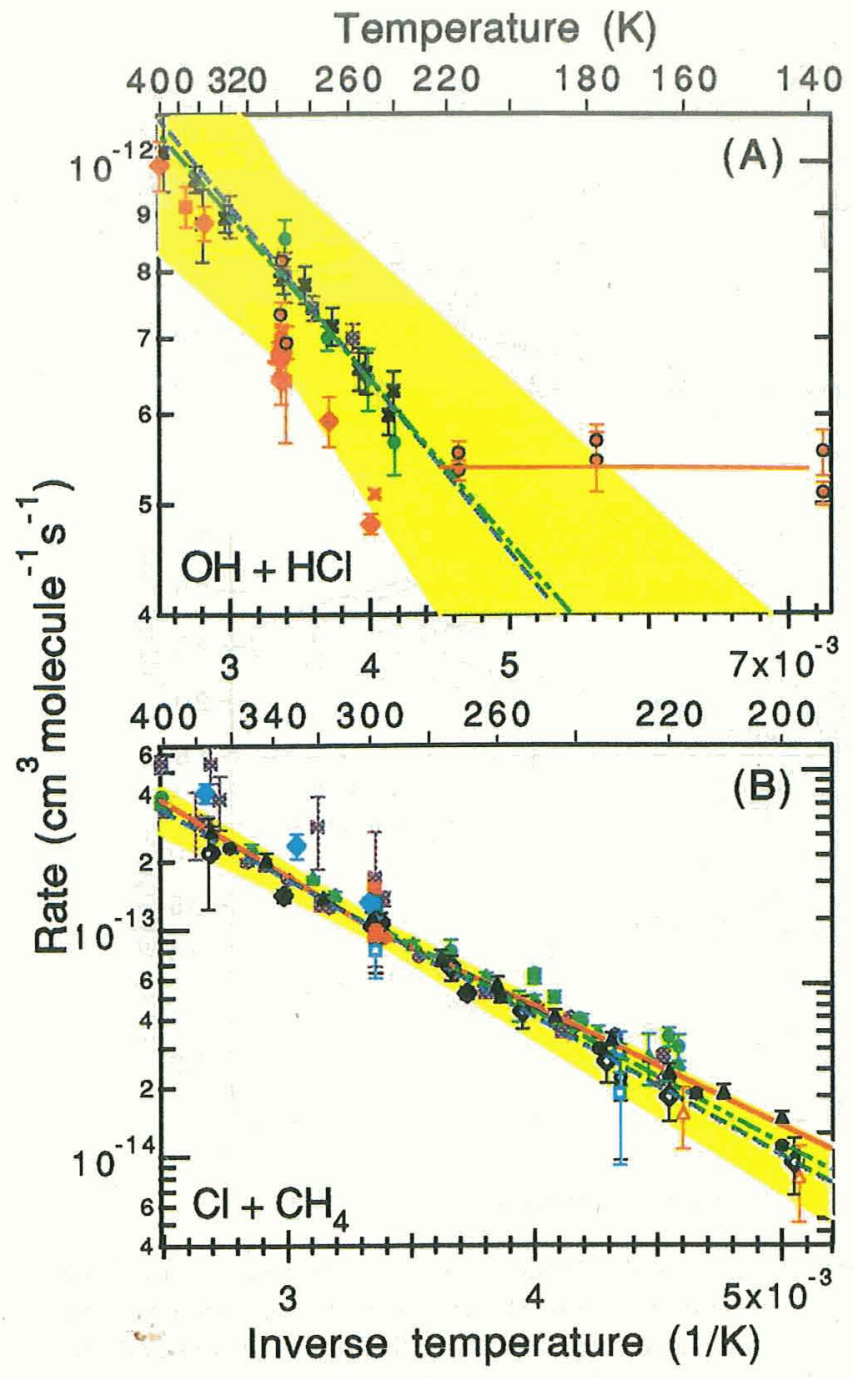

Figure 2. Measured and recommended rates. Recommended rates are from NASA94 (dashed blue lines with shaded yellow $1 \sigma$ uncertainty), NIST92 (dot-dashed green lines), and this study (red lines). Data shown with $1 \sigma$ error bars: (A) $\boldsymbol{O H}+\boldsymbol{H C l}$ MTakacs \& Glass [1973], S Smith \& Zellner [1974], Ravishankara et al. [1977], Husain et al. (1981), X Ravishankara et al. [1983], + Husain et al. (1984), , Keyser [1984], Molina et al. [1984], X Ravishankara et al. (1985), $\nabla$ Smith et al. [1985], Sharkey \& Smith [1993]; (B) $\boldsymbol{C l}+\boldsymbol{C H}_{4} \bigcirc$ Pritchard et al. [1955], $\square$ Knox \& Nelson [1959], Davis et al. [1970], Clyne \& Walker [1973], Poulet et al. [1974], Watson et al. [1976], $\triangle$ Manning \& Kurylo [1977], $\nabla$ Michael \& Lee [1977], Whytock et al. [1977], Keyser [1978], $\diamond$ Lin et al. [1978], - Zahniser et al. [1978], Ravishankara \& Wine [1980], $\triangle$ DeMore [1991], Dobis \& Benson [1987], Sawersyn et al. [1987], A Beichert et al. [1995]. Red symbols indicate data not used by NASA94 and NIST92; open symbols, rates inferred from competitive chlorination measurements relative to recommended rates from NASA94. We regret that limited space prohibits inclusion of a full reference list; see NASA94 and NIST 92 and references therein.

Acknowledgments. We thank M. Allen, J. G. Anderson, W. B. DeMore, S. P. Sander, and S. C. Wofsy for valuable discussions, and M. H. Sze for help preparing figures. This work was supported by the National Science Foundation through a postdoctoral fellowship for
HAM and the National Aeronautics and Space Administration (NASA) grant NAS1-19955. Research at JPL, California Institute of Technology, is performed under contract to NASA.

\section{References}

Abrams, M. C., et al., On the assessment and uncertainty of atmospheric trace gas burden measurements with high resolution infrared solar occultation spectra from space, Geophys. Res. Lett., this issue.

Allen, M., and M. L. Delitsky, Inferring the abundances of $\mathrm{ClO}$ and $\mathrm{HO}_{2}$ from Spacelab 3 Atmospheric Trace Molecule Spectroscopy observations, J. Geophys. Res., 96, 2913-2919, 1991.

Atkinson, R., et al., Evaluated kinetic and photochemical data for atmospheric chemistry, J. Phys. Chem. Ref. Data, 21, 1992.

Brasseur, G., A. D. Rudder, and C. Tricot, Stratospheric response to chemical perturbations, J. Atmos. Chem., 3, 261-288, 1985.

Chang, A. Y., et al., A comparison of ATMOS and the ER-2: Halogenated gases, Geophys. Res. Lett., this issue.

Croskey, C. L., et al., The Millimeter Wave Atmospheric Sounder (MAS): A shuttle-based remote sensing experiment, IEEE Trans. Microwave Theory Tech., 40, 1090-1100, 1992.

DeMore, W. B., et al., Chemical kinetics and photochemical data for use in stratospheric modeling, Evaluation number 11, 1994.

Dessler, A. E., et al., Correlated observations of $\mathrm{HCl}$ and $\mathrm{ClONO}_{2}$ from UARS and implications for stratospheric chlorine partitioning, Geophys. Res. Lett., 22, 1721-1724, 1995.

Hanson, D. R., and A. R. Ravishankara, Heterogeneous chemistry of bromine species in sulfuric acid under stratospheric conditions Geophys. Res. Lett., 22, 385-388, 1995.

Hills, A. J., and C. J. Howard, Rate coefficient temperature dependence and branching ratio for the $\mathrm{OH}+\mathrm{ClO}$ reaction, J. Chem. Phys., 81, 4458-4465, 1984.

Leu, M. T., and C. L. Lin, Rate constants for the reactions of $\mathrm{OH}$ with $\mathrm{ClO}, \mathrm{Cl}_{2}$ and $\mathrm{Cl}_{2} \mathrm{O}$ at $298 \mathrm{~K}$, Geophys. Res. Lett., 6, 425-428, 1979.

McElroy, M. B., and R. J. Salawitch, Changing composition of the global stratosphere, Science, 243, 763-770, 1989.

Michelsen, H. A., et al., Production of $\mathrm{O}\left({ }^{1} \mathrm{D}\right)$ from photolysis of $\mathrm{O}_{3}$, Geophys. Res. Lett., 21, 2227-2230, 1994.

Natarajan, M., and L. B. Callis, Stratospheric photochemical studies with Atmospheric Trace Molecule Spectroscopy (ATMOS) measurements, J. Geophys. Res., 96, 9361-9370, 1991.

Poulet, G., G. Laverdet, and G. L. Bras, Rate constant and branching ratio for the reaction of $\mathrm{OH}$ with $\mathrm{ClO}, J$. Phys. Chem., 90, 159-165, 1986.

Ravishankara, A. R., and P. H. Wine, A laser flash photolysis-resonance fluorescence kinetics study of the reaction $\mathrm{Cl}\left({ }^{2} \mathrm{P}\right)+\mathrm{CH}_{4} \rightarrow \mathrm{CH}_{3}+\mathrm{HCl}$, J. Chem. Phys., 72, 25-30, 1980.

Rinsland, C. P., et al., ATMOS/ATLAS-3 measurements of stratospheric chlorine and reactive nitrogen partitioning inside and outside the November 1994 Antarctic vortex, Geophys. Res. Lett., this issue.

Sharkey, P., and I. W. M. Smith, Kinetics of elementary reactions at low temperatures: Rate constants for the reactions of $\mathrm{OH}$ with $\mathrm{HCl}(298 \geq \mathrm{T} / \mathrm{K} \geq 138), \mathrm{CH}_{4}(298 \geq \mathrm{T} / \mathrm{K} \geq 178)$ and $\mathrm{C}_{2} \mathrm{H}_{6}(298 \geq \mathrm{T} / \mathrm{K} \geq 138), J$. Chem. Soc. Faraday Trans., 89, 631-638, 1993.

Stachnik, R. A., et al., Submillimeterwave heterodyne measurements of stratospheric $\mathrm{ClO}, \mathrm{HCl}, \mathrm{O}_{3}$, and $\mathrm{HO}_{2}$ : First results, Geophys. Res. Lett., 19, 1931-1934, 1992.

Woodbridge, E. L., et al., Estimates of total organic and inorganic chlorine in the lower stratosphere from in situ and flask measurements during AASE II, J. Geophys. Res., 100, 3057-3064, 1995.

Zander, R., et al., The 1994 northern midlatitude budget of stratospheric chlorine derived from ATMOS/ATLAS-3 observations, Geophys. Res. Lett., this issue.

H. A. Michelsen, ARP, Harvard Univ., 12 Oxford St., Cambridge, MA 02138. (e-mail: ham@io.harvard.edu)

(Received September 27, 1995; revised January 4, 1996; accepted March 5, 1996) 


\title{
ATMOS/ATLAS-3 measurements of stratospheric chlorine and reactive nitrogen partitioning inside and outside the November 1994 Antarctic vortex
}

\author{
C. P. Rinsland, ${ }^{1}$ M. R. Gunson, ${ }^{2}$ R. J. Salawitch, ${ }^{2}$ H. A. Michelsen, ${ }^{3}$ R. Zander, ${ }^{4}$ \\ M. J. Newchurch, ${ }^{5}$ M. M. Abbas, ${ }^{6}$ M. C. Abrams, ${ }^{7}$ G. L. Manney, ${ }^{2}$ A. Y. Chang, ${ }^{2}$ \\ F. W. Irion, ${ }^{8}$ A. Goldman, ${ }^{9}$ and E. Mahieu ${ }^{4}$
}

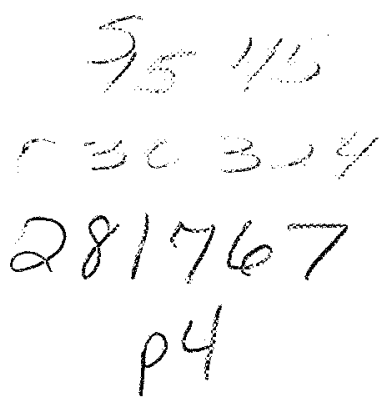

\begin{abstract}
Partitioning between $\mathrm{HCl}$ and $\mathrm{ClONO}_{2}$ and among the main components of the reactive nitrogen family ( $\mathrm{NO}, \mathrm{NO}_{2}$, $\mathrm{HNO}_{3}, \mathrm{ClONO}_{2}, \mathrm{~N}_{2} \mathrm{O}_{5}$, and $\mathrm{HO}_{2} \mathrm{NO}_{2}$ ) has been studied inside and outside the Antarctic stratospheric vortex based on ATMOS profiles measured at sunrise during the 3-12 November 1994 ATLAS-3 Shuttle mission. Elevated mixing ratios of $\mathrm{HCl}$ in the lower stratosphere with a peak of -2.9 ppbv $\left(10^{-9}\right.$ parts per volume) were measured inside the vortex near $500 \mathrm{~K}$ potential temperature $(\sim 19 \mathrm{~km})$. Maximum $\mathrm{ClONO}_{2}$ mixing ratios of $\sim 1.2$, $\sim 1.4$, and $\sim 0.9 \mathrm{ppbv}$ near $700 \mathrm{~K}(-25 \mathrm{~km})$ were measured inside, at the edge, and outside the vortex, respectively. Model calculations reproduce the higher levels of $\mathrm{HCl}$ and $\mathrm{NO}_{\mathrm{x}}$ $\left(\mathrm{NO}+\mathrm{NO}_{2}\right.$ ) inside the lower strato spheric vortex both driven by photochemical processes initiated by low $\mathrm{O}_{3}$. The high $\mathrm{HCl}$ at low $\mathrm{O}_{3}$ results from chemical production of $\mathrm{HCl}$ via the reaction of enhanced $\mathrm{Cl}$ with $\mathrm{CH}_{4}$, limited production of $\mathrm{ClONO}_{2}$, and the descent of inorganic chlorine from higher altitudes.
\end{abstract}

\section{Introduction}

The critical role of active chlorine in the winter and springtime catalytic destruction of stratospheric $\mathrm{O}_{3}$ in polar regions has been well established through ground-, aircraft-, and satellite-based measurements [cf. World Meteorological Organization (WMO), 1995, Chapt. 3]. Reactive nitrogen is also important in polar $\mathrm{O}_{3}$ losses, especially over Antarctica where it is irreversibly removed over large portions of the vortex through the sedimentation of polar stratospheric cloud (PSC) particles (denitrification) [Fahey et al., 1990]. Low levels of reactive nitrogen inhibit the formation of $\mathrm{ClONO}_{2}$ when sunlight returns in spring, thereby allowing active chlorine to persist and catalytic $\mathrm{O}_{3}$ destruction to continue inside the vortex [e.g., Brune et al., 1991].

A primary objective of the Atmospheric Laboratory for Applications and Science (ATLAS)-3 Shuttle mission from 3-12 November 1994, was to obtain sets of volume mixing ratio

\footnotetext{
${ }^{1}$ NASA Langley Research Center, Hampton, Virginia.

2 Jet Propulsion Laboratory, California Institute of Technology.

${ }^{3}$ Harvard University, Cambridge, Massachusetts.

${ }_{5}^{4}$ Institute of Astrophysics, University of Liège.

${ }^{5}$ University of Alabama, Huntsville.

${ }^{6}$ NASA Marshall Space Flight Center, Huntsville, Alabama.

${ }^{7}$ Science Applications International Corporation.

${ }^{8}$ California Institute of Technology, Pasadena.

${ }^{9}$ Department of Physics, University of Denver.
}

Copyright 1996 by the American Geophysical Union.

Paper number 96GL01474

0094-8534/96/96GL-01474\$05.00
(VMR) profiles at high southern latitudes during the photochemical recovery phase of the Antarctic ozone hole. We report here Atmospheric Trace MOlecule Spectroscopy (ATMOS) observations of inorganic chlorine and reactive nitrogen species inside and outside the vortex. A photochemical model was used to interpret the measurements.

\section{Observations and Spectral Analysis}

The ATMOS/ATLAS-3 Antarctic measurements were recorded during sunrises between $64.5^{\circ} \mathrm{S}$ and $72.4^{\circ} \mathrm{S}$ latitude. The measurements reported here were obtained by combining nearly spatially and temporally coincident observations with filters 3 $\left(1580-3420 \mathrm{~cm}^{-1}\right)$ and $12\left(625-1400 \mathrm{~cm}^{-1}\right)$. The 6 principal components of the reactive nitrogen ( $\mathrm{NO}_{\mathrm{y}}$ ) family, $\mathrm{NO}, \mathrm{NO}_{2}$, $\mathrm{HNO}_{3}, \mathrm{ClONO}_{2}, \mathrm{~N}_{2} \mathrm{O}_{5}$, and $\mathrm{HO}_{2} \mathrm{NO}_{2}$, plus $\mathrm{HCl}, \mathrm{N}_{2} \mathrm{O}$, and $\mathrm{O}_{3}$ were measured.

Diurnal corrections were included in the $\mathrm{NO}$ and $\mathrm{NO}_{2}$ retrievals presented here [Newchurch et al., 1996]. Random error bars, which vary with altitude and species, are shown in the plots. Total systematic errors (1 sigma) were $\mathrm{HCl}(5 \%), \mathrm{ClONO}_{2}$ $(20 \%)$, NO (5\%), $\mathrm{NO}_{2}(6 \%), \mathrm{HNO}_{3}(16 \%), \mathrm{N}_{2} \mathrm{O}_{5}(16 \%)$, and $\mathrm{HO}_{2} \mathrm{NO}_{2}(20 \%)$ [Abrams et al., 1996a]. The effective vertical resolution of the ATMOS measurements was -2 to $4 \mathrm{~km}$.

\section{Measurement Results}

Figure 1 presents an overview of the Antarctic measurements of $[\mathrm{HCl}]$ (top), $\left[\mathrm{ClONO}_{2}\right]$ (middle), and $\left[\mathrm{O}_{3}\right]$ (bottom) (where $[\mathrm{x}]$ denotes the species VMR) plotted versus longitude and potential temperature $(\Theta)$. Longitude is a useful coordinate because the measurements were recorded over a narrow latitude range, and the vortex remained at similar longitudes throughout the mission $\left(\sim 240^{\circ}\right.$ to $\left.315^{\circ} \mathrm{E}\right)$ [Manney et al., 1996]. The vortex is apparent in the $\left[\mathrm{N}_{2} \mathrm{O}\right]$ contours overlayed in all of the panels.

Above $900 \mathrm{~K}$, no $[\mathrm{HCl}]$ variations with longitude are apparent. Zonal mean [HCl] decreased from $3.35 \pm 0.42 \mathrm{ppbv}$ at $2000 \mathrm{~K}$ $(-50 \mathrm{~km})$ to $3.09 \pm 0.17 \mathrm{ppbv}$ at $1550 \mathrm{~K}(-44 \mathrm{~km})$, and $2.73 \pm$ $0.09 \mathrm{ppbv}$ at $1000 \mathrm{~K}(\sim 34 \mathrm{~km})$. Error limits denote stan dard deviations of the measurements. Below $800 \mathrm{~K}(\sim 28 \mathrm{~km})$, [HCl] inside the vortex was systematically higher than outside. A shallow minimum in [HCl] occurred inside at $725 \mathrm{~K}(-26 \mathrm{~km})$ followed by a sharp rise to a maximum of $-2.9 \mathrm{ppbv}$ at $500 \mathrm{~K}$ $(\sim 19 \mathrm{~km}) .[\mathrm{HCl}]$ remained elevated inside the vortex at least down to $400 \mathrm{~K}(\sim 15 \mathrm{~km})$.

Peak $\left[\mathrm{ClONO}_{2}\right]$ occurred at $\sim 700 \mathrm{~K}(25 \mathrm{~km})$ at all longitudes. Maximum values, $-1.4 \mathrm{ppbv}$, were measured at the vortex edge, as compared to $-1.2 \mathrm{ppbv}$ inside and $0.9 \mathrm{ppbv}$ outside the vortex. The $\left[\mathrm{ClONO}_{2}\right]$ decline below its peak was much sharper inside the vortex than outside. Inside and outside VMRs were equal at $550 \mathrm{~K}(\sim 21 \mathrm{~km})$; below $450 \mathrm{~K}(\sim 17 \mathrm{~km}),\left[\mathrm{ClONO}_{2}\right]$ in the vortex interior was $<0.1$ ppbv. 


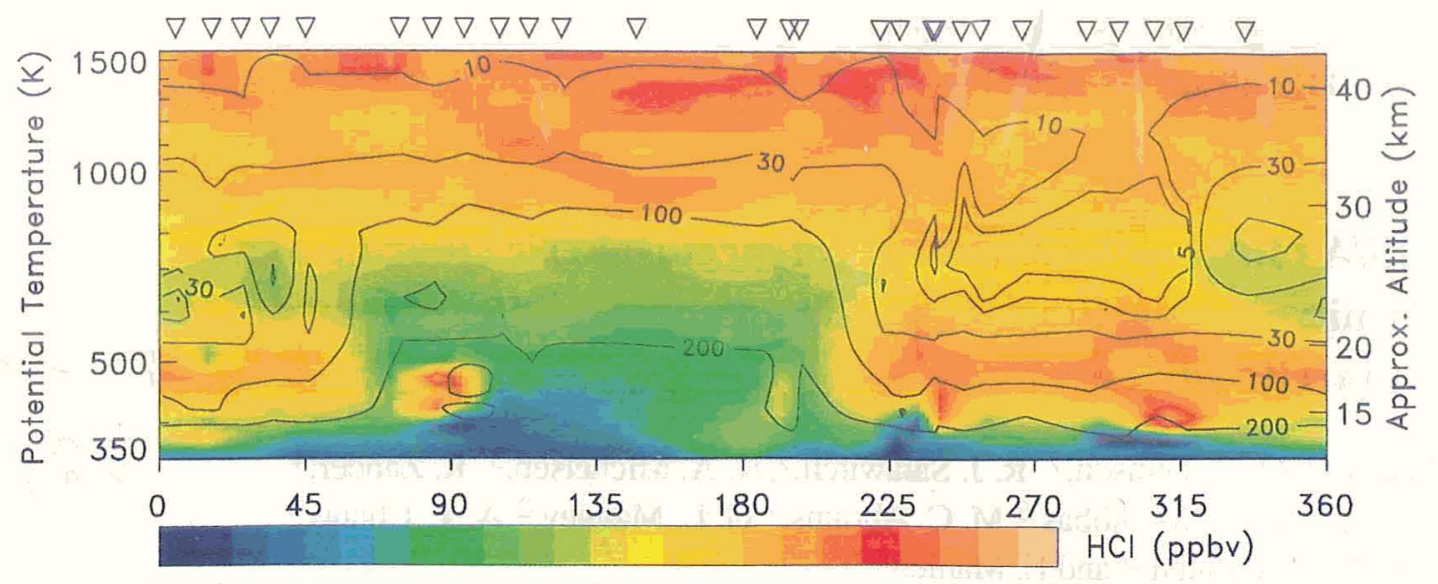

0 .

1.

2.

3.

4.
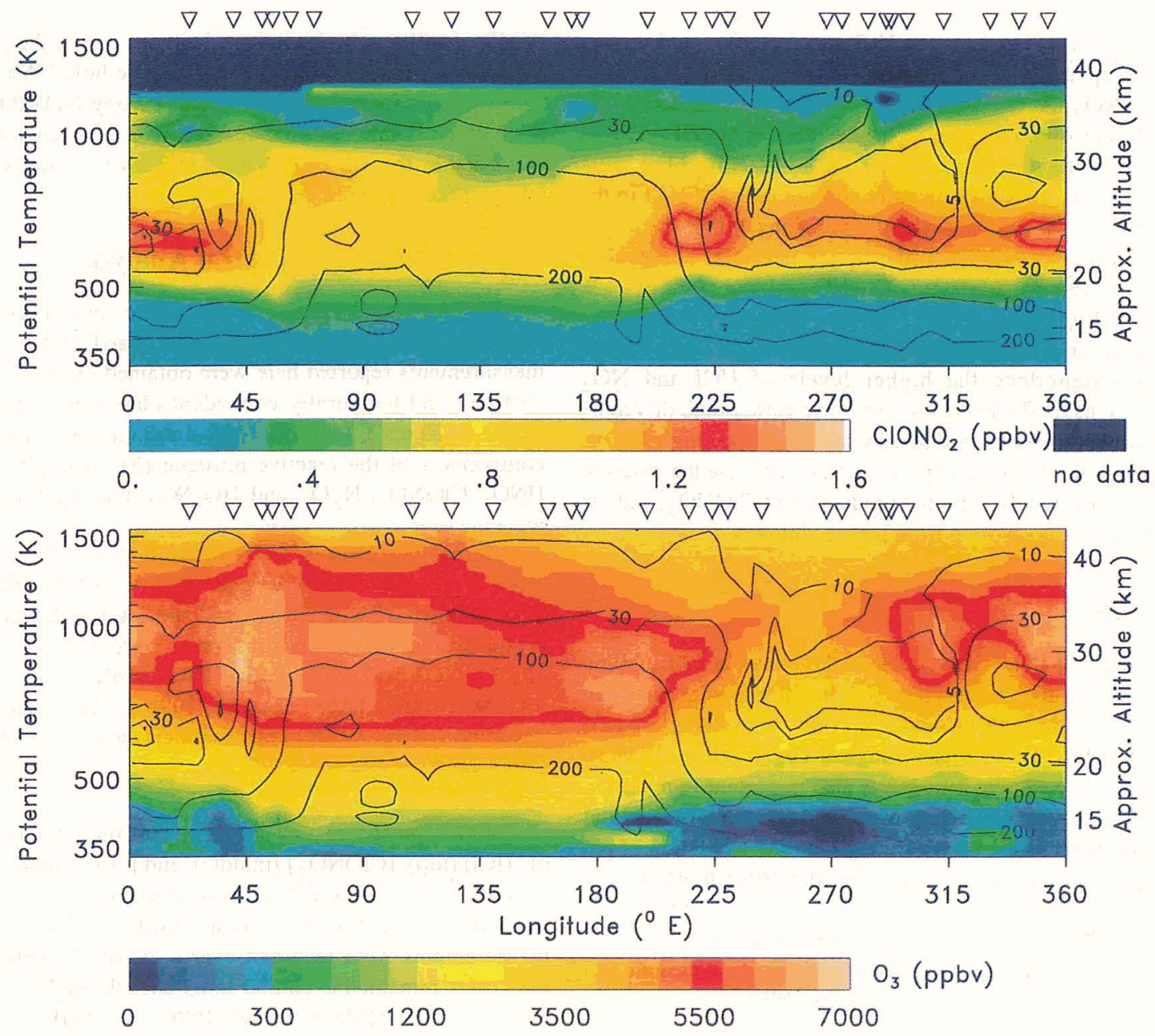

Figure 1. Distributions of Antarctic filter $3[\mathrm{HCl}]$ (top), filter $12\left[\mathrm{ClONO}_{2}\right]$ (middle), and filter $12\left[\mathrm{O}_{3}\right]$ (bottom) versus longitude ( ${ }^{\circ} \mathrm{E}$ ) and $\Theta$. Inverted triangles mark measurement locations. Contours show filter $3\left[\mathrm{~N}_{2} \mathrm{O}\right]$ (ppbv) superimposed on each panel.

Table 1 lists mean $[\mathrm{HCl}]$ and $\left[\mathrm{ClONO}_{2}\right]$ profiles measured in the interior and outside the vortex. The standard deviations provide an indication of the variability among the measurements. The classifications are based on scaled potential vorticity (sPV) derived with -PV in the southern hemisphere so that values increase toward the vortex center. The criteria for clas sification are reported by Rinsland et al. [1996]. Gradients in sPV suggest that the vortex extended down to about $400 \mathrm{~K}$ [Abrams et al., 1996b].

The ATMOS/ATLAS-3 lower stratospheric measurements inside the vortex contrast sharply with late winter and early spring values inside the Arctic vortex where peak $\left[\mathrm{ClONO}_{2}\right]$ is $\sim 2 \mathrm{ppbv}$ [e.g., Rinsland et al., 1995]. [HCl] exceeded $\left[\mathrm{ClONO}_{2}\right]$ throughout the Antarctic stratosphere during ATLAS 3.

\section{Analysis}

The ATLAS-3 measurements inside the Antarctic lower stratospheric vortex were obtained during conditions of exceptionally low $\left[\mathrm{O}_{3}\right]$ and reduced levels of $\left[\mathrm{NO}_{\mathrm{y}}\right]$ [Rinsland et al., 1996]. Theory predicts the partitioning of $\mathrm{Cl}-\mathrm{ClO}-\mathrm{ClONO}_{2}$ shifts in favor of $\mathrm{Cl}$ due to a decrease in the rate of the reaction of $\mathrm{Cl}$ with $\mathrm{O}_{3}$ and an increase in the rate of the reaction of $\mathrm{ClO}$ with $\mathrm{NO}$, which is elevated with respect to $\mathrm{NO}_{2}$ due to reduced daytime $\mathrm{NO}_{2}$ production via $\mathrm{NO}+\mathrm{O}_{3}$. Enhanced levels of $\mathrm{HCl}$ in the vortex result from production via $\mathrm{Cl}+\mathrm{CH}_{4}$ and the descent of $\mathrm{Cl}_{\mathrm{y}}$-rich air [Prather and Jaffe, 1990; Douglass et al., 1995].

The ATMOS observations of high $[\mathrm{HCl}]$ in the Antarctic lower stratospheric vortex are consistent with previous $\mathrm{HCl}$ measurements: (1) ground-based IR total columns from McMurdo, 
Table 1. ATMOS Antarctic $\mathrm{HCl}$ and $\mathrm{ClONO}_{2}$ VMRs (ppbv) Versus $\Theta$ Inside and Outside the Vortex*

\begin{tabular}{rlllll}
\hline & \multicolumn{2}{c}{$\mathrm{HCl}$} & & \multicolumn{2}{c}{$\left[\mathrm{ClONO}_{2}\right]$} \\
\cline { 2 - 3 } \cline { 5 - 6 }$\Theta(\mathrm{k})$ & Inside & Outside & & Inside & Outside \\
\hline $750-800$ & 2.34 & $1.98(12)$ & & $0.94(18)$ & $0.89(30)$ \\
$700-750$ & $2.20(10)$ & $1.79(9)$ & & $1.22(13)$ & $0.93(20)$ \\
$650-700$ & $2.32(14)$ & $1.68(7)$ & & $1.20(17)$ & $0.90(15)$ \\
$600-650$ & $2.49(21)$ & $1.60(10)$ & & $1.22(17)$ & $0.85(17)$ \\
$575-600$ & $2.51(38)$ & $1.63(4)$ & & $1.04(10)$ & $0.89(13)$ \\
$550-575$ & $2.64(19)$ & $1.57(8)$ & & $0.87(13)$ & $0.82(10)$ \\
$525-550$ & $2.63(36)$ & $1.38(14)$ & & $0.64(10)$ & $0.74(11)$ \\
$500-525$ & $2.71(38)$ & $1.32(17)$ & & $0.49(10)$ & $0.60(3)$ \\
$475-500$ & $2.90(9)$ & $1.23(12)$ & & $0.30(9)$ & $0.49(8)$ \\
$450-475$ & $2.79(22)$ & $1.17(15)$ & & $0.18(8)$ & $0.35(8)$ \\
$425-450$ & $2.73(49)$ & $0.95(16)$ & & $0.10(8)$ & $0.21(10)$ \\
$400-425$ & $2.11(72)$ & $1.07(58)$ & & $0.06(51)$ & -- \\
\hline
\end{tabular}

*Measurements are classified as inside the vortex if $\mathrm{sPV}>2$, outside if $s P V<1$. Units are $10^{-4} \mathrm{~s}^{-1}$. Values in parenthesis are VMR standard deviations in units of the last quoted digit. The values are derived from filter $3 \mathrm{HCl}$ measurements and filter $12 \mathrm{ClONO}_{2}$ measurements.

Antarctica in October 1989 [Liu et al., 1992], (2) HALOE VMRs at $\Theta=460 \mathrm{~K}$ in October 1992 [Douglass et al., 1995], and (3) ASHOE-MAESA mission ER-2 VMRs on 10 and 13 October, 1994 , characterized by low [ClO], low $\left[\mathrm{O}_{3}\right]$, high $[\mathrm{HCl}]$, and high [NO] (R. C. Wamsley et al., manuscript in preparation, 1996).

In Figure 2, ATMOS $\mathrm{HCl}$ and $\mathrm{ClONO}_{2}$ profiles and their sum are shown for correlative occultations typical of the outside (top panel) and inside (bottom panel) the vortex measurements (Table 1). Dashed lines show total organic chlorine $\left(\mathrm{CCl}_{\mathrm{y}}\right)$ profiles calculated from simultaneous $\mathrm{N}_{2} \mathrm{O}$ measurements and the relationship between $\left[\mathrm{CCl}_{\mathrm{y}}\right]$ and $\left[\mathrm{N}_{2} \mathrm{O}\right]$ derived from 1994 midlatitude northern hemisphere measurements, primarily the ATMOS/ATLAS 3 dataset [Zander et al., 1996]. The curve labeled $\mathrm{Cl}_{\text {TOT }}$ shows total chlorine, computed by summing [HCl], $\left[\mathrm{ClONO}_{2}\right]$, and $\left[\mathrm{CCl}_{\mathrm{y}}\right]$. Average and standard deviations of $\left[\mathrm{C}_{\mathrm{TOT}}\right]$ are $3.54 \pm 0.16 \mathrm{ppbv}$ (top) and $3.28 \pm 0.36 \mathrm{ppbv}$ (bottom). The agreement of $\left[\mathrm{Cl}_{\mathrm{TOT}}\right]$ and $[\mathrm{HCl}]$ of $3.35 \pm$ $0.42 \mathrm{ppbv}$ at $2000 \mathrm{~K}$, where all other components are minor contributors [Zander et al., 1996], demonstrates the consistency of the ATMOS measurements. The minimum ratio of $\left[\mathrm{O}_{3}\right]$ inside the vortex to $\left[\mathrm{O}_{3}\right]$ outside was 0.16 at $395 \mathrm{~K}(\sim 15 \mathrm{~km})$ where $\left[\mathrm{O}_{3}\right]=89 \pm 29 \mathrm{ppbv}$ inside the vortex. South-viewing $\mathrm{ClO}$ measurements by the UARS Microwave Limb Sounder indicate that enhanced $\mathrm{ClO}$ conditions inside the Antarctic vortex had ended and chlorine was deactivated during ATLAS 3 (L. Froidevaux, private communication, 1996).

Figure 3 illustrates measurements of $\mathrm{NO}, \mathrm{NO}_{2}, \mathrm{HNO}_{3}$, and $\mathrm{N}_{2} \mathrm{O}_{5}$. Measurements obtained outside the vortex (upper panel) show that $\mathrm{HNO}_{3}$ and $\mathrm{NO}_{\mathrm{x}}\left(\mathrm{NO}+\mathrm{NO}_{2}\right)$ were the most abundant $\mathrm{NO}_{\mathrm{y}}$ components in the middle and lower stratosphere. The ratio $\left[\mathrm{HNO}_{3}\right] /\left[\mathrm{NO}_{\mathrm{y}}\right]$ increases at lower $\Theta$, reaching $85 \%$ at $400 \mathrm{~K}$ $(\sim 15 \mathrm{~km})$. In the vortex interior (lower panel) $\mathrm{NO}_{\mathrm{x}}$ and $\mathrm{HNO}_{3}$ each account for $\sim 50 \%$ of $\mathrm{NO}_{\mathrm{y}}$ below $700 \mathrm{~K}(-25 \mathrm{~km})$.

A photochemical model [Salawitch et al., 1994] was used to compute the partitioning of total inorganic chlorine $\left(\mathrm{Cl}_{\mathrm{y}}\right)$ and total reactive nitrogen $\left(\mathrm{NO}_{\mathrm{y}}\right.$ ) for the sets of ATMOS measurements in Figures 2 and 3 . The calculations were constrained by the ATMOS profiles of temperature, pressure, $\left[\mathrm{O}_{3}\right],\left[\mathrm{H}_{2} \mathrm{O}\right],\left[\mathrm{CH}_{4}\right]$, $\left[\mathrm{C}_{2} \mathrm{H}_{6}\right],\left[\mathrm{NO}_{y}\right],[\mathrm{CO}]$, and the sum of $\left[\mathrm{ClONO}_{2}\right]$ and $[\mathrm{HCl}]$. Model [ClO], $[\mathrm{HOCl}]$, and $\left[\mathrm{Cl}_{2} \mathrm{O}_{2}\right]$ were added to $\left[\mathrm{ClONO}_{2}\right]$ and
$\mathrm{HCl}]$ to estimate $\left[\mathrm{Cl}_{\mathrm{y}}\right]$. Aerosol surface area profiles were taken from correlative SAGE II measurements (G.K. Yue and $L . W$. Thomason, private communication, 1995).

Two sets of model calculations were performed. In the first, reaction rates and absorption cross sections from DeMore et al. [1994] were assumed. In the second, the data of DeMore et al. were again assumed except for modifications deduced from reanalysis of laboratory reaction rate data and comparisons between model calculations and ATMOS arctic, northern midlatitude, and tropical inorganic chlorine measurements [Michelsen et al., 1996]. For the present study, the most important change is the assumption of a branching ratio of $7 \%$ for the $\mathrm{ClO}+\mathrm{OH}$ reaction, leading to production of $\mathrm{HCl}$.

The model calculations in Figures 2 and 3 reproduce the main fea tures of the measurements, highlighted by elevated $[\mathrm{HCl}]$ and $\left[\mathrm{NO}_{\mathrm{x}}\right] /\left[\mathrm{NO}_{\mathrm{y}}\right]$, both driven by low $\left[\mathrm{O}_{3}\right]$, and very low $\left[\mathrm{N}_{2} \mathrm{O}_{5}\right]$ at all altitudes inside the vortex. The low $\left[\mathrm{N}_{2} \mathrm{O}_{5}\right]$ inside relative to outside the vortex is a consequence of the difference in the lengths of the night. For the air sampled inside the vortex, the stratospheric solar zenith angle never exceeds $92^{\circ}$, preventing the nighttime buildup of $\left[\mathrm{N}_{2} \mathrm{O}_{5}\right]$. The outside-the-vortex occultations were recorded $\sim 4^{\circ}$ equatorward, where the night was $\sim 4$ hours long, allowing $\left[\mathrm{N}_{2} \mathrm{O}_{5}\right]$ to accumulate rapidly in darkness.

The inorganic chlorine measurements inside the vortex are better reproduced by the modified model calculations (labeled revised) than by the standard run (labeled JPL94) based on the data of DeMore et al. [1994]. The improvements, which are largest near $700 \mathrm{~K}(-25 \mathrm{~km})$, are consistent with the results of

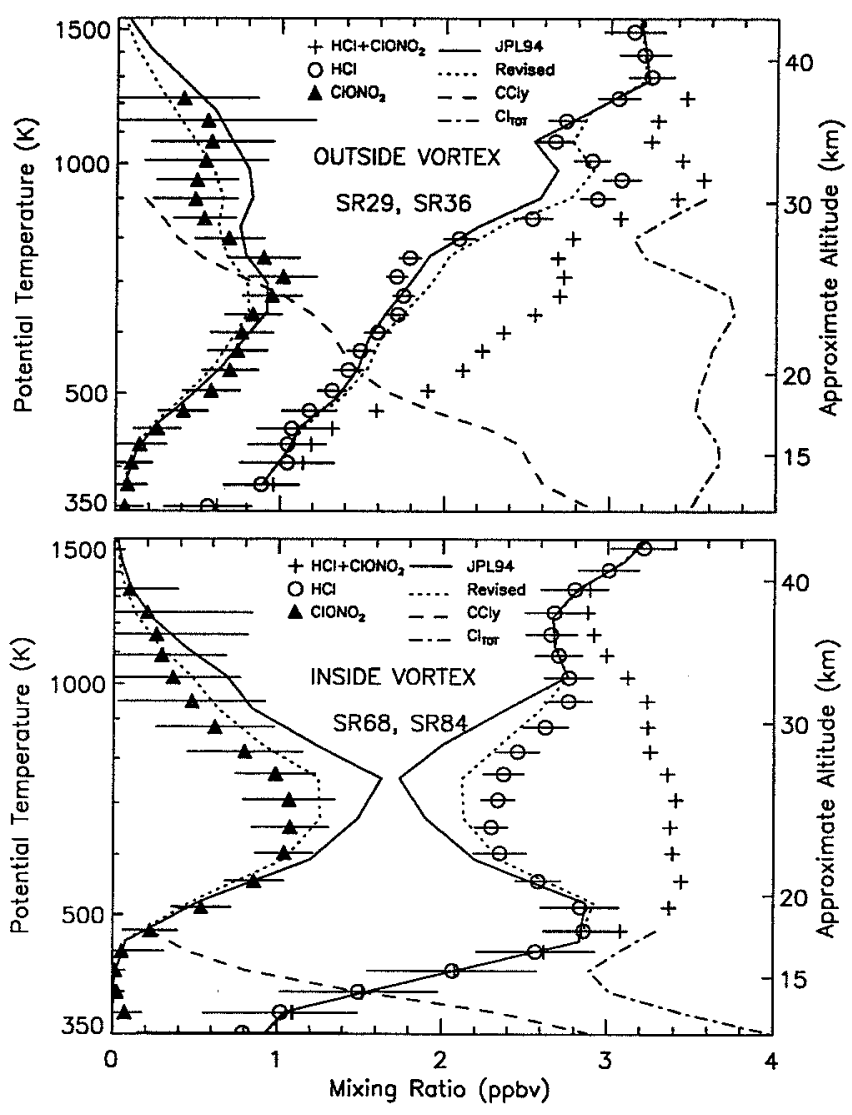

Figure 2. Correlative ATMOS/ATLAS 3 inorganic chlorine measurements (symbols) and corresponding model values (lines) outside (top) and inside (bottom) the Antarctic vortex versus $\Theta$. Filter number (see text), latitude, and longitude are $3,68.2^{\circ} \mathrm{S}$, and $146.6^{\circ} \mathrm{E}$ for SR29 and $12,69.3^{\circ} \mathrm{S}, 139.6^{\circ} \mathrm{E}$ for SR36 (top); 12 , $72.2^{\circ} \mathrm{S}$, and $243.0^{\circ} \mathrm{E}$ for SR84 and $3,71.7^{\circ} \mathrm{S}, 253.0^{\circ} \mathrm{E}$ for SR68 (bottom). Error bars show 1- $\sigma$ measurement precisions. 

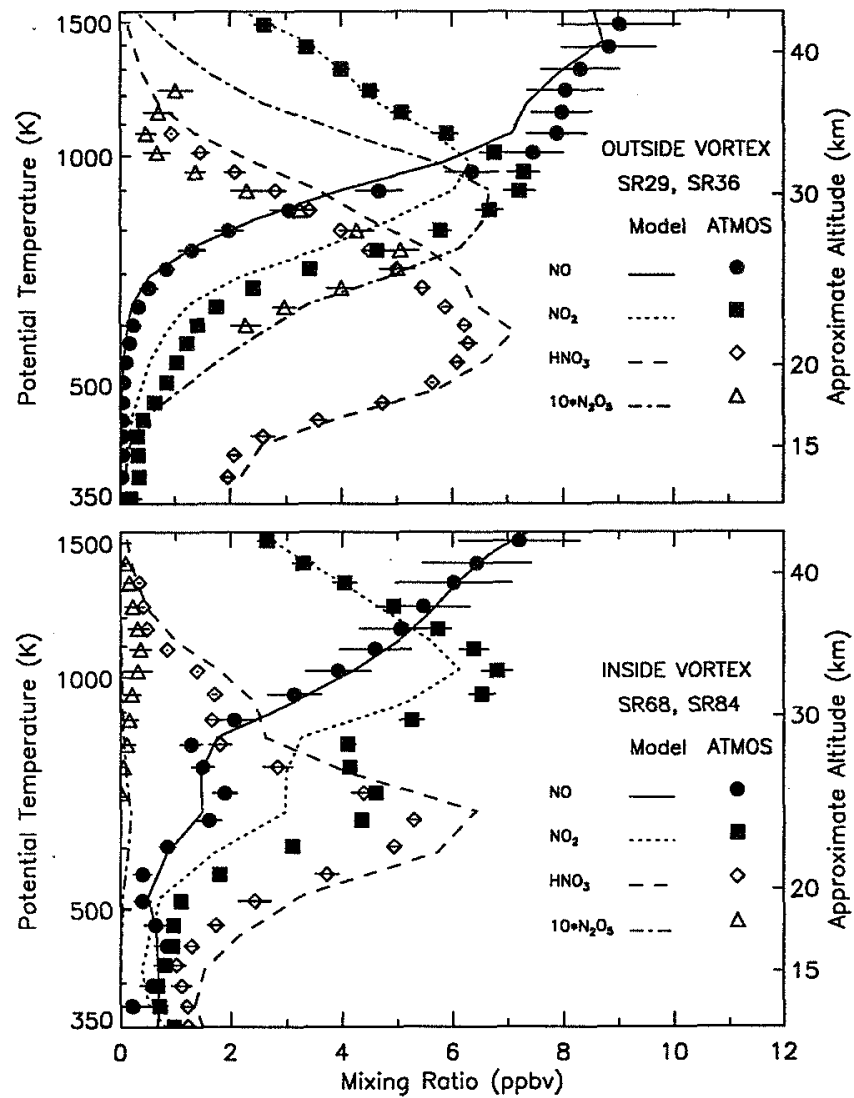

Figure 3. Correlative ATMOS/ATLAS 3 reactive nitrogen measurements and corresponding model values outside and inside the Antarctic vortex versus $\Theta$, for the same occultations as Figure 2. Only a single set of model calculations is shown since the 2 cases (described in the text) gave identical results except for $\mathrm{ClONO}_{2}$. Measured $\mathrm{HO}_{2} \mathrm{NO}_{2}$ mixing ratios (not shown) were less than about $0.2 \mathrm{ppbv}$.

Michelsen et al. [1996]. The differences between the two sets of calculations for the outside-the-vortex case are smaller, and it is not possible to distinguish between them given the measurement uncertainties.

The comparisons in Figure 3 show several systematic discrepancies: (1) the $\left[\mathrm{NO}_{2}\right] /[\mathrm{NO}]$ ratio in the mid- to lower stratosphere was underpredicted by up to $50 \%$ both inside and outside the vortex, (2) $\left[\mathrm{N}_{2} \mathrm{O}_{5}\right]$ was overpredicted outside the vortex above $800 \mathrm{~K}$, and (3) the $\left[\mathrm{NO}_{x}\right] /\left[\mathrm{NO}_{y}\right]$ ratio was underpredicted in the lower stratosphere both inside and outside the vortex. The discrepancy for $\mathrm{NO}_{2} / \mathrm{NO}$ is consistent with either slower photolysis of $\mathrm{NO}_{2}$ than calculated, a faster rate for the reaction of $\mathrm{NO}$ wth $\mathrm{O}_{3}$, or some other process that rapidly converts $\mathrm{NO}$ to $\mathrm{NO}_{2}$. The remaining discrepancies suggest a faster rate for the conversion of $\mathrm{N}_{2} \mathrm{O}_{5}$ to $\mathrm{HNO}_{3}$ than calculated from heterogeneous and gas phase chemistry.

\section{Conclusions}

ATMOS/ATLAS 3 measurements obtained in November 1994 confirm previous observations of high $[\mathrm{HCl}]$ and high $[\mathrm{NO}]$ in the interior of the Antarctic lower stratospheric vortex during its recovery phase. Model calculations initialized with ATMOS species and temperature measurements plus correlative SAGE II aerosol surface areas successfully reproduce these key features of $\mathrm{Cl}_{\mathrm{y}}$ and $\mathrm{NO}_{\mathrm{y}}$ partitioning, confirming a basic understanding of the chemistry, which is driven by very low $\left[\mathrm{O}_{3}\right]$. However, significant model-measurement discrepancies exist for both the inside and outside the vortex cases. These differences are being investigated in the context of the larger set of ATMOS measurements.

Acknowledgments. Research at the Jet Propulsion Laboratory (JPL) was performed under contract to the National Aeronautics and Space Administration (NASA). We thank the ATMOS data processing team at JPL and L. Chiou of Science Applications International Corporation (SAIC), Hampton, Virginia, for their help.

\section{References}

Abrams, M. C., et al., On the assessment of atmospheric trace gas burdens with high resolution infrared solar occultation measurements from space, Geophys. Res. Lett., this issue, 1996a.

Abrams, M. C., et al., ATMOS/ATLAS 3 observations of trace gas transport in the Antarctic vortex of 1994, Geophys. Res. Lett., this issue, $1996 \mathrm{~b}$.

Brune, W. H., et al., The potential for ozone depletion in the Arctic polar stratosphere, Science, 252, 1260-1266, 1991

DeMore, W. B., et al., Chemical kinetics and photochemical data for use in stratospheric modeling, Evaluation No. 11, JPL Publication 94-26, Jet Propulsion Lab, Pasadena, CA, 1994

Douglass, A. R., et al., Interhemispheric differences in springtime production of $\mathrm{HCl}$ and $\mathrm{ClONO}_{2}$ in polar vortices, J. Geophys. Res., 100, $13,967-13,978,1995$.

Fahey, D. W., et al., Observations of denitrification and dehydration in winter polar stratospheres, Nature, 344, 321-324, 1990.

Liu, X., et al., Measurements and model calculations of $\mathrm{HCl}$ column amounts and related parameters over McMurdo during the Austra spring in 1989, J. Geophys. Res., 97, 20,795-20,804, 1992.

Manney, G. L. R. Swinbank, and A. O'Neill, Stratospheric meteorological conditions for the 3-12 Nov. 1994 ATMOS/ATLAS 3 measurements, Geophys. Res. Lett., this issue, 1996.

Michelsen, H. A., et al. Chlorine partitioning in the stratosphere: Evidence from ATMOS measurements for an additional source of $\mathrm{HCl}$, Geophys. Res. Lett. , this issue, 1996.

Newchurch, M. J., et al., Diurnally corrected stratospheric NO and NO abundances from ATMOS solar-occultation measurements, Geophys. Res. Lett. , this issue, 1996.

Prather, M., and A. H. Jaffe, Global impact of the Antarctic ozone hole: Chemical propagation, J. Geophys. Res., 95, 3471-3492, 1990.

Rinsland, C. P., et al., April 1993 arctic profiles of stratospheric $\mathrm{HCl}$, $\mathrm{ClONO}_{2}$, and $\mathrm{CCl}_{2} \mathrm{~F}_{2}$ from atmospheric trace molecule spectroscopy/ATLAS 2 infrared solar occultation spectra, J. Geophys. Res., 100, 14,019-14,027, 1995.

Rinsland, C. P., et al., ATMOS measurements of $\mathrm{H}_{2} \mathrm{O}+2 \mathrm{CH}_{4}$ and total reactive nitrogen in the November 1994 Antarctic stratosphere: Dehydration and denitrification in the vortex, Geophys. Res. Lett., this issue, 1996.

Salawitch, R. J., et al., The diurnal variation of hydrogen, nitrogen, and chlorine radicals: Implications for the heterogeneous production of $\mathrm{HNO}_{2}$, Geophys. Res. Lett., 21, 2551-2554, 1994.

World Meteorological Organization (WMO) Report No. 37, Scientific assessment of stratospheric ozone: 1994, Geneva, 1995.

Zander, R., et al., The 1994 northern midlatitude budget of stratospheric chlorine derived from ATMOS/ATLAS 3 observations, Geophys. Res. Lett., this issue, 1996.

M. M. Abbas, SSL, NMSFC, Huntsville, AL 35889.

M. C. Abrams, Science Applications International Corporation, Hampton, VA 23666.

A. Y. Chang, M. R. Gunson, G. L. Manney and R. J. Salawitch, JPL Mail Stop 183-301, 4800 Oak Grove Drive, Pasadena, CA 91109.

A. Goldman, Department of Physics, University of Denver, Denver, CO 80208.

E. Mahieu, R. Zander and Institute of Astrophysics, University of Liège, 4000 Liège-Cointe, Belgium.

H. A. Michelsen, Division of Applied Sciences and Department of Earth and Planetary Sciences, Harvard University, Cambridge, MA 02138.

F. W. Irion, Department of Chemical Engineering, Caltech, Pasadena, CA 91125.

M. J. Newchurch, University of Alabama at Huntsville, Huntsville, AL 35899.

C. P. Rinsland, NASA LaRC, Mail Stop 401A, Hampton, VA 23681-0001.

(Received October 6, 1995; revised March 7, 1996; accepted April 25, 1996). 


\title{
$\mathrm{NO}_{\mathrm{y}}$ correlation with $\mathrm{N}_{2} \mathrm{O}$ and $\mathrm{CH}_{4}$ in the midlatitude stratosphere
}

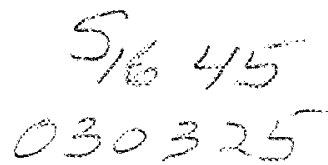

\author{
Y. Kondo, ${ }^{1}$ U. Schmidt, ${ }^{2}$ T. Sugita, ${ }^{1}$ A. Engel, ${ }^{3}$ M. Koike, ${ }^{1}$ P. Aimedieu, ${ }^{4}$ M.R. Gunson, ${ }^{5}$ and J. Rodriguez ${ }^{6}$

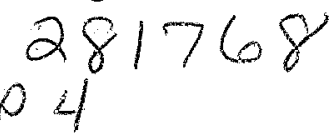

\begin{abstract}
Total reactive nitrogen $\left(\mathrm{NO}_{\mathrm{y}}\right)$, nitrous oxide $\left(\mathrm{N}_{2} \mathrm{O}\right)$, methane $\left(\mathrm{CH}_{4}\right)$, and ozone $\left(\mathrm{O}_{3}\right)$ were measured on board a balloon launched from Aire sur l'Adour $\left(44^{\circ} \mathrm{N}\right.$, $0^{\circ} \mathrm{W}$ ), France on October 12,1994 . Generally, $\mathrm{NO}_{\mathrm{y}}$ was highly anti-correlated with $\mathrm{N}_{2} \mathrm{O}$ and $\mathrm{CH}_{4}$ at altitudes between 15 and $32 \mathrm{~km}$. The linear $\mathrm{NO}_{\mathrm{y}}-\mathrm{N}_{2} \mathrm{O}$ and $\mathrm{NO}_{\mathrm{y}}-$ $\mathrm{CH}_{4}$ relationships obtained by the present observations are very similar to those obtained on board ER-2 and DC-8 aircraft previously at altitude below $20 \mathrm{~km}$ in the northern hemisphere. They also agree well with the data obtained by the Atmospheric Trace Molecule Spectroscopy (ATMOS) instrument at $41^{\circ} \mathrm{N}$ in November 1994. Slight departures from linear correlations occurred around $29 \mathrm{~km}$, where $\mathrm{N}_{2} \mathrm{O}$ and $\mathrm{CH}_{4}$ mixing ratios were larger than typical midlatitude values, suggesting horizontal transport of tropical airmasses to northern midlatitudes in a confined altitude region.
\end{abstract}

\section{Introduction}

Reactive nitrogen in the stratosphere is produced from $\mathrm{N}_{2} \mathrm{O}$ via the following reaction.

$$
\mathrm{N}_{2} \mathrm{O}+\mathrm{O}\left({ }^{1} \mathrm{D}\right) \rightarrow 2 \mathrm{NO}
$$

Total reactive nitrogen, defined as $\mathrm{NO}_{\mathrm{y}}=\mathrm{NO}+\mathrm{NO}_{2}+$ $\mathrm{NO}_{3}+2 \mathrm{~N}_{2} \mathrm{O}_{5}+\mathrm{HNO}_{3}+\mathrm{HO}_{2} \mathrm{NO}_{2}+\mathrm{ClONO}_{2}+\mathrm{BrONO}_{2}+$ aerosol nitrate has a very long photochemical lifetime in the middle and lower stratosphere. However, in the tropical upper stratosphere, $\mathrm{NO}_{\mathrm{y}}$ is removed via the following reaction [Fahey et al., 1990b] and photochemical lifetime becomes much shorter.

$$
\mathrm{NO}+\mathrm{N} \rightarrow \mathrm{N}_{2}+\mathrm{O}
$$

where $\mathrm{N}$ is produced by photolysis of NO.

\footnotetext{
${ }^{1}$ Solar-Terrestrial Environment Laboratory, Nagoya University, Toyokawa, Japan

${ }^{2}$ Institute for Meteorology and Geophysics, Johann Wolfgang Goethe-University, Frankfurt am Main, Germany

${ }^{3}$ Institute for Stratospheric Chemistry, Forschungszentrum Jülich, Jülich, Germany

${ }^{4}$ Service d'Aèronomie, CNRS, Verrières le Buisson, France

${ }^{5}$ Jet Propulsion Laboratory, Pasadena, CA

${ }^{6}$ Atmospheric and Environmental Research, Inc., Cambridge, MA
}

Copyright 1996 by the American Geophysical Union.
It was found that the $\mathrm{NO}_{\mathrm{y}}$ mixing ratio has a tight linear anti-correlation with $\mathrm{N}_{2} \mathrm{O}$ in the lower stratosphere in the absence of irreversible removal processes for $\mathrm{NO}_{y}$, e.g. the sedimentation of aerosol particles containing $\mathrm{NO}_{\mathrm{y}}$ species [Fahey et al., 1990a]. This correlation is a very useful tool in quantitatively investigating the budget of $\mathrm{NO}_{\mathrm{y}}$. Fahey et al. [1990b] also found a departure of the $\mathrm{NO}_{\mathrm{y}}-\mathrm{N}_{2} \mathrm{O}$ relationship from linearity in the upper stratosphere using the data set of major $\mathrm{NO}_{\mathrm{y}}$ species, namely, $\mathrm{NO}, \mathrm{NO}_{2}$, $\mathrm{HNO}_{3}, 2 \mathrm{~N}_{2} \mathrm{O}_{5}, \mathrm{HO}_{2} \mathrm{NO}_{2}$, and $\mathrm{ClONO}_{2}$ obtained from the Atmospheric Trace Molecule Spectroscopy (ATMOS) instrument on board Spacelab 3 [Russell III et al., 1998]. The observed departure was interpreted as due to net loss of $\mathrm{NO}_{\mathrm{y}}$ via $\mathrm{R} 2$.

In situ $\mathrm{NO}_{\mathrm{y}}$ measurements are not influenced with the uncertainty of the measurement of each $\mathrm{NO}_{\mathrm{y}}$ species and can be done with higher spatial resolution. However, no direct in situ simultaneous measurements of $\mathrm{NO}_{\mathrm{y}}$ and $\mathrm{N}_{2} \mathrm{O}$ has been made above $20 \mathrm{~km}$, so far. In order to investigate the relationship between $\mathrm{NO}_{\mathrm{y}}$ and long-lived trace gases up to middle stratosphere, we made balloon-borne measurements of $\mathrm{NO}_{\mathrm{y}}, \mathrm{N}_{2} \mathrm{O}$, and $\mathrm{CH}_{4}$ at midlatitude in the fall of 1994 as a part of the Second European Stratospheric Arctic and Midlatitude Experiment (SESAME). These extended measurements of the known correlations are important for the interpretation of polar observations, as to identify airmasses perturbed by polar chemistry.

\section{Balloon Experiments}

The simultaneous balloon-borne profile measurements of $\mathrm{NO}_{y}, \mathrm{~N}_{2} \mathrm{O}, \mathrm{CH}_{4}$, and $\mathrm{O}_{3}$ were made on October 12, 1994, from Aire sur l'Adour, France $\left(44^{\circ} \mathrm{N}, 0^{\circ} \mathrm{W}\right)$, using a 150 , $000-\mathrm{m}^{3}$ balloon. The integrated gondola was launched at 1015 universal time (UT) and reached a maximum altitude of $33.5 \mathrm{~km}(7.1 \mathrm{hPa})$ at $1200 \mathrm{UT}$. The slow descent started at 1240 UT and 12 air samples were taken by a neon-cooled cryogenic sampler [Schmidt et al., 1985] at altitudes between 32 and $15 \mathrm{~km}$ during descent. $\mathrm{NO}_{\mathrm{y}}$ was measured above $14 \mathrm{~km}$ throughout the flight and $\mathrm{O}_{3}$ was measured during ascent.

$\mathrm{NO}_{y}$ was measured with a chemiluminescence NO detector combined with a gold catalytic converter heated to $300{ }^{\circ} \mathrm{C}$. Detailed descriptions of the $\mathrm{NO}_{\mathrm{y}}$ instrument are given in Kondo et al. [1992 and references therein]. The sample air was drawn through the Teflon coated inlet tube with an inner diameter of $3 \mathrm{~cm}$. For the present observations, the inlet tube was shortened to $17 \mathrm{~cm}$ to minimize loss of reactive nitrogen. The conversion efficiency for $\mathrm{NO}_{2}$ at a pressure of $7 \mathrm{hPa}$ was measured to be $0.99 \pm 0.02$ both before and after the balloon flights. The conversion efficiency for $\mathrm{HNO}_{3}$ was measured after the balloon flights in a similar way as described in Kondo et al. [1996] and was found to be higher than $95 \%$. Mass flow rates of the sample air for $\mathrm{NO}_{y}$ measurements were recalibrated in the laboratory in 1994 and their uncertainties have been reduced to $3 \%$ up to $7 \mathrm{hPa}$. Overall errors of the $\mathrm{NO}_{\mathrm{y}}$ measurements, including uncertainties in the NO 
calibration and zero level determination, are estimated to be $\pm 15 \%$ between 100 and $7 \mathrm{hPa}$.

The air samples collected by the cryogenic air sampler were analyzed in the laboratory at Forschungszentrum in Jülich employing different gas chromatographic techniques. The accuracies of the $\mathrm{CH}_{4}$ and $\mathrm{N}_{2} \mathrm{O}$ measurements are 5 and $7 \%$, respectively.

$\mathrm{O}_{3}$ was measured with two electro-chemical concentration cell (ECC) sondes flown on the same gondola. The values obtained by the two ozonesondes agreed to within $3 \%$ for pressures lower than $140 \mathrm{hPa}$. The estimated uncertainty of the $\mathrm{O}_{3}$ measurements ranged from 3 to $8 \%$ between 100 and $7 \mathrm{hPa}$.

\section{Results and Discussion}

The altitude of the tropopause was determined to be 13.5 $\mathrm{km}$ based on the observed temperature. The observed $\mathrm{NO}_{\mathrm{y}}$ and $\mathrm{O}_{3}$ profiles are shown in Figure 1. It can be seen that the $\mathrm{NO}_{\mathrm{y}}$ values obtained during ascent and descent agree quite well. The $\mathrm{NO}_{\mathrm{y}}$ values increased with altitude up to 33 $\mathrm{km}$, and they were about $1 \mathrm{ppbv}$ at $15 \mathrm{~km}$ and reached 20 ppbv at $33 \mathrm{~km}$. The $\mathrm{O}_{3}$ profile correlated well with that of $\mathrm{NO}_{y}$ up to $23 \mathrm{~km}$. Good correlation between $\mathrm{NO}_{\mathrm{y}}$ and $\mathrm{O}_{3}$ in the lower stratosphere was reported by Murphy et al. [1993]. Between 23 and $32 \mathrm{~km}$, the vertical gradient of $\mathrm{O}_{3}$ became greater than that of $\mathrm{NO}_{\mathrm{y}}$.

The $\mathrm{N}_{2} \mathrm{O}$ and $\mathrm{CH}_{4}$ profiles are shown in Figure 2. The mean $\mathrm{N}_{2} \mathrm{O}$ and $\mathrm{CH}_{4}$ profiles derived from eight balloon observations are plotted as "reference" midlatitude profiles. These measurements were made at $44^{\circ} \mathrm{N}$ in the fall of 1979 , $1982,1985,1988,1990$, and 1993. The present $\mathrm{N}_{2} \mathrm{O}$ and $\mathrm{CH}_{4}$ mixing ratios generally decreased with altitude and the

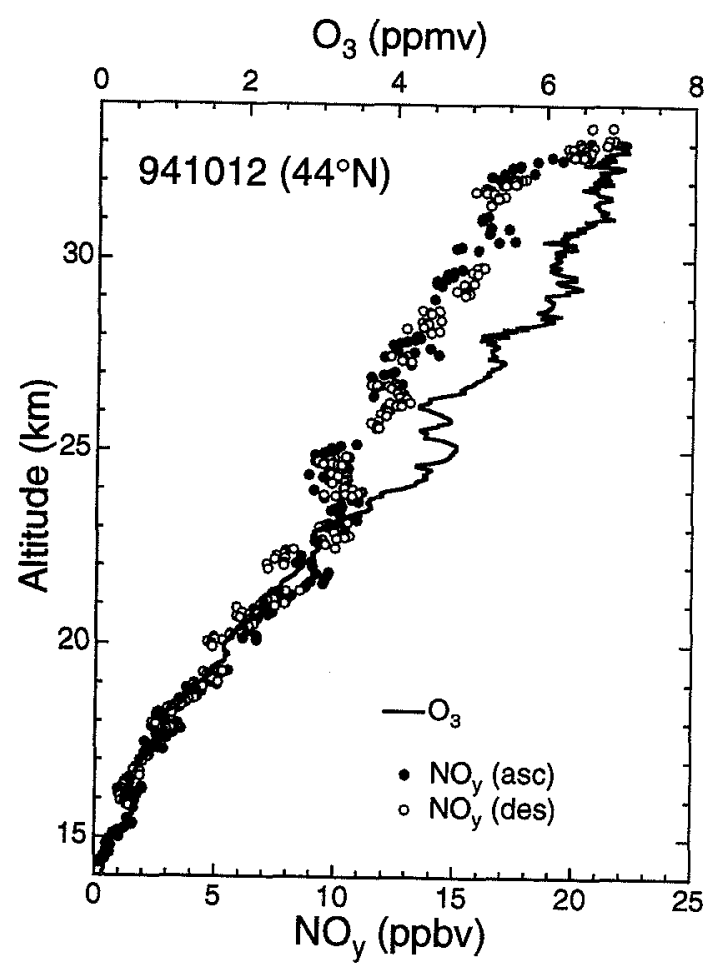

Figure 1. Vertical profiles of the $\mathrm{NO}_{y}$ and $\mathrm{O}_{3}$ mixing ratios observed on October 12, 1994. Closed and open circles indicate the $\mathrm{NO}_{\mathrm{y}}$ values obtained during ascent and descent, respectively. The $\mathrm{O}_{3}$ measurements were made during ascent.

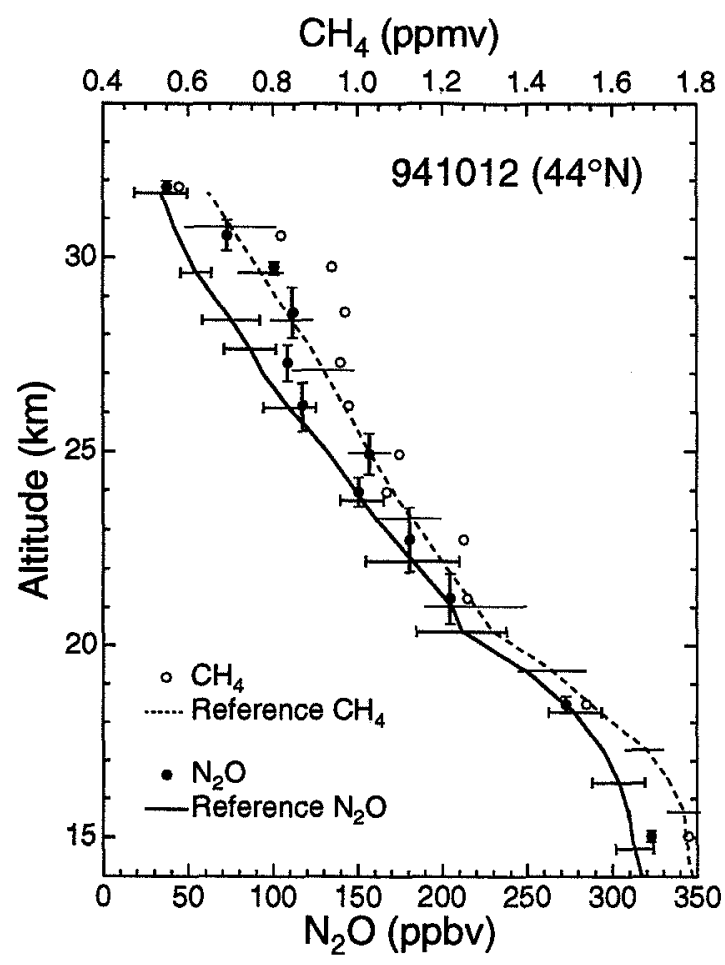

Figure 2. Vertical profiles of the $\mathrm{N}_{2} \mathrm{O}$ and $\mathrm{CH}_{4}$ mixing ratios observed on October 12,1994 . The vertical bar indicates the range of the altitude where each sample was collected. The solid line indicates the mean "reference" $\mathrm{N}_{2} \mathrm{O}$ profile for autumn at $44^{\circ} \mathrm{N}$. The horizontal bar represents the one $\sigma$ standard deviation of the values used for the reference profile.

minimum $\mathrm{N}_{2} \mathrm{O}$ and $\mathrm{CH}_{4}$ values of $38 \mathrm{ppbv}$ and $0.58 \mathrm{ppmv}$ were obtained at the highest altitude of $32 \mathrm{~km}$, respectively.

The $\mathrm{N}_{2} \mathrm{O}$ values up to $26 \mathrm{~km}$ were very close to the "reference" mean values. However, at altitudes between 28 and $30.5 \mathrm{~km}$, the $\mathrm{N}_{2} \mathrm{O}$ values were considerably larger than the mean values. Considering that $\mathrm{N}_{2} \mathrm{O}$ and $\mathrm{CH}_{4}$ mixing ratios at these altitudes are larger in the tropics than at midlatitudes [Jones and Pyle, 1984; Podolske et al., 1993], it is consistent to interpret that the airmasses measured at these altitudes originated from lower latitudes. At $32 \mathrm{~km}$, the $\mathrm{N}_{2} \mathrm{O}$ value was again close to the mean value. Isentropic ten-day back trajectories do not indicate that the airmasses measured between 28 and $30.5 \mathrm{~km}$ were transported from lower latitudes within 10 days (R. Kawa, private communication). This is not necessarily inconsistent with the observations considering that $\mathrm{N}_{2} \mathrm{O}$ and $\mathrm{CH}_{4}$ have lifetimes much longer than 10 days as discussed below.

The fine structures of the $\mathrm{N}_{2} \mathrm{O}$ and $\mathrm{CH}_{4}$ profiles are very similar as can be seen from Figure 2. In fact, $\mathrm{N}_{2} \mathrm{O}$ and $\mathrm{CH}_{4}$ were correlated very well with a correlation coefficient of 0.995 . A relationship between $\mathrm{N}_{2} \mathrm{O}$ and $\mathrm{CH}_{4}$ has been derived by Kawa et al. [1993] based on a much larger data set obtained by in situ measurements.

$$
\left[\mathrm{CH}_{4}\right](\mathrm{ppmv})=0.50+0.00382\left[\mathrm{~N}_{2} \mathrm{O}\right](\mathrm{ppbv})
$$

This relationship holds also for the present data. $\mathrm{N}_{2} \mathrm{O}$ and $\mathrm{CH}_{4}$ are transported from the troposphere and destroyed photochemically in the upper stratosphere. The photochemical lifetimes of $\mathrm{N}_{2} \mathrm{O}$ at the altitude of 40,32 , 
and $25 \mathrm{~km}$ at midlatitude in summer are 1 month, 6 months and 10 years, respectively [Solomon and Garcia, 1984]. The photochemical lifetimes of $\mathrm{CH}_{4}$ at the same altitudes are 2 month, 12 months, and 10 years. The long photochemical lifetimes relative to transport time determine the compactness of the correlation [Plumb and Ko, 1992].

In order to investigate the correlation between $\mathrm{NO}_{\mathrm{y}}$ and $\mathrm{N}_{2} \mathrm{O}$, the $\mathrm{NO}_{\mathrm{y}}$ values are plotted versus the $\mathrm{N}_{2} \mathrm{O}$ values in Figure 3. For comparison, the data obtained by the ATMOS at $41^{\circ} \mathrm{N}$ between November 4 and 6 in 1994 are plotted as open circles in Figure 3. A linear least-square fit to the balloon data points is expressed as

$$
\left[\mathrm{NO}_{\mathrm{y}}\right](\mathrm{ppbv})=19.9-0.0595\left[\mathrm{~N}_{2} \mathrm{O}\right](\mathrm{ppbv})
$$

and is shown as the straight line in the figure. The dotted line indicates the linear relationship expressed as

$$
\left[\mathrm{NO}_{\mathrm{y}}\right](\mathrm{ppbv})=20.7-0.0644\left[\mathrm{~N}_{2} \mathrm{O}\right](\mathrm{ppbv})
$$

obtained by the ER-2 measurements for $\mathrm{N}_{2} \mathrm{O}$ mixing ratios larger than 90 ppbv during AASE and AASE II in winter [Fahey et al., 1990b; Loewenstein et al., 1993]. The latitude covered by these measurements was $22-90^{\circ} \mathrm{N}$.

Generally, the present observations show that $\mathrm{NO}_{\mathrm{y}}$ was linearly correlated with $\mathrm{N}_{2} \mathrm{O}$ up to $32 \mathrm{~km}$ where the $\mathrm{N}_{2} \mathrm{O}$ mixing ratio was $38 \mathrm{ppbv}$ and the agreement with the ATMOS data is good. The present $\mathrm{NO}_{\mathrm{y}}$ maximum value of $20 \mathrm{ppbv}$ was observed at $33.5 \mathrm{~km}$, at altitudes where unfortunately $\mathrm{N}_{2} \mathrm{O}$ and $\mathrm{CH}_{4}$ were not measured.

A slight deviation from this linearity is observed at altitudes between 28.6 and $30.6 \mathrm{~km}$, where the $\mathrm{N}_{2} \mathrm{O}$ values were between 70 and $110 \mathrm{ppbv}$. In this region, the $\mathrm{NO}_{\mathrm{y}}$ mixing ratio is larger than that expected from the equations (2) and (3). Some differences in the $\mathrm{NO}_{y}-\mathrm{N}_{2} \mathrm{O}$ relationship depending on latitude are predicted by a two

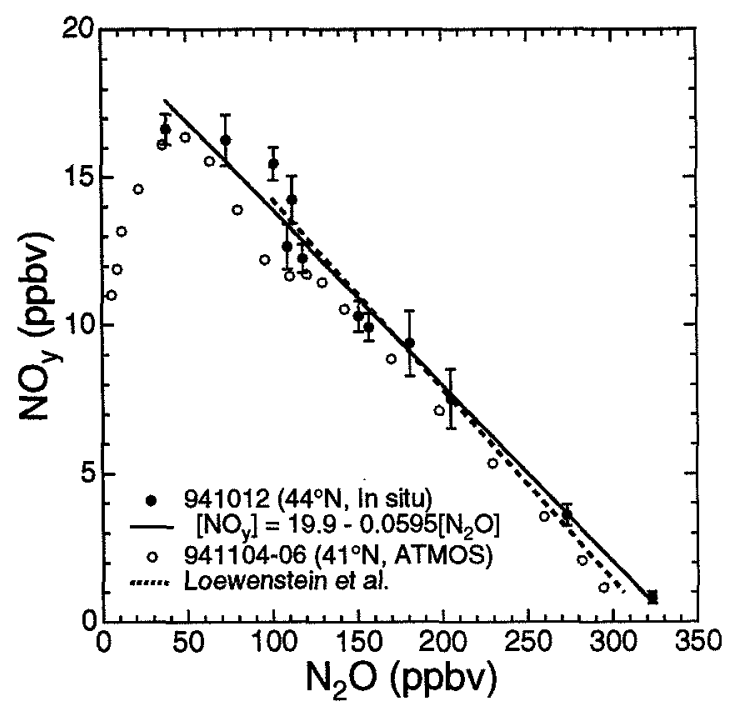

Figure 3. $\mathrm{NO}_{\mathrm{y}}$ mixing ratio plotted versus $\mathrm{N}_{2} \mathrm{O}$ mixing ratio. The bar indicates the one $\sigma$ standard deviation of the $\mathrm{NO}_{\mathrm{y}}$ values in the altitude where each sample was collected. The straight line is the linear least-square fit to the data points. The dotted line is the relationship obtained by Loewenstein et al. [1993] using the $\mathrm{N}_{2} \mathrm{O}$ and $\mathrm{NO}_{\mathrm{y}}$ data obtained on board the ER-2 in the Arctic stratosphere unperturbed by heterogeneous processes. The ATMOS data obtained at $41^{\circ} \mathrm{N}$ are shown as open circles.

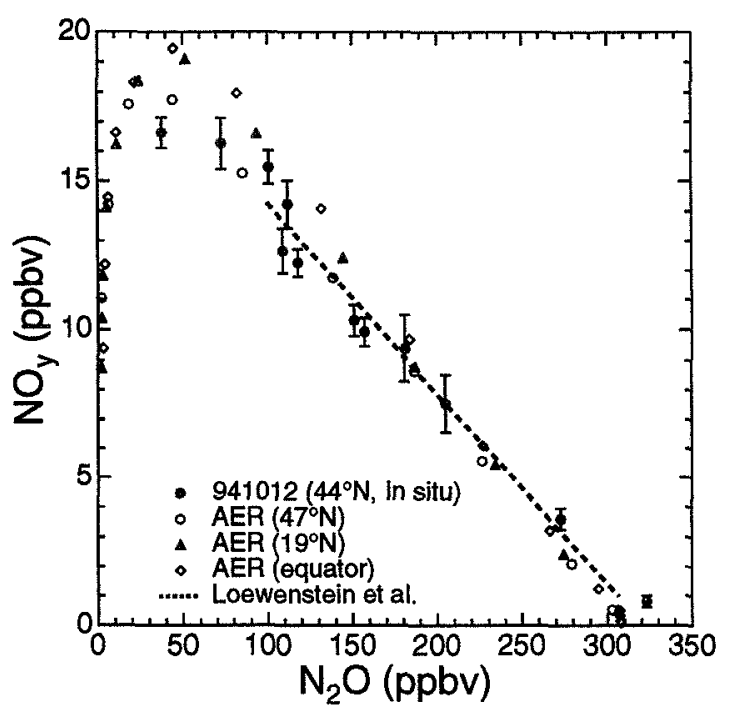

Figure 4. Comparison of the observed $\mathrm{NO}_{\mathrm{y}}-\mathrm{N}_{2} \mathrm{O}$ correlation with that calculated by the 2 dimensional model for $47^{\circ} \mathrm{N}, 19^{\circ} \mathrm{N}$, and equator.

dimensional photochemical-dynamical model [Rodriguez et al., 1994]. $\mathrm{NO}_{\mathrm{y}}-\mathrm{N}_{2} \mathrm{O}$ correlation calculated by the model from mid to low latitudes in October are shown in Figure 4. The observed $\mathrm{NO}_{\mathrm{y}}$ values are generally close to those calculated at $47^{\circ} \mathrm{N}$. The calculated $\mathrm{NO}_{\mathrm{y}}$ values for the equator and $19^{\circ} \mathrm{N}$ are slightly higher than those at $47^{\circ} \mathrm{N}$ and agree with the $\mathrm{NO}_{y}$ values observed between 28.6 and 30.6 $\mathrm{km}$. In this way, the air parcels in this altitude region are consistent with transport from lower latitudes based on the model correlations.

The ATMOS 1994 data indicate that $\mathrm{NO}_{\mathrm{y}}-\mathrm{N}_{2} \mathrm{O}$ relationship started to deviate from linearity at $\mathrm{N}_{2} \mathrm{O}$ mixing ratios less than $35 \mathrm{ppbv}$ (Figure 3). The deviation is prominent for $\mathrm{N}_{2} \mathrm{O}$ mixing ratios below $20 \mathrm{ppbv}$. Deviation from the linearity was also observed by the ATMOS at $28^{\circ} \mathrm{N}$ and $48^{\circ} \mathrm{S}$ in May 1985 at altitudes where $\mathrm{N}_{2} \mathrm{O}$ mixing ratios were less than 25 ppbv [Fahey et al., 1990b]. This deviation from linearity is considered to be caused by photochemical loss of $\mathrm{NO}_{\mathrm{y}}$ via the reaction $\mathrm{R} 2$.

The $\mathrm{NO}_{y}$ values are plotted versus $\mathrm{CH}_{4}$ in Figure 5. As expected from the tight correlation between $\mathrm{N}_{2} \mathrm{O}$ and $\mathrm{CH}_{4}$, $\mathrm{NO}_{\mathrm{y}}$ is also well anti-correlated with $\mathrm{CH}_{4}$ with a correlation coefficient of -0.964 . A linear least-square fit to the data points is expressed as

$$
\left[\mathrm{NO}_{\mathrm{y}}\right](\mathrm{ppbv})=27.2-14.93\left[\mathrm{CH}_{4}\right](\mathrm{ppmv})
$$

and is shown as the straight line in the figure. For comparison, the data obtained by ATMOS at $41^{\circ} \mathrm{N}$ are plotted as open circles in Figure 5. As expected from the comparison of the $\mathrm{NO}_{y}-\mathrm{N}_{2} \mathrm{O}$ correlations shown in Figure 3 , the correlations obtained by the present measurements and ATMOS are very similar.

Weinheimer et al. [1993] measured $\mathrm{NO}_{y}$ and $\mathrm{CH}_{4}$ on board the DC-8 during the NASA Airborne Arctic Stratospheric Expedition (AASE) II in January, February, and March 1992. The latitude covered ranges between 40 and $90^{\circ} \mathrm{N}$. The linear relationship between $\mathrm{NO}_{y}$ and $\mathrm{CH}_{4}$ obtained for $\mathrm{NO}_{y}$ values smaller than $5 \mathrm{ppbv}$ and $\mathrm{CH}_{4}$ values larger than $1.45 \mathrm{ppmv}$ is shown in Figure 5 for comparison.

$\mathrm{NO}_{y}$ is linked with $\mathrm{N}_{2} \mathrm{O}$ photochemically since $\mathrm{NO}_{\mathrm{y}}$ is produced from $\mathrm{N}_{2} \mathrm{O}$ via $\mathrm{R} 1$. On the other hand, there is no 


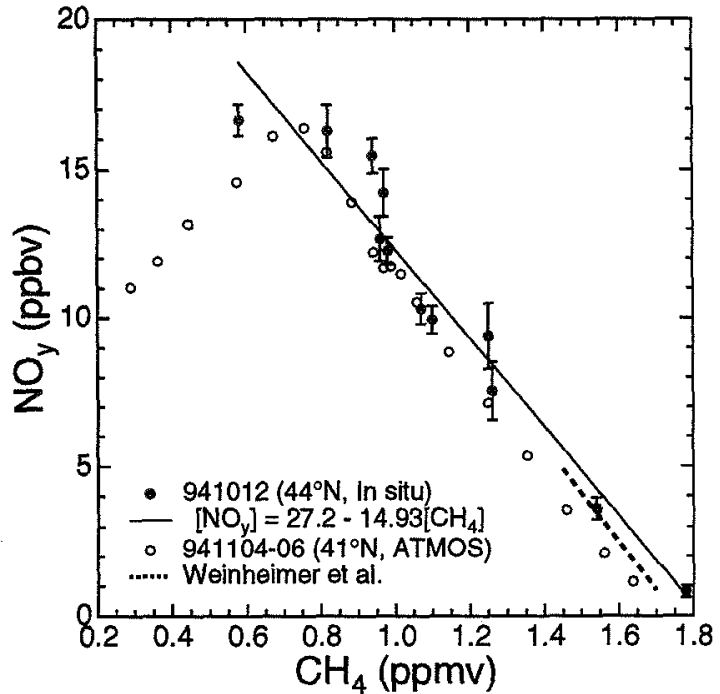

Figure 5. $\mathrm{NO}_{\mathrm{y}}$ mixing ratio plotted versus $\mathrm{CH}_{4}$ mixing ratio. The straight line is the linear least-square fit to the data points. The dotted line is the relationship obtained by Weinheimer et al. [1993] using the $\mathrm{CH}_{4}$ and $\mathrm{NO}_{\mathrm{y}}$ data obtained on board the DC- 8 during AASE II. The ATMOS data obtained at $41^{\circ} \mathrm{N}$ in November 1994 are shown as open circles.

direct photochemical connection between $\mathrm{NO}_{\mathrm{y}}$ and $\mathrm{CH}_{4}$. The tight correlation between $\mathrm{NO}_{\mathrm{y}}$ and $\mathrm{CH}_{4}$ holds because the lifetime of $\mathrm{CH}_{4}$ is longer than the transport time and because the $\mathrm{CH}_{4}$ mixing ratio has a negative gradient with altitude due to the low altitude source and high altitude sink [Plumb and Ko, 1992]. The agreement of the present $\mathrm{NO}_{\mathrm{y}}-$ $\mathrm{CH}_{4}$ correlation with that derived by other measurements indicates that $\mathrm{CH}_{4}$ as well as $\mathrm{N}_{2} \mathrm{O}$ can be used as a good tracer up to $32 \mathrm{~km}$ at midlatitudes.

\section{Summary}

By the simultaneous balloon-borne measurements of $\mathrm{NO}_{\mathrm{y}}$, $\mathrm{N}_{2} \mathrm{O}$, and $\mathrm{CH}_{4}$ at $44^{\circ} \mathrm{N}$ in October, 1994, correlations among these species have been constructed extending above $20 \mathrm{~km}$. $\mathrm{NO}_{\mathrm{y}}$ has revealed tight correlations with $\mathrm{N}_{2} \mathrm{O}$ and $\mathrm{CH}_{4}$ up to $32 \mathrm{~km}$ where $\mathrm{N}_{2} \mathrm{O}$ and $\mathrm{CH}_{4}$ mixing ratios were $38 \mathrm{ppbv}$ and $0.58 \mathrm{ppmv}$, respectively. These correlations are in good agreement with those observed by Loewenstein et al. [1993] and by the ATMOS observed at $41^{\circ} \mathrm{N}$ in November, 1994 up to $32 \mathrm{~km}$. Slight departures from linear correlations occurred around $29 \mathrm{~km}$, suggesting horizontal transport of tropical airmasses to midlatitudes in this altitude region.

Acknowledgments. We wish to thank CNES for the excellent balloon operation and logistical support at Aire sur l'Adour. Partial funding by DG XII of the CEC, the Japanese MESC, the German BMBF, and the French CNRS are gratefully acknowledged. 10 -day back trajectories were calculated by $\mathrm{R}$. Kawa.

\section{References}

Fahey, D.W., et al., Observations of denitrification and dehydration in the winter polar stratospheres, Nature, 344, 321-324, 1990a.
Fahey, D.W., et al., A diagnostic for denitrification in the winter polar stratospheres, Nature, 345, 698-702, $1990 \mathrm{~b}$.

Jones, R.L., and J.A. Pyle, Observations of $\mathrm{CH}_{4}$ and $\mathrm{N}_{2} \mathrm{O}$ by the Nimbus 7 SAMS: A comparison with in situ data and twodimensional numerical model calculations, J. Geophys. Res., 89, 5263-5279, 1984.

Kawa, S.R., R.A. Plumb, and U. Schmidt, Simultaneous observations of long-lived species, Chapter $H$, The atmospheric effects of stratospheric aircraft: Report of the 1992 models and measurements workshop, NASA Ref. Pub. 1292, 352 pp., 1993.

Kondo, Y., et al., Reactive nitrogen, ozone, and nitrate aerosols observed in the Arctic stratosphere in January 1990, $J$. Geophys. Res., 97, 13025-13038, 1992.

Kondo, Y., et al., Reactive nitrogen over the Pacific Ocean during PEM-West-A, J. Geophys. Res., 101, 1809-1828, 1996.

Loewenstein, M., et al., New observations of the $\mathrm{NO}_{y} / \mathrm{N}_{2} \mathrm{O}$ correlation in the lower stratosphere, Geophys. Res. Lett., 20, 2531-2534, 1993.

Murphy, D.M., et al., Reactive odd nitrogen and its correlation with ozone in the lower stratosphere and upper troposphere, $J$. Geophys. Res., 98, 8751-8773, 1993.

Plumb, R.A., and M.K.W. Ko, Interrelationships between mixing ratios of long-lived stratospheric constituents, J. Geophys. Res., 97, 10145-10156, 1992.

Podolske, J.R., M. Loewenstein, A. Weaver, S.E. Strahan, and K.R. Chan, Northern hemisphere nitrous oxide morphology during the 1989 AASE and the 1991-1992 AASE II campaigns, Geophys. Res. Lett., 20, 2535-2538, 1993.

Rodriguez, J.M., M.K.W. Ko, N.D. Sze, C.W. Heisey, G.K. Yue, and M.P. McCormick, Ozone response to enhanced heterogeneous processing after the eruption of Mt. Pinatubo, Geophys. Res. Lett., 21, 209-212, 1994.

Russell III, J.M., et al., Measurements of odd nitrogen compounds in the stratosphere by the ATMOS experiment on Spacelab 3, J. Geophys. Res., 93, 1718-1736, 1988.

Schmidt, U., D. Knapska, and S.A. Penkett, A study of the vertical distribution of methyl chloride in the midlatitude stratosphere, J. Atmos. Chem., 3, 363-376, 1985.

Solomon, S., and R.R. Garcia, On the distributions of long-lived tracers and chlorine species in the middle atmosphere, $J$. Geophys. Res., 89, 11633-11644, 1984.

Weinheimer, A.J., et al., Stratospheric $\mathrm{NO}_{\mathrm{y}}$ measurements on the NASA DC-8 during AASE II, Geophys. Res. Lett., 20, 25632566, 1993.

Y. Kondo, T. Sugita, and M. Koike, Solar-Terrestrial Environment Laboratory, Nagoya University, Toyokawa, Aichi 442, Japan. (e-mail: kondo@stelab.nagoya-u.ac.jp)

U. Schmidt, Institute for Meteorology and Geophysics, Johann Wolfgang Goethe-University, D-60054 Frankfurt, Germany.

A. Engel, Forschungszentrum Jülich $\mathrm{GmbH}$, Institute for Stratospheric Chemistry, D-52425 Jülich, Germany.

P. Aimedieu, Service d'Aèronomie, CNRS, BP3 91370 Verrières le Buisson, France.

M.R. Gunson, Jet Propulsion Laboratory, 4800 Oak Grove Drive, Mail Stop 183-301, Pasadena, CA91109.

J. Rodriguez, Atmospheric and Environmental Research, Inc., 840 Memorial Drive, Cambridge, MA 02139.

(Received September 20, 1995; revised January 5, 1996; accepted March 5, 1996) 


\title{
Stratospheric $\mathrm{NO}$ and $\mathrm{NO}_{2}$ abundances from ATMOS solar-occultation measurements
}

\author{
M. J. Newchurch', M. Allen², M. R. Gunson'2, R. J. Salawitch', G. B. Collins', \\ K. H. Huston ${ }^{1}$, M. M. Abbas ${ }^{3}$ M. C. Abrams ${ }^{5}$ A. Y. Chang ${ }^{2}$, D. W. Fahey ${ }^{6}$, R. S. Gao ${ }^{6}$, \\ F. W. Irion ${ }^{4}$, M. Loewenstein ${ }^{7}$, G. L. Manney ${ }^{2}$, H. A. Michelsen ${ }^{8}$, J. R. Podolske ${ }^{7}$, \\ C. P. Rinsland ${ }^{9}$, R. Zander $^{10}$
}

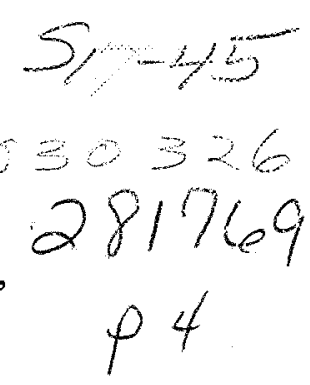

\begin{abstract}
Using results from a time-dependent photochemical model to calculate the diurnal variation of $\mathrm{NO}$ and $\mathrm{NO}_{2}$, we have corrected Atmospheric Trace MOlecule Spectroscopy (ATMOS) solar-occultation retrievals of the $\mathrm{NO}$ and $\mathrm{NO}_{2}$ abundances at $90^{\circ}$ solar zenith angle. Neglecting to adjust for the rapid variation of these gases across the terminator results in potential errors in retrieved profiles of $\sim 20 \%$ for $\mathrm{NO}_{2}$ and greater than $100 \%$ for NO at altitudes below $25 \mathrm{~km}$. Sensitivity analysis indicates that knowledge of the local $\mathrm{O}_{3}$ and temperature profiles, rather than zonal mean or climatological conditions of these quantities, is required to obtain reliable retrievals of $\mathrm{NO}$ and $\mathrm{NO}_{2}$ in the lower stratosphere. Extremely inaccurate $\mathrm{O}_{3}$ or temperature values at 20 $\mathrm{km}$ can result in $50 \%$ errors in retrieved $\mathrm{NO}$ or $\mathrm{NO}_{2}$. Mixing ratios of NO in the mid-latitude, lower stratosphere measured by ATMOS during the November 1994 ATLAS-3 mission compare favorably with in situ ER-2 observations, providing strong corroboration of the reliability of the adjusted space-borne measurements.
\end{abstract}

\section{Introduction}

The rapid temporal variation in the concentrations of NO and $\mathrm{NO}_{2}$ at sunrise and sunset, if not properly accounted for, will produce errors in the retrieval of these gases from solaroccultation measurements [Kerr et al., 1977; Boughner et al., 1980; Roscoe and Pyle, 1987; Russell et al., 1988]. We demonstrate that the correction for temporal variation is sufficiently sensitive to profiles of temperature and $\mathrm{O}_{3}$ that local values of these quantities, rather than zonal or climatological means, are necessary to return accurate profiles of NO and $\mathrm{NO}_{2}$. A coincidence between ATMOS and the NOAA NO$\mathrm{NO}_{\mathrm{y}}$ sensor on the ER-2 during November 1994 reveals good agreement between corrected NO profiles measured by ATMOS and in situ measurements in the lower stratosphere.

\footnotetext{
${ }^{1}$ University of Alabama in Huntsville, Huntsville, AL

${ }^{2}$ Jet Propulsion Laboratory, California Institute of Technology, Pasadena, CA

${ }^{3}$ Marshall Space Flight Center, Huntsville, AL

${ }^{4}$ California Institute of Technology, Pasadena, CA

${ }^{5}$ SAIC - NASA Langley Research Center, Hampton, VA

${ }^{6}$ NOAA Aeronomy Laboratory, Boulder, $\mathrm{CO}$

${ }^{7}$ NASA Ames Research Center, Moffett Field, CA

${ }^{8}$ Harvard University, Cambridge, MA

${ }^{9}$ NASA Langley Research Center, Hampton, VA

${ }^{10}$ University of Liège, Liège, Belgium
}

Copyright 1996 by the American Geophysical Union.

Paper number 96GL01196

0094-8534/96/96GL-01196\$05.00

\section{Sensitivity of correction factors to adopted parameters}

The variation of $\mathrm{NO}$ and $\mathrm{NO}_{2}$ near the terminator is governed primarily by the reaction of $\mathrm{NO}$ with $\mathrm{O}_{3}$ and by the photolysis of $\mathrm{NO}_{2}$. Hence, accurate knowledge of the local values of $\mathrm{O}_{3}$, temperature (due to the temperature dependence of $\mathrm{NO}+\mathrm{O}_{3}$ ), and the radiation field is required to calculate the variation of NO and $\mathrm{NO}_{2}$ across the terminator. We used the Caltech/JPL onedimensional, time-dependent photochemical model [Allen and Delitsky, 1990, 1991] to calculate the diurnal variation of NO and $\mathrm{NO}_{2}$. As a validation of our model calculations, we compared the computed variation of $\mathrm{NO}$ and $\mathrm{NO}_{2}$ with observed diurnal variation at several altitudes and geophysical conditions [Kondo et al., 1989, 1990; Kawa et al., 1990; Webster et al., 1990]. In all cases, the agreement was very good. For example, Figure 1 shows a comparison of NO measurements at $20 \mathrm{~km}$ [Kawa et al., 1990 ] with the results of the model constrained by the measurements of $\mathrm{O}_{3}$ and temperature simultaneous with NO. With both model results and measurements normalized by the volume mixing ratio (VMR) at solar zenith angle (SZA) of $90^{\circ}$, the agreement is excellent. The accuracy with which the model calculates the relative variation of $\mathrm{NO}$ and $\mathrm{NO}_{2}$ is the critical element for correcting solar-occultation retrievals. We calculated factors describing the diurnal variation of $\mathrm{NO}$ and $\mathrm{NO}_{2}$ relative to the values at $\mathrm{SZA}=90^{\circ}$ (either sunrise or sunset) at $0.01^{\circ}$ increments and subsequently smoothed to $0.1^{\circ}$ increments for use in the ATMOS onion-peeling reduction algorithm [Norton and Rinsland, 1991] following the methodology of Murcray et al. [1978] and Rinsland et al. [1984]. This methodology applies the calculated factors to the measured slant columns during the onion-peeling retrieval process.

Sample model calculations show that the variation of NO and $\mathrm{NO}_{2}$ at the terminator is not sensitive to assumptions concerning the local values of $\mathrm{NO}_{\mathrm{y}}, \mathrm{H}_{2} \mathrm{O}, \mathrm{CH}_{4}$, the rates of heterogeneous reactions, or albedo variation. The sensitivities to prescribed $\mathrm{O}_{3}$ and temperature, however, are large enough to require using $\mathrm{O}_{3}$ and temperature measurements obtained simultaneously with the $\mathrm{NO}_{\mathrm{x}}\left(=\mathrm{NO}+\mathrm{NO}_{2}\right)$ measurements to yield accurate, retrieved profiles. For example, an error of $+1-50 \%$ in model $\mathrm{O}_{3}$ at all altitudes results in an error in retrieved NO at $\mathrm{SZA}=90^{\circ}$ of $-10 /-55 \%$ [NO] and $+12 /-55 \%\left[\mathrm{NO}_{2}\right]$ at $20 \mathrm{~km}$. Model errors of $+/-25 \%$ in temperature result in somewhat smaller retrieval errors. These perturbations encompass potentially real deviations from climatological or zonal mean conditions; however, in the winter vortex, larger excursions may occur. The sensitivity to uncertainty in the adopted values for model calculations of both $\mathrm{O}_{3}$ and temperature increases with decreasing altitude below $30 \mathrm{~km}$. Because of the convolved effects of kinetics, photolysis, and slant-column geometry, however, the sensitivity is not always monotonic in 


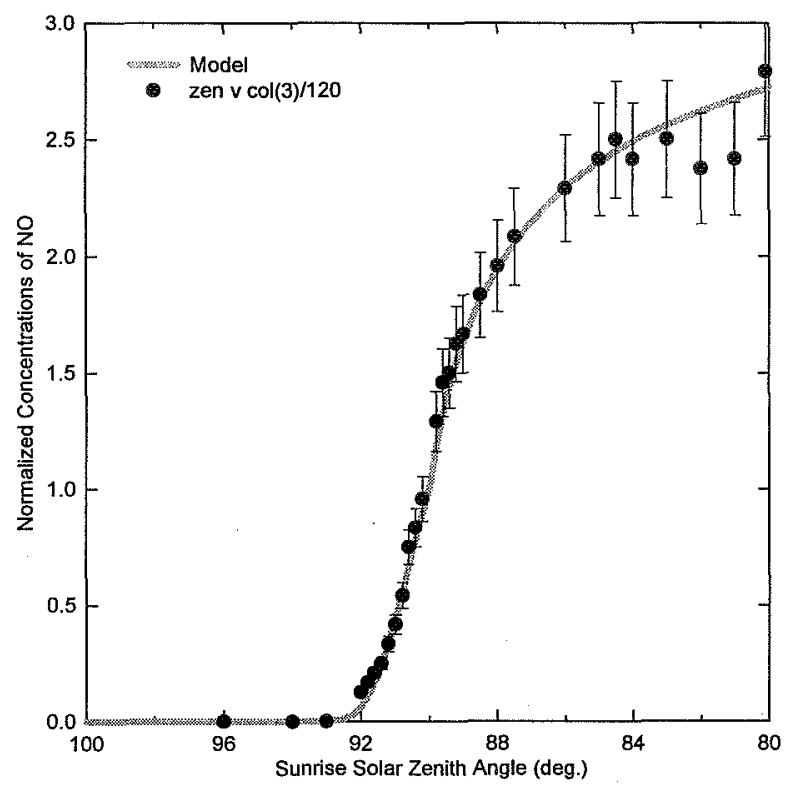

Figure 1. Kawa et al., [1990] ER-2 measurements (circles with $1 \sigma$ error bars) of NO VMR normalized to the $90^{\circ}$ solar zenith angle compared with results of a model simulation (grey line) The measurements occurred at $20 \mathrm{~km}$ and $39^{\circ} \mathrm{N}$.

altitude. Simultaneous departures in both $\mathrm{O}_{3}$ and temperature generally compound the resulting error in the gas profile, but not necessarily in a simple fashion. We note that some solar occultation experiments either ignore the effects of diurnal variation (e.g., SAGE $\mathrm{NO}_{2}$ [Chu and McCormick, 1986 and Kerr et al., 1977]) or use a monthly, zonal-mean atmosphere for the correction computation (e.g., HALOE NO and $\mathrm{NO}_{2}$ [J. M. Russell III, personal communication]).

\section{Implications for ATMOS retrievals}

\section{NO profiles}

Figure $2 \mathrm{a}$ displays the profiles of NO using three different treatments of the diurnal correction. ATLAS-3/SS01 (first sunset occultation) obtained at $49^{\circ} \mathrm{N}$ is a typical mid-latitude sunset occultation with an $\mathrm{O}_{3}$ profile significantly different from the zonalmean $\mathrm{O}_{3}$, but with temperatures similar to the zonal mean. The retrieval that ignores the effects of diurnal variation (denoted NC) is the lowest of the three in the 10 to $30-\mathrm{km}$ region. The retrieval that uses correction factors from a model employing simultaneously acquired ATMOS $\mathrm{O}_{3}$ and temperature profiles (denoted PC) produces the highest values of NO. A retrieval using model results based on zonal-mean profiles of $\mathrm{O}_{3}$ and temperature (denoted ZC) is intermediate to the other NO profiles. The PC retrieval abundances exceed $1 \sigma \mathrm{NC}$ measurement uncertainties between 16 and $18 \mathrm{~km}$ and also between 24 and $30 \mathrm{~km}$. Figure $2 \mathrm{~b}$ shows retrieved profiles of NO for sunrise occultation ATLAS3/SR09 (sunrise number 9) in the southern hemisphere vortex. The NC retrieval yields roughly $50 \%$ of the amount of NO at 20 $\mathrm{km}$ compared to the $\mathrm{PC}$ retrieval. The $\mathrm{ZC}$ retrieval profile is quite similar to, and equally inaccurate as, the NC profile. The failure of the $\mathrm{ZC}$ retrieval is due to the extreme zonal variation of $\mathrm{O}_{3}$ and temperature at these latitudes, which encompass the polar vortex [Manney et al., this issue]. As shown in the fractional difference between $\mathrm{PC}$ and $\mathrm{NC}$, the correction exceeds the 1-sigma measurement uncertainty (dotted line) between 16 and $28 \mathrm{~km}$. We also used the photochemical reconstruction model of Salawitch et al. [1994] to compute correction factors and obtained retrieved profiles of $\mathrm{NO}$ and $\mathrm{NO}_{2}$ that differ from the $\mathrm{PC}$ values shown in Figures 2 and 3 by no more than $20 \%$, significantly less than the uncertainty of the ATMOS measurement.

Similar analysis of other sunrise and sunset occultations reveals that the difference between $\mathrm{PC}$ and $\mathrm{NC}$ retrievals vary widely among the occultations, but that significant corrections do not occur above $30 \mathrm{~km}$. Below $25 \mathrm{~km}$, the true NO values may be as much as a factor of 5 larger than uncorrected values. Under some circumstances (e.g., at high latitudes, $72.3^{\circ} \mathrm{S}$, in SR68 where the maximum SZA is only $91.7^{\circ}$ ) the PC profile values are actually less than the uncorrected profile values. No clear differences between sunrise and sunset corrections emerge.

\section{$\mathrm{NO}_{2}$ profiles}

The effect of ignoring the diurnal variation of $\mathrm{NO}_{2}$ in the retrieval process is in the opposite sense and of smaller magnitude
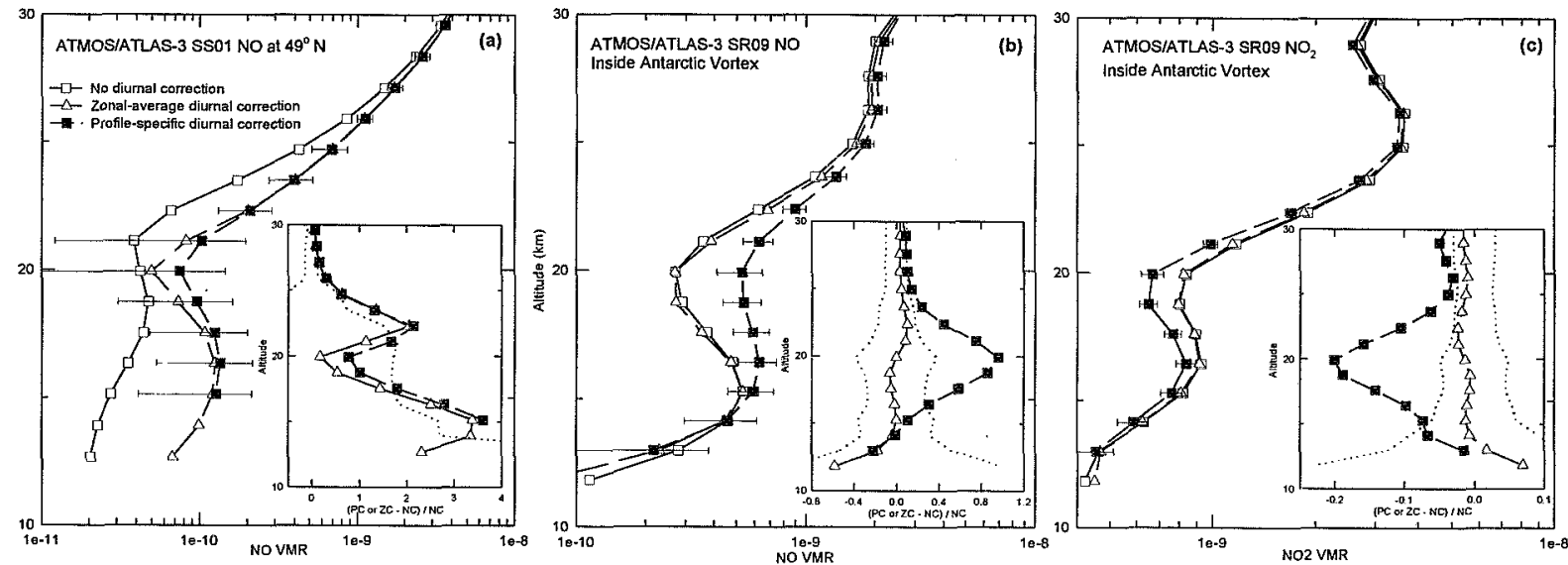

Figure 2. Retrieved profiles for $\mathrm{NO}$ and $\mathrm{NO}_{2}$ without correction ( $\mathrm{NC}$ denoted with open squares), with corrections computed from zonal-mean conditions (ZC denoted with open triangles), and with corrections computed from simultaneously measured conditions (PC denoted with solid squares) $\pm 1 \sigma$ precision. The insets show $\mathrm{ZC}$ and $\mathrm{PC}$ values relative to the NC values with dotted lines representing $1 \sigma \mathrm{NC}$ measurement uncertainties. Panel 2(a) shows the sunrise 09 profile inside the SH polar vortex. Panel 2(b) shows the sunset 01 profile with significant corrections of $200 \%$ and $350 \%$ at $22 \mathrm{~km}$ and $15 \mathrm{~km}$, respectively. Panel 2(c) shows PC, ZC, and $\mathrm{NC}$ profiles for $\mathrm{NO}_{2}$ in occultation SR09. Note that these corrections are in the direction opposite to the NO corrections. 
than for NO. For example, as illustrated in Figure $2 \mathrm{c}$ for ATMOS/ATLAS-3 SR09, the accurately corrected $\mathrm{NO}_{2}$ abundances (PC) are typically 10-20\% lower than uncorrected amounts (NC), well in excess of the measurement uncertainties. Also, the approximate correction, $\mathrm{ZC}$, is nearly the same as the $\mathrm{NC}$ profile. In another case, ATMOS/ATLAS-3 SS01 (not shown), both PC and $\mathrm{ZC}$ profiles are $\sim 20 \%$ lower than the NC profile near $20 \mathrm{~km}$. These corrections are in the same sense, but of greater magnitude, than previous estimates of approximately $5-10 \%$ [Kerr et al., 1977; Russell et al., 1988], possibly because of differences in time (or SZA) resolution of the model calculations. While the ZC corrections are typically $0-50 \%$ of the $\mathrm{PC}$ adjustments, sometimes the $\mathrm{ZC}$ correction exceeds the $\mathrm{PC}$ adjustment. Above $40 \mathrm{~km}$, we find that $\mathrm{PC}$ profiles for $\mathrm{NO}_{2}$ are typically lower that $\mathrm{NC}$ profiles (as has been illustrated for the lower stratosphere), but the corrections (less than 10\%) are smaller than in the lower stratosphere. In addition, the corrections in the upper stratosphere are typically smaller than the measurement uncertainties at those altitudes and smaller than one previous estimate [Russell et al., 1988].

\section{NO profiles}

The sum of diurnally corrected $\mathrm{NO}$ and $\mathrm{NO}_{2}$ amounts does not necessarily equal the sum of uncorrected $\mathrm{NO}_{\mathrm{x}}$. This difference is due to uncertainties in the inversion process; it is not a property of the model chemistry, which does conserve $\mathrm{NO}_{\mathrm{x}}$ to better than $3 \%$ at $20 \mathrm{~km}$, for example. Typically, the difference between corrected and uncorrected $\mathrm{NO}_{\mathrm{x}}$ profiles varies by $\pm 15 \%$, roughly the measurement precision at $20 \mathrm{~km}$. This difference diminishes with increasing altitude, approaching zero at $30 \mathrm{~km}$ (above which altitude both $\mathrm{NO}$ and $\mathrm{NO}_{2}$ corrections become insignificant.) Below $20 \mathrm{~km}$, where both NO and $\mathrm{NO}_{2}$ corrections increase in magnitude, the difference

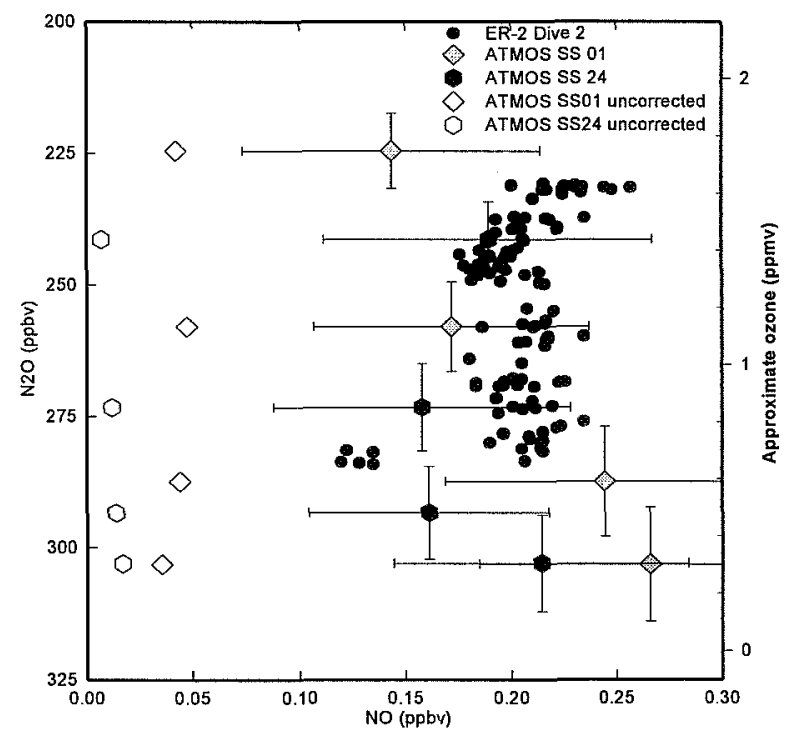

Figure 3. Comparison of ATMOS/ATLAS-3 NO measurements with results of the NO-NO $\mathrm{NOAA}$ instrument on board the ER-2 during ASHOE/MAESA (dive 2) on November 4, 1994. Both SS01 and SS24 have been diurnally corrected as described in the text and scaled to the ER-2 solar zenith angle of $72^{\circ}$. Error bars on ATMOS NO and $\mathrm{N}_{2} \mathrm{O}$ reflect estimates of the 1- $\sigma$ measurement precision between corrected and uncorrected $\mathrm{NO}_{\mathrm{x}}$ can be as large as $40 \%$ in the few occultations we have examined. However, the absolute magnitude of the correction does not exceed 0.2 ppbv at any altitude, a small fraction of the $\mathrm{NO}_{\mathrm{y}}\left(=\mathrm{NO}+\mathrm{NO}_{2}\right.$ $+\mathrm{HNO}_{3}+\mathrm{ClONO}_{2}+\mathrm{HNO}_{4}+2 * \mathrm{~N}_{2} \mathrm{O}_{5}$ ) budget.

\section{ER-2 comparisons}

Some of the sunset occultation measurements obtained during the early days of the ATMOS ATLAS-3 mission occurred within 1 day and roughly coincident in space with in situ measurements of NO on 4 November 1994, during the ASHOE/MAESA ER-2 campaign. SS01 and SS24, while respectively 1 day before and 1 day after the ER-2 observations on 4 November, yielded correlations of $\mathrm{O}_{3}$ and $\mathrm{N}_{2} \mathrm{O}$ very similar to the in situ observations [Chang et al., this issue]. Chang et al. demonstrate good agreement between ATMOS and in-situ measurements of correlations between $\mathrm{NO}_{y}$ and $\mathrm{N}_{2} \mathrm{O}$. Figure 3 illustrates a comparison of the space-borne ATMOS and in situ measurements of NO [Fahey et al., 1989] as a function of the long-lived tracer $\mathrm{N}_{2} \mathrm{O}$ [Lowenstein et al., 1989] to account for variations in the precursors $\left(\mathrm{O}_{3}\right.$ and $\mathrm{NO}_{y}$ ) that regulate levels of $\mathrm{NO}_{\mathrm{x}}$ in the sampled air masses. The NC ATMOS profile values (open symbols) are shown for reference. We used the photochemical model to adjust the PC profiles (not shown) for SS01 and SS24 to account for the change in NO from $\mathrm{SZA}=90^{\circ}$ to the ER-2 solar conditions of mid-day at $\mathrm{SZA}=72^{\circ}$. The scaling from $90^{\circ}$ to mid-day is approximately a factor of two at all altitudes reported in figure 3 . The good agreement between calculated and observed variation in NO with changing solar illumination illustrated in Figure 1 provides confidence in the fidelity of the scaling used to estimate the mid-day values of NO from the ATMOS sunset measurements. The agreement between NO measured by ATMOS and the NOAA NO-NO ure 3 is well within the ATMOS $1-\sigma$ precision error bars. The systematic error in these measurements is estimated to be $5 \%$ for NO. The accuracy of the ER-2 NO measurements is $15 \%$ with precision of $0.02 \mathrm{ppbv}$.

The ATMOS temperatures were approximately 5-10 K lower than the ER-2 temperatures at all altitudes. Adjustment for this temperature difference would lower the ATMOS NO values by approximately $10-30 \%$ due to the temperature dependence of the rate of $\mathrm{NO}+\mathrm{O}_{3}$, which increases with increasing temperature. This adjustment would degrade the agreement somewhat. At the lowest part of dive 2 (pressures greater than $100 \mathrm{mb}$ ), the ER-2 encountered higher temperatures and lower values of NO. These lower NO values cluster around an average value of $0.1 \mathrm{ppbv}$ at $280 \mathrm{ppbv} \mathrm{N}_{2} \mathrm{O}$. In situ measurements with temperatures greater than $223 \mathrm{~K}$ are unrepresentative of the comparable air mass and, therefore, are not shown in Figure 3. Five points of the low-NO cluster remain in Figure 3. 12-Day back trajectory calculations initialized at 420 and $465 \mathrm{~K}$ indicate that air at the locations of SS01 and SS24, and at a number of ER-2 measurement locations, has similar history, having been drawn into midlatitudes from the subtropics. However, at $465 \mathrm{~K}$, air from some of the ER-2 measurement locations compared here, appears to have come from mid-latitudes around the developing vortex. A more complete comparison of spaceborne and in situ measurements of NO requires accounting for the latitude and temperature histories of the air parcels, an analysis beyond the scope of this paper. 


\section{Conclusions}

Adjustments to NO profiles retrieved from solar-occultation observations for the effects of rapid abundance variation near the terminator become significant below $\sim 25-28 \mathrm{~km}$, typically $100-$ $200 \%$, but sometimes as large as $500 \%$. The adjustment to $\mathrm{NO}_{2}$ solar-occultation profiles is smaller than for NO; the adjustment becomes significant below $\sim 30 \mathrm{~km}$ and is $\sim 20 \%$ at $20 \mathrm{~km}$ (well in excess of the $5-10 \%$ estimate currently accepted.) These results use model calculations adopting profiles for $\mathrm{O}_{3}$ and temperature measured simultaneously with the $\mathrm{NO}$ and $\mathrm{NO}_{2}$ observations. $\mathrm{Be}-$ cause the adjustment for diurnal variation is a strong function of atmospheric ozone amount and temperature (and the resulting species concentrations as a function of solar zenith angle) for a wide range of $\mathrm{NO}_{x}$ levels, adjustments to the $\mathrm{NO}$ and $\mathrm{NO}_{2}$ retrievals using model calculations initialized with zonal mean or climatological $\mathrm{O}_{3}$ and temperature profiles may be quite different (and erroneous). $\mathrm{NO}_{\mathrm{x}}$ profiles based on the sum of corrected retrieved $\mathrm{NO}$ and $\mathrm{NO}_{2}$ are typically different from $\mathrm{NO}_{\mathrm{x}}$ based on uncorrected retrievals, $\sim 15 \%$ at $20 \mathrm{~km}$, but sometimes as high as $40 \%$ at $16 \mathrm{~km}$. When adjusted for differences in solar illumination at times of measurement, corrected ATMOS/ATLAS-3 values for NO between 17 and $20 \mathrm{~km}$ are in good agreement with in situ, ER-2 measurements. This agreement suggests that the remotely sensed measurement of NO is reliable within its stated error bars, even at a mixing-ratio level two orders of magnitude and $25 \mathrm{~km}$ below the peak in the stratospheric profile.

Acknowledgments. This work was supported in part by the NASA Atmospheric Chemistry Modeling and Analysis Program, NASA/MSFC Earth Sciences and Applications Division contract NAS8-38609, and JPL-959894. Work at the Jet Propulsion Laboratory, California Institute of Technology was done under contract with the National Aeronautics and Space Administration. Partial support was received from NASA grant NAGW-1538 to the California Institute of Technology.

\section{References}

Allen, M., and M. L. Delitsky, Stratospheric NO, $\mathrm{NO}_{2}$, and $\mathrm{N}_{2} \mathrm{O}_{5}$ : A comparison of model results with Spacelab 3 Atmospheric Trace Molecule Spectroscopy measurements, J. Geophys. Res., 95, 14,077-14,082, 1990.

Allen, M., and M. L. Delitsky, A test of odd-oxygen photochemistry using Spacelab 3 Atmospheric Trace Molecule Spectroscopy observations, J. Geophys. Res., 96, 12,883-12,891, 1991.

Boughner, R., J. C. Larsen, and M. Natarajan, The influence of NO and $\mathrm{ClO}$ variations at twilight on the interpretation of solar occultation measurements, Geophys. Res. Lett., 7, 231-234, 1980.

Chang et al., A comparison of measurements from ATMOS and the ER-2: Tracers of atmospheric transport, Geophys. Res. Lett., this issue.

Chu, W. P., and M. P. McCormick, SAGE observations of stratospheric nitrogen dioxide, J. Geophys. Res., 91, 5465-5476, 1986.

Fahey, D. W. et al., In situ measurements of total reactive nitrogen, total water, and aerosol in a polar stratospheric cloud in the Antarctic, J. Geophys. Res., 94,11,299-11,315, 1989.
Kawa, S. R. et al., Interpretation of aircraft measurements of NO, $\mathrm{ClO}$, and $\mathrm{O}_{3}$ in the lower stratosphere, J. Geophys. Res., 95, $18,597-18,609,1990$.

Kerr, J. B., W. F. J. Evans, and J. C. McConnell, The effects of $\mathrm{NO}_{2}$ changes at twilight on tangent ray $\mathrm{NO}_{2}$ measurements, Geophys. Res. Lett., 4, 577-579, 1977.

Kondo, Y., N. Toriyama, W. A. Matthews, and P. Aimedieu, Calibration of the balloon-borne NO instrument, J. Geomag. Geoelectr., 41, 507-523, 1989.

Kondo, Y. et al., Diurnal variation of nitric oxide in the upper stratosphere, J. Geophys. Res., 95, 22,513-22,522, 1990.

Loewenstein, M., J. R. Podolske, K. R. Chan, and S. E. Strahan, Nitrous oxide as a dynamical tracer in the 1987 Airborne Antarctic Ozone Experiment, J. Geophys. Res., 94, 11,589-11,598, 1989.

Manney, G. L., R. Swinbank, and A. O'Neill, Stratospheric Meteorological conditions for the 3-12 Nov. 1994 ATMOS/ATLAS3 measurements, Geophys. Res. Lett. ,this issue.

Michelangeli, D. V., M. Allen, and Y. L. Yung, El Chichon volcanic aerosols: Impact of radiative, thermal and chemical perturbations, J. Geophys. Res., 94, 18,429-18,443, 1989.

Murcray, D. G., A. Goldman, G. R. Cook, D. K. Rolens, and L. R. Megill, On the interpretation of infrared solar spectra for altitude distribution of atmospheric trace constituents, FAA-EE-7830, Department of Transportation Federal Aviation Administration Office of Environment and Energy, University of Denver, Denver, Colorado, 1978.

Norton, R. H. , and C. P. Rinsland, ATMOS data processing and science analysis methods, Appl. Opt., 30, 389-400, 1991.

Rinsland, C. P., R. E. Boughner, J. C. Larsen, G. M. Stokes, and J. W. Brault, Diurnal variations of atmospheric nitric oxide: Ground-based infrared spectroscopic measurements and their interpretation with time-dependent photochemical model calculations, J. Geophys. Res., 89, 9613-9622, 1984.

Roscoe, H. K. and J. A. Pyle, Measurements of solar occultation: the error in a naive retrieval if the constituent's concentration changes, J. Atmos. Chem., 5, 323-341, 1987.

Russell III, J. M. et al., Measurements of odd nitrogen compounds in the stratosphere by the ATMOS experiment on Spacelab 3, J. Geophys. Res., 93, 1718-1736, 1988.

Salawitch, R. J. et al., The diurnal variation of hydrogen, nitrogen, and chlorine radicals: Implications for the heterogeneous production of $\mathrm{HNO}_{2}$, Geophys. Res. Lett., 21, 2551-2554, 1994.

Webster, C. R., R. D. May, R. Toumi, and J. A. Pyle, Active nitrogen partitioning and the nighttime formation of $\mathrm{N}_{2} \mathrm{O}_{5}$ in the stratosphere: Simultaneous in situ measurements of $\mathrm{NO}, \mathrm{NO}_{2}$, $\mathrm{HNO}_{3}, \mathrm{O}_{3}$, and $\mathrm{N}_{2} \mathrm{O}$ using the BLISS diode laser spectrometer, J. Geophys. Res., 95, 13,851-13, 866, 1990.

M. J. Newchurch, Earth System Science Laboratory, U. Alabama in Huntsville, Huntsville, AL, 35899, USA.(mike@ozone.atmos.uah.edu)

(Received October, 17, 1995; revised March, 8, 1996; accepted March 22, 1996.) 


\section{Heavy ozone enrichments from ATMOS infrared solar spectra}

Abstract. Vertical enrichment profiles of stratospheric $16 \mathrm{O}^{16} \mathrm{O}^{18} \mathrm{O}$ and ${ }^{16} \mathrm{O}^{18} \mathrm{O}^{16} \mathrm{O}$ (hereafter referred to as ${ }^{668} \mathrm{O}_{3}$ and ${ }^{686} \mathrm{O}_{3}$ respectively) have been derived from space-based solar occultation spectra recorded at $0.01 \mathrm{~cm}^{-1}$ resolution by the ATMOS (Atmospheric Trace MOlecule Spectroscopy) Fouriertransform infrared (FTIR) spectrometer. The observations, made during the Spacelab 3 and ATLAS-1, -2 , and -3 shuttle missions, cover polar, mid-latitude and tropical regions between 26 to $2.6 \mathrm{mb}$ inclusive $(\approx 25$ to $41 \mathrm{~km})$. Average enrichments, weighted by molecular $48 \mathrm{O}_{3}$ density, of $(15 \pm 6) \%$ were found for $668 \mathrm{O}_{3}$ and $(10 \pm 7) \%$ for $686 \mathrm{O}_{3}$. Defining the mixing ratio of $50 \mathrm{O}_{3}$ as the sum of those for ${ }^{668} \mathrm{O}_{3}$ and ${ }^{686} \mathrm{O}_{3}$, an enrichment of $(13 \pm 5) \%$ was found for ${ }^{50} \mathrm{O}_{3}$ ( $1 \sigma$ standard deviation). No latitudinal or vertical gradients were found outside this standard deviation. From a series of ground-based measurements by the ATMOS instrument at Table Mountain, California $\left(34.4^{\circ} \mathrm{N}\right)$, an average total column ${ }^{668} \mathrm{O}_{3}$ enrichment of $(17 \pm 4) \%(1 \sigma$ standard deviation) was determined, with no significant seasonal variation discernable. Possible biases in the spectral intensities that affect the determination of absolute enrichments are discussed.

\section{Introduction}

Stratospheric enrichment of ${ }^{50} \mathrm{O}_{3}$ was first reported by Mauersberger [1981], who found enrichments ranging from $0 \%$ to $40 \%$ using a balloon-borne mass spectrometer. (For the purposes of this paper, \% enrichment $=\left[R_{\mathrm{obs}} / R_{\mathrm{std}}-1\right] \times 100$, where $R_{\mathrm{obs}}$ is the observed abundance ratio of the heavy isotopomer to the regular isotopomer, and $R_{\text {std }}$ is the standard ratio. For $50 \mathrm{O}_{3}$ enrichment, $R_{\text {std }}=6.01 \times 10^{-3}$, about three times the natural abundance ratio of $18 \mathrm{O}$ to $16 \mathrm{O}$ ignoring a very small abundance of ${ }^{16} \mathrm{O}^{17} \mathrm{O}^{17} \mathrm{O}$. See IUPAC [1983].) Further stratospheric enrichment of $50 \mathrm{O}_{3}$ has been reported based on mass spectrometry [Mauersberger, 1987], far-infrared emission spectroscopy [Abbas et al., 1987; Carli and Park, 1988], and cryogenic grab-sampling followed by mass spectrometry [Schueler et al., 1990]. These measurements have shown varied consistency with each other. Figure 1 summarizes previous measurements of $50 \mathrm{O}_{3}$ enrichment profiles and averaged results of the analyses presented here.

'California Institute of Technology, Pasadena, California 91125

2 Jet Propulsion Laboratory, California Institute of Technology, Pasadena, California 91109

${ }^{3}$ Atmospheric Sciences Division, NASA Langley Research Center, Hampton, Virginia 23681

${ }^{4}$ SAIC - NASA Langley Research Center, Hampton, Virginia 23681

${ }^{5}$ Department of Physics, University of Denver, Denver, Colorado 80210

Copyright 1996 by the American Geophysical Union.

Paper number 96GL01695

0094-8534/96/96GL-01695\$05.00
Mid-infrared solar absorption Fourier-transform spectrometry has been used to determine column enrichments of $668 \mathrm{O}_{3}$ and $686 \mathrm{O}_{3}$. From Kitt Peak, Arizona $\left(31.9^{\circ} \mathrm{N}\right)$, Rinsland et al. [1985] determined a column enrichment of $(11 \pm 11) \%$ for ${ }^{668} \mathrm{O}_{3}$ and $(5 \pm 7) \%$ for $686 \mathrm{O}_{3}$. Goldman et al. [1989], from two balloon-based observations, found column enrichments above $37 \mathrm{~km}$ of $(20 \pm 14) \%$ and $(40 \pm 18) \%$ for ${ }^{668} \mathrm{O}_{3}$, and $(16 \pm 8) \%$ and $(25 \pm 12) \%$ for ${ }^{686} \mathrm{O}_{3}$. From April 1994 to August 1995, Meier and Notholt [1996] took measurements from a high northern latitude of $79^{\circ} \mathrm{N}$; they reported average column enrichments of

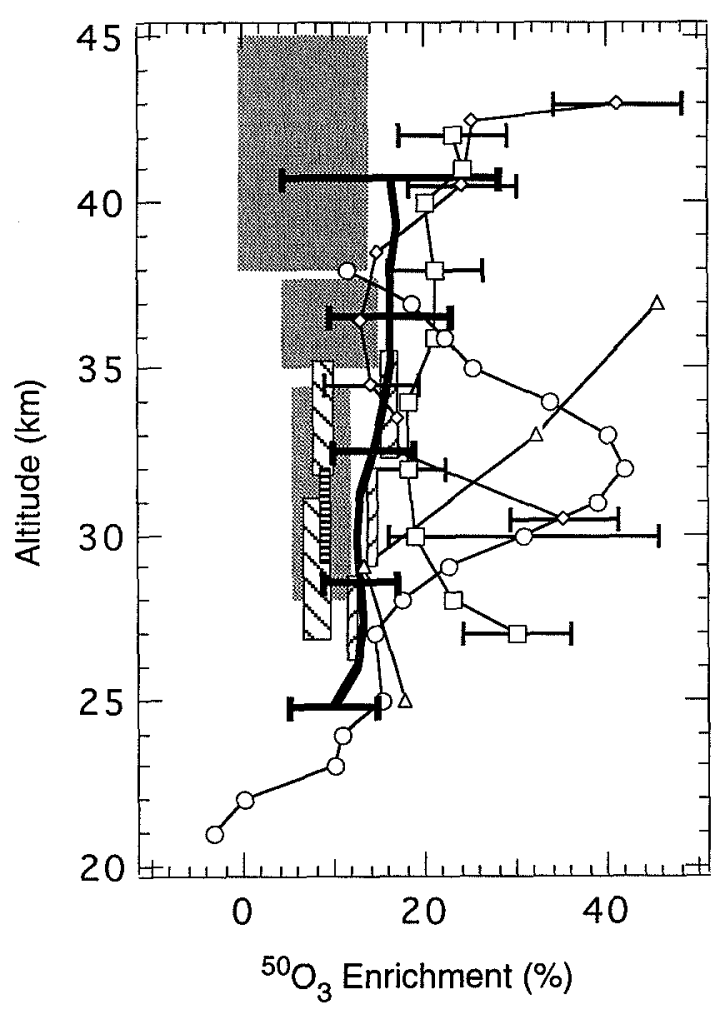

Figure 1. Previously reported and globally averaged ATMOS space-borne measurements of ${ }^{50} \mathrm{O}_{3}$ enrichment profiles. ATMOS altitudes are approximate and ATMOS ${ }^{50} \mathrm{O}_{3}$ enrichments are assumed to be the sum of two-thirds the ${ }^{668} \mathrm{O}_{3}$ enrichment and onethird the ${ }^{686} \mathrm{O}_{3}$ enrichment (c. f. Figure 2). The error bars on the ATMOS measurements are the $1 \sigma$ standard deviations, and do not include systematic error. For clarity, error bars for many of the data points have been omitted, and altitudes for Mauersberger [1987], flight a, have been shifted upwards by $0.5 \mathrm{~km}$. Data from Carli and Park [1988] are the range of their measurements. Mauersberger [1981]: O; Mauersberger [1987], flight a: $\diamond$ flight b: $\square ;$ Abbas et al. [1987]: $\triangle$; Carli and Park [1988]: : Scheueler et al. [1990] flight I: $\circlearrowright \backslash$ flight II: $\square$ flight III: $\equiv ;$ ATMOS Spacelab 3 and ATLAS- $1,-2$ and -3 average: $=$ 
$(15.4 \pm 0.9) \%$ for ${ }^{668} \mathrm{O}_{3}$ and $(11.2 \pm 1.4) \%$ for $686 \mathrm{O}_{3}$ from solar absorption spectra, and $(9.0 \pm 3.3) \%$ for ${ }^{668} \mathrm{O}_{3}$ and $(8.5 \pm 2.5) \%$ for $686 \mathrm{O}_{3}$ from lunar absorption spectra recorded during the polar night. Using mass spectrometry, enrichment of $50 \mathrm{O}_{3}$ from about 8 to $10 \%$ in tropospheric urban air was reported by Krankowsky et al. [1995]; no apparent variation in the enrichment with tropospheric $\mathrm{O}_{3}$ mixing ratio was found.

Heavy ozone enrichment has also been observed in laboratory measurements. Anderson et al. [1989] found ozone created using an electric discharge (which can produce excited states of $\mathrm{O}$ and $\mathrm{O}_{2}$ ) was enriched primarily in the asymmetric isotopomers, such as $668 \mathrm{O}_{3}$. Morton et al. [1990] found that enrichment can occur via the reaction of ground-state atomic and molecular oxygen, $\mathrm{O}\left({ }^{3} \mathrm{P}\right)+\mathrm{O}_{2}\left({ }^{3} \sum_{\mathrm{g}}\right)+\mathrm{M} \rightarrow \mathrm{O}_{3}+\mathrm{M}$, the socalled Chapman formation reaction; under these conditions, Mauersberger et al. [1993] found enrichment only in the asymmetric isotopomers. Miller et al. [1994] proposed that the reaction of vibrationally hot $\mathrm{O}_{2}(\mathrm{v} \geq 26)$ with another $\mathrm{O}_{2}$ molecule can lead to heavy ozone enrichment via preferential potential energy curve-crossing of heteronuclear $\mathrm{O}_{2}$ from the $\mathrm{O}_{2}\left({ }^{1} \Delta_{\mathrm{g}}\right)$ to the $\mathrm{O}_{2}\left({ }^{3} \sum_{\mathrm{g}}\right)$ state (see Valentini [1987]). However, appreciable production of such vibrationally hot $\mathrm{O}_{2}$ was predicted to occur only above about $35 \mathrm{~km}$, with the enrichment produced less than $5 \%$ at $40 \mathrm{~km}$.

While Miller et al. [1994] may have provided an explanation for some of the enrichment in the mid-stratosphere, the bulk of the enrichment lacks a generally accepted theoretical explanation. Indeed, detailed statistical mechanical analyses by Kaye and Strobel [1983] and Kaye [1986] predicted a slight depletion of ${ }^{50} \mathrm{O}_{3}$ under stratospheric conditions. This discrepancy between observation and theory indicates that current understanding of ozone formation is incomplete (see, for example, Anderson et al. [1992]). However, a promising advance in finding an enrichment mechanism is the recent discovery of several electronically excited states of ozone near the dissociation threshold [Anderson and Mauersberger, 1995]. It is possible that the formation of ozone via the Chapman mechanism goes through one or more of these states whose quantum properties (such as lifetime) are dependent on the isotopic composition of the reactants.

From Figure 1, no vertical gradient consistently appears within the assembly of previous measurements, and data are lacking on latitudinal gradients in the stratosphere. In this study, we take advantage of the high vertical and latitudinal range of the ATMOS data set from the Spacelab3 and ATLAS series missions to constrain any vertical or latitudinal variability. We also evaluate ${ }^{668} \mathrm{O}_{3}$ column enrichments from ground-based spectra taken from Table Mountain Facility (TMF), Wrightwood, California, $\left(34.4^{\circ} \mathrm{N}, 117.7^{\circ} \mathrm{W}, 2.2 \mathrm{~km}\right.$ altitude) to examine seasonal variations in the column enrichment from a mid-latitude site.

\section{Data Acquisition}

Information about the ATMOS instrument and its use on the shuttle can be found in Gunson et al. [this issue]. The spectral filters used for analyses described here, filters 1,9 and 12, had ranges of $650-1100 \mathrm{~cm}^{-1}, 650-2450 \mathrm{~cm}^{-1}$, and $625-1400 \mathrm{~cm}^{-1}$ respectively. The number of vertical profiles used for this study were 4 from Spacelab 3, 39 from ATLAS-1, 29 from ATLAS-2 and 87 from ATLAS-3. Using the ATMOS instrument from Table Mountain Facility (TMF), ground-based total column measurements were made on 48 separate days from
Table 1. Spectral intervals used for heavy ozone profile analyses from space

\begin{tabular}{|c|c|c|c|c|c|}
\hline $\begin{array}{l}\text { Window } \\
\text { center } \\
\text { frequency } \\
\left(\mathrm{cm}^{-1}\right)\end{array}$ & $\begin{array}{l}\text { Window } \\
\text { width } \\
\left(\mathrm{cm}^{-1}\right)\end{array}$ & $\begin{array}{l}\text { Line } \\
\text { centers } \\
\left(\mathrm{cm}^{-1}\right)\end{array}$ & $\begin{array}{l}\text { Line intensity } \\
\text { at } 296 \mathrm{~K} \\
\left(\times 10^{22} \mathrm{~cm}\right. \\
\text { molecule-1) }\end{array}$ & $\begin{array}{l}\text { Ground } \\
\text { state } \\
\text { energy } \\
\left(\mathrm{cm}^{-1}\right)\end{array}$ & $\begin{array}{l}\text { Temperature } \\
\text { sensitivity of } \\
\text { intensity } \\
(\% / \mathrm{K} \text { at } \\
220 \mathrm{~K})\end{array}$ \\
\hline $\begin{array}{l}{ }^{6} 68 \mathbf{O}_{3} \\
1090.445\end{array}$ & 0.55 & unresolved & $2.14(a)$ & 203(b) & -0.1 \\
\hline $\begin{array}{l}\mathbf{6 8 6} \mathbf{O}_{3} \\
975.27\end{array}$ & 0.12 & $\begin{array}{l}975.2503 \\
975.2838\end{array}$ & $\begin{array}{l}0.169 \\
0.230\end{array}$ & $\begin{array}{l}546 \\
495\end{array}$ & $\begin{array}{l}1.0 \\
0.7\end{array}$ \\
\hline 981.715 & 0.14 & $\begin{array}{l}981.6756 \\
981.7107\end{array}$ & $\begin{array}{l}3.54 \\
4.46\end{array}$ & $\begin{array}{l}363 \\
328\end{array}$ & $\begin{array}{l}0.4 \\
0.3\end{array}$ \\
\hline 985.09 & 0.44 & $\begin{array}{l}984.9062 \\
984.9062 \\
985.0321 \\
985.1031 \\
985.1578 \\
985.2171\end{array}$ & $\begin{array}{l}4.32 \\
5.48 \\
2.20 \\
4.78 \\
3.32 \\
5.10\end{array}$ & $\begin{array}{l}298 \\
264 \\
395 \\
279 \\
334 \\
268\end{array}$ & $\begin{array}{l}0.2 \\
0.1 \\
0.5 \\
0.1 \\
0.3 \\
0.1\end{array}$ \\
\hline 990.422 & 0.14 & $\begin{array}{l}990.3889 \\
990.3895 \\
990.3918\end{array}$ & $\begin{array}{l}1.55 \\
1.74 \\
1.74\end{array}$ & $\begin{array}{l}493 \\
567 \\
567\end{array}$ & $\begin{array}{l}1.3 \\
1.3 \\
1.1\end{array}$ \\
\hline
\end{tabular}

Notes: (a) Sum of intensities between 1090.1 and $1090.6 \mathrm{~cm}^{-1}$ (b) Average weighted by the intensity of the individual lines.

October, 1985 through July, 1990, usually at solar zenith angles corresponding to about 1,5 and 10 airmasses. Further discussion of ATMOS measurements from TMF may be found in Gunson and Irion [1991].

\section{Data Analysis}

ATMOS analysis techniques for space-based observations have been described by Norton and Rinsland [1991]. For ${ }^{48} \mathrm{O}_{3}$, large spectral windows (from 14.5 to $16 \mathrm{~cm}^{-1}$ wide) covering regions of the $v_{1}, v_{2}$, and $v_{3}$ bands were used for analyses, while additionally for the broadband filter 9 , smaller windows $\left(\leq 1 \mathrm{~cm}^{-1}\right.$ wide) containing lines from the $v_{1}+v_{2}, v_{1}+v_{3}$, and $2 \mathrm{v}_{2}$ bands were used. (A fuller discussion of ATMOS ${ }^{48} \mathrm{O}_{3}$ retrievals and comparison with other instruments may be found in M. C. Abrams et al., "An evaluation of stratospheric ozone observed by the Atmospheric Trace Molecule Spectroscopy (ATMOS) Experiment During April 1992," in preparation for $J$. Geophys. Res., 1996.) For $668 \mathrm{O}_{3}$, the narrow, unresolved $v_{1}$ band Q-branch centered at $1090.35 \mathrm{~cm}^{-1}$ was analyzed, as were several lines of the $v_{3}$ band P-branch for ${ }^{686} \mathrm{O}_{3}$ (see Table 1 ). Spectral parameters for all lines were from the ATMOS linelist [Brown et al., 1996], which currently incorporates the ozone parameters given by Flaud et al. [1986; 1990] and Camy-Peyret et al. [1986]. Error sources for stratospheric measurements are discussed by Abrams et al. [this issue]. However, as discussed by Meier and Notholt [1996], an uncertainty exists in the spectral line intensities of $668 \mathrm{O}_{3}$ due to what may have been an incorrect assumption for the isotopic makeup of the reference gas used in the experiments of Flaud et al. [1986] and CamyPeyret et al. [1986]. Furthermore, any errors in the line intensities of ${ }^{686} \mathrm{O}_{3}$ (which were derived from theory) will impact those of ${ }^{668} \mathrm{O}_{3}$. As will be seen, these uncertainties can affect the interpretation of our results. The average $1 \sigma$ random errors for an enrichment from a single spectrum, mostly from signalto-noise error and interfering lines for spectral windows featuring the heavy isotopomers, are $20 \%$ for ${ }^{666} \mathrm{O}_{3}$ and $18 \%$ for $668 \mathrm{O}_{3}$. 
Table 2. Spectral intervals used for column density analyses from Table Mountain

\begin{tabular}{|c|c|c|c|c|}
\hline $\begin{array}{l}\text { Line Center (a) } \\
\left(\mathrm{cm}^{-1}\right)\end{array}$ & $\begin{array}{l}\text { Line Inten- } \\
\text { sity }\left(\times 10^{22}\right. \\
\mathrm{cm} \text { molec.-1) }\end{array}$ & $\begin{array}{c}\text { Ground State } \\
\text { Energy } \\
\left(\mathrm{cm}^{-1}\right)\end{array}$ & $\begin{array}{c}\text { Temperature } \\
\text { Sensitivity of } \\
\text { Intensity } \\
(\% / \mathrm{K} \text { at } 220 \mathrm{~K})\end{array}$ & $\begin{array}{c}\text { Airmass } \\
\text { Range }\end{array}$ \\
\hline \multicolumn{5}{|l|}{${ }^{48} \mathrm{O}_{3}$} \\
\hline 1095.1008 & 5.19 & 310.3 & 0.2 & $<1.5$ \\
\hline 1114.8233 & 1.03 & 77.1 & -0.5 & $3-10$ \\
\hline 1123.4234 & 6.85 & 120.3 & -0.4 & $<3$ \\
\hline 1126.2511 & 2.49 & 42.9 & -0.5 & $<5$ \\
\hline 1140.9448 & 1.02 & 190.2 & -0.1 & $3-10$ \\
\hline 1163.4222 & 2.57 & 253.9 & 0.04 & $<5$ \\
\hline 1176.1047 & 1.41 & 353.3 & 0.3 & $3-10$ \\
\hline${ }^{668} \mathrm{O}_{3}$ & $2.14(c)$ & $203(d)$ & -0.1 (d) & all \\
\hline
\end{tabular}

Notes: (a) Interval width for all ${ }^{48} \mathrm{O}_{3}$ windows was $0.16 \mathrm{~cm}^{-1}$. The window for ${ }^{668} \mathrm{O}_{3}$ was $1090.35 \mathrm{~cm}^{-1}$ with a width of $0.5 \mathrm{~cm}^{-1}$. (b) Several unresolved lines between 1090.1 and $1090.6 \mathrm{~cm}^{-1}$ (c) Sum of intensities between 1090.1 and $1090.6 \mathrm{~cm}^{-1}$

(d) Average weighted by the intensities of the individual lines.

For the TMF ground-based retrievals of $48 \mathrm{O}_{3}$ and $668 \mathrm{O}_{3}$, an assumed vertical ozone mixing ratio profile was scaled by a single multiplicative factor until a best fit was obtained between observed and calculated spectra. Assumed vertical ozone profiles were created using monthly-averaged profiles from the JPL Lidar on TMF [McDermid, 1993]. Vertical temperature and pressure profiles were adapted from daily National Meteorological Center data and merged with the U.S. Standard Atmosphere [1976] for higher altitudes. These profiles were then adjusted so that the atmosphere was in hydrostatic equilibrium. To achieve better fits and improved consistency in the column retrievals among the different airmasses sampled within a day, the height registration of the assumed ozone profiles was shifted vertically up to $\pm 3 \mathrm{~km}$. Measurement of column ${ }^{48} \mathrm{O}_{3}$ by the ATMOS instrument at TMF was previously reported by Gunson and Irion [1991], however, as some of the ${ }^{48} \mathrm{O}_{3}$ lines used in that study may have been saturated at high airmasses, the ${ }^{48} \mathrm{O}_{3}$ columns have been re-analyzed for this study. Table 2 describes the lines used for TMF retrievals. The $686 \mathrm{O}_{3}$ column from TMF was not retrieved because interference by neighboring ${ }^{48} \mathrm{O}_{3}, \mathrm{H}_{2} \mathrm{O}$ and $\mathrm{CO}_{2}$ lines was too large to achieve acceptable fits. Random error for TMF retrievals, mostly from errors in the pressure/temperature profile and the assumed $\mathrm{O}_{3}$ profiles, averaged about $9 \%$ for the ${ }^{668} \mathrm{O}_{3}$ enrichments.

\section{Results}

The solid lines in Figure 2 illustrate average vertical enrichment profiles for ${ }^{668} \mathrm{O}_{3}$ and ${ }^{686} \mathrm{O}_{3}$ measured by ATMOS over the four shuttle missions, while the dashed lines indicate $1 \sigma$ standard deviations. (The standard error for the average is nowhere more than $2 \%$.) The individual points on Figure 2 show enrichments averaged for latitude and mission and weighted by inverse square random error. For these latitudinal averages, the standard errors average $8 \%$ for ${ }^{668} \mathrm{O}_{3}$ and $10 \%$ for $686 \mathrm{O}_{3}$. No significant variation with latitude or altitude could be determined. Figure 1 illustrates previously published and the average ATMOS $50 \mathrm{O}_{3}$ enrichment profiles determined by averaging results across constant pressure surfaces and assigning an approximate altitude. Weighted by the $48 \mathrm{O}_{3}$ density, the globally averaged ${ }^{50} \mathrm{O}_{3}$ enrichment between 2.6 and $26 \mathrm{mb}$ inclusive is
$(13 \pm 5) \%$, while that for ${ }^{668} \mathrm{O}_{3}$ and $686 \mathrm{O}_{3}$ are $(15 \pm 6) \%$ and $(10 \pm 7) \%$, respectively ( $1 \sigma$ standard deviation).

Figure 3 shows the ${ }^{668} \mathrm{O}_{3}$ enrichment measured above TMF. The average random error for the data is $9 \%$. No seasonal variation in the enrichment can be discerned. Ignoring systematic error, the average column enrichment is $(17 \pm 4) \%$ ( $1 \sigma$ standard deviation), in good agreement with the $668 \mathrm{O}_{3}$ enrichment derived from the ATMOS stratospheric profiles. This is also in agreement with Meier and Notholt [1996] for their solar spectra, $(15.4 \pm 0.9) \%$, but higher than their average of $(9.0$ $\pm 3.3) \%$ for lunar spectra taken during the polar night.

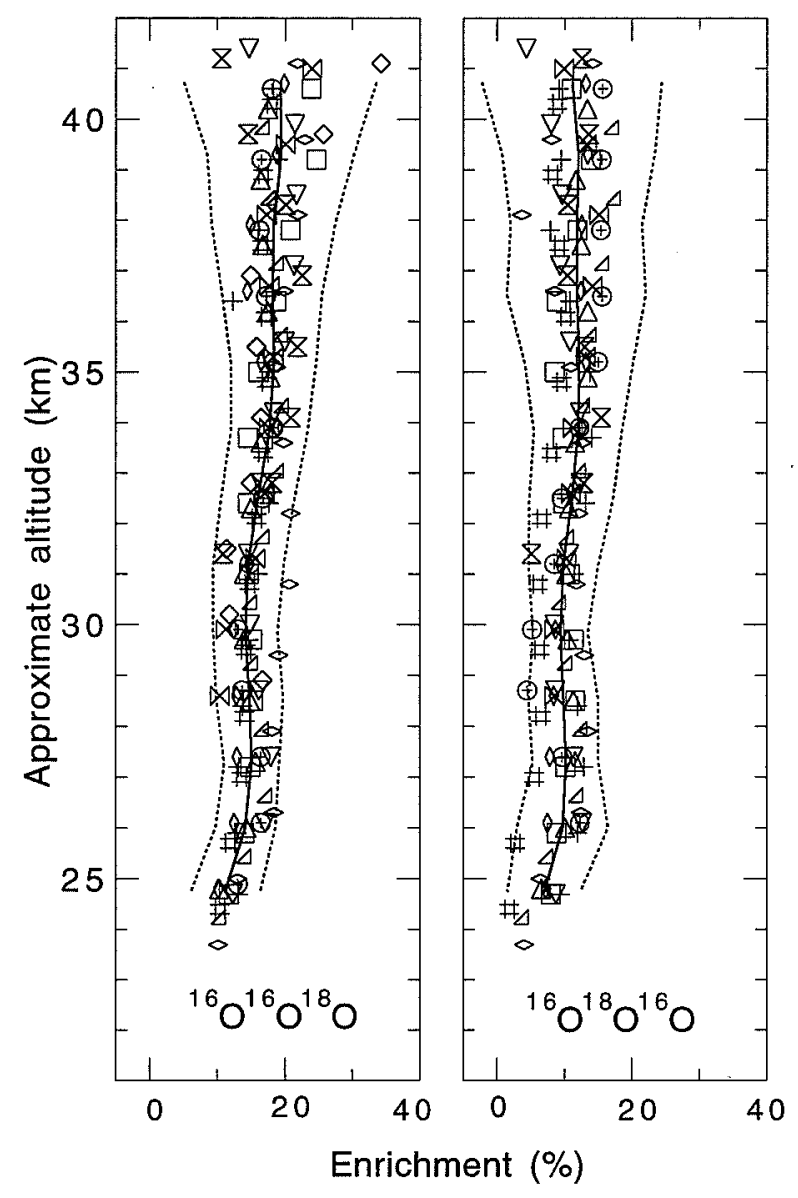

Figure 2. ${ }^{668} \mathrm{O}_{3}$ and ${ }^{686} \mathrm{O}_{3}$ global and latitudinally-averaged enrichments from ATMOS space observations. All averages were done on a fixed pressure scale; the altitude shown is the average altitude at each pressure level in its particular latitude bin. All latitudinal averages were weighted by the inverse random error of the measurement and at least three observations were averaged for each point. The error ranges for the globally averaged profiles are the $1 \sigma$ standard deviations. See text and Abrams et al. [this issue] for discussion of systematic errors. ATLAS-3 (Nov. 1994): $\nabla 80^{\circ} \mathrm{S}-$ $60^{\circ} \mathrm{S}$, outside vortex; $-80^{\circ} \mathrm{S}-60^{\circ} \mathrm{S}$, inside vortex; $+0^{\circ}-10^{\circ} \mathrm{N}$; $\square 10^{\circ} \mathrm{N}-30^{\circ} \mathrm{N} ; \triangle 30^{\circ} \mathrm{N}-60^{\circ} \mathrm{N}$. ATLAS-2 (May 1993): $\oplus 60^{\circ} \mathrm{S}-$ $30^{\circ} \mathrm{S}$; \# $60^{\circ} \mathrm{N}-80^{\circ} \mathrm{N}$, outside vortex; $\triangle 60^{\circ} \mathrm{N}-80^{\circ} \mathrm{N}$, inside vortex. ATLAS-1 (Apr 1992): $60^{\circ} \mathrm{S}-30^{\circ} \mathrm{S} ; \diamond 30^{\circ} \mathrm{S}-10^{\circ} \mathrm{S} ; \bowtie 10^{\circ} \mathrm{S}$ $10^{\circ} \mathrm{N} ; 810^{\circ} \mathrm{N}-30^{\circ} \mathrm{N}$. Spacelab-3 (Apr 1985): $\nabla \approx 50^{\circ} \mathrm{S} ; \Delta \approx 30^{\circ} \mathrm{N}$.

Global average; ……... $1 \sigma$ standard deviation. 


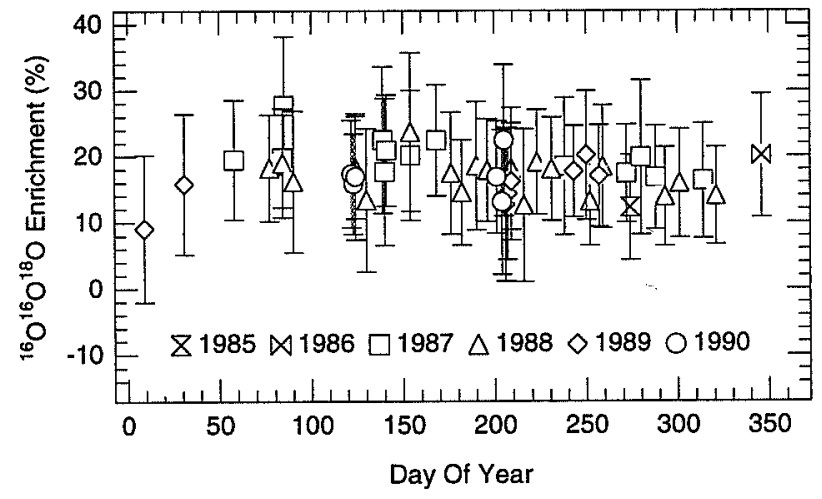

Figure 3. Column ${ }^{668} \mathrm{O}_{3}$ enrichments from Table Mountain. The error bars do not include systematic error.

\section{Conclusions}

We have analyzed ATMOS stratospheric spectra for the enrichments of ${ }^{668} \mathrm{O}_{3}$ and ${ }^{686} \mathrm{O}_{3}$, and ground-based spectra for the column enrichment of ${ }^{668} \mathrm{O}_{3}$. While some previous studies, most notably Mauersberger $[1981 ; 1987]$ and Abbas et al. [1987], have shown large vertical gradients, results here indicate that the vertical and latitudinal variability of the enrichment is generally small. We can find no discernable seasonal variability in the ${ }^{668} \mathrm{O}_{3}$ column enrichment over Table Mountain. These observations suggest the enrichment of heavy ozone in the sunlit stratosphere is relatively constant, and perhaps only weakly regulated by temperature and pressure.

Although previous studies have not ruled out other enrichment processes, laboratory measurements have shown the most significant enrichment in the Chapman reaction, with $\mathrm{O}$ and $\mathrm{O}_{2}$ reactants in the electronic ground state. Noting that the Chapman mechanism has been shown to produce no enrichment for the ${ }^{686} \mathrm{O}_{3}$ isotopomer, our determination of a $686 \mathrm{O}_{3}$ enrichment of $(10 \pm 7) \%$ ( $1 \sigma$ standard deviation) suggests that the $686 \mathrm{O}_{3}$ line intensities may be biased too low by $10 \%$ with respect to the line intensities of ${ }^{48} \mathrm{O}_{3}$. However, it may be possible that other unknown processes are operating to enrich stratospheric $686 \mathrm{O}_{3}$, much as ozone produced in an electric discharge becomes enriched in ${ }^{686} \mathrm{O}_{3}$. Furthermore, since the ${ }^{668} \mathrm{O}_{3}$ line intensities derived by Camy-Peyret et al. [1986] were determined making use of the ${ }^{686} \mathrm{O}_{3}$ line intensities, then if these are incorrect, the ${ }^{668} \mathrm{O}_{3}$ line intensities may also be too low. Laboratory measurement of the $668 \mathrm{O}_{3}$ and $686 \mathrm{O}_{3}$ spectral line intensities are necessary for calibration of the absolute stratospheric enrichments described here and the interpretation thereof. Since Flaud et al. [1986] and Camy-Peyret et al. [1986] reported good model fittings to their laboratory spectra, such laboratory measurements would probably produce a constant, corrective scaling of the line intensities (though not necessarily the same amount for both isotopomers), and would not affect the precision of the enrichments described here.

Acknowledgements. We thank L. R. Brown, W. B. DeMore, J. Closs, C. B. Farmer, J. C. Foster, P. L. Houston, H. Pickett, I. S. McDermid and G. C. Toon for their assistance. The research reported herein was performed at the Jet Propulsion Laboratory, California Institute of Technology under contract to National Aeronautics and Space Administration.

\section{References}

Abbas, M. M. et al., Heavy ozone distribution in the stratosphere from far-infrared observations, J. Geophys. Res., 92, 13231-13239, 1987.

Abrams, M. C. et al., On the assessment and uncertainty of atmospheric trace gas burden measurements with high resolution infrared solar occultation spectra from space, Geophys. Res. Lett., this issue.
Anderson, S. M. et al., Laboratory measurements of ozone isotopomers by tunable diode absorption spectroscopy, Chem. Phys. Lett., 156, 175-180, 1989.

Anderson, S. M. et al., "Heavy ozone anomaly: Evidence for a mysterious mechanism" in Isotope Effects in Gas-Phase Chemistry, Jack Kaye, ed., American Chemical Society, 1992.

Anderson, S. M. and K. Mauersberger, Ozone absorption spectroscopy in search of low-lying electronic states, J. Geophys. Res., 100, 3033 $3048,1995$.

Brown, L. R. et al., The 1995 Atmospheric Trace Molecule Spectroscopy (ATMOS) Linelist, Appl. Opt., in press.

Camy-Peyret, $C$. et al., The hybrid-type bands $v_{1}$ and $v_{3}$ of ${ }^{668} \mathrm{O}_{3}$ : line positions and intensities, J. Mol. Spectrosc., 118, 345-354, 1986.

Carli, B. and J. H. Park, Simultaneous measurement of minor stratospheric constituents with emission far-infrared spectroscopy, $J$. Geophys. Res., 93, 3851-3865, 1988.

Flaud, J.-M. et al., The $v_{1}$ and $v_{3}$ bands of ${ }^{686} \mathrm{O}_{3}$ : line positions and intensities, J. Mol. Spectrosc., 118, 334-344, 1986.

Flaud, J.-M. et al., Atlas of Ozone Spectral Parameters from Microwave to Medium Infrared, Academic Press, San Diego CA, 1990.

Goldman, A. et al., Isotopic abundances of stratospheric ozone from balloon-borne high resolution infrared solar spectra, J. Geophys. Res., 94, 8467-8473, 1989.

Gunson, M. R. and F. W. Irion, "Measurement of atmospheric composition by the ATMOS instrument from Table Mountain Observatory" in Remote Sensing of Atmospheric Chemistry, edited by J. L. McElroy and R. J. McNeal, pg.335-346, Proc. SPIE vol. 1491, 1991.

Gunson, M. R. et al., Measurements of $\mathrm{CH}_{4}, \mathrm{~N}_{2} \mathrm{O}, \mathrm{CO}, \mathrm{H}_{2} \mathrm{O}$, and $\mathrm{O}_{3}$ in the middle atmosphere by the Atmospheric Trace Molecule Spectroscopy experiment on Spacelab 3, J. Geophys. Res., 95, 13867-13882, 1992.

Gunson, M. R. et al., The Atmospheric Trace Molecule Spectroscopy (ATMOS) experiment: Deployment on the ATLAS-3 Space Shuttle missions, Geophys. Res. Lett. [this issue].

I.U.P.A.C., Isotopic composition of the elements, Pure. Appl. Chem., 55, 1119-1136, 1983.

Kaye, J. A. and D. F. Strobel, Enhancement of heavy ozone in earth's atmosphere?, J. Geophys. Res., 88, 8447-8452, 1983.

Kaye, J. A., Theoretical analysis of isotope effects on ozone formation in oxygen photochemistry, J. Geophys. Res., 97, 7865-7874, 1986.

Krankowsky, D. et al., Measurement of heavy isotope enrichment in tropospheric ozone, Geophys. Res. Lett., 22, 13, 1713-1716, 1995.

Mauersberger, K., Measurement of heavy ozone in the stratosphere, Geophys. Res. Lett., 8, 935-939, 1981.

Mauersberger, K., Ozone isotope measurements in the stratosphere, Geophys. Res. Lett., 14, 80-83, 1987.

Mauersberger, K. et al., Multi-isotope study of ozone: implications for the heavy ozone anomaly, Geophys. Res. Lett., 20, 1031-1034, 1993.

McDermid, I. S., A 4-year climatology of stratospheric ozone from lidar measurements at Table Mountain, $34.4^{\circ} \mathrm{N}, J$. Geophys. Res., 98, 10509-10515, 1993

Meier, A. and J. Notholt, Determination of the isotopic abundances of heavy $\mathrm{O}_{3}$ as observed in arctic ground-based FTIR-spectra, Geophys. Res. Lett., 23, 551-554, 1996.

Miller, R. L. et al., The "ozone deficit" problem: $\mathrm{O}_{2}(X, \mathrm{v} \geq 26)+\mathrm{O}\left({ }^{3} P\right)$ from 226-nm ozone photodissociation, Science, 265, 1831-1838, 1994.

Morton, J. et al., Laboratory studies of heavy ozone, J. Geophys. Res., 95, 901-907, 1990.

Norton, R. H. and C. P. Rinsland, ATMOS data processing and science analysis methods, Appl. Opt., 30, 389-400, 1991.

Rinsland, C. P. et al., Identification of ${ }^{18} \mathrm{O}$-isotopic lines of ozone in infrared ground-based solar absorption spectra, J. Geophys. Res., 90, 10719-10725, 1985

Schueler, B. et al., Measurement of isotopic abundances in collected stratospheric ozone samples, Geophys. Res. Lett., 17, 1295-1298, 1990.

U.S. Standard Atmosphere, 1976, National Oceanic and Atmospheric Administration, National Aeronautics and Space Administration, and United States Air Force, Washington, D. C., 1976.

Valentini, J. J., Mass-independent isotopic fractionation in nonadiabatic molecular collisions, J. Chem. Phys., 86, 6755-6765, 1987.

F. W. Irion, Department of Planetary Science, California Institute of Technology, Pasadena CA 91125. (email: fwi@cco.caltech.edu)

(Received: October 6, 1995; revised April 22, 1996; accepted May 7, 1996.) 


\title{
Stratospheric observations of $\mathrm{CH}_{3} \mathrm{D}$ and HDO from ATMOS infrared solar spectra: Enrichments of deuterium in methane and implications for $\mathrm{HD}$
}

\author{
F. W. Irion,1 E. J. Moyer, 1 M. R. Gunson,2 C. P. Rinsland, ${ }^{3}$ Y.L. Yung, ${ }^{1}$ H. A. \\ Michelsen,4 R. J. Salawitch,2 A. Y. Chang2, M. J. Newchurch,5 M. M. Abbas, 6 M. C. \\ Abrams, ${ }^{7}$ and R. Zander 8
}

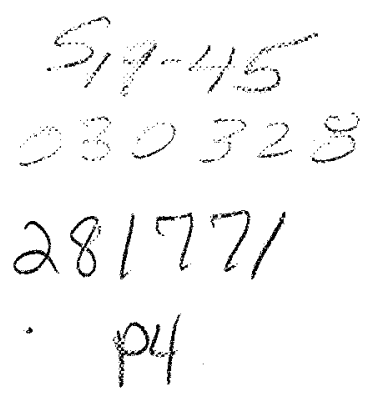

\begin{abstract}
Stratospheric mixing ratios of $\mathrm{CH}_{3} \mathrm{D}$ from $100 \mathrm{mb}$ to $17 \mathrm{mb}(\approx 15$ to $28 \mathrm{~km})$ and $\mathrm{HDO}$ from $100 \mathrm{mb}$ to $10 \mathrm{mb}(\approx 15$ to 32 $\mathrm{km}$ ) have been inferred from high resolution solar occultation infrared spectra from the Atmospheric Trace MOlecule Spectroscopy (ATMOS) Fourier-transform interferometer. The spectra, taken on board the Space Shuttle during the Spacelab 3 and ATLAS- $1,-2$, and -3 missions, extend in latitude from $70^{\circ} \mathrm{S}$ to $65^{\circ} \mathrm{N}$. We find $\mathrm{CH}_{3} \mathrm{D}$ entering the stratosphere at an average mixing ratio of $(9.9 \pm 0.8) \times 10^{-10}$ with a $\mathrm{D} / \mathrm{H}$ ratio in methane (7.1 \pm 7.4$) \%$ less than that in Standard Mean Ocean Water (SMOW) ( $1 \sigma$ combined precision and systematic error). In the mid to lower stratosphere, the average lifetime of $\mathrm{CH}_{3} \mathrm{D}$ is found to be (1.19 \pm 0.02$)$ times that of $\mathrm{CH}_{4}$, resulting in an increasing $\mathrm{D} / \mathrm{H}$ ratio in methane as air "ages" and the methane mixing ratio decreases. We find an average of $(1.0 \pm 0.1)$ molecules of stratospheric $\mathrm{HDO}$ are produced for each $\mathrm{CH}_{3} \mathrm{D}$ destroyed ( $1 \sigma$ combined precision and systematic error), indicating that the rate of HDO production is approximately equal to the rate of $\mathrm{CH}_{3} \mathrm{D}$ destruction. Assuming negligible amounts of deuterium in species other than $\mathrm{HDO}, \mathrm{CH}_{3} \mathrm{D}$ and $\mathrm{HD}$, this limits the possible change in the stratospheric HD mixing ratio below about $10 \mathrm{mb}$ to be \pm 0.1 molecules HD created per molecule $\mathrm{CH}_{3} \mathrm{D}$ destroyed.
\end{abstract}

\section{Introduction}

In the stratosphere, $\mathrm{H}_{2} \mathrm{O}$ is created primarily by oxidation of $\mathrm{CH}_{4}$ and $\mathrm{H}_{2}$ by $\mathrm{OH}, \mathrm{Cl}$ and $\mathrm{O}\left({ }^{\prime} \mathrm{D}\right)$. Likewise, oxidation of $\mathrm{CH}_{3} \mathrm{D}$ and $\mathrm{HD}$ creates $\mathrm{HDO}$, but these deuterated species show a sufficiently different reactivity with surrounding chemical species so that their measurement can provide additional insight into the stratospheric hydrogen budget. In understanding the deuterium budget of the stratosphere and relating it to the hydrogen budget, several questions arise. First, what is the lifetime of $\mathrm{CH}_{3} \mathrm{D}$ compared to that of $\mathrm{CH}_{4}$, or put another way, how does the $\mathrm{D} / \mathrm{H}$ ratio

\footnotetext{
${ }^{1}$ California Institute of Technology, Pasadena CA 91125.

2Jet Propulsion Laboratory, California Institute of Technology, Pasadena CA 91109.

3NASA Langley Research Center, Hampton VA 23681.

4Harvard University, Cambridge, MA 02138.

sUniversity of Alabama in Huntsville, Huntsville AL 35899.

6NASA Marshall Space Flight Center, Huntsville AL 35812.

7SAIC - NASA Langley Research Center, Hampton VA 23681.

8Institute of Astrophysics, University of Liège, 4000 Liège-Cointe, Belgium.
}

Copyright 1996 by the American Geophysical Union.

Paper number 96GL01402

0094-8534/96/96GL-01402\$05.00 in methane vary with the $\mathrm{CH}_{4}$ mixing ratio? Second, is the rate of stratospheric HDO production balanced by that of $\mathrm{CH}_{3} \mathrm{D}$ oxidation? If not, could there be a net production or destruction of $\mathrm{HD}$, and possibly a change in the $\mathrm{D} / \mathrm{H}$ ratio of molecular hydrogen, due to a difference in the rates between $\mathrm{CH}_{3} \mathrm{D}$ oxidation to $\mathrm{HD}$ and $\mathrm{HD}$ oxidation to HDO? (Discussion of the D/H ratio in stratospheric water is in an accompanying paper by Moyer et al. [this issue].)

We address these questions using spectroscopic measurements of stratospheric $\mathrm{CH}_{3} \mathrm{D}$ and $\mathrm{HDO}$ mixing ratios using data from the ATMOS instrument. ATMOS, described in detail by Farmer et al. [1987], is a Fourier-transform infrared interferometer that gathered spectral absorption measurements at $0.01 \mathrm{~cm}^{-1}$ resolution from solar occultations on board four Space Shuttle missions (see Gunson et al. [this issue]). A previous report of $\mathrm{HDO}$ and $\mathrm{CH}_{3} \mathrm{D}$ results from ATMOS was made by Rinsland et al. [1991] with data from the Spacelab 3 mission; however, their report did not relate changes in the $\mathrm{CH}_{3} \mathrm{D}$ mixing ratio to those in HDO. With the combined Spacelab 3 and ATLAS-1, -2, and -3 missions, a much broader latitudinal coverage and many more vertical mixing ratio profiles were obtained, allowing characterization of the $\mathrm{CH}_{3} \mathrm{D}$ and $\mathrm{HDO}$ budget on a more global basis. An important addition to previous reports is measurement of $\mathrm{CH}_{3} \mathrm{D}$ inside the Arctic and Antarctic vortices. Above $28 \mathrm{~km}$, the molecular density of $\mathrm{CH}_{3} \mathrm{D}$ is normally too low to gain an adequate spectral absorption signal with the ATMOS instrument. However, the descent of upper stratospheric air to altitudes below $28 \mathrm{~km}$ inside the vortices [Abrams et al., this issue a,b] allows measurement of $\mathrm{CH}_{3} \mathrm{D}$ in "old" air that would be otherwise impossible to measure. As stratospheric dehydration is not the focus of this letter, we do not report measurements of HDO inside the polar vortices. Analyses are continuing on these vortex HDO measurements, and they will be the focus of an upcoming paper.

In this paper, the delta notation is used to describe isotopic fractionation as the difference in parts per thousand of an isotopically labelled species with respect to a standard, e. g.,

$$
\delta D_{\text {sampic }}(\%)=1000 \times \frac{(\mathrm{D} / \mathrm{H})_{\text {sample }}-(\mathrm{D} / \mathrm{H})_{\text {standard }}}{(\mathrm{D} / \mathrm{H})_{\text {standard }}} .
$$

Standard Mean Ocean Water (SMOW) is used for the standard D/H ratio (see IUPAC [1983]) and we use the recommended value of (155.76 \pm 0.05$) \times 10^{-6}$ [Hagemann et al., 1970].

\section{Observations and data analysis}

Information about ATMOS on the shuttle missions, and discussion of the mixing ratio retrieval process, can be found in Gunson et al. [this issue]. For the results described here, spectral lines of the $v_{2}$ band of $\mathrm{HDO}$ and the $v_{1}$ band of $\mathrm{CH}_{3} \mathrm{D}$ were analyzed, and spectral intervals used for these analyses are described in Table 1. HDO is best observed in filters 2 (1100- 
Table 1. Spectral intervals and lines used for $\mathrm{HDO}$ and $\mathrm{CH}_{3} \mathrm{D}$ analyses

\begin{tabular}{|c|c|c|c|c|c|c|c|}
\hline $\begin{array}{c}\text { Spectral } \\
\text { interval } \\
\text { center } \\
(\mathrm{cm} \cdot 1)\end{array}$ & $\begin{array}{c}\text { Interval } \\
\text { width } \\
\text { (cm-l) }\end{array}$ & $\begin{array}{c}\text { Altitude } \\
\text { range } \\
(\mathrm{km})\end{array}$ & $\begin{array}{l}\text { Line } \\
\text { center } \\
(\mathrm{cm}-1)\end{array}$ & $\begin{array}{c}\text { Line } \\
\text { intensity } \\
(x 1023 \mathrm{~cm} \\
\left.\text { molec. } .^{-1}\right)\end{array}$ & $\begin{array}{c}\text { Air- } \\
\text { broadened } \\
\text { half-width } \\
\text { (cm-1/atm } \\
\text { at } 296 \mathrm{~K})\end{array}$ & $\begin{array}{l}\text { Ground- } \\
\text { state } \\
\text { energy } \\
(\mathrm{cm} \cdot 1)\end{array}$ & $\begin{array}{l}\text { Temperature } \\
\text { sensitivity } \\
\text { of line } \\
\text { intensity at } \\
230 \mathrm{~K}(\% \mathrm{~K} \cdot 1)\end{array}$ \\
\hline \multicolumn{8}{|l|}{$\mathrm{CH}_{3} \mathrm{D}$} \\
\hline 2950.84 & 0.24 & $10-28$ & 2950.8514 & 2.81 & 0.072 & 266.3 & 0.07 \\
\hline 3061.56 & 0.20 & $15-36$ & 3061.4148 & 5.21 & 0.077 & 89.9 & -0.4 \\
\hline \multirow{2}{*}{3078.34} & 0.17 & $15-28$ & 3078.3125 & 1.61 & 0.070 & 217.1 & -0.07 \\
\hline & & & 3078.3551 & 2.09 & 0.072 & 184.7 & -0.2 \\
\hline \multicolumn{7}{|l|}{ HDO } & 0.3 \\
\hline 1408.35 & 0.25 & $10-34$ & 1408.3914 & 0.962 & 0.102 & 29.8 & -0.6 \\
\hline 1421.62 & 0.40 & $13-42$ & 1421.6073 & 1.26 & 0.077 & 233.1 & 0.02 \\
\hline 1439.93 & 0.32 & $15-40$ & 1439.8887 & 1.54 & 0.095 & 150.1 & -0.2 \\
\hline 1451.40 & 0.34 & $10-29$ & 1451.4597 & 1.21 & 0.093 & 265.2 & 0.07 \\
\hline 1469.43 & 0.23 & $17-40$ & 1469.3658 & 2.53 & 0.095 & 156.4 & -0.2 \\
\hline 1474.09 & 0.31 & $19-40$ & 1474.1110 & 1.30 & 0.094 & 156.4 & -0.2 \\
\hline 1475.62 & 0.31 & $20-33$ & 1475.5917 & 1.37 & 0.096 & 150.2 & -0.2 \\
\hline 1479.96 & 0.42 & $10-26$ & 1480.0941 & 0.581 & 0.093 & 225.9 & -0.04 \\
\hline 1484.11 & 0.25 & $15-40$ & 1484.1065 & 2.44 & 0.092 & 225.9 & -0.04 \\
\hline \multirow[t]{2}{*}{1488.16} & 0.48 & $20-40$ & 1488.0252 & 1.09 & 0.093 & 221.9 & .0 .04 \\
\hline & & & 1488.1937 & 1.33 & 0.100 & 32.5 & -0.6 \\
\hline 1494.86 & 0.29 & $15-29$ & 1494.8598 & 0.834 & 0.095 & 221.8 & -0.05 \\
\hline 1497.85 & 0.34 & $15-40$ & 1497.8807 & 2.08 & 0.085 & 308.6 & 0.02 \\
\hline
\end{tabular}

Note: The temperature dependence for air-broadened half-widths is $T-1.75$ for $\mathrm{CH}_{3} \mathrm{D}$ and $\mathrm{T}-0.64$ for HDO. Line parameters for $\mathrm{CH}_{3} \mathrm{D}$ are from Rinsland et al. [1991]. HDO line parameters are discussed in Brown et al. [1995].

$\left.2000 \mathrm{~cm}^{-1}\right)$ and $9\left(600-2450 \mathrm{~cm}^{-1}\right)$, while $\mathrm{CH}_{3} \mathrm{D}$ could only be observed in filter $3\left(1580-3400 \mathrm{~cm}^{-1}\right)$. However, $\mathrm{CH}_{4}$ can be analyzed in all of these filters and, with a correlation of $\mathrm{CH}_{3} \mathrm{D}$ to $\mathrm{CH}_{4}$ in filter 3 , we are able infer the mixing ratio of $\mathrm{CH}_{3} \mathrm{D}$ in filters 2 and 9 from $\mathrm{CH}_{4}$ mixing ratios. At polar latitudes, determination as to whether a $\mathrm{CH}_{3} \mathrm{D}$ measurement was inside or outside the vortex was based on visual inspection of co-located mixing ratio profiles of $\mathrm{CH}_{4}$ and $\mathrm{N}_{2} \mathrm{O}$, as these gases show markedly smaller mixing ratios inside the vortex than outside at similar altitudes (see Abrams et al., this issue a,b). "Vortex edge" observations were not used. $\mathrm{CH}_{3} \mathrm{D}$ results are presented from tangent pressures of $100 \mathrm{mb}$ to $17 \mathrm{mb}$, and HDO results are from 100 $\mathrm{mb}$ to $10 \mathrm{mb}$. Before final analyses, data were filtered by rejecting any observations with an estimated random error greater than $30 \%$ for $\mathrm{CH}_{3} \mathrm{D}$ and $\mathrm{HDO}$, or $10 \%$ for $\mathrm{CH}_{4}$. This step eliminated about $16 \%$ of the filter $3 \mathrm{CH}_{3} \mathrm{D}$ data and about $19 \%$ of the filter 2 and 9 HDO data. Systematic biases, mostly from errors in line intensities, are estimated to be $7 \%$ for $\mathrm{CH}_{3} \mathrm{D}, 6 \%$ for $\mathrm{HDO}$, and $5 \%$ for $\mathrm{CH}_{4}$ (see Abrams et al., this issue, c).

\section{$\mathrm{CH}_{3} \mathrm{D}$ measurement and the $\mathrm{D} / \mathrm{H}$ ratio in methane}

Rate constants for reactions of $\mathrm{OH}$ or $\mathrm{Cl}$ with $\mathrm{CH}_{3} \mathrm{D}$ are lower than those for $\mathrm{CH}_{4}$ [DeMore et al., 1994; Wallington and Hurley, 1992], but any isotope effect between the rate constants of the excited $\mathrm{O}\left({ }^{1} \mathrm{D}\right)+\mathrm{CH}_{3} \mathrm{D}$ and that of $\mathrm{O}\left({ }^{1} \mathrm{D}\right)+\mathrm{CH}_{4}$ is expected to be relatively minor [Kaye, 1987]. To estimate these combined effects on the $\mathrm{D} / \mathrm{H}$ ratio of methane, we begin by assuming that in the mid to lower stratosphere $\mathrm{CH}_{4}$ and $\mathrm{CH}_{3} \mathrm{D}$ are only destroyed by $\mathrm{OH}, \mathrm{Cl}$, and $\mathrm{O}\left({ }^{1} \mathrm{D}\right)$ oxidation, and photolysis is negligible. The oxidation of $\mathrm{CH}_{3} \mathrm{D}$ and $\mathrm{CH}_{4}$ can then be described by:

$$
\frac{1}{\left[\mathrm{CH}_{3} \mathrm{D}\right]} \frac{\mathrm{d}\left[\mathrm{CH}_{3} \mathrm{D}\right]}{\mathrm{dt}}=-\mathrm{k}_{\mathrm{OH}}^{*}[\mathrm{OH}]-\mathrm{k}_{\mathrm{Cl}}^{*}[\mathrm{Cl}]-\mathrm{k}_{\mathrm{O}\left({ }^{\prime} \mathrm{D}\right)}^{*}\left[\mathrm{O}\left({ }^{\prime} \mathrm{D}\right)\right] \text {, }
$$

$$
\frac{1}{\left[\mathrm{CH}_{4}\right]} \frac{\mathrm{d}\left[\mathrm{CH}_{4}\right]}{\mathrm{dt}}=-\mathrm{k}_{\mathrm{OH}}[\mathrm{OH}]-\mathrm{k}_{\mathrm{Cl}}[\mathrm{Cl}]-\mathrm{k}_{\left.\mathrm{O}^{\prime} \mathrm{D}\right)}\left[\mathrm{O}\left({ }^{\prime} \mathrm{D}\right)\right] .
$$

Let $\gamma_{\mathrm{i}}(\mathrm{T})$ equal the ratios of the rate constants. That is, $\gamma_{\mathrm{OH}}(\mathrm{T})=\mathrm{k}_{\mathrm{OH}}^{\cdot} / \mathrm{k}_{\mathrm{OH}}, \gamma_{\mathrm{Cl}}(\mathrm{T})=\mathrm{k}_{\mathrm{Cl}}^{\cdot} / \mathrm{k}_{\mathrm{Cl}}$ and $\gamma_{\mathrm{O}{ }^{\prime} \mathrm{D},}(\mathrm{T})=\mathrm{k}_{\mathrm{O}(\mathrm{D})}^{*} / \mathrm{k}_{\mathrm{O}\left({ }^{\mathrm{D}} \mathrm{D}\right)}$, where $\mathrm{T}$ is temperature. Subtraction of Equation (3) from (2) gives:

$$
\begin{aligned}
& \frac{1}{\left[\mathrm{CH}_{3} \mathrm{D}\right]} \frac{\mathrm{d}\left[\mathrm{CH}_{3} \mathrm{D}\right]}{\mathrm{dt}}-\frac{1}{\left[\mathrm{CH}_{4}\right]} \frac{\mathrm{d}\left[\mathrm{CH}_{4}\right]}{\mathrm{dt}} \\
& \quad=-\left(\gamma_{\mathrm{OH}}-1\right) \mathrm{k}_{\mathrm{OH}}[\mathrm{OH}]-\left(\gamma_{\mathrm{Cl}}-1\right) \mathrm{k}_{\mathrm{Cl}}[\mathrm{Cl}]-\left(\gamma_{\mathrm{O}\left({ }^{1} \mathrm{D}\right)}-1\right) \mathrm{k}_{\mathrm{O}\left({ }^{1} \mathrm{D}\right)}\left[\mathrm{O}\left({ }^{1} \mathrm{D}\right)\right] .
\end{aligned}
$$

But we note that

$$
\mathrm{k}_{\mathrm{OH}}[\mathrm{OH}]=-\frac{-1}{\left[\mathrm{CH}_{4}\right]} \frac{\mathrm{d}\left[\mathrm{CH}_{4}\right]}{\mathrm{dt}} \mathrm{f}_{\mathrm{OH}}(\mathrm{z}),
$$

where $\mathrm{f}_{\mathrm{OH}}(\mathrm{z})$ is the fraction of all $\mathrm{CH}_{4}$ destroyed at altitude $\mathrm{z}$ that is destroyed by $\mathrm{OH}$. With similar definitions for $\mathrm{f}_{\mathrm{Cl}}(\mathrm{z})$ and $\mathrm{f}_{\mathrm{O}\left({ }^{1} \mathrm{D}\right)}(\mathrm{z})$, substitution in (4) for $\mathrm{k}_{\mathrm{OH}}, \mathrm{k}_{\mathrm{Cl}}$, and $\mathrm{k}_{\mathrm{O}\left({ }^{\prime} \mathrm{D}\right)}$ (noting that they sum to unity) and rearrangement yield:

$$
\begin{aligned}
& \frac{1}{\left[\mathrm{CH}_{3} \mathrm{D}\right]} \frac{\mathrm{d}\left[\mathrm{CH}_{3} \mathrm{D}\right]}{\mathrm{dt}} \\
& =\left(\gamma_{\mathrm{OH}} \mathrm{f}_{\mathrm{OH}}+\gamma_{\mathrm{G}} \mathrm{f}_{\mathrm{O}}+\gamma_{\mathrm{O}\left({ }^{1} \mathrm{D}\right)} \mathrm{f}_{\mathrm{O}(\mathrm{D})}\right) \frac{1}{\left[\mathrm{CH}_{4}\right]} \frac{\mathrm{d}\left[\mathrm{CH}_{4}\right]}{\mathrm{dt}},
\end{aligned}
$$

or:

$$
\frac{1}{\left[\mathrm{CH}_{3} \mathrm{D}\right]} \frac{\mathrm{d}\left[\mathrm{CH}_{3} \mathrm{D}\right]}{\mathrm{dt}}=\kappa(\mathrm{T}, \mathrm{z}) \frac{1}{\left[\mathrm{CH}_{4}\right]} \cdot \stackrel{\mathrm{d}\left[\mathrm{CH}_{4}\right]}{\mathrm{dt}},
$$

where $\kappa(T, z)$ is the term inside the parentheses of (6). Note that the left hand side of Equation (7) is the time constant for destruction of $\mathrm{CH}_{3} \mathrm{D}$, or equivalently, its inverse lifetime. Thus, for a $\kappa(T, z)<1$, the lifetime of $\mathrm{CH}_{3} \mathrm{D}$ is longer than that of $\mathrm{CH}_{4}$. We estimate an "average" $K$ in the mid to lower stratosphere by assuming average fractions for destruction (i.e., $f_{\mathrm{OH}}, f_{\mathrm{Cl}}$, and $\mathrm{f}$ $O\left({ }^{1} D\right)$ ), and assuming that the ratios of the rate constants, $\gamma$, are constant with the temperature range in the mid to lower stratosphere and that mixing effects are minor. Integrating (6), assigning boundary conditions, and dividing by total molecular concentration to get mixing ratios $(\chi)$ yield:

$$
\ln \chi\left(\mathrm{CH}_{3} \mathrm{D}\right)=\ln \chi_{0}\left(\mathrm{CH}_{3} \mathrm{D}\right)+\kappa_{\mathrm{av}} \ln \left\lfloor\frac{\chi\left(\mathrm{CH}_{4}\right)}{\chi_{0}\left(\mathrm{CH}_{4}\right)}\right\rfloor,
$$

or equivalently,

$$
\frac{\chi\left(\mathrm{CH}_{3} \mathrm{D}\right)}{\chi_{0}\left(\mathrm{CH}_{3} \mathrm{D}\right)}=\left(\frac{\chi\left(\mathrm{CH}_{4}\right)}{\chi_{0}\left(\mathrm{CH}_{4}\right)}\right)^{\mathrm{K}},
$$

where $\chi_{0}\left(\mathrm{CH}_{3} \mathrm{D}\right)$ and $\chi_{0}\left(\mathrm{CH}_{4}\right)$ are the initial mixing ratios of these gases as they enter the stratosphere. A rough estimate of $\kappa_{\mathrm{av}}$ can be made if we set $\gamma_{\mathrm{OH}}=0.67$ (averaged from 190K to $250 \mathrm{~K}$ using the rate constants reported by DeMore et al. [1994]), $\gamma_{\mathrm{Cl}}=0.735$ (from the evaluation by Wallington and Hurley [1992] at 295K), and $\gamma_{O(1 D)} \approx 1$ (see Kaye [1987]). Model calculations by one of us (RJS) indicate that between about $16 \mathrm{~km}$ and $30 \mathrm{~km}$, about $50 \%$ of the destruction of $\mathrm{CH}_{4}$ is by $\mathrm{OH}, 29 \%$ by $\mathrm{Cl}$ and $21 \%$ by $\mathrm{O}\left({ }^{1} \mathrm{D}\right)$. Substituting these laboratory data and model results, $\kappa_{\mathrm{av}} \approx 0.78$, suggesting that the lifetime of $\mathrm{CH}_{3} \mathrm{D}$ is significantly longer than that of $\mathrm{CH}_{4}$, and enrichment of deuterium in methane can occur as the $\mathrm{CH}_{4}$ mixing ratio decreases. We emphasize that this is only a rough estimation as none of the experiments measuring the kine- 


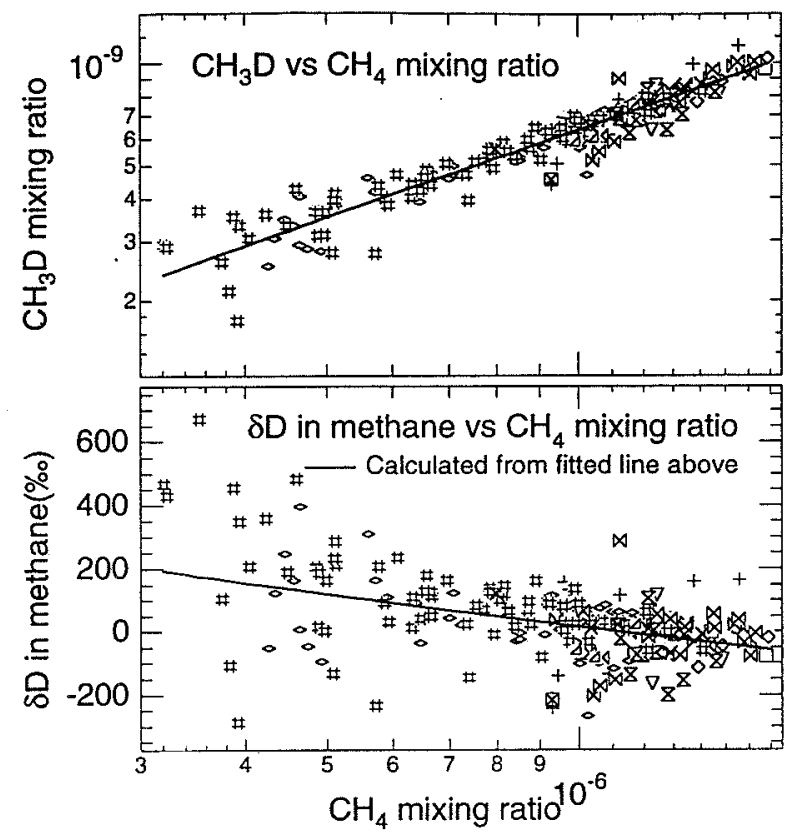

Figure 1. $\mathrm{CH}_{3} \mathrm{D}$ mixing ratio vs $\mathrm{CH}_{4}$ mixing ratio (upper panel) and $\delta \mathrm{D}$ in methane vs $\mathrm{CH}_{4}$ mixing ratio (lower panel). For clarity, only one-sixth the data in extra-vortex regions are displayed. Spacelab 3: $\otimes 48^{\circ} \mathrm{S}, \times 26^{\circ} \mathrm{N}-31^{\circ} \mathrm{N} ;$ ATLAS-1: $+50^{\circ} \mathrm{S}-20^{\circ} \mathrm{S}$; ATLAS-2: $\nabla 50^{\circ} \mathrm{S}-25^{\circ} \mathrm{S}, \triangle 65^{\circ} \mathrm{N}-70^{\circ} \mathrm{N}$ outside vortex, $\circ 65^{\circ} \mathrm{N}$ $70^{\circ} \mathrm{N}$ inside vortex; ATLAS-3: \& $75^{\circ} \mathrm{S}-65^{\circ} \mathrm{S}$ outside vortex,

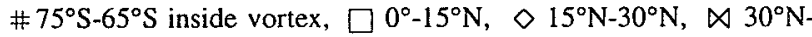
$50^{\circ} \mathrm{N}$. Note: Some polar profiles had regions inside and some regions outside the vortex.

tic rate constants of $\mathrm{OH}$ or $\mathrm{Cl}$ with $\mathrm{CH}_{3} \mathrm{D}$ were made at the cold temperatures typical of the lower stratosphere, and we are unaware of any laboratory measurements of the $\mathrm{O}\left({ }^{1} \mathrm{D}\right)+\mathrm{CH}_{3} \mathrm{D}$ rate constant.

ATMOS measurements of the mixing ratios of $\mathrm{CH}_{3} \mathrm{D}$ are plotted against co-located measurements of $\mathrm{CH}_{4}$ in the upper panel of Figure 1. We assume that $\chi_{0}\left(\mathrm{CH}_{4}\right)=(1.71 \pm 0.03) \times 10^{-6}$ (from global 1992 tropospheric measurements reported in WMO [1995], and where we have assumed a $2 \%$ error). From a best fit line of In $\chi\left(\mathrm{CH}_{3} \mathrm{D}\right)$ vs in $\chi\left(\mathrm{CH}_{4}\right)$, we observe $\kappa_{\mathrm{av}}=(0.84 \pm 0.02)(1 \sigma$ combined precision and systematic error), which is higher than our initial estimate of 0.78 . Note that the error in $\kappa_{a v}$ is not affected by systematic errors in the measured stratospheric mixing ratios of $\mathrm{CH}_{3} \mathrm{D}$ and $\mathrm{CH}_{4}$ (which instead affect the error in the intercept). On average, the stratospheric lifetime of $\mathrm{CH}_{3} \mathrm{D}$ should be $\mathrm{K}_{\mathrm{av}}^{-1}$ $(=1.19 \pm 0.02)$ times that of $\mathrm{CH}_{4}$, or about 190 years based on a stratospheric $\mathrm{CH}_{4}$ lifetime of 160 years [Prather and Spivakovsky, 1990]. From the fitted line at a tropospheric $\mathrm{CH}_{4}$ mixing ratio of $(1.71 \pm 0.03) \times 10^{-6}$, we find the mixing ratio of $\mathrm{CH}_{3} \mathrm{D}$ entering the stratosphere to be $(9.9 \pm 0.8) \times 10^{-10}$ (16 combined precision and systematic error).

The lower panel of Figure 1 shows the enrichment of $\mathrm{CH}_{3} \mathrm{D}$ with $\mathrm{CH}_{4}$ mixing ratio, as well as the average enrichment calculated using the fitted line from the upper panel. Although there is considerable scatter in the data, it is seen that methane becomes progressively enriched in deuterium as the mixing ratio of $\mathrm{CH}_{3} \mathrm{D}$ decreases. With a tropospheric $\mathrm{CH}_{4}$ mixing ratio of $(1.71 \pm$ $0.03) \times 10^{-6}$, the average $\delta \mathrm{D}$ in methane entering the stratosphere is $-(71 \pm 74) \% o$ ( $1 \sigma$ combined precision and systematic error).
This is lower than, but within error of the ATMOS Spacelab 3 measurements of Rinsland et al. [1991] who found the average $\delta D$ in methane from 18 to $28 \mathrm{~km}$ near latitudes of $30^{\circ} \mathrm{N}$ and $49^{\circ} \mathrm{S}$ to be $-(49 \pm 44) \% o$ and $+(24 \pm 125) \%$ respectively. This is also within error of free tropospheric measurements by Ehhalt [1973], who reported values of $-86 \%$ and $-94 \%$, and Wahlen et al. [1987], who reported $-(80 \pm 8) \%$.

\section{Measurement of $\mathrm{HDO}$ ws $\mathrm{CH}_{3} \mathrm{D}$ and implications for HD}

In the mid to lower stratosphere, atomic $\mathrm{H}$ is distributed primarily among $\mathrm{CH}_{4}, \mathrm{H}_{2} \mathrm{O}$ and $\mathrm{H}_{2}$, with negligible amounts among other species. It appears that oxidation of $\mathrm{H}_{2}$ is roughly balanced by its production via oxidation of $\mathrm{CH}_{4}$ through a shortlived $\mathrm{CH}_{2} \mathrm{O}$ intermediate [Dessler et al, 1994; Abbas et al., this issue]. Similarly, deuterium is most likely distributed mainly among $\mathrm{CH}_{3} \mathrm{D}, \mathrm{HDO}$, and $\mathrm{HD}$ (with $\mathrm{HD}$ produced by oxidation of $\mathrm{CH}_{3} \mathrm{D}$ and destroyed by oxidation to HDO). However, in the mid to lower stratosphere, it may not necessarily be the case that the mixing ratio of $\mathrm{HD}$ is as weakly varying as that of $\mathrm{H}_{2}$. As suggested by Ehhalt et al. [1989], the lower reaction rate constant of $\mathrm{OH}$ and $\mathrm{HD}$ compared to that of $\mathrm{OH}$ and $\mathrm{H}_{2}$ may serve to enrich tropospheric hydrogen gas in deuterium. However, stratospheric destruction of $\mathrm{HD}$ is regulated not only by rates of $\mathrm{OH}$ attack, but also to a large extent by those of $O\left({ }^{l} D\right)$, and the rate constant of the $O\left({ }^{1} \mathrm{D}\right)$ reaction is not expected to be significantly affected by deuterium substitution [Kaye, 1987]. (Reaction with $\mathrm{Cl}$ is a much more minor sink for hydrogen than for methane using the rate constants given by DeMore et al. [1994].) For production of HD, account must be taken of not only the oxidation rates of $\mathrm{CH}_{3} \mathrm{D}$, but also the partitioning of $\mathrm{D}$ in the short-lived species in the reaction pathway from $\mathrm{CH}_{3} \mathrm{D}$ to $\mathrm{HD}$ (e.g. the yield of $\mathrm{CH}_{2} \mathrm{D}$ vs $\mathrm{CH}_{3}$, or that of $\mathrm{CHDO}$ vs $\mathrm{CH}_{2} \mathrm{O}$ ). In the absence of direct measure-

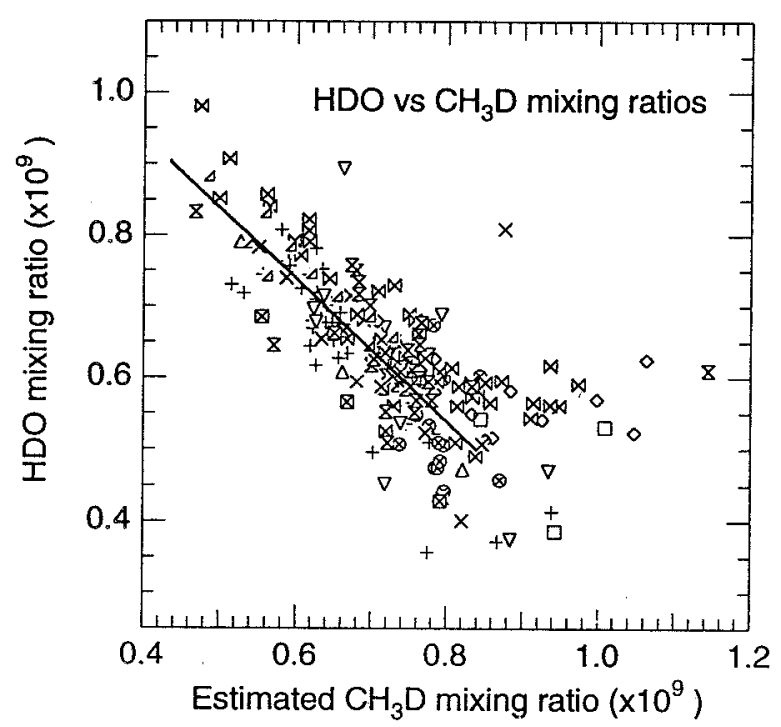

Figure 2. HDO mixing ratio vs $\mathrm{CH}_{3} \mathrm{D}$ mixing ratio. The line is fitted only where the $\mathrm{CH}_{4}$ mixing ratio is less than $1.4 \times 10^{-6}$. For clarity, only one-third the data for each latitude bin are shown. Spacelab 3: $\otimes 49^{\circ} \mathrm{S}, \times 26^{\circ} \mathrm{N}-31^{\circ} \mathrm{N}$; ATLAS-1: $+55^{\circ} \mathrm{S}-30^{\circ} \mathrm{S}$, $\otimes 10^{\circ} \mathrm{S}-15^{\circ} \mathrm{N}, \triangle 15^{\circ} \mathrm{N}-30^{\circ} \mathrm{N}$; ATLAS- $2: \nabla 50^{\circ} \mathrm{S}-30^{\circ} \mathrm{S}, \triangle 65^{\circ} \mathrm{N}-$ $70^{\circ} \mathrm{N}$, outside vortex; ATLAS-3: \& $75^{\circ} \mathrm{S}-65^{\circ} \mathrm{S}$, outside vortex, $\square 12^{\circ} \mathrm{N}, \diamond 15^{\circ} \mathrm{N}-30^{\circ} \mathrm{N}, \bowtie 30^{\circ} \mathrm{N}-50^{\circ} \mathrm{N}$. 
ment of HD, a test for changes in the HD mixing ratio is examination of those for $\mathrm{HDO}$ and $\mathrm{CH}_{3} \mathrm{D}$. Assuming the sum of the mixing ratios of $\mathrm{HDO}, \mathrm{CH}_{3} \mathrm{D}$, and $\mathrm{HD}$ is constant, then:

$$
\frac{\mathrm{d} \chi(\mathrm{HDO})}{\mathrm{d} \chi\left(\mathrm{CH}_{3} \mathrm{D}\right)}+\frac{\mathrm{d} \chi(\mathrm{HD})}{\mathrm{d} \chi\left(\mathrm{CH}_{3} \mathrm{D}\right)}=-1 \text {, }
$$

and any deviation of $\mathrm{d} \chi(\mathrm{HDO}) / \mathrm{d} \chi\left(\mathrm{CH}_{3} \mathrm{D}\right)$ from -1 through the mid and lower stratosphere implies a changing mixing ratio of HD.

In order to compare $\mathrm{HDO}$ and $\mathrm{CH}_{3} \mathrm{D}$ mixing ratios, which are not measured simultaneously, we use the relationship in Equation 8 , with $\kappa=0.84 \pm 0.02, \chi_{0}\left(\mathrm{CH}_{4}\right)=(1.71 \pm 0.03) \times 10^{-6}$, and $\chi_{0}\left(\mathrm{CH}_{3} \mathrm{D}\right)=(9.9 \pm 0.8) \times 10^{-10}$, to estimate the $\mathrm{CH}_{3} \mathrm{D}$ mixing ratio from measurements of $\mathrm{CH}_{4}$. Figure 2 is a scatter plot of the HDO mixing ratio in filters 2 and 9 versus this derived $\mathrm{CH}_{3} \mathrm{D}$ mixing ratio. We calculate $\mathrm{d} \chi(\mathrm{HDO}) / \mathrm{d} \chi\left(\mathrm{CH}_{3} \mathrm{D}\right)$ by a least-squares straight line fit to data where $\mathrm{CH}_{4}$ mixing ratios were less than $1.4 \times 10^{-6}$ to avoid seasonal effects in HDO in the lower stratosphere. The calculated slope, $-(1.0 \pm 0.1)$ ( $1 \sigma$ combined precision and systematic error), indicates that HDO production is in near balance with $\mathrm{CH}_{3} \mathrm{D}$ destruction. The error in the slope constrains the production of $\mathrm{HD}$ to be \pm 0.1 molecules $\mathrm{HD}$ created for each $\mathrm{CH}_{3} \mathrm{D}$ molecule destroyed; however, as only about $10 \%$ of stratospheric deuterium is in $\mathrm{HD}$, significant changes in the HD mixing ratio are still possible within this error. Large effects may also occur in the $\mathrm{D} / \mathrm{H}$ ratio of stratospheric molecular hydrogen, but this would also be dependent on even minor changes in the $\mathrm{H}_{2}$ mixing ratio due to $\mathrm{H}_{2}$ photolysis or $\mathrm{CH}_{4}$ oxidation (see, for example, Dessler et al. [1994]). The results in this report provide constraints for changes in HD below about $10 \mathrm{mb}$. To illustrate this, we assume a constant $\mathrm{H}_{2}$ mixing ratio of $0.5 \times 10^{-6}$ and a $\delta \mathrm{D}$ in hydrogen entering the stratosphere of $+(70 \pm 30) \%$ [ Friedman and Scholz, 1974], which gives an initial HD mixing ratio of (1.67 \pm 0.05$) \times 10^{-10}$. Assuming that $\mathrm{CH}_{3} \mathrm{D}$ enters the stratosphere at a mixing ratio of $9.9 \times 10^{-10}$, then results here indicate that for each $1 \%$ decrease in the $\mathrm{CH}_{3} \mathrm{D}$ mixing ratio, the percentage change in the HD mixing ratio from its tropospheric value is constrained to be $\pm 0.6 \%$ and the change in $\delta D$ in molecular hydrogen can be constrained to $\pm 6 \%$ ( $1 \sigma)$.

\section{Conclusions}

We have analyzed ATMOS spectra for mid to lower stratospheric $\mathrm{HDO}$ and $\mathrm{CH}_{3} \mathrm{D}$ mixing ratios. The average $\mathrm{D} / \mathrm{H}$ ratio in methane entering the stratosphere was found to be $-(71 \pm 74) \%$, but as the lifetime for $\mathrm{CH}_{3} \mathrm{D}$ is greater than that of $\mathrm{CH}_{4}$ by a factor of $(1.19 \pm 0.02)$, this $\mathrm{D} / \mathrm{H}$ ratio increases as methane becomes oxidized. Stratospheric production of HDO is (1.0 \pm 0.1 ) times that of $\mathrm{CH}_{3} \mathrm{D}$ loss, and assuming deuterated species other than $\mathrm{HDO}, \mathrm{CH}_{3} \mathrm{D}$ and $\mathrm{HD}$ are in negligible abundance, changes in HD abundance are thus constrained to be \pm 0.1 molecules HD per molecule $\mathrm{CH}_{3} \mathrm{D}$ destroyed ( $1 \sigma$ combined precision and systematic error). To better understand these phenomena, research is warranted into the partitioning of deuterium in $\mathrm{CH}_{3} \mathrm{D}$ destruction products (including a comparison of the photolysis rates of $\mathrm{CHDO}$ vs that of $\mathrm{CH}_{2} \mathrm{O}$ ), and direct stratospheric measurements of the HD mixing ratio.

Acknowledgements. We thank L. R. Brown, T. L. Brown, J. C. Foster, C. B. Farmer, G. D. Lynch, O. F. Raper, and G. C. Toon for their assistance. Research was performed at the Jet Propulsion Laboratory, California
Institute of Technology, under contract to the National Aeronautics and Space Administration.

\section{References}

Abbas, M. M. et al., Seasonal cycle of water vapor entry into the stratosphere from ATMOS/ATLAS-3 measurements, this issue.

Abrams, M. C. et al., ATMOS/ATLAS-3 observations of trace gas transport in the Antarctic vortex in November 1994, Geophys. Res. Lett., this issue, a.

Abrams, M. C. et al., Trace gas transport in the Arctic vortex inferred from ATMOS ATLAS-2 observations during April 1993, Geophys. Res. Lett., this issue, $b$.

Abrams, M. C. et al., On the assessment and uncertainty of atmospheric trace gas burden measurements with high resolution infrared solar occultation spectra from space, Geophys. Res. Lett., this issue, c.

Brown, L. R. et al., The 1995 Atmospheric Trace Molecule Spectroscopy (ATMOS) Linelist, submitted to Applied Optics, 1995.

DeMore, W. B. et al., Chemical Kinetics and Photochemical Data for Use in Stratospheric Modelling - Evaluation Number 11, JPL Publication 94-26, 273 pp., Jet Propulsion Laboratory, California Institute of Technology, Pasadena CA, 1994.

Dessler, A. E. et al., An examination of the total hydrogen budget of the lower stratosphere, Geophys. Res. Lett., 21, 2563-2566, 1994.

Ehhalt, D. H., Methane in the atmosphere, in Carbon and the Biosphere, edited by G. M. Woodwell and E. V. Pecan, pp. 144-157, U. S. Atomic Energy Commission, Washington, D. C., 1973

Fhhalt, D. H., et al., The kinetic isotope effect in the reaction of $\mathrm{H}_{2}$ with OH, J. Geophys. Res., 94, 9831-9836, 1989.

Farmer, C. B., O. F. Raper, and F.G. O'Callaghan, Final report on the first flight of the ATMOS instrument during the Spacelab 3 mission, April 29 through May 6, 1985, JPL Publication 87-32, 45 pp., Jet Propulsion Laboratory, California Institute of Technology, Pasadena CA, 1987.

Friedman, I. and T. G. Scholz, Isotopic composition of atmospheric hydrogen 1967-1969, J. Geophys. Res., 79, 783-786, 1974.

Gunson, M. R. et al.. The Atmospheric Trace Molecule Spectroscopy (ATMOS) experiment deployment on the ATLAS-3 Space Shuttle Mission, Geophys. Res. Lett., this issue.

Hagemann, R., G. Nief, and E. Roth, Absolute isotopic scale for deuterium analysis in natural waters. Absolute $\mathrm{D} / \mathrm{H}$ ratio of SMOW, Tellus, 22, $712-715,1970$

IUPAC commission on Atomic Weights and Isotopic Abundances, Isotopic composition of the elements 1981, Pure Appl. Chem., 55, 1119-1136, 1983.

Kaye, J. A., Mechanisms and observations for isotope fractionation of molecular species in planetary atmospheres, Rev. Geophys., 25, 1609-1658, 1987.

Moyer, E. J., F. W. Irion, Y. L. Yung and M. R. Gunson, Implications of stratospheric deuterated water for troposphere-stratosphere transport, Geophys. Res. Lett., this issue.

Prather, M. and C. M. Spivakovsky, Tropspheric $\mathrm{OH}$ and the lifetimes of hydrochlorofluorocarbons, J. Geophys. Res., 95, 18723-18729, 1990.

Rinsland, C. P., M. R. Gunson, J. C. Foster, R. A. Toth, C. B. Farmer and R. Zander, Stratospheric profiles of heavy water isotopes and $\mathrm{CH}_{3} \mathrm{D}$ from analysis of the ATMOS Spacelab 3 infrared solar spectra, J. Geophys. Res., 96, 1057-1068, 1991.

Wahlen, M. et al., $13 \mathrm{C}, \mathrm{D}$, and $14 \mathrm{C}$ in methane (abstract), Eos. Trans. $A G U$, $68,1220,1987$.

Wallington, T. J. and M. D. Hurley, A kinetic study of the reaction of chlorine atmos with $\mathrm{CF}_{3} \mathrm{CHCl}_{2}, \mathrm{CF}_{3} \mathrm{CH}_{2} \mathrm{~F}, \mathrm{CFCl}_{2} \mathrm{CH}_{3}, \mathrm{CF}_{2} \mathrm{ClCH}_{3}$, $\mathrm{CHF}_{2} \mathrm{CH}_{3}, \mathrm{CH}_{3} \mathrm{D}, \mathrm{CH}_{2} \mathrm{D}_{2}, \mathrm{CHD}_{3}, \mathrm{CD}_{4}$, and $\mathrm{CD}_{3} \mathrm{Cl}$ at $295 \pm 2 \mathrm{~K}$, Chem. Phys. Lett., 189, 437-442, 1992.

WMO, Scientific Assessment of Ozone Depletion: 1994, World Meteorological Organization Global Ozone Research and Monitoring Project - Report No. 37, Geneva, 1995.

F. W. Irion, Division of Geological and Planetary Sciences, California Institute of Technology, Pasadena CA 91125. (e-mail: fwi@cco.caltech.edu)

(Received November 3, 1995; revised April 3, 1996; accepted April 12, 1996) 


\title{
ATMOS stratospheric deuterated water and implications for troposphere-stratosphere transport
}

\author{
Elisabeth J. Moyer ${ }^{1}$, Fredrick W. Irion ${ }^{2}$, Yuk L. Yung ${ }^{1}$, and Michael R. \\ Gunson $^{3}$
}

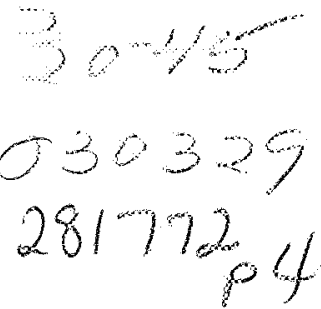

\begin{abstract}
Measurements of the isotopic composition of stratospheric water by the ATMOS instrument are used to infer the convective history of stratospheric air. The average water vapor entering the stratosphere is found to be highly depleted of deuterium, with $\delta \mathrm{D}_{\mathrm{w}}$ of $670 \pm 80$ (67\% deuterium loss). Model calculations predict, however, that under conditions of thermodynamic equilibrium, dehydration to stratospheric mixing ratios should produce stronger depletion to $\delta \mathrm{D}_{\mathrm{w}}$ of -800 to 900 (80-90\% deuterium loss). Deuterium enrichment of water vapor in ascending parcels can occur only in conditions of rapid convection; enrichments persisting into the stratosphere require that those conditions continue to near-tropopause altitudes. We conclude that either the predominant source of water vapor to the uppermost troposphere is enriched convective water, most likely evaporated cloud ice, or troposphere-stratosphere transport occurs closely associated with tropical deep convection.
\end{abstract}

\section{Introduction}

The original Brewer-Dobson proposal for the circulation between troposphere and stratosphere involved slow ascent throughout the tropics, where the tropopause is highest and coldest, with the cell extending some distance into the troposphere [Brewer, 1949]. While subsequent research has confirmed the tropics as the location of most troposphere-stratosphere transport (abbrev. STE by convention) [e.g. Rosenlof and Holton, 1993], the speed and scale of the processes which move air across the tropopause and into the stratosphere are not yet well known. Tropopause temperature measurements suggest that the scale of STE may be more localized or episodic, since much of the tropical tropopause is too warm to freeze-dry air to observed stratospheric values [e.g. Frederick and Douglass, 1983]. Theories of STE now span a large range of temporal and spatial scales, from extremely rapid injection during isolated convective events which perturb the local temperature

\footnotetext{
${ }^{1}$ Division of Geological and Planetary Sciences, Caltech

${ }^{2}$ Department of Chemistry and Chemical Engineering, Caltech

${ }^{3}$ Jet Propulsion Laboratory
}

Copyright 1996 by the American Geophysical Union.

Paper number 96GL01489

0094-8534/96/96GL-01489\$05.00 structure $\left(1-10^{3} \mathrm{~km}^{2}\right)$ [e.g. Danielsen, 1982], to slower seasonal ascent over the coldest subregion of the tropics $\left(\approx 10^{7} \mathrm{~km}^{2}\right)$ [Newell and Gould-Stewart, 1981], with proposals for dehydration in the stratosphere itself during gravity-wave temperature fluctuations allowing ascent over even larger areas [Potter and Holton, 1994].

Upward mass transport in the underlying tropical troposphere, on the other hand, is believed to be highly localized and inhomogeneous, occurring primarily in convective cumulus towers [Riehl and Malkus, 1958]. STE may result simply from the extension of some cumuli into the stratosphere proper, or it may represent a qualitatively different process, with a transition between a middle troposphere in which upward motion is dominated by localized convection and an uppermost troposphere where slower mean motions are also important [e.g. Houze, 1989]. Observations of stratospheric water vapor content have not provided a means of resolving between these scenarios.

These scenarios differ in the process by which ascending air is dehydrated. During gradual ascent, dehydration must proceed by simple condensation and fallout of moisture. Dehydration in convective systems can be more complicated, since cumulus towers can carry with them enormous quantities of water as ice - neartropopause ice:vapor ratios can exceed 100 [Knollenberg et al., 1993] - and final water vapor mixing ratios may be net of both evaporation and condensation. Dehydration in stratospheric waves may also involve cycles of condensation and re-evaporation, but without significant transport of ice particles. Determination of not only the final water content of stratospheric air, but of the process by which that content is reached, can thus discriminate between transport scenarios, providing insight into the larger question of how air is exchanged between troposphere and stratosphere.

We propose that the isotopic composition of stratospheric water vapor is a useful tracer for this purpose, because it records the condensation and evaporation experienced by each air parcel that crosses the tropopause. Whenever several phases of water are present in thermodynamic equilibrium, the heavier isotopes partition preferentially into the condensed phases. As water condenses out of an air parcel, then, deuterated water is preferentially removed and the residual vapor is progressively lightened. The degree of preference, or fractionation factor, $\alpha$ ( $\alpha \equiv \mathrm{D}: \mathrm{H}_{\text {condensate }} / \mathrm{D}: \mathrm{H}_{\text {vapor }}$ ), is quite strong for deuterated water, with $\alpha$ ranging from 1.08 at room temperature to over 1.4 for ice condensation at the $\approx 190 \mathrm{~K}$ tropical tropopause [Majoube, 1971; Merlivat and Nief, 1967]. Stratospheric air, with 
a water vapor concentration four orders of magnitude less than that at sea surface, should be highly depleted in deuterium, but the exact degree of depletion will depend on the convective history of that air.

\section{ATMOS observations of $\delta \mathbf{D}_{\mathrm{w}}$}

Observations of stratospheric deuterated water by the Atmospheric Trace Molecular Spectroscopy (ATMOS) Fourier transform infrared spectrometer over the last decade provide the first large database of isotopic compositions that can be applied to the problem of STE. There have been few previous reported measurements of $\mathrm{HDO}$ and $\mathrm{H}_{2} \mathrm{O}$ in the lower stratosphere, where accurate spectroscopic measurement of both species is difficult, with none in the tropics, the presumed source region for stratospheric air and water; nor have there been simultaneous measurements of $\mathrm{CH}_{4}$ and $\mathrm{CH}_{3} \mathrm{D}$. Observations in the mid-latitudes stratosphere have found water vapor strongly depleted of deuterium, but with $\delta \mathrm{D}_{\mathrm{w}}$ increasing with altitude from $\approx-600 \%$ at $20 \mathrm{~km}$ to $-350-450 \%$ at $35 \mathrm{~km}$. [Rinsland et al., 1984, 1991; Dinelli et al., 1991; Carli and Park, 1988; Pollock et al., 1980]. (Deuterium content is given in $\delta$ notation, where $\delta \mathrm{D}_{\mathrm{w}}$ is the fractional difference, in per mil, of the $\mathrm{D} / \mathrm{H}$ ratio of measured water from that of standard mean ocean water (SMOW), at $1.5576 \times 10^{-4}$ [Hageman et al., 1970].) This increase has been presumed to be the result of oxidation of isotopically heavier atmospheric methane. [e.g. Ehhalt, 1973; Rinsland et al., 1991].

This analysis uses data from all four ATMOS missions from 1985-1994, a total of 68 occultations in which $\mathrm{HDO}, \mathrm{H}_{2} \mathrm{O}$, and $\mathrm{CH}_{4}$ were retrieved (filters 2,9), and 67 in which $\mathrm{CH}_{3} \mathrm{D}$ was retrieved (filter 3 ). Only occulta-

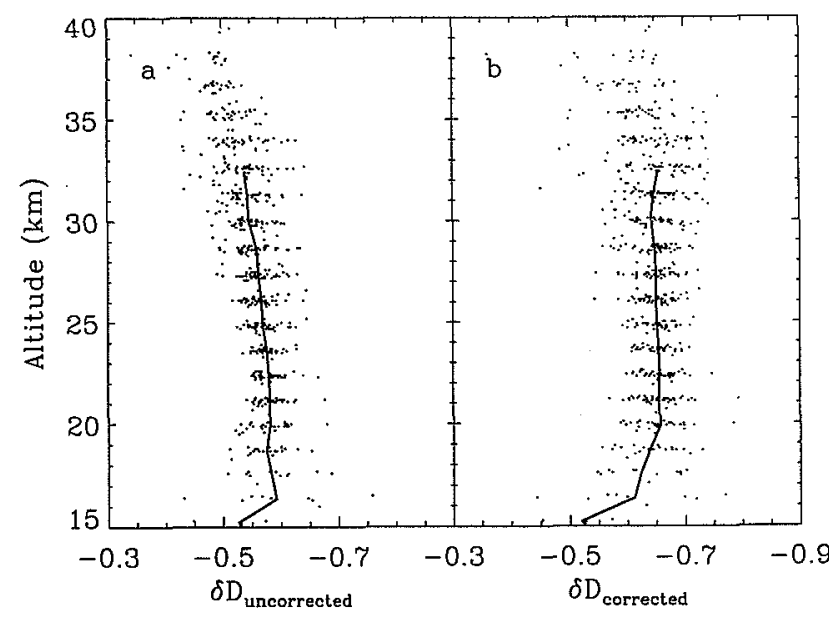

Figure 1. ATMOS measurements of the deuterium content of stratospheric water. The left panel shows uncorrected observations; the right panel the same data with the estimated contribution of $\mathrm{H}_{2} \mathrm{O}$ and $\mathrm{HDO}$ from methane oxidation subtracted. Data shown are those with error $\mathrm{H}_{2} \mathrm{O} \leq 10 \%$, error $\mathrm{CH}_{4} \leq 10 \%$, and error $\mathrm{HDO}$ $\leq 30 \%, \approx 75 \%$ of the total dataset. Solid lines represent weighted mean profiles from $15-32 \mathrm{~km}$. Distribution $\sigma$ for corrected $\delta D_{w}$ is $35 \%$; total error with systematic errors is $80 \%$. tions in the polar vortices, where dehydration on polar stratospheric clouds produces additional isotopic effects, have been excluded. Latitudinal coverage is nearglobal ( $24 \%$ tropical, $18 \%$ mid-latitudes, $58 \%$ high latitudes). The ATMOS instrument, coverage, and data reduction procedure are described in detail elsewhere [Gunson et al., 1996, and references therein].

Water vapor isotopic compositions are corrected for changes occurring in the stratosphere by subtraction of methane-derived contributions of $\mathrm{HDO}$ and $\mathrm{H}_{2} \mathrm{O}$ at each data point; conservation of hydrogen and deuterium between water and methane in the stratosphere is demonstrated from these same data by Irion et al. and $A b b a s$ et al. [1996]. Initial concentrations of $\mathrm{CH}_{4}$ and $\mathrm{CH}_{3} \mathrm{D}$ are taken as $1.7 \times 10^{-6}$ and $9.9 \times 10^{-10}$, respectively, from $W M O$ [1994] and the filter $3 \ln \left[\mathrm{CH}_{3} \mathrm{D}\right]$ : $\ln \left[\mathrm{CH}_{4}\right]$ relationship [Irion et al., 1996]. $\mathrm{CH}_{3} \mathrm{D}$ concentrations are inferred from observed $\mathrm{CH}_{4}$ in filters 2 and 9 using this relationship. This correction yields the isotopic composition of water as it first crosses the tropopause, with no assumptions as to the initial concentration of that water.

Figure 1 shows measurements of the isotopic composition of stratospheric water from all four ATMOS missions, before (1a) and after (1b) correction for methane contribution. After correction there is no trend in composition with altitude above $20 \mathrm{~km}$. Slight deviations below $20 \mathrm{~km}$ are most likely due to the increased error in retrieval of water concentrations. The mean stratospheric water is highly depleted, with a $\overline{\delta D_{w}}$ of -670 $\pm 80 \%$, or $67 \%$ loss of deuterium (weighted mean of all extravortex observations from $18-32 \mathrm{~km}$; error represents $1 \sigma$ of the distribution + systematic error.)

There is no significant variation in $\overline{\delta D}_{\mathrm{w}}$ with mission, filter, or latitude, over the 9 year span of these measurements, nor in comparison with previous measurements dating to 1980 [e.g. Pollock et al., 1980]. Mesoscale or seasonal variability in $\delta \mathrm{D}_{\mathrm{w}}$ of entering stratospheric air should be discernible only in the lower tropical stratosphere, where ATMOS HDO data is limited to only four occultations (28 observations). The distribution of $\delta \mathrm{D}_{\mathrm{w}}$ values in these data is similar to that of the whole dataset, a Gaussian distribution with a peak at $-680 \%$ and a width of $90 \%$, with no outliers that might suggest contributions from isotopically distinct processes. Although isentropic mixing should erase much spatial inhomogeneity, this consistency suggests that stratospheric water enters with a characteristic isotopic signature similar to the mean stratospheric value.

\section{Implications of stratospheric $\delta \mathbf{D}_{\mathrm{w}}$}

To explore the implications of the isotopic signature of stratospheric water, we have developed a multi-phase cloud model that computes isotopic trajectories during the ascent of air to the tropopause. The model represents the one-dimensional, pseudoadiabatic lifting of air parcels, with the concentrations and isotopic compositions of vapor, liquid, and ice tracked throughout. Air parcels are stepped upward until the water vapor mixing ratio equals the mean lower stratospheric value in 


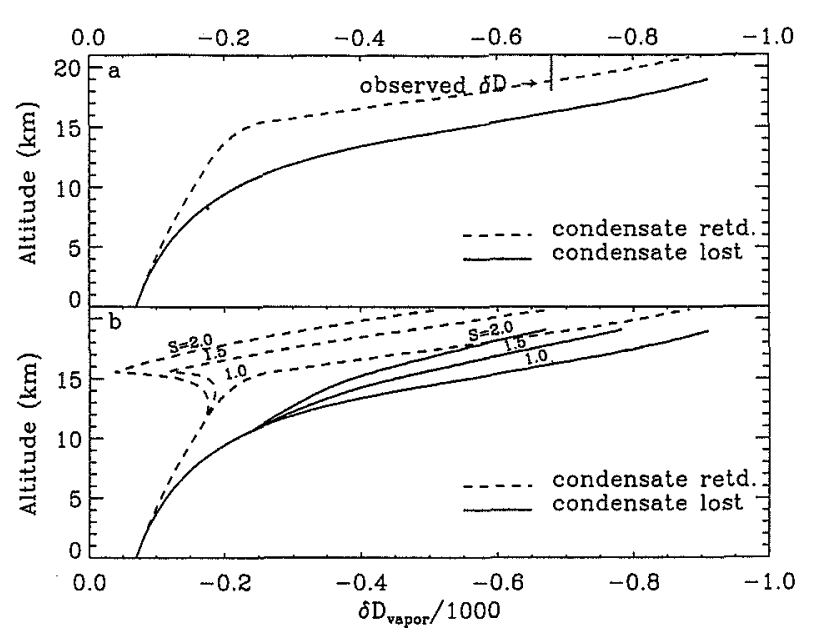

Figure 2. Model calculations showing the isotopic composition of water vapor during ascent to the stratosphere: upper panel, for conditions of thermodynamic equilibrium; lower panel, with fractionation factor modified by supersaturated conditions. Solid/dashed lines represent immediate removal/total retention of liquid condensate, and bracket the full range of $\delta \mathrm{D}$ values possible in these scenarios. The strong enhancements at $10-12 \mathrm{~km}$ in high- $S$ water-retaining cases occur because glaciation is allowed to proceed by evaporation of droplets and recondensation as ice: at high $S, \alpha_{\text {vapor-ice }}$ is less than $\alpha_{\text {vapor-liquid }}$ and deuterium is pumped into the vapor. Complete vapor deposition of ice is not realistic and is an endmember case only.

these data of $3.6 \mathrm{ppm}$. (Model results are robust with respect to this value; variations in water vapor of $\pm 20 \%$ produce only minor isotopic changes). Isotopic fractionation occurs during the initial evaporation of seawater, the condensation of liquid and ice, and the conversion of liquid to ice as the cloud glaciates. Cloud liquid is allowed to re-equilibrate with cloud vapor, while ice is effectively removed from the vapor. Free parameters of the model are: surface temperature and relative humidity, temperatures of the onset of ice nucleation and the completion of glaciation, the relative importance of droplet freezing to evaporation and re-deposition during glaciation, the degree of supersaturation over ice in the final stages of ascent, and the precipitation (or lack thereof) in all condensing stages. Although simplified, the model captures the full range of possible conditions for a simple convective updraft.

In the first set of model runs isotopic partitioning was assumed to occur at thermodynamic equilibrium in all stages, with the temperature dependence of $\alpha$ taken from Majoube [1971] and Merlivat and Nief [1967]. In these conditions, model-generated final isotopic compositions at the tropical tropopause in these conditions are all considerably lighter than observed stratospheric water (Figure 2a). Stratospheric water vapor is highly depleted of deuterium, but it is less depleted than would be expected given the extent of dehydration experienced. While at lower altitudes, where liquid is present, vapor isotopic compositions can be substantially altered by choice of model parameters, all trajectories converge during the nearly $7 \mathrm{~km}$ of ascent in ice-only conditions from the homogeneous freezing point of liquid water at $\approx 233 \mathrm{~K}$ to the tropical tropopause at $\approx 190 \mathrm{~K}$. Dehydration in those last kilometers strips out virtually all deuterium: vapor concentration must drop by a factor of over a hundred, with strong fractionation at $\alpha$ $=1.3-1.4$, so vapor $\mathrm{D} / \mathrm{H}$ must drop by over $80 \%$ over this altitude range alone. Even if no depletion occurred until the onset of ice condensation, the final $\delta \mathrm{D}$ under these conditions would still be less than -800 .

The deuterium content of stratospheric water can be increased only if we postulate that (1) air parcels in this $10-17 \mathrm{~km}$ region receive additional contributions from sources that are not in equilibrium with the vapor, or (2) isotopic fractionation is weaker than equilibrium values imply. For postulate (1), lofted cloud ice is the only plausible source of non-equilibrated water in the upper troposphere or lower stratosphere. While liquid water can rapidly exchange and equilibrate with its environment, the isotopic composition of ice remains essentially fixed [Jouzel and Merlivat, 1984]. Ice particles carried upwards from their altitudes of condensation thus preserve anomalously heavy isotopic compositions in relation to their surrounding vapor, and, if later evaporated, serve to enrich the vapor $\mathrm{D} / \mathrm{H}$ ratio.

The second postulate, that isotopic fractionation has been reduced, is possible during ice deposition in highly supersaturated air parcels, where kinetic effects prevent the vapor and condensate from achieving their equilibrium isotopic partitioning [Jouzel and Merlivat, 1984]. Figure $2 \mathrm{~b}$ shows isotopic trajectories calculated using this kinetic fractionation, for a range of supersaturations $(S)$. Because the condensing material extracts less deuterium, the residual vapor remains heavier; at $S$ of 1.5 to $2(150-200 \%)$, depending on convective parameters, sufficient deuterium is left at the tropopause to match the observed stratospheric isotopic composition.

Either of these scenarios is possible only in strong convective systems. Supersaturations of $1.5-2$ can be sustained only by updraft velocities typical of the strongest cumulus cores; in steady state, approximately $20-50 \mathrm{~m} / \mathrm{s}$ for the ice particle size distribution observed during the STEP campaign in tropical near-tropopause cloud systems [Knollenberg et al., 1993; Rogers and Yau, 1989; Pruppacher and Klett, 1980]. Ice crystal evaporation, on the other hand, can significantly alter the isotopic composition of vapor only if the ice crystals are substantially out of equilibrium with that vapor, i.e. if evaporation occurs at altitudes significantly higher than the level of condensation, again a condition possible only in strong convective updrafts. (Temperature fluctuations of 5-8 degrees in stratospheric waves can produce only minor enrichments.)

Deuterium enrichment by either of these processes must occur near the tropopause in order for some effect to persist into the stratosphere. Water vapor in a rising air parcel is depleted from its original composition to observed stratospheric values in less than $3 \mathrm{~km}$ of ascent at upper tropospheric temperatures, so non-equilibrium deuterium contributions must be occurring in the last 
$3 \mathrm{~km}$ beneath the tropopause. Most water entering the stratosphere must experience convective conditions to at least the uppermost troposphere.

\section{Conclusions}

ATMOS deuterated water data constrain the process of STE to one of two scenarios. In the first, STE is closely associated with deep convection. Actual crosstropopause transport may occur either as penetrative convection which deposits air above the tropopause or as more gradual ascent in and above mesoscale convective systems; in the latter case the convective systems contributing to STE must reach altitudes of at least 14 to $15 \mathrm{~km}$ (for a $17 \mathrm{~km}$ tropopause). In the second, STE occurs over wider parts of the tropics, less directly associated with convective systems, but here water in the uppermost troposphere consists almost exclusively of enriched water from convective systems, most likely evaporated cloud ice. Again, this evaporation must occur at $14-15 \mathrm{~km}$ altitude or higher.

Further observations in the tropical lower stratosphere and upper troposphere are necessary for discriminating between these possibilities. Isotopic data from the stratosphere alone may be sufficient to demonstrate whether the final dehydration of stratospheric air occurs in slow ascent rather than in convective penetration with admixture of evaporated ice, if a clear seasonal cycle in stratospheric $\delta \mathrm{D}_{\mathrm{w}}$ is detectable. Upper tropospheric data are necessary to determine spatial scales of transport. We conclude that a high priority should be placed on obtaining further tropical measurements of $\mathrm{HDO}$ and $\mathrm{H}_{2} \mathrm{O}$ at these altitudes, and that these observations should provide substantial additional insight into mechanisms of troposphere-stratosphere transport.

Acknowledgments. We thank Michael Brown for his comments and suggestions on this manuscript, and David Keith and Nilton Renno for useful discussions. EJM acknowledges the support of a National Science Foundation Graduate Fellowship and a NASA Global Change Graduate Fellowship. This research was supported in part by NASA grant NAGW-413 to the California Institute of Technology.

\section{References}

Abbas, M. M. et al., Seasonal variations of water vapor in the lower stratosphere inferred from ATMOS/ATLAS-3 measurements of $\mathrm{H}_{2} \mathrm{O}$ and $\mathrm{CH}_{4}$, Geophys. Res. Lett., this issue, 1996.

Brewer, A. W., Evidence for a world circulation provided by the measurements of helium and water vapour distributions in the stratosphere, Quart. J. R. Met. Soc., 75, $187,1949$.

Carli, B. and J. Park, Simultaneous measurement of minor stratospheric constituents with emission far-infrared spectroscopy, J. Geophys. Res., 93, 33851, 1988.

Danielsen, E. F., A dehydration mechanism for the stratosphere, Geophys. Res. Lett., 9, 605, 1982.

Dinelli, B. M. et al., Measurement of stratospheric distributions of $\mathrm{H}_{2}^{16} \mathrm{O}, \mathrm{H}_{2}^{18} \mathrm{O}, \mathrm{H}_{2}^{17} \mathrm{O}$, and $\mathrm{HD}^{16} \mathrm{O}$ from far-infrared spectra, J. Geophys. Res., 96, 7509, 1991.
Ehhalt, D. H. Methane in the atmosphere, in Carbon and the Biosphere, G. M. Woodwell and E. V. Pecan, eds., Atomic Energy Commission, 1973.

Frederick, J. E. and A. R. Douglass, Atmospheric temperatures near the tropical tropopause: temporal variations, zonal asymmetry, and implications for stratospheric water vapor, Mon. Weather Rev., 111, 1397, 1983.

Gunson, M. R. et al., The Atmospheric Trace Molecular Spectroscopy (ATMOS) experiment deployment on the ATLAS-3 space shuttle mission, Geophys. Res. Lett., this issue, 1996.

Hageman, R. et al., Absolute D/H ratio for SMOW, Tellus, 22, 712,1970 .

Houze, R. A., Observed structure of mesoscale convective systems and implications for large-scale heating, $Q . J . R$. Meteo. Soc., 115, 425, 1989.

Irion, F. W. et al., Stratospheric observations of $\mathrm{C}_{3} \mathrm{D}$ and HDO from ATMOS infrared solar spectra: enrichments of deuterium in methane and implications for $\mathrm{HD}$, Geophys. Res. Lett., this issue, 1996.

Jouzel, J. and L. Merlivat, Deuterium and oxygen-18 in precipitation: modeling of the isotopic effects during snow formation, J. Geophys. Res., 89, 11749, 1984.

Knollenberg, R. G. et al., Measurements of high number densities of ice crystals in the tops of tropical cumulonimbus, J. Geophys. Res., 98, 8639, 1993.

Majoube, M., Fractionation of oxygen 18 and of deuterium between water and its vapor, J. Chem. Phys., 68, 1423, 1971.

Merlivat, L. and G. Nief, Isotopic fractionation of the solidvapor and liquid-vapor changes of state of water at temperatures below 0C, Tellus, 19, 122, 1967.

Newell, R. E. and S. Gould-Stewart, A stratospheric fountain? J. Atmos. Sci., 38, 2789, 1981.

Pollock, W. et al., Measurement of stratospheric water vapor by cryogenic collection, J. Geophys. Res., 85, 5555, 1980.

Potter, B. E. and J. R. Holton, The role of monsoon convection in dehydration of the lower tropical stratosphere, J. Atmos. Sci., 52, 1034, 1994.

Pruppacher, H. R. and J. D. Klett, Microphysics of clouds and precipitation, D. Reidel Co., 1980.

Riehl, H. and J. S. Malkus, On the heat balance in the equatorial trough zone, Geophysica, 6, 503, 1958.

Rinsland, C. P. et al., Stratospheric profiles of heavy water isotopes and $\mathrm{CH}_{3} \mathrm{D}$ from analysis of the ATMOS Spacelab 3 infrared solar spectra, J. Geophys. Res., 96, 1057, 1991.

Rinsland, C. P. et al., Simultaneous stratospheric measurements of $\mathrm{H}_{2} \mathrm{O}, \mathrm{HDO}$, and $\mathrm{CH}_{4}$ from balloon-borne and aircraft infrared solar absorption spectra and tunable diode laser laboratory spectra of HDO, J. Geophys. Res., 89, $7259,1984$.

Rogers, R. R. and M. K. Yau, A short course in cloud physics, Pergamon Press, 1989.

World Meteorological Organization, Scientific Assessment of Ozone Depletion, 1994.

E. J. Moyer and Y. L. Yung, Division of Geological and Planetary Sciences, Caltech, Pasadena, CA 91125 (email: moyer@earth1.gps.caltech.edu)

Fredrick W. Irion, Dept. of Chemistry and Chemical

Engineering, Caltech, Pasadena, CA 91125.

M. R. Gunson, Jet Propulsion Laboratory, Pasadena, CA 91109

(received November 3, 1995; revised January 23, 1996; accepted April 4, 1996.) 


\title{
A comparison of measurements from ATMOS and instruments aboard the ER-2 aircraft: Tracers of atmospheric transport
}

\author{
A. Y. Chang, ${ }^{1}$ R. J. Salawitch, ${ }^{1}$ H. A. Michelsen, ${ }^{2}$ M. R. Gunson, ${ }^{1}$ M. C. Abrams, ${ }^{3}$ \\ R. Zander, ${ }^{4}$ C. P. Rinsland, ${ }^{5}$ M. Loewenstein, ${ }^{6}$ J. R. Podolske, ${ }^{6}$ M. H. Proffitt, ${ }^{7,8}$ \\ J. J. Margitan, ${ }^{1}$ D. W. Fahey, ${ }^{7}$ R.-S. Gao, ${ }^{7}$ K. K. Kelly, ${ }^{7}$ J. W. Elkins, ${ }^{9}$ \\ C. R. Webster, ${ }^{1}$ R. D. May, ${ }^{1}$ K. R. Chan, ${ }^{6}$ M. M. Abbas, ${ }^{10}$ A. Goldman, ${ }^{11}$ \\ F. W. Irion, ${ }^{12}$ G. L. Manney, ${ }^{1}$ M. J. Newchurch, ${ }^{13}$ and G. P. Stiller ${ }^{14}$
}

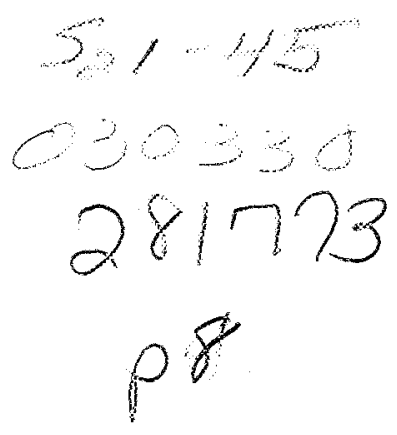

\begin{abstract}
We compare volume mixing ratio profiles of $\mathrm{N}_{2} \mathrm{O}$, $\mathrm{O}_{3}, \mathrm{NO}_{\mathrm{y}}, \mathrm{H}_{2} \mathrm{O}, \mathrm{CH}_{4}$, and $\mathrm{CO}$ in the mid-latitude lower stratosphere measured by the ATMOS Fourier transform spectrometer on the ATLAS-3 Space Shuttle Mission with in situ measurements acquired from the NASA ER-2 aircraft during Nov 1994. ATMOS and ER-2 observations of $\left[\mathrm{N}_{2} \mathrm{O}\right]$ show good agreement, as do measured correlations of $\left[\mathrm{O}_{3}\right]$, $\left[\mathrm{NO}_{\mathrm{y}}\right],\left[\mathrm{H}_{2} \mathrm{O}\right]$, and $\left[\mathrm{CH}_{4}\right]$ with $\left[\mathrm{N}_{2} \mathrm{O}\right]$. Thus a consistent measure of the hydrogen $\left(\mathrm{H}_{2} \mathrm{O}, \mathrm{CH}_{4}\right)$ content of the lower stratosphere is provided by the two platforms. The similarity of $\left[\mathrm{NO}_{y}\right]$ determined by detection of individual species by ATMOS and the total $\left[\mathrm{NO}_{\mathrm{y}}\right]$ measurement on the ER-2 provides strong corroboration for the accuracy of both techniques. A $25 \%$ discrepancy in lower stratospheric [CO] observed by ATMOS and the ER-2 remains unexplained. Otherwise, the agreement for measurements of long-lived tracers demonstrates the ability to combine ATMOS data with in situ observations for quantifying atmospheric transport.
\end{abstract}

\section{Introduction}

A near coincidence between the deployment of ATMOS (Atmospheric Trace MOlecule Spectroscopy) on the ATLAS-3 (ATmospheric Laboratory for Applications and Science) mission of Nov 1994 (STS-66) and the final two flights of the NASA ER-2 aircraft during ASHOE/MAESA (Airborne Southern Hemisphere Ozone Experiment / Measurements for Assessing the Effects of Stratospheric Aircraft) provides a valuable opportunity for the intercomparison of observations

\footnotetext{
${ }^{1}$ Jet Propulsion Laboratory, California Institute of Technology, Pasadena, CA.

${ }^{2}$ Harvard University, Cambridge, MA

${ }^{3}$ SAIC, NASA Langley Research Center, Hampton, VA.

${ }^{4}$ Institute of Astrophysics, Univ. of Liège, Liège-Cointe, Belgium.

${ }^{5}$ NASA Langley Research Center, Hampton, VA.

${ }^{6}$ NASA Ames Research Center, Moffett Field, CA.

${ }^{7}$ NOAA Aeronomy Laboratory, Boulder, CO.

${ }^{8}$ Cooperative Institute for Research in Environmental Sciences, University of Colorado, Boulder, $\mathrm{CO}$.

${ }^{9}$ NOAA Climate Monitoring and Diagnostics Lab., Boulder, CO.

${ }^{10}$ NASA Marshall Space Flight Center, Huntsville, AL.

${ }^{11}$ Department of Physics, University of Denver, Denver, CO

${ }^{12}$ California Institute of Technology, Pasadena, CA.

${ }^{13}$ University of Alabama in Huntsville, Huntsville, AL

${ }^{14}$ IMK, Forschungszentrum Karlsruhe, Karlsruhe, Germany.

Copyright 1996 by the American Geophysical Union.
}

by these vastly different measurement techniques. The ATMOS infrared Fourier transform spectrometer records highresolution solar occultation spectra to derive volume mixing ratios (VMRs) of more than 30 gaseous constituents over a wide range of altitudes and latitudes [Gunson et al., 1996]. The suite of in situ instruments aboard the ER-2 also measure VMRs of numerous gases, for altitudes below $\sim 20 \mathrm{~km}$ and geographic regions accessible to the aircraft. The ability to combine space-based and in situ measurements of radical precursors and tracers of atmospheric transport is important for understanding processes controlling the composition of the atmosphere. In this paper we compare VMRs of $\mathrm{N}_{2} \mathrm{O}, \mathrm{O}_{3}$, $\mathrm{NO}_{\mathrm{y}}$ (total nitrogen oxides: $\mathrm{NO}+\mathrm{NO}_{2}+\mathrm{HNO}_{3}+\mathrm{ClONO}_{2}+$ $\left.2 \mathrm{~N}_{2} \mathrm{O}_{5}+\mathrm{HNO}_{4}\right), \mathrm{H}_{2} \mathrm{O}, \mathrm{CH}_{4}$, and $\mathrm{CO}$. A companion paper examines halogenated species [Chang et al., 1996].

\section{ATMOS / ER-2 Flight Coincidence}

The ATMOS instrument observed solar occultations from Space Shuttle Atlantis for a 10 day period beginning on 4 Nov 1994. The first sunset occultation (SS01) occurred at $49^{\circ} \mathrm{N}, 126^{\circ} \mathrm{W}$, and subsequent sunsets were recorded at latitudes progressively southward and westward, separated in longitude by $\sim 23^{\circ}$. Each ATMOS occultation consists of a progression of atmospheric transmission spectra obtained at different tangent heights. Vertical VMR profiles are retrieved by fitting absorption features of a target gas in one or several spectral intervals using an 'onion-peeling' algorithm [Gunson et al., 1996]. The retrieval process is iterated three times to minimize propagation of errors and a priori assumptions. During the spectral-fitting, the background transmission is itself a fitted parameter, so that ATMOS retrievals are largely unaffected by changes in background transmission due to broadband absorption by aerosols. Retrieval of constituents is preceded by a combination of pressure and temperature sounding, using an assumed vertical profile of $\mathrm{CO}_{2}$ [Gunson et al., 1996]. In the ATLAS-3 data, the tangent height spacing varied from 1 to $4 \mathrm{~km}$, with the smaller separations occurring at lower tangent heights and more tropical latitudes. The vertical resolution, set by the instrument field-of-view, is 3-4 $\mathrm{km}$. ATMOS profiles are reported on a pressure grid of 12 points per decade.

The ER-2 aircraft was deployed over an eight month period in mid to late 1994 in support of the ASHOE/MAESA campaign, designed to investigate the chemistry and transport of a vast region of the lower stratosphere. Although most flights of ASHOE/MAESA occurred in either the tropics or the southern hemisphere, the second-to-last flight on 2 Nov 1994 marked the return of the ER-2 from Hawaii to its 
within $15^{\circ}$ latitude and $30^{\circ}$ longitude of Moffett Field. Optical bandpass filters, used to improve the signal-to-noise of the ATMOS measurement, determine which set of species were measured in each occultation. The coincidence group includes five Filter $3\left(1580-3340 \mathrm{~cm}^{-1}\right)$, one Filter 4 (3150$\left.4800 \mathrm{~cm}^{-1}\right)$, two Filter $9\left(600-2450 \mathrm{~cm}^{-1}\right)$, and four Filter 12 $\left(600-1400 \mathrm{~cm}^{-1}\right)$ observations. All ATMOS observations presented here are Version 2 data [Gunson et al., 1996].

\section{Measurement Comparison}

Measurements of long-lived constituents are better compared by examining correlations with gases, rather than vertical profiles, to account for variations in dynamical history of differing air masses [Loewenstein et al., 1989]. $\mathrm{N}_{2} \mathrm{O}$ is a suitable reference for comparing measurements of a variety of tracers of atmospheric transport, as it has a long stratospheric lifetime, is retrieved from all ATMOS filters, and is measured aboard the ER-2 at high temporal frequency and accuracy. To provide context to the correlation of other gases with $\left[\mathrm{N}_{2} \mathrm{O}\right.$ ] (throughout, [] denotes VMR), vertical profiles of $\left[\mathrm{N}_{2} \mathrm{O}\right]$ obtained by ATMOS and the ER-2 are shown in Fig. 2.

ATMOS observes $\left[\mathrm{N}_{2} \mathrm{O}\right]$ with a $1 \sigma$ accuracy (systematic uncertainty) of $5 \%$ and estimated precisions (random errors) of better than $5 \%$ at altitudes above that corresponding to a pressure of 200 mbar. In Fig. 2, error bars indicate estimated $1 \sigma$ precisions for a single ATMOS profile of $\left[\mathrm{N}_{2} \mathrm{O}\right]$. Random errors in the vertical coordinate have been folded into the horizontal (constituent) errors. Estimated precisions reported by ATMOS are calculated $a$ priori during the retrieval process and are based on considerations such as residual errors in spectral fitting. The reported precision for many ATMOS gases is comparable to the standard deviation of an ensemble of profiles observed in cases where the natural variability is small [Abrams et al., 1996]. Accuracies are derived by combining uncertainties in the spectroscopic band intensities of the measured species with those of $\mathrm{CO}_{2}$, whose retrieval is used to assign tangent pressures for each spectra. The precision and accuracy of ATMOS retrievals for all species are discussed at greater length in Abrams et al. [1996]. Systematic uncertainties are not included in the plotted ATMOS error bars, as these reflect possible biases which are best considered separately when comparing measurements obtained by different techniques.

The Airborne Tunable Laser Absorption Spectrometer (ATLAS) [Loewenstein et al., 1989] measures $\left[\mathrm{N}_{2} \mathrm{O}\right.$ ] on the ER2 by monitoring absorption at $2230 \mathrm{~cm}^{-1}$, at a sampling interval of $1 \mathrm{~Hz}$ and a $1 \sigma$ total uncertainty of $3 \%$. The Meteorological Measurement System (MMS) [Chan et al., 1989] aboard the ER-2 measures pressure, at a frequency of 5 $\mathrm{Hz}$, to accuracies of 0.5 mbar. Profiles of $\left[\mathrm{N}_{2} \mathrm{O}\right]$ vs pressure from the ER-2 are plotted in Fig. 2. In this study, all in situ observations obtained at a sampling rate of $>0.1 \mathrm{~Hz}$ have been median filtered to $0.1 \mathrm{~Hz}$. Uncertainties for the in situ data are reported but not plotted for clarity.

Figure 2 demonstrates that the ER-2 aircraft is able to sample a wide range of $\left[\mathrm{N}_{2} \mathrm{O}\right]$ despite its altitude limitations. In fact, the ER-2 encounters its smallest $\left[\mathrm{N}_{2} \mathrm{O}\right]$ air at lower altitudes than comparable observations by ATMOS. Similar levels of dynamical variability are revealed by both sets of measurements (one ATMOS profile in particular shows considerable descent, but exhibits correlations indistinguishable from the other profiles). The variability exhibited in Fig. 2 obscures any direct comparison, although data for $\left[\mathrm{N}_{2} \mathrm{O}\right]$ near the tropopause suggest a $+2 \%$ bias of ATMOS compared with the ER-2 measurements (throughout, $\%$ biases are computed as [ATMOS - ER-2] / ATMOS). Worth noting is that ATMOS and ER-2 sample different spatial scales; while ATMOS effectively averages over an optical path several hundred kilometers long and a few kilometers in diameter, the ER-2, traveling at $\sim 200 \mathrm{~m} / \mathrm{s}$, integrates over relatively short distances along the flight path only.

The ACATS-IV (Airborne Chromatograph for Atmospheric Trace Species) gas chromatograph on the ER-2 reports $\left[\mathrm{N}_{2} \mathrm{O}\right]$ every 6 minutes with a $1 \sigma$ precision of $0.9 \%$ and an accuracy of $2 \%$ [Elkins et al., 1996], and is calibrated by running a sample of air at mixing ratios near stratospheric levels once every 4 ambient samples or $30 \mathrm{~min}$. Although not shown, measurements of $\left[\mathrm{N}_{2} \mathrm{O}\right]$ by ACATS-IV agree to better than $1 \%$ with those of ATLAS for the flight segments considered here. All subsequent correlations of ER-2 data in this study use the more frequent ATLAS observations. Observations of $\left[\mathrm{N}_{2} \mathrm{O}\right]$ (and $\left[\mathrm{CH}_{4}\right]$ ) from the ALIAS instrument [Webster et al., 1994] were unavailable for these flights.

Figure 3a shows a comparison of measured correlations of $\left[\mathrm{O}_{3}\right]$ with $\left[\mathrm{N}_{2} \mathrm{O}\right]$. ATMOS reports $\left[\mathrm{O}_{3}\right]$ for Filters 3, 9, and 12 with a $1 \sigma$ systematic uncertainty of $6 \%$ and estimated precisions of better than $5 \%$ at altitudes above 70 mbar, degrading with lower altitude to $15 \%$ at 120 mbar. The NOAA dual-beam ultraviolet absorption photometer obtains in situ measurements of $\left[\mathrm{O}_{3}\right]$ on board the ER-2 at $1 \mathrm{~Hz}$ with a $1 \sigma$ total uncertainty of better than 5\% [Proffitt et al., 1990]. Variability in the correlation is evident in both sets of measurements, especially in the ATMOS data in the mid and upper stratosphere, indicative of mixing with ozone-rich air from tropical latitudes. The ATMOS and ER-2 correlations of $\left[\mathrm{O}_{3}\right]$ vs $\left[\mathrm{N}_{2} \mathrm{O}\right]$ differ in mean from $+28 \%\left(+0.9\right.$ ppmv of $\left.\left[\mathrm{O}_{3}\right]\right)$ at $\left[\mathrm{N}_{2} \mathrm{O}\right]=170 \mathrm{ppbv}$ to $-28 \%\left(-0.2 \mathrm{ppmv}\right.$ of $\left.\left[\mathrm{O}_{3}\right]\right)$ at $\left[\mathrm{N}_{2} \mathrm{O}\right]=280$ ppbv. The computed difference at low $\left[\mathrm{N}_{2} \mathrm{O}\right]$ is exaggerated by a few high ATMOS values; nevertheless a residual difference is apparent. Since $\left[\mathrm{N}_{2} \mathrm{O}\right] \approx 170$ ppbv was sampled at different altitudes by the two platforms, this difference may be due to the altitude dependence of $\left[\mathrm{O}_{3}\right]$ vs $\left[\mathrm{N}_{2} \mathrm{O}\right]$ observed in previous ER-2 campaigns [e.g., Fig. 1c of Proffitt et al., 1990]. A comparison of $\left[\mathrm{O}_{3}\right]$ measured from ATMOS and the space-based SAGE II (Stratospheric Aerosol and Gas Experiment) and MLS (Microwave Limb Sounder) instruments shows agreement within $5 \%$ for the mid and upper stratosphere [Abrams et al., in preparation].

Correlations of $\left[\mathrm{NO}_{y}\right]$ vs $\left[\mathrm{N}_{2} \mathrm{O}\right]$ are shown in Fig. $3 \mathrm{~b}$. ATMOS independently measures the VMR of each primary species in the $\mathrm{NO}_{\mathrm{y}}$ family: $\mathrm{NO}, \mathrm{NO}_{2}, \mathrm{HNO}_{3}, \mathrm{ClONO}_{2}, \mathrm{~N}_{2} \mathrm{O}_{5}$, and $\mathrm{HNO}_{4}$. Profiles of $[\mathrm{NO}],\left[\mathrm{NO}_{2}\right]$, and $\left[\mathrm{HNO}_{3}\right]$ are each measured in Filters 3 and 9 and together comprise more than $85 \%$ of $\left[\mathrm{NO}_{\mathrm{y}}\right]$. In the present analysis, ATMOS $\left[\mathrm{NO}_{\mathrm{y}}\right]$ is determined by combining $[\mathrm{NO}],\left[\mathrm{NO}_{2}\right]$, and $\left[\mathrm{HNO}_{3}\right]$ measured in each Filter 3 and 9 .occultation with average profiles of $\left[\mathrm{ClONO}_{2}\right]$, $\left[\mathrm{N}_{2} \mathrm{O}_{5}\right]$, and $\left.\mathrm{HNO}_{4}\right]$, from the four Filter 12 occultations (averaged on isobaric surfaces). Estimated accuracies and precisions for the individual components of the $\mathrm{NO}_{\mathrm{y}}$ family are described in Abrams et al. [1996]. Based on weighting the uncertainties of each $\mathrm{NO}_{y}$ species, the ATMOS measurement of total $\left[\mathrm{NO}_{\mathrm{y}}\right]$ is estimated to have $1 \sigma$ precisions of better than $10 \%$ for altitudes between 2 and 50 mbar (rapidly degrading at lower altitudes), and systematic uncertainties of $15 \%$ near 50 mbar (where $\mathrm{HNO}_{3}$ dominates the budget of $\mathrm{NO}_{y}$ ), 


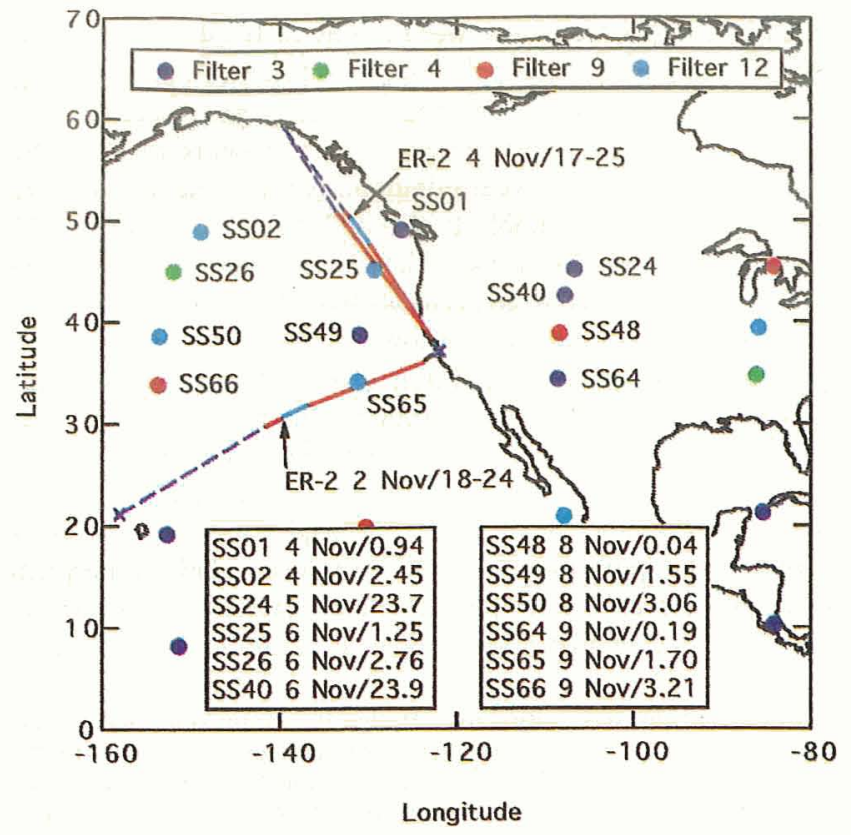

Figure 1. ATMOS/ATLAS-3 observations coincident with ER2 aircraft flights of 2 and 4 Nov 1994. The 12 labeled ATMOS occultations (symbols) and ER-2 flight segments denoted by solid lines $\left(30^{\circ}\right.$ to $\left.51^{\circ} \mathrm{N}\right)$ are considered in this study. The location of the ER-2 dives are indicated by the short segments at $31^{\circ}$ and $49^{\circ} \mathrm{N}$. Dates and universal time (hr) are indicated.

base at Moffett Field, CA $\left(37^{\circ} \mathrm{N}, 122^{\circ} \mathrm{W}\right)$. The last flight, on 4 Nov, was a round trip from Moffett Field which extended to the southern coast of Alaska. The trajectories of these flights and locations of nearby ATMOS observations are illustrated in Fig. 1. To focus the present comparisons on mid-latitudes, only the ER-2 flight segments between $30^{\circ}$ and $51^{\circ} \mathrm{N}$ are considered. For the 2 Nov flight, this includes half of the cruise portion, a dive near $31^{\circ} \mathrm{N}$, and the descent over Moffett Field; for 4 Nov this encompasses both ascent and descent, half of the outgoing and incoming cruise legs, and a dive on the return route at $49^{\circ} \mathrm{N}$.

The twelve ATMOS coincidences identified in Fig. 1 occur $\sim 1$ day earlier to 4 days later than the 4 Nov flight of the ER-2,

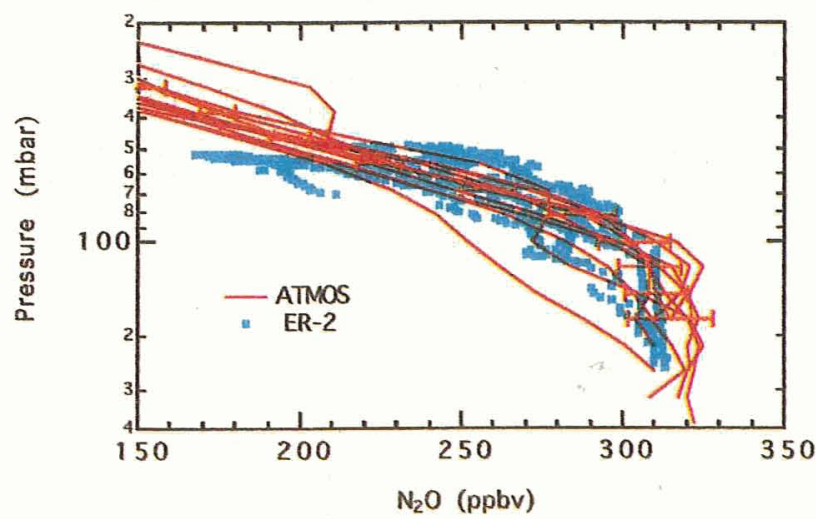

Figure 2. Vertical profiles of $\left[\mathrm{N}_{2} \mathrm{O}\right]$ measured from ATMOS and instruments aboard the ER-2 aircraft: $\mathrm{N}_{2} \mathrm{O}$ (ATLAS), Loewenstein et al.; pressure (MMS), Chan et al. Estimated $1 \sigma$ precision error bars for one ATMOS occultation are shown.
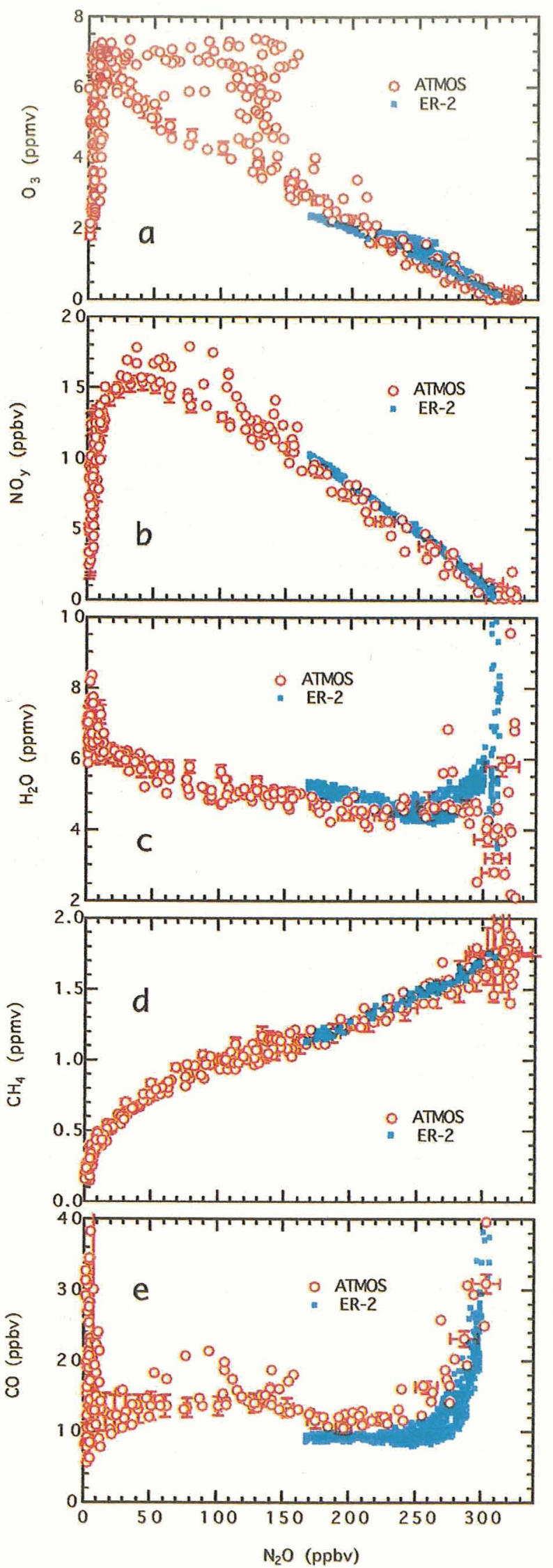

Figure 3. Correlations of $\left[\mathrm{O}_{3}\right],\left[\mathrm{NO}_{\mathrm{y}}\right],\left[\mathrm{H}_{2} \mathrm{O}\right],\left[\mathrm{CH}_{4}\right]$, and $[\mathrm{CO}]$ vs $\left[\mathrm{N}_{2} \mathrm{O}\right]$ measured from ATMOS and instruments aboard the ER-2 aircraft: $\mathrm{N}_{2} \mathrm{O}$ (ATLAS), Loewenstein et al.; $\mathrm{O}_{3}$, Proffitt et al.; $\mathrm{NO}_{\mathrm{y}}$, Fahey et al.; $\mathrm{H}_{2} \mathrm{O}$, Kelly et al.; $\mathrm{CH}_{4}$ (ACATS-IV), Elkins et al.; CO (ALIAS), Webster et al. 
improving to $\sim 6 \%$ in the upper stratosphere (where NO and $\mathrm{NO}_{2}$ are the primary components). ATMOS data presented here were retrieved without accounting for the variation of the concentration of $\mathrm{NO}$ and $\mathrm{NO}_{2}$ across the day-night terminator, which could result in errors in $\left[\mathrm{NO}_{\mathrm{y}}\right]$ of $<0.2 \mathrm{ppbv}$ in the lower stratosphere [Newchurch et al., 1996].

The NOAA NO-NO $\mathrm{N}_{\mathrm{y}}$ chemiluminescence detector obtains $\left[\mathrm{NO}_{\mathrm{y}}\right]$ measurements on the ER-2 at a frequency of $1 \mathrm{~Hz}$ with a $1 \sigma$ total uncertainty of better than $10 \%$ [Fahey et al., 1989]. A gold catalyst converts all forms of $\mathrm{NO}_{\mathrm{y}}$ to $\mathrm{NO}$ prior to detection, with equal sensitivity to the nitrogen abundance of all gas-phase $\mathrm{NO}_{\mathrm{y}}$ species. The mean difference between ATMOS and ER-2 measurements varies from $-8 \%(-0.7 \mathrm{ppbv}$ of $\left.\left[\mathrm{NO}_{\mathrm{y}}\right]\right)$ at $\left[\mathrm{N}_{2} \mathrm{O}\right]=170 \mathrm{ppbv}$ to $-13 \%\left(-0.3 \mathrm{ppbv}\right.$ of $\left.\left[\mathrm{NO}_{\mathrm{y}}\right]\right)$ at $\left[\mathrm{N}_{2} \mathrm{O}\right]=280 \mathrm{ppbv}$, with an average deviation of $-0.6 \mathrm{ppbv}$ for all values of $\left[\mathrm{N}_{2} \mathrm{O}\right]$. The consistency of $\left[\mathrm{NO}_{\mathrm{y}}\right]$ determined by observation of each individual species by ATMOS with values of total $\left[\mathrm{NO}_{\mathrm{y}}\right]$ measured by chemiluminescence following chemical conversion of each $\mathrm{NO}_{\mathrm{y}}$ component to NO provides strong corroboration for the accuracy of both methods. ATMOS measurements extend the $\left[\mathrm{NO}_{\mathrm{y}}\right]$ and $\left[\mathrm{N}_{2} \mathrm{O}\right]$ relation to high altitude, and show the decrease in $\left[\mathrm{NO}_{\mathrm{y}}\right]$ above $40 \mathrm{~km}$ due to the reaction $\mathrm{N}+\mathrm{NO}$.

Figure $3 \mathrm{c}$ shows observations of $\left[\mathrm{H}_{2} \mathrm{O}\right]$ vs $\left[\mathrm{N}_{2} \mathrm{O}\right]$. ATMOS retrieves $\left[\mathrm{H}_{2} \mathrm{O}\right]$ in Filters 3,4 , and 9 with a $1 \sigma$ systematic uncertainty of $6 \%$ and estimated precisions of better than $5 \%$ at altitudes above 200 mbar. The NOAA Lyman- $\alpha / \mathrm{OH}$ resonance fluorescence hygrometer measures $\left[\mathrm{H}_{2} \mathrm{O}\right]$ on the ER2 at a frequency of $1 \mathrm{~Hz}$ and a $1 \sigma$ total uncertainty of $5 \%$ [Kelly et al., 1989]. ATMOS Version 2 retrievals of $\left[\mathrm{H}_{2} \mathrm{O}\right]$ occasionally suffer instabilities due to the sudden change in the slope of the profile at the hygropause, as particularly evident by one occultation in the coincidence group. Otherwise, the agreement between the ATMOS and NOAA measurements of $\left[\mathrm{H}_{2} \mathrm{O}\right]$ is good; differences in mean range from $-8 \%\left(-0.4 \mathrm{ppmv}\right.$ of $\left.\left[\mathrm{H}_{2} \mathrm{O}\right]\right)$ at $\left[\mathrm{N}_{2} \mathrm{O}\right]=170 \mathrm{ppbv}$ to $+6 \%$ $\left(+0.2 \mathrm{ppmv}\right.$ of $\left.\left[\mathrm{H}_{2} \mathrm{O}\right]\right)$ at $\left[\mathrm{N}_{2} \mathrm{O}\right]=260$ ppbv. A second ER-2 Lyman- $\alpha$ hygrometer, not deployed during ASHOE/MAESA, observed VMRs of $\left[\mathrm{H}_{2} \mathrm{O}\right] \sim 15 \%$ higher than the NOAA instrument [Hintsa et al., 1994], a difference larger than seen in the present comparison.

Correlations of $\left[\mathrm{CH}_{4}\right]$ vs $\left[\mathrm{N}_{2} \mathrm{O}\right]$ are shown in Fig. 3 d. ATMOS measures $\left[\mathrm{CH}_{4}\right]$ in all filters, with a $1 \sigma$ systematic uncertainty of $5 \%$ and estimated precisions of better than $5 \%$ at altitudes above 150 mbar. ACATS-IV measures $\left[\mathrm{CH}_{4}\right]$ on the ER-2 at a sampling interval of 3 min with a $1 \sigma$ precision of $1.5 \%$ and an accuracy of $2 \%$ [Elkins et al., 1996]. Agreement in mean between the ATMOS and ACATS-IV measurements of $\left[\mathrm{CH}_{4}\right]$ is better than $\pm 3 \%\left( \pm 0.05 \mathrm{ppmv}\right.$ of $\left[\mathrm{CH}_{4}\right]$ at $\left[\mathrm{N}_{2} \mathrm{O}\right]=300$ ppbv) for all values of $\left[\mathrm{N}_{2} \mathrm{O}\right]$. Further analysis of ATMOS data shows variations of the $\left[\mathrm{CH}_{4}\right]$ vs $\left[\mathrm{N}_{2} \mathrm{O}\right]$ correlation depending on geographic region due to differences in the latitudinal dependence of the photochemical sinks, suggesting this correlation can be used as an indicator of mixing between air of tropical and mid-latitude origin.

Figure $3 \mathrm{e}$ shows correlations of $[\mathrm{CO}]$ vs $\left[\mathrm{N}_{2} \mathrm{O}\right]$. ATMOS observes [CO] in Filters 3 and 9 with a $1 \sigma$ systematic uncertainty of $5 \%$ and estimated precisions of better than $10 \%$ below altitudes of $20 \mathrm{mbar}$, improving to $5 \%$ at $100 \mathrm{mbar}$. The ALIAS (Aircraft Laser Infrared Absorption Spectrometer) diode laser instrument [Webster et al., 1994] provides [CO] measurements on the ER-2 at an interval of $3.5 \mathrm{~s}$ with a $1 \sigma$ precision of $10 \%$ and an accuracy of $5 \%$. The observations of
[CO] by ALIAS are $23 \%$ lower ( 3 ppbv of [CO]) than those of ATMOS at $\left[\mathrm{N}_{2} \mathrm{O}\right]<220 \mathrm{ppbv}$ and $41 \%$ lower (7 ppbv of [CO]) at $\left[\mathrm{N}_{2} \mathrm{O}\right]=270 \mathrm{ppbv}$, a difference larger than expected based on the respective uncertainties. Measurements of [CO] by ALIAS in Nov 1995 reveal minimum [CO] in the stratosphere of 12-13 ppbv, comparable to the ATMOS observations of 12 ppbv obtained in Nov 1994. The cause of the discrepancy in [CO] in Nov 1994 between ATMOS and ALIAS is unclear.

In this paper we have shown good agreement among measurements of $\left[\mathrm{N}_{2} \mathrm{O}\right],\left[\mathrm{O}_{3}\right],\left[\mathrm{NO}_{\mathrm{y}}\right],\left[\mathrm{H}_{2} \mathrm{O}\right]$, and $\left[\mathrm{CH}_{4}\right]$ obtained by ATMOS and instruments aboard the ER-2. The comparisons presented here demonstrate that high resolution in situ measurements of tracers can be combined with spacebased measurements to study atmospheric transport over broad geographic regions and altitudes. This synergism enhances our ability to quantify the dynamical and chemical processes that regulate the abundance of stratospheric ozone.

Acknowledgments. Research at the Jet Propulsion Laboratory, California Institute of Technology, is performed under contract with the National Aeronautics and Space Administration (NASA). ATMOS and ER-2 studies are supported in part by NASA's Upper Atmosphere Research Program and Atmospheric Effects of Aviation Project. We thank the Shuttle astronauts and ER-2 pilots for making these observations possible. The helpful comments of the referees are appreciated.

\section{References}

Abrams, M. C. et al., On the assessment and uncertainty of atmospheric trace gas burden measurements with high resolution infrared solar occultation spectra from space, Geophys. Res. Lett., this issue, 1996.

Chan, K. R., S. G. Scott, T. P. Bui, S. W. Bowen, and J. Day, Temperature and horizontal wind measurements on the ER-2 aircraft during the 1987 Airborne Antarctic Ozone Experiment, J. Geophys. Res., 94, 11573-11587, 1989.

Chang, A. Y. et al., A comparison of measurements from ATMOS and instruments aboard the ER-2 aircraft: Halogenated gases, Geophys. Res. Lett., this issuc, 1996

Elkins, J. W. et al., Airborne gas chromatograph for in situ measurements of long-lived species in the upper troposphere and lower stratosphere, Geophys. Res. Lett., 23, 347-350, 1996.

Fahey, D. W. et al., In situ measurements of total reactive nitrogen, total water, and aerosol in a polar stratospheric cloud in the Antarctic, $J$. Geophys. Res., 94, 11299-11315, 1989.

Gunson, M. R. et al., The Atmospheric Trace Molecule Spectroscopy (ATMOS) experiment: Deployment on the ATLAS Space Shuttle missions, Geophys. Res. Lett., this issue, 1996.

Hintsa, E. J. et al., SPADE $\mathrm{H}_{2} \mathrm{O}$ measurements and the seasonal cycle of stratospheric water vapor, Geophys. Res. Lett., 21, 2559-2562, 1994.

Kelly, K. K. et al., Dehydration in the lower Antarctic stratosphere during late winter and early spring, 1987, J. Geophys. Res., 94, 11317-11357, 1989.

Loewenstein, M., J. R. Podolske, K. R. Chan, and S. E. Strahan, Nitrous oxide as a dynamical tracer in the 1987 Airborne Antarctic Ozone Experiment, J. Geophys. Res., 94, 11589-11598, 1989.

Newchurch, M. J. et al., Stratospheric $\mathrm{NO}$ and $\mathrm{NO}_{2}$ abundances from ATMOS solar-occultation measurements, Geophys. Res. Lett., this issue, 1996.

Proffitt, M. H., J. J. Margitan, K. K. Kelly, M. Loewenstein, J. R. Podolske, and K. R. Chan, Ozone loss in the Arctic polar vortex inferred from high-altitude aircraft measurements, Nature, 347, 31$36,1990$.

Webster C. R., R. D. May, C. A. Trimble, R. G. Chave, and J. Kendall, Aircraft laser infrared absorption spectrometer for in situ stratospheric measurements of $\mathrm{HCl}, \mathrm{N}_{2} \mathrm{O}, \mathrm{CH}_{4}, \mathrm{NO}_{2}$, and $\mathrm{HNO}_{3}$, Appl. Opt., 33, 454-472, 1994.

A. Y. Chang , Jet Propulsion Laboratory, 4800 Oak Grove Drive, M. S.183-301, Pasadena CA 91109 (email: aychang@cacsar.jpl.nasa.gov).

(Received October 6, 1995; revised April 25, 1996;

accepted May $7,1996$. 


\title{
A comparison of measurements from ATMOS and instruments aboard the ER-2 aircraft: Halogenated gases
}

\author{
A. Y. Chang, ${ }^{1}$ R. J. Salawitch, ${ }^{1}$ H. A. Michelsen, ${ }^{2}$ M. R. Gunson, ${ }^{1}$ M. C. Abrams, ${ }^{3}$ \\ R. Zander, ${ }^{4}$ C. P. Rinsland, ${ }^{5}$ J. W. Elkins, ${ }^{6}$ G. S. Dutton, ${ }^{6,7}$ C. M. Volk, ${ }^{6,7}$ \\ C. R. Webster, ${ }^{1}$ R. D. May, ${ }^{1}$ D. W. Fahey,${ }^{8}$ R.-S. Gao,${ }^{8}$ M. Loewenstein, ${ }^{9}$ \\ J. R. Podolske, ${ }^{9}$ R. M. Stimpfle, ${ }^{2}$ D. W. Kohn, ${ }^{2}$ M. H. Proffitt, ${ }^{6,7}$ J. J. Margitan, ${ }^{1}$ \\ K. R. Chan, ${ }^{9}$ M. M. Abbas, ${ }^{10}$ A. Goldman, ${ }^{11}$ F. W. Irion, ${ }^{12}$ G. L. Manney, \\ M. J. Newchurch, ${ }^{13}$ and G. P. Stiller ${ }^{14}$
}

\begin{abstract}
We compare volume mixing ratio profiles of $\mathrm{N}_{2} \mathrm{O}$, $\mathrm{CFC}-11, \mathrm{CFC}-12, \mathrm{CCl}_{4}, \mathrm{SF}_{6}$, and $\mathrm{HCl}$ in the mid-latitude lower stratosphere measured by the ATMOS Fourier transform spectrometer on the ATLAS-3 Space Shuttle Mission with in situ measurements acquired from the NASA ER-2 aircraft during Nov 1994. Good agreement is found between ATMOS and in situ correlations of [CFC-11], [CFC-12], and [ $\left.\mathrm{SF}_{6}\right]$ with $\left[\mathrm{N}_{2} \mathrm{O}\right]$. ATMOS measurements of $\left[\mathrm{CCl}_{4}\right]$ are $15 \%$ high compared to ER-2 data, but agree within the systematic uncertainties. ATMOS observations of $[\mathrm{HCl}]$ vs $\left[\mathrm{N}_{2} \mathrm{O}\right]$ are within $\sim 10 \%$ of ER-2 data for $[\mathrm{HCl}]>1 \mathrm{ppbv}$, but exceed in situ measurements by larger fractional amounts for smaller $[\mathrm{HCl}]$. ATMOS measurements of $\left[\mathrm{ClONO}_{2}\right]$ agree well with values inferred from in situ observations of $[\mathrm{ClO}]$, [NO], and $\left[\mathrm{O}_{3}\right]$. The sum of $[\mathrm{HCl}]$ and $\left[\mathrm{ClONO}_{2}\right]$ observed by ATMOS, supplemented by a minor contribution from [ClO] estimated with a photochemical model, is consistent with the levels of inorganic chlorine inferred from in situ measurements of chlorine source gases.
\end{abstract}

\section{Introduction}

The ATMOS (Atmospheric Trace MOlecule Spectroscopy) Fourier transform spectrometer uses solar occultation measurements to derive volume mixing ratio (VMR) profiles of more than 30 constituents in Earth's atmosphere. During the ATMOS/ATLAS-3 (ATmospheric Laboratory for Applications and Science) Space Shuttle mission of Nov

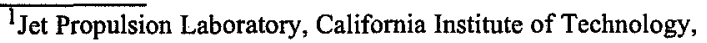
Pasadena, CA.

${ }^{2}$ Harvard University, Cambridge, MA

${ }^{3}$ SAIC, NASA Langley Research Center, Hampton, VA.

${ }^{4}$ Institute of Astrophysics, Univ. of Liège, Liège-Cointe, Belgium.

${ }^{5}$ NASA Langley Research Center, Hampton, VA.

${ }^{6}$ NOAA Climate Monitoring and Diagnostics Lab., Boulder, CO.

${ }^{7}$ Cooperative Institute for Research in Environmental Sciences,

University of Colorado, Boulder, $\mathrm{CO}$.

${ }^{8}$ NOAA Aeronomy Laboratory, Boulder, $\mathrm{CO}$.

${ }^{9}$ NASA Ames Research Center, Moffett Field, CA.

10NASA Marshall Space Flight Center, Huntsville, AL.

${ }^{11}$ Department of Physics, University of Denver, Denver, $\mathrm{CO}$

${ }^{12}$ California Institute of Technology, Pasadena, CA.

${ }^{13}$ University of Alabama in Huntsville, Huntsville, AL.

${ }^{14}$ IMK, Forschungszentrum Karlsruhe, Karlsruhe, Germany.
}

Copyright 1996 by the American Geophysical Union.
1994, twelve ATMOS occultations, occurring between 4 and 9 Nov 1994, were near-coincident with the mid-latitude (30-51 N) segments of the flights of the NASA ER-2 aircraft on 2 and 4 Nov, during ASHOE/MAESA (Airborne Southern Hemisphere Ozone Experiment / Measurements for Assessing the Effects of Stratospheric Aircraft). Chang et al. [1996] discuss more fully the geographic coincidence of the observations, compare vertical profiles of $\left[\mathrm{N}_{2} \mathrm{O}\right]$, and demonstrate good agreement for correlations of long-lived tracers $\left[\mathrm{O}_{3}\right],\left[\mathrm{NO}_{\mathrm{y}}\right],\left[\mathrm{H}_{2} \mathrm{O}\right]$, and $\left[\mathrm{CH}_{4}\right]$ with $\left[\mathrm{N}_{2} \mathrm{O}\right]$ observed from the two platforms (throughout, [] denotes VMR). This paper presents correlations of $[\mathrm{CFC}-11],[\mathrm{CFC}-12],\left[\mathrm{CCl}_{4}\right],\left[\mathrm{SF}_{6}\right]$, $\left[\mathrm{ClONO}_{2}\right],[\mathrm{HCl}]$, and $\left[\mathrm{Cl}_{\mathrm{y}}\right]$ (total inorganic chlorine) with $\left[\mathrm{N}_{2} \mathrm{O}\right]$ measured or inferred from ATMOS and the ER-2, and compares measurements of inorganic chlorine species with results of photochemical model simulations.

All comparisons in this study are presented as correlations with the long-lived tracer $\mathrm{N}_{2} \mathrm{O}$ to account for the dynamical histories of different air masses. For the ER-2 flight segments considered here, $\left[\mathrm{N}_{2} \mathrm{O}\right]$ measured by ATLAS (Airborne Tunable Laser Absorption Spectrometer) [Loewenstein et al., 1989] are within $1 \%$ of those observed by ACATS-IV (Airborne Chromatograph for Atmospheric Trace Species) [Elkins et al., 1996]. Because of the higher sampling rate, the ATLAS data for $\left[\mathrm{N}_{2} \mathrm{O}\right]$ are used for ER-2 correlations shown here. Version 2 ATMOS data are used throughout [Gunson et al., 1996].

\section{Halogen Source Gases}

ATMOS measures VMRs of CFC-11 $\left(\mathrm{CCl}_{3} \mathrm{~F}\right) ; \mathrm{CFC}-12$ $\left(\mathrm{CCl}_{2} \mathrm{~F}_{2}\right), \mathrm{HCFC}-22\left(\mathrm{CHClF}_{2}\right), \mathrm{CH}_{3} \mathrm{Cl}$, and $\mathrm{CCl}_{4}$, which together constitute $\sim 80 \%$ of total tropospheric organic chlorine [Zander et al., 1996]. The ACATS-IV gas chromatograph on the ER-2 measures CFC-11, CFC-12, CFC$113\left(\mathrm{CCl}_{2} \mathrm{~F}-\mathrm{CClF} 2\right), \mathrm{CH}_{3} \mathrm{CCl}_{3}, \mathrm{CCl}_{4}$, and Halon-1211 $\left(\mathrm{CBrClF}_{2}\right)$, which likewise comprise $\sim 80 \%$ of tropospheric chlorine. Halogenated gases are measured by ACATS-IV with precisions and accuracies of better than $1 \%$ and $2 \%$ respectively, at intervals of $3 \mathrm{~min}$, except $\mathrm{SF}_{6}$, which is measured every $6 \mathrm{~min}$. ACATS-IV is calibrated on the ground against the same standards used in the National Oceanic and Atmospheric Administration / Climate Monitoring and Diagnostic Laboratory network of tropospheric measuring stations [Elkins et al., 1993], and is calibrated in flight by running a sample of air with mixing ratios near stratospheric levels every 4 ambient samples, or 15 to $30 \mathrm{~min}$.

Optical bandpass filters, used to improve the signal-tonoise ratio (SNR) of measured spectra, determine the set of 


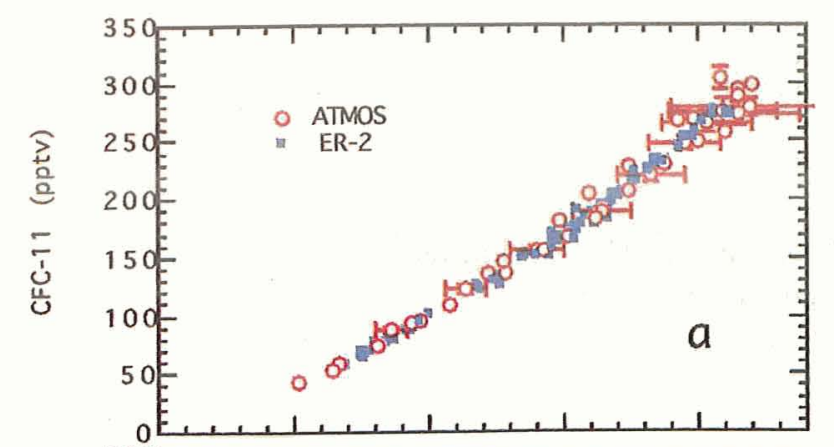

wavenumbers, such as CFC-11, CFC-12, $\mathrm{CCl}_{4}, \mathrm{ClONO}_{2}$, and $\mathrm{SF}_{6}$. For these species, only Filter 12 measurements are considered in the present paper. Gases measured in Filter 9 based on fitting either features at higher wavenumbers or an

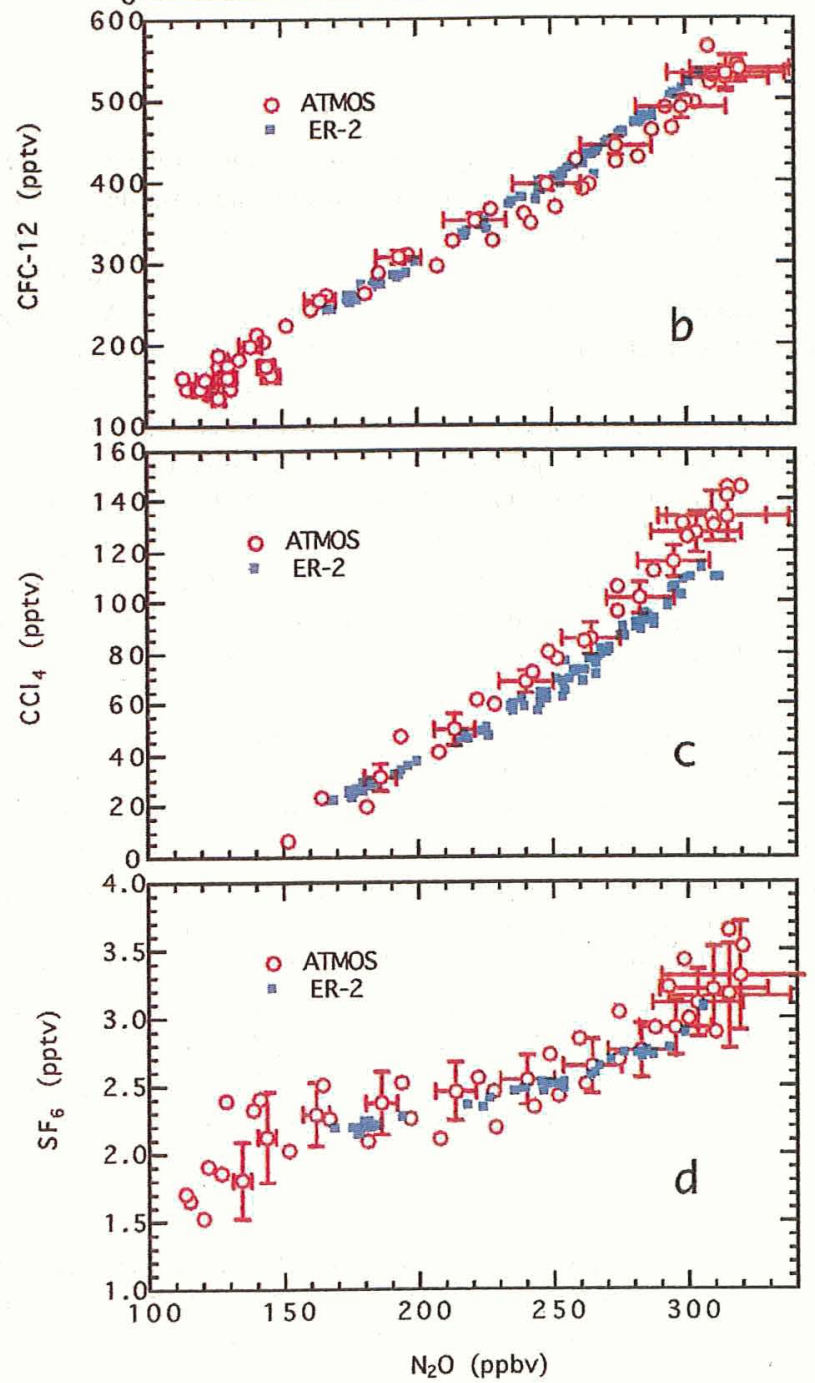

Figure 1. Correlations of [CFC-11], [CFC-12], [CCl 4 , and $\left[\mathrm{SF}_{6}\right]$ vs $\left[\mathrm{N}_{2} \mathrm{O}\right]$ measured by ATMOS and instruments aboard the ER-2 aircraft: $\left[\mathrm{N}_{2} \mathrm{O}\right.$ ] (ATLAS), Loewenstein et al.; [CFC11], [CFC-12], [CCl $]$, and [SF 6 (ACATS-IV), Elkins et al. [1996]. ATMOS error bars reflect estimated $1 \sigma$ precision; systematic uncertainties are discussed in the text.

species measured in each ATMOS occultation [Gunson et al., 1996]. All species examined in this paper, except for $\mathrm{HCl}$ and $\mathrm{N}_{2} \mathrm{O}$, are detected using transitions below $1000 \mathrm{~cm}^{-1}$. In this wavenumber region, spectra from Filter $9\left(600-2450 \mathrm{~cm}^{-1}\right)$ exhibit degraded SNR relative to Filter $12\left(600-1400 \mathrm{~cm}^{-1}\right)$, particularly during ATLAS-3 due to lowered instrumental gain settings. This problem adversely affects VMRs retrieved in Filter 9 using a single spectral window at low

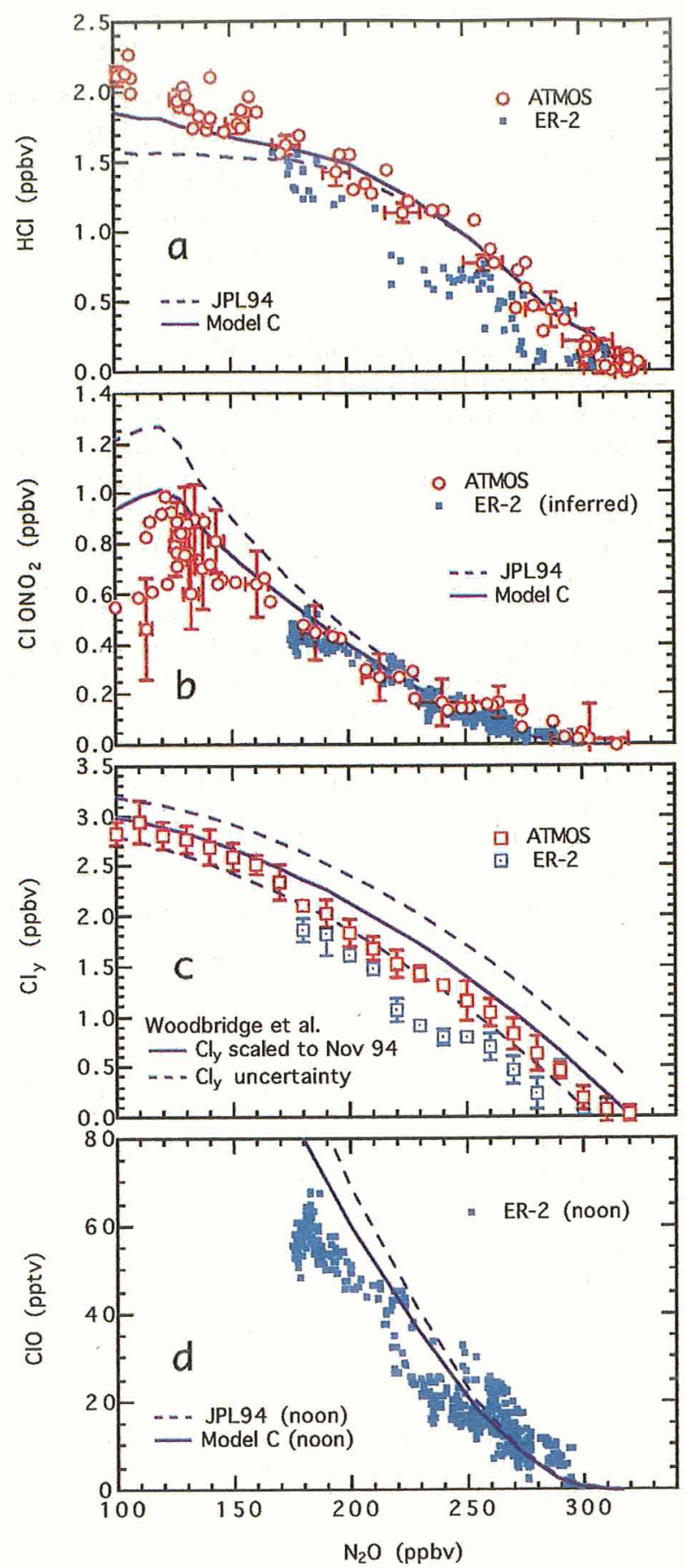

Figure 2. Correlations of $[\mathrm{HCl}],\left[\mathrm{ClONO}_{2}\right]$ or $\left[\mathrm{ClONO}_{2}\right]^{*}$, $\left[\mathrm{Cl}_{\mathrm{y}}\right]=[\mathrm{HCl}]+\left[\mathrm{ClONO}_{2}\right]+[\mathrm{ClO}]$, and $[\mathrm{ClO}]$ vs $\left[\mathrm{N}_{2} \mathrm{O}\right]$ from ATMOS and ER-2 measurements: [ $\left.\mathrm{N}_{2} \mathrm{O}\right]$ (ATLAS), Loewenstein et al.; [HCl] (ALIAS), Webster et al.; [ClO], Stimpfle et al. ER$2\left[\mathrm{ClONO}_{2}\right]^{*}$ is inferred for sunset from noontime [ClO], [NO] (Fahey et al.), and $\left[\mathrm{O}_{3}\right]$ (Proffitt et al.). ATMOS $\left[\mathrm{Cl}_{\mathrm{y}}\right]$ includes calculated $[\mathrm{ClO}]$. ATMOS error bars reflect estimated precision, except for the third panel, where ATMOS and ER-2 error bars represent the $1 \sigma$ deviation of the components of $\left[\mathrm{Cl}_{\mathrm{y}}\right]$, averaged over equally spaced intervals of $\left[\mathrm{N}_{2} \mathrm{O}\right]$. JPL94 [DeMore et al.] and Model C [Michelsen et al.] assume yields of $0 \%$ and $7 \%$, respectively, for $\mathrm{HCl}$ from $\mathrm{ClO}+\mathrm{OH}$. 
ensemble of spectral windows (e.g., $\mathrm{O}_{3}, \mathrm{~N}_{2} \mathrm{O}, \mathrm{CH}_{4}, \mathrm{H}_{2} \mathrm{O}$, $\mathrm{NO}$, $\mathrm{NO}_{2}$ ) are unaffected by these difficulties.

Figure 1a illustrates the agreement of ATMOS and ER-2 measurements of [CFC-11] with $\left[\mathrm{N}_{2} \mathrm{O}\right]$. The horizontal axis corresponds to altitudes from the upper troposphere to $\sim 30$ $\mathrm{km}$. ATMOS measures [CFC-11] with estimated $1 \sigma$ precisions (random errors) of better than $5 \%$ and an accuracy (systematic uncertainty) of $11 \%$, and detects $\left[\mathrm{N}_{2} \mathrm{O}\right]$ with $1 \sigma$ precisions and accuracies of $5 \%$ each. ATMOS makes a priori estimates of precision during each VMR retrieval from considerations such as residual errors in the fitting of spectra; systematic uncertainties are determined by combining uncertainties in the spectroscopic band intensities [Brown et al., 1996] with those related to the tangent pressure assignment. More details on ATMOS accuracies and precisions are presented in Abrams et al. [1996]. The mean difference in [CFC-11] measured by ATMOS and the ER- 2 is $+4 \%(+11$ pptv of [CFC11] at $\left.\left[\mathrm{N}_{2} \mathrm{O}\right]=300 \mathrm{ppbv}\right)$, well within the uncertainties of the measurements, with no apparent dependence on $\left[\mathrm{N}_{2} \mathrm{O}\right]$ (throughout, \% biases are computed as [ATMOS - ER-2] $\%$ ATMOS). Correlations of [CFC-12] with $\left[\mathrm{N}_{2} \mathrm{O}\right]$ are shown in Fig. 1b. ATMOS measures [CFC-12] with a $1 \sigma$ accuracy of $9 \%$ and estimated precisions of better than $5 \%$. In mean, good agreement is obtained in [CFC-12] between the ATMOS and ER-2, with differences ranging from $+5 \%(+13$ pptv of [CFC12]) at $\left[\mathrm{N}_{2} \mathrm{O}\right]=170 \mathrm{ppbv}$ to $-6 \%(-26 \mathrm{pptv}$ of [CFC-12]) at $\left[\mathrm{N}_{2} \mathrm{O}\right]=280$ ppbv.

Figure 1c compares observations of $\left[\mathrm{CCl}_{4}\right]$ vs $\left[\mathrm{N}_{2} \mathrm{O}\right]$. ATMOS measures $\left[\mathrm{CCl}_{4}\right]$ with a $1 \sigma$ accuracy of $20 \%$ and estimated precisions of better than $10 \%$. The ATMOS values of $\left[\mathrm{CCl}_{4}\right]$ are $15 \%$ higher $\left(20\right.$ pptv of $\left[\mathrm{CCl}_{4}\right]$ at $\left[\mathrm{N}_{2} \mathrm{O}\right]=300$ ppbv) compared to the in situ data. ATMOS retrieves [CCl $\left.\mathrm{CC}_{4}\right]$ using the $v_{3}, v_{1}+v_{4}$ bandhead at $796 \mathrm{~cm}^{-1}$, with temperaturedependent absorption cross-sections and band intensities from Orlando et al. [1992]. The deviation of the ATMOS measurements is consistent with the estimated uncertainty in the bandstrength, whose value varies considerably in the literature [Brown et al., 1996]. Figure 1d shows comparisons of $\left[\mathrm{SF}_{6}\right]$ with $\left[\mathrm{N}_{2} \mathrm{O}\right]$. ATMOS measures $\left[\mathrm{SF}_{6}\right]$, a good indicator of the age of stratospheric air owing to its long photochemical lifetime and known tropospheric growth rate, with a accuracy of $11 \%$ and estimated $1 \sigma$ precisions of $\sim 10 \%$. The ATMOS data are on average $5 \%$ higher $\left(0.16\right.$ pptv of $\left[\mathrm{SF}_{6}\right]$ at $\left[\mathrm{N}_{2} \mathrm{O}\right]=300 \mathrm{ppbv}$ ) than the in situ observations, demonstrating quite good agreement over a wide range of $\left[\mathrm{N}_{2} \mathrm{O}\right]$.

\section{Inorganic Chlorine}

The coincidence between ATMOS and ER-2 measurements in Nov 1994 provides a test of our understanding of the partitioning of inorganic chlorine at mid-latitudes. ATMOS measures $[\mathrm{HCl}]$ and $\left[\mathrm{ClONO}_{2}\right]$, which are the dominant forms of $\mathrm{Cl}_{\mathrm{y}}$ in the lower stratosphere for air unaffected by polar stratospheric clouds. ATMOS observes $[\mathrm{HCl}]$ and $\left[\mathrm{ClONO}_{2}\right]$ using different optical filters and thus never in the same occultation; however, species such as $\mathrm{O}_{3}$ and $\mathrm{N}_{2} \mathrm{O}$ measured in multiple filters provide a framework for combining nearby observations. Instruments aboard the ER-2 observe [ $\mathrm{HCl}]$ and [ClO], the latter being the reactive form of $\mathrm{Cl}_{\mathrm{y}}$ that photochemically removes ozone. Although in situ measurements of $\left[\mathrm{ClONO}_{2}\right]$ have never been obtained, its concentration can be inferred from data of $[\mathrm{ClO}]$ and $\left[\mathrm{NO}_{2}\right]$, or $[\mathrm{ClO}],\left[\mathrm{O}_{3}\right]$, and $[\mathrm{NO}]$.

Measured correlations of $[\mathrm{HCl}]$ vs $\left[\mathrm{N}_{2} \mathrm{O}\right]$ are shown in Fig. 2a. ATMOS observes $[\mathrm{HCl}]$ in Filter 3 with a $1 \sigma$ accuracy of $5 \%$ and estimated precisions of better than $5 \%$ at altitudes above $50 \mathrm{mbar}$, degrading to $15 \%$ at 150 mbar. The ALIAS (Aircraft Laser Infrared Absorption Spectrometer) diode laser instrument provides in situ observations of [HCl] using transitions in the same spectral band used by ATMOS [Webster et al., 1994]. For the Nov 1994 flights, ALIAS reports $[\mathrm{HCl}]$ at 6 minute intervals with a $1 \sigma$ precision of 0.07 ppbv (e.g., $5 \%$ at $1.5 \mathrm{ppbv}, 20 \%$ at $0.35 \mathrm{ppbv}$ ) and an accuracy of $5 \%$. For $[\mathrm{HCl}]>1 \mathrm{ppbv}$, the ER-2 observations are $13 \%$ lower $(0.2 \mathrm{ppbv}$ of [HCl]) than the ATMOS data, but the measurements agree within their combined uncertainties. For $[\mathrm{HCl}]<1 \mathrm{ppbv}$, the ER-2 mixing ratios are lower by $40 \%(0.4$ ppbv of $[\mathrm{HCl}]$ ), although the upper range of variability of the ALIAS data overlaps the ATMOS data. ALIAS observations of [HCl] at a given $\left[\mathrm{N}_{2} \mathrm{O}\right]$ during Nov 1994 were $\sim 60 \%$ higher than seen by ALIAS in May 1993, when northern hemisphere mid-latitude $[\mathrm{HCl}] /\left[\mathrm{Cl}_{\mathrm{y}}\right]$ ratios were observed to be $\sim 0.4$, compared to model calculations of $\sim 0.7$ [Webster et al., 1994]. This evolution of $[\mathrm{HCl}] /\left[\mathrm{Cl}_{\mathrm{y}}\right]$ sampled by the ER-2 appears correlated with decreasing sulfate aerosol loading [Webster et al., in preparation].

The correlations of $\left[\mathrm{ClONO}_{2}\right]$ vs $\left[\mathrm{N}_{2} \mathrm{O}\right]$ measured by ATMOS and inferred from ER-2 measurements are shown in Fig. 2b. ATMOS detects $\left[\mathrm{ClONO}_{2}\right]$ with a accuracy of $20 \%$ and estimated $1 \sigma$ precisions of $10-20 \%$. The peak mixing ratio of $\mathrm{ClONO}_{2}$ equals 0.9 ppbv and occurs at $\sim 26 \mathrm{~km}$ altitude $\left(\left[\mathrm{N}_{2} \mathrm{O}\right]\right.$ $\approx 120 \mathrm{ppbv}$ ), leading to a maximum of $\sim 0.5$ for the $\left[\mathrm{ClONO}_{2}\right] /[\mathrm{HCl}]$ ratio. As measurements of $\left[\mathrm{NO}_{2}\right]$ on the ER-2 are unavailable for the flights under consideration, $\left[\mathrm{ClONO}_{2}\right]$ is inferred from noon-time measurements of [ClO], [NO], and $\left[\mathrm{O}_{3}\right]$, using the steady-state expressions [Kawa et al., 1992],

$$
\begin{gathered}
{\left[\mathrm{ClONO}_{2}\right]^{*}(\text { noon })=\frac{\mathrm{k}_{\mathrm{ClO}+\mathrm{NO}_{2}+\mathrm{M}}[\mathrm{ClO}]\left[\mathrm{NO}_{2}\right][\mathrm{M}]}{\mathrm{J}_{\mathrm{ClONO}_{2}}}} \\
{\left[\mathrm{NO}_{2}\right]^{*}=[\mathrm{NO}] \frac{\mathrm{k}_{\mathrm{NO}+\mathrm{O}_{3}}\left[\mathrm{O}_{3}\right]+\mathrm{k}_{\mathrm{NO}+\mathrm{ClO}}[\mathrm{ClO}]}{\mathrm{J}_{\mathrm{NO}_{2}}},}
\end{gathered}
$$

where ${ }^{*}$ denotes an inferred quantity and here [] refers to concentration. Photolysis rates for $\mathrm{ClONO}_{2}$ and $\mathrm{NO}_{2}$ are computed using a radiative transfer model constrained by planetary reflectivity from the Total Ozone Mapping Spectrometer (TOMS) and vertical profiles of $\mathrm{O}_{3}$ derived by combining in situ observations with total column measurements from TOMS [Salawitch et al., 1994]. ATMOS measurements are obtained at sunset, where models indicate $\left[\mathrm{ClONO}_{2}\right]$ should fall midway between values at noon and night. Figure $2 \mathrm{~b}$ shows $\left[\mathrm{ClONO}_{2}\right]^{*}$ (sunset) inferred for the $\mathrm{ER}-2$, calculated from in situ measurements of [ClO], [NO], and $\left[\mathrm{O}_{3}\right]$ obtained within $\pm 2 \mathrm{hr}$ of local noon using Eqns. (1), $(2)$, and $\left[\mathrm{ClONO}_{2}\right]^{*}$ (sunset) $=\left[\mathrm{ClONO}_{2}\right]^{*}$ (noon) $+0.5[\mathrm{ClO}]$ (noon). The $1 \sigma$ uncertainty in $\left[\mathrm{ClONO}_{2}\right]^{*}$ estimated from the propagation of errors in the kinetic parameters and observed quantities is $\pm 90 \%$ [Kawa et al., 1992]. For $\left[\mathrm{N}_{2} \mathrm{O}\right]<250 \mathrm{ppbv}$, the ATMOS measurements of $\left[\mathrm{ClONO}_{2}\right]$ and values inferred from the in situ data agree on average within $\pm 10 \%( \pm 0.04$ ppbv of $\left.\left[\mathrm{ClONO}_{2}\right]\right)$. Systematic discrepancies of $+45 \%(+0.06$ ppbv of $\left.\left[\mathrm{ClONO}_{2}\right]\right)$ are exhibited at higher values of $\left[\mathrm{N}_{2} \mathrm{O}\right]$, where the fractional uncertainties in $\left[\mathrm{ClONO}_{2}\right]$ measured by ATMOS become large due to its decreasing abundance.

Figure 2c compares $\left[\mathrm{Cl}_{\mathrm{y}}\right]$ vs $\left[\mathrm{N}_{2} \mathrm{O}\right]$ from ATMOS and ER-2 data. The ATMOS values of $\left[\mathrm{Cl}_{\mathrm{y}}\right]$ were constructed by summing the measured $[\mathrm{HCl}]$ and $\left[\mathrm{ClONO} \mathrm{O}_{2}\right]$, averaged on a 10 
ppbv wide grid of $\left[\mathrm{N}_{2} \mathrm{O}\right]$, with [ClO] calculated for sunset using the assumptions for 'Model $\mathrm{C}^{\prime}$ described below. The ER-2 values of $\left[\mathrm{Cl}_{y}\right]$ were determined by averaging on the same $\left[\mathrm{N}_{2} \mathrm{O}\right]$ grid measurements of $[\mathrm{HCl}],\left[\mathrm{ClONO}_{2}\right]^{*}$ (noon), and [ClO] collected within $\pm 2 \mathrm{hr}$ of local noon. The contribution of $[\mathrm{ClO}]$ to $\left[\mathrm{Cl}_{\mathrm{y}}\right]$ is less than $5 \%$ for all cases considered here. Observations [Zander et al., 1996] and photochemical simulations indicate that other gases, such as $\mathrm{HOCl}$, contribute negligibly to $\left[\mathrm{Cl}_{\mathrm{y}}\right]$ at these altitudes.

Figure $2 \mathrm{c}$ includes another estimate of inorganic chlorine, denoted here as $\left[\mathrm{Cl}_{\mathrm{y}}\right]^{\dagger}$, determined by subtracting total organic chlorine, measured by ACATS and the Whole Air Sampler during the 1992 ER-2 campaign, from the total organic chlorine loading in the troposphere [Woodbridge et al, 1995]. The $\left[\mathrm{Cl}_{\mathrm{y}}\right]^{\dagger}$ relation shown here has been increased by $6.7 \%$ relative to the published relation of Woodbridge et al. to account for changes in stratospheric chlorine between 1992 and Nov 1994 [Zander et al., 1996]. ATMOS [ $\mathrm{Cl}_{\mathrm{y}}$ ] are on average $0.25 \mathrm{ppbv}$ lower than levels predicted from in situ measurements of organic source gases for $\left[\mathrm{N}_{2} \mathrm{O}\right]>180 \mathrm{ppbv}$, with better agreement at lower $\left[\mathrm{N}_{2} \mathrm{O}\right]$. Inferred $\left[\mathrm{Cl}_{\mathrm{y}}\right]$ from the ER-2 are on average $0.58 \mathrm{ppbv}$ lower than $\left[\mathrm{Cl}_{\mathrm{y}}\right]^{\dagger}$. The smaller abundance of $\left[\mathrm{Cl}_{\mathrm{y}}\right]$ from the in situ measurements of inorganic chlorine compared to $\left[\mathrm{Cl}_{\mathrm{y}}\right]$ from ATMOS is due to lower values of $[\mathrm{HCl}]$ that are not balanced by higher $\left[\mathrm{ClONO}_{2}\right]^{*}$. Trajectory calculations indicate that air parcels along these ER-2 flights have recently undergone large excursions in temperature $(\sim 15 \mathrm{~K})$ and latitude $\left(\sim 15^{\circ}\right)$ [P. Newman, private communication, 1996]. However, our model simulations show the partitioning of $[\mathrm{HCl}]$ and $\left[\mathrm{ClONO}_{2}\right]$ is insensitive to the changes in temperature and latitude along these trajectories, provided precursor (i.e., $\mathrm{Cl}_{\mathrm{y}}, \mathrm{NO}_{\mathrm{y}}, \mathrm{H}_{2} \mathrm{O}, \mathrm{O}_{3}$ ) levels remain unchanged. It is unlikely that the air sampled by ATMOS and the ER-2 could have large differences in $\left[\mathrm{Cl}_{\mathrm{y}}\right]$ for the same $\left[\mathrm{N}_{2} \mathrm{O}\right]$, given the similarity of source gases displayed in Fig. 1. The cause of the discrepancy between ATMOS and ER-2 measurements of $[\mathrm{HCl}]$ remains unclear.

Photochemical simulations [Salawitch et al., 1994; Michelsen et al., 1996] are used to test our understanding of partitioning of inorganic chlorine gases. The model is constrained to match calculated $[\mathrm{HCl}]+\left[\mathrm{ClONO}_{2}\right]$ to the sum measured by ATMOS. Two sets of kinetic parameters are considered: the first uses reaction rate constants and photolytic cross sections from the JPL 1994 compilation [DeMore et al., 1994] and a $0 \%$ yield of $\mathrm{HCl}$ from the reaction $\mathrm{ClO}+\mathrm{OH}$; the second ('Model $\mathrm{C}^{\prime}$ of Michelsen et al. [1996]) incorporates several changes of which the most significant with respect to partitioning of $\left[\mathrm{ClONO}_{2}\right]$ and $[\mathrm{HCl}]$ is an assumed $7 \%$ yield of $\mathrm{HCl}$ from the reaction $\mathrm{ClO}+\mathrm{OH}$. The sensitivity of model results to each kinetic parameter is discussed in Michelsen et al. [1996].

Model results for $[\mathrm{HCl}]$ and $\left[\mathrm{ClONO}_{2}\right]$ at local sunset are compared with ATMOS observations in Figs. $2 a$ and $2 b$. For $\left[\mathrm{N}_{2} \mathrm{O}\right]>200 \mathrm{ppbv}$, both models predict similar levels of [HCl] and are consistent with the partitioning of $\left[\mathrm{ClONO}_{2}\right]$ and [HCl] observed by ATMOS. At lower levels of $\left[\mathrm{N}_{2} \mathrm{O}\right]$, the model allowing for production of $\mathrm{HCl}$ from $\mathrm{ClO}+\mathrm{OH}$ results in better agreement with the ATMOS data. Figure $2 \mathrm{~d}$ shows a comparison of [ClO] calculated subject to constraints imposed by the ATMOS data, but corresponding to midafternoon solar conditions sampled by the ER-2, where in situ measurements of $[\mathrm{ClO}]$ are obtained with a $1 \sigma$ accuracy of $15 \%$ [Stimpfle et al., 1994]. Although both models overestimate [ClO] for $\left[\mathrm{N}_{2} \mathrm{O}\right]<240 \mathrm{ppbv}$, data for [ClO] agree more closely with the model allowing for production of $\mathrm{HCl}$ from $\mathrm{ClO}+\mathrm{OH}$.

The decade-long record of ATMOS observations of organic and inorganic chlorine allows quantification of distributions and trends for gases that are precursors of ozone destroying radicals and significant contributors to greenhouse warming [Zander et al., 1996]. ATMOS measurements provide a selfconsistent picture of the organic and inorganic chlorine budgets and, together with in situ data, provide valuable constraints on our understanding of chlorine chemistry and the effects of anthropogenic chlorine compounds on ozone.

Acknowledgments. Research at the Jet Propulsion Laboratory, California Institute of Technology, is performed under contract with the National Aeronautics and Space Administration (NASA). ATMOS and ER-2 studies are supported in part by NASA's Upper Atmosphere Research Program and Atmospheric Effects of Aviation Project. We thank the Shuttle astronauts and ER-2 pilots for making these observations possible. The helpful comments of the referees are appreciated.

\section{References}

Abrams, M. C. et al., On the assessment and uncertainty of atmospheric trace gas burden measurements with high resolution infrared solar occultation spectra from space, Geophys. Res. Lett., this issue, 1996.

Brown, L. R., M. R. Gunson, R. A. Toth, F. W. Irion, and C. P. Rinsland, The 1995 Atmospheric Trace Molecule Spectroscopy (ATMOS) linelist, Appl. Opt., in press, 1996.

Chang, A. Y. et al., A comparison of measurements from ATMOS and instruments aboard the ER-2 aircraft: Tracers of atmospheric transport, Geophys. Res. Lett., this issue, 1996.

DeMore, W. B. et al., Chemical kinetics and photochemical data for use in stratospheric modeling, Evaluation number 11,JPL Publication 9496, Jet Propulsion Laboratory, Pasadena, CA, 1994

Elkins, J. W. et al., Decrease in the growth rates of atmospheric chlorofluorocarbons 11 and 12, Nature, 364, 780-783, 1993.

Elkins, J. W. et al., Airborne gas chromatograph for in situ measurements of long-lived species in the upper troposphere and lower stratosphere, Geophys. Res. Lett., 23, 347-350, 1996.

Fahey, D. W. et al., In situ measurements of total reactive nitrogen, total water, and aerosol in a polar stratospheric cloud in the Antarctic, $J$. Geophys. Res., 94, 11299-11315, 1989.

Gunson, M. R. et al., The Atmospheric Trace Molecule Spectroscopy (ATMOS) experiment: Deployment on the ATLAS Space Shuttle missions, Geophys. Res. Lett., this issue, 1996.

Kawa, S. R. et al., Photochemical partitioning of the reactive nitrogen and chlorine reservoirs in the high-latitude stratosphere, $J$. Geophys. Res., 97, 7905-7923, 1992.

Loewenstein, M., J. R. Podolske, K. R. Chan, and S. E. Strahan, Nitrous oxide as a dynamical tracer in the 1987 Airborne Antarctic Ozone Experiment, J. Geophys. Res., 94, 11589-11598, 1989.

Michelsen, H. A. et al., Stratospheric chlorine partitioning: Constraints from shuttle-borne measurements of $[\mathrm{HCl}],\left[\mathrm{ClNO}_{3}\right]$, and $[\mathrm{ClO}]$ Geophys. Res. Lett., this issue, 1996.

Orlando, J. J., G. S. Tyndall, A. Huang, and J. G Calvert, Temperature dependence of the infrared absorption cross sections of carbon tetrachloride, Geophys. Res. Lett., 19, 1005-1008, 1992.

Proffitt, M. H. et al., In situ ozone measurements within the 1987 Antarctic ozone hole from a high-altitude ER-2 aircraft, J. Geophys. Res., 94, 16547-16555, 1989.

Salawitch, R. J. et al., The distribution of hydrogen, nitrogen, and chlorine radicals in the lower stratosphere: Implications for changes in $\mathrm{O}_{3}$ due to emission of $\mathrm{NO}_{\mathrm{y}}$ from supersonic aircraft, Geophys. Res. Lett., 21, 2547-2550, 1994.

Stimpfle, R. M. et al., The response of ClO radical concentrations to variations in $\mathrm{NO}_{2}$ radical concentrations in the lower stratosphere Geophys. Res. Lett., 21, 2543-2546, 1994.

Webster, C. R. et al., Hydrochloric acid and the chlorine budget of the lower stratosphere, Geophys. Res. Lett., 21, 2575-2578, 1994.

Woodbridge, E. L. et al., Estimates of total organic and inorganic chlorine in the lower stratosphere from in situ and flask measurements during AASE II, J. Geophys. Res., 100, 3057-3064, 1995.

Zander, R. et al., The 1994 northern midlatitude budget of stratospheric chlorine derived from ATMOS/ATLAS 3 observations, Geophys. Res. Lett., this issue, 1996.

A. Y. Chang, Jet Propulsion Laboratory, 4800 Oak Grove Drive, M. S. 183-301, Pasadena, CA 91109 (email: aychang@caesar.jpl.nasa.gov)

(Received October 17, 1995; revised April 25, 1996;

accepted May 8, 1996.) 


\title{
ATMOS measurements of $\mathrm{H}_{2} \mathrm{O}+2 \mathrm{CH}_{4}$ and total reactive nitrogen in the November 1994 Antarctic stratosphere: Dehydration and denitrification in the vortex
}

\author{
C. P. Rinsland, ${ }^{1}$ M. R. Gunson, ${ }^{2}$ R. J. Salawitch, ${ }^{2}$ M. J. Newchurch, ${ }^{3}$ R. Zander, ${ }^{4}$ \\ M. M. Abbas, ${ }^{5}$ M. C. Abrams, ${ }^{6}$ G. L. Manney, ${ }^{2}$ H. A. Michelsen, ${ }^{7}$ A. Y. Chang, \\ and A. Goldman 8
}

\begin{abstract}
Simultaneous stratospheric volume mixing ratios (VMRs) measured inside and outside the Antarctic vortex by the Atmospheric Trace Molecule Spectroscopy (ATMOS) instrument in November 1994 reveal previously unobserved features in the distributions of total reactive nitrogen $\left(\mathrm{NO}_{\mathrm{y}}\right)$ and total hydrogen $\left(\mathrm{H}_{2} \mathrm{O}+2 \mathrm{CH}_{4}\right)$. Maximum removal of $\mathrm{NO}_{y}$ due to sedimentation of polar stratospheric clouds (PSCs) inside the vortex occurred at a potential temperature $(\Theta)$ of $500-525 \mathrm{~K}(\sim 20 \mathrm{~km})$, where values were 5 times smaller than measurements outside. Maximum loss of $\mathrm{H}_{2} \mathrm{O}+2 \mathrm{CH}_{4}$ due to PSCs occurred in the vortex at $425-450 \mathrm{~K}$, $\sim 3 \mathrm{~km}$ lower than the peak $\mathrm{NO}_{\mathrm{y}}$ loss. At that level, $\mathrm{H}_{2} \mathrm{O}+2 \mathrm{CH}_{4}$ VMRs inside the vortex were $\sim 70 \%$ of corresponding values outside. The Antarctic and April 1993 Arctic measurements by ATMOS show no significant differences in $\mathrm{H}_{2} \mathrm{O}+2 \mathrm{CH}_{4}$ VMRs outside the vortices in the two hemispheres. Elevated $\mathrm{NO}_{\mathrm{y}}$ VMRs were measured inside the vortex near $700 \mathrm{~K}$. Recent model calculations indicate that this feature results from downward transport of elevated $\mathrm{NO}_{\mathrm{y}}$ produced in the thermosphere and mesosphere.
\end{abstract}

\section{Introduction}

Cold temperatures inside the Antarctic vortex during austral winter promote the condensation of polar stratospheric cloud (PSC) particles that provide surfaces for the heterogeneous activation of reactive chlorine and bromine, leading to catalytic $\mathrm{O}_{3}$ destruction in sunlit regions [cf. World Meteorological Organization (WMO), 1990]. Wide-spread, permanent removal of $\mathrm{NO}_{\mathrm{y}}$ (denitrification) and $\mathrm{H}_{2} \mathrm{O}$ (dehydration) by sedimentation of PSCs is a regular feature of the Antarctic vortex. These losses may lead to lower $\mathrm{NO}_{y}$ and $\mathrm{H}_{2} \mathrm{O}$ mixing ratios over broad regions of the mid-latitude southern hemisphere [Kelly et al., 1990;

\footnotetext{
${ }^{1}$ Atmospheric Sciences Division, NASA Langley Research Center, Hampton, Virginia

2 Jet Propulsion Laboratory, California Institute of Technology, Pasadena, Califormia

${ }^{3}$ University of Alabama, Huntsville

${ }^{4}$ Institute of Astrophysics, University of Liège, 4000 Liège-Cointe, Belgium

${ }^{5}$ Space Science Laboratory, NASA Marshall Space Flight Center, Huntsville, Alabama

${ }^{6}$ Systems and Applied Sciences Corporation, Hampton, Virginia

${ }^{7}$ Harvard University, Cambridge, Massachusetts

${ }^{8}$ Department of Physics, University of Denver, Denver, Colorado
}

Copyright 1996 by the American Geophysical Union.

Paper number 96GL00048

0094-8534/96/96GL-00048\$05.00

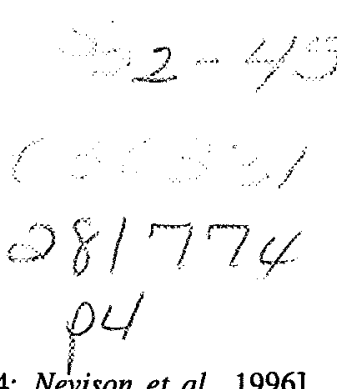

Pierce et al., 1994; Tuck et al., 1994; Nevison et al., 1996]. Ozone losses following PSC formation also occur in the Arctic vortex but less often and on smaller spatial scales [e.g., Fahey et al., 1990a]. The possibility that PSC formation temperatures may rise as a result of $\mathrm{H}_{2} \mathrm{O}$ or $\mathrm{HNO}_{3}$ increases [Newman et al., 1993] underscores the importance of understanding these processes.

We report here an analysis of simultaneous volume mixing ratios (VMRs) of total reactive nitrogen, $\mathrm{NO}_{y}$, and $\mathrm{H}_{2} \mathrm{O}+2 \mathrm{CH}_{4}$ (total hydrogen less $\mathrm{H}_{2}$ ), as a function of potential temperature $(\Theta)$ and $\left[\mathrm{N}_{2} \mathrm{O}\right]$ (where $[\mathrm{x}]$ denotes the VMR of species $\mathrm{x}$ ) derived from Atmospheric Trace Molecule Spectroscopy (ATMOS) $0.01-\mathrm{cm}^{-1}$ resolution IR solar occultation spectra recorded near or over Antarctica during the ATLAS-3 shuttle mission of 3-12 November 1994. The ATMOS measurements cover a wide $\Theta$ range inside and outside the vortex and reveal previously unobserved features in the distributions of $\left[\mathrm{NO}_{\mathrm{y}}\right]$ and $\left[\mathrm{H}_{2} \mathrm{O}\right]+2\left[\mathrm{CH}_{4}\right]$.

Minimum Antarctic stratospheric temperatures of $\sim 200 \mathrm{~K}$ imply that PSCs had evaporated prior to the mission. However, the vortex remained intact in the lower stratosphere with very low $\mathrm{O}_{3}$ levels [Manney et al., 1996].

\section{Measurements}

The ATMOS measurements analyzed here were recorded with filter $3\left(1580-3420 \mathrm{~cm}^{-1}\right)$ or $9\left(650-2450 \mathrm{~cm}^{-1}\right)$ during sunrises at $64.5^{\circ} \mathrm{S}$ to $72.4^{\circ} \mathrm{S}$ latitude. Simultaneous $\mathrm{H}_{2} \mathrm{O}, \mathrm{CH}_{4}$, and $\mathrm{N}_{2} \mathrm{O}$ profiles were derived from measurements with both filters. All the gases used to define $\mathrm{NO}_{\mathrm{y}}$, calculated from $\left[\mathrm{NO}_{\mathrm{y}}\right]=[\mathrm{NO}]+\left[\mathrm{NO}_{2}\right]$ $+\left[\mathrm{HNO}_{3}\right]+2\left[\mathrm{~N}_{2} \mathrm{O}_{5}\right]+\left[\mathrm{HO}_{2} \mathrm{NO}_{2}\right]+\left[\mathrm{Cl} \mathrm{ONO}_{2}\right]$ were measured only with filter 9 .

Version 2 profiles are presented here with no diurnal corrections applied in the $\mathrm{NO}$ and $\mathrm{NO}_{2}$ retrievals [Abrams et al., 1996a]. Photochemical model studies show differences of $<0.2$ ppbv $\left(10^{-9}\right.$ by volume) between $[\mathrm{NO}]+\left[\mathrm{NO}_{2}\right]$ obtained with and without diurnal correction factors for a wide range of conditions in the lower stratosphere [Newchurch et al., 1996], producing a negligible error in $\left[\mathrm{NO}_{\mathrm{y}}\right]$. Additionally, $\left[\mathrm{NO}_{\mathrm{y}}\right]$ was calculated assuming a $3-\mathrm{km}$ scale height decreases in $\left[\mathrm{N}_{2} \mathrm{O}_{5}\right]$, [CONO$\left.{ }_{2}\right]$, $\left[\mathrm{HO}_{2} \mathrm{NO}_{2}\right],\left[\mathrm{NO}_{2}\right]$, and $\left[\mathrm{HNO}_{3}\right]$ above and below the altitude range of the measurements. Our $\mathrm{NO}_{\mathrm{y}}$ values are not sensitive to this assumption because the extrapolated VMRs are small relative to those of the measured species.

The estimate uncertainty in $\left[\mathrm{NO}_{\mathrm{y}}\right]$ determined by combining the systematic uncertainties of the individual constitutents is $\pm 15 \%$. ATMOS and correlative ER-2 measurements of $\mathrm{NO}_{\mathrm{y}}$, $\mathrm{H}_{2} \mathrm{O}$, and $\mathrm{CH}_{4}$ show excellent agreement. Hence, the true systematic error in the ATMOS measurements may be less than the estimated values [Chang et al., 1996]. 

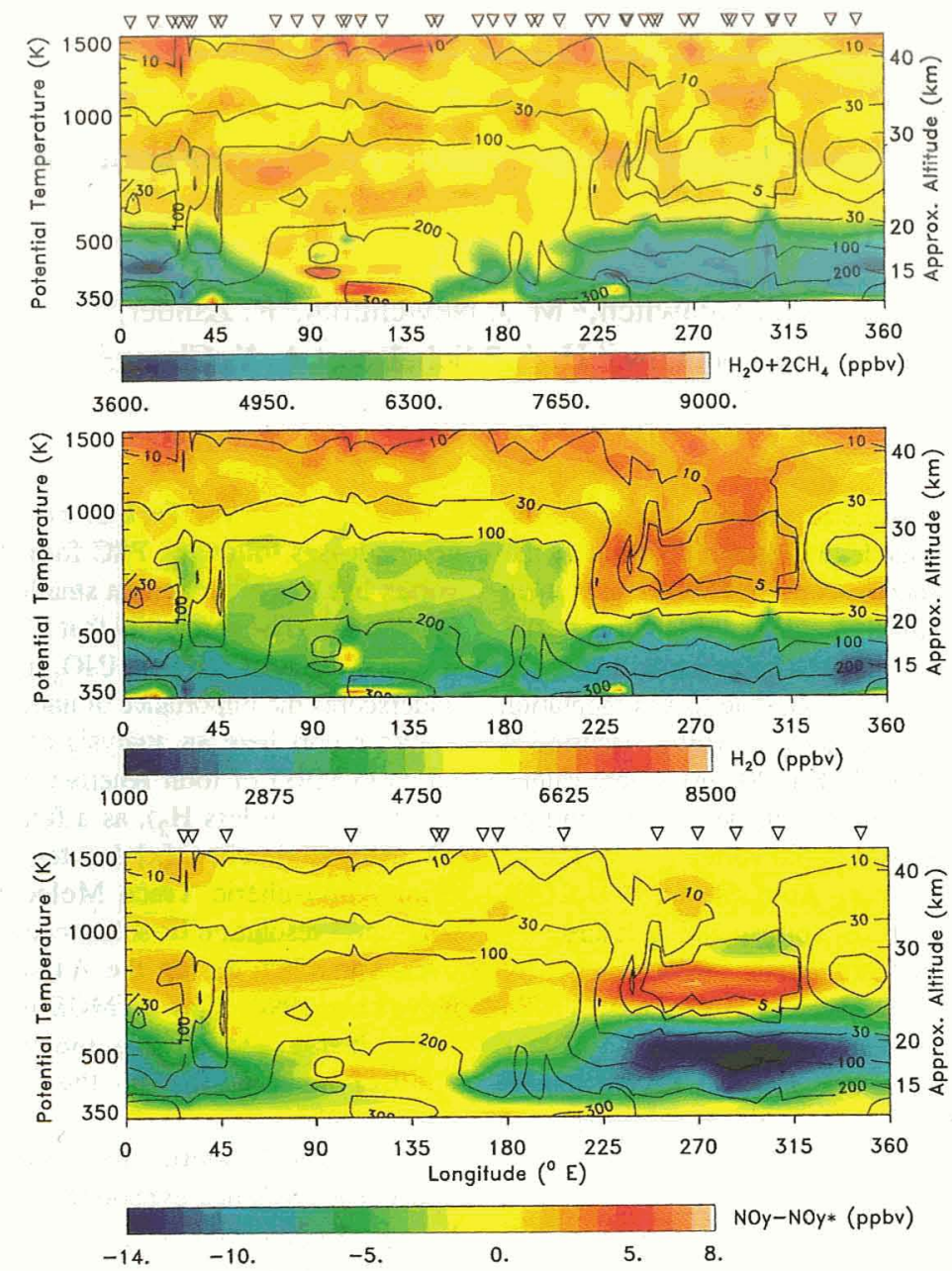

Figure 1. Distributions of $[\mathrm{H}]$ (top), $\left[\mathrm{H}_{2} \mathrm{O}\right]$ (middle), and $[\Delta \mathrm{N}]$ (bottom) versus $\Theta$ and longitude $\left({ }^{\circ} \mathrm{E}\right.$ ). Inverted triangles mark the longitudes of the measurements. Contours of $\left[\mathrm{N}_{2} \mathrm{O}\right]$ (ppbv) are superimposed.

\section{Analysis}

ATMOS Antarctic measurements of $\left[\mathrm{H}_{2} \mathrm{O}\right]+2\left[\mathrm{CH}_{4}\right]$ (denoted $[\mathrm{H}]),\left[\mathrm{H}_{2} \mathrm{O}\right]$, and $\left[\mathrm{NO}_{\mathrm{y}}\right]-\left[\mathrm{NO}_{\mathrm{y}}{ }^{*}\right]$ (denoted $[\Delta \mathrm{N}]$ ) are displayed versus $\Theta$ and longitude in Fig. 1. $\left[\mathrm{NO}_{\mathrm{y}}{ }^{*}\right]$, the $\mathrm{NO}_{\mathrm{y}} \mathrm{VMR}$ expected in the absence of denitrification, was derived from a fit to $\left[\mathrm{NO}_{\mathrm{y}}\right]$ versus $\left[\mathrm{N}_{2} \mathrm{O}\right]$ measured outside the vortex. Longitude is a useful coordinate since the measurements were obtained over a narrow latitude range and the vortex was at similar longitudes throughout the mission [Manney et al., 1996]. Contours of [ $\left.\mathrm{N}_{2} \mathrm{O}\right]$ (overlayed) show the clear signature of deep descent between longitudes of $\sim 230^{\circ} \mathrm{E}$ to $30^{\circ} \mathrm{E}$.

Above $~ 550 \mathrm{~K}$, measurements of $[\mathrm{H}]$ (top) are characterized by values between 7.0 and $7.5 \mathrm{ppmv}\left(10^{-6}\right.$ by volume), a small increase with $\Theta$, and no obvious longitude dependence despite differences of two orders of magnitude between $\left[\mathrm{N}_{2} \mathrm{O}\right]$ inside and outside the vortex near $700 \mathrm{~K}$. Below $550 \mathrm{~K}$, [H] decreases sharply to a minimum of $4 \mathrm{ppmv}$ in the vortex interior. Minimum $[\mathrm{H}]$ and the corresponding $\Theta$ agree with measurements by the Halogen Occultation Experiment during September and October 1992 [Pierce et al., 1994].

The distribution of $\left[\mathrm{H}_{2} \mathrm{O}\right]$ (middle) is $\sim 1.5 \mathrm{ppmv}$ higher inside the vortex than outside between 550 and $900 \mathrm{~K}$, due to downward transport of moist air from the upper stratosphere and lower mesosphere. Similar $\left[\mathrm{H}_{2} \mathrm{O}\right]$ observations in the November 1991 southern vortex were reported by Harwood et al. [1993].
Minimum $\left[\mathrm{H}_{2} \mathrm{O}\right]$ of $\sim 2 \mathrm{ppmv}$ in the vortex is also consistent with previous measurements [Kelly et al., 1990; Pierce et al., 1994; Santee et al., 1995].

The ATMOS $[\Delta N]$ measurements (bottom) reveal massive denitrification within the vortex between 400 and $600 \mathrm{~K}$. The signature of denitrification extends to extra-vortex air near $180^{\circ} \mathrm{E}$ for $\Theta<400 \mathrm{~K}$. However, the vast majority of extra-vortex air shows no significant denitrification. Enhanced $\left[\mathrm{NO}_{\mathrm{y}}\right]$ exists in the vortex between 700 and $900 \mathrm{~K}$, for air characterized by exceedingly low $\left[\mathrm{N}_{2} \mathrm{O}\right]$.

Values of scaled potential vorticity (sPV) in $10^{-4} \mathrm{~s}^{-1}$ [Manney et al., 1996] were used to classify each measurement as inside the vortex $(\mathrm{sPV}>2)$, outside $(\mathrm{sPV}<1)$, or as intermediate "edge" air $(1<\mathrm{sPV}<2) . \quad\left[\mathrm{N}_{2} \mathrm{O}\right]$ is well correlated with sPV values above $\sim 400 \mathrm{~K}$; below this level, $\left[\mathrm{N}_{2} \mathrm{O}\right]$ is relatively well-mixed with respect to longitude, while $\mathrm{SPV}$ gradients for the same locations and times suggest that the vortex extended down at least to $375 \mathrm{~K}$ [Abrams et al., 1996b; Manney et al., 1996].

Figure 2 shows $[\mathrm{H}]$ versus the $\log$ of $\left[\mathrm{N}_{2} \mathrm{O}\right]$ for measurements between 320 and $2050 \mathrm{~K}$. The solid line, a linear fit to the outof-vortex measurements (filled circles), defines $\left[\mathrm{H}^{*}\right]$ versus $\left[\mathrm{N}_{2} \mathrm{O}\right]$, the out-of-vortex reference relation. [ $\left.\mathrm{H}^{*}\right]$ decrease from 7.70 ppmv at $\left[\mathrm{N}_{2} \mathrm{O}\right]$ of 2 ppbv to $7.00 \mathrm{ppmv}$ at $\left[\mathrm{N}_{2} \mathrm{O}\right]$ of $300 \mathrm{ppbv}$; increases at low $\left[\mathrm{N}_{2} \mathrm{O}\right]$ are presumably due to the oxidation of $\mathrm{H}_{2}$. Values of $[\mathrm{H}]$ inside the vortex (open circles) lie below the reference relation, especially for $\left[\mathrm{N}_{2} \mathrm{O}\right]$ between 20 and 


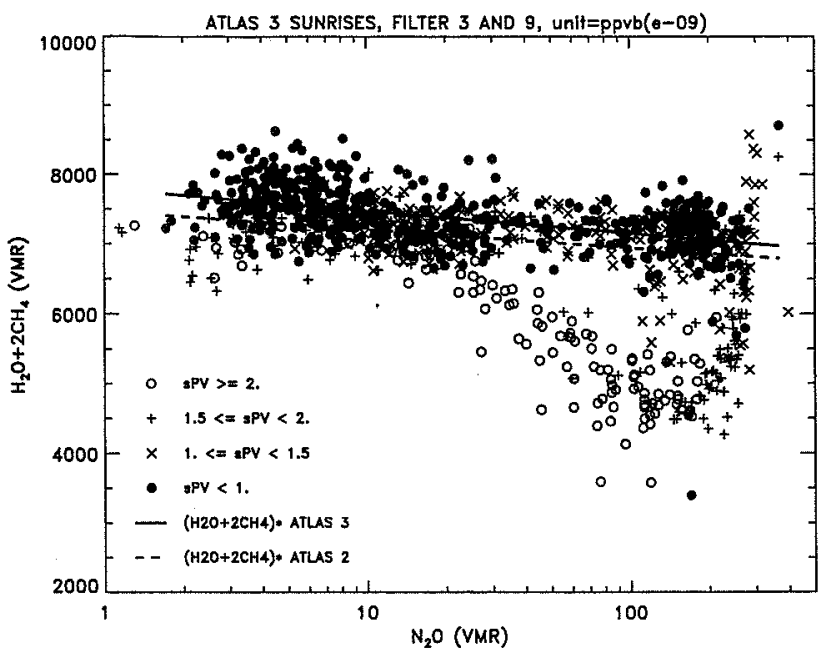

Figure 2. ATMOS/ATLAS 3 [H] (ppbv) vs. [ $\left.\mathrm{N}_{2} \mathrm{O}\right]$ (ppbv) from Antarctic measurements between $\Theta$ of $320 \mathrm{~K}$ and $2050 \mathrm{~K}$. The symbols show classifications based on SPV. A solid line shows the fit to the solid circle measurements $(\mathrm{SPV}<1)$. The dashed line shows the fit to ATMOS/ATLAS 2 Arctic measurements (not shown) with sPV $>2$. The lines are defined by $\left[\mathrm{H}^{*}\right]=\mathrm{a}+\mathrm{b}^{*} \log _{10}\left[\mathrm{~N}_{2} \mathrm{O}\right]$ with $\mathrm{a}=7800.6228$ and $\mathrm{b}=-323.6982$ for the Antarctic and $a=7478.1497$ and $b=-267.50353$ for the Arctic data.

250 ppbv. This difference defines the level of dehydration in the vortex since similar relations would be expected in both regions due to the long lifetimes of the gases.

The dashed line in Fig. 2 shows a fit to ATMOS/ATLAS 2 Arctic extra-vortex $(\mathrm{sPV}>2)\left[\mathrm{H}^{*}\right]$ measurements from filters 2 $\left(1100-2000 \mathrm{~cm}^{-1}\right)$ and 3 . The observations were recorded 8-17 April 1993. Antarctic extra-vortical [H*] averages 0.2-0.3 ppmv higher than values in the Arctic for the same $\left[\mathrm{N}_{2} \mathrm{O}\right]$. In contrast, $\sim 1 \mathrm{ppmv}$ lower $\left[\mathrm{H}_{2} \mathrm{O}\right]$ was measured outside the vortex in the Antarctic than in the Arctic from the ER-2 aircraft [Kelly et al., 1990; Fahey et al., 1990a]. The cause of this difference is not clear.

In Fig. 3, simultaneous ATMOS measurements of [ $\left.\mathrm{NO}_{\mathrm{y}}\right]$ and $\left[\mathrm{N}_{2} \mathrm{O}\right]$ in the Antarctic are shown. The filled circles $(\mathrm{sPV}<1)$ define a compact out-of-vortex relation over the full measurement range. A least-squares fit to these measurements (solid curve) defines $\left[\mathrm{NO}_{\mathrm{y}}{ }^{*}\right]$ vs. $\left[\mathrm{N}_{2} \mathrm{O}\right]$. The substantial change in the slope of the reference curve at low $\left[\mathrm{N}_{2} \mathrm{O}\right]$ reflects the upper atmospheric sink of $\mathrm{NO}_{\mathrm{y}}$ due to the $\mathrm{NO}+\mathrm{N}$ loss reaction [Fahey et al., 1990b]. The dashed curve shows the average out-of-vortex relation derived equatorward of the vortex boundary from 1987 Antarctic and 1989 Arctic ER-2 measurements [Fahey et al., 1990a]. Evidence for a nonlinear correlation between $\left[\mathrm{NO}_{\mathrm{y}}\right]$ and $\left[\mathrm{N}_{2} \mathrm{O}\right]$, not due to denitrification, was reported during AASE II [Loewenstein et al., 1993], consistent with other $\mathrm{NO}_{\mathrm{y}}$ measurements at low $\left[\mathrm{N}_{2} \mathrm{O}\right]$ [e.g., Fahey et al., 1990b].

Measurements of $\left[\mathrm{NO}_{\mathrm{y}}\right]$ inside the vortex (open circles, plus symbols) in Fig. 3 diverge from the reference relation at $\left[\mathrm{N}_{2} \mathrm{O}\right] \approx 270 \mathrm{ppbv}$. These differences define the extent of denitrification due to sedimentation of PSCs. The minimum $\left[\mathrm{NO}_{\mathrm{y}}\right] /\left[\mathrm{NO}_{\mathrm{y}}{ }^{*}\right]$ ratio of 0.2 occurred at $\left[\mathrm{N}_{2} \mathrm{O}\right] \approx 100 \mathrm{ppbv}$. The minimum [NO $\mathrm{NO}_{\mathrm{y}}$ ] of $\sim 3 \mathrm{ppbv}$ measured by ATMOS inside the vortex agrees with the lowest levels of $\sim 2 \pm 1 \mathrm{ppbv}$ measured in the Antarctic vortex during August 1987 [Fahey et al., 1989].

Based on the ATLAS 3 measurements and the corresponding out-of-vortex reference curves in Figs. 2 and 3, $[\Delta \mathrm{H}]$, defined as

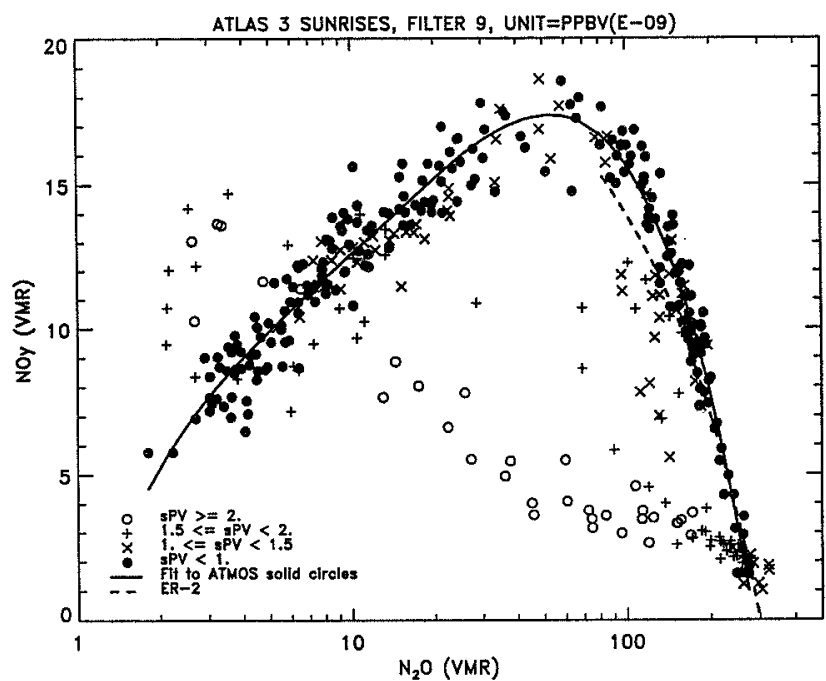

Figure 3. $\left[\mathrm{NO}_{\mathrm{y}}\right]$ (ppbv) vs. [NO] (ppbv) from ATLAS 3 Antarctic filter 9 measurements between $\Theta$ of $320 \mathrm{~K}$ and $2050 \mathrm{~K}$. The solid curve, determined from a least-squares fit to the solid circles, is given by $\left[\mathrm{NO}_{\mathrm{y}}^{*}\right]=\mathrm{a}+\mathrm{bx}+\mathrm{cx}^{2}+\mathrm{dx}^{3}+\mathrm{ex}^{4}$ with $a=-1.3820071, b=30.022251, c=-32.447532, d=21.963289$, $\mathrm{e}=-5.5570435$, and $\mathrm{x}=\log _{10}\left[\mathrm{~N}_{2} \mathrm{O}\right]$. The dashed curve shows the linear $\left[\mathrm{NO}_{\mathrm{y}}{ }^{*}\right]-\left[\mathrm{N}_{2} \mathrm{O}\right]$ relation derived from Arctic and Antarctic ER-2 aircraft measurements [Fahey et al., 1990a].

$[\mathrm{H}]-\left[\mathrm{H}^{*}\right]$, and $[\Delta \mathrm{N}]$ have been calculated as a function of $\Theta$. Values for measurements with $\mathrm{sPV}>2$ (vortex interior air) are given in Table 1 . The minimum $[\Delta \mathrm{H}]$ is reached at $425-450 \mathrm{~K}$, $\sim 17 \mathrm{~km}$ altitude, whereas the minimum $[\Delta \mathrm{N}]$ occurred at $500-525 \mathrm{~K}, \sim 20 \mathrm{~km}$ altitude.

The results in Table 1 indicate that the maximum $\mathrm{NO}_{\mathrm{y}}$ removal occurred at a higher altitude than that of $\mathrm{H}_{2} \mathrm{O}+2 \mathrm{CH}_{4}$. These results are consistent with Antarctic aircraft measurements which observed type I PSCs above the altitude range containing type II PSCs [Kinne et al., 1989]. The altitude distributions of $\mathrm{NO}_{\mathrm{y}}$ and $\mathrm{H}_{2} \mathrm{O}+2 \mathrm{CH}_{4}$ in Table 1 provide a unique observational constraint for understanding the microphysical processes that result in PSC formation, denitrification, and dehydration.

Antarctic PSCs are observed between altitudes of 14 and $28 \mathrm{~km}$ with a peak sighting frequency at $\sim 19 \mathrm{~km}$ during austral late winter and early spring [Poole and Pitts, 1994]. The $[\Delta \mathrm{H}]$ and $[\Delta N]$ changes with $\Theta$ are roughly consistent with this

Table 1. ATMOS Antarctic $[\Delta \mathrm{H}]$ and $[\Delta \mathrm{N}]$ Measurements with sPV $<2.0$ versus $\Theta$

\begin{tabular}{ccc}
$\Theta(\mathrm{K})$ & $\begin{array}{c}{[\Delta \mathrm{H}]} \\
(\mathrm{ppmv})\end{array}$ & $\begin{array}{c}{[\Delta \mathrm{N}]} \\
(\mathrm{ppbv})\end{array}$ \\
\hline $750-800$ & $-0.66(0.05)$ & 3.21 \\
$700-750$ & $-0.63(0.35)$ & $5.84(0.24)$ \\
$650-700$ & $-0.46(0.25)$ & $3.36(2.03)$ \\
$600-650$ & $-0.43(0.24)$ & $-0.92(3.03)$ \\
$575-600$ & $-0.49(0.13)$ & $-6.31(0.36)$ \\
$550-575$ & $-0.88(0.44)$ & $-9.88(1.32)$ \\
$525-550$ & $-1.11(0.15)$ & -12.00 \\
$500-525$ & $-1.75(0.41)$ & $-13.07(0.63)$ \\
$475-500$ & $-1.81(0.29)$ & $-12.44(1.24)$ \\
$450-475$ & $-2.22(0.30)$ & $-10.18(2.07)$ \\
$425-450$ & $-2.42(0.47)$ & $-8.97(1.64)$ \\
$400-425$ & $-2.25(0.58)$ & \\
\hline
\end{tabular}

Values in parentheses are standard deviations. 
distribution below and near its peak altitude, but the variations above $575 \mathrm{~K}$ are not: $[\Delta \mathrm{H}]$ reaches a minimum at $600-700 \mathrm{~K}$ and [ $\Delta \mathrm{N}]$ is positive above $650 \mathrm{~K}$ with a peak of $+5.6 \mathrm{ppbv}$ at 700-750 K, where the maximum descent indicated by $\left[\mathrm{N}_{2} \mathrm{O}\right]$ (Fig. 1) occurs and high altitude $\mathrm{O}_{3}$ depletion has been reported [Hofmann et al., 1991].

We hypothesize that the positive $[\Delta N]$ inside the vortex result from downward transport of elevated $\mathrm{NO}_{y}$ produced in the thermosphere and mesosphere. Evidence for such transport during polar night was reported by Russell et al. [1984]. Model simulations incorporating the winter descent of $\mathrm{NO}_{y}$-enriched air produced in the thermosphere [Solomon et al., 1982] predict a pocket of elevated $\mathrm{NO}_{\mathrm{y}}$ near $30 \mathrm{~km}$ at polar latitudes [Nevison et al., 1996]. Callis et al. [1996] considered this source plus relativistic electron precipitation in the mesosphere and lower thermosphere. Their simulations indicate that both sources contributed significantly to the elevated $\mathrm{NO}_{\mathrm{y}}$ levels measured by ATMOS.

Aside from dehydration, $[\Delta \mathrm{H}]$ is determined by the chemistry of $\mathrm{CH}_{4}$ oxidation and the descent of air. In particular, high $\left[\mathrm{H}_{2}\right]$ and low $\left[\mathrm{H}_{2} \mathrm{O}\right]$ are calculated for this region during high latitude winter [Le Texier et al., 1988]. We suggest that $[\Delta \mathrm{H}]$ above $\sim 575 \mathrm{~K}$ may have resulted from the descent in the vortex of $\mathrm{H}_{2} \mathrm{O}$-poor $/ \mathrm{H}_{2}$-rich air produced in the lower mesosphere during winter. As temperatures below $195 \mathrm{~K}$ occurred up to $\sim 15$ mbar during the 1994 Antarctic winter, dehydration and denitrification may also have played a role in determining $[\Delta \mathrm{H}]$ and $[\Delta \mathrm{N}]$ at the higher altitudes.

Acknowledgments. Research at the Jet Propulsion Laboratory (JPL) is performed under contract with the National Aeronautics and Space Administration (NASA). The authors thank the ATMOS data processing team at JPL and L. Chiou of Science Applications International Corporation (SAIC), Hampton, Virginia, for their help.

\section{References}

Abrams, M. C., et al., On the assessment of atmospheric trace gas burdens with high resolution infrared solar occultation measurements from space, Geophys. Res. Lett., this issue, 1996a.

Abrams, M. C., et al., ATMOS/ATLAS 3 observations of trace gas transport in the Antarctic vortex of 1994, Geophys. Res. Lett., this issue, $1996 \mathrm{~b}$.

Callis, L. B., et al., A 2-D model simulation of downward transport of $\mathrm{NO}_{\mathrm{y}}$ into the stratosphere: Effects on 1994 austral spring $\mathrm{O}_{3}$ and NO ${ }_{y}$, Geophys. Res. Lett., this issue, 1996.

Chang, A. Y., et al., A comparison of measurements from ATMOS and the ER-2: Tracers of atmospheric transport, Geophys. Res. Lett., this issue, 1996.

Fahey, D. W., et al., Measurements of nitric oxide and total reactive nitrogen in the antarctic stratosphere: Observations and chemical implications, J. Geophys. Res., 94, 16,665-16,681, 1989.

Fahey, D. W., et al., Observations of denitrification and dehydration in the winter polar stratospheres, Nature, 344, 321-324, 1990a

Fahey, D. W., et al., A diagnostic for denitrification in winter polar stratospheres, Nature, 345, 698-702, $1990 \mathrm{~b}$.
Harwood, R. S., et al., Springtime stratospheric water vapour in the southern hemisphere as measured by MLS, Geophys. Res. Lett., 20, 1235-1238, 1993.

Hofmann, D. J, et al., Observation and possible causes of new ozone depletion in Antarctica in 1991, Nature, 359, 283-287, 1992.

Kelly, K. K., et al., A comparison of the ER-2 measurements of stratospheric water vapor between the 1987 antarctic and 1989 arctic airborne missions, Geophys. Res. Lett., 17, 465-468, 1990.

Kinne, S., et al., Measurements of size and composition of particles in polar stratospheric clouds from infrared solar absorption spectra, $J$. Geophys. Res., 94, 16,481-16,491, 1989.

Le Texier, H., S. Solomon, and R. R. Garcia, The role of molecular hydrogen and methane oxidation in the water vapour budget of the stratosphere, Q. J. R. Meteorol. Soc., 114, 281-295, 1988.

Loewenstein, M., et al., New observations of the $\mathrm{NO}_{\mathrm{y}} / \mathrm{N}_{2} \mathrm{O}$ correlation in the lower stratosphere, Geophys. Res. Lett., 20, 2531-2534, 1993.

Manney, G. L., R. Swinbank, and A. O'Neill, Stratospheric meteorological conditions for the 3-12 Nov. 1994 ATMOS/ATLAS 3 measurements, Geophys. Res. Lett., this issue, 1996.

Nevison, C. D., et al., $\mathrm{NO}_{\mathrm{y}} / \mathrm{N}_{2} \mathrm{O}$ correlations in the lower stratosphere: Two-dimensional model seasonal and latitudinal patterns, I. Geophys. Res., submitted, 1996.

Newchurch, M. J., et al., Stratospheric $\mathrm{NO}$ and $\mathrm{NO}_{2}$ derived from diurnally corrected solar-occultation measurements of ATMOS, Geophys. Res. Lett., this issue, 1996.

Newman, P. A., et al., Stratospheric meteorological conditions in the arctic polar vortex, 1991 to 1992, Science, 261, 1143-1145, 1993.

Pierce, R. B., et al, Spring dehydration in the antarctic stratospheric vortex observed by HALOE, J. Atmos. Sci., 51, 2931-2941, 1994.

Poole, L. R., and M. C. Pitts, Polar stratospheric cloud climatology based on stratospheric aerosol measurement II observations frorr 1978 to 1989, J. Geophys. Res., 99, 13,083-13,089, 1994.

Russell III, J. M., et al., The variability of stratospheric and mesospheric $\mathrm{NO}_{2}$ in the polar winter night, J. Geophys. Res., 89, 7267-7275, 1984.

Santee, M. L., et al., Interhemispheric differences in polar stratospheric $\mathrm{HNO}_{3}, \mathrm{H}_{2} \mathrm{O}, \mathrm{ClO}$, and $\mathrm{O}_{3}$, Science, 267, 849-852, 1995.

Solomon, S., P. J. Crutzen, and R. G. Roble, Photochemical coupling between the thermosphere and lower atmosphere 1. Odd nitrogen from 50 to $120 \mathrm{~km}, J$. Geophys. Res., 87, 7206-7220, 1982.

Tuck, A. F., et al., Spread of denitrification from 1987 Antarctic and 1988-1989 arctic stratospheric vortices, J. Geophys. Res., 99, 20,573-20,583, 1994.

World Meteorological Organization (WMO) Report No. 20, Scientific assessment of stratospheric ozone: 1989, Geneva, 1990.

C. P. Rinsland, NASA LaRC, Mail Stop 401A, Hampton, VA 23681-0001

A. C. Chang, M. R. Gunson, and R. J. Salawitch, JPL, Mail Stop 183-

301, 4800 Oak Grove Drive, Pasadena, CA 91109

M. J. Newchurch, UAH, Huntsville, AL 35899

R. Zander, Institute of Astrophysics, University of Liège, 4000 LiègeCointe, Belgium

M. M. Abbas, SSL, NMSFC, Huntsville, AL 35889

M. C. Abrams, NASA LaRC, Mail Stop 475, Hampton, VA 23681-0001

H. A. Michelsen, Division of Applied Sciences and Department of Earth and Planetary Sciences, Harvard University, Cambridge, MA 02138

A. Goldman, Department of Physics, University of Denver, Denver, CO 80208

(Received September 27, 1995; revised November 21, 1995;

accepted December 6, 1995.) 


\title{
Seasonal variations of water vapor in the lower stratosphere inferred from ATMOS/ATLAS-3 measurements of $\mathrm{H}_{2} \mathrm{O}$ and $\mathrm{CH}_{4}$
}

\author{
M. M. Abbas, ${ }^{1}$ H. A. Michelsen, ${ }^{2}$ M. R. Gunson, ${ }^{3}$ M. C. Abrams, ${ }^{4}$ M. J. Newchurch, ${ }^{5} 08 / 778$ \\ R. J. Salawitch, ${ }^{3}$ A. Y. Chang, ${ }^{3}$ A. Goldman, ${ }^{6}$ F. W. Irion, ${ }^{7}$ G. L. Manney, ${ }^{3}$ E. J. \\ Moyer, ${ }^{7}$ R. Nagaraju, ${ }^{5}$ C. P. Rinsland, ${ }^{4}$ G. P. Stiller, ${ }^{8}$ and R. Zander ${ }^{9}$
}

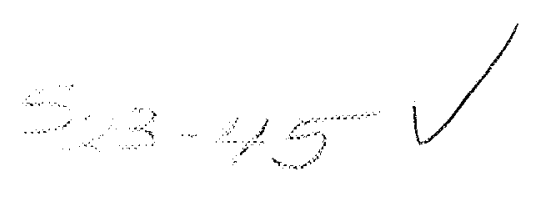

\begin{abstract}
Stratospheric measurements of $\mathrm{H}_{2} \mathrm{O}$ and $\mathrm{CH}_{4}$ by the Atmospheric Trace Molecule Spectroscopy (ATMOS) Fourier transform spectrometer on the ATLAS-3 shuttle flight in November 1994 have been examined to investigate the altitude and geographic variability of $\mathrm{H}_{2} \mathrm{O}$ and the quantity $\boldsymbol{H}=\left(\mathrm{H}_{2} \mathrm{O}+2 \mathrm{CH}_{4}\right)$ in the tropics and at mid-latitudes (8 to $49^{\circ} \mathrm{N}$ ) in the northern hemisphere. The measurements indicate an average value of $7.24 \pm 0.44$ ppmv for $\boldsymbol{H}$ between altitudes of about 18 to $35 \mathrm{~km}$, corresponding to an annual average water vapor mixing ratio of $3.85 \pm 0.29 \mathrm{ppmv}$ entering the stratosphere. The $\mathrm{H}_{2} \mathrm{O}$ vertical distribution in the tropics exhibits a wave-like structure in the $16-$ to $25-\mathrm{km}$ altitude range, suggestive of seasonal variations in the water vapor transported from the troposphere to the stratosphere. The hygropause appears to be nearly coincident with the tropopause at the time of observations. This is consistent with the phase of the seasonal cycle of $\mathrm{H}_{2} \mathrm{O}$ in the lower stratosphere, since the ATMOS observations were made in November when the $\mathrm{H}_{2} \mathrm{O}$ content of air injected into the stratosphere from the troposphere is decreasing from its seasonal peak in JulyAugust.
\end{abstract}

\section{Introduction}

The stratospheric water vapor distribution is determined by a combination of chemical and dynamical processes. Chemical processes involving the oxidation of $\mathrm{CH}_{4}$ enhance the abundance of $\mathrm{H}_{2} \mathrm{O}$, producing approximately two molecules of $\mathrm{H}_{2} \mathrm{O}$ for each molecule of $\mathrm{CH}_{4}$ and leading to higher $\mathrm{H}_{2} \mathrm{O}$ mixing ratios with altitude relative to air entering the stratosphere. Since $\mathrm{H}_{2} \mathrm{O}$ is a major source of the reactive odd hydrogen species, and is considered to be an important tracer of transport from the troposphere to the stratosphere, measurements of its

\footnotetext{
${ }^{1}$ NASA Marshall Space Flight Center.

${ }^{2}$ Harvard University.

3 Jet Propulsion Laboratory, California Institute of Technology.

${ }^{4}$ SAIC-NASA Langley Research Center.

${ }^{5}$ University of Alabama at Huntsville.

${ }^{6}$ Denver University.

${ }^{7}$ California Institute of Technology.

${ }^{8}$ Forschungszentrum Karlsruhe/Universitaet Karlsruhe.

${ }^{9}$ Institute of Astrophysics, University of Liege.
}

Copyright 1996 by the American Geophysical Union.

Paper number 96GL01321

0094-8534/96/96GL-01321\$05.00 stratospheric distribution have been extensive and have been carried out with a variety of techniques including ground-based, and aircraft-, balloon-, and satellite-borne instruments [e.g., Hansen and Robinson, 1989; Gunson et al., 1990; McCormick et al., 1993; Oltmans and Hofmann, 1995].

The ATMOS/ATLAS-3 limb viewing solar occultation observations of stratospheric trace constituents provide simultaneous measurements of $\mathrm{H}_{2} \mathrm{O}$ and $\mathrm{CH}_{4}$ distributions over the 8 to $49^{\circ} \mathrm{N}$ and 67 to $72^{\circ} \mathrm{S}$ latitude ranges during November 3 to 12,1994 [Gunson et al., 1996]. This paper focuses on a portion of the retrieved data for the $16-$ to $35-\mathrm{km}$ altitude range at northern mid-latitudes and tropics. We test the conservation of $\boldsymbol{H}=\left(\mathrm{H}_{2} \mathrm{O}+2 \mathrm{CH}_{4}\right)$, deduce the annual average $\mathrm{H}_{2} \mathrm{O}$ mixing ratio transported to the stratosphere, and examine profiles of $\mathrm{H}_{2} \mathrm{O}$ for evidence of seasonal variation of water vapor entering into the stratosphere.

\section{Methane Oxidation and the Hydrogen Budget of the Lower and Middle Stratosphere}

Methane is oxidized in the stratosphere mainly by reactions with $\mathrm{OH}$ and $\mathrm{O}\left({ }^{1} \mathrm{D}\right)$ and to a lesser extent $\mathrm{Cl}$. The net result of a series of reactions leads to formation of approximately two molecules of $\mathrm{H}_{2} \mathrm{O}$ for each molecule of $\mathrm{CH}_{4}$ :

$$
\mathrm{CH}_{4}+2 \mathrm{O}_{2} \rightarrow \mathrm{CO}_{2}+2 \mathrm{H}_{2} \mathrm{O} \text {. }
$$

In addition to $\mathrm{CH}_{4}$ and $\mathrm{H}_{2} \mathrm{O}, \mathrm{H}_{2}$ is the only other abundant hydrogen-containing species in the stratosphere. Available in situ balloon-borne/rocket measurements of molecular hydrogen in the stratosphere indicate nearly constant mixing ratio with altitude, in accordance with a near balance between production by methane oxidation and loss by oxidation of $\mathrm{H}_{2}$ [e.g., Ehhalt and Tonnisen, 1980; Brasseur and Solomon, 1986]. A major source of variability of $\mathrm{H}_{2} \mathrm{O}$ in the lower stratosphere is related to changes in $\mathrm{CH}_{4}$, owing to different photolytic histories of individual air masses. Simultaneous observations of $\mathrm{H}_{2} \mathrm{O}$ and $\mathrm{CH}_{4}$ enable us to assess whether changes in $\mathrm{H}_{2} \mathrm{O}$ are related to oxidation of $\mathrm{CH}_{4}$, or some other process, and recent ATMOS measurements in the 16- to $35-\mathrm{km}$ altitude range for a wide range of latitudes are examined to test our general understanding of the conservation of $\boldsymbol{H}$ and the hydrogen budget of the lower and middle stratosphere.

A correlation plot of $\mathrm{H}_{2} \mathrm{O}$ versus $\mathrm{CH}_{4}$ for 47 profiles in the 8 to $49^{\circ} \mathrm{N}$ latitude range is shown in Figure 1. The line represents an average linear fit to the measurements (closed symbols) over the $18-$ to $35-\mathrm{km}$ range $\left(\mathrm{CH}_{4} \sim 0.5\right.$ to $\left.1.6 \mathrm{ppmv}\right)$ given by

$$
\left[\mathrm{H}_{2} \mathrm{O}\right]=(-1.99 \pm 0.04)\left[\mathrm{CH}_{4}\right]+7.24 \pm 0.04 \mathrm{ppmv},
$$

with the uncertainties representing the standard deviation of 


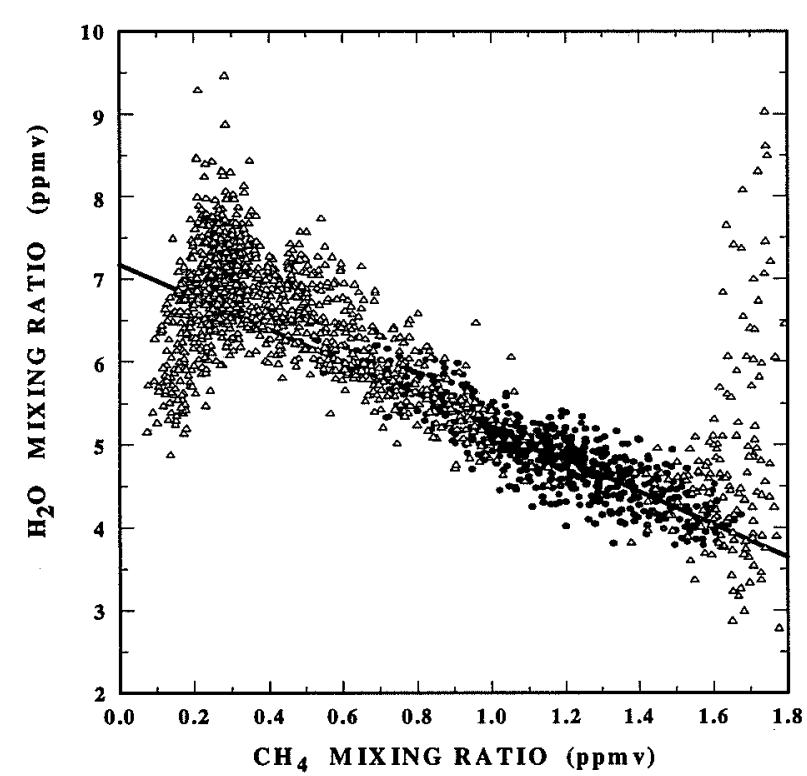

Figure 1. A scatter plot of ATMOS measurements of $\mathrm{H}_{2} \mathrm{O}$ versus $\mathrm{CH}_{4}$ for 47 sunset occultations in the 8 to $49^{\circ} \mathrm{N}$ latitude range. The solid line represents a least square fit (equations given in text) to data obtained over the 18 - to $35-\mathrm{km}$ altitude range $\left(\mathrm{CH}_{4} \sim 0.6\right.$ to $\left.1.6 \mathrm{ppmv}\right)$.

the linear regression. The systematic uncertainty in the measurements of $\mathrm{H}_{2} \mathrm{O}$ and $\mathrm{CH}_{4}$ lead to total uncertainties of \pm 0.16 and $\pm 0.44 \mathrm{ppmv}$ in slope and intercept, respectively. The inferred slope $\partial \mathrm{H}_{2} \mathrm{O} / \partial \mathrm{CH}_{4} \cong-2$ implies that each $\mathrm{CH}_{4}$ molecule is converted into two molecules of $\mathrm{H}_{2} \mathrm{O}$, whereas the intercept $7.24 \pm 0.44$ indicates approximately the average $\boldsymbol{H}$ in the 18- to $35-\mathrm{km}$ region. A precise determination of this slope and its deviation from -2 is important because of its implication for the distribution of $\mathrm{H}_{2}$ in the stratosphere [Dessler et al., 1994; Abbas et al., 1996].

Observed mixing ratio of $\mathrm{H}_{2}$ by balloon-borne instruments suggest a small decrease with height in the $16-$ to $35-\mathrm{km}$ range, varying from $\sim 0.55 \mathrm{ppmv}$ at the tropopause to $\sim 0.5$ at $30 \mathrm{~km}$ [e.g., Ehhalt and Tonnisen, 1980]. Assuming conservation of $\boldsymbol{H}$ and $\mathrm{H}_{2}$, this would correspond to a slope $\partial \mathrm{H}_{2} \mathrm{O} / \partial \mathrm{CH}_{4}$ $\sim-2.06$. Dessler et al. [1994] inferred a value of $\partial \mathrm{H}_{2} \mathrm{O} / \partial \mathrm{CH}_{4}=$ $-1.94 \pm 0.27$ in the lower stratosphere from measurements on the ER-2 aircraft. The aircraft, balloon-borne, and ATMOS data in the 16 - to $35-\mathrm{km}$ altitude range are all consistent statistically with conservation of $\boldsymbol{H}$ and with a $2 \mathrm{H}_{2} \mathrm{O} / 2 \mathrm{CH}_{4}$ of -2 . The distribution of $\boldsymbol{H}$ with altitude and its deviation from a constant value in the upper stratosphere and the mesosphere is discussed in another paper [Abbas et al., 1996].

Measurements of $\boldsymbol{H}$ by other experiments for comparison are: 6.0 ppmv from LIMS and SAMS data [Jones et al., 1986]; $7.0 \pm 0.6 \mathrm{ppmv}$ at $30 \mathrm{~km}$ from the ATMOS experiment on Spacelab 3 [Gunson et al., 1990]; $7.6 \pm 0.6 \mathrm{ppmv}$ from data collected on the ER-2 aircraft [Dessler et al., 1994]. It is unlikely that an atmospheric process could be responsible for the large differences in $\boldsymbol{H}$ observed by ATMOS and the combination of LIMS and SAMS. The Nimbus 7 satellite measurements of $\boldsymbol{H}$ have large systematic errors, as discussed by Hansen and Robinson [1989]. A small part of the difference between values of $\boldsymbol{H}$ measured by the present ATMOS observations and the ATMOS data for April 1985 reported by Gunson et al. [1990] may be accounted for by long-term increases in $\mathrm{H}_{2} \mathrm{O}$ and $\mathrm{CH}_{4}$ [Oltmans and Hofmann, 1995; WMO, 1995].
Differences in the determination of $\boldsymbol{H}$ based on the present ATMOS observations and the in situ data reported by Dessler et al. [1994] lie within the systematic uncertainties of both sets of measurements, although the ATMOS value is lower by about 0.4 ppmv. A comparison of ATMOS observations of $\mathrm{H}_{2} \mathrm{O}$, $\mathrm{CH}_{4}$, and $\mathrm{N}_{2} \mathrm{O}$ with nearly coincident in situ measurements obtained in the lower stratosphere during November 1994 shows excellent agreement for each species, yielding virtually identical values of $\boldsymbol{H}$ [Chang et al., 1996]. For the November 1994 coincidence, the only available in situ measurements of $\mathrm{H}_{2} \mathrm{O}$ and $\mathrm{CH}_{4}$ were obtained by the NOAA Lyman $\alpha$ hygrometer and the NOAA Airborne Chromatograph for Atmospheric Trace Species (ACATS) gas chromatograph, respectively. Dessler et al.'s [1994] estimate of $\boldsymbol{H}$ was based on observations of $\mathrm{H}_{2} \mathrm{O}$ and $\mathrm{CH}_{4}$ from the Harvard Lyman $\alpha$ hygrometer and the Aircraft Laser Infrared Absorption Spectrometer (ALIAS) instrument, respectively. During May 1993 , measurements of $\mathrm{H}_{2} \mathrm{O}$ by the Harvard hygrometer exceeded those obtained by the NOAA instrument by $\sim 15 \%$ [Hintsa et al., 1994], and measurements of $\mathrm{CH}_{4}$ by ALIAS were $10 \%$ lower than measurements by ACATS [Dessler et al., 1994]. Consequently, the lower value of $\boldsymbol{H}$ measured by ATMOS relative to the in situ determination of Dessler et al. [1994] may be primarily due to systematic differences in the measurement of $\mathrm{H}_{2} \mathrm{O}$, offset slightly by the differences in observations of $\mathrm{CH}_{4}$.

The value of $\boldsymbol{H}$ is important for assessing the average mixing ratio of $\mathrm{H}_{2} \mathrm{O}$ injected from the troposphere into the stratosphere. Our estimate for the annual average amount of $\mathrm{H}_{2} \mathrm{O}$ entering the stratosphere is based on least square fits to ATMOS measurements of $\mathrm{H}_{2} \mathrm{O}$ and $\mathrm{CH}_{4}$ collected over a wide range of altitudes, rather than observations at the tropopause, to reduce the sensitivity of our result to seasonal and episodic variations in $\mathrm{H}_{2} \mathrm{O}$. ATMOS observations used in the fit were obtained in the 18 - to $35-\mathrm{km}$ altitude range, representing an average over a period of $\sim 18$ months, assuming upward velocities of $\sim 7$ to $8 \mathrm{~km}$ per year (see discussion below). Since tropo-

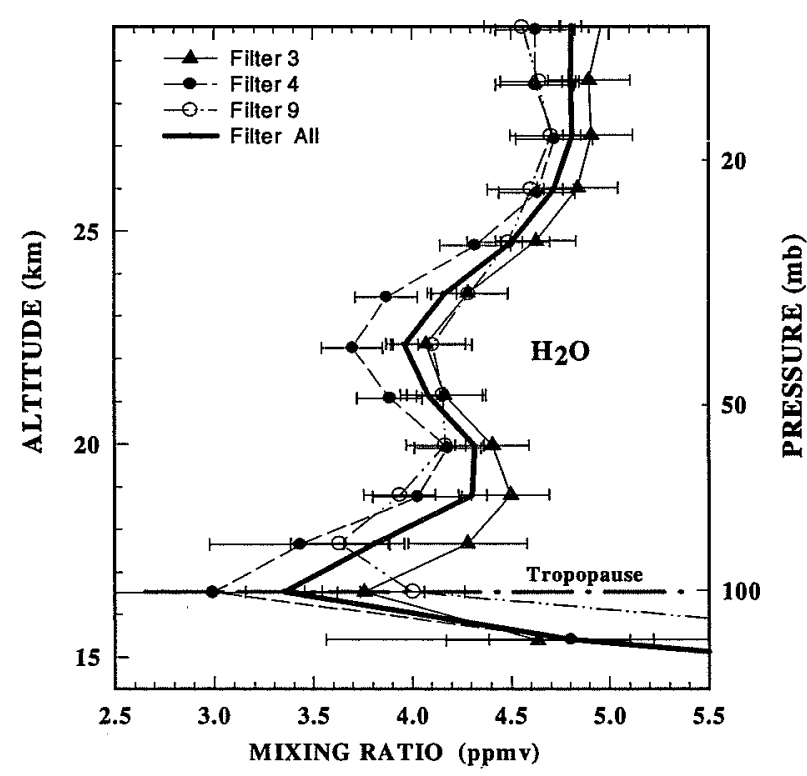

Figure 2. Average profiles of $\mathrm{H}_{2} \mathrm{O}$ in the tropics data obtained by filters 3,4 , and 9 separately, and an average of profiles obtained using all filters for latitude range of 8 to $28^{\circ} \mathrm{N}$. The tropopause height, as determined from the National Meteorological Center data, is indicated by the horizontal line. 
spheric methane has small seasonal variations $(\sim 3 \%)$, we assume a constant mixing ratio of $1.70 \mathrm{ppmv}$ for $\mathrm{CH}_{4}$ for air entering the stratosphere. This leads to an annually averaged $\mathrm{H}_{2} \mathrm{O}$ mixing ratio of $3.84 \pm 0.29 \mathrm{ppmv}$ for air injected into the stratosphere.

Previous in situ and satellite observations of this quantity are: Remsberg et al. [1995] estimated an annual average value in the range of 3.2 to $3.7 \mathrm{ppmv}$ the HALOE data; Dessler et al. [1994] determined a value of $4.2 \pm 0.5 \mathrm{ppmv}$ from ER-2 data; Hansen and Robinson [1989] reported 3.25 ppmv from LIMS/SAMS data; while Jones et al. [1986] reported $2.7 \mathrm{ppmv}$ from an earlier analysis of the same satellite data. We note again the possibility of large systematic errors that have not been fully quantified in the LIMS/SAMS data [Hansen and Robinson, 1989]. The discrepancy between ATMOS and in situ estimates of $\mathrm{H}_{2} \mathrm{O}$ entering the stratosphere lies with the systematic uncertainty of both sets of measurements. The in situ estimate, although based on measurements over the 17- to 20 $\mathrm{km}$ altitude range, included a wide range of $\mathrm{CH}_{4}$ mixing ratios ( 0.9 to $1.5 \mathrm{ppmv}$ ) and should have a minor sensitivity to seasonal variations in $\mathrm{H}_{2} \mathrm{O}$. Systematic differences between measurements of $\mathrm{H}_{2} \mathrm{O}$ and $\mathrm{CH}_{4}$ obtained by ATMOS and the in situ observations of Dessler et al. [1994] may be primarily responsible for the offset.

\section{Seasonal Variations in Water Vapor}

Since observations of the hygropause (a minimum in the stratospheric water vapor mixing ratio) by Kley et al. in 1979 , the nature and mechanism of its formation have been the subject of active discussion in the literature. The amount of water vapor entering the stratosphere may be influenced by temperatures at the tropopause and within upper troposphere. With the injection limited to the tropical regions, the hygropause altitude was expected to be coincident with the tropopause at tropical latitudes. Numerous measurements have indicated the hygropause to be located above the tropopause by 2 to $3 \mathrm{~km}$ [e.g., Russell et al., 1984; Jones et al., 1986; Kelly et al., 1989, 1993]. The tropical tropopause in the northern hemisphere has a maximum monthly mean temperature in the sum-

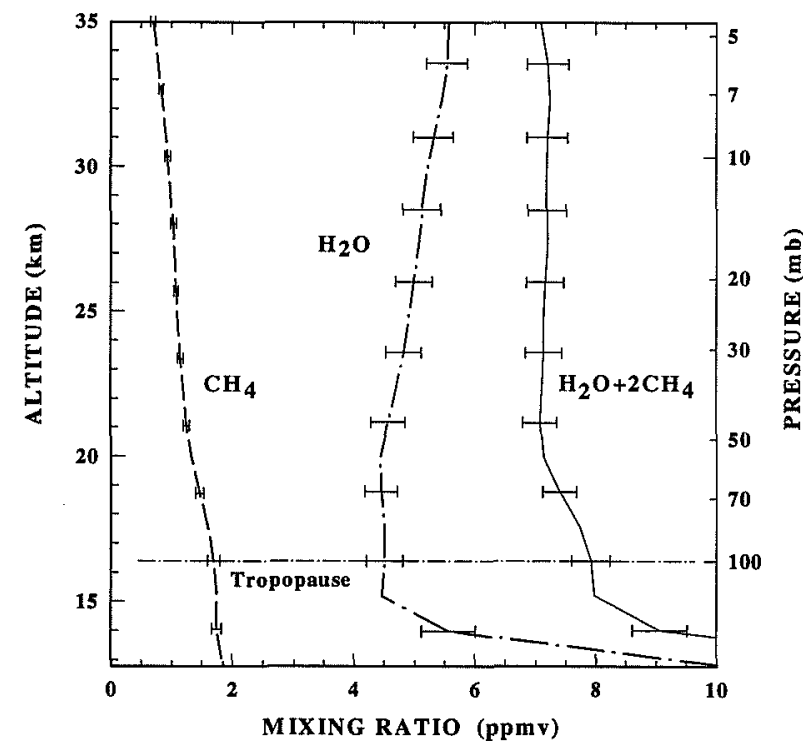

Figure 3. The vertical profiles of $\mathrm{CH}_{4}, \mathrm{H}_{2} \mathrm{O}$, and $\mathrm{H}=\left(\mathrm{H}_{2} \mathrm{O}+2 \mathrm{CH}_{4}\right)$ from a zonal average of 27 sunset occultations for midlatitudes in the latitude 32 to $49^{\circ} \mathrm{N}$ range. The tropopause height, as determined from the National Meteorological Center data, is indicated by the horizontal line.

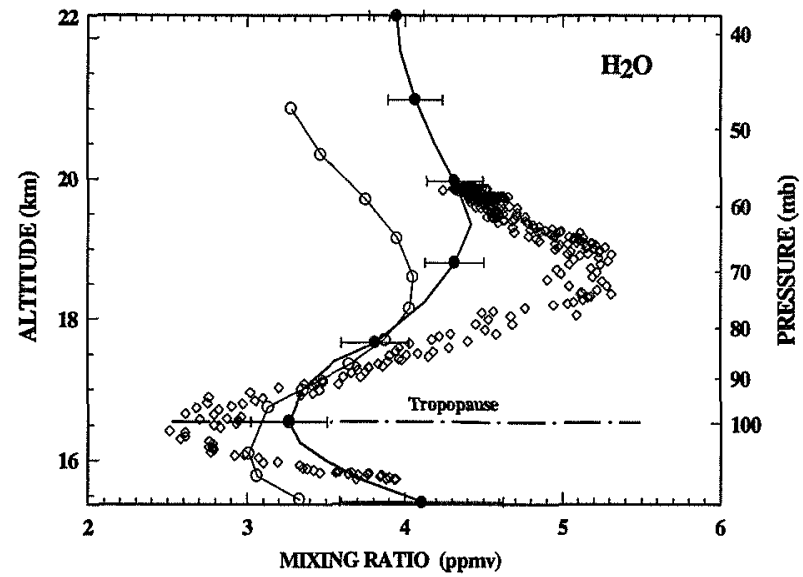

Figure 4. Comparison of the average profile of $\mathrm{H}_{2} \mathrm{O}$ retrieved from 18 sunset occultations by ATMOS in the 8 to $28^{\circ} \mathrm{N}$ latitude range on November 3 to 12,1994 , (filled circles) with: NOAA Lyman- $\alpha$ hygrometer measurements (diamonds) over $2^{\circ} \mathrm{S}$ to $2^{\circ} \mathrm{N}$ latitudes made on ER-2 aircraft on October 29, 1994 [K. Kelly, private communication]; average of HALOE measurements (open circles) over $5^{\circ} \mathrm{S}$ to $5^{\circ} \mathrm{N}$ latjtudes made on November 1 to 13, 1994 [Mote et al., 1996].

mer and occurs at a lower altitude, and has a minimum mean temperature in the winter occurring at a higher altitude. The variation in tropopause temperature from summer to winter is $\sim 5 \mathrm{~K}$, with a change in altitude of $\sim 1 \mathrm{~km}$ [e. g., Reid and Gage, 1981]. Several recent measurements at both tropical and middle latitudes have provided convincing evidence of seasonal variations in lower stratospheric water vapor, in phase with changes in annual tropopause temperatures [McCormick, 1993; Hintsa et al., 1994; Boering et al., 1995; Mote et al., 1995].

The simultaneous ATMOS measurements of $\mathrm{H}_{2} \mathrm{O}$ and $\mathrm{CH}_{4}$ made during the ATLAS-3 mission in November 1994 are examined for a record of seasonal variations of $\mathrm{H}_{2} \mathrm{O}$ entry into the stratosphere. The vertical profiles of $\mathrm{H}_{2} \mathrm{O}$ retrieved from a single occultation exhibit periodic structures of \pm 3 to $4 \%$ arising from random errors in the retrieval process which may be due to tangent pressure/altitude assignments for the limb-viewing geometry. This structure, however, is largely smoothed out when averaged over a sufficient number of occultations. In addition, there are systematic differences between the results obtained from the three different optical bandpass filters employed in the observations. To show this variability, we plot in Figure 2 the profiles of $\mathrm{H}_{2} \mathrm{O}$ retrieved from data obtained during November 3 to 12,1994 , from three different optical band-pass filters, zonally averaged over: (i) nine occultations $\left(8\right.$ to $\left.28^{\circ} \mathrm{N}\right)$, with filter 3 in the $1580-3340 \mathrm{~cm}^{-1}$ region, (ii) four occultations $\left(9\right.$ to $\left.22^{\circ} \mathrm{N}\right)$, with filter 4 in the $3150-4800 \mathrm{~cm}^{-1}$ region, (iii) five occultations $\left(12\right.$ to $\left.26^{\circ} \mathrm{N}\right)$, with filter 9 in the 600 to $2,450 \mathrm{~cm}^{-1}$ region, and (iv) 18 occultations $\left(8\right.$ to $\left.28^{\circ} \mathrm{N}\right)$ using data from all filters. The error bars shown are the quadrature sum of the weighted standard deviation of the mean and systematic spectral errors of $\pm 6 \%$. The tropopause height indicated in Figure 2 (and all subsequent figures) is based on an analysis of NMC temperature profiles for the relevant region.

The tropical $\mathrm{H}_{2} \mathrm{O}$ profiles exhibit characteristic features with two minima occurring at $\sim 16.5 \mathrm{~km}$ and $22.5 \mathrm{~km}$, a maximum at $\sim 19.0 \mathrm{~km}$, and a weak maximum $\sim 27 \mathrm{~km}$. These features are suggestive of seasonal variations in the $\mathrm{H}_{2} \mathrm{O}$ entry into the stratosphere; simultaneous measurements of profiles of $\mathrm{CH}_{4}$ (not shown) are relatively featureless, demonstrating that the variability in profiles of $\mathrm{H}_{2} \mathrm{O}$ does not originate from oxida- 
tion of $\mathrm{CH}_{4}$. Seasonal variations in $\mathrm{H}_{2} \mathrm{O}$ at lower stratospheric altitudes below $\sim 19 \mathrm{~km}$ have also been observed by SAGE II [Rind et al., 1993], HALOE and MLS [Mote et al., 1995], and instruments aboard the ER-2 aircraft [Boering et al., 1995]. The average profiles of $\mathrm{H}_{2} \mathrm{O}, \mathrm{CH}_{4}$, and $\boldsymbol{H}$ obtained from 33 occultations at a mean value of $\sim 42^{\circ} \mathrm{N}$ are shown in Figure 3 . The $\mathrm{H}_{2} \mathrm{O}$ profile is relatively featureless above $\sim 19 \mathrm{~km}$, with mixing ratio increasing from a minimum of $4.5 \mathrm{ppmv}$ at the tropopause to about $5.6 \mathrm{ppmv}$ at $35 \mathrm{~km}$. By the time air reaches the mid-latitude region from the tropics, seasonal variations in $\mathrm{H}_{2} \mathrm{O}$ appear to have smoothed out. Figure 4 shows a comparison of the average $\mathrm{H}_{2} \mathrm{O}$ profile of Figure 2 (mean latitude $\sim 42^{\circ} \mathrm{N}$ ) with the results obtained from near simultaneous observations by the HALOE solar occultation experiment on UARS made during November 1 to 13,1994 , in the $5^{\circ} \mathrm{S}$ to $5^{\circ} \mathrm{N}$ latitude range [Mote et al., 1995], and the NOAA Lyman $\alpha$ hygrometer measurements obtained on ER-2 aircraft on October 29,1994 , from $2.2^{\circ} \mathrm{S}$ to $2.2^{\circ} \mathrm{N}$ [K. Kelly, private communication]. Considering the $0.2-\mathrm{km}$ vertical resolution of the ER-2 measurements relative to the ATMOS field-of-view of $\sim 1.5$ to $2 \mathrm{~km}$, Figure 4 shows good agreement at lower altitudes between ATMOS, HALOE, and in situ measurements of $\mathrm{H}_{2} \mathrm{O}$. The discrepancy between ATMOS and HALOE measurements of $\mathrm{H}_{2} \mathrm{O}$ mixing ratios at higher altitudes could be partly due to latitudinal differences.

In view of seasonal variations in the water vapor entry into the stratosphere with a minimum in January and maximum in July [e.g., Rind et al., 1993; Boering et al., 1995; Mote et al., 1995], a close examination of ATMOS profiles of $\mathrm{H}_{2} \mathrm{O}$ leads to the following conclusions:

(i) The minimum mixing ratio of $\mathrm{H}_{2} \mathrm{O}$ shown in Figures 2 to 4 is coincident with the tropopause within the uncertainty of the field-of-view of ATMOS (with the exception of filter 9 measurements), consistent with the phase of the seasonal cycle, i.e., decreasing water vapor entry in November during the observational period; a separation of the hygropause from the tropopause would be expected in observations obtained a month or two after the minimum water vapor entry in JanuaryFebruary to a month or two after the maximum in July-August.

(ii) Assuming that the local maximum in the mixing ratio of $\mathrm{H}_{2} \mathrm{O}$ at $19 \mathrm{~km}$ represents the upward transport of air that entered the stratosphere in July 1994 and the local minimum at $22.5 \mathrm{~km}$ reflects entry in January 1994, the inferred annually averaged vertical velocity is estimated to be $\sim 7$ to $8 \mathrm{~km}$ /year $\left(2.4 \times 10^{-2} \mathrm{~cm} \mathrm{~s}^{-1}\right)$ near $22 \mathrm{~km}$. This analysis strongly suggests that ATMOS measurements of $\mathrm{H}_{2} \mathrm{O}$ and $\mathrm{CH}_{4}$ are consistent with water vapor entry into the stratosphere in all seasons and air with minimum $\mathrm{H}_{2} \mathrm{O}$ mixing ratio enters in the winter (January-February) in the northern hemisphere and maximum in the summer (July-August).

\section{Conclusions}

An examination of the simultaneous measurements of $\mathrm{H}_{2} \mathrm{O}$ and $\mathrm{CH}_{4}$ made by ATMOS/ATLAS-3 indicates near conservation of the quantity $\boldsymbol{H}=\left(\mathrm{H}_{2} \mathrm{O}+2 \mathrm{CH}_{4}\right)$ below altitudes of about $35 \mathrm{~km}$ at midlatitudes. The data indicate that air enters the stratosphere with an average $\mathrm{H}_{2} \mathrm{O}$ mixing ratio of $\sim 3.84 \pm$ 0.29 ppmv. Vertical structure in averaged tropical profiles of $\mathrm{H}_{2} \mathrm{O}$ suggests seasonal variations in the mixing ratio of $\mathrm{H}_{2} \mathrm{O}$ for air entering the stratosphere, and an average velocity of $\sim 7$ to $8 \mathrm{~km} /$ year in the lower stratosphere. The hygropause is observed to be within $\sim 2 \mathrm{~km}$ of the tropopause at tropical latitudes and is expected to be above the tropopause only when observed in the January-February to July-August period, when the $\mathrm{H}_{2} \mathrm{O}$ content of the air entering the stratosphere reaches its seasonal maximum.
Acknowledgment. Research at the Jet Propulsion Laboratory, California Institute of Technology was performed under contract to the National Aeronautics and Space Administration.

\section{References}

Abbas M. M., et al., The hydrogen budget of the stratosphere inferred from ATMOS measurements of $\mathrm{H}_{2} \mathrm{O}$ and $\mathrm{CH}_{4}$, this issue.

Bithell, M., et al., Synoptic interpretation of measurements from HALOE, J. Atmos. Sci., 51, 2942-2956, 1994.

Boering, K. A., et al., Measurements of stratospheric carbon dioxide and water vapor at northern midlatitudes: Implications for troposphere to stratosphere transport, Geophys. Res. Lett., submitted for publication, 1995.

Brasseur, G., and S. Solomon, Aeronomy of the Middle Atmosphere, D. Reidel Publishing, Holland, 1986.

Carr, E. S., et al., Tropical stratospheric water vapor measured by the microwave limb sounder (MLS), Geophys. Res. Lett., 22, 691-694, 1995.

Chang, A. Y., et al., A comparison of ATMOS and ER-2 measurements from ASHOE/MAESA: Reactive species, this issue.

Dessler, A. E., et al., An examination of the total hydrogen budget of the lower stratosphere, 21, 2563-2566, 1994.

Ehhalt, D. H., and A. Tonnisen, Hydrogen and carbon compound in the stratosphere, 129-151, in Proc. NATO Advan. Study Inst. on Atmos. Ozone, eds., M Nicolet and A. C. Aikin, U. S. Dept of Transportation, Washington, D. C., 1980.

Gunson, M. R., et al., Measurements of $\mathrm{CH}_{4}, \mathrm{~N}_{2} \mathrm{O}, \mathrm{CO}, \mathrm{H}_{2} \mathrm{O}$, and $\mathrm{O}_{3}$ in the middle atmosphere by the Atmospheric Trace Molecule Spectroscopy experiment on Spacelab 3,J. Geophys. Res., 95, $13,867-13,882,1990$.

Gunson, M. R,, et al., The Atmospheric Trace Molecule Spectroscopy experiment deployment on the ATLAS-3 space shuttle mission, this issue.

Hansen A. R., and G. D. Robinson, Water vapor and methane in the upper stratosphere: An examination of some of the Nimbus measurements, J. Geophys. Res., 94, 8474-8484, 1989.

Hintsa, E.J., et al., SPADE $\mathrm{H}_{2} \mathrm{O}$ measurements and the seasonal cycle of stratospheric water vapor, Geophys. Res. Lett., 21, 2559-2562, 1994.

Jones, R.L., et al., The water vapor budget of the stratosphere studied using LIMS and the SAMS satellite data, Q.J.R. Meteorol. Soc., 112, $1127-1143,1986$.

Kelly, K.K., et al., Dehydration in the lower Antarctic stratosphere during the winter and the early spring, 1987, J. Geophys. Res., 94, $11,317-11,357,1989$

Kelly, K.K., et al., Water vapor and cloud measurements over Darwin during the STEP 1987 tropical mission, J. Geophys. Res., 98, 8713-8723, 1993

Kley, D., et al., In situ measurements of the mixing ratio of the water vapor in the stratosphere, J. Atmos. Sci., 36, 2513-2534, 1979.

McCormick, M. P., et al., Annual variations of water vapor in the stratosphere and upper stratosphere observed by the stratospheric aerosol and gas experiment II, J. Geophys. Res., 98, 4867-4874, 1993.

Mote P. M., et al., An atmospheric tape recorder: The imprint of tropical tropopause temperature on stratospheric water vapor, J. Geophys. Res., submitted for publication, 1995.

Oltmans, S. J., and D. J, Hofmann, Increase in lower-stratospheric wate vapor at a mid-latitude northern hemisphere site from 1981 to 1994 , Nature, 374, 146-149, 1995.

Reid, G. C., and K. Gage, On the annual variation of the tropical tropopause, J. Atmos. Sci., 38, 1928-1938, 1981.

Remsberg, E. E., P. P. Bhatt, and J. M. Russell III, Estimates of water vapor budget of the stratosphere from UARS HALOE data, $J$. Geophys. Res., in press, 1995.

Rind, D., et al., Overview of the stratospheric aerosol gas experiment II water vapor observations: Method, validation, and data characteristics, J. Geophys. Res., 98, 4835-4856, 1993.

WMO, Scientific Assessment of Ozone Depletion: 1994, World Meteorological Organization, Global Ozone Research and Monitoring Project - No. 37, 1995.

M. M. Abbas, Mail Code ES41, Space Sciences Laboratory, NASA Marshall Space Flight Center, AL 35812. (e-mail:mian.abbas@msfc. nasa.gov)

(Received October 17, 1995; revised January 24, 1996; accepted March 26, 1996.) 


\title{
The hydrogen budget of the stratosphere inferred from ATMOS measurements of $\mathrm{H}_{2} \mathrm{O}$ and $\mathrm{CH}_{4}$
}

\author{
M. M. Abbas, ${ }^{1}$ M. R. Gunson, ${ }^{2}$ M. J. Newchurch, ${ }^{3}$ H. A. Michelsen, ${ }^{4}$ R. J. Salawitch, ${ }^{2}$ \\ M. Allen, ${ }^{5}$ M. C. Abrams, ${ }^{6}$ A. Y. Chang, ${ }^{2}$ A. Goldman, ${ }^{7}$ F. W. Irion, ${ }^{5}$ E. J. Moyer, ${ }^{5}$ \\ R. Nagaraju, ${ }^{3}$ C. P. Rinsland, ${ }^{6}$ G. P. Stiller, ${ }^{8}$ and R. Zander ${ }^{9}$
}

\begin{abstract}
The total hydrogen budget of the stratosphere and lower mesosphere has been examined using vertical mixing ratio profiles of $\mathrm{H}_{2} \mathrm{O}$ and $\mathrm{CH}_{4}$ measured by the Atmospheric Trace Molecule Spectroscopy (ATMOS) experiment from four space shuttle missions. The oxidation of $\mathrm{CH}_{4}$ and $\mathrm{H}_{2}$ is investigated by evaluating the quantity $\mathrm{H}\left(=\mathrm{H}_{2} \mathrm{O}+2 \mathrm{CH}_{4}\right)$ entering the stratosphere, and examining its conservation with altitude in the upper atmosphere. Data from all four ATMOS missions indicate $\boldsymbol{H}$ to be nearly conserved in the lower stratosphere and to exhibit a broad maximum in the $35-$ to $65-\mathrm{km}$ range. The observations provide evidence of a secondary source of $\mathrm{H}_{2} \mathrm{O}$ from $\mathrm{H}_{2}$ oxidation at altitudes from 35 to $55 \mathrm{~km}$, and net production of $\mathrm{H}_{2}$ at altitudes above $\sim 55 \mathrm{~km}$. ATMOS measurements of $\mathrm{H}_{2} \mathrm{O}$ and $\mathrm{CH}_{4}$ permit the first evaluation of a sickle-shaped vertical profile of $\mathrm{H}_{2}$ that is qualitatively consistent with profiles calculated using two-dimensional models.
\end{abstract}

\section{Introduction}

Stratospheric and lower mesospheric hydrogen resides mostly in $\mathrm{H}_{2} \mathrm{O}, \mathrm{CH}_{4}$, and $\mathrm{H}_{2}$ with negligible contributions to the total budget expected from all other constituents. In the stratosphere, the oxidation of $\mathrm{CH}_{4}$ produces approximately two molecules of $\mathrm{H}_{2} \mathrm{O}$ for each molecule of $\mathrm{CH}_{4}$ that is removed, and the gradient $2 \mathrm{H}_{2} \mathrm{O} / \partial \mathrm{CH}_{4}$ is expected to be nearly equal to -2 . Production of $\mathrm{H}_{2}$ from oxidation of $\mathrm{CH}_{4}$ is nearly balanced by oxidation of $\mathrm{H}_{2}$, so that the mixing ratio of $\mathrm{H}_{2}$ remains nearly constant with altitude in the lower stratosphere. Model calculations [Le Texier et al., 1988] suggest the mixing ratio of $\mathrm{H}_{2}$ reaches a broad minimum in the midstratosphere, where oxidation begins to proceed more rapidly, and increases at higher altitudes due to production from photolysis of $\mathrm{H}_{2} \mathrm{O}$. The few measurements of stratospheric $\mathrm{H}_{2}$ to date have been acquired by rockets or from balloons using grab sampling techniques and are limited to altitudes below $\sim 40 \mathrm{~km}$ [e.g., Ehhalt and Tonnisen, 1980; Schmidt et al., 1974; Fabian et al., 1979).

In this paper, vertical profiles of $\mathrm{H}_{2} \mathrm{O}$ and $\mathrm{CH}_{4}$ measured by ATMOS are examined to evaluate the hydrogen budget of the

\footnotetext{
1 NASA Marshall Space Flight Center.

2 Jet Propulsion Laboratory, California Institute of Technology.

3 University of Alabama at Huntsville.

${ }^{4}$ Harvard University.

5 California Institute of Technology.

${ }^{6}$ SAIC - NASA Langley Research Center.

${ }^{7}$ Denver University.

${ }^{8}$ Forschungszentrum Karlsruhe/Universitaet Karlsruhe.

${ }^{9}$ Institute of Astrophysics, University of Liege.
}

Copyright 1996 by the American Geophysical Union.

Paper number 96GL01320

0094-8534/96/96GL-01320\$05.00 stratosphere and lower mesosphere. The conservation of hydrogen between $\mathrm{H}_{2} \mathrm{O}$ and $\mathrm{CH}_{4}$ is examined for different altitude regions. The distribution of $\mathrm{H}_{2}$ is inferred by calculating the average $\mathrm{H}_{\mathrm{tot}}$ entering the lower stratosphere, and requiring the total hydrogen $\mathrm{H}_{\mathrm{tot}}=\mathrm{H}+\mathrm{H}_{2}$ to be conserved with altitude.

\section{Photochemistry of Stratospheric Hydrogen Compounds}

The distribution of $\mathrm{H}_{2} \mathrm{O}$ in the stratosphere is maintained by oxidation of $\mathrm{CH}_{4}$ by reaction with $\mathrm{OH}, \mathrm{O}\left({ }^{\mathrm{l}} \mathrm{D}\right)$, and to a lesser extent $\mathrm{Cl}$, producing formaldehyde $\left(\mathrm{CH}_{2} \mathrm{O}\right)$ with a chain of reactions [e.g., Bates and Nicolet, 1965; Ehhalt and Tonnisen, 1980; Brasseur and Solomon, 1986]. Formaldehyde is converted into $\mathrm{H}_{2}, \mathrm{CO}$, and $\mathrm{H}_{2} \mathrm{O}$ by photolysis and reaction with $\mathrm{OH}$. The end products of the above chain of reactions are hydrogen radicals $\left(\mathrm{H}, \mathrm{OH}\right.$, and $\left.\mathrm{HO}_{2}\right)$ with lifetimes of less than a day, which quickly recombine to form $\mathrm{H}_{2} \mathrm{O}$ mainly with the reaction

$$
\mathrm{OH}+\mathrm{HO}_{2} \rightarrow \mathrm{H}_{2} \mathrm{O}+\mathrm{O}_{2} \text {, }
$$

and constitute the main photochemical source of water vapor in the stratosphere. Water vapor is lost in the stratosphere by

$$
\mathrm{H}_{2} \mathrm{O}+\mathrm{O}\left({ }^{1} \mathrm{D}\right) \rightarrow 2 \mathrm{OH},
$$

and in the mesosphere by photolysis

$$
\mathrm{H}_{2} \mathrm{O}+\mathrm{hv} \rightarrow \mathrm{H}+\mathrm{OH} \text {. }
$$

$\mathrm{H}_{2}$ is produced by

$$
\begin{gathered}
\mathrm{CH}_{2} \mathrm{O}+\mathrm{h} v \rightarrow \mathrm{H}_{2}+\mathrm{CO}, \\
\mathrm{CH}_{4}+\mathrm{O}\left({ }^{\mathrm{l} D}\right) \rightarrow \mathrm{H}_{2}+\mathrm{CH}_{2} \mathrm{O}, \\
\mathrm{H}+\mathrm{HO}_{2} \rightarrow \mathrm{H}_{2}+\mathrm{O}_{2}, \\
\mathrm{H}_{2} \mathrm{O}+\mathrm{hv} \rightarrow \mathrm{H}_{2}+\mathrm{O}\left({ }^{1} \mathrm{D}\right),
\end{gathered}
$$

and is lost by oxidation due to $\mathrm{OH}$ and $\mathrm{O}\left({ }^{\mathrm{l}} \mathrm{D}\right)$, similar to methane,

$$
\begin{aligned}
& \mathrm{H}_{2}+\mathrm{OH} \rightarrow \mathrm{H}_{2} \mathrm{O}+\mathrm{H}, \\
& \mathrm{H}_{2}+\mathrm{O}\left({ }^{\mathrm{l}} \mathrm{D}\right) \rightarrow \mathrm{OH}+\mathrm{H} .
\end{aligned}
$$

One-dimensional photochemical model calculations using the ATMOS atmospheric profiles indicate that the major source ( 80 to $95 \%$ ) of $\mathrm{H}_{2}$ in the stratosphere and the lower mesosphere up to altitudes of $\sim 55 \mathrm{~km}$ is the photolysis of $\mathrm{CH}_{2} \mathrm{O}$ (Eq. 4) with a minor contribution from reaction (5). In the mesosphere at altitudes above $55 \mathrm{~km}, \mathrm{H}+\mathrm{HO}_{2}$ and the photolysis of $\mathrm{H}_{2} \mathrm{O}$ (Eqs. 6 and 7) dominate the production of $\mathrm{H}_{2}$. Loss of $\mathrm{H}_{2}$ in the stratosphere occurs by reaction with $\mathrm{OH}(\mathrm{Eq} .8)$, while loss in the mesosphere is dominated by reaction with $\mathrm{O}\left({ }^{1} \mathrm{D}\right)(\mathrm{Eq} .9)$. 
The three species $\mathrm{CH}_{4}, \mathrm{H}_{2} \mathrm{O}$, and $\mathrm{H}_{2}$ are rather long lived in the lower stratosphere with similar photochemical lifetimes of many years; lifetimes decrease to only a few months at $40 \mathrm{~km}$. In the upper stratosphere and lower mesosphere, the lifetime of $\mathrm{H}_{2}$ increases progressively back to a few years. The time constants for transport for the three species at stratospheric altitudes are comparable to the photochemical lifetimes [e.g., Le Texier et al., 1988]; consequently, the effect of transport processes is significant in determining global distributions.

\section{Water Vapor and Methane Distributions}

High-resolution, infrared, limb-viewing, solar-occultation observations by ATMOS have been carried out on four shuttle missions starting with Spacelab 3 in May 1985, and ATLAS 1 to 3 in April 1992, April 1993, and November 1994, respectively. Data from each mission with the same instrument have been processed in a similar manner using ATMOS version 2 processing algorithms [Gunson et al., 1996]. These observations provide simultaneous measurements of mixing ratio profiles of $\mathrm{H}_{2} \mathrm{O}$ and $\mathrm{CH}_{4}$ over various latitudes, employing optical bandpass filters in the 1,100 to $2,000 \mathrm{~cm}^{-1}, 650$ to 2,450 $\mathrm{cm}^{-1}$, and 1,580 to $3,420 \mathrm{~cm}^{-1}$ spectral regions. ATMOS retrieves mixing ratios of $\mathrm{H}_{2} \mathrm{O}$ and $\mathrm{CH}_{4}$ with estimated total uncertainties of $\sim 6 \%$ and $5 \%$, respectively [Gunson et al., 1996]. A comparison of ATMOS observations of $\mathrm{H}_{2} \mathrm{O}, \mathrm{CH}_{4}$, and $\mathrm{N}_{2} \mathrm{O}$ with nearly coincident in situ measurements obtained in the lower stratosphere during November 1994 demonstrates the high degree of accuracy and precision of the space-borne observations [Chang et al., 1996]. The current analyses examine the $\mathrm{H}_{2} \mathrm{O}$ and $\mathrm{CH}_{4}$ measurements obtained from the four ATMOS missions, with a particular emphasis on the comprehensive data provided by the ATLAS-3 mission obtained over the 8 to $49^{\circ} \mathrm{N}$ latitude range. The high accuracy and precision of these measurements permits a qualitative evaluation of the total hydrogen budget of the upper atmosphere.

Figure 1 shows correlation plots of $\mathrm{H}_{2} \mathrm{O}$ versus $\mathrm{CH}_{4}$ for all four ATMOS missions, restricted to data obtained out of the polar vortex regions indicated by a marked decrease in $\mathrm{CH}_{4}$ and $\mathrm{N}_{2} \mathrm{O}$ profiles, with the open circles representing data obtained in the $18-$ to $35-\mathrm{km}$ range and the crosses representing data outside this range. The dotted lines in Figure 1 with fixed slopes of -2 represent expected distributions if oxidation of each $\mathrm{CH}_{4}$ molecule were to produce two molecules of $\mathrm{H}_{2} \mathrm{O}$. Deviations of the data from a slope of -2 provide information about the total hydrogen budget of the stratosphere. The solid lines in Figure 1 show a linear least square fit to all measurements obtained between altitudes of 18 and $35 \mathrm{~km}$. This altitude region was chosen to restrict our analysis to stratospheric air, for regions where the mixing ratio of $\mathrm{H}_{2}$ is not expected to vary appreciably [e.g., Le Texier et al., 1988].

The slopes of the linear fits in Figure 1 representing averages of four values derived from data with lower altitude varying from 18 to $21 \mathrm{~km}$, and the upper altitude at $35 \mathrm{~km}$, are: $-2.09 \pm$ 0.13 for Spacelab 3; and $-2.11 \pm 0.08,-2.14 \pm 0.08$, and 1.99 \pm 0.07 for ATLAS 1 to 3 missions, respectively, with the uncertainties indicating the $1 \sigma$ standard deviation of the linear regression. The estimated total uncertainties in the slope, accounting for the total uncertainty in the measurements of $\mathrm{H}_{2} \mathrm{O}$ and $\mathrm{CH}_{4}$, are: \pm 0.21 for Spacelab 3; and $\pm 0.18,0.19$, and 0.17 for ATLAS 1 to 3 , respectively. The weighted mean slope $\partial \mathrm{H}_{2} \mathrm{O} / \partial \mathrm{CH}_{4}=-2.05 \pm 0.16$ for the four ATMOS missions indicates near conservation of $\boldsymbol{H}$ in the lower and middle stratosphere. The region above altitudes at which $\mathrm{CH}_{4}$ mixing ratios are less than $\sim 0.8$ ppmv, however, exhibits excess $\mathrm{H}_{2} \mathrm{O}$ and

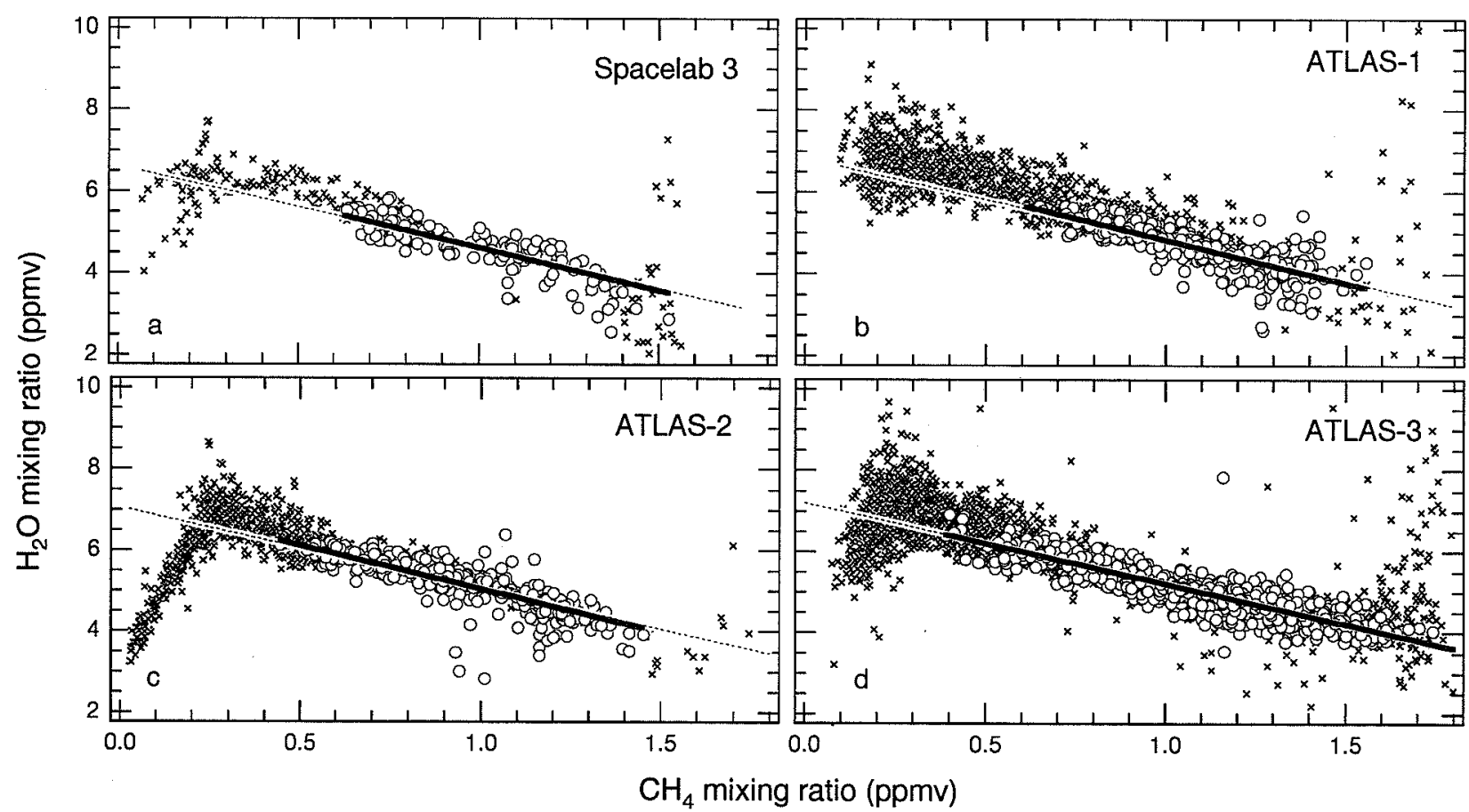

Figure 1. Plots of $\mathrm{H}_{2} \mathrm{O}$ versus $\mathrm{CH}_{4}$ for: (a) 8 occultations for Spacelab-3 (April, 1985) over $50^{\circ} \mathrm{S}$ to $31^{\circ} \mathrm{N}$, (b) 50 occultations for ATLAS-1 (March 1992) over $54^{\circ} \mathrm{S}$ to $28^{\circ} \mathrm{N}$, (c) 26 occultations of ATLAS-2 (October 1993) over $50^{\circ} \mathrm{S}$ to $67^{\circ} \mathrm{N}$, (d) 88 occultations of ATLAS-3 (November 1994) in the $72^{\circ} \mathrm{S}$ to $54^{\circ} \mathrm{N}$ latitude range. Data obtained at altitudes between 18 and $35 \mathrm{~km}$ are indicated by open circles, crosses represent data obtained outside this range. Only data obtained outside polar vortices are shown. The solid lines represent linear least square fits to the data in the $18-$ to $35-\mathrm{km}$ range (equations given in the text), and the dotted lines represent fits to the same data of lines with a fixed slope of -2 . 

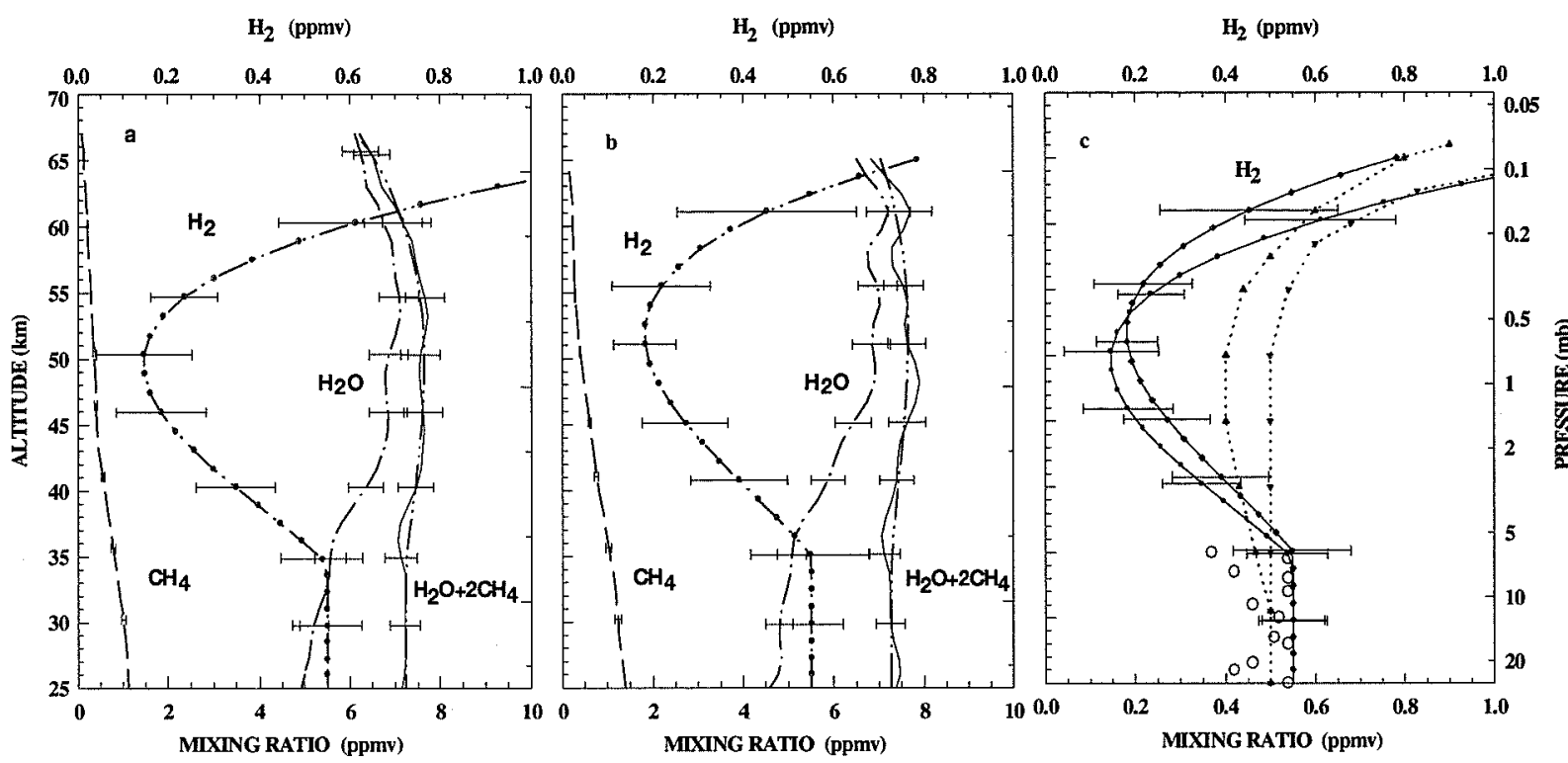

Figure 2. (a) The vertical profiles of $\mathrm{CH}_{4}, \mathrm{H}_{2} \mathrm{O}, \boldsymbol{H}=\left(\mathrm{H}_{2} \mathrm{O}+2 \mathrm{CH}_{4}\right)$, and inferred $\mathrm{H}_{2}$ from a zonal average of 33 sunset occultations over 31 to $49^{\circ} \mathrm{N}$. The dash-dot.dot curve shows a polynomial fit for $\boldsymbol{H}$ with a linear fit in the region below $-35 \mathrm{~km}$, (b) for 14 occultations over 8 to $28^{\circ} \mathrm{N}$, (c) comparison of inferred $\mathrm{H}_{2}$ distributions for midlatitudes (filled circles) and low latitudes (diamonds); two-dimensional model calculations of $L e$ Texier et al. [1988] for midlaitudes (triangles up) and low latitudes (triangles down); observed values of $\mathrm{H}_{2}$ for $3^{\circ} \mathrm{N}$ are shown in open circles [Ehhalt and Tonnisen, 1980].

more negative slope for all four missions, and will be discussed further in the next section.

The intercepts of the linear least square fits with fixed slopes of -2 represent the average $\boldsymbol{H}$ in the $18-$ to $35-\mathrm{km}$ region: 6.70 $\pm 0.03 \mathrm{ppmv}$ for Spacelab 3; and $6.86 \pm 0.01,7.10 \pm 0.02$, and $7.23 \pm 0.02$ ppmv for ATLAS 1 to 3 , respectively, with the uncertainties indicating the $1 \sigma$ standard deviation of the linear regression. With estimated systematic errors of $6 \%$ for $\mathrm{H}_{2} \mathrm{O}$ and $5 \%$ for $\mathrm{CH}_{4}$, the total uncertainties in the intercept are calculated to be: \pm 0.43 ppmv for Spacelab 3; and $\pm 0.43,0.41$, and $0.40 \mathrm{ppmv}$ for ATLAS 1 to 3, respectively. Our estimate for the amount of $\mathrm{H}_{2} \mathrm{O}$ entering the stratosphere is based on linear least square fits to ATMOS measurements of $\mathrm{H}_{2} \mathrm{O}$ and $\mathrm{CH}_{4} \mathrm{col}$ lected over a wide range of altitudes and latitudes, rather than actual observations at the tropopause, to minimize the sensitivity of our results to seasonal variations on $\mathrm{H}_{2} \mathrm{O}$. Seasonal oscillations present in individual ATMOS occultations are likely to be averaged out by this process, since air with a variety of ages is examined [Dessler et al., 1994]. Using a $\mathrm{CH}_{4}$ mixing ratio of $1.70 \mathrm{ppmv}$ for 1994 at the tropopause, and assuming an average yearly $\mathrm{CH}_{4}$ increase of $0.46 \%$ /year between 1990 and 1994 and a $0.73 \% / y e a r$ increase over the period 1985 to 1989 [WMO, 1994], we deduce the $\mathrm{H}_{2} \mathrm{O}$ content of air entering the stratosphere for the four missions to be: $3.48 \pm 0.26 \mathrm{ppmv}$ for Spacelab 3; and 3.51 $\pm 0.27,3.72 \pm$ 0.28 , and $3.83 \pm 0.29$ ppmv for ATLAS 1 to 3 , respectively. A mean value of $3.70 \pm 0.28$ ppmv of $\mathrm{H}_{2} \mathrm{O}$ entry into the stratosphere, weighted to the number of observed occultations, is thus inferred from the data base of the four ATMOS missions.

The mean value of $\mathrm{H}_{2} \mathrm{O}$ entering the stratosphere compares with annual average values in the range of 3.2 to $3.7 \mathrm{ppmv}$ estimated by Remsberg et al. [1995] from the data set obtained by HALOE from 1992 to 1994 , and the value $4.2 \pm 0.5 \mathrm{ppmv}$ obtained by in situ measurements during May 1993 [Dessler et $a l ., 1994]$; the discrepancy between these values, however, is within the uncertainty of measurements. A more detailed comparison of the hydrogen budget inferred by ATMOS with analy- ses based on other remote and in situ measurements is given in Abbas et al. [1996], which focuses on the seasonal variation of $\mathrm{H}_{2} \mathrm{O}$ in the tropical lower stratosphere observed by ATMOS. The lower stratosphere observed by ATMOS during the Spacelab 3 mission in April 1985 appears dry relative to the later ATMOS measurements. It is unclear what significance, if any, to attach to the apparent trend in the $\mathrm{H}_{2} \mathrm{O}$ content of air entering the stratosphere inferred from the four missions, which with ATLAS 1 data excepted, appears to be of the same order as a $0.8 \%$ per year trend in stratospheric $\mathrm{H}_{2} \mathrm{O}$ that has been inferred from a 10 -year time series of frost-point hygrometer measurements at Boulder, CO [Oltmans and Hofmann, 1995]. A more comprehensive analysis to address the significance of the apparent trend should account fully for the different latitudinal and seasonal coverage of the four missions, possible biases associated with the ATMOS retrievals of $\mathrm{H}_{2} \mathrm{O}$ using its various optical filters, as well as an examination of satellite, balloon-borne, and in situ measurements of $\mathrm{CH}_{4}$ and $\mathrm{H}_{2} \mathrm{O}$ over the past decade.

\section{Hydrogen Budget of the Stratosphere and Lower Mesosphere}

Simultaneous measurements of $\mathrm{H}_{2} \mathrm{O}$ and $\mathrm{CH}_{4}$ provided by ATMOS permit an evaluation of the partitioning of hydrogen species in the stratosphere and lower mesosphere. The correlation plots (Figure 1) for all four missions indicate an excess of $\mathrm{H}_{2} \mathrm{O}$ relative to the amount supplied by oxidation of $\mathrm{CH}_{4}$, for $\mathrm{CH}_{4}$ less than $\sim 0.5 \mathrm{ppmv}$ (corresponding to altitudes of $\sim 35$ $\mathrm{km}$ ). The excess $\mathrm{H}_{2} \mathrm{O}$ present in this region of the correlation plots is evidence of a source of $\mathrm{H}_{2} \mathrm{O}$ from oxidation of $\mathrm{H}_{2}$. For $\mathrm{CH}_{4}$ mixing ratios less than $\sim 0.2$ ppmv (altitudes of $\sim 50 \mathrm{~km}$ ), $\mathrm{H}_{2} \mathrm{O}$ and $\mathrm{CH}_{4}$ are positively correlated, implying the conversion of $\mathrm{H}_{2} \mathrm{O}$ and $\mathrm{CH}_{4}$ to $\mathrm{H}_{2}$ by reactions $\mathrm{H}+\mathrm{HO}_{2}$ and photolysis of $\mathrm{H}_{2} \mathrm{O}$ (Eqs. 6 and 7).

A vertical distribution for $\mathrm{H}_{2}$ can be inferred from ATMOS measurements of $\mathrm{H}_{2} \mathrm{O}$ and $\mathrm{CH}_{4}$ by assuming total hydrogen 
$\mathrm{H}_{\text {tot }}=\left(\boldsymbol{H}+\mathrm{H}_{2}\right)$ transported to the lower stratosphere from the troposphere is conserved with altitude. Figures $2 a-b$ show the average vertical profiles of $\mathrm{CH}_{4}, \mathrm{H}_{2} \mathrm{O}$, and $\boldsymbol{H}$ obtained from 27 ATLAS-3 representative sunset occultations for midlatitudes in the 28 to $49^{\circ} \mathrm{N}$ range, and from 13 occultations for low latitudes in the 8 to $28^{\circ} \mathrm{N}$ range, respectively. The error bars indicate the quadrature sum of $1 \sigma$ standard deviation of the mean values and systematic errors arising from $6 \%$ and $5 \%$ uncertainties in the spectral parameters of $\mathrm{H}_{2} \mathrm{O}$ and $\mathrm{CH}_{4}$. The vertical profiles of $\boldsymbol{H}$ have been smoothed using polynomial curves above $\sim 35 \mathrm{~km}$ and linear fits in the lower region, in order to remove smallscale structures. It is clear from both the observed and smoothed plots that $\boldsymbol{H}$ is not conserved with altitude. The maximum of the quantity $\boldsymbol{H}$ at altitudes higher than $50 \mathrm{~km}$ indicates that production of $\mathrm{H}_{2}$ by $\mathrm{H}+\mathrm{HO}_{2}$ and photolysis of $\mathrm{H}_{2} \mathrm{O}$ becomes important at these altitudes.

Distributions of $\mathrm{H}_{2}$ have been derived by subtracting the smoothed curve fit for $\boldsymbol{H}$ from a constant value for $\mathrm{H}_{\text {tot }}$ of 7.80 ppmv assuming a tropospheric mixing ratio of $\mathrm{H}_{2}=0.55 \mathrm{ppmv}$ [Ehhalt and Tonnisen, 1980] and a value of $\boldsymbol{H}=7.25 \mathrm{ppmv}$ based on ATMOS/ATLAS-3 observations in the $\mathrm{NH}$ at midlatitudes and the tropics. The minimum mixing ratio inferred for $\mathrm{H}_{2}$ occurs at $-50 \mathrm{~km}$ with values of $0.15 \pm 0.1 \mathrm{ppmv}$ at mid-latitudes and $0.18 \pm 0.1 \mathrm{ppmv}$ at the tropics. The uncertainty in this case represents the standard deviation of the mean profile for $\boldsymbol{H}$, and does not include systematic errors in the measurements of $\mathrm{H}_{2} \mathrm{O}$ and $\mathrm{CH}_{4}$. The inferred profile for $\mathrm{H}_{2}$ is sensitive also to seasonal and episodic variations in $\boldsymbol{H}$, neither of which are accounted for in the present analysis. For example, profiles for $\mathrm{H}_{2}$ inferred from ATLAS 1 measurements (not shown) exhibit a shallower minimum (less variation with altitude) than profiles shown in Figure 2.

A comparison of the plots in Figures $2 a-b$ shows that the mixing ratios of $\mathrm{H}_{2} \mathrm{O}$ are higher and of $\mathrm{CH}_{4}$ are lower at the midlatitudes for altitudes below $\sim 50 \mathrm{~km}$, as expected from the Brewer circulation. Consistent with the indicated latitudinal differences in $\mathrm{H}_{2} \mathrm{O}$ and $\mathrm{CH}_{4}$ distributions, the inferred $\mathrm{H}_{2}$ mixing ratios are higher in the midlatitude stratosphere below 50 $\mathrm{km}$ where $\mathrm{CH}_{4}$ mixing ratios are higher and $\mathrm{H}_{2}$ is produced by oxidation of $\mathrm{CH}_{4}$ via $\mathrm{C} \mathrm{H}_{2} \mathrm{O}$. In the mesosphere above $50 \mathrm{~km}$, $\mathrm{H}_{2}$ produced by $\mathrm{H}+\mathrm{HO}_{2}$ and photolysis of $\mathrm{H}_{2} \mathrm{O}$ leads to higher values in the tropics compared with the midlatitudes.

Figure $2 \mathrm{c}$ shows a comparison of the inferred $\mathrm{H}_{2}$ distributions with the profiles for midlatitudes and the tropics calculated by Le Texier et al. [1988] using a two-dimensional model with coupled photochemistry and dynamics. Also shown by open circles are the observed values of $\mathrm{H}_{2}$ limited to an altitude of $35 \mathrm{~km}$, at $32^{\circ} \mathrm{N}$ [Ehhalt and Tonnisen, 1980]. Although the inferred $\mathrm{H}_{2}$ distributions for midlatitudes appear to be qualitatively similar to the model calculations, the minimum inferred values are lower than the model values. The altitude of minimum $\mathrm{H}_{2}$, however, appears to be in general agreement with the two-dimensional model. Model calculations by Le Texier et al. indicate that mesospheric transport effects are important for high latitudes in the winter where air rich in $\mathrm{H}_{2}$ is transported to lower altitudes in the stratosphere. Since such large values of $\mathrm{H}_{2}$ have not been inferred for the low and midlatitude regions considered here, consistent with the model calculations of Le Texier et al., the effect of mesospheric transport does not appear to be evident. In a recent work brought to our attention, Harries et al. [1996] have employed a similar technique for inferring mixing rations of $\mathrm{H}_{2}$ in the mesosphere from HALOE measurements. Although the high mixing ratios above $0.5 \mathrm{mb}$ levels inferred in their study are qualitatively consistent with the results presented here, a comparison for lower levels cannot be made at this time because of different assumptions employed in the two studies.

\section{Conclusions}

An examination of measurements of $\mathrm{H}_{2} \mathrm{O}$ and $\mathrm{C} \mathrm{CH}_{4}$ made by ATMOS indicates that the quantity $\boldsymbol{H}=\left(\mathrm{H}_{2} \mathrm{O}+2 \mathrm{CH}_{4}\right)$ is conserved in the lower and middle stratosphere and yields an average water vapor entry into the stratosphere of $3.70 \pm 0.28$ ppmv. $\boldsymbol{H}$ is not conserved in the upper stratosphere and lower mesosphere; it reaches a maximum at $\sim 50$ to $55 \mathrm{~km}$, then decreases with height. This structure is evident in the correlation plots of $\mathrm{H}_{2} \mathrm{O}$ versus $\mathrm{CH}_{4}$ for all four missions. The region near $-50 \mathrm{~km}$ is characterized by an excess abundance of $\mathrm{H}_{2} \mathrm{O}$ $\sim 0.4 \mathrm{ppmv}$, providing evidence of a source of $\mathrm{H}_{2} \mathrm{O}$ from oxidation of $\mathrm{H}_{2}$, whereas the decrease in $\boldsymbol{H}$ observed at higher altitudes implies net production of $\mathrm{H}_{2}$ from $\mathrm{H}_{2} \mathrm{O}$ and $\mathrm{CH}_{4}$. The distribution of $\mathrm{H}_{2}$ inferred from variation of $\boldsymbol{H}$ with altitude indicates a sickle-shaped profile with a constant mixing ratio at altitudes of $\leq 35 \mathrm{~km}$, a minimum at $\sim 50 \mathrm{~km}$, and increasing to $>1 \mathrm{ppmv}$ above $65 \mathrm{~km}$. The inferred distribution of $\mathrm{H}_{2}$ is in qualitative agreement with the values obtained from a twodimensional coupled photochemical-dynamical model.

Acknowledgments. Research at the Jet Propulsion Laboratory (JPL), California Institute of Technology is performed under contract to the National Aeronautics and Space Administration (NASA).

\section{References}

Abbas, M. M., et al., Stratospheric measurements of water vapor and methane by ATMOS/ATLAS-3: Methane oxidation and troposphere to stratosphere transport, Geophys. Res. Lett., this issue, 1996.

Bates, D. R., and M. Nicolet, Atmospheric hydrogen, Plan. Spa. Sci., 13, 905-909, 1965.

Brasseur, G., and S. Solomon, Aeronomy of the Middle Atmosphere, D. Reidel Publishing, Holland, 1986.

Chang, A. Y., et al., A comparison of ATMOS and ER-2 measurements from ASHOE/MAESA: Reactive species, this issue.

Dessler, A. E., et al., An examination of the total hydrogen budget of the lower stratosphere, $21,2563-2566,1994$.

Ehhalt, D. H., and A. Tonnisen, Hydrogen and carbon compound in the stratosphere, 129-151, in Proc. NATO Advan. Study Institute on Atmospheric Ozone, eds., M. Nicolet and A. C. Aiken, U. S. Department of Transportation, Washington, D. C., 1980.

Fabian, P., et al., Simultaneously measured vertical profiles of $\mathrm{H}_{2}, \mathrm{CH}_{4}$, $\mathrm{CO}, \mathrm{N}_{2} \mathrm{O}, \mathrm{CFCl}_{3}$, and $\mathrm{CF}_{2} \mathrm{Cl}_{2}$ in the midlatitude stratosphere and troposphere, J. Geophys. Res., 84, 3149, 1979.

Gunson, M. R., et al., The Atmospheric Trace Molecule Spectroscopy experiment deployment on the ATLAS-3 Space Shuttle Mission, this issue.

Harries, J. E., S. Ruth, and J. M. Russell III, On the distribution of mesospheric molecular hydrogen inferred from HALOE measurements of $\mathrm{H}_{2} \mathrm{O}$ and $\mathrm{CH}_{4}$, Geophys. Res. Lett., in press, 1996.

Le Texier, H., S. Solomon, and R.R. Garcia, The role of molecular hydrogen and methane oxidation in the water vapor budget of the stratosphere, Q.J.R. Meteorol., Soc., 114, 281-295, 1988.

Oltmans, S. J., and D. J. Hofmann, Increase in lower-stratospheric water vapor at a mid-latitude northern hemisphere site from 1981 to 1994 Nature, 374, 146-149, 1995.

Remsberg, E. E., P. P. Bhatt, and J. M. Russell III, Estimates of water vapor budget of the stratosphere from UARS HALOE data, $J$. Geophys. Res., in press, 1995.

Schmidt, U., Molecular hydrogen in the atmosphere, Tellus, 26, 78, 1974 WMO, Scientific Assessment of Ozone Depletion: World Meteorological Organization, Global Research and Monitoring Project, No. 37, 1995.

M. M. Abbas, Mail Code ES41, Space Sciences Laburatory, NASA Marshall Space Flight Center, AL 35812. (e-mail:mian.abbas@msfc. nasa.gov)

(Received October 17, 1995; revised March 7, 1996, (accepted April 9, 1996) 


\title{
Stratospheric meteorological conditions for the 3-12 Nov 1994 ATMOS/ATLAS-3 measurements
}

\author{
G.L. Manney \\ Jet Propulsion Laboratory/California Institute of Technology, Pasadena
}

R. Swinbank

Meteorological Office, Bracknell, United Kingdom

\section{A. O'Neill}

Centre for Global Atmospheric Modelling, Reading, United Kingdom

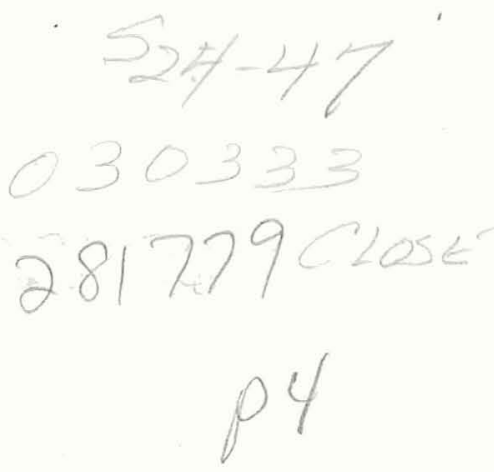

\begin{abstract}
During the ATLAS-3 mission (3-12 Nov 1994), United Kingdom Meteorological Office fields show that the SH vortex was still strong below about $700 \mathrm{~K}(\sim 16 \mathrm{hPa})$, with coherent vortex fragments apparent up to about $1300 \mathrm{~K}(\sim 3 \mathrm{hPa})$. The $\mathrm{SH}$ vortex was shifted off the pole toward $270^{\circ} \mathrm{E}$ throughout ATLAS-3, although its shape varied from day to day. SH temperatures were increasing during ATLAS3 ; temperatures below $188 \mathrm{~K}$ were last seen $35-45$ days before the mission, but were below $195 \mathrm{~K}$ as late as 2 Nov 1994. The NH polar vortex had developed at levels above about $550 \mathrm{~K}(\sim 35 \mathrm{hPa})$. Simulated highresolution potential vorticity (PV) fields clearly show low latitude air being drawn up around the polar vortices in both hemispheres. These fields indicate that meteorological analyses underestimate the amount of atmospheric variability, including the strength of local PV gradients, and small-scale structure. Structure such as that in the simulated fields can result in apparent discrepancies between tracer measurements and PV fields.
\end{abstract}

\section{Introduction}

The Atmospheric Trace Molecule Spectroscopy (ATMOS) instrument measured a large number of stratospheric trace gas concentrations during the ATLAS3 mission, 3-12 Nov 1994. Southern hemisphere (SH) measurements were made at high latitudes $\left(\sim 64^{\circ}-73^{\circ} \mathrm{S}\right)$, in and near the polar vortex. Northern hemisphere (NH) measurements were from the tropics to mid-latitudes $\left(\sim 5^{\circ}-50^{\circ} \mathrm{N}\right)$. We summarize here the meteorological conditions before and during ATLAS-3, knowledge of which is essential to analysis of the ATMOS/ATLAS3 data.

Geopotential heights, temperatures and horizontal winds are from the United Kingdom Meteorological Office (UKMO) stratosphere-troposphere data assimilation system [Swinbank and O'Neill, 1994]. PV is calcu-

Copyright 1996 by the American Geophysical Union.

Paper number 96GL00774

0094-8534/96/96GL-00774\$05.00 lated from these data and is scaled in "vorticity units" (sPV) [e.g., Dunkerton and Delisi, 1986; Manney et al., 1994] to give a similar range of values at all levels; sPV is given in units of $10^{-4} \mathrm{~s}^{-1}$. Strong horizontal sPV gradients on isentropic (constant potential temperature, $\theta$ ) surfaces are associated with a barrier to transport, and thus can be used to identify the approximate size, shape, location and evolution of the polar vortex.

The extent of the $\mathrm{NH}$ and $\mathrm{SH}$ polar vortices during the ATLAS-3 mission is shown in Fig. 1, $|\mathrm{sPV}|$ and the sPV gradient plotted as a function of $\theta$ and equivalent latitude (EqL) [e.g., Butchart and Remsberg, 1986] on 6 Nov 1994. EqL is the latitude that would enclose the same area as each sPV contour for which it is evaluated; sPV plotted in this coordinate thus indicates the size of the vortex, independent of whether it may be distorted or shifted off the pole. In the SH, strong sPV gradients associated with the polar vortex extend from below 375 $\mathrm{K}$ up to about $1000 \mathrm{~K}$; above this level gradients are much weaker, but some indication of a vortex remnant is seen up to about $1300 \mathrm{~K}$. The vortex is still large and strong below about $600 \mathrm{~K}$, with both size and strength decreasing rapidly with height above that level. In the $\mathrm{NH}$, a region of strong sPV gradients associated with the developing polar vortex is apparent above about $550 \mathrm{~K}$.

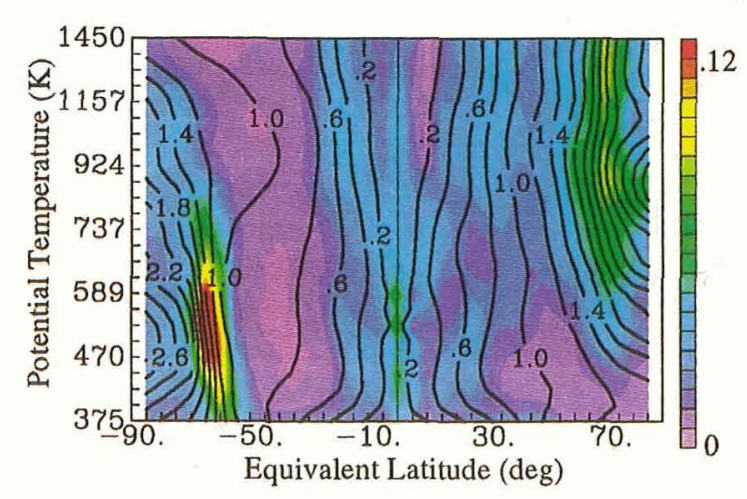

Figure 1. $|\mathrm{sPV}|$ (contours, $10^{-4} \mathrm{~s}^{-1}$ ) and sPV gradient (colors, $10^{-4} \mathrm{~s}^{-1} /{ }^{\circ} \mathrm{EqL}$ ) on $6 \mathrm{Nov} 1994$, as a function of EqL and $\theta$. 


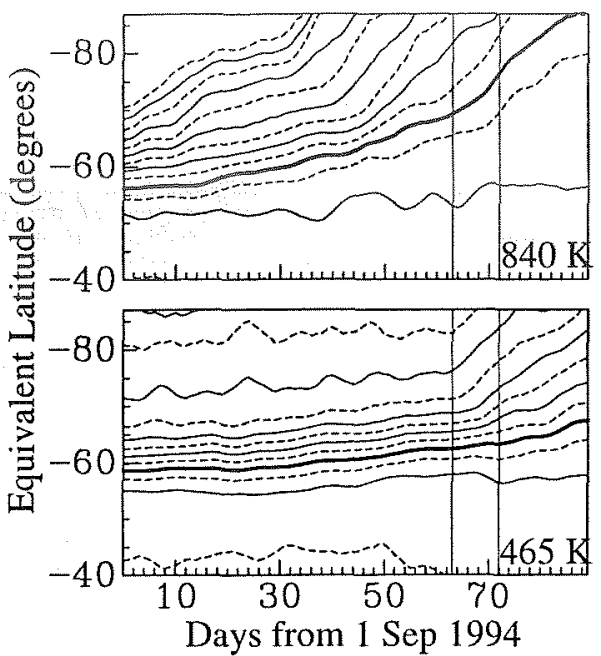

Figure 2. Area integrals of sPV for 1 Sep to $30 \mathrm{Nov}$ 1994 in the $\mathrm{SH}$, at $840(\sim 10 \mathrm{hPa})$ and $465 \mathrm{~K}(\sim 50$ $\mathrm{hPa}$ ). Contour interval is 0.2 , and bold contour is -1.4 $\times 10^{-4} \mathrm{~s}^{-1}$. Vertical lines show the ATLAS-3 period.

\section{Southern Hemisphere}

Fig. 2 shows area integrals [Butchart and Remsberg, 1986] of sPV as a function of EqL during SH late winter/spring 1994, including the ATLAS-3 period. The vortex has already broken down in the upper stratosphere (Fig. 1) and is being substantially eroded in the middle stratosphere (Fig. 2a), with rapid weakening of sPV gradients during ATLAS-3. The lower stratospheric vortex (Fig. 2b) remains strong, with sPV gradients weakening rapidly after mid-November. This evolution is typical, and similar to that in the previous two SH winters [e.g., Manney et al., 1994]. As noted by Manney et al. [1994], at the lowest levels $(\sim 375-420$ $\mathrm{K})$, the vortex remains strong into December.

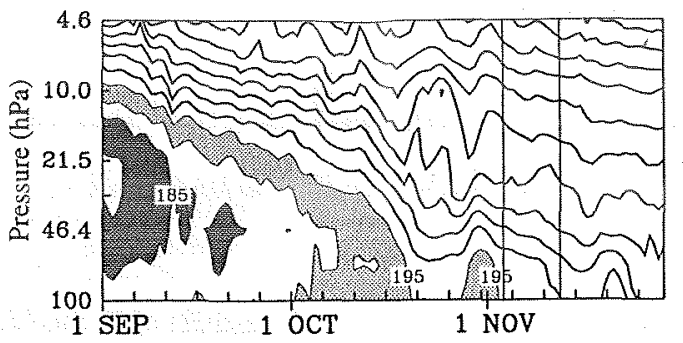

Figure 3. Minimum temperature south of $-40^{\circ}$, for 1 Sep to 30 Nov 1994. Dark shading is $180-185 \mathrm{~K}$ and light shading 190-195 K. Vertical lines show the ATLAS-3 period.

SH winter lower stratospheric vortex temperatures are typically low enough to form both type I and type II polar stratospheric clouds (PSCs) for several months, with important consequences for ozone depletion, dehydration and denitrification. The $1994 \mathrm{SH}$ winter was fairly typical in this respect, and very similar to the 1992 SH winter [Manney and Zurek, 1993]. Temperatures first dropped below $195 \mathrm{~K}$ (a convenient approximation to the type I PSC threshold) around 10 May and below $188 \mathrm{~K}$ (approximately the type II PSC threshold) around 5 June. In late July, temperatures less than $195 \mathrm{~K}$ extended from $\sim 100-7 \mathrm{hPa}$ and temperatures less than $188 \mathrm{~K}$ from $\sim 90-10 \mathrm{hPa}$. Cold regions appear first near $20 \mathrm{hPa}$, and persist latest near 100-50 hPa. Fig. 3 shows temperatures below $188 \mathrm{~K}$ until late Sep, and below $195 \mathrm{~K}$ as late as $2 \mathrm{Nov} 1994$. The persistent region of temperatures below $195 \mathrm{~K}$ disappeared around 20 Oct, but a slight cooling led to a small region of temperatures below $195 \mathrm{~K}$ over the Palmer peninsula between 28 Oct and 2 Nov. The SH ATLAS-3 measurements were thus made $35-45$ days after the last occurrence of type II PSC temperatures, and only a few days after the last occurrence of type I PSC temperatures.
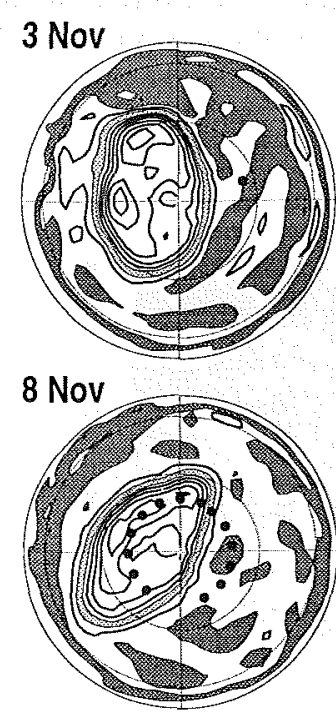
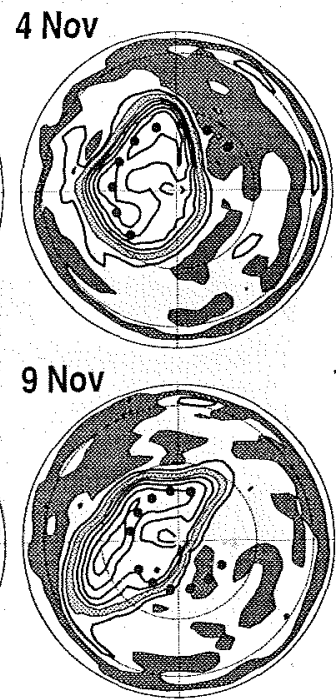
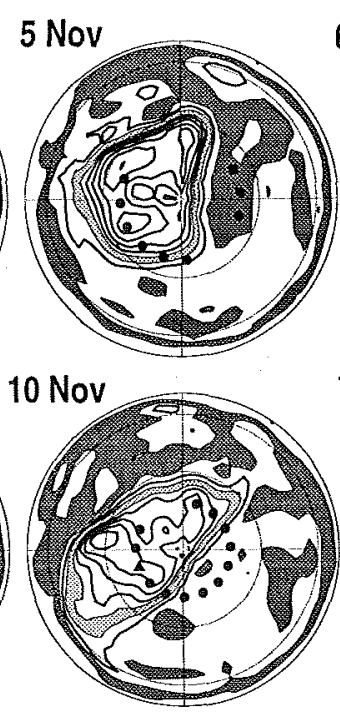
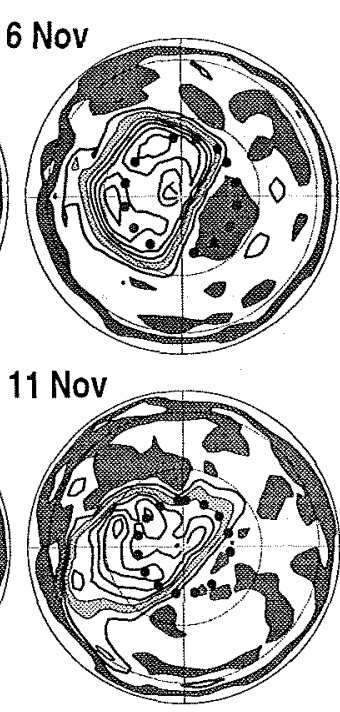
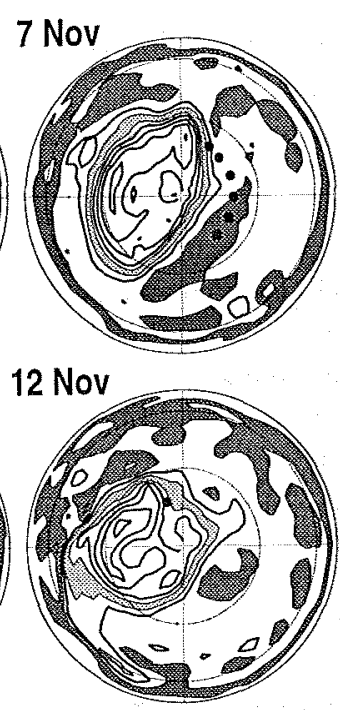

Figure 4. SH sPV at $655 \mathrm{~K}(\sim 20 \mathrm{hPa})$ on each day of ATLAS-3, at 12 GMT. Black dots are locations of ATMOS measurements. The projection is orthographic, with $0^{\circ}$ at the top and $90^{\circ} \mathrm{E}$ to the right; thin dashed lines are $30^{\circ}$ and $60^{\circ} \mathrm{S}$. Contour interval is 0.2 , light shading from 1.2 to 1.4 and dark shading from 0.6 to $0.8 \times 10^{-4} \mathrm{~s}^{-1}$. 

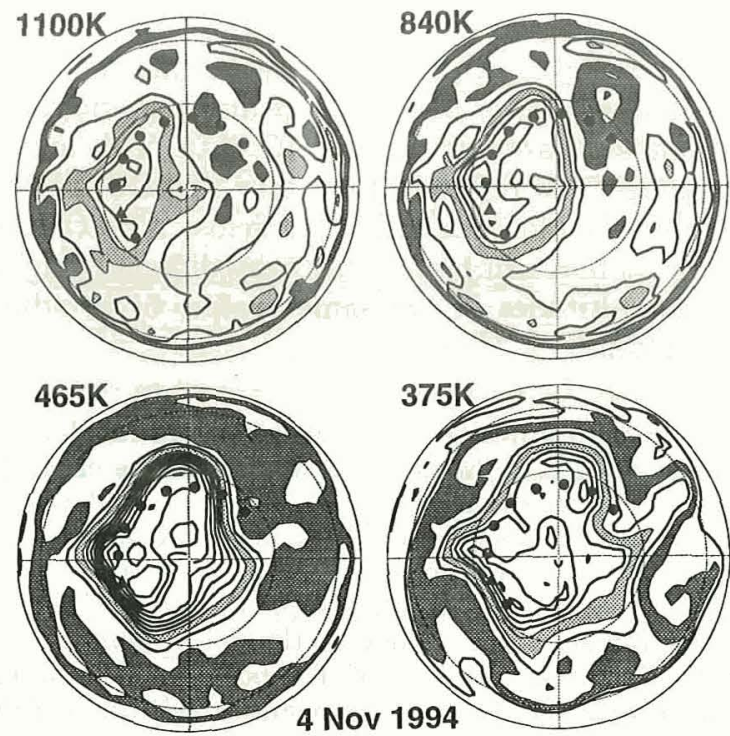

Figure 5. SH sPV maps at $1100,840,465$, and $375 \mathrm{~K}$ on 6 Nov 1994. Layout is as in Fig. 4.

Fig. 4 shows $\mathrm{SH} \mathrm{PV}$ at $655 \mathrm{~K}(\sim 20 \mathrm{hPa})$ throughout ATLAS-3, in relation to ATMOS measurements. The vortex shape changes during ATLAS-3, but it remains shifted off the pole towards $\sim 270^{\circ} \mathrm{E}$, so most of the ATMOS measurements between $180^{\circ}$ and $360^{\circ} \mathrm{E}$ fall within the vortex at this level; thus, cross-sections of trace species constructed from ATMOS measurements over the 10 day period [e.g., Abrams et al., 1996; Rinsland et al., 1996] give a reasonable picture of the longitudinal variations in the observations. The location shown by a triangle on 6 Nov is used by Rinsland et al. [1996] as an example of an extra-vortex measurement; at $655 \mathrm{~K}$, this is in fact subtropical air (dark shading) that has been
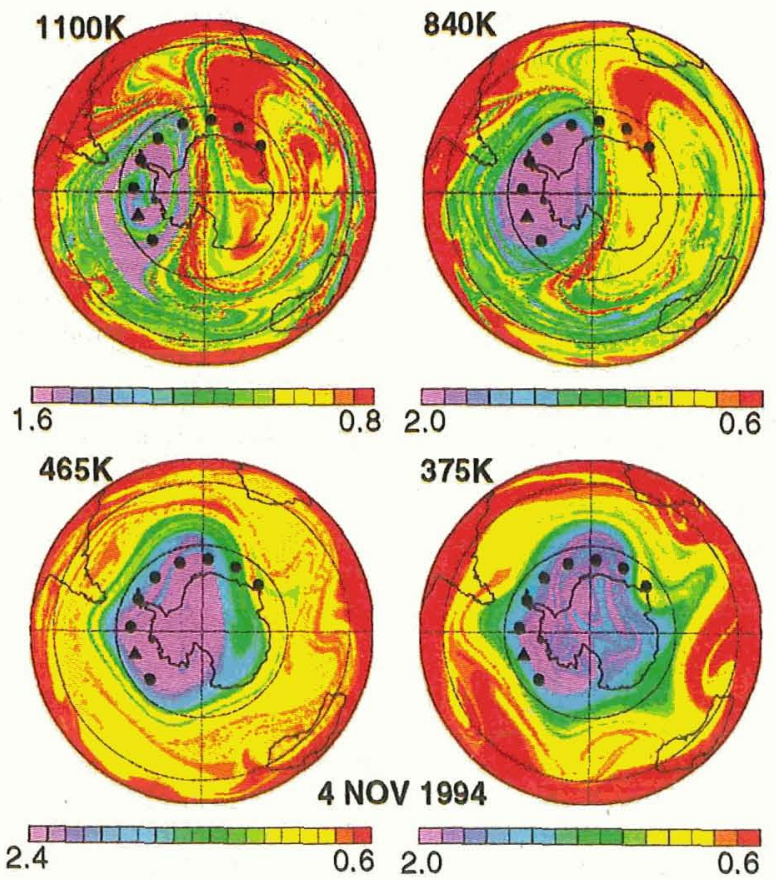

Figure 6. High-resolution sPV fields from 10 day reverse trajectory calculations, for 4 Nov 1994 in the SH, at $1100,840,465$ and 375 K. Layout is as in Fig. 4.

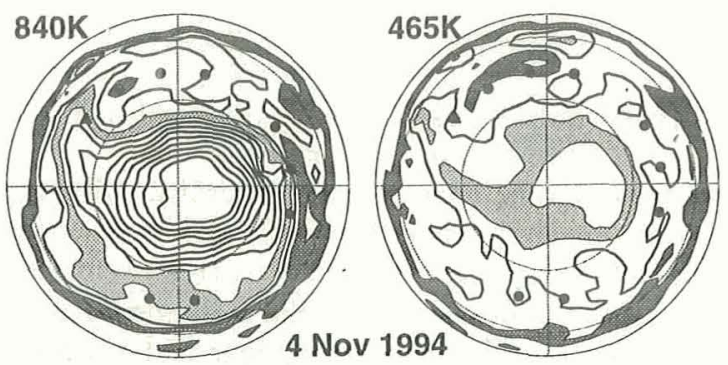

Figure 7. As in Fig. 5, but in the NH at 840 and 465 $\mathrm{K}$. $0^{\circ}$ longitude is at the bottom and $\mathrm{sPV}$ values are positive.

drawn up around the vortex. The triangle on 10 Nov is used as an example of a vortex interior measurement.

Fig. 5 shows sPV at several other levels on 4 Nov 1994. The vortex is shifted in the same direction at all levels, with higher levels farther off the pole. The measurement shown by a triangle is examined in detail by Newchurch et al. [1996] and is within the vortex at all levels. As shown in Fig. 1, sPV gradients at $1100 \mathrm{~K}$ (and above) are weak, and only a small remnant of vortex air is seen. At $375 \mathrm{~K}$, although strong sPV gradients exist, gradients are weak at some longitudes, suggesting that the average strong gradients (Fig. 1) at this level may indicate a weaker transport barrier than at higher levels where strong sPV gradients are continuous around the circumference of the vortex.

Although the sPV fields shown above suggest material may be drawn up from low latitudes and pulled off the vortex, the extent of this behavior is not obvious in the relatively low resolution $\mathrm{SPV}$ fields calculated from the UKMO data. To get a more complete picture of the circulation outside the vortex, we use reverse trajectory calculations [Sutton et al., 1994] (done on an equal area grid with $0.8^{\circ}$ latitude spacing and $0.8^{\circ}$ longitude spacing at the equator, and run isentropically for 10 days using UKMO winds) to generate simulated high-resolution sPV fields. Fig. 6 shows such fields for 4 Nov 1994. The vortex remnant at $1100 \mathrm{~K}$ is still separated from the exterior flow; however, lower sPV material characteristic of the vortex exterior is being mixed with the remaining "vortex" air. At both 1100 and $840 \mathrm{~K}$, long tongues of material with sPV typical of the vortex edge are drawn out into low latitudes and coiled up with very low sPV air drawn up around the vortex. Calculations for vertical sections show that these tongues are relatively deep, extending down to at

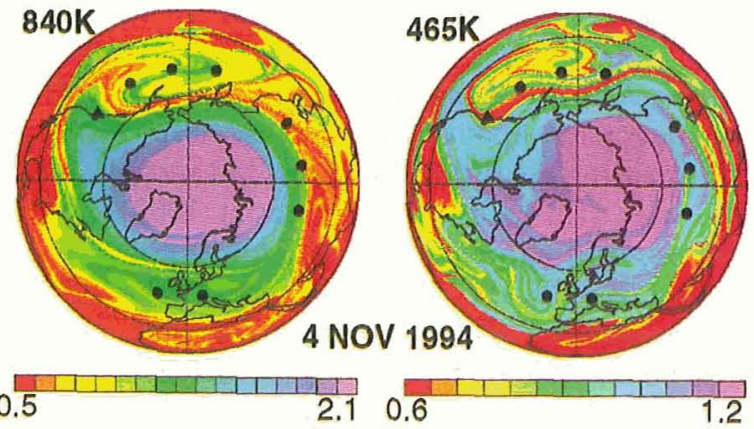

Figure 8. As in Fig. 6, but in the $\mathrm{NH}$ at 840 and 585 $\mathrm{K}$. Layout is as in Fig. 7. 
least $700 \mathrm{~K}$. At some locations (e.g., near $0^{\circ}$ longitude crossing the pole), extremely strong horizontal tracer gradients would be expected, as material from low latitudes is wrapped around the vortex.

At lower levels, narrow (and generally quite shallow) filaments are frequently drawn off the vortex edge, but are usually wrapped around the vortex, as opposed to extending to low latitudes. Such filaments may lead to tracer measurements that appear to be inconsistent with the fields in Fig. 5 . At $375 \mathrm{~K}$, material with sPV characteristic of the vortex edge appears to be entrained deep into the vortex; this supports our earlier suggestion that the transport barrier is not as strong here.

\section{Northern Hemisphere}

Fig. 7 shows sPV fields in the NH middle and lower stratosphere on 4 Nov 1.994. As was seen in Fig. 1, the vortex is strong at and above $840 \mathrm{~K} .465 \mathrm{~K}$ is below the level where the vortex has formed, and PV gradients are weak. The vortex is shifted slightly off the pole towards $0^{\circ}$ longitude; this is before the formation of the climatological "Aleutian high" which shifts the vortex further off the pole in that direction [e.g., Juckes and O'Neill, 1988].

Simulated high-resolution sPV fields (Fig. 8) show more clearly the material being drawn up around the vortex from low latitudes and coiling up with air from the vortex edge. As is typical in $\mathrm{NH}$ early winter, material is drawn in from low latitudes and off the vortex nearly continuously. This is thought to contribute to forming the main vortex/surf zone [e.g., Juckes and O'Neill, 1988] by strengthening PV and tracer gradients both along the vortex edge and in the subtropics. Fig. 8 shows that adjacent ATMOS measurements could sample very different tracer values, e.g., at $840 \mathrm{~K}$, the measurement shown by a triangle (discussed by Newchurch et al. [1996] and Chang et al. [1996]) near $230^{\circ} \mathrm{E}$ may sample air characteristic of the vortex edge, while the adjacent measurement near $210^{\circ} \mathrm{E}$ may sample subtropical air. Although the vortex is just forming at $465 \mathrm{~K}$, material is being drawn up from the tropics, so a measurement that samples vortex edge air at higher levels may sample subtropical air at this level (e.g., triangle). The complexity of the air motion over the Pacific and western US at this time makes it especially important to carefully interpret analyses and intercomparisons [e.g., Chang et al., 1996] of measurements in this region in view of the variety of meteorological conditions in a small area.

\section{Summary}

During ATLAS-3, the SH polar vortex was still large and strong below about $655 \mathrm{~K}$. Its size decreased rapidly with increasing altitude above this, but strong sPV gradients were still evident below about $1000 \mathrm{~K}$ and coherent vortex fragments apparent up to about $1300 \mathrm{~K}$. Throughout ATLAS-3, the SH vortex was shifted off the pole toward $270^{\circ} \mathrm{E}$. SH lower stratospheric temperatures below $195 \mathrm{~K}$ were observed a few days before ATLAS-3, which was 35-45 days after the last occurrence of temperatures less than $188 \mathrm{~K}$. In the $\mathrm{NH}$, the vortex was developing during ATLAS-3, and a region of strong sPV gradients was apparent above about $550 \mathrm{~K}$.
High-resolution sPV fields for both hemispheres show that the ATMOS/ATLAS-3 measurements are obtained in conditions of considerably more atmospheric variability than is apparent in the UKMO data. This may lead to apparent discrepancies between ATMOS tracer observations and low-resolution PV fields. ATMOS mneasurements may sample air with very different origins at different altitudes in the same profile, or in adjacent observations.

Acknowledgments. We thank $\mathrm{T}$. Luu for data management at JPL, P. Newman for supplying the original PV routines, P. Connew and D. Podd for helping to develop and run the UKMO assimilation system. Work at the UKMO is supported by the European Commission under contract EV5V-CT94-0441. G. L. M. is supported by a UARS Theoretical Investigation. Work at the Jet Propulsion Laboratory, California Institute of Technology was done under contract with the National Aeronautics and Space Administration.

\section{References}

Abrams, M. C., et al., ATMOS/ATLAS-3 observations of long-lived tracers and descent in the Antarctic vortex in November 1994, Geophys. Res. Lett., this issue, 1996.

Butchart, N., and E. E. Remsberg, The area of the stratospheric polar vortex as a diagnostic for tracer transport on an isentropic surface, J. Atmos. Sci., 43, 1319-1339, 1986.

Chang, A. Y., et al., A comparison of ATMOS and ER-2 measurements from ASHOE/MAESA, Geophys. Res. Lett., this issue, 1996.

Dunkerton, T. J., and D. P. Delisi, Evolution of potential vorticity in the winter stratosphere of January-February 1979, J. Geophys. Res., 91, 1199-1208, 1986.

Juckes, M. N., and A. O'Neill, Early winter in the northern hemisphere, Q. J. R. Meteorol. Soc., 114, 1111-1125, 1988.

Manney, G. L., and R. W. Zurek, Interhemispheric comparison of the development of the stratospheric polar vortex during fall: A 3-dimensional perspective for 1991-1992, Geophys. Res. Lett., 20, 1275-1278, 1993.

Manney, G. L., et al., On the motion of air through the stratospheric polar vortex, J. Atmos. Sci., 51, 2973-2994, 1994.

Newchurch, M. J., et al., Stratospheric $\mathrm{NO}$ and $\mathrm{NO}_{2}$ derived from diurnally corrected solar occultation measurements of ATMOS, Geophys. Res. Lett., this issue, 1996.

Rinsland, C. P., et al., ATMOS measurements of $\mathrm{H}_{2} \mathrm{O}+$ $2 \mathrm{CH}_{4}$ and reactive nitrogen in the November 1994 Antarctic stratosphere: dehydration and denitrification in the vortex, Geophys. Res. Lett., this issue, 1996.

Sutton, R. T., et al., High-resolution stratospheric tracer fields estimated from satellite observations using Lagrangian trajectory calculations, J. Atmos. Sci., 51, 2995 $3005,1994$.

Swinbank, R., and A. O'Neill, A Stratosphere-troposphere data assimilation system, Mon. Weather Rev., 122, 686$702,1994$.

G. L. Manney (manney@camel.jpl.nasa.gov), M/S 183-701, Jet Propulsion Laboratory, Pasadena, CA 91109. R. Swinbank, Meteorological Office, CR Division, London Road, Bracknell RG12 2SZ United Kingdom A. O'Neill, Centre for Global Atmospheric Modelling, University of Reading, 2 Earley Gate, Whiteknights, Reading RG6 2AU United Kingdom.

(received September 20, 1995; revised January 18, 1996; accepted March 5, 1996.) 
ISBN-0-87590-893-4 